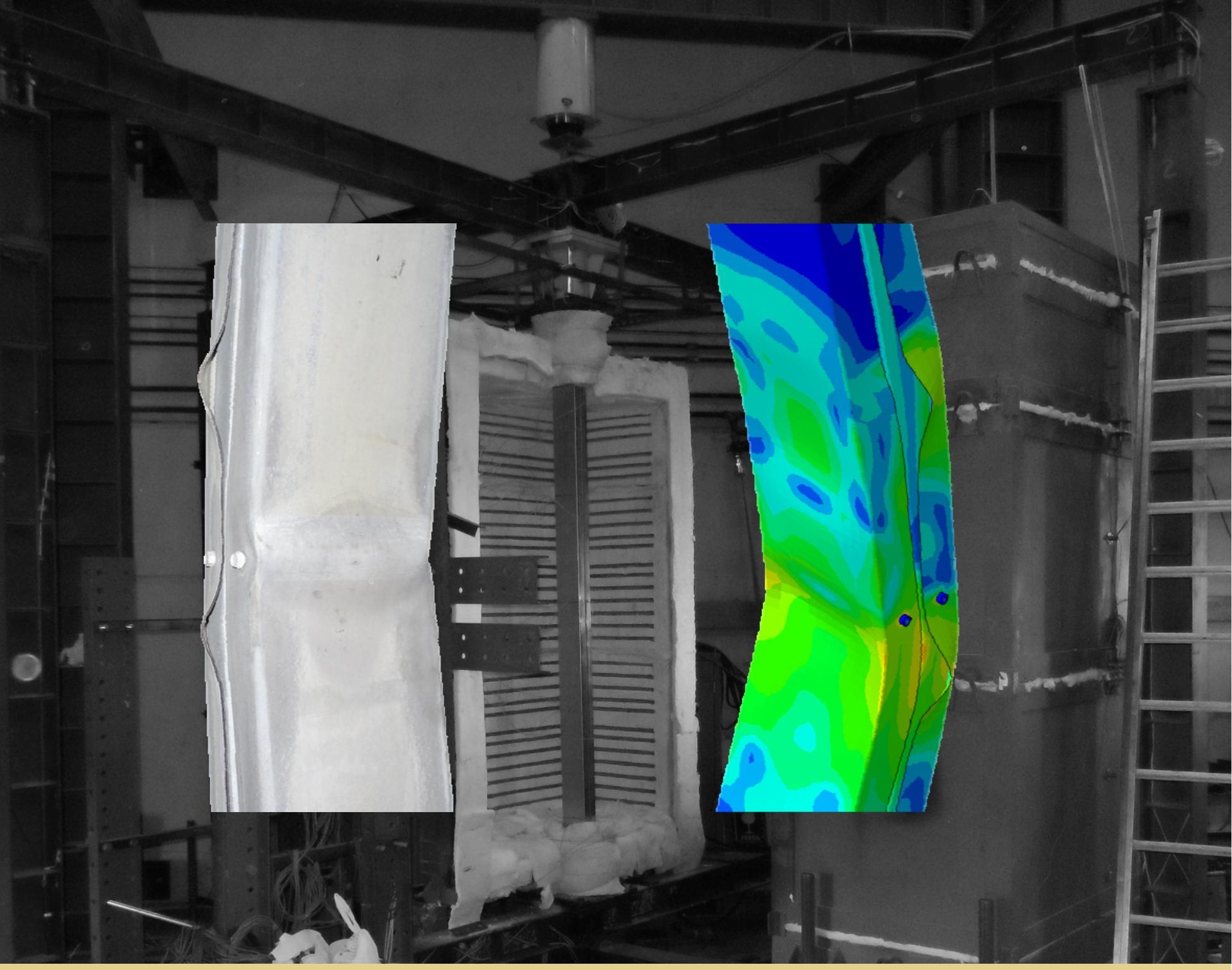

Hélder David da Silva Craveiro

\title{
FIRE RESISTANCE OF COLD-FORMED STEEL COLUMNS
}

Tese de doutoramento em Engenharia de Segurança ao Incêndio, orientada por João Paulo Correia Rodrigues e coorientada por Luís Miguel dos Santos Laím e apresentada à Faculdade de Ciências e Tecnologia da Universidade de Coimbra

\section{Setembro/2015}

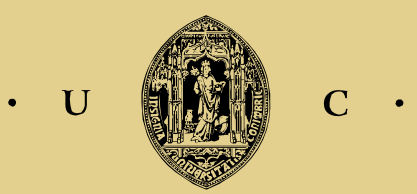




\section{FIRE RESISTANCE OF COLD-FORMED STEEL COLUMNS}

Thesis presented in fulfilment of the requirements for the degree of Doctor of Philosophy in "Engenharia de Segurança ao Incêndio"

\section{Author}

\section{Hélder David da Silva Craveiro}

Supervisor

Prof. Doutor João Paulo Correia Rodrigues

Co-Supervisor

Luís Miguel dos Santos Laím 

"However difficult life may seem there is always something you can do and succeed at." Stephen Hawking

"Scientists are the true driving force of civilization." James Burke 



\section{ACKOWLEDGEMENTS}

First and foremost, I would like to express my deep and sincere gratitude to my supervisors, Professor João Paulo Correia Rodrigues and Professor Luís Miguel dos Santos Laím, for all the support, guidance and encouragement throughout these last three years.

My sincere thanks to the Portuguese Foundation for Science and Technology (FCT) for the financial support under the scope of a National Research Project FireColdFSteel with reference PTDC/ECM/116859/2010. Thanks also to PERFISA S.A. for providing all the cold-formed steel profiles used in this research.

I also would like to express my gratitude to the University of Coimbra, especially to the Department of Civil Engineering and to the research unit ISISE, and all the staff members that always supported this investigation. Special thanks to José Rafael Campos, who was directly involved in the execution of the experimental tests, Ricardo Oliveira, Dulce Marques, David Rodrigues, Edmundo Pais, Ilídio Santos, Luís Gaspar, for all support and encouragement.

To my longtime friends (Mariano Veiga, Nuno Amaral, Paulo Gonçalves, Carlos Miranda, Ana Teresa Ferreira, Laura Resende, Alexandra Pereira, Susana Lages, Joana Pocinho, Tiago Orfão, Miguel Cortez, David Moreira, David Paiva, João Baltazar and Sérgio Martins) a special thanks for helping me survive all the stress and not letting me give up. To all my colleagues at ISISE for the support and friendship in difficult times. To my friends João Ribeiro, Luís Laím, Hugo Caetano and Ricardo Breda for the encouragement in the final steps of my thesis. Thank you, especially for all fun moments.

My deep love and appreciation goes to my girlfriend, Mafalda Chicória, for all the love, support, encouragement, patience and optimism showed during all this process.

Finally, I would like to express my love and infinite gratitude to my parents (Ilídio Craveiro Feitor and Maria Otília S. S. Craveiro), my brother (Rui Craveiro), my sister-in-law (Margarida Martins) and nephew (Diogo Craveiro) for the endless support and encouragement.

Thank you all for helping me in this wonderful and challenging journey. 



\section{RESUMO}

A construção de edifícios com estrutura constituída por perfis de aço galvanizado enformados a frio aumentou significativamente nos últimos anos, especialmente em edifícios com utilização residencial e de média altura. $\mathrm{O}$ aumento da sua quota de mercado no ramo da construção devese às inúmeras vantagens que esta solução apresenta em comparação com outras mais tradicionais, nomeadamente o peso reduzido, eficiência estrutural traduzida pelo elevado rácio entre a sua resistência mecânica e o seu peso, pré-fabricação e rapidez de montagem. No entanto, em resultado da reduzida espessura dos perfis utilizados surgem problemas adicionais no âmbito do projeto devido à maior suscetibilidade a fenómenos de instabilidade local e distorcional. A investigação e desenvolvimento de produto conduzido no passado levou à publicação da EN 1993-1-3:2006. Existem, no entanto, algumas limitações, nomeadamente em relação ao dimensionamento elementos estruturais de secção transversal composta (dois ou mais perfis acoplados entre si) tradicionalmente utilizados na construção.

Incêndios em edifícios ocorrem com alguma regularidade, causando inúmeros danos materiais e num cenário mais gravoso a perda de vidas. Para combater este cenário a Engenharia de Segurança ao Incêndio passou a ter uma importância fulcral no âmbito do dimensionamento estrutural. Diversos trabalhos de investigação de relevo foram realizados no campo da segurança de estruturas em situação de incêndio, no entanto, na sua maioria focados em estruturas de aço laminado a quente. A investigação em elementos estruturais constituídos por perfis de aço enformado a frio em situação de incêndio é ainda escassa, e além disso os modos de instabilidade caraterísticos deste tipo de perfis foram, na maioria dos casos, estudados de forma individual, sem considerar a sua possível interação. A influência da estrutura envolvente no comportamento de colunas constituídas por perfis de aço enformado a frio em situação de incêndio também ainda não havia sido investigada. Também o material, aço estrutural enformado a frio $\mathrm{S} 280 \mathrm{GD}+\mathrm{Z}$, carece de investigação aprofundada a temperaturas elevadas. Para responder aos problemas apresentados uma extensa análise experimental e numérica foi realizada em colunas de aço enformado a frio, quer à temperatura ambiente, quer em situação de incêndio com restrição à expansão térmica da coluna de aço. Este trabalho foi desenvolvido no âmbito do projeto de investigação nacional FireColdFSteel - Análise experimental $e$ numérica de elementos estruturais de aço enformado a frio sujeito a incêndio PTDC/ECM/116859/2010.

O aço estrutural S280GD+Z foi devidamente caraterizado através de ensaios experimentais de tração axial para determinação das suas propriedades mecânicas quer à temperatura ambiente 
quer a elevadas temperaturas. As propriedades térmicas do aço foram também avaliadas experimentalmente.

Numa segunda fase foi conduzida uma extensa investigação experimental em colunas de perfis de aço enformado a fio à temperatura ambiente e em situação de incêndio. Foram realizados 24 ensaios para avaliação da capacidade de suporte de carga de colunas com diferentes secções transversais e diferentes condições de suporte à temperatura ambiente. Em situação de incêndio com dilatação térmica da coluna restringida por ação da estrutura envolvente, foram realizados 96 ensaios. Foram avaliadas diferentes secções transversais, a influência do nível de carregamento, do nível de restrição à dilatação térmica imposto pela estrutura circundante e diferentes condições de apoio.

Todos os resultados obtidos na campanha experimental foram posteriormente utilizados como dados a introduzir nos modelos de elementos finitos ou como dados para validação do próprio modelo de elementos finitos desenvolvido com recurso ao software Abaqus. Os modelos desenvolvidos e validados poderão ser usados no futuro para realização de extensos estudos paramétricos para avaliar a influência de diversos parâmetros fundamentais no comportamento de colunas de perfis de aço enformado a frio.

Globalmente, observou-se que colunas de perfis de aço enformado a frio, sem proteção externa, apresentam uma reduzida resistência ao fogo. A restrição à expansão térmica de colunas tem um papel relevante no comportamento global de colunas isoladas de perfis de aço enformado a frio em situação de incêndio. Por exemplo, observou-se que aumentando o nível de restrição à expansão térmica o comportamento da coluna é controlado pelo aumento das forças axiais geradas devido à restrição imposta, enquanto para níveis de restrição baixos o comportamento da coluna é controlado pelo aumento de temperatura e pela consequente degradação das propriedades mecânicas do aço S280GD+Z. Concluiu-se, portanto, que níveis elevados de restrição à expansão térmica de colunas conduzem a valores mais elevados de forças de restrição geradas e eventualmente a menores valores de temperatura e tempo crítico.

Palavras Chave: fogo, perfis de aço enformado a frio, coluna, mecânica, térmica, propriedades, encurvadura, experimental, numérico, restrição à expansão térmica 


\section{ABSTRACT}

The market share of light steel framing using cold-formed steel profiles has increased significantly in the past few years since it has been recognized they can be used effectively as primary structural elements, especially for residential and mid-rise buildings. Its growing popularity in building construction industry is due to several advantages over other construction materials such as lightness, high strength to weight ratio and ease of fabrication and transportation. However, the use of thinner sections may lead to additional design problems not commonly found in traditional structural steel design, since thinner sections are traditionally susceptible to various buckling modes including local and distortional buckling that may govern its ultimate strength. Research and product development carried out in the past lead to the implementation of design specifications for cold-formed steel structures at ambient temperature, such as the EN 1993-1-3:2006. However, some limitations still exist, especially regarding the design of built-up cold-formed steel members, commonly used in the building construction industry.

Fire is an accidental action that regularly affects buildings, causing significant loss of properties and in the worst case scenario loss of lives. Hence, structural fire safety design has received greater attention in the past few decades. Relevant research has been conducted, however mainly on hot-rolled steel members. This led to the assumption that the developed design guidelines, based on research conducted on hot-rolled steel, may also be used in fire safety design of cold-formed steel structures. Moreover, the available research on cold-formed steel members under fire conditions is usually focused on individual buckling modes, without considering their possible interaction and without taking into consideration the influence of the surrounding structure on the overall behaviour of the cold-formed steel member. Also the physical properties of steel itself are not accurately characterized in the literature at elevated temperatures, since the available data still show some significant scatter. In order to address some of the mentioned issues regarding the behavior of cold-formed steel members an extensive experimental and numerical analysis on single and built-up cold-formed steel columns at both ambient and elevated temperatures was undertaken in the scope of a National Research Project entitled FireColdFSteel - Experimental and Numerical Analysis of Cold-Formed Steel Members Under Fire Conditions - PTDC/ECM/116859/2010. 
An experimental investigation on the material characterization was conducted in order to determine accurately both mechanical and thermal properties of the S280GD $+Z$ steel at both ambient and elevated temperatures.

In a second phase an extensive experimental research on the behaviour of single and built-up cold-formed steel columns at both ambient and elevated temperature was undertaken. Four different cross-section shapes were tested. At ambient temperature twenty-four buckling tests were undertaken to assess the buckling load of the tested columns. Under fire conditions ninetysix fire tests with restraint to thermal elongation were conducted. In these tests the influence of the cross-section shape, initial applied load level, end-support conditions and levels of restraint to thermal elongation imposed by the surrounding structure to the cold-formed steel columns were investigated.

All experimental test results available were then used as input or as data for validation of the finite element models developed to accurately reproduce the behaviour of the tested coldformed steel columns at both ambient and fire conditions. At this point the developed finite element model was validated against the experimental results. Using the available data and finite element models it is intended to undertake in the near future extensive parametric studies. The ultimate goal of the overall investigation is to improve/develop available/new design methodologies for cold-formed steel columns at both ambient and elevated temperatures.

Generally, it was found the cold-formed steel columns commonly used in the building construction industry, without any external protection, present low fire resistance. It seems that restraint to thermal elongation play a relevant role on the behavior of isolated cold-formed steel columns. For instance, it was observed that increasing the level of restraint to thermal elongation the failure of cold-formed steel columns may be controlled by the generated axial restraining forces, whereas for lower levels of restraint the failure may be controlled by temperature increase and consequent degradation of the mechanical properties. Hence, higher levels of imposed restraint to thermal elongation will lead to higher values of generated restraining forces and eventually to lower values of critical temperature and time.

Keywords: fire, cold-formed steel, column, mechanical, thermal, properties, buckling, experimental, numerical simulations, restraining, thermal elongation 


\section{TABLE OF CONTENTS}

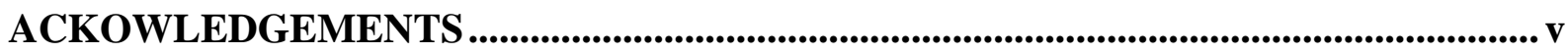

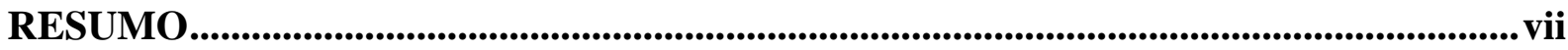

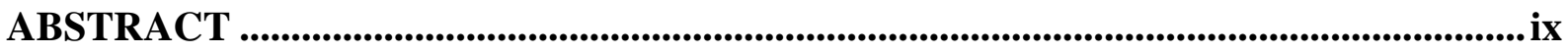

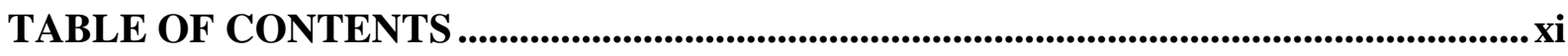

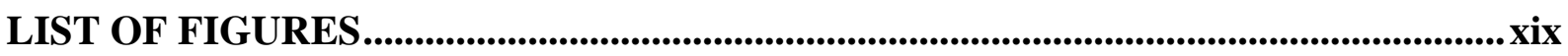

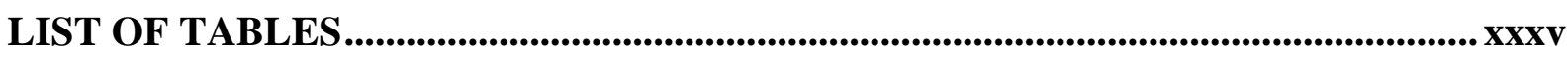

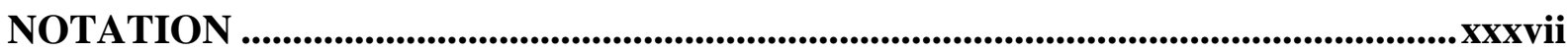

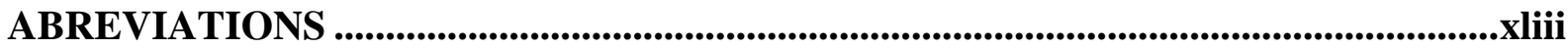

1 INTRODUCTION ..........................................................................................1

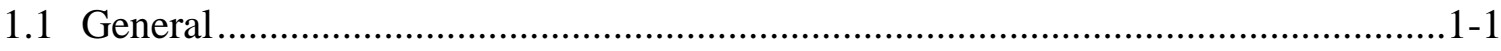

1.2 Motivation / Problem Definition........................................................................ 1-5

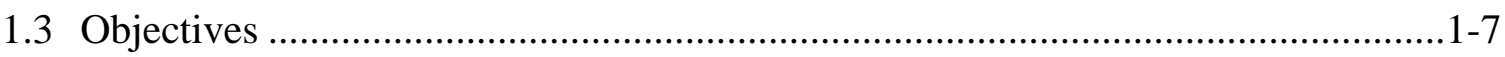

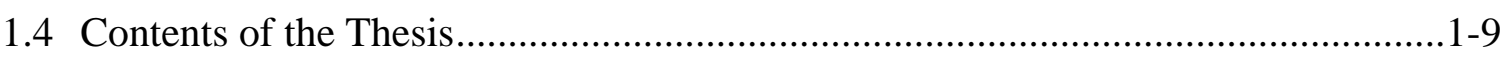

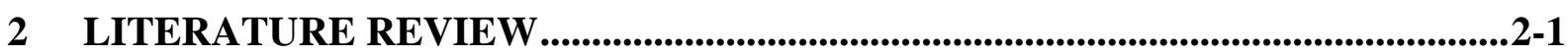

2.1 Mechanical and Thermal Properties of Cold-Formed Steels ..................................2-1

2.1.1 Mechanical properties at ambient temperatures .........................................2-1

2.1.2 Mechanical properties at elevated temperatures..........................................2-2

2.1.3 Thermal properties of cold-formed steel .................................................2-14

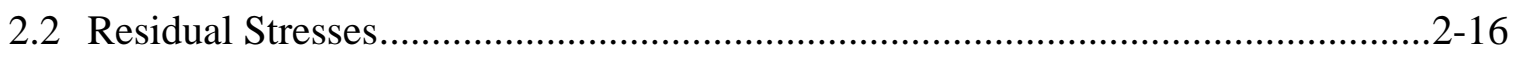

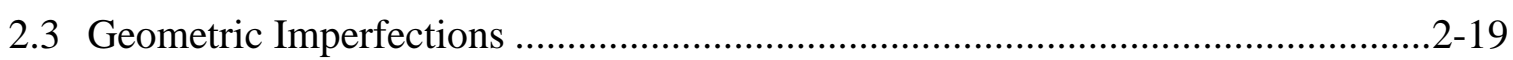

2.4 Cold-Formed Steel Columns at Ambient Temperature .......................................2-22

2.4.1 General buckling modes ....................................................................2-22

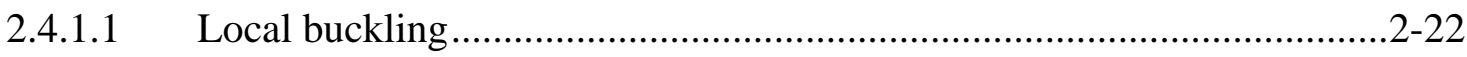

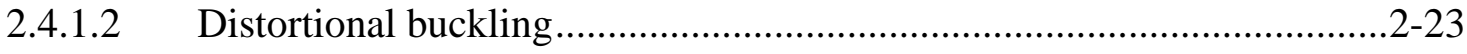

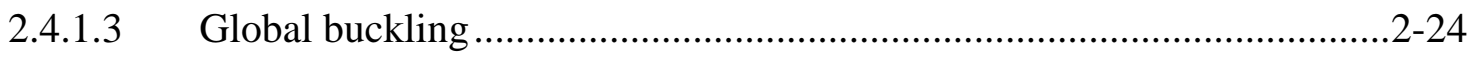

2.4.2 Previous research on cold-formed steel columns at ambient temperature ......2-24

2.4.2.1 Single section cold-formed steel columns.........................................2-25

2.4.2.2 Open and closed built-up cross-sections ..........................................2-2-32 
2.4.3 Design rules at ambient temperature........................................................ 2-36

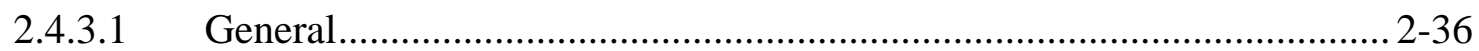

2.4.3.2 Design according to EN 1993-1-3:2006 …......................................... 2-40

2.4.3.3 Design of cold-formed steel columns according to the DSM established in

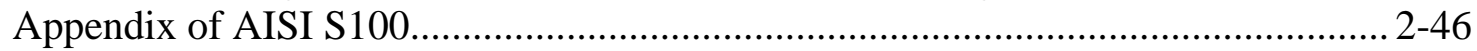

2.5 Cold-Formed Steel Columns under Fire Conditions ......................................... 2-48

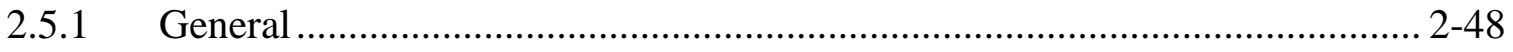

2.5.2 Research on hot-rolled steel columns under fire conditions with restraint to

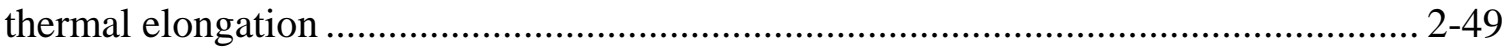

2.5.3 Research on cold-formed steel columns at elevated temperatures ................ 2-50

2.5.4 Design rules according to the EN 1993-1-2:2005 ......................................... 2-54

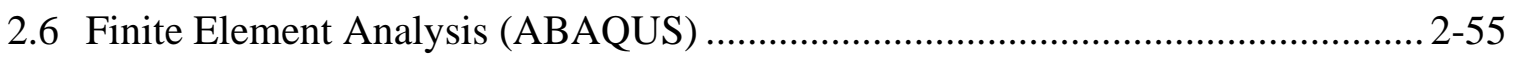

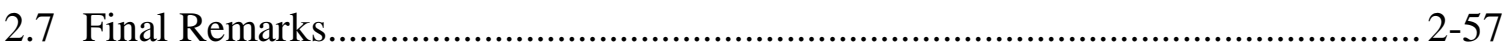

3 MATERIAL CHARACTERIZATION ............................................................... 3-1

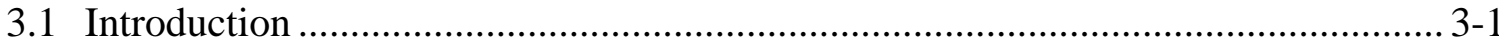

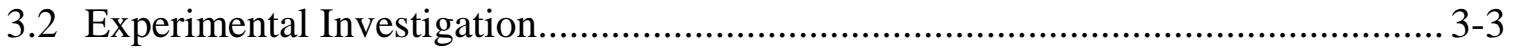

3.2.1 Mechanical properties of the S280GD+Z steel at ambient and elevated

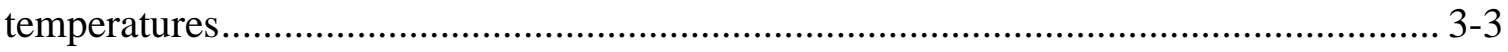

3.2.1.1 Experimental test set-up .............................................................. 3-3

3.2.1.2 Test specimens............................................................................. $3-5$

3.2.2 Thermal properties of the S280GD+Z steel at ambient and elevated

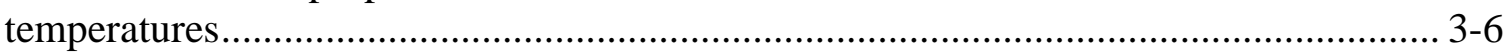

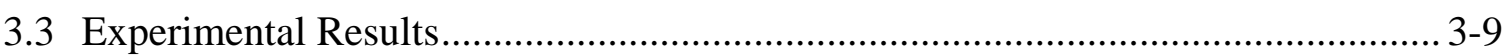

3.3.1 Mechanical properties of the S280GD+Z steel at ambient and elevated

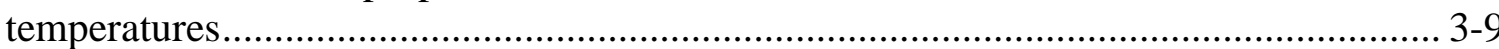

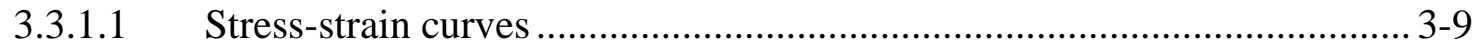

3.3.1.2 Mechanical properties reduction factors.......................................... 3-12

3.3.1.3 Comparison with available results in the literature ................................ 3-14

3.3.1.4 Comparison with available design standards....................................... 3-16

3.3.1.5 Predictive equations for mechanical properties .................................. 3-18

3.3.1.6 Stress-strain relationship to Ramberg-Osgood methodology ...................3-19

3.3.1.7 Stress-strain relationship of EN 1993-1-2 …....................................... 3-22

3.3.2 Thermal properties of the S280GD+Z steel............................................. 3-29

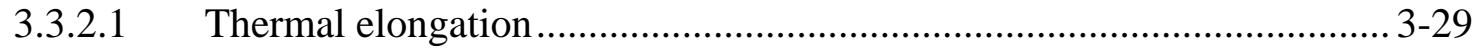


3.3.2.2 Thermal conductivity .....................................................................

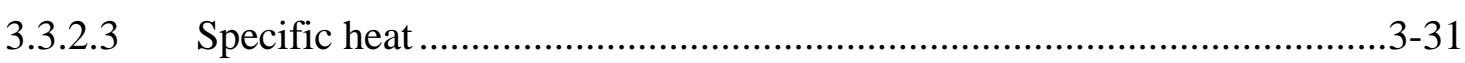

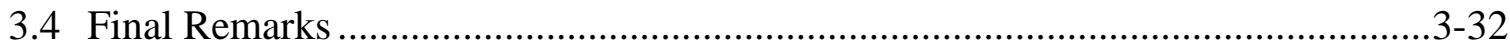

4 EXPERIMENTAL ANALYSIS ON COLD-FORMED STEEL COLUMNS ...........4-1

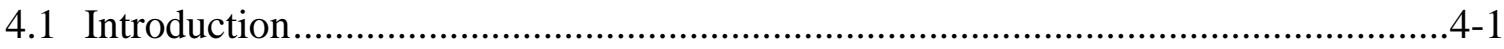

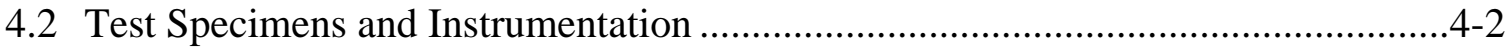

4.3 Test Set-Up .........................................................................................

4.3.1 Test set-up for buckling tests at ambient temperature ........................................4-4

4.3.2 Test set-up for fire tests with restraint to thermal elongation............................. 4-8

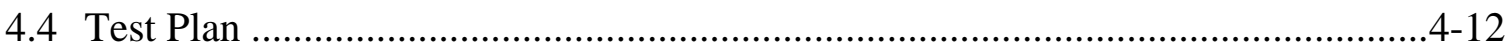

4.4.1 Buckling tests at ambient temperature........................................................4-12

4.4.2 Fire tests with restraint to thermal elongation .................................................4-13

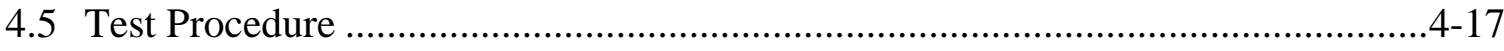

4.5.1 Buckling tests at ambient temperature.......................................................4-17

4.5.2 Fire tests with restraint to thermal elongation ................................................ 4 -17

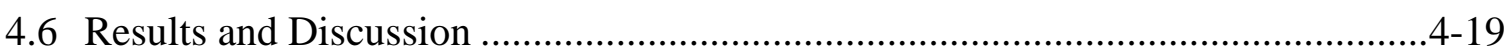

4.6.1 Buckling tests at ambient temperature..........................................................4-19

4.6.2 Fire tests with restraint to thermal elongation .................................................4-33

4.6.2.1 Temperature evolution ....................................................................4-33

4.6.2.2 Evolution of restraining forces .............................................................4-39

4.6.2.3 Lateral and axial displacements ……………................................... $4-55$

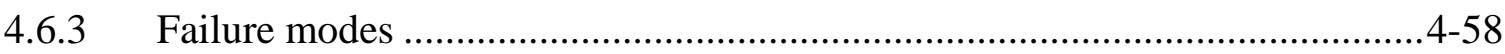

4.6.3.1 Buckling tests at ambient temperature ……………............................ $4-58$

4.6.3.2 Fire tests with restraint to thermal elongation.........................................4-65

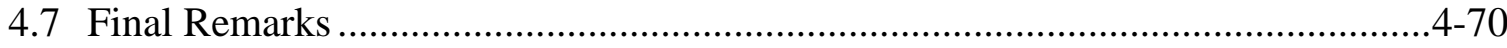

5 NUMERICAL MODELLING OF COLD-FORMED STEEL COLUMNS...............5-1

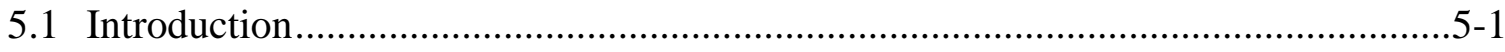

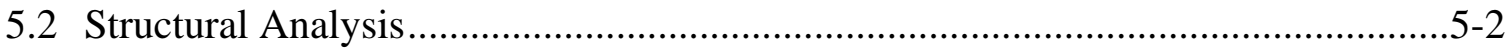

5.2.1 Selection of finite element type ………………........................................ $5-2$

5.2.2 Material modelling …………………………......................................

5.2.3 Finite element mesh.............................................................................

5.2.4 Loading, boundary conditions and contact conditions …………......................5-6 


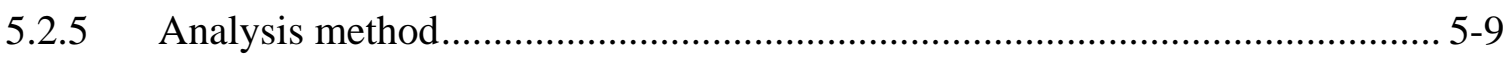

5.3 Validation of Finite Element Model ................................................................. 5-10

5.3.1 Validation of the finite element model for buckling tests of CFS columns at ambient temperature

5.3.2 Validation of the finite element model for fire tests with restraint to thermal elongation

5.4 Validation of the Finite Element Models in Terms of the Observed Final Deformed Shape

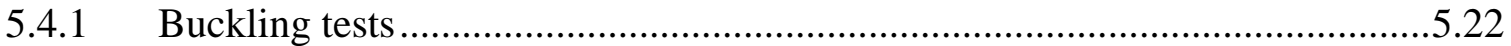

5.4.2 Fire tests with restraint to thermal elongation.......................................... 5-24

5.5 Heat Transfer Analysis .................................................................................. 5-27

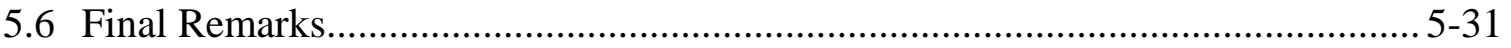

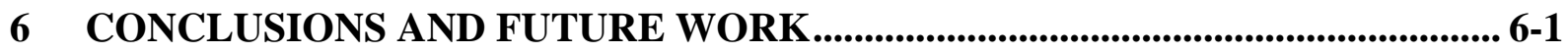

6.1 Mechanical and Thermal Properties of the S280GD+Z Steel ................................. 6-2

6.2 Experimental Analysis of Cold-Formed Steel Columns ......................................... 6-3

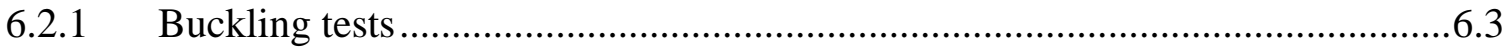

6.2.2 Fire tests with restraint to thermal elongation............................................ 6-5

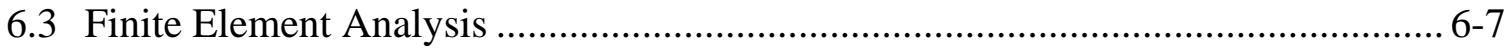

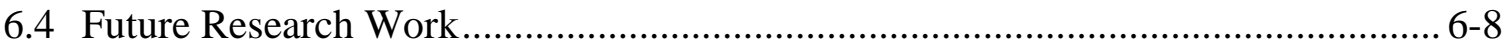

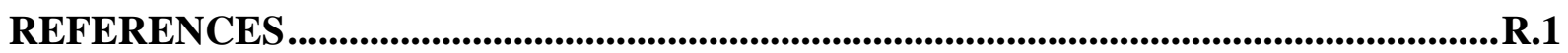

APPENDIX A MECHANICAL AND THERMAL PROPERTIES ..................................1

A.1 Tensile Coupon Tests at both Ambient and Elevated Temperatures for the Structural

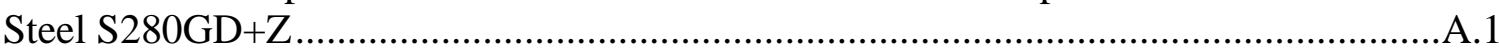

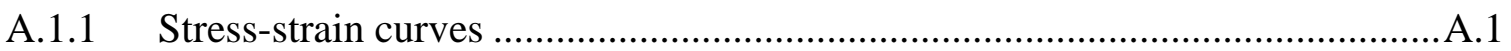

A.2 Thermal Properties of the Structural Steel S280GD+Z .........................................4

\section{APPENDIX B AXIAL AND ROTATIONAL STIFFNESS OF THE RESTRAINING} FRAMES USED IN THE FIRE TESTS ................................................................................1

APPENDIX C DETERMINATION OF THE DESIGN BUCKLING LOADS ...............C.1

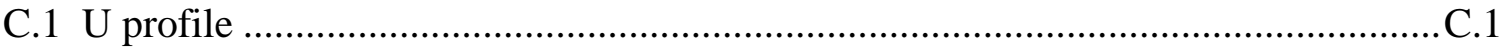

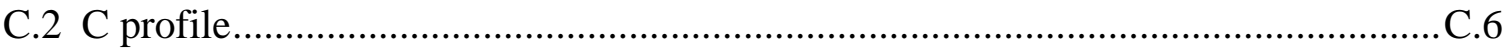

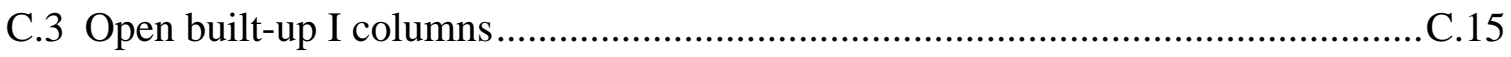




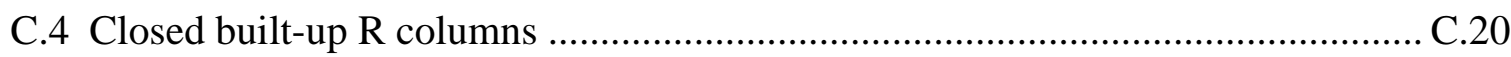

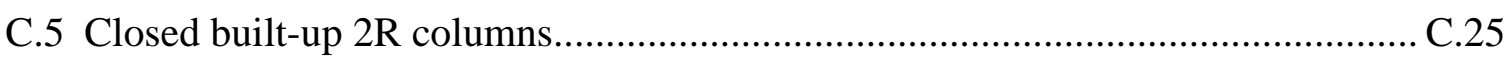

APPENDIX D IMPROVEMENT OF THE END-SUPPORT DEVICES ...................... D.1

APPENDIX E BUCKLING TESTS AT AMBIENT TEMPERATURE.......................... E.1

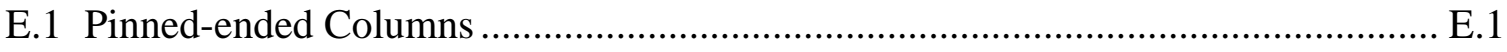

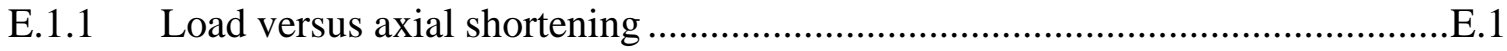

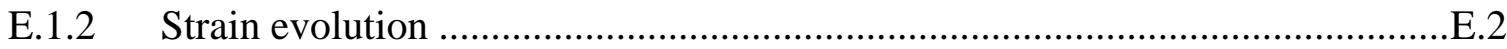

E.2 Fixed-ended Columns .............................................................................. E. 4

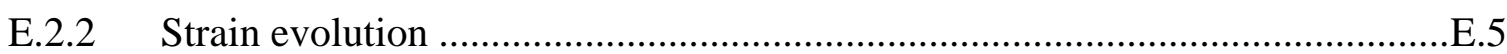

E.3 Photographs of the Final Deformed Shapes............................................................. E.

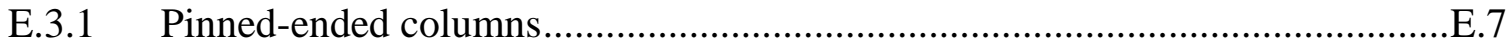

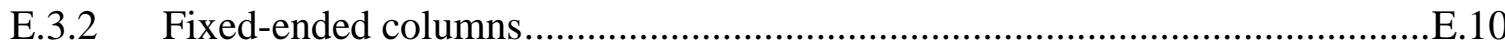

APPENDIX F FIRE RESISTANCE TESTS .....................................................................

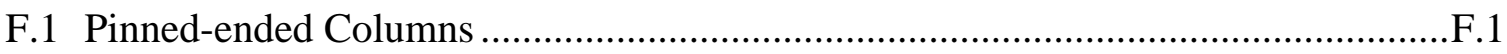

F.1.1 Lipped channel columns - C_PP_30LL_K1-i ........................................... 1

F.1.2 Lipped channel columns - C_PP_50LL_K1-i ............................................. 2

F.1.3 Lipped channel columns - C_PP_30LL_K2-i .............................................. 2

F.1.4 Lipped channel columns - C_PP_50LL_K2-i ............................................... 3

F.1.5 Open built-up I columns - I_PP_30LL_K1-i _........................................... 4

F.1.6 Open built-up I columns - I_PP_50LL_K1-i _.......................................... 4

F.1.7 Open built-up I columns - I_PP_30LL_K2-i ............................................... 5

F.1.8 Open built-up I columns - I_PP_50LL_K2-i .......................................... F.6

F.1.9 Closed built-up R columns - R_PP_30LL_K1-i...........................................6

F.1.10 Closed built-up R columns - R_PP_50LL_K1-i........................................... 7

F.1.11 Closed built-up R columns - R_PP_30LL_K2-i........................................ 8

F.1.12 Closed built-up R columns - R_PP_50LL_K2-i..................................... 8

F.1.13 Closed built-up 2R columns - 2R_PP_30LL_K1-i ......................................9

F.1.14 Closed built-up 2R columns - 2R_PP_50LL_K1-i ..................................... 10

F.1.15 Closed built-up 2R columns - 2R_PP_30LL_K2-i ....................................... 10

F.1.16 Closed built-up 2R columns - 2R_PP_50LL_K2-i ....................................... 11

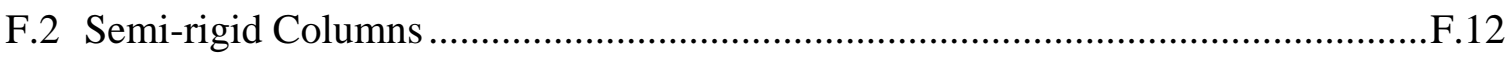


F.2.1 Lipped channel columns - C_SR_30LL_K3-i........................................ F. 12

F.2.2 Lipped channel columns - C_SR_50LL_K3-i......................................... F.13

F.2.3 Lipped channel columns - C_SR_30LL_K4-i.......................................... F.13

F.2.4 Lipped channel columns - C_SR_50LL_K4-i........................................... F.14

F.2.5 Open built-up I columns - I_SR_30LL_K3-i ........................................... F.15

F.2.6 Open built-up I columns - I_SR_50LL_K3-i .......................................... F.15

F.2.7 Open built-up I columns - I_SR_30LL_K4-i .......................................... F.16

F.2.8 Open built-up I columns - I_SR_50LL_K4-i .......................................... F. 17

F.2.9 Closed built-up R columns - R_SR_30LL_K3-i ....................................... F.17

F.2.10 Closed built-up R columns - R_SR_30LL_K3-i...................................... F.18

F.2.11 Closed built-up R columns - R_SR_30LL_K4-i....................................... F.19

F.2.12 Closed built-up R columns - R_SR_50LL_K4-i ........................................ F.19

F.2.13 Closed built-up 2R columns - 2R_SR_30LL_K3-i ................................... F.20

F.2.14 Closed built-up 2R columns - 2R_SR_50LL_K3-i .................................... F.21

F.2.15 Closed built-up 2R columns - 2R_SR_30LL_K4-i ...................................... F.21

F.2.16 Closed built-up 2R columns - 2R_SR_50LL_K4-i ..................................... F.22

APPENDIX G PHOTOGRAPHS OF COLUMNS AFTER FIRE CONDITIONS....... G.1

G.1 Lipped Channel Columns ...................................................................................

G.1.1 Pinned-end support condition and K1 level of restraint to thermal elongation G.1

G.1.2 Pinned-end support condition and K2 level of restraint to thermal elongation G.2

G.1.3 Semi rigid end-support condition and K1 level of restraint to thermal elongation

G.1.4 Semi rigid end-support condition and K2 level of restraint to thermal elongation

G.2 Open Built-Up I Columns

G.2.1 Pinned-end support condition and K1 level of restraint to thermal elongation G.5

G.2.2 Pinned-end support condition and K2 level of restraint to thermal elongation G.6

G.2.3 Semi rigid end-support condition and K3 level of restraint to thermal elongation

G.2.4 Semi rigid end-support condition and K4 level of restraint to thermal elongation .....

G.3 Closed Built-Up R Columns

G.3.1 Pinned-end support condition and K1 level of restraint to thermal elongation G.9 
G.3.2 Pinned-end support condition and K2 level of restraint to thermal elongation

G.3.3 Semi rigid end-support condition and K3 level of restraint to thermal elongation

G.3.4 Semi rigid end-support condition and K4 level of restraint to thermal elongation.....

G.4 Closed Built-Up 2R Columns

G.4.1 Pinned-end support condition and K1 level of restraint to thermal elongation

G.4.2 Pinned-end support condition and K2 level of restraint to thermal elongation

G.4.3 Semi-rigid end-support condition and K3 level of restraint to thermal elongation

G.4.4 Semi-rigid end-support condition and K4 level of restraint to thermal elongation 



\section{LIST OF FIGURES}

Figure 1.1. Use of cold-formed steel products. a) CFS building (Industrial machinery News@, 2015). b) Composite slab with profiled steel sheeting (Steel Construction Info@,2010). c) CFS portal frames (Shed Quarters Company@, 2009). d) Truss members (LTH steel structures@, 2015)

Figure 1.2. a) Galvanized steel coils (Aço Potiguar@, 2015). b) Roll forming machine (DreiStern@, 2015).

Figure 1.3. Stages in roll forming a simple section (Rhodes, 1991)

Figure 1.4. Press braking machine (Amada America, Inc@, 2015)

Figure 1.5. Typical CFS cross-section shapes used in building construction industry. a1) Plain channel (U). a2) Lipped channel (C). a3) Double lipped channel (C). a4) Double lipped U. a5) $\Sigma$ section. a6) Z section. a7) $\Omega$ section. b1) and b2) Open built-up I cross-sections. b3) and b4) Closed built-up cross-sections. .1-4

Figure 2.1. Effects of strain hardening and strain aging on stress-strain characteristics of structural steels (Yu, 2010; Chajes et al., 1963; Rondal and Dubina, 2005; Moen et al., 2008).

Figure 2.2. a) Stress-strain curve of hot-rolled steels. b) Stress-strain curve for cold-worked steels (Yu, 2010).

Figure 2.3. Yield strength of structural steel S350GD+Z and comparison with different design codes (Outinen et al., 2000).

Figure 2.4. Elastic modulus of structural steel S350GD+Z and comparison with different design

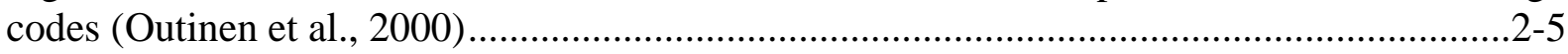

Figure 2.5. High temperature testing device (Outinen and Makelainen, 2002). $.2-6$

Figure 2.6. Tensile test results for specimens taken before and after high temperature compression tests (Outinen and Makelainen, 2002)

Figure 2.7. Comparison between test results and design standards for the reduction factors. a) Yield strength ( $0.2 \%$ proof stress). b) Elastic modulus for both transient and steady state test method. (Chen and Young, 2007).

Figure 2.8. Test set-up (Ranawaka and Mahendran, 2009) .............................................2-10

Figure 2.9. Yield strength reduction factors based on the $0.2 \%$ proof stress (Ranawaka .....2-11

Figure 2.10. Elastic modulus reduction factors (Ranawaka and Mahendran, 2009). . -12

Figure 2.11. Comparison of yield strength reduction factors with those obtained by other researchers (Kankanamge and Mahendran, 2011). $2-13$ 
Figure 2.12. Comparison of elastic modulus reduction factors with those obtained by other researchers (Kankanamge and Mahendran, 2011)......................................................... 2-13

Figure 2.13. Relative thermal elongation of steel as a function of temperature. .................2-15

Figure 2.14. a) Thermal conductivity of steel. b) Specific heat of steel. (EN 1993-1-2, 2005). 2-16

Figure 2.15. Definition of flexural and membrane residual stress (Schafer and Peköz, 1998)..

Figure 2.16. Average bending residual stress as \% fy. a) Roll-formed. b) Press-braked. .... 2-17

Figure 2.17. Influence of sheet thickness and yield stress on through-thickness longitudinal

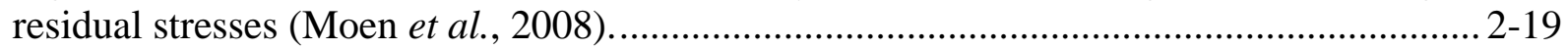

Figure 2.18. Sectional geometrical imperfections (Schafer and Peköz, 1998)...................2-20

Figure 2.19. Ultimate loads for FE-models with local imperfection $\mathrm{h} / 200$ and different global imperfection values (Kaitila, 2002).

Figure 2.20. Effect of initial geometric imperfection on failure loads (Ranawaka and Mahendran, 2010). $2-22$

Figure 2.21. a) Local buckling of compression members (Ranawaka, 2006). b) Local buckling of component walls of a cold-formed steel section (Dubina et al, 2012). c) Experimental. 2-23 Figure 2.22 Distortional buckling of compression members. a) (Ranawaka, 2006). b) Experimental.

Figure 2.23 Global buckling modes. a) Flexural buckling. b) Torsional buckling. c) Flexuraltorsional buckling (Dubina et al, 2012).

Figure 2.24 a) Failure of fixed-ended lipped channel. b) Failure of fixed-ended plain channel (Young and Rasmussen, 1999). b1) Deformed shape for the finite element model (Young and Yan, 2002a).

Figure 2.25 a) Local buckling of short columns (Popovic et al, 2001). b) Failure mode for both experimental and numerical analysis (Dhanalakshmi and Shanmugam 2001).....

Figure 2.26 a) Configuration of the tested cross-section. b) Experimental results for three specimens with different lengths (Yan and Young (2002).............................................2-30

Figure 2.27 Cross-sections tested (Kwon et al, 2009).

Figure 2.28 Buckling stress versus half-wavelength/column length curves for a lipped channel with intermediate stiffeners in the flanges (Kwon et al, 2009). 2-31

Figure 2.29 Failure observed for the open built-up I cross-section (Stone and LaBoube, 2005). 2-33

Figure 2.30 Connection pieces: a) column base. B) intermediate spacers. C) Built-up column (Georgieva et al, 2012b). 2-35

Figure 2.31 Built-up innovative cross-sections tested (Georgieva et al 2012a). 2-35 
Figure 2.32. a) Non-uniform stress distribution (real situation). b) Uniform stress distribution over the effective width. 2-36

Figure 2.33 The cross-section stability "signature curve" from an FSM solution (Li and Schafer, 2010). 2-39

Figure 2.34 Signature curve with proposed methodology (FSM and cFSM) (Li and Schafer,

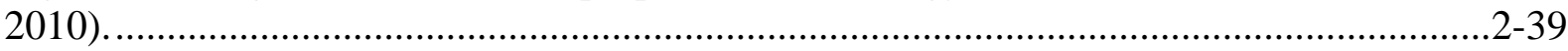

Figure 2.35 Edge stiffener of a lipped channel section (EN 1993-1-3, 2006). $.2-43$

Figure 2.36 a) Cross-sections tested. b) Experimental test set-up. c) Final deformed shape of a Type A specimen at $650^{\circ} \mathrm{C}$. (Ranawaka and Mahendran, 2009).......................................2-52

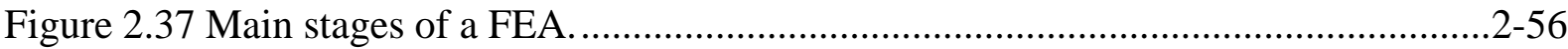

Figure 3.1 a) High temperature extensometer Epsilon HI 3548. b) Test set-up for ambient temperature testing.

Figure 3.2 Test set-up. a) Global view. b) Detail of the high temperature extensometer. c) Electrical tubular split furnace and high temperature extensometer. ................................... 3-4

Figure 3.3 Dimensions of the tensile test specimens.........................................................

Figure 3.4 a) TPS 2500S equipment. b) Hot Disk Kapton sensor with cable (Hot Disk AB,

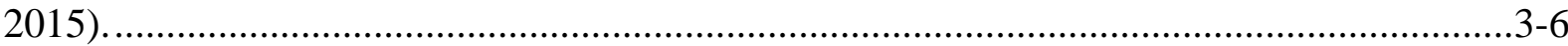

Figure 3.5 Hot disk sensors used in this investigation. a) Kapton sensor model 4922. b) Mica

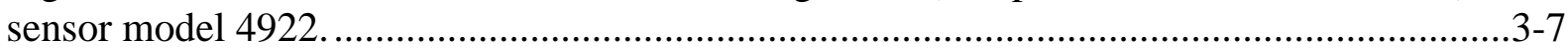

Figure 3.6 Ambient temperature sample support with slab sample holder setup...................3-8

Figure 3.7 a) High temperature sensor holder. b) 4 wires Macor probe and connection interface.

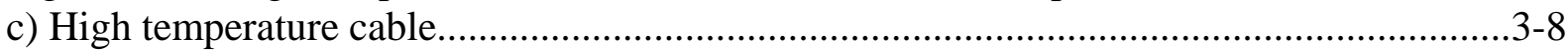

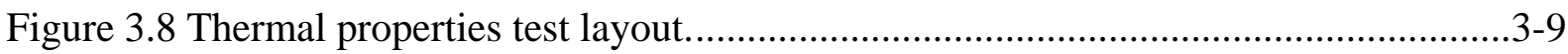

Figure 3.9 Comparison between the experimental results obtained with the high temperature extensometer Epsilon HI3548 and the extensometer FLA-6-11. .3-10

Figure 3.10 Stress-strain curves of the S280GD+Z steel; temperatures ranging from 20 to $800^{\circ} \mathrm{C}$.

Figure 3.11 Failure modes observed in the tensile tests both at ambient and elevated temperatures ranging from 20 to $800^{\circ} \mathrm{C}$

Figure 3.12 Reduction factors taken from the experimental tests. a) Yield strength. b) Elastic modulus. c) Ultimate strength and proportional limit. 3-14

Figure 3.13 Comparison of the yield strength reduction factors at elevated temperatures determined in the scope of this investigation and with other authors. .3-15

Figure 3.14 Comparison of the modulus of elasticity reduction factors at elevated temperatures determined in the scope of this investigation and with other authors. 3-16 
Figure 3.15 Comparison of the ultimate strength reduction factors at elevated temperatures determined in the scope of this investigation and with other authors. 3-16

Figure 3.16 Comparison between the reduction factors determined in this research with the ones available in the current design standards. a) Yield strength reduction factors. b) Modulus of elasticity reduction factors. c) Proportional limit reduction factors. 3-17

Figure 3.17 Comparison between proposed models and experimental results. a) Yield strength. b) Modulus of elasticity. 3-19

Figure 3.18 Comparison between experimental stress-strain curves and the proposed stressstrain model at elevated temperatures based on the Ramberg-Osgood model. 3-21

Figure 3.19 Comparison of the proposed stress-strain model with other predictive stress-strain models proposed by other authors. 3-22

Figure 3.20 Comparison of stress-strain curves predicted by EN 1993-1-2:2005 model with test results. a) Stress-strain model without strain hardening. b) Stress-strain model with strain hardening.

Figure 3.21 Comparison between the first modified proposed model based on the EN 1993-12:2005 formulation with test results. Complete stress-strain curves.

Figure 3.22 Comparison between the second modified proposed model based on the EN 19931-2:2005 formulation with test results. Complete stress-strain curves.

Figure 3.23 Comparison between both proposed models and the obtained results for different temperature levels (initial part of the stress-strain curves).

Figure 3.24 Thermal elongation as a function of temperature. Comparison between experimental results and proposed model with available design standards provisions and other proposed models. 3-30

Figure 3.25 Thermal conductivity test results and new predictive model. Comparison with the EN 1993-1-2:2005 model.. 3-31

Figure 3.26 Specific heat experimental results and comparison with the model presented in the EN 1993-1-2:2005 (2005)..... 3-32

Figure 4.1 a) and b) Dimensions of the single profiles used. a1), b1), c1) and d1) Dimensions of the cross-sections tested in the scope of this investigation (C, I, R, 2R).

Figure 4.2 CFS cross-sections tested and positioning of the strain gauges used in buckling tests. 4-3

Figure 4.3 CFS cross-sections tested and positioning of the thermocouples used in the fire tests. 4-4

Figure 4.4 Experimental test set-up designed and built for buckling tests. a) Test set-up. b) Schematic of the test set-up. 4-5 
Figure 4.5 a) Concrete footing. b) Concrete footing with hydraulic jack and end-support device. c) Concrete footing with hydraulic jack, end-support device and steel plates used to prevent any type of rotation while applying the compressive load. ..5 5

Figure 4.6 a) Concrete footing view cut. b) Concrete footing with hydraulic jack and endsupport device.

Figure 4.7 a) End-support devices. Schematics of the end-support device. b) Adjustable system adopted to fix each one of tested cross-sections. c) Pinned support. d) Fixed support. .4-7

Figure 4.8 a) Positioning of the LWT along the length of the column in both directions. b) Load cell. c) Positioning of the LVDT in the end-support device. 4-7

Figure 4.9 Schematic view of the experimental set-up. 4-9

Figure 4.10 Global view of the experimental set-up. 4-10

Figure 4.11 Device for measuring the restraining forces. 4-11

Figure 4.12 a) Positioning of LVDT on top and bottom of the CFS column. b) Location of thermocouples and LWT along the column length in both directions (about minor and major axis) c) Connections between peripheral columns and top beams. 4-12

Figure 4.13 Positioning of the strain gauges in all cross-sections tested. 4-17

Figure 4.14 Load-axial displacement diagrams for the compression tests undertaken on CFS columns with C, I, R and $2 \mathrm{R}$ cross-sections. a) Results for the pinned-end support condition. b) Results for the fixed-end support condition.

Figure 4.15 Evolution of the relative compressive loads $\left(P / P_{\max }\right)$ as a function of the lateral deflection at mid-height.

Figure 4.16 Rotations of the pinned-end support devices determined using the measurements obtained during each experimental test. a) C_PP_3. b) I_PP_3. c) R_PP_3.d) 2R_PP_2...4-22

Figure 4.17 Evolution of lateral deflections about the minor axis along the of the column for the lipped channel (C) cross-section. a) Pinned-end. b) Fixed-end. 4-23

Figure 4.18 Evolution of lateral deflections about the minor axis along the of the column for the open built-up (I) cross-section. a) Pinned-end. b) Fixed-end. 4-23

Figure 4.19 Evolution of lateral deflections about the minor axis along the of the column for the closed built-up (R) cross-section. a) Pinned-end. b) Fixed-end. .4-24

Figure 4.20 Evolution of lateral deflections about the minor axis along the of the column for the closed built-up (2R) cross-section. a) Pinned-end. b) Fixed-end. .4-24

Figure 4.21 Load vs strain curves obtained from the strain gauges. a) C_FF_3. b) C_PP_3. 4-25

Figure 4.22 Load vs strain curves obtained from the strain gauges. a1) and a2) I_FF_2. ....4-26 Figure 4.23 Load vs strain curves obtained from the strain gauges for the pinned-end column. a1) and a2) I_PP_3. 
Figure 4.24 Load vs strain curves obtained from the strain gauges for the fixed-end column. a1) and a2) R_FF_2.

Figure 4.25 Load vs strain curves obtained from the strain gauges for the pinned-end column. a1) and a2) R_PP_3...

Figure 4.26 Load vs strain curves obtained from the strain gauges for the fixed-end column. a1) and a2) 2R_FF_2.

Figure 4.27 Load vs strain curves obtained from the strain gauges for the pinned-end column. a1) and a2) 2R_PP_2. 4-31

Figure 4.28 Stress field determined for the maximum axial load for lipped channel columns considering both pinned and fixed-end support conditions. a) Fixed-end. b) Pinned-end... 4-32

Figure 4.29 Stress field determined for the maximum axial load for open built-up I columns considering both pinned and fixed-end support conditions. a) Fixed-end. b) Pinned-end... 4-32

Figure 4.30 Stress field determined for the maximum axial load for closed built-up R columns considering both pinned and fixed-end support conditions. a) Fixed-end. b) Pinned-end. .. 4-33

Figure 4.31 Stress field determined for the maximum axial load for closed built-up 2R columns considering both pinned and fixed-end support conditions. a) Fixed-end. b) Pinned-end. .. 4-33

Figure 4.32 Comparison between temperature evolution of the furnace in the experimental tests and ISO 834 fire curve. a) $i \_P P \_30 L L \_K 1$. b) $i \_P P \_30 L L \_K 2$. c) $i \_S R \_30 L L \_K 3$. D)

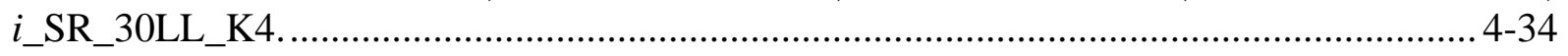

Figure 4.33 Evolution of temperature in the cross-section TS3 for the test column C_SR_30LL_K3-1 as a function of time. ................................................................ 4-36

Figure 4.34 Evolution of temperature in the cross-section TS3 for the test column I_SR_30LL_K3-2 as a function of time. ................................................................ 4-37

Figure 4.35 Evolution of temperature in the cross-section TS3 for the test column R_SR_30LL_K3-2 as a function of time.................................................................. 4-37

Figure 4.36 Evolution of temperature in the cross-section TS3 for the test column

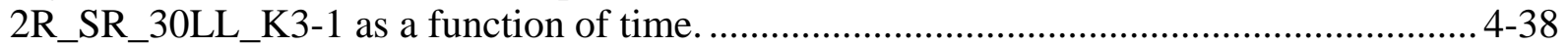

Figure 4.37 Evolution of the mean temperature of the column for all tested cross-sections as a function of time. 4-38

Figure 4.38 Evolution of temperature along the length of the column. a) C_SR_30LL_K3-1.b) I_SR_30LL_K3-2.c) R_SR_30LL_K3-2.d) 2R_SR_30LL_K3-1.

Figure 4.39 Non-dimensional restraining forces ratio for lipped and open built-up I columns. a) $\mathrm{C}$ cross-section with pinned-end support. b) $\mathrm{C}$ cross-section with semi-rigid end support. c) I cross-section with pinned-end support. d) I cross-section with semi-rigid end support....... 4-40

Figure 4.40 Non-dimensional restraining forces ratio for closed built-up CFS columns. a) $R$ cross-section with pinned-end support. b) $\mathrm{R}$ cross-section with semi-rigid end support. c) $2 \mathrm{R}$ cross-section with pinned-end support. d) $2 \mathrm{R}$ cross-section with semi-rigid end support. .. 4-46 
Figure 4.41 Evolution of the non-dimensional restraining forces ratio as a function of the mean temperature of the columns. a) $\left.i \_P P \_30 L L \_K 1-j . b\right) i \_P P \_50 L L \_K 1-j$. c) $i \_P P \_30 L L \_K 2-j . d$ ) $i \_$PP_50LL_K2-j. ( $i$ is the type of cross-section; $j$ is the presented test result).

Figure 4.42 Evolution of the non-dimensional restraining forces ratio as a function of the mean temperature of the columns. a) $\left.i \_S R \_30 L L \_K 3-j . b\right) i \_S R \_50 L L \_K 3-j$.c $) i \_S R \_30 L L \_K 3-j$.d) $i \_S R \_50 L L \_K 3-j$. ( $i$ is the type of cross-section; $j$ is the presented test result). 4-53

Figure 4.43 Variation of critical temperatures $\left(\theta_{c r}\right)$ as a function of the non-dimensional axial restraint ratio $(\alpha k)$. a) Pinned-end support. b) Fixed-end support. 4-55

Figure 4.44 Lateral deformations about the minor axis for lipped channels and open built-up I cross-sections for both end-support conditions tested. a) C_PP_30LL_K1-3. b) C_SR_30LL_K3-1.c) I_PP_30LL_K1-1.d) I_SR_30LL_K3-2 ....................................4-56

Figure 4.45 Lateral deformations about the minor axis for lipped channels and open built-up I cross-sections for both end-support conditions tested. a) R_PP_30LL_K1-1. b) R_SR_30LL_K3-2.c) 2R_PP_30LL_K1-3.d) 2R_SR_30LL_K3-1...............................4-57

Figure 4.46 Axial displacements. a) Lipped and open built-up I cross-section columns with pinned-end supports. b) Lipped and open built-up I cross-section columns with semi-rigid end supports. c) Closed built-up $\mathrm{R}$ and $2 \mathrm{R}$ cross-section columns with pinned-end supports. d) Closed built-up $\mathrm{R}$ and $2 \mathrm{R}$ cross-section columns with semi-rigid end supports. 4-58

Figure 4.47 Failure modes of the test column C_PP_3 at ambient temperature. a1) and a3) Distortional buckling. a2) and a5) Flexural buckling. a4) Rotation of the end-support........4-59

Figure 4.48 Failure modes of the test column C_FF_3 at ambient temperature. a1), a3) and a4) Distortional buckling. a2) and a5) Flexural buckling.

Figure 4.49 Failure modes of the test column I_PP_3 at ambient temperature. a1) and a3) Distortional buckling. a2) and a5) Flexural buckling. a4) Rotation of the end-support........4-61

Figure 4.50 Failure modes of the test column I_SR_2 at ambient temperature. a1) and a3) Distortional buckling. a2) Flexural buckling. a4) End-support device.

Figure 4.51 Failure modes of the test column R_PP_3 at ambient temperature. a1) Distortional buckling. a2) and a4) Flexural buckling. a3) and a5) Local buckling. 4-62

Figure 4.52 Failure modes of the test column R_FF_2 at ambient temperature. a1), a3) and a5) Local buckling. a2) Flexural buckling. a4) Distortional buckling.

Figure 4.53 Failure modes of the test column 2R_PP_2 at ambient temperature. a1) and a3) Local and distortional buckling. a2) and a5) Flexural buckling. a4) Rotation of the support. .4-64

Figure 4.54 Failure modes of the test column 2R_FF_2 at ambient temperature. a1), a4) and a5) Local and distortional buckling. a2) and a3) Flexural buckling. 4-64

Figure 4.55 Failure modes for the specimen C_PP_50LL_K1-2 and C_PP_50LL_K1-3.a1) and b1) Flexural buckling. a2) and b2) Minor distortional buckling. 4-65 
Figure 4.56 Failure modes for the specimen C_SR_50LL_K3-1. a1), a3) and a4) Distortional buckling. a2) Flexural buckling. 4-66

Figure 4.57 Failure modes for the specimen I_PP_50LL_K1-1. a1) and a3) Minor distortional buckling. a2) Flexural buckling 4-67

Figure 4.58 Failure modes for the specimen I_SR_50LL_K3-2. a1) and a3) Distortional buckling. a2) Flexural buckling. 4-67

Figure 4.59 Failure modes for the specimen R_PP_30LL_K1-1. a1) Local buckling. a2)

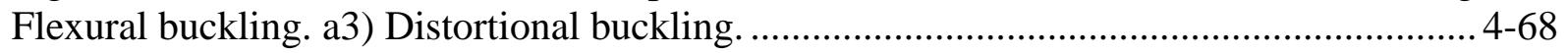

Figure 4.60 Failure modes for the specimen R_SR_30LL_K3-2. a1) and a3) Local and distortional buckling. a2) Flexural buckling................................................................... 4-69

Figure 4.61 Failure modes for the specimen 2R_PP_30LL_K1-3. a1), a2) and a3) Local and distortional buckling. a3) Flexural buckling ..................................................................... 4-69

Figure 4.62 Failure modes for the specimen 2R_SR_30LL_K3-1. a1) and a3) Local and distortional buckling. a2) Flexural buckling. 4-70

Figure 5.1 Schematic representation of the S4R element (ABAQUS Analysis - User's manual, 2012.)

Figure 5.2 Schematic representation of the C3D8R element (ABAQUS Analysis - User's Manual, 2012).

Figure 5.3 Experimental stress-strain curves and proposal based on the Ramberg-Osgood model.......

Figure 5.4 True stress and logarithmic plastic strain curves used as input in the finite element

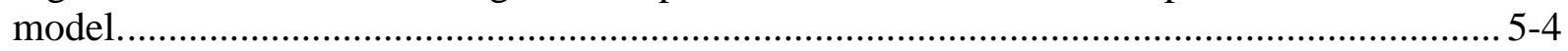

Figure 5.5 Detail of the finite element mesh used in all CFS columns. a) C. b) I. c) R. d) 2R.

Figure 5.6 Predicted load for the lipped channel column at ambient temperature using different mesh sizes. $5-6$

Figure 5.7 Finite element model developed for each type of columns tested. Model for CFS columns under simulated fire conditions with restraint to thermal elongation. a) Lipped channel column. b) Column with open built-up I cross-section. c) Column with closed built-up R crosssection. d) Column with closed built-up 2R cross-section..... 5-8

Figure 5.8 Comparison of FEA and experimental axial load versus axial shortening curves for all tested cross-sections and pinned-end supports. a) C. b) I. c) R. d) 2 R cross-section. ..... 5-11

Figure 5.9 Comparison of FEA and experimental axial load versus axial shortening curves for all tested cross-sections and fixed-ended supports. a) C. b) I. c) R. d) 2 R cross-section. .... 5-11 Figure 5.10 Comparison between experimental and FEA results for pinned-ended C columns. 
Figure 5.11 Comparison between experimental and FEA results for semi-rigid lipped channel columns

Figure 5.12 Comparison between experimental and FEA results for pinned-ended columns with open built-up I cross-section.

Figure 5.13 Comparison between experimental and FEA results for semi-rigid columns with open built-up I cross-section.

Figure 5.14 Comparison between experimental and FEA results for pinned-ended columns with closed built-up R cross-section.

Figure 5.15 Comparison between experimental and FEA results for semi-rigid columns with closed built-up R cross-section.

Figure 5.16 Comparison between experimental and FEA results for pinned-ended columns with closed built-up $2 \mathrm{R}$ cross-section.

Figure 5.17 Comparison between experimental and FEA results for semi-rigid columns with closed built-up $2 \mathrm{R}$ cross-section.

Figure 5.18 FEA (a) and experimental (b) failure modes for pinned-ended lipped channel columns.

Figure 5.19 FEA (a) and experimental (b) failure modes for pinned-ended columns with open built-up I cross-section.

Figure 5.20 FEA (a) and experimental (b) failure modes for pinned-ended columns with closed built-up R cross-section.

Figure 5.21 FEA (a) and experimental (b) failure modes for pinned-ended columns with closed built-up $2 \mathrm{R}$ cross-section.

Figure 5.22 FEA (a) and experimental (b) failure modes for semi-rigid lipped channel columns.

Figure 5.23 FEA (a) and experimental (b) failure modes for semi-rigid columns with open builtup I cross-section.

Figure 5.24 FEA (a) and experimental (b) failure modes for semi-rigid columns with closed built-up R cross-section.

Figure 5.25 FEA (a) and experimental (b) failure modes for semi-rigid columns with closed built-up $2 \mathrm{R}$ cross-section.

Figure 5.26 Thermal properties of air at one atmospheric pressure as a function of temperature.

a) Thermal conductivity. b) Specific heat. $.5-28$

Figure 5.27 Comparison between test and FEA temperature evolution. C_SR_30LL_K3-1. $.5-28$

Figure 5.28 Comparison between test and FEA temperature evolution. I_SR_30LL_K3-2.5-29 Figure 5.29 Comparison between test and FEA temperature evolution. R_SR_30LL_K3-2... 
Figure 5.30 Comparison between test and FEA temperature evolution. 2R_SR_30LL_K3-1. .5-30

Figure 5.31 Comparison between test and FEA temperature evolution using the provisions presented in the EN 1991-1-2:2002 (2002) (ISO 834 as thermal action and coefficient of heat transfer by convection equal to $25 \mathrm{~W} / \mathrm{m}^{2} \mathrm{~K}$. a) C. b) I. c) R. d) $2 \mathrm{R}$ cross-section. 5-30

Figure A.1 Stress vs strain curves for the structural steel S280GD+Z. a) $20^{\circ} \mathrm{C}$. b) $100^{\circ} \mathrm{C}$. c) $200^{\circ} \mathrm{C}$. d) $300^{\circ} \mathrm{C}$.

Figure A.2 Stress vs strain curves for the structural steel S280GD+Z. a) $400^{\circ} \mathrm{C}$. b) $500^{\circ} \mathrm{C}$. c) $600^{\circ} \mathrm{C}$.d) $700^{\circ} \mathrm{C}$. e) $800^{\circ} \mathrm{C}$.

Figure B.1 3D restraining frame modelled using the finite element model and the finite element software ABAQUS. B-1

Figure B.2 Mesh detail of the developed finite element model. B-1

Figure B.3 Deformed shape of the 3D restraining frame due to a vertical force applied in the centre of the massive steel column. B-2

Figure B.4 Deformed shape of the 3D restraining frame due to a horizontal force applied in the massive steel column in order to induce rotation of the top beams. B-3

Figure C.1 U gross cross sectional scheme formed by flat plane elements. C-1

Figure C.2 Effective section scheme of the U cross-section.

Figure C.3 Scheme of the lipped channel gross cross-section formed by flat plane elements and boundary conditions assumed. C-6

Figure C.4 Effective cross-section scheme of the lipped channel. C-9

Figure C.5 Reduction factor for the distortional buckling resistance of the edge stiffener. C-11 Figure C.6 Scheme of the open built-up I gross cross-section formed by flat plane elements and boundary conditions assumed. C-15

Figure C.7 Effective section scheme of the open built-up I cross-section. C-17

Figure C. 8 Gross cross sectional scheme of the R cross-section formed by flat plane elements. C-20

Figure C.9 Effective cross-section scheme for the closed built-up R cross-section.

Figure C.10 Gross cross sectional scheme of the closed built-up 2R cross-section formed by flat plane elements.

Figure C.11 Effective section scheme of the closed built-up 2R cross-section. 
Figure D.1 Influence of Teflon (PTFE) in the hinge of the end-support device. D-1

Figure E.1 Axial load versus axial shortening curves for all tested cross-sections considering the pinned-ended support condition. a) Columns with lipped channel cross-section. b) Columns with open built-up I cross-section. c) Columns with closed built-up R cross-section. d) Columns with closed built-up $2 \mathrm{R}$ cross-section. E-1

Figure E.2 Load-strain curves obtained from strain gauges placed in different points of the lipped channel cross-section. C_PP-2. E-2

Figure E.3 Load-strain curves obtained from strain gauges placed in different points of the open built-up I cross-section. a1) and a2) I_PP-2.

Figure E.4 Load-strain curves obtained from strain gauges placed in different points of the closed built-up R cross-section. a1) and a2) R_PP-2. E-3

Figure E.5 Load-strain curves obtained from strain gauges placed in different points of the closed built-up 2R cross-section. a1) and a2) 2R_PP-3. E.4

Figure E.6 Axial load versus axial shortening curves for all tested cross-sections considering the fixed-ended support condition. a) C. b) I. c) R. d) 2R columns. E-4

Figure E.7 Load-strain curves obtained from strain gauges placed in different points of the lipped channel cross-section. C_FF-2. E-5

Figure E. 8 Load-strain curves obtained from strain gauges placed in different points of the open built-up I cross-section. a1) and a2) I_FF-3.

Figure E.9 Load-strain curves obtained from strain gauges placed in different points of the closed built-up R cross-section. a1) and a2) R_FF-3. E-6

Figure E.10 Load-strain curves obtained from strain gauges placed in different points of the closed built-up 2R cross-section. a1) and a2) 2R_FF-3.

Figure E.11 Observed final deformed shape. a1), a2) and a3) C_PP-1. b1) and b2) C_PP_2...

Figure E.12 Observed final deformed shape. a1), a2) and a3) I_PP-1.b1) and b2) I_PP-2. .E-8

Figure E.13 Observed final deformed shape. a1) and a2) R_PP-1.b1) and b2) R_PP_2.....E-8

Figure E.14 Observed final deformed shape. a1), a2) and a3) 2R_PP-1. b1), b2) and b3) R_PP_3. E-9

Figure E.15 Observed final deformed shape. a1) and a2) C_FF-1.b1) and b2) C_FF_2....E-10 Figure E.16 Observed final deformed shape. a1) and a2) I_FF-1.b1) and b2) I_FF_3......E-10 Figure E.17 Observed final deformed shape. a1) R_FF-3. b1), b2) and b3) R_FF_3 .........E-11 Figure E.18 Observed final deformed shape. a1) and a2) 2R_FF-1.b1) and b2) 2R_FF_3.E-11 
Figure F.1 Evolution of restraining forces with temperature increase for lipped channel columns considering a pinned-ended support condition a 30\% load level, and a K1 level of restraint to thermal elongation.

Figure F.2 Evolution of the mean temperature of the CFS column with temperature increase as well as the furnace temperature and ISO 834 fire curve. F-1

Figure F.3 Evolution of restraining forces with temperature increase for C columns. F-2

Figure F.4 Evolution of the mean temperature of the CFS column with temperature increase as well as the furnace temperature and ISO 834 fire curve. F-2

Figure F.5 Evolution of restraining forces with temperature increase for C columns. F-2

Figure F.6 Evolution of the mean temperature of the CFS column with temperature increase as well as the furnace temperature and ISO 834 fire curve......................................................

Figure F.7 Evolution of restraining forces with temperature increase for C columns............F-3

Figure F.8 Evolution of the mean temperature of the CFS column with temperature increase as well as the furnace temperature and ISO 834 fire curve ................................................

Figure F.9 Evolution of restraining forces with temperature increase for I columns. ...........F-4

Figure F.10 Evolution of the mean temperature of the CFS column with temperature increase as well as the furnace temperature and ISO 834 fire curve. ...............................................F-4

Figure F.11 Evolution of restraining forces with temperature increase for I columns. ..........F-4

Figure F.12 Evolution of the mean temperature of the CFS column with temperature increase as well as the furnace temperature and ISO 834 fire curve. F-5

Figure F.13 Evolution of restraining forces with temperature increase for I columns. F-5

Figure F.14 Evolution of the mean temperature of the CFS column with temperature increase as well as the furnace temperature and ISO 834 fire curve. F-5

Figure F.15 Evolution of restraining forces with temperature increase for I columns. F-6 Figure F.16 Evolution of the mean temperature of the CFS column with temperature increase as well as the furnace temperature and ISO 834 fire curve. .......................................... -6

Figure F.17 Evolution of restraining forces with temperature increase for R columns..........F-6 Figure F.18 Evolution of the mean temperature of the CFS column with temperature increase as well as the furnace temperature and ISO 834 fire curve. ..............................................

Figure F.19 Evolution of restraining forces with temperature increase for R columns..........F-7

Figure F.20 Evolution of the mean temperature of the CFS column with temperature increase as well as the furnace temperature and ISO 834 fire curve. ..............................................

Figure F.21 Evolution of restraining forces with temperature increase for R columns..........F-8

Figure F.22 Evolution of the mean temperature of the CFS column with temperature increase as well as the furnace temperature and ISO 834 fire curve. F-8 
Figure F.23 Evolution of restraining forces with temperature increase for R columns. ........ F-8

Figure F.24 Evolution of the mean temperature of the CFS column with temperature increase as well as the furnace temperature and ISO 834 fire curve. .......................................... F-9

Figure F.25 Evolution of restraining forces with temperature increase for $2 \mathrm{R}$ columns. ...... F-9

Figure F.26 Evolution of the mean temperature of the CFS column with temperature increase as well as the furnace temperature and ISO 834 fire curve........................................... F-9

Figure F.27 Evolution of restraining forces with temperature increase for $2 \mathrm{R}$ columns. .... F-10

Figure F.28 Evolution of the mean temperature of the CFS column with temperature increase as well as the furnace temperature and ISO 834 fire curve. ....................................... F-10

Figure F.29 Evolution of restraining forces with temperature increase for $2 \mathrm{R}$ columns. .... F-10

Figure F.30 Evolution of the mean temperature of the CFS column with temperature increase as well as the furnace temperature and ISO 834 fire curve........................................ F-11

Figure F.31 Evolution of restraining forces with temperature increase for $2 \mathrm{R}$ columns. .... F-11

Figure F.32 Evolution of the mean temperature of the CFS column with temperature increase as well as the furnace temperature and ISO 834 fire curve............................................... F-11

Figure F.33 Evolution of restraining forces with temperature increase for C columns. ...... F-12

Figure F.34 Evolution of the mean temperature of the CFS column with temperature increase as well as the furnace temperature and ISO 834 fire curve. ....................................... F-12

Figure F.35 Evolution of restraining forces with temperature increase for C columns. ...... F-13

Figure F.36 Evolution of the mean temperature of the CFS column with temperature increase as well as the furnace temperature and ISO 834 fire curve........................................... F-13

Figure F.37 Evolution of restraining forces with temperature increase for C columns. ...... F-13

Figure F.38 Evolution of the mean temperature of the CFS column with temperature increase as well as the furnace temperature and ISO 834 fire curve. ........................................ F-14

Figure F.39 Evolution of restraining forces with temperature increase for C columns. ...... F-14

Figure F.40 Evolution of the mean temperature of the CFS column with temperature increase as well as the furnace temperature and ISO 834 fire curve ........................................... F-14

Figure F.41 Evolution of restraining forces with temperature increase for I columns......... F-15

Figure F.42 Evolution of the mean temperature of the CFS column with temperature increase as well as the furnace temperature and ISO 834 fire curve.......................................... F-15

Figure F.43 Evolution of restraining forces with temperature increase for I columns......... F-15

Figure F.44 Evolution of the mean temperature of the CFS column with temperature increase as well as the furnace temperature and ISO 834 fire curve.......................................... F-16

Figure F.45 Evolution of restraining forces with temperature increase for I columns......... F-16 
Figure F.46 Evolution of the mean temperature of the CFS column with temperature increase as well as the furnace temperature and ISO 834 fire curve. F-16

Figure F.47 Evolution of restraining forces with temperature increase for I columns. F-17

Figure F.48 Evolution of the mean temperature of the CFS column with temperature increase as well as the furnace temperature and ISO 834 fire curve. F-17

Figure F.49 Evolution of restraining forces with temperature increase for R columns. F-17

Figure F.50 Evolution of the mean temperature of the CFS column with temperature increase as well as the furnace temperature and ISO 834 fire curve. F-18

Figure F.51 Evolution of restraining forces with temperature increase for R columns........F-18

Figure F.52 Evolution of the mean temperature of the CFS column with temperature increase as well as the furnace temperature and ISO 834 fire curve. ..........................................

Figure F.53 Evolution of restraining forces with temperature increase for R columns........F-19

Figure F.54 Evolution of the mean temperature of the CFS column with temperature increase as well as the furnace temperature and ISO 834 fire curve. .........................................

Figure F.55 Evolution of restraining forces with temperature increase for R columns........F-19

Figure F.56 Evolution of the mean temperature of the CFS column with temperature increase as well as the furnace temperature and ISO 834 fire curve.

Figure F.57 Evolution of restraining forces with temperature increase for $2 \mathrm{R}$ columns......F-20

Figure F.58 Evolution of the mean temperature of the CFS column with temperature increase as well as the furnace temperature and ISO 834 fire curve.

Figure F.59 Evolution of restraining forces with temperature increase for $2 \mathrm{R}$ columns......F-21

Figure F.60 Evolution of the mean temperature of the CFS column with temperature increase as well as the furnace temperature and ISO 834 fire curve.

Figure F.61 Evolution of restraining forces with temperature increase for $2 \mathrm{R}$ columns......F-21

Figure F.62 Evolution of the mean temperature of the CFS column with temperature increase as well as the furnace temperature and ISO 834 fire curve.

Figure F.63 Evolution of restraining forces with temperature increase for $2 \mathrm{R}$ columns......F-22

Figure F.64 Evolution of the mean temperature of the CFS column with temperature increase as well as the furnace temperature and ISO 834 fire curve.

Figure G.1 Final deformed shapes for pinned-ended lipped channel columns. a1), a2) and a3) C_PP_30LL_K1-1, 2 and 3.b1), b2) and b3) C_PP_50LL_K1-1, 2 and 3.

Figure G.2 Final deformed shapes for pinned-ended lipped channel columns. a1), a2) and a3) C_PP_30LL_K2-1, 2 and 3.b1), b2) and b3) C_PP_50LL_K2-1, 2 and 3. G-2 
Figure G.3 Final deformed shapes for semi-rigid lipped channel columns. a1), a2) and a3) C_SR_30LL_K3-1, 2 and 3.b1), b2) and b3) C_SR_50LL_K3-1, 2 and 3 .

Figure G.4 Different configurations due to distortional buckling observed in the experimental tests.

Figure G.5 Final deformed shapes for semi-rigid lipped channel columns. a1), a2) and a3) C_PP_30LL_K4-1, 2 and 3. b1), b2) C_PP_50LL_K4-1, 2.

Figure G.6 Final deformed shapes for pinned-ended open built-up I columns. a1), a2) and a3) I_PP_30LL_K1-1, 2 and 3.b1), b2) I_PP_50LL_K1-1, 2 and 3.

Figure G.7 Final deformed shapes for pinned-ended open built-up I columns. a1), a2) and a3) I_PP_30LL_K2-1, 2 and 3. b1), b2) I_PP_50LL_K2-1, 2 and 3.

Figure G.8 Final deformed shapes for semi-rigid open built-up I columns. a1), a2) and a3) I_SR_30LL_K3-1, 2 and 3.b1), b2) I_FF_50LL_K3-1, 2 and 3.

Figure G.9 Different configurations due to sectional buckling modes (distortional buckling) observed in the experimental tests.

Figure G.10 Final deformed shapes for semi-rigid open built-up I columns. a1), a2) and a3) I_SR_30LL_K4-1, 2 and 3.b1), b2) I_FF_30LL_K4-1, 2 and 3.

Figure G.11 Different configurations due to sectional buckling modes (distortional buckling) observed in the experimental tests

Figure G.12 Final deformed shapes for pinned-ended closed built-up R columns. a1), a2) and a3) R_PP_30LL_K1-1, 2 and 3.b1), b2) R_PP_50LL_K1-1, 2 and 3 .

Figure G.13 Different configurations due to sectional buckling modes (local and distortional buckling) observed in the experimental tests. G-9

Figure G.14 Final deformed shapes for pinned-ended closed built-up R columns. a1), a2) and a3) R_PP_30LL_K2-1, 2 and 3. b1), b2) R_PP_50LL_K2-1, 2 and 3.

Figure G.15 Different configurations due to sectional buckling modes (local and distortional buckling) observed in the experimental tests. G-10

Figure G.16 Final deformed shapes for semi-rigid closed built-up R columns. a1), a2) and a3) R_SR_30LL_K3-1, 2 and 3.b1), b2) R_SR_50LL_K3-1, 2 and 3. G-11

Figure G.17 Different configurations due to sectional buckling modes (local and distortional buckling) observed in the experimental tests. G-11

Figure G.18 Final deformed shapes for semi-rigid closed built-up R columns. a1), a2) and a3) R_SR_30LL_K4-1, 2 and 3.b1), b2) R_SR_50LL_K4-1, 2 and 3. G-12

Figure G.19 Different configurations due to sectional buckling modes (local and distortional buckling) observed in the experimental tests. G-12

Figure G.20 Final deformed shapes for pinned-ended closed built-up R columns. a1), a2) and a3) 2R_PP_30LL_K1-1, 2 and 3.b1), b2) 2R_PP_50LL_K1-1, 2 and 3. G. 13 
Figure G.21 Different configurations due to sectional buckling modes (local and distortional buckling) observed in the experimental tests. G-13

Figure G.22 Final deformed shapes for pinned-ended closed built-up R columns. a1), a2) and a3) 2R_PP_30LL_K2-1, 2 and 3.b1), b2) 2R_PP_50LL_K2-1, 2 and 3. G-14

Figure G.23 Different configurations due to sectional buckling modes (local and distortional buckling) observed in the experimental tests. G-14

Figure G.24 Final deformed shapes for pinned-ended closed built-up R columns. a1), a2) and a3) 2R_SR_30LL_K3-1, 2 and 3.b1), b2) 2R_SR_50LL_K3-1, 2 and 3. G-15

Figure G.25 Different configurations due to sectional buckling modes (local and distortional buckling) observed in the experimental tests. G-15

Figure G.26 Final deformed shapes for pinned-ended closed built-up R columns. a1), a2) and a3) 2R_SR_30LL_K4-1, 2 and 3.b1), b2) 2R_SR_50LL_K4-1, 2 and 3. G-16

Figure G.27 Different configurations due to sectional buckling modes (local and distortional buckling) observed in the experimental tests. G-16

Figure H.1 Comparison between experimental and FEA using material properties assessed in experimental tests and using material properties presented in the EN 1993-1-2:2005. H-16 


\section{LIST OF TABLES}

Table 2.1. Reduction factors for mechanical properties of corner coupon specimens (Chen and Young, 2006).

Table 2.2 Effective widths of internal compression elements (EN 1993-1-5:2006)............2-42

Table 2.3 Effective widths of outstand compression elements (EN 1993-1-5:2006). $.2-42$

Table 3.1 Chemical composition of the $\mathrm{S} 280 \mathrm{GD}+\mathrm{Z}$ steel

Table 3.2 Mean values of the experimental results.

Table 3.3 Yield strength reduction factors for different strain values.

Table 3.4 Modulus of elasticity, ultimate strength and proportional limit reduction factors....

Table $3.5 n$ parameter determined for each temperature level.

Table 4.1 Test programme and predicted values for CFS columns.

Table 4.2 Test programme and predicted values for CFS columns with single and open builtup cross-section.

Table 4.3 Test programme and predicted values for CFS columns with closed built-up crosssection.

Table 4.4 Test results for pinned-end columns. 4-20

Table 4.5 Test results for fixed-end columns.

Table 4.6 Results for the lipped channel (C) cross-section specimens - pin-ended support. 4-41

Table 4.7 Results for the lipped channel (C) cross-section specimens - semi-rigid end support.

Table 4.8 Results for the built-up I (2C) cross-section specimens - pin-ended support.

Table 4.9 Results for the built-up I (2C) cross-section specimens - semi-rigid ended support.

Table 4.10 Results for the closed built-up R column with pinned-end supports. -4-48

Table 4.11 Results for the closed built-up R columns with semi-rigid end support. 4-49

Table 4.12 Results for the closed built-up 2R columns with pin-ended supports. .4-50

Table 4.13 Results for the closed built-up 2R columns with semi-rigid end supports. 4-51

Table 5.1 Experimental and numerical buckling load of lipped channel CFS columns. 
Table 5.2 Experimental and numerical buckling load of open built-up I CFS columns....... 5-12 Table 5.3 Experimental and numerical buckling load ofclosed built-up R CFS columns.... 5-13 Table 5.4 Experimental and FEA buckling load of closed built-up 2R CFS columns......... 5-13 Table 5.5 Experimental and FEA critical temperature and maximum axial load for pinnedended lipped channel columns. $5-18$

Table 5.6 Experimental and FEA critical temperature and maximum axial load for semi-rigid lipped channel columns. 5-18

Table 5.7 Experimental and FEA critical temperature and maximum axial load for pinnedended columns with open built-up I cross-section.

Table 5.8 Experimental and FEA critical temperature and maximum axial load for semi-rigid columns with open built-up I cross-section. 5-19

Table 5.9 Experimental and FEA critical temperature and maximum axial load for pinnedended columns with closed built-up R cross-section. $5-20$

Table 5.10 Experimental and FEA critical temperature and maximum axial load for semi-rigid columns with closed built-up R cross-section.

Table 5.11 Experimental and FEA critical temperature and maximum axial load for pinnedended columns with closed built-up $2 \mathrm{R}$ cross-section.

Table 5.12 Experimental and FEA critical temperature and maximum axial load for semi-rigid columns with closed built-up R cross-section.

Table A.1 Experimental results A-3

Table A.2 Experimental results

Table B.1 Determined values for the imposed levels of axial and rotational restraint to the tested CFS columns. B-3 


\section{NOTATION}

\section{Roman upper case letters}

A

$A_{c}$

$A_{c, e f f}$

$A_{\text {eff }}$

$A_{g}$

$A_{s}$

$A_{s, \text { red }}$

$E, E_{\text {normal }}, E_{20^{\circ} \mathrm{C}}$

$E_{T}$ and $E_{\theta}$

$F_{n}$

$F_{y}$

G

I

$I_{s}$

$I_{T}$

IW

Ieff, $x$

$I_{x}$

$I_{\text {eff }, x y}$

$I_{x y}$

K

$K_{a, c}$

$K_{a, s}$

$K_{i}$

$K_{r, c}$

$K_{r, s}$

$L$

$L_{e}$

$N_{b, R d}$

$N_{c r}$ cross-sectional area

gross area of the compression element

Effective area of a flat compression element

effective cross-sectional area

Gross cross-section area

effective cross-sectional area of the edge stiffener

reduced effective cross-sectional area of the edge stiffener

modulus of elasticity at ambient temperature

modulus of elasticity at temperature $T$ or $\theta$

critical buckling stress

yield stress

shear modulus

second moment about the strong axis of a cross-section

second moment of effective area of the edge stiffener

torsional constant

warping constant

second moment of effective area with respect to $\mathrm{x}$-axis

second moment of area with respect to $\mathrm{x}$-axis

product moment of effective area with respect to $x$ - and $y$-axis

product moment of area with respect to $\mathrm{x}$ - and $\mathrm{y}$-axis

spring stiffness of the edge stiffener per unit length

axial stiffness of the column

axial stiffness of the surrounding structure

level $i$ of stiffness of the surrounding structure to the cold-formed steel columns in the experimental tests

rotational stiffness of the column

rotational stiffness of the surrounding structure

length of the column

effective length

design buckling resistance of a compression member

elastic critical force for the relevant buckling mode 


$\begin{array}{ll}N_{c, R d} & \text { design cross-sectional resistance of the section to uniform compression } \\ P & \text { force } \\ & \text { axial restraining force generated in the column in the fire tests } \\ & \text { or } \\ & \text { axial compressive force in the buckling tests } \\ P_{0} & \text { initial applied load on the cold-formed steel column } \\ P_{c r d} & \text { critical elastic distortional column buckling load } \\ P_{c r e} & \text { minimum of the critical elastic column buckling load in flexural, } \\ P_{c r l} & \text { torsional, or flexural-torsional buckling } \\ P_{F E A} & \text { critical elastic local column buckling load } \\ P_{m a x} & \text { maximum numerical load-carrying capacity of the column } \\ & \text { maximum axial force generated in the column in fire tests } \\ P_{n d} & \text { or } \\ P_{n e} & \text { maximum buckling load in buckling tests } \\ P_{n l} & \text { axial strength for distortional buckling } \\ R F . i & \text { axial strength of a column for flexural, torsional or flexural-torsional } \\ S_{x} & \text { buckling } \\ T . i & \text { axial strength for local buckling } \\ T_{S} & \text { Restraining frame number i } \\ U_{S C} & \text { first moment of area with respect to x-axis } \\ W_{e f f} & \text { thermocouple i } \\ W_{y} & \text { temperature of steel } \\ X_{e f f, G C} & \text { shear centre co-ordinate with respect to u-axis } \\ X_{G C} & \text { effective section modulus of the beam } \\ & \text { section modulus of a cross-section } \\ \text { effective gravity centre co-ordinate with respect to x-axis } & \text { gravity centre co-ordinate with respect to x-axis }\end{array}$

\section{Roman lower case letters}

$a$

$b$

$b_{1}$

$b_{\text {eff }}$

$b_{p}$

$c$

$c_{a}$

$c_{e f f}$
Intermediate fastener or spot welding spacing

width of the flange

or

width of the plate

distance from the web-to-flange junction to the centre of the effective area of the edge stiffener (including effective part $b_{\text {eff }}$ of the flange)

effective flange width

width of the flange taking into account the influence of rounded corners width of the edge stiffener

specific heat of steel according to the EN 1993-1-2:2005

effective width of the edge stiffener 


\begin{tabular}{|c|c|}
\hline$c_{p}$ & $\begin{array}{l}\text { width of the edge stiffener taken into account the influence of rounded } \\
\text { corners }\end{array}$ \\
\hline$d a$ & axial displacement of the cold-formed steel columns in fire tests \\
\hline$d_{1}$ & maximum local imperfection in a stiffened element \\
\hline$d_{2}$ & $\begin{array}{l}\text { maximum deviation from straightness for a lip stiffened or unstiffened } \\
\text { flange }\end{array}$ \\
\hline$d h$ & $\begin{array}{l}\text { horizontal deflections of the cold-formed steel columns in both } \\
\text { buckling tests and fire tests }\end{array}$ \\
\hline$d_{h, P \max }$ & $\begin{array}{l}\text { horizontal deflections of the cold-formed steel columns in buckling } \\
\text { tests at ambient temperature when maximum axial load is reached }\end{array}$ \\
\hline$d_{v}$ & axial shortening of the cold-formed steel columns in buckling tests \\
\hline$d_{v, P \max }$ & $\begin{array}{l}\text { axial shortening of the cold-formed steel columns in buckling tests at } \\
\text { ambient temperature when maximum axial load is reached }\end{array}$ \\
\hline$f_{0.2 p}, f_{0.2,20^{\circ} \mathrm{C}}$ & $0.2 \%$ yield strength at ambient temperature \\
\hline$f_{0.2, \theta}$ & $0.2 \%$ yield strength at temperature $\theta$ \\
\hline$f_{0.5}$ & $0.5 \%$ yield strength \\
\hline$f_{p}$ & proportional stress limit \\
\hline$f_{p, \theta}$ & Proportional stress limit at temperature $\theta$ \\
\hline$f_{u}$ & ultimate strength \\
\hline$f_{u, \theta}$ & ultimate strength at temperature $\theta$ considering strain hardening \\
\hline$f_{y}, f_{y, \text { normal }}, f_{y, 20}$ & yield strength at ambient temperature \\
\hline$f_{y a}$ & Average yield strength \\
\hline$f_{y b}$ & basic yield strength \\
\hline$f_{y, \theta}$ & yield strength at temperature $\theta$ \\
\hline$h$ & height of the section \\
\hline$h_{w}$ & web depth \\
\hline$h_{\text {eff }}$ & effective web width \\
\hline$h_{p}$ & width of the web taken into account the influence of rounded corners \\
\hline$i_{u}$ & radius of gyration with respect to $\mathrm{u}$-axis \\
\hline io & polar radius of gyration about shear centre \\
\hline$k_{E, \theta}$ & reduction factor for the modulus of elasticity of steel at temperature $\theta$ \\
\hline$k_{p, \theta}$ & reduction factor for the proportional limit of steel at temperature $\theta$ \\
\hline$k_{u, \theta}$ & reduction factor for the ultimate strength of steel at temperature $\theta$ \\
\hline$k_{u, \theta^{*}}$ & $\begin{array}{l}\text { reduction factor for the ultimate strength in relation to the yield strength } \\
\text { of steel at temperature } \theta\end{array}$ \\
\hline$k_{v}, k_{y}$ & effective length factor \\
\hline$k_{W}$ & warping effective length factor \\
\hline$k_{y, \theta}$ & reduction factor for the yield strength of steel at the steel temperature $\theta$ \\
\hline
\end{tabular}




\begin{tabular}{|c|c|}
\hline$k_{\sigma}$ & plate local buckling factor \\
\hline$n \theta$ & Ramberg-Osgood coefficient \\
\hline$r_{i}$ & $\begin{array}{l}\text { Minimum radius of gyration of full unreduced cross-sectional area of } \\
\text { an individual shape in a built-up member }\end{array}$ \\
\hline$t$ & thickness \\
\hline & $\begin{array}{l}\text { or } \\
\text { time }\end{array}$ \\
\hline$t_{c r}$ & critical time of the column \\
\hline$t_{n}$ & nominal thickness \\
\hline$t_{\text {peak }}$ & time when the maximum restraining force in the column is reached \\
\hline$u_{\max }$ & maximum co-ordinate of the plate with respect to u-axis \\
\hline$U_{\text {min }}$ & minimum co-ordinate of the plate with respect to $u$-axis \\
\hline$v_{\max }$ & maximum co-ordinate of the section with respect to $\mathrm{v}$-axis \\
\hline$v_{\min }$ & minimum co-ordinate of the section with respect to $\mathrm{v}$-axis \\
\hline
\end{tabular}

\section{Greek upper case letters}

$\begin{array}{ll}\Delta l / l & \text { relative thermal elongation } \\ \Delta p & \text { probing depth } \\ \Delta X_{G C} & \text { distance in } \mathrm{x} \text {-direction from gravity centre to effective gravity centre of } \\ & \text { the cross-sectional area }\end{array}$

\section{Greek lower case letters}

$\alpha$

$\alpha_{c}$

$\alpha_{K, 20^{\circ} \mathrm{C}}$

$\beta$

$\gamma_{M O}$

$\varepsilon$

$\varepsilon_{\text {eng }}$

$\varepsilon T$

$\varepsilon_{t h}$

$\varepsilon$ true

$\varepsilon \theta$ residual stress reduction factor

or

Imperfection factor, corresponding to the appropriate buckling curve, according to the type of cross-section, axis of buckling and yield strength Heat transfer coefficient

level of axial restraint to the cold-formed steel column

parameter defining the Ramberg-Osgood stress-strain model

or

rotation of the end-support devices in the buckling tests

partial factor for resistance of cross-sections

longitudinal strain

or

elastic strain

engineering (nominal) strain

strain at a given stress at temperature $\mathrm{T}$

Coefficient of thermal expansion

true (logarithmic) strain

strain at temperature $\theta$ 


\begin{tabular}{|c|c|}
\hline$\varepsilon_{r, \theta}$ & strain at rupture \\
\hline$\varepsilon_{u s, \theta}$ & plastic strain at maximum tension load \\
\hline$\eta^{T}$ & parameter defining the Ramberg-Osgood stress-strain model \\
\hline $\bar{\theta}_{C}$ & mean temperature of the column \\
\hline$\theta_{a}$ & steel temperature \\
\hline$\theta_{c r}$ & critical temperature of the column \\
\hline$\theta_{\text {peak }}$ & column temperature when the maximum restraining force is reached \\
\hline$\theta_{S}$ & steel temperature \\
\hline $\bar{\theta}_{S}$ & mean temperature of the column cross-section \\
\hline$\lambda_{a}$ & thermal conductivity of steel according to the EN 1993-1-2:2005 \\
\hline $\bar{\lambda}_{c}, \lambda_{c}$ & nondimensional slenderness \\
\hline $\bar{\lambda}_{d}$ & edge stiffener slenderness for the distortional buckling \\
\hline $\bar{\lambda}_{p}$ & plate slenderness \\
\hline $\bar{\lambda}_{p, \text { red }}$ & reduced plate slenderness \\
\hline$\mu$ & mean value \\
\hline$v$ & poisson ratio \\
\hline$\rho$ & reduction factor for plate buckling \\
\hline$\rho_{c}$ & $\begin{array}{l}\text { or } \\
\text { degree of rotational restraint } \\
\text { rotational stiffness of the column }\end{array}$ \\
\hline$\rho_{i}$ & non-dimensional rotational restrain ratio in direction $\mathrm{i}$ \\
\hline$\rho_{s}$ & rotational stiffness of the surrounding structure \\
\hline$\sigma$ & $\begin{array}{l}\text { stress } \\
\text { or } \\
\text { standard deviation }\end{array}$ \\
\hline$\sigma_{c o m, E d}$ & $\begin{array}{l}\text { maximum design compressive stress in the plate } \\
\text { or } \\
\text { reduced compression stress } \\
\text { elastic buckling stress }\end{array}$ \\
\hline$\sigma_{c r, s}$ & elastic critical buckling stress of the edge stiffener \\
\hline$\sigma_{\text {eng }}$ & engineering (nominal) stress \\
\hline$\sigma_{\text {true }}$ & true stress \\
\hline$\sigma_{y, T}$ & yield strength at elevated temperatures \\
\hline$\phi$ & resistance factor \\
\hline$\chi_{d}$ & $\begin{array}{l}\text { reduction factor for the distortional buckling resistance of the edge } \\
\text { stiffener }\end{array}$ \\
\hline$\psi$ & stress ratio in the plate \\
\hline
\end{tabular}





\section{ABREVIATIONS}

$\begin{array}{ll}\text { CFS } & \text { Cold-Formed Steel } \\ \text { cFSM } & \text { constrained Finite Strip Method } \\ \text { CV } & \text { Coefficient of Variation } \\ \text { DEC } & \text { Department of Civil Engineering } \\ \text { DSM } & \text { Direct Strength Method } \\ \text { EAM } & \text { Effective Area Method } \\ \text { EDM } & \text { Electrical Discharge Machining } \\ \text { EC3 } & \text { Eurocode 3 } \\ \text { ECBL } & \text { Erosion of Critical Bifurcation Load } \\ \text { ECCS } & \text { European Convention for Constructional Steelwork } \\ \text { ESM } & \text { Effective Section Method } \\ \text { EWM } & \text { Effective Width Method } \\ \text { FCT } & \text { Portuguese Foundation for Science and Technology } \\ \text { FCTUC } & \text { Faculty of Sciences and Technology of the University of Coimbra } \\ \text { FEA } & \text { Finite Element Analyses } \\ \text { FEM } & \text { Finite Element Method } \\ \text { FSM } & \text { Finite Strip Method } \\ \text { GBT } & \text { Generalized Beam Theory } \\ \text { GBTUL } & \text { Generalized Beam Theory at the University of Lisbon } \\ \text { LSF } & \text { Light Steel Framing } \\ \text { LVDT } & \text { Linear Variable Displacement Transducer } \\ \text { LWT } & \text { Linear Wire Transducer } \\ \text { PP } & \text { Pinned } \\ \text { FF } & \text { Fixed } \\ \text { SR } & \text { Semi-Rigid } \\ \text { TPS } & \text { Transient Plane Source } \\ \text { SG } & \text { Strain Gauges } \\ & \end{array}$





\section{INTRODUCTION}

\subsection{General}

Cold-formed steel (CFS) is a term commonly used to identify products manufactured by pressing, or more commonly, by rolling of the steel at ambient temperature taking advantage of the ductility of steel. Cold formed steel products are widely used in different fields such as the building construction industry and automotive industry. In the building construction industry cold-formed steel products are commonly used as primary load bearing members (beams in floor assemblies, columns in wall assemblies, individual beams and columns, truss members, panels and decks) and non-loading bearing members (members in partition walls) (Fig.1.1).
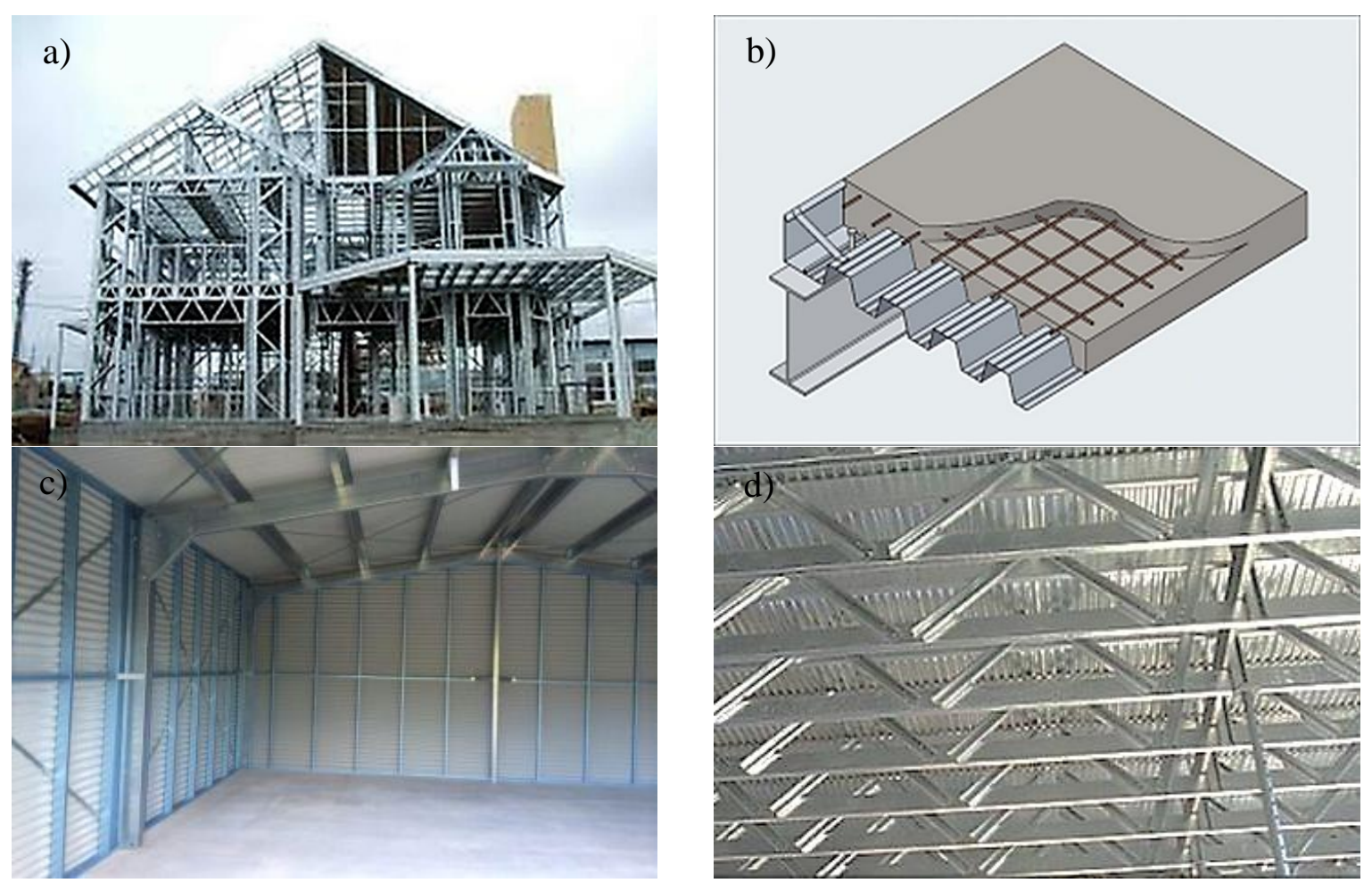

Figure 1.1. Use of cold-formed steel products. a) CFS building (Industrial machinery News@, 2015). b) Composite slab with profiled steel sheeting (Steel Construction Info@,2010). c)

CFS portal frames (Shed Quarters Company@, 2009). d) Truss members (LTH steel structures@,2015).

The use of cold-formed steel elements in the building construction industry started in the 1850s in both United States and Great Britain but its acceptance was still limited since there was no 
accurate technical information on the material use in building codes. Based on the research work developed by Professor George Winter the first edition of the Specification for the Design of Light Gauge Steel Structural members was published by the American Iron and Steel Institute (AISI) in 1946. Based on continuous research the specification has been updated to the most recent version AISI S100-12 - North American Specification for the Design of ColdFormed Steel Structural Members (AISI S100, 2012). In Europe the most recent document was published in 2006 as the European Standard Eurocode 3: Design of steel structures. Part 1-3: General rules. Supplementary rules for cold-formed thin gauges members and sheeting (EN 1993-1-3, 2006). This document was based on the European Recommendations for the design of light gauge steel members in 1987 by the ECCS Committee TC7 in 1987 (ECCS, 1987). Regarding the fire design of cold-formed steel structural elements there are no specific guidelines to this type of structural members. Currently the available guidelines in the EN 19931-2 (2005) for hot-rolled steels are also applicable to CFS members with class 4 cross-section.

As previously mentioned cold-formed steel products are manufactured by roll forming or folding and press-braking. Roll forming consists of a continuous bending operation of a long strip of coiled sheet metal, at ambient temperature, into a desired cross-section (Fig. 1.2.). A roll forming machine has a set of consecutive rolls. Each pair of opposite rolls is called a stand and each stand has specific roller shape. The more complex the desired cross-section shape the bigger the number of stands needed to obtain the final cross-section shape (Fig. 1.3.).

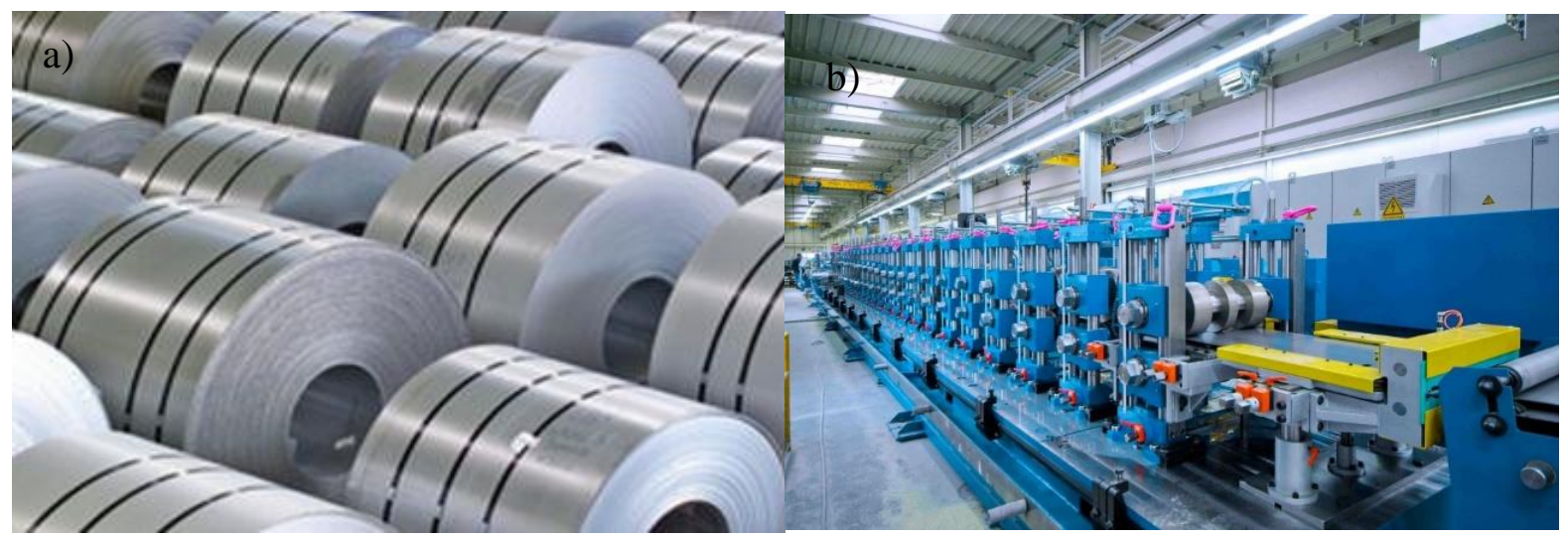

Figure 1.2. a) Galvanized steel coils (Aço Potiguar@, 2015). b) Roll forming machine (DreiStern@, 2015). 


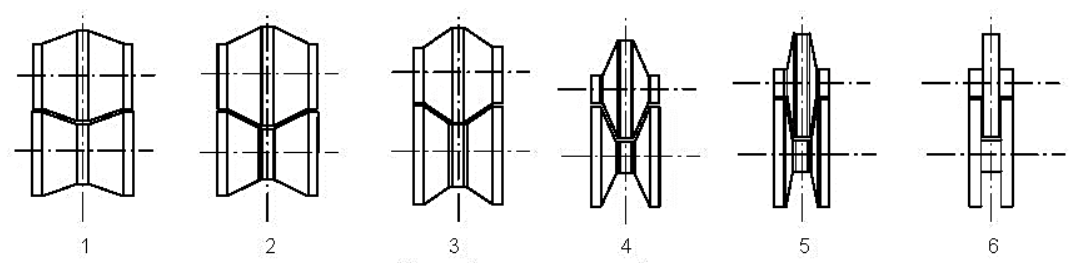

Roller shapes at each stage

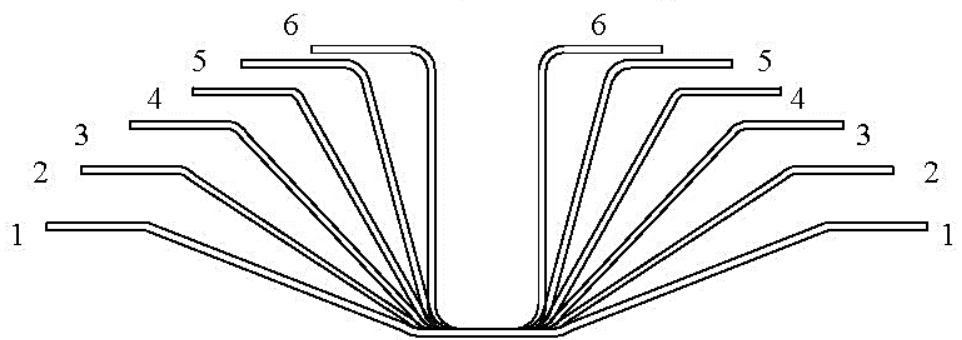

Figure 1.3. Stages in roll forming a simple section (Rhodes, 1991).

Press-braking is traditionally used to manufacture small quantities of a specified product, since the maximum length of the product to be manufactured depends on the length of the press brake. In this process a section is formed from a length of strip by pressing the strip between shaped dies to form the profile shape (Fig. 1.4.).
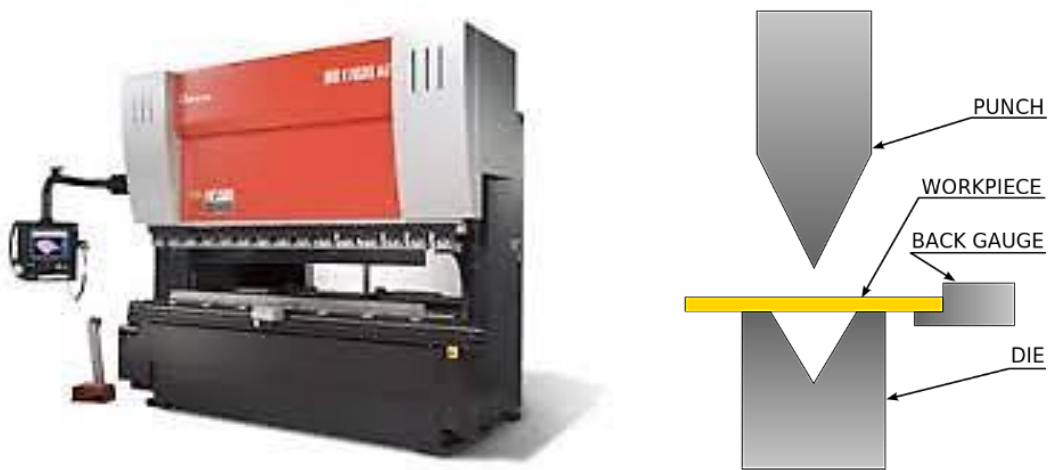

Figure 1.4. Press braking machine (Amada America, Inc@, 2015).

Currently, several commercial sections that may be used as individual structural framing members are available, such as C-sections, U-sections, Z-sections, $\Sigma$-sections, angles, hatsections, etc. (Fig. 1.5.). Moreover, the available single sections may be combined in order to fabricate open built-up and closed built-up cross-sections. This translates in a major economic advantage since the available resources are being used to provide additional possibilities to designers without changing the manufacture process (Georgieva et al, 2012a). Nowadays open and closed built-up cross-sections are widely used in structures built with CFS members since these cross-sections are able to span more distance and to present a higher buckling load. 


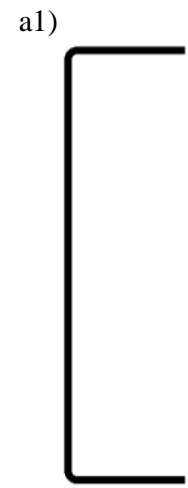

a2)

a3)

a4)

a5)

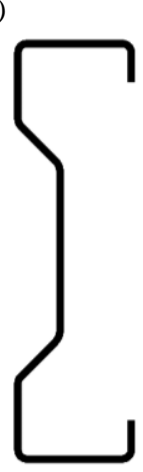

b3) a6)

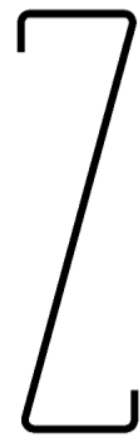

a7) b1)

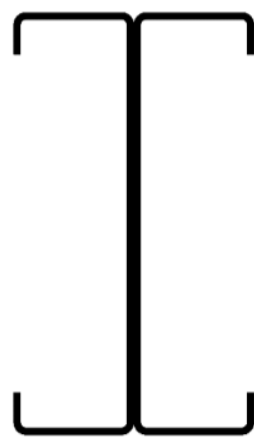

b2)

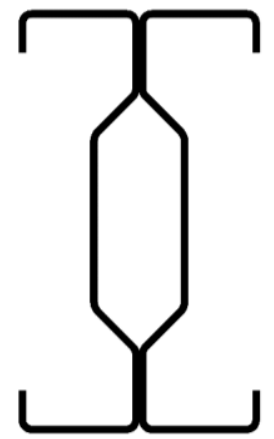

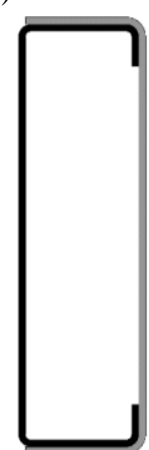

b4)

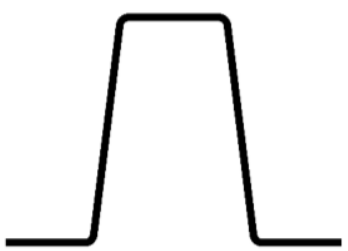

Figure 1.5. Typical CFS cross-section shapes used in building construction industry. a1) Plain channel (U). a2) Lipped channel (C). a3) Double lipped channel (C). a4) Double lipped U. a5) $\Sigma$ section. a6) Z section. a7) $\Omega$ section. b1) and b2) Open built-up I cross-sections. b3) and b4)

Closed built-up cross-sections $\mathrm{R}$ and $2 \mathrm{R}$.

In steel construction there are two main types of structural members, namely hot-rolled and cold formed steel sections. CFS steel members are used in Light Steel Frame (LSF) construction, especially for low and mid-rise buildings. Comparing both solutions CFS members present some advantages (Yu, 2010) such as lightness, possibility to manufacture members to relatively light loads and/or short spans, high strength-to-weight ratios, mass production, nestable sections allowing for compact packaging and transportation, fast and ease erection and installation. Due to the presented advantages the demand for structures built using CFS members has increased significantly in the past few years. However, the use of thin sections and cold-forming process brings additional challenges to designers and researchers. CFS members are usually subjected to several types of buckling modes, such as local, distortional, flexural, flexural-torsional buckling and their combinations, have low torsional rigidity and low fire resistance. Research and product development are still needed in order to fully understand the structural behaviour of CFS members. There is still a lack of research in built-up CFS members, that are widely used in the building construction industry, translated in the absence of specific design guidelines in the EN 1993-1-3 (2006). Also the fire behaviour of 
this type structural members is still an open research field. So far, few studies on CFS structural elements in fire situation have been reported, and as a consequence there are no specific guidelines for structural fire design available in the current standards, namely in the EN 19931-2 (2005). Consequently, the design provisions for hot-rolled steels are still applicable for cold-formed steels.

\subsection{Motivation / Problem Definition}

Cold-formed steel market share has increased significantly in the past few years all over the world due to its advantages when comparing to other construction materials. Also the extensive range of use, the constantly improving technology of manufacture and the corrosion protection add some extra value to this solution. Bear in mind that CFS members are pre-galvanised steel sections with a standard (Z275) zinc coating thickness of $0.04 \mathrm{~mm}\left(275 \mathrm{~g} / \mathrm{m}^{2}\right)$ in the case of the S280GD $+Z$ steel. However, for structural engineers thinner sections brings additional design problems that are not usually found in traditional structural steel design (Dubina et al, 2012), since the philosophy behind cold formed steel members is to use shape instead of thickness to provide optimal structural performance. Due to the reduced thickness of the individual plates of CFS members in relation to their widths, buckling at stress levels below the yield stress of the material may occur. Hence CFS members are highly susceptible to several instability phenomena such as local buckling (particularly prevalent in CFS members), distortional buckling, flexural buckling, flexural-torsional buckling, lateral-torsional buckling and the interaction between the mentioned buckling modes. Additional complexity is introduced due to the fact that the great majority of single members have open sections and asymmetric sections leading to low torsional stiffness, since the centroid and the shear centre do not coincide. Another common problem is the web crippling at points of concentrated load and supports.

With increasing popularity new challenges and demands come along. For instance, in order to respond to the necessity to design structures with CFS members with higher spans and higher buckling load, the use of built-up (open and closed) members has become common in CFS building construction industry. However, the available research on built-up CFS members is scarce and the available guidelines for designers are vague. For instance, there is no reliable data available about the influence of fasteners or seam weld spacing in the buckling load of a built-up member.

Another key issue regarding structures using CFS members is their structural fire performance. Structural fire safety design has become a key subject in the past few years due to continuous loss of lives and properties. This specific area is vital to guarantee adequate evacuation times, 
minimum safety conditions to firefighters, as well as to minimize property, economic and live losses. It is accepted that the fire resistance of unprotected CFS members is low due to high section factor and high thermal conductivity of steel. Hence in light steel framing (LSF) fire resistant plasterboards are used to protect structural members. Depending on the adopted thickness a fire resistance of 120 minutes can be achieved. However, due to pleasing aesthetic appearance the demand for unprotected CFS members has also increased. This brings additional problems to structural engineers since there is a relevant lack of knowledge on the behaviour of CFS members in fire situation as well as on the mechanical properties of this type of steel at elevated temperatures. So far the conducted research in the field of structural fire safety has focused predominantly on hot-rolled steel members and not on CFS members.

Currently the available design guidelines presented in the EN 1993-1-2 (2005) for hot-rolled steel members are also applicable to CFS members with class 4 cross-section, establishing the same reduction factors for the yield strength of steel and limiting the temperatures to $350^{\circ} \mathrm{C}$. However, some authors stated that the strength reduction factors of CFS steels at elevated temperatures may be 10-20\% higher than for hot-rolled steels (Sidey and Teague, 1988), hence this is a particular area where additional research contributions are needed. Also the material characterization in terms of thermal properties may be crucial for an accurate design of this type of structural elements. For example, properties such as thermal conductivity, specific heat and thermal elongation should be studied and compared with the available ones in the design standards for hot-rolled steels.

Regarding the behaviour of CFS compression members in fire situation it was found that the majority of the available research was focused on individual predominant buckling modes without considering their possible interaction. Other important variable that has been neglected, so far, in research of CFS compression members in fire situation is the influence of restraint to thermal elongation imposed by the surrounding structure to the CFS member. The assessment of the influence of restraint to thermal elongation on the behaviour of hot-rolled steel columns has already been studied, but for CFS columns nothing has been found, to the best of the author's knowledge. This variable is crucial to understand the actual behaviour of a CFS column inserted in a real building and subjected to fire. Hence it is urgent to conduct experimental and numerical research on CFS compression members in fire situation with restraint to thermal elongation in order collect relevant data for the development/improvement of new/available design methods for CFS single and built-up columns.

To achieve a good understanding on the structural behaviour of CFS columns at both ambient and fire conditions an extensive experimental and numerical research is needed. Some key 
aspects need particular attention, namely, material characterization at elevated temperatures (mechanical and thermal properties), assessment of buckling load of CFS columns and finally the assessment of the behaviour of those columns in fire situation considering restraint to thermal elongation.

This thesis is intended to provide relevant scientific data regarding the behaviour of CFS columns at both ambient and elevated temperatures as well as suitable advanced analysis methods based on the finite element method (FEM), capable of accurately reproducing and predicting the behaviour of CFS columns. Considering the data presented in this document as well as the developed and validated finite element models it will be possible, in the near future, to undertake large parametric studies in order to gather enough information so that new/existent accurate design methods may be developed/improved.

\subsection{Objectives}

The overall objective of this research work was to investigate the behaviour of cold-formed steel (CFS) columns, commonly used in the building construction industry, at both ambient and fire conditions, from material characterization to structural solution. This research was conducted in the scope of a National Research Project entitled FireColdFSteel - Experimental and Numerical Analyses of Cold-Formed Steel Members Under Fire Conditions funded by the National Foundation for Science and Technology - PTDC/ECM/116859/2010 (FCT). Based on the experimental and numerical investigation carried out in the scope of this thesis and on future parametric studies to be conducted it is intended to present accurate design methodologies for both ambient and fire situations that eventually may be incorporated in future revisions of the current standards, namely EN 1993-1-3 (2006) and EN 1993-1-2 (2005).

To achieve this goal, the conducted research was divided in two large stages, namely experimental and numerical research. The objectives for each one of the defined stages can be detailed individually.

Within the experimental research three major steps were performed, namely material characterization, assessment of buckling load of CFS single, open built-up and closed built-up columns at ambient temperature, and finally assessment of the behaviour of CFS columns subjected to fire with restraint to thermal elongation provided by the surrounding structure just like in a real building. 
Regarding material characterization, the objectives were:

- Determination of mechanical properties of the steel S280GD+Z with $2.5 \mathrm{~mm}$ thickness at both ambient and elevated temperatures $\left(20\right.$ to $\left.800^{\circ} \mathrm{C}\right)$ through tensile coupon tests;

- Determination and comparison of the deterioration of mechanical properties (reduction factors) with the available design standards, namely yield stress, modulus of elasticity, ultimate stress and proportional limit;

- Propose new predictive equations to determine stress-strain curves for the S280GD+Z steel at both ambient and elevated temperatures;

- Determination of thermal properties of the S280GD+Z steel using the Transient Plane Source (TPS) technique (thermal conductivity and specific heat) (Hot Disk, 2015);

- Determination of thermal elongation using the high temperature extensometer Epsilon HI3548 (Epsilon, 2011);

Regarding the experimental campaign on the assessment of the buckling load of CFS columns with single ( $\mathrm{C}$ - lipped channel), open built-up (I - 2 lipped channel back-to back) and closed built-up cross-sections ( $\mathrm{R}$ - 1 lipped channel and 1 plain channel; $2 \mathrm{R}-2$ lipped channels backto-back and 2 plain channels) the objectives were:

- to assess the buckling load of the tested CFS columns considering different end-support conditions. In this experimental campaign it was attempted to simulate both pinned and fixed end-support conditions;

- to investigate and characterize the observed failure modes. Overall characterization of the structural response of the tested columns (axial displacements, lateral displacements and rotations at the end-supports;

- to compare the structural performance of different types of columns and compare the buckling load with the determined design buckling loads.

Regarding the experimental campaign on the assessment of the fire behaviour of CFS columns with restrained thermal elongation the main objectives were:

- to assess the fire behaviour of CFS columns with restrained thermal elongation;

- to assess the influence of the cross-section shape, influence of the initial applied service load level, influence of the level of restraint to thermal elongation imposed to the CFS column (the restraint used intended to reproduce as faithfully as possible the actual boundary conditions of a CFS column inserted in a real building) and influence of the adopted end-support condition on the overall behaviour of this type of columns; 
- Monitor and record critical temperatures, critical times, generated restraining forces, axial and lateral displacements during each experimental test;

- Based on the experimental results assess the influence of the level of restraint to thermal elongation on the overall performance of the tested CFS columns, comparing critical temperatures recorded for each level of restraint as well as the amount of generated axial restraining forces.

Within the numerical research the defined objectives in the scope of this research work were:

- To develop finite element numerical models capable of accurately reproducing the behaviour of CFS columns at both ambient and fire conditions with restraint to thermal elongation, namely in terms of the observed buckling modes;

- Validate the developed finite element model using the previously obtained experimental results. Use the obtained results in the material characterization as input data in the model and use the obtained results in the structural tests to compare with the ones obtained in the numerical simulations, and consequently validate the finite element model.

Finally, whenever possible and based on observations, it is intended to propose issues of good practice for CFS building construction industry. To achieve the detailed objectives this research was based on a thorough literature review, on careful design, assembly and execution of each experimental test and finally on the developed finite element models validated against experimental tests.

\subsection{Contents of the Thesis}

The outline of this thesis is as follows:

- Chapter 1 presents a general introduction about structures built-using cold-formed steel members, their applications in the building construction industry as well as its advantages and peculiarities when compared with other existent solutions. Also briefly presents the available design standards for both ambient and elevated temperatures. Then a detailed description is presented on the motivation and problem definition. Finally, the objectives of this research are thoroughly described as well as the adopted methodology to achieve them.

- Chapter 2 presents the most relevant literature review findings on the subject, namely material characterization, experimental analysis on CFS columns at both ambient and elevated temperatures, numerical and analytical investigations conducted by other researchers. It is focused particularly on the mechanical properties of CFS steels and on 
buckling behaviour of CFS compression members (single, open built-up and closed built-up members) at ambient and fire conditions, including global flexural, local, distortional, torsional buckling and their interactions. Further it describes the available design methods for cold-formed steel compression members. Finally, some general considerations are presented. The available findings in the literature review are analysed and based on them, strengths and weaknesses are identified.

- Chapter 3 presents the experimental study carried out to assess mechanical and thermal properties of the S280GD+Z steel at both ambient and elevated temperatures. To evaluate the mechanical properties of the $\mathrm{S} 280 \mathrm{GD}+\mathrm{Z}$ steel tensile coupon tests were conducted at both ambient and elevated temperatures $\left(20\right.$ to $\left.800^{\circ} \mathrm{C}\right)$. The entire test setup and experimental programme is detailed. Elevated temperature tests were conducted using the steady state test method using the high temperature extensometer Epsilon HI 3548 (Epsilon, 2011). The obtained results are compared with the ones available in the literature and the ones available in the design standards. New predictive models are presented for the stress-strain relationship at both ambient and elevated temperatures, as well as new predictive methods for the S280GD+Z steel reduction factors for the different mechanical properties. Thermal elongation was also experimentally determined and compared with the available models presented in the design standards. Also thermal properties of the S280GD+Z steel were determined, namely thermal conductivity and specific heat using the Transient Plane source technique and the Transient Plane Source equipment TPS 2500s from Hot Disk (Hot Disk, 2015). All test set-up is thoroughly described and test results will be presented and compared with the available provisions presented in the design standards.

- Chapter 4 presents the experimental investigation conducted on CFS columns at both ambient temperature and simulated fire conditions with restraint to thermal elongation. The tests at ambient temperature were undertaken in order to evaluate the buckling load of different types of columns considering different boundary conditions and to characterize the observed failure modes. In the fire tests it was intended to assess the fire performance of CFS columns with restraint to thermal elongation. To achieve a good understanding on this matter different types of columns were tested considering different boundary conditions, levels of initial applied service level, and levels of restraint imposed to the CFS column in fire. All obtained results and observed failure modes are presented and characterized in this chapter. The tested specimens are thoroughly detailed in this chapter as well as the entire experimental test set-ups and instrumentation used in each experimental test. The experimental programme and testing procedures are also detailed. The obtained results in this investigation are 
presented and whenever possible compared with the results obtained using the provisions presented in the design standards.

- Chapter 5 presents the finite element modelling of CFS compression members at both ambient and elevated temperatures and the validation of those models against the obtained results in the experimental research. It was intended to thoroughly describe the developed finite element model, showing the most relevant details of the finite element model and presenting the adopted assumptions in order to accurately reproduce the experimental tests. Then the obtained results were compared with the ones obtained in the experimental investigation in order to validate the developed finite element model.

- Chapter 6 presents the relevant findings from this research and also presents some paths for future investigation within this research work, namely in terms of future parametric studies using the developed and validated finite element models and finally, in terms of proposals of new/improved design methods for CFS columns at both ambient and fire conditions. Moreover, some recommendations are presented for future research work on CFS columns in order to study and develop new solutions. 



\section{LITERATURE REVIEW}

\subsection{Mechanical and Thermal Properties of Cold-Formed Steels}

\subsubsection{Mechanical properties at ambient temperatures}

Knowing exactly the mechanical behavior of steel, or any other material, is crucial to a good understanding and prediction of the behavior of structures. Material properties play an important role in finite element analysis as the more accurate are the used mechanical properties as input, the more realistic the results will be.

The properties of structural steel result from both its chemical composition and manufacturing method. Mechanical properties of hot-rolled steels are well known and the available design standards provide accurate mechanical properties at ambient and elevated temperatures. However, mechanical properties of cold-formed steels can be significantly different from those of the virgin steel sheet before forming and hot-rolled steels due to cold-forming procedure at ambient temperature. The cold-forming process changes the mechanical properties of the steel from the original steel sheet, since both yield strength and ultimate tensile strength increases while reducing the ductility of steel (Yu, 2010, Chen and Young, 2006). The deformations imposed during fabrication process at the flat parts may be elastic whereas at the corners the deformations are essentially plastic. Hence the mechanical properties vary over the crosssection (Karren and Winter, 1967).

Basically three phenomena are responsible for the changes in the mechanical properties during cold-forming, namely strain hardening (strengthening of steel by plastic deformation), strain aging (when steel has been deformed plastically and then allowed to age; strength of the material increases and the ductility decreases) and Bauschinger effect that refers to the fact that the longitudinal compression yield strength of the stretched steels is smaller than the longitudinal tension yield strength (Yu, 2010, Karren and Winter, 1967, Karren , 1967, and Chajes et al, 1963 ). In Figure 2.1. it is shown that the influence of cold-forming in the mechanical properties of steel are mainly due to strain hardening and strain aging phenomena.

Due to the differences between the manufacture process for hot-rolled and cold-formed steels there are two types of stress-strain curves of steel, namely sharp-yielding (Fig. 2.2.a)) and gradual yielding type (Fig. 2.2.b)). Steels produced by hot-rolling are usually sharp yielding whereas steels produced by cold-forming are gradual yielding. For hot-rolled steels the yield 
point is defined by the level at which the stress-strain curve becomes horizontal whereas for cold-formed steels the yield strength is the stress at strain levels of $0.2 \%(0.2 \%$ proof stress) or $0.5 \%$.

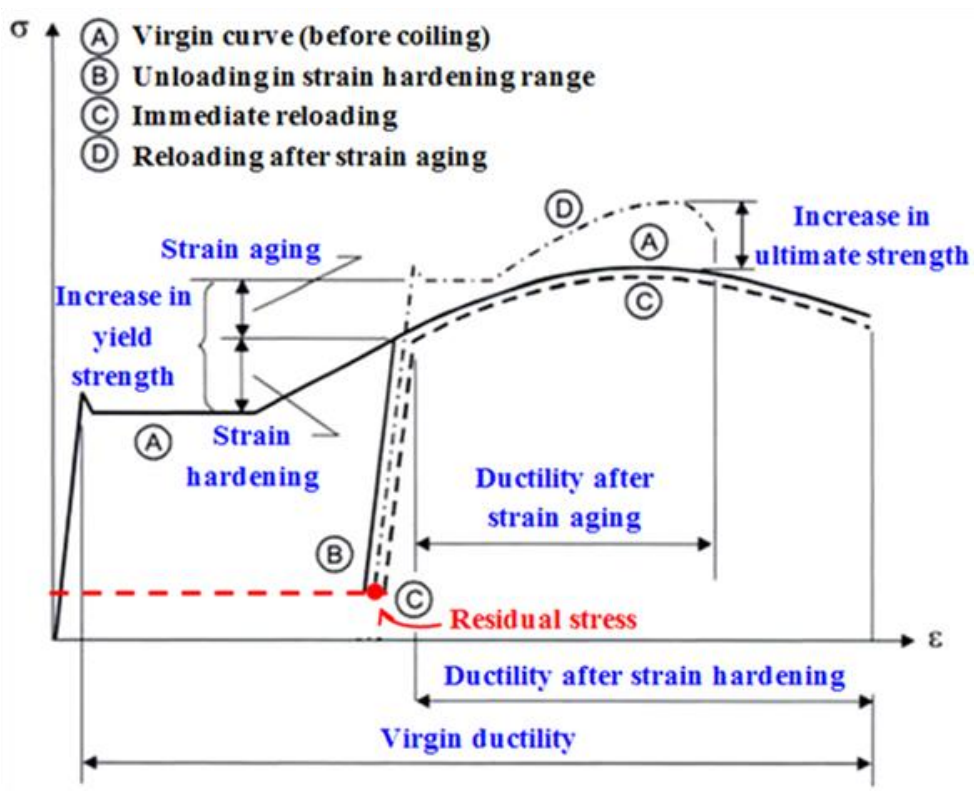

Figure 2.1. Effects of strain hardening and strain aging on stress-strain characteristics of structural steels (Yu, 2010; Chajes et al., 1963; Rondal and Dubina, 2005; Moen et al., 2008).
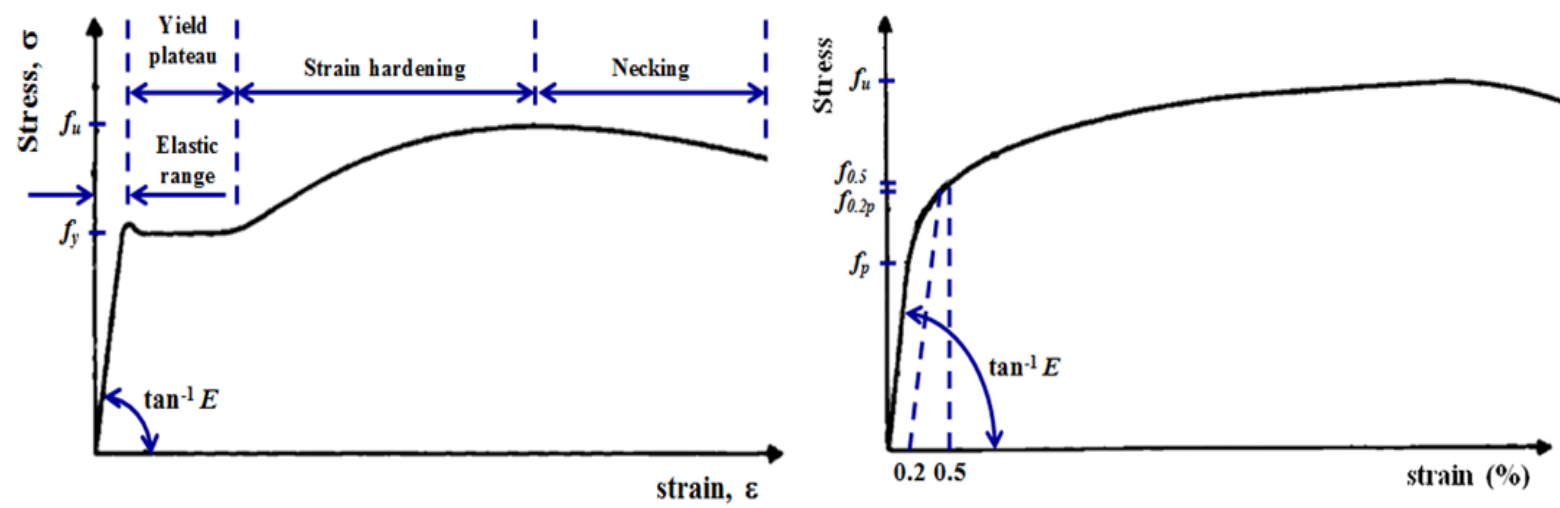

Figure 2.2. a) Stress-strain curve of hot-rolled steels. b) Stress-strain curve for cold-worked steels (Yu, 2010).

\subsubsection{Mechanical properties at elevated temperatures}

The deterioration of mechanical properties, such as yield strength and modulus of elasticity is a key issue in the assessment of the performance of CFS structural elements in fire. The deterioration of mechanical properties with increasing temperature results in a significant loss 
of buckling load of CFS structural elements. The currently available design codes such as BS 5950 Part 8 (1990) and the EN 1993-1-2:2005 (2005) provide reduction factors for mechanical properties for cold-formed steels at elevated temperatures but with some limitations. For instance, BS 5950 Part 8 (1990) only provides reduction factors for yield strengths corresponding to $0.5 \%, 1.5 \%$ and $2.0 \%$ strain levels whereas the EN 1993-1-2:2005 (2005) provides the same reduction factors used for class 4 hot-rolled steels. However, Sidey and Teague (1988) stated that the strength reduction of cold-formed steels at elevated temperatures may be 10-20\% higher than that of hot-rolled steels due to metallurgical composition and molecular surface effects. So far some relevant research has been published on the mechanical properties of cold-formed steels at elevated temperatures.

Makelainen and Miller (1983) reported an investigation on the mechanical properties of the cold-formed galvanised steel sheet Z32 at elevated temperatures. In the tensile tests both transient and steady state methods were used and based on the obtained results new predictive equations were proposed for the reduction factors of the elastic modulus and yield strength. In the transient-state test the specimen is subjected to a constant load under a constant rate of temperature increase, whereas in the steady-state test method the specimen is heated up to a specific temperature and then the tensile test is carried out. Based on the obtained results using the steady state method the following equation (Equation 2.1) for the reduction factors of the elastic modulus was presented.

$$
\frac{E_{T}}{E_{20^{\circ} C}}=-0.46 \tanh \left(\frac{T_{S}-550}{250}\right)+0.56
$$

For the transient state tests the reduction factors for yield strength and elastic modulus were fitted to the following equations (Equations 2.2 and 2.3):

$$
\begin{gathered}
\frac{\sigma_{y, T}}{\sigma_{y, 20^{\circ} C}}=1.008-0.1314 \exp \left(0.0047\left(T_{S}-148.3^{\circ} C\right)\right) \quad 20 \leq T_{S} \leq 500^{\circ} C \\
\frac{\sigma_{y, T}}{\sigma_{y, 20^{\circ} C}}=\frac{104^{\circ} \mathrm{O} C\left(1-T_{S} / 1135^{\circ} \mathrm{o} C\right)}{T_{S}-356^{\circ} \mathrm{C}} T_{S}>500^{\circ} \mathrm{\circ} C \\
\frac{E_{T}}{E_{20^{\circ} \mathrm{o}}}=1.01-0.139 \exp \left(0.007\left(T_{S}-346^{\circ} \mathrm{o}\right)\right)
\end{gathered}
$$

Outinen et al (2000) presented an extensive experimental research for the investigation of the mechanical properties of different types of structural steels, namely S350GD+Z, S355 and 
$\mathrm{S} 460 \mathrm{M}$, at elevated temperatures using the transient and steady state test method. It is stated that transient state test method provides more realistic results as it simulates a structural member under static loading subjected to fire since creep effects are also taken into account. The test piece of the S350GD+Z steel were cut from a virgin steel plate with nominal thickness of 2 $\mathrm{mm}$, longitudinally to rolling direction. Specifically, for the S350GD+Z steel it was found that the reduction factors for the elastic modulus are similar for the transient state and steady state test method except at 400 and $500^{\circ} \mathrm{C}$ (Fig. 2.4). Both elastic modulus and yield strength reduction factors do not agree with the EN 1993-1-2:2005 (2005) predictions for hot-rolled steels (Figure 2.3 and 2.4). The most recent version of the EN 1993-1-2:2005 (2005) provide new yield strength reduction factors for cold-formed steels (the same presented for hot-rolled steels with class 4 cross-section), however they still do not agree with the reduction factor proposed by Outinen et al. (2000).

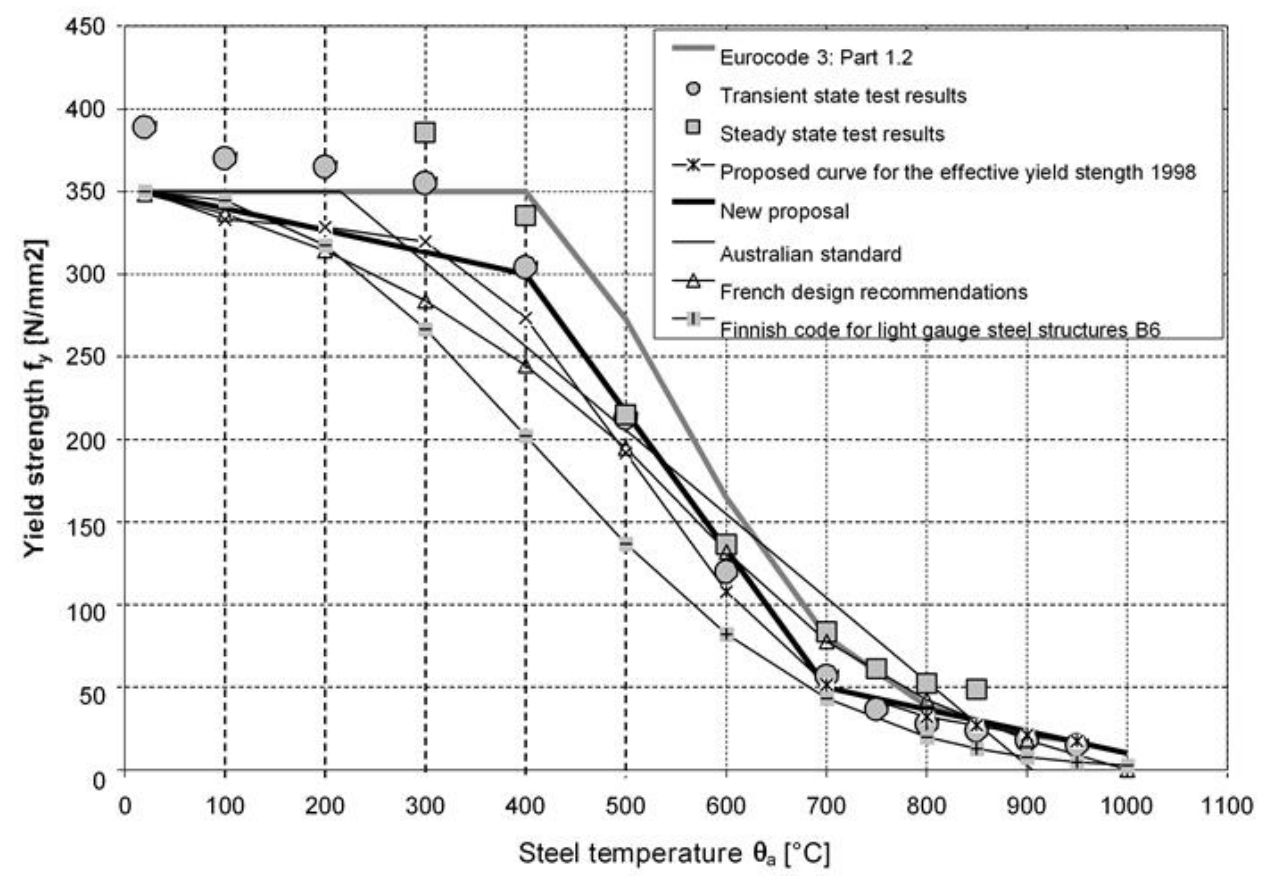

Figure 2.3. Yield strength of structural steel S350GD $+Z$ and comparison with different design codes (Outinen et al., 2000) 


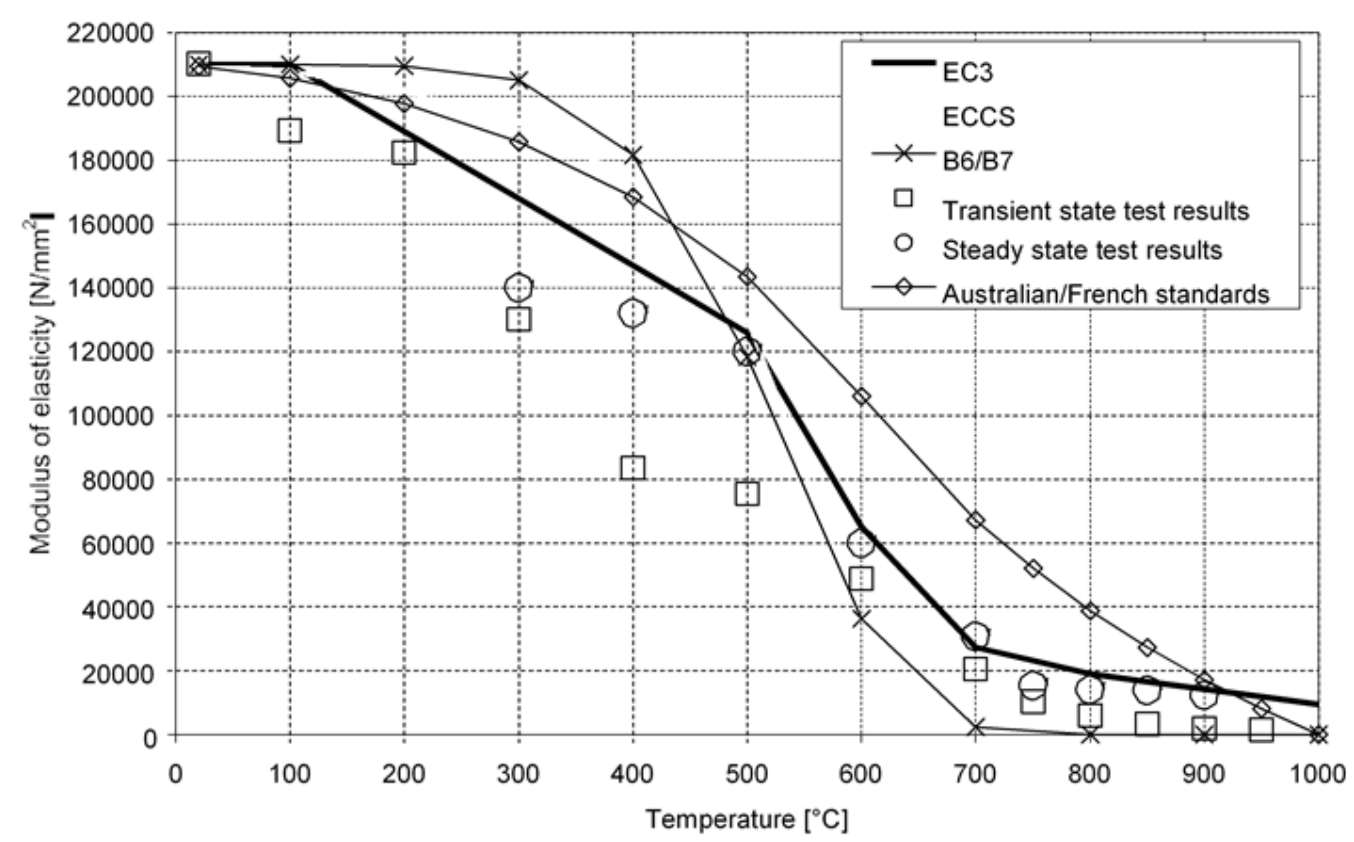

Figure 2.4. Elastic modulus of structural steel S350GD+Z and comparison with different design codes (Outinen et al., 2000)

Outinen and Makelainen (2002) reported an experimental research on the mechanical properties of structural steel at elevated temperatures and after cooling down. Among others the S350GD+Z steel was tested. Once again the transient state test method was used, hence the specimen is subjected to a constant tensile load and to a constant temperature rise. Also some steady state tests were also performed as reference tests. In Figure 2.5 the used test set-up is presented. A comparison is established between the obtained results in terms of reduction factors for the yield strength and the available provisions presented in the EN 1993-1-2:2005 (2005) for hot-rolled steels. In the EN 1993-1-2:2005 (2005) the yield strength is assumed to be constant up to $400^{\circ} \mathrm{C}$ whereas in the experimental results the yield strength starts to decrease earlier. Also in the scope of this investigation some tensile tests were conducted on test pieces taken before and after high temperature (up to $950^{\circ} \mathrm{C}$ ) compression tests. It was observed that mechanical properties after heating seemed to be near the nominal values of the material (Fig. 2.6). This observation may be very important when considering the buckling load of a steel structure after fire. Hence the steel structure may still be usable after fire if the distortions are within the shape and straightness tolerances of the structure. 


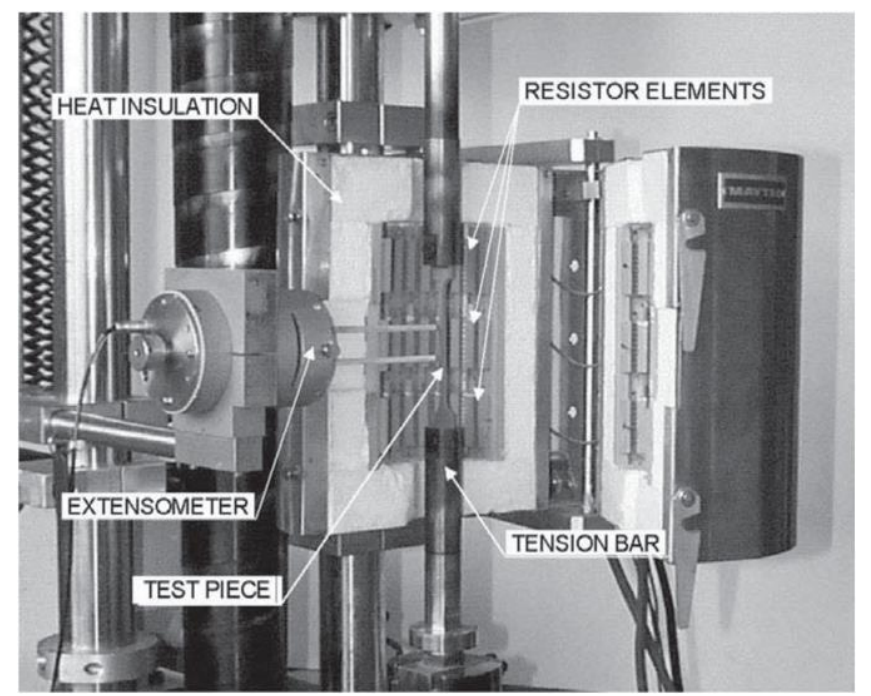

Figure 2.5. High temperature testing device (Outinen and Makelainen, 2002).

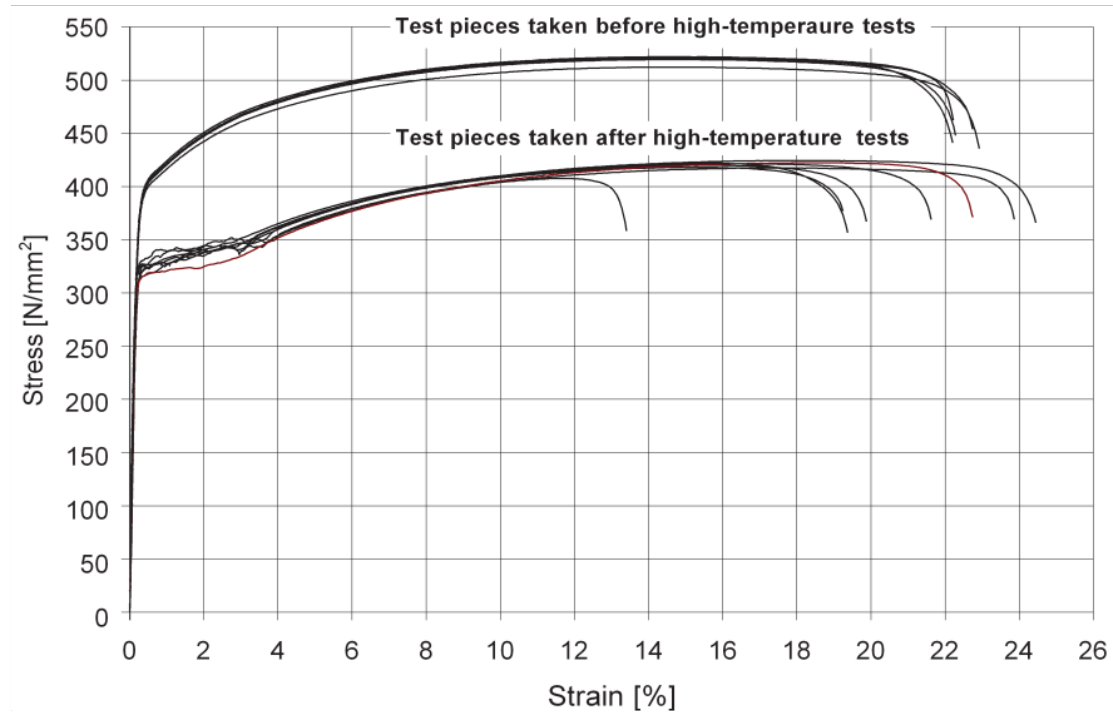

Figure 2.6. Tensile test results for specimens taken before and after high temperature compression tests (Outinen and Makelainen, 2002)

Lee et al. (2003) conducted an experimental study on the deterioration of the mechanical properties of low and high strength cold-formed steels. Three steel grades (G300, G500 and G550; minimum yield stress of 300, 500 and $550 \mathrm{MPa}$ ) and six thicknesses were tested, ranging from $0.4 \mathrm{~mm}$ to $1.2 \mathrm{~mm}$. Due to its simplicity and accuracy of the results the steady state test method was used in this investigation. The obtained results showed that thickness has very little influence on the mechanical properties. Further, some empirical equations for the reduction factors of mechanical properties for cold-formed steels were presented, namely for yield strength (Equation 2.4) and elastic modulus (Equation 2.5). Finally, it was found that the 
reduction factors given in the currently available design standards for hot-rolled steels are unconservative for cold-formed steels.

$$
\begin{aligned}
& \frac{f_{y, T}}{f_{y, 20}}=1.0 \\
& 20^{\circ} \mathrm{C} \leq T<100^{\circ} \mathrm{C} \\
& \frac{f_{y, T}}{f_{y, 20}}=0.964+0.00045 T-3.08 .10^{-6} T^{2}+1.969 .10^{-9} T^{3} \quad 100^{\circ} C \leq T<350^{\circ} \mathrm{C} \\
& \frac{f_{y, T}}{f_{y, 20}}=1.514-\frac{0.0144 \times T}{f_{y, 20}^{1 / 5}+4.72} \quad 400^{\circ} C \leq T<750^{\circ} \mathrm{o} C \\
& \frac{f_{y, T}}{f_{y, 20}}=0.1 \quad T=800^{\circ} \mathrm{O} C \\
& \frac{E_{T}}{E_{20}}=1.0 \quad 20^{\circ} \mathrm{O} C \leq T<100^{\circ} \mathrm{O} C \\
& \frac{E_{T}}{E_{20}}=1-0.0014(T-100) \quad 100^{\circ} \mathrm{C} \leq T<500^{\circ} \mathrm{C} \\
& \frac{E_{T}}{E_{20}}=\frac{1-\frac{T}{1200}}{0.00122 T+0.3}-0.203 \quad 500^{\circ} \mathrm{o} C \leq T<800^{\circ} \mathrm{C}
\end{aligned}
$$

Chen and Young (2006) presented the detail of an experimental research on mechanical properties of corner parts and flat parts of cold-formed steel profiles (steel grade G500 with nominal thickness of $1.9 \mathrm{~mm}$ ) at elevated temperatures, ranging from 20 to $1000^{\circ} \mathrm{C}$. The specimens were cut from a sigma $(\Sigma)$ shaped cross-section. Flat, inner and outer corner specimens were tested at elevated temperatures. Also is worth mentioning that in this experimental research the influence of cold-forming on the mechanical properties over the cross-section is clear. For instance, the obtained yield strength for flat coupon specimens was $524 \mathrm{MPa}$ whereas for the outer corner coupon specimens the yield strength was $573 \mathrm{MPa}$. The results for flat and corner specimens were compared and a unified equation for reduction factors of the elastic modulus, yield strength, ultimate strength and ultimate strain of the corner parts is proposed. In Table 2.1 the proposed equations as well as the correspondent coefficients are presented. In this investigation it was found that the reduction factors of yield strength, elastic modulus and ultimate strength of the corner coupon specimens are similar to those of the flat coupon specimens. 
Table 2.1. Reduction factors for mechanical properties of corner coupon specimens (Chen and Young, 2006).

\begin{tabular}{|c|c|c|c|c|c|}
\hline & Temperature $\left[{ }^{\circ} \mathrm{C}\right]$ & $\mathrm{a}$ & $\mathrm{b}$ & $\mathrm{c}$ & $\mathrm{n}$ \\
\hline Yield strength & $22 \leq T<300$ & 1.0 & 22 & $5.56 \times 10^{3}$ & 1 \\
\hline$f_{y, T}-(T-b)^{n}$ & $300 \leq T<650$ & 0.95 & 300 & $1.45 \times 10^{5}$ & 2 \\
\hline$\overline{f_{y, \text { normal }}}=a$ & $650 \leq T<1000$ & 0.105 & 650 & $5.00 \times 10^{3}$ & 1 \\
\hline Elastic modulus & $22 \leq T<450$ & 1.0 & 22 & $1.25 \times 10^{3}$ & 1 \\
\hline$\frac{E_{T}}{E_{\text {normal }}}=a-\frac{(I-b)^{n}}{c}$ & $450 \leq T<650$ & -0.11 & 860 & $-2.20 \times 10^{5}$ & 2 \\
\hline Ultimate strength & $22 \leq T<450$ & 1.0 & 22 & $5.6 \times 10^{8}$ & 3 \\
\hline$\frac{f_{u, T}}{f_{u, \text { normal }}}=a-\frac{(T-b)^{n}}{c}$ & $450 \leq T<1000$ & 0.043 & 1000 & $-1.12 x^{11}$ & 4 \\
\hline $\begin{array}{c}\text { Ultimate strain } \\
\frac{\varepsilon_{u, T}}{\varepsilon_{u, \text { normal }}}=a-\frac{(T-b)^{n}}{c}\end{array}$ & $22 \leq T<1000$ & 1.0 & 22 & $1.0 \times 10^{6}$ & 2 \\
\hline
\end{tabular}

Chen and Young (2007a) presented another experimental study on the deterioration of the mechanical properties of cold-formed steel grades G550 (1 mm thickness) and G450 (1.9 mm thickness) at elevated temperatures $\left(20\right.$ to $\left.1000^{\circ} \mathrm{C}\right)$. Both steady state and transient state test methods were used in this investigation. Test results for different mechanical properties such as elastic modulus, yield strength $(0.2 \%$ proof stress, $0.5 \%, 1.5 \%$ and $2 \%)$, ultimate strength, ultimate strain and thermal elongation are presented and compared with available design standards and with the results presented by other researchers (Lee et al., 2003). The reduction factor of $0.2 \%$ yield strength were compared with the Australian Standard AS4100 (1998) and with the test results presented by Lee et al. (2003) (Figure 2.7a), whereas the reduction factors for the strain levels of $0.5 \%, 1.5 \%$ and $2 \%$ were compared with the EN 1993-1-2:2005 (2005) and BS 5950 Part 8 (1990). It was observed that the AS 4100 (1998) provides conservative predictions from 220 to $400^{\circ} \mathrm{C}$ and unconservative predictions from $450^{\circ}$ to $800^{\circ} \mathrm{C}$. Regarding the results presents by Lee et al. (2003) some significant differences were found for temperatures ranging from 450 to $800^{\circ} \mathrm{C}$. Also it was found that the reduction factors of $2 \%$ yield strength predicted in the EN 1993-1-2:2005 (2005) were conservative for G450 and G550 steels for temperatures ranging from 20 to $550^{\circ} \mathrm{C}$ and 20 to $400^{\circ} \mathrm{C}$, respectively. For temperatures above $660^{\circ} \mathrm{C}$ the test results for the G450 steel were very different from the predictions presented in the EN 1993-1-2:2005 (2005). The same was observed for the G550 steel for temperatures ranging from 450 to $800^{\circ} \mathrm{C}$. Regarding the reduction factor of the modulus of elasticity it was found that the obtained results using the transient state test method are very close to the EN 1993-1-2:2005 (2005) prediction. Other interesting observation was the significant difference between the reduction factors of the elastic modulus obtained from the 
transient state test method and the steady state test method (Fig. 2.7b). Also in the scope of this investigation some tests were conducted in order to determine the thermal elongation of the steel at elevated temperatures.
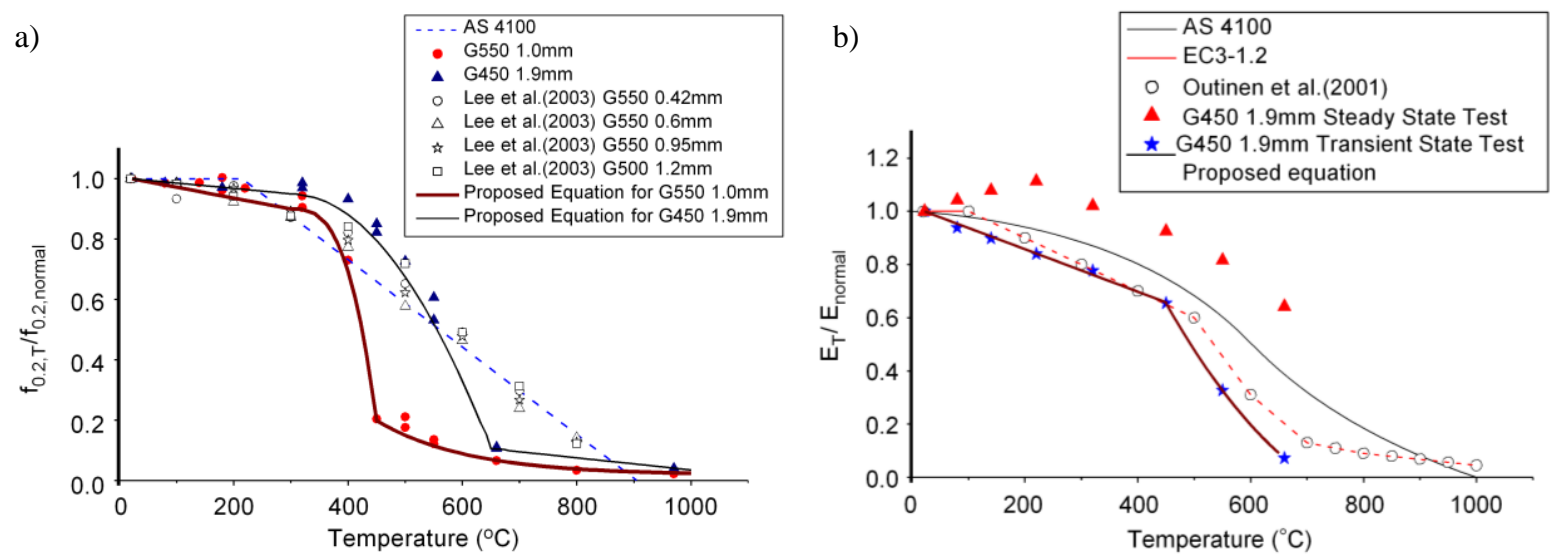

Figure 2.7. Comparison between test results and design standards for the reduction factors. a) Yield strength ( $0.2 \%$ proof stress). b) Elastic modulus for both transient and steady state test method. (Chen and Young, 2007).

Ranawaka and Mahendran (2009) reported an experimental study on the mechanical properties of cold-formed steels at elevated temperatures. Low and high strength steels were tested, namely G250 and G550 with three nominal thicknesses of 0.6, 0.8 and $0.95 \mathrm{~mm}$. In this research the steady state test method was used. Since both steady state and transient state tests are usually completed within an hour the amount of creep effect is limited, and as a consequence the difference between the two methods is very small. In the Figure 2.8 the experimental test setup is presented.

Analysing the obtained results, it was found that the low strength steel lost its strength more rapidly than high strength steels for temperatures ranging from 200 to $500^{\circ} \mathrm{C}$. However, for temperatures beyond $400^{\circ} \mathrm{C}$ a sudden drop on the yield strength was observed for the high strength steel. The different rates of strength degradation are due to the higher level of coldworking used in high strength steels. Also it was found that thickness does not have significant influence on the reduction factors. Regarding the elastic modulus, it was found that the reduction factors were similar for low and high strength steels. In Figure 2.9 and Figure 2.10 the yield strength and elastic modulus reduction factors are presented for both steel grades and thicknesses tested. Based on the yield strength and elastic modulus results empirical predictive equations for mechanical properties were proposed for each steel grade tested (Equations 2.6, 
2.7 and 2.8). Equations 2.6 are recommended for the determination of the yield strength reduction factors for the G550 steel.
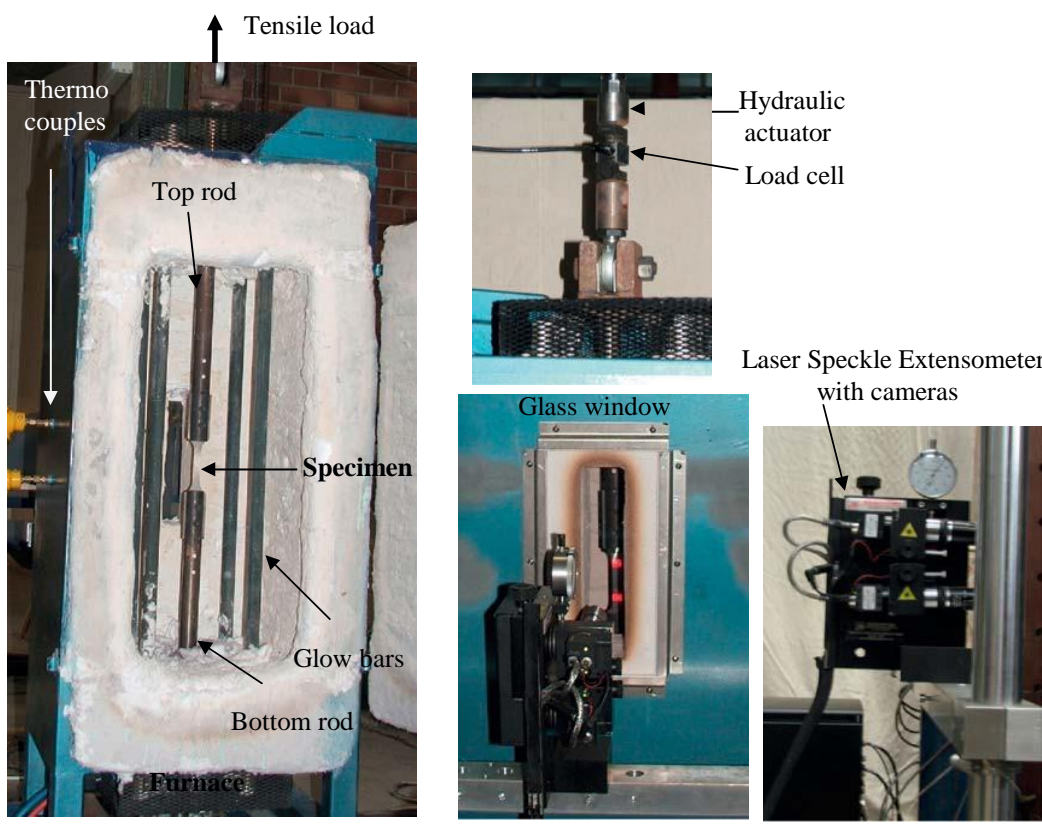

Figure 2.8. Test set-up (Ranawaka and Mahendran, 2009).

$$
\begin{array}{ll}
\frac{f_{y, T}}{f_{y, 20}}=-0.00016 T+1.0003 & 20^{\circ} C \leq T \leq 200^{\circ} C \\
\frac{f_{y, T}}{f_{y, 20}}=0.97-\frac{(T-200)^{1.81}}{58500} & 200^{\circ} C \leq T \leq 600^{\circ} C \\
\frac{f_{y, T}}{f_{y, 20}}=-0.00037 T+0.3363 & 600^{\circ} C \leq T \leq 800^{\circ} C
\end{array}
$$

Equations 2.7 are recommended for the determination of the yield strength reduction factors for the G250 steel.

$$
\begin{array}{ll}
\frac{f_{y, T}}{f_{y, 20}}=-0.0007 T+1,014 & 20^{\circ} C \leq T \leq 200^{\circ} C \\
\frac{f_{y, T}}{f_{y, 20}}=3.7 T-\frac{(T-74)^{0.15}}{0,736} & 200^{\circ} C \leq T \leq 800^{\circ} C
\end{array}
$$


Equations 2.8 are recommended for the determination of the elastic modulus reduction factors for both G250 and G550 steels.

$$
\begin{array}{cc}
\frac{E_{T}}{E_{20}}=1 & 20^{\circ} \mathrm{C} \leq T \leq 100^{\circ} \mathrm{C} \\
\frac{E_{T}}{E_{20}}=-0.0013 T+1,1297 & 100^{\circ} \mathrm{\circ} \leq T \leq 800^{\circ} \mathrm{C}
\end{array}
$$

Moreover a stress strain model was developed for each steel grade tested, based on the Ramberg and Osgood (1943) stress-strain model, represented by the Equation 2.9, where $\varepsilon_{T}$ is the strain corresponding to a given stress $f_{T}$ at temperature T, $E_{T}$ and $f_{y, T}$ are the elastic modulus and yield strength, respectively, and $\beta$ and $\eta_{T}$ are the two parameters defining the stress-strain model.

$$
\varepsilon_{T}=\frac{f_{T}}{E_{T}}+\beta\left(\frac{f_{y, T}}{E_{T}}\right)\left(\frac{f_{T}}{f_{y, T}}\right)^{\eta_{T}}
$$

The parameter $\beta$ was defined as 0.86 while the parameter $\eta_{T}$ is defined in Equation 2.9a), for the G550 steel, and Equation 2.9b) for the G250 steel.

$$
\begin{array}{ccc}
\eta_{T}=-3.05 \times & 10^{-7} T^{3}+0.0005 T^{2}-0.2615 T & 20^{\circ} C \leq T \leq 800^{\circ} C \\
& +62.653 & \\
& \eta_{T}=0.000138 T^{2}-0.085468 T+19.212 & 350^{\circ} C \leq T \leq 800^{\circ} C
\end{array}
$$

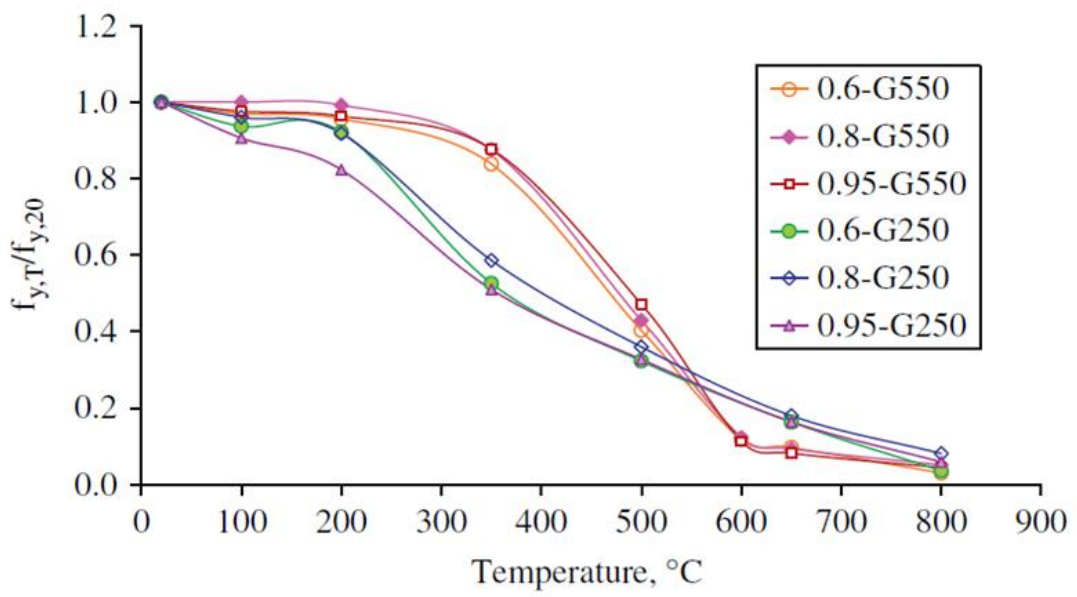

Figure 2.9. Yield strength reduction factors based on the $0.2 \%$ proof stress (Ranawaka 
and Mahendran, 2009).

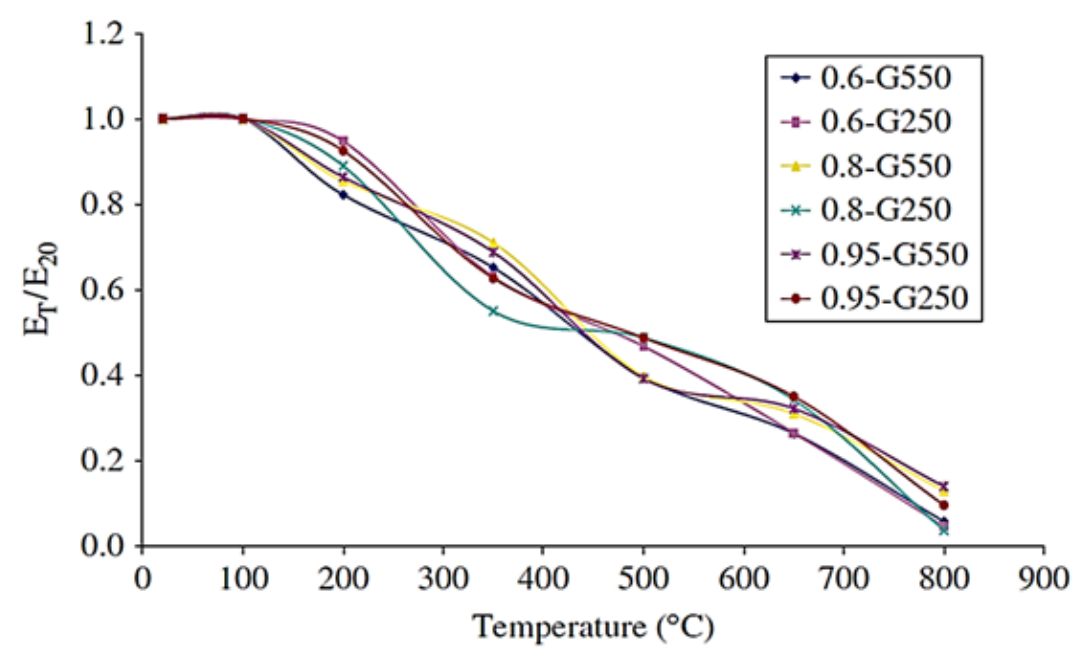

Figure 2.10. Elastic modulus reduction factors (Ranawaka and Mahendran, 2009).

Kankanamge and Mahendran (2011) performed an experimental study to investigate the elevated temperature mechanical properties of low (G250) and high strength steels (G450) with two thicknesses. This investigation is very similar to the one presented by Ranawaka and Mahendran (2009) in terms of test set-up and test methodology. Also some of the most relevant conclusions of this study validate the ones reported in the study presented by Ranawaka and Mahendran (2009). Further new improved equations to predict the yield strength and elastic modulus reduction factors and stress-strain curves using the Ramberg and Osgood (1943) formulation are presented. In this study one of the more interesting observations is related with the significant scatter that exists in terms of yield strength and elastic modulus reduction factors, when all the available results are compared. In Figure 2.11 and Figure 2.12 the obtained results by Kankanamge and Mahendran (2011) for the yield strength and elastic modulus reduction factors, respectively, are presented and compared with other researchers.

Wei and Jihong (2012) reported an experimental study of the material properties of the G550 cold-formed steel with $1 \mathrm{~mm}$ thickness at elevated temperatures. In this research both steady and transient state test methods were used and the results were then compared. It was found that the steady state test method is not equivalent to the transient state test method. Regarding the elastic modulus reduction factors it is clear that the ones obtained using the transient state test method are clearly lower than those obtained using the steady state test method. Regarding the yield strength $(0.2 \%$ proof stress) reduction factors according to the transient state test method are slightly lower than those according to the steady state test method from $300^{\circ} \mathrm{C}$ to $500^{\circ} \mathrm{C}$. However, beyond $500^{\circ} \mathrm{C}$ the difference between the reduction factors increase significantly, this means that the reduction factors according to the transient state test method are significantly higher than the ones according to the steady state test method. Finally, the stress-strain curves 
were compared with the stress-strain model presented in the EN 1993-1-2:2005 (2005). The results show that the stress-strain curves are quite different.

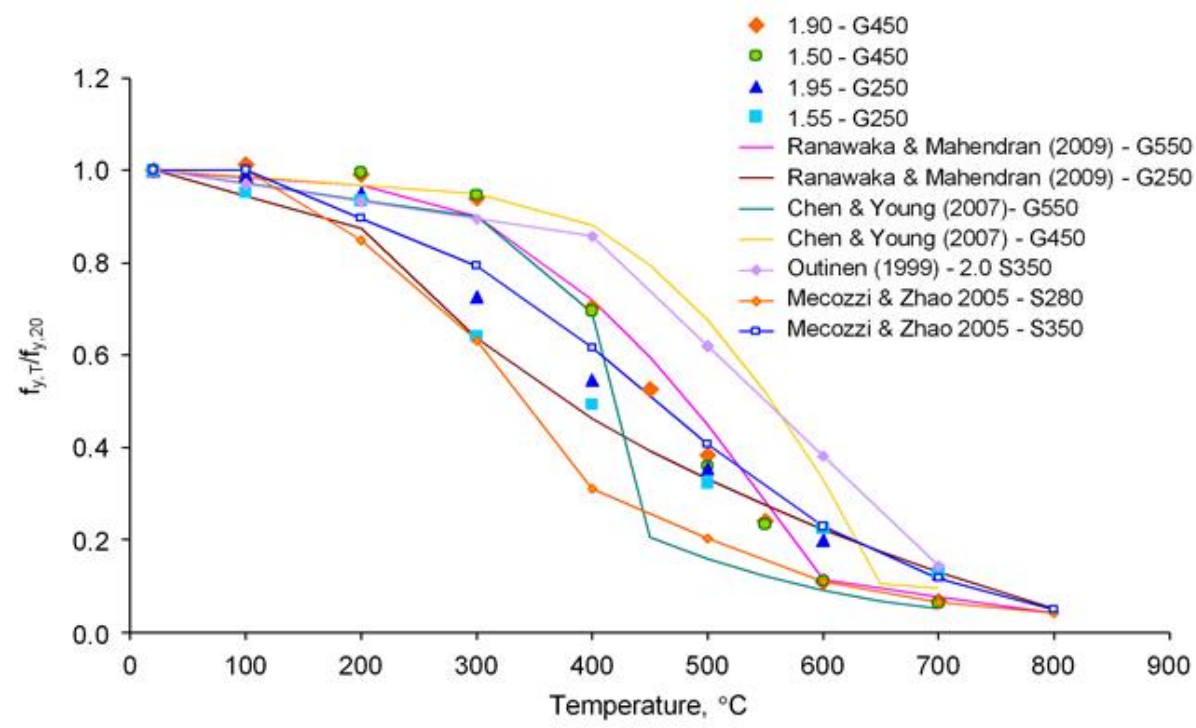

Figure 2.11. Comparison of yield strength reduction factors with those obtained by other researchers (Kankanamge and Mahendran, 2011).

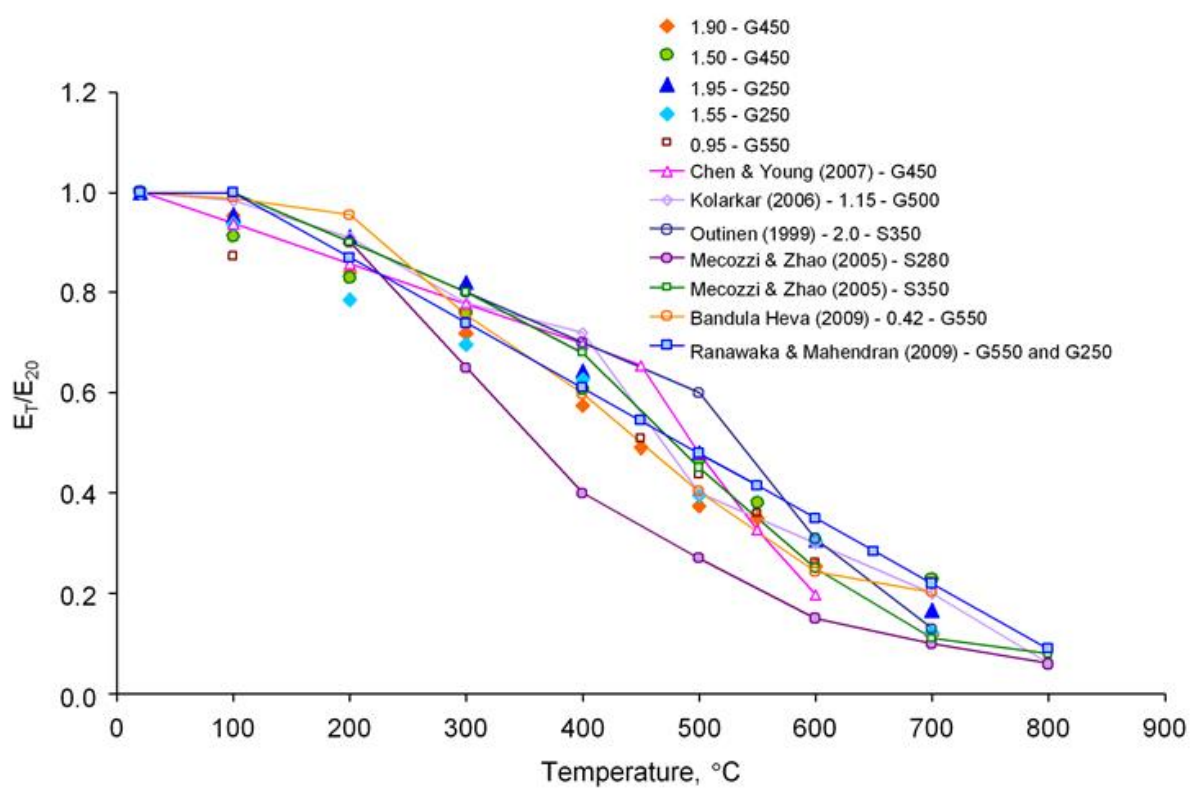

Figure 2.12. Comparison of elastic modulus reduction factors with those obtained by other researchers (Kankanamge and Mahendran, 2011). 


\subsubsection{Thermal properties of cold-formed steel}

Thermal properties such as thermal conductivity $\left(\lambda_{a}\right)$, specific heat $\left(c_{a}\right)$, coefficient of thermal expansion $\left(\varepsilon_{\mathrm{th}}\right)$, and emissivity $(\varepsilon)$ govern heat transfer and thermal deformations. The temperature rise for a steel member depends on its thermal conductivity and specific heat. The thermal expansion controls the thermal strain field of structural members and depending on the boundary conditions of that structural member it may also control the magnitude of thermal deformations. Hence these properties are very important, especially nowadays, for numerical applications using the finite element method. The accuracy of the developed numerical models depends on the input data available, namely predictive models regarding thermal properties. However, research on this subject performed by the civil engineering community is still scarce.

Thermal conductivity $\left(\lambda_{a}\right)[\mathrm{W} / \mathrm{mK}]$ is defined as the amount of heat flux that would pass through a certain material from its warmer surface to its colder surface, per unit of time and per unit of surface area, depending on the temperature gradient over the material.

Specific heat $\left(\mathrm{c}_{\mathrm{a}}\right)\left[\mathrm{J} / \mathrm{kgK}\right.$ and $\left.\mathrm{J} / \mathrm{kg}^{\circ} \mathrm{C}\right]$ is an intensive property which means that it independent of the mass of a substance. Specific heat is defined as the amount of heat per unit mass required to raise the temperature of a substance by one degree Celsius.

Coefficient of thermal elongation $\left(\varepsilon_{\mathrm{th}}\right)\left[\mathrm{mm} / \mathrm{mm} /{ }^{\circ} \mathrm{C}\right]$ is defined as the increase or elongation in length occurring in a member per unit increase in temperature.

Emissivity $(\varepsilon)$ [-- of a material is defined as the ratio of energy radiated to energy radiated by a black body at the same temperature.

The EN 1993-1-2 (2005) presents the thermal properties of steel used in structural fire design. The relative thermal elongation of steel $\Delta l / l$ should be determined from the following (Equation 2.10):

$$
\begin{array}{cc}
\frac{\Delta l}{l}=1.2 \times 10^{-5} \theta_{a}+0.4 \times 10^{-8} \theta_{a}^{2}-2.416 \times 10^{-4} & 20^{\circ} C \leq \theta_{a}<750^{\circ} C \\
\frac{\Delta l}{l}=1.1 \times 10^{-2} & 750^{\circ} C \leq \theta_{a} \leq 860^{\circ} C \\
\frac{\Delta l}{l}=2 \times 10^{-5} \theta_{a}-6.2 \times 10^{-3} & 860^{\circ} C<\theta_{a} \leq 1200^{\circ} C
\end{array}
$$


Where:
$l \quad$ is the length at $20^{\circ} \mathrm{C}$;
$\Delta l \quad$ is the temperature induced elongation;
$\theta_{a} \quad$ is the steel temperature $\left[{ }^{\circ} \mathrm{C}\right]$.

Some experimental tests have already been conducted by some researchers to assess the relative thermal elongation of some steels, namely structural steel S355 (hot-rolled) and G450 (coldformed). In Figure 2.13 the obtained results by Outinen et al. (2002) and Chen and Young (2007) are presented and compared with the predictions presented in the EN 1993-1-2 (2005) and BS 5950-8 (1990). Some design standards such as AS 4100 (1998) and AISC-Appendix 4 (2010) simply ignore the temperature dependence of the thermal elongation.

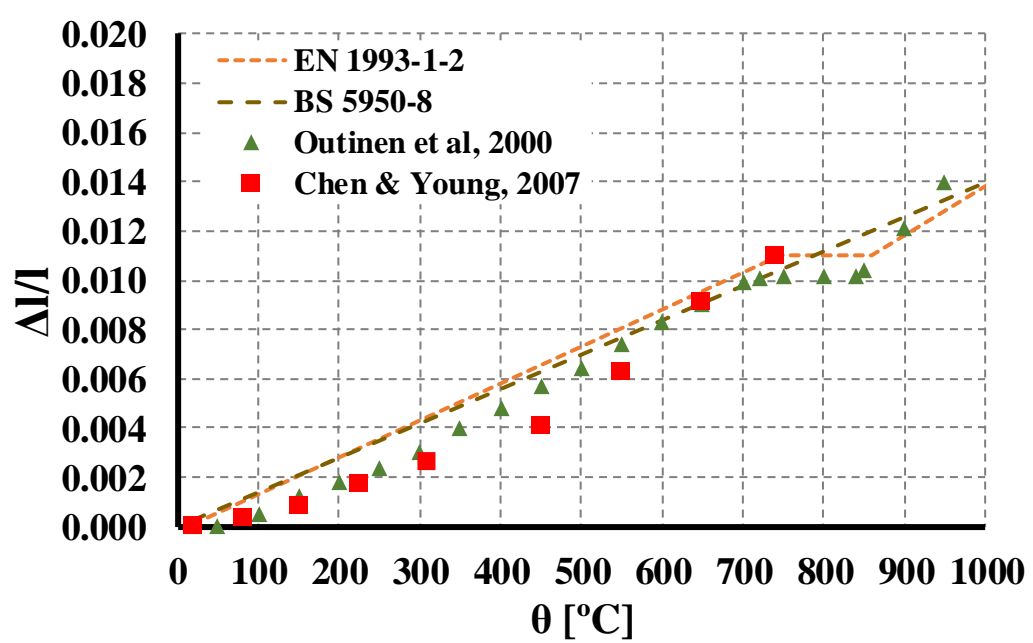

Figure 2.13. Relative thermal elongation of steel as a function of temperature.

The specific heat of steel, $c_{a}[\mathrm{~J} / \mathrm{kgK}]$, according to the EN 1993-1-2 (2005) is defined as follows (Equations 2.11):

$$
\begin{array}{cr}
c_{a}=425+7.73 \times 10^{-1} \theta_{a}-1.69 \times 10^{-3} \theta_{a}^{2}+2.22 \times 10^{-6} \theta_{a}^{3} & 20^{\circ} C \leq \theta_{a}<600^{\circ} C \\
c_{a}=666+\frac{13002}{738-\theta_{a}} & 600^{\circ} C \leq \theta_{a}<735^{\circ} \mathrm{o} C \\
c_{a}=545+\frac{17820}{\theta_{a}-731} & 735^{\circ} \mathrm{o} C \theta_{a}<900^{\circ} \mathrm{o} C
\end{array}
$$


$c_{a}=650$

$900^{\circ} \mathrm{C} \leq \theta_{a} \leq 1200^{\circ} \mathrm{C}$

The thermal conductivity of steel, $\lambda_{a}[\mathrm{~W} / \mathrm{mK}]$, according to the EN 1993-1-2 (2005) is defined as follows (Equations 2.12):

$$
\begin{array}{cc}
\lambda_{a}=54-3.33 \times 10^{-2} \theta_{a} & 20^{\circ} C \leq \theta_{a}<800^{\circ} C \\
\lambda_{a}=27.3 & 800^{\circ} C \leq \theta_{a}<1200^{\circ} \mathrm{C} C
\end{array}
$$

In Figure 2.14 both thermal conductivity and specific heat models are presented.
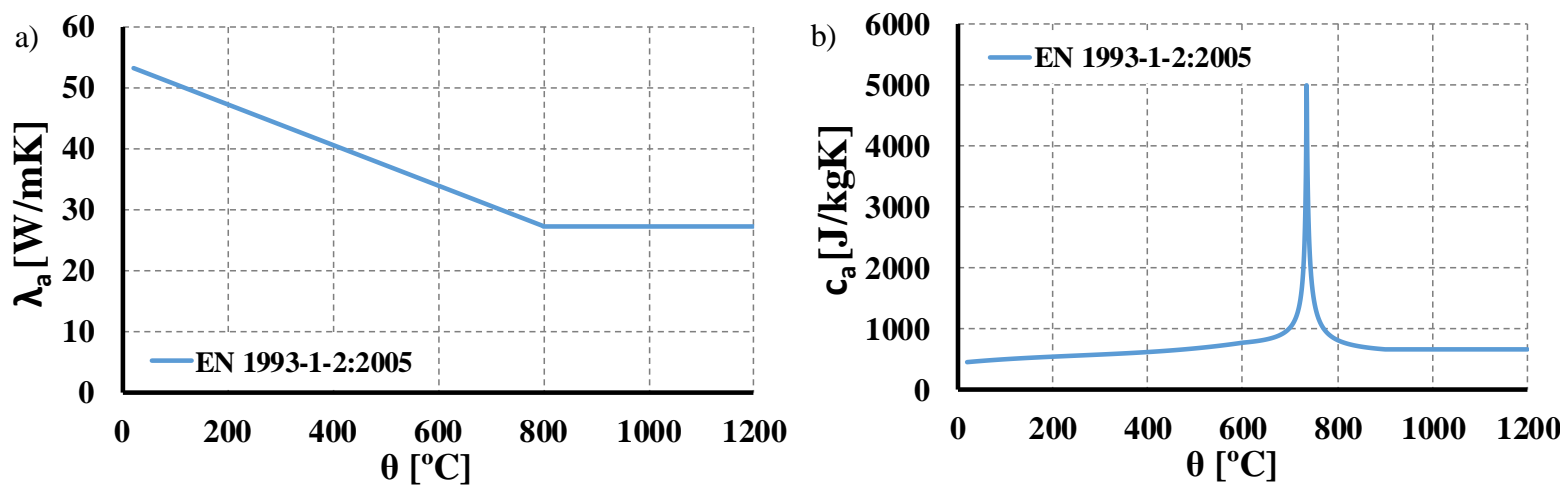

Figure 2.14. a) Thermal conductivity of steel. b) Specific heat of steel. (EN 1993-1-2, 2005).

Thermal properties such as thermal conductivity, thermal diffusivity and specific heat may be determined using the Transient Plane Source method (He, 2005; Zhang, 2014; Kodur, 2013; Gustavsson, 1994). The Transient Plane Source equipment is nowadays widely used to measure thermal conductivity of bulk and slab specimens. Among the existing techniques to determine thermal conductivity, such as hot wire and laser flash, the Hot Disk (Hot Disk AB, 2014) technique is probably the faster and more accurate thermal conductivity technique. This subject will be further discussed in the chapter concerning the experimental tests on thermal properties of the S280GD+Z steel tested in the scope of this research work.

\subsection{Residual Stresses}

Residual stresses are a result of manufacturing processes. For hot-rolled steels residual stresses result from uneven air cooling after hot-rolling or welding (assumed to be uniform across the thickness of the member - membrane type residual stresses) whereas for cold-formed steels the residual stresses are mainly of flexural or through thickness variation and result from the cold- 
forming process and coiling and uncoiling process (Yu, 2010). To measure residual stresses destructive and non-destructive methods are used, namely strip sectioning, saw cutting method and the Electrical Discharge Machining (EDM) (Weng et al., 1990).

Schafer and Peköz (1998a) reported a study on the characterization of residual stresses. Based on collected data and experiments simple rules were presented so they could be used as input in computational models. Residuals stresses in cold-formed steel members are dominated by a flexural or through thickness variation which leads to early yielding of cold-formed steel plates. Since it is not possible to measure the through thickness residual stress variation a simplification was adopted. It was assumed that the residual stresses are the summation of two types, namely flexural and membrane (Figure 2.15). It was found that the membrane residual stresses are significantly lower than the flexural residual stresses. Hence membrane residual stresses can be ignored especially in press-braked members, since membrane residual stresses are more prevalent in rolled-formed members. Based on this investigation two residual stress models for rolled-formed and press-braked members are presented in Figure 2.16.

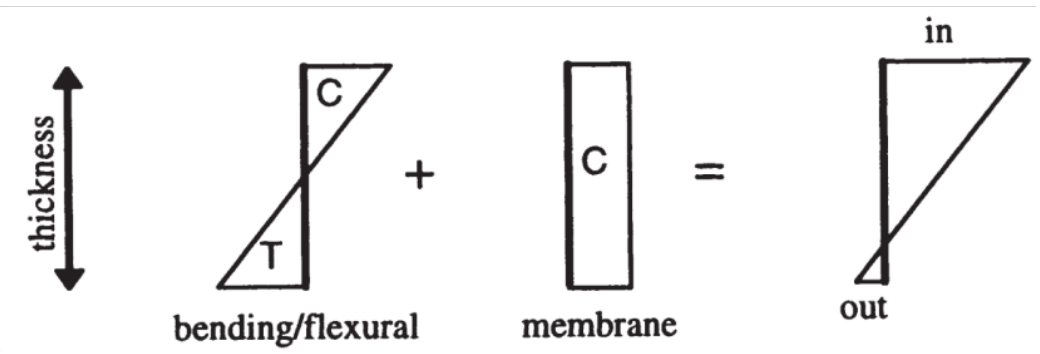

Figure 2.15. Definition of flexural and membrane residual stress (Schafer and Peköz, 1998).

a)

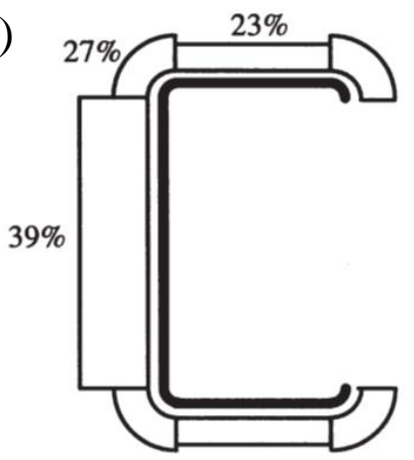

b)

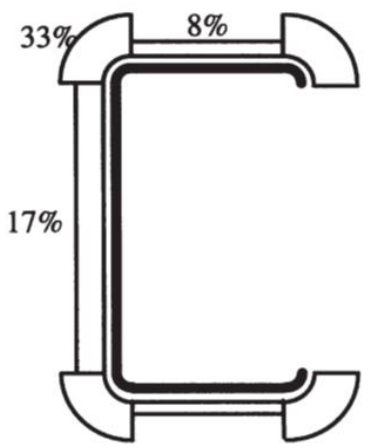

Figure 2.16. Average bending residual stress as \% fy (Schafer and Peköz, 1998). a) Rollformed. b) Press-braked.

Quach et al. (2004) presented an analytical solution and finite element simulation for the residual stresses that arise from the coiling-uncoiling process experienced by steel sheets before 
cold-forming. It was found that through-thickness variations of residual stresses are non-linear and that the magnitude of the residual stresses depend on the coiling radius and yield strength of steel.

Quach et al. (2006) presented a finite element-based method for predicting the residual stresses in press-braked thin-walled sections considering the effects of coiling and uncoiling of the steel sheet. The finite element code ABAQUS was used and the finite element model was validated against the experiments conducted by Weng and Peköz (1990), in press-braked channel sections, and against the experiments conducted by Weng and White (1990) on cold-bent thick plates. The developed finite element model agrees well with the laboratory measurements. It was found that the maximum residual stresses in a press-braked section occur in the corner region and away from the surfaces. Hence, measuring the surface residual stresses and assuming a linear variation across the thickness of the steel plate may underestimate residual stresses. Moreover, it was showed that residual stresses in the flat zones are highly dependent on the diameter of the coil. This may be the reason for the significant scatter in the assessment of buckling load of CFS members.

Moen et al. (2008) presented a mechanics-based prediction method for determination of initial residual stresses and effective plastic strains in CFS members. It was found that both transverse and longitudinal residual stress distributions and shapes of these distributions are nonlinear through the thickness. Also this study shows that stress and strain magnitudes increase with decreasing steel yield strength and increasing sheet thickness. In Figure 2.17 it is possible to observe the influence of sheet thickness and virgin yield stress on the longitudinal residual stress distributions in flats and corners.

With increasing temperature, the influence of residual stresses decrease (Lee, 2004 and Vila Real et al., 2004). For this reason, Lee (2004) proposed an equation to find the reduced residual stress at elevated temperatures (Equation 2.13). It was assumed that for a steel containing $0.2 \%$ of carbon the residual stresses are fully relieved at $800^{\circ} \mathrm{C}$ and that the reducing rate at different temperature levels is linear.

$$
\alpha=1.0181-0.00128 T \quad 20^{\circ} C \leq T \leq 800^{\circ} C
$$

Where

$\alpha \quad$ is the residual stress reduction factor;

$T \quad$ is the temperature. 


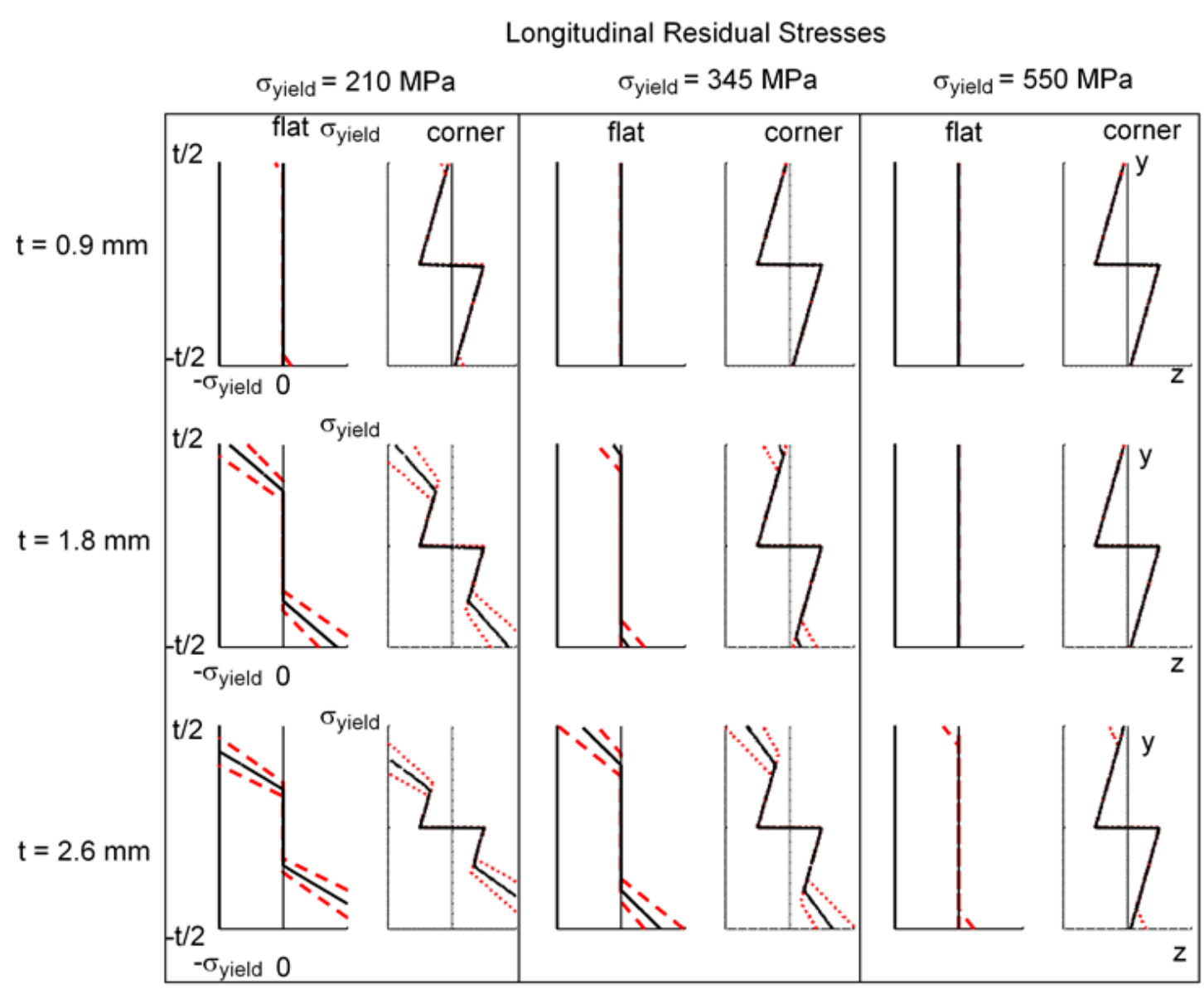

Positive stress is tension, negative stress is compression

Figure 2.17. Influence of sheet thickness and yield stress on through-thickness longitudinal residual stresses (Moen et al., 2008).

\subsection{Geometric Imperfections}

Due to fabrication process, storage, transport and installation all CFS members have some deviations from the idealised geometry. In a CFS member the geometric imperfections include bowing, warping, twisting and local deviations (Schafer and Peköz, 1998). There are two types of imperfections, namely local and global imperfection. The first influence the local or distortional buckling capacities and the second influence the buckling load of the member. One of the biggest problems in modelling CFS members using the finite element method is the definition of the initial state of the element in terms of geometric imperfections. Some relevant studies were conducted to provide more accurate information on magnitudes to be used for modelling geometric imperfections of CFS members. 
Schafer and Peköz (1998) presented a study on the characterization of geometric imperfections of CFS members. The collected data was organized in two different categories: type 1, maximum local imperfection in a stiffened element (local buckling) and type 2, maximum deviation from straightness for a lip stiffened or unstiffened flange (distortional buckling) as detailed in Figure 2.18. The defined rules of thumb present some limitations for the width to thickness ratio $(w / t)$ and for the thickness. For type 1 imperfections the ratio $(w / t)$ should be less than 200 and less than 100 for type 2 imperfections, while the thickness should be less than $3 \mathrm{~mm}$. For type 1 and type 2 imperfections the following equations were proposed (Equations 2.14).

$$
\begin{aligned}
d_{1} \approx 0.006 w \quad \text { or } \quad d_{1} & \approx 6 t e^{-2 t}\left(d_{1} \text { and } t \text { in } \mathrm{mm}\right) \\
d_{2} & \approx t
\end{aligned}
$$

Using a probabilistic treatment, it is stated that a typical member is expected to have imperfections less than $1.35\left(d_{1} / t\right)$ for type 1 and $3.44\left(d_{2} / t\right)$ for type 2 imperfections $95 \%$ of the time.
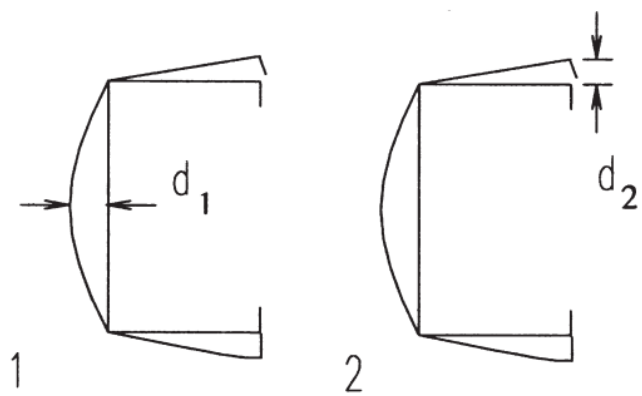

Figure 2.18. Sectional geometrical imperfections (Schafer and Peköz, 1998).

Kaitila (Kaitila, 2002) reported an imperfection sensitivity analysis of lipped channel columns. One of the goals of this investigation was to evaluate the influence of initial geometric imperfections on the modelled behaviour of columns. It was observed a clear relation between the magnitude of initial imperfections and the ultimate load as it can be observed in Figure 2.19. In this case the ultimate loads were determined considering an initial local imperfection of $h / 200$ and different global imperfection values ranging from $L / 1000$ up to $L / 400$. 


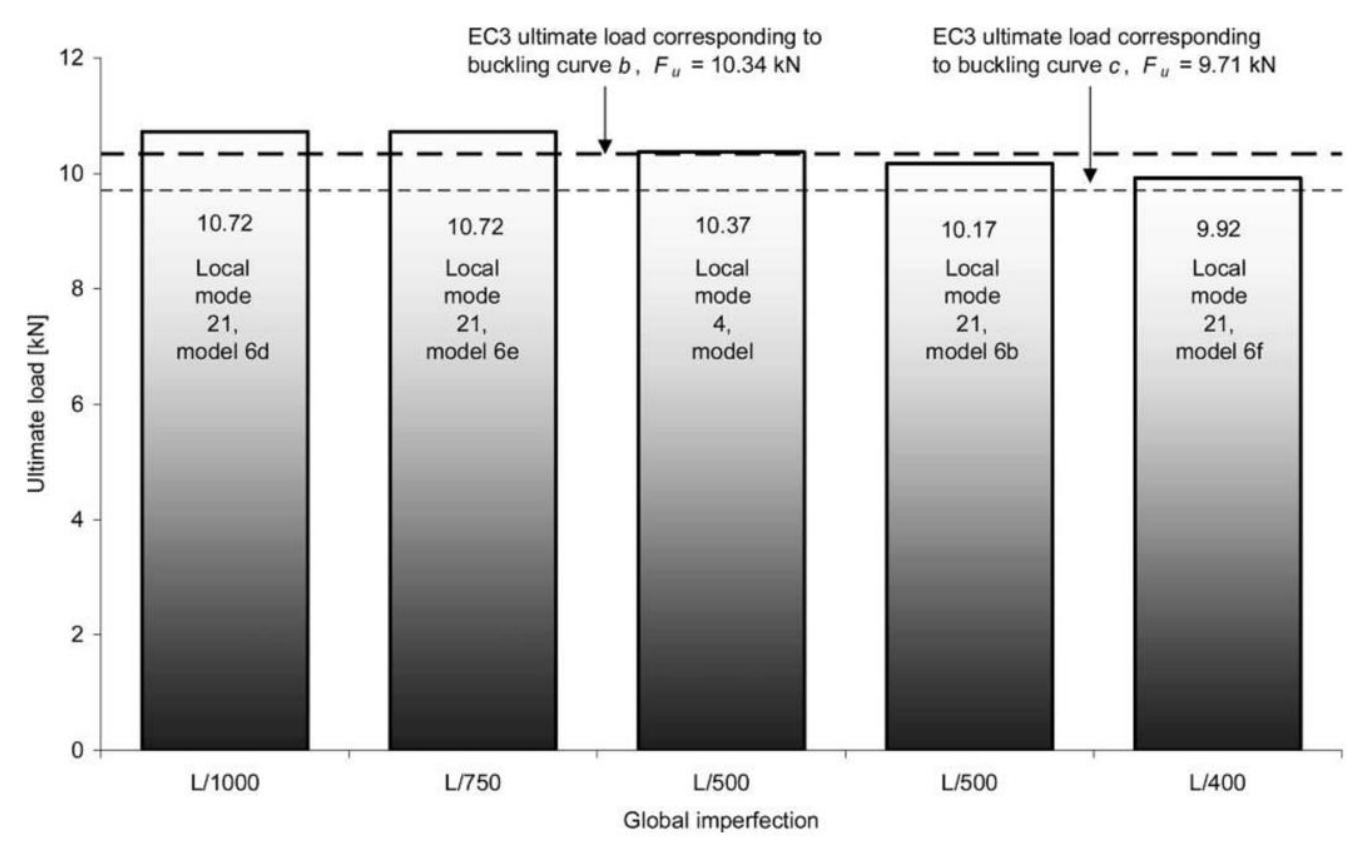

Figure 2.19. Ultimate loads for FE-models with local imperfection $\mathrm{h} / 200$ and different global imperfection values (Kaitila, 2002).

Feng et al. (2004) conducted a research to evaluate the sensitivity of column failure strength to initial imperfections. In this investigation the following values of $\mathrm{h} / 200, \mathrm{t}, 10 \% \mathrm{t}, 50 \% \mathrm{t}$ were used for local imperfections whereas for global buckling values of L/1000 and L/500 were used. It was found that for short columns where the predominant failure mode is local buckling the initial local buckling imperfection has significant influence on the ultimate strength. For long columns the influence of local imperfection on the ultimate strength was negligible. On the other hand, global imperfections have little influence on short columns and great influence on long columns.

Ranawaka and Mahendran (2010) using a validated numerical model tested the influence of geometric imperfections on the distortional buckling behaviour and strength of CFS columns. In this study the geometric imperfection magnitude ranged from $-1.9 \mathrm{~mm}(-2 \mathrm{t})$ to $1.9 \mathrm{~mm}(2 \mathrm{t})$ at $0.25 \mathrm{t}$ intervals and two temperature levels were tested $\left(20^{\circ} \mathrm{C}\right.$ and $\left.500^{\circ} \mathrm{C}\right)$. It was found that initial geometric imperfection magnitude significantly influences the ultimate load as it can be observed in Figure 2.20. Increasing the geometric imperfection to $2 t$ lead to a reduction on the ultimate load of about 20 to $30 \%$. Also it was found that the influence of the imperfection direction is very small $(<3 \%)$. 


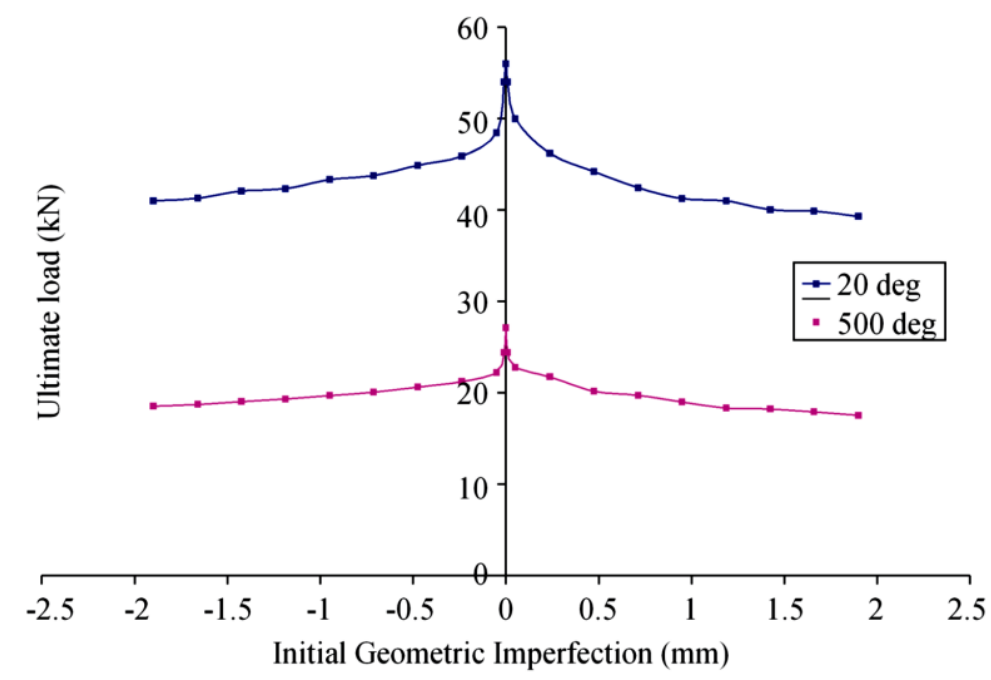

Figure 2.20. Effect of initial geometric imperfection on failure loads (Ranawaka and Mahendran, 2010)

Regarding overall sinusoidal imperfection (global imperfection), which is expressed in terms of member length, it is common to use the standard value of L/1000 (Dubina et al, 2012).

Finally, it is worth mentioning that some researchers had the possibility to measure the geometric imperfections (Ranawaka, 2006; Sivakumaran and Abdel Rahman, 1998; Dubina and Ungureanu, 2002) however most researchers had to use standard values since measurement of local imperfection require accurate instrumentation.

\subsection{Cold-Formed Steel Columns at Ambient Temperature}

\subsubsection{General buckling modes}

\subsubsection{Local buckling}

Local buckling is a sectional instability mode because it affects shape and resistance of member cross-section (Dubina et al., 2012). Local buckling is characterized by the deflection of individual plate elements without changing the position of longitudinal edges of compression members and is particularly prevalent in members with large width to thickness ratios. The typical configuration of the failure mode is presented in Figure 2.21. The half wavelength of local buckling is usually the shortest among the general buckling modes. 

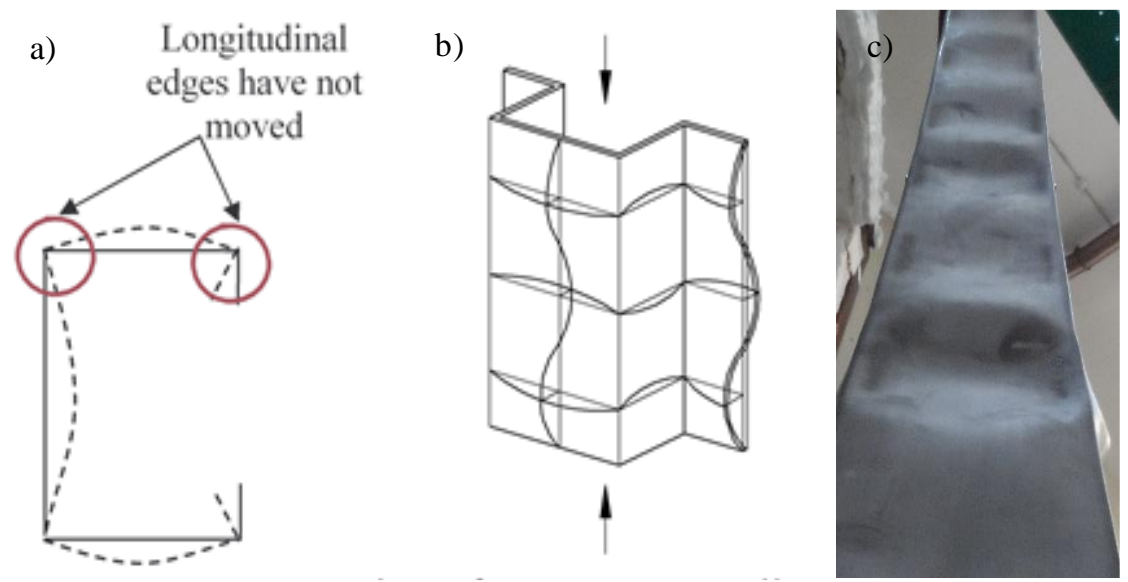

Figure 2.21. a) Local buckling of compression members (Ranawaka, 2006). b) Local buckling of component walls of a cold-formed steel section (Dubina et al, 2012). c) Experimental test.

\subsubsection{Distortional buckling}

Considering a lipped channel, distortional buckling involves the rotation of each flange and lip about the flange-web junction, hence distortional buckling of compression lipped channel members is controlled by the rotational stiffness at the web-flange junction (Figure 2.22). Distortional buckling depends on the width of the flanges. For members with narrow flanges failure may be due to local buckling since the web is much slender and buckles first, while for members with wide flanges distortional buckling may be the predominant failure mode (wider flanges leads to lower values of rotational stiffness at the web-flange junction). The half wavelength of distortional buckling is usually in between that of local buckling and flexural buckling.

a)

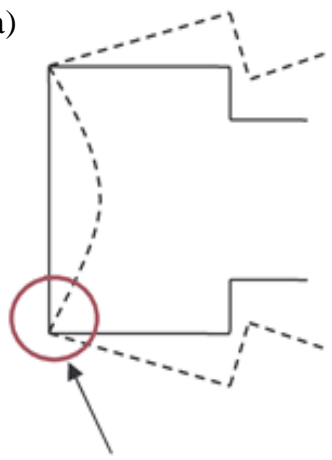

Rotation of flange at flangeweb junction b)

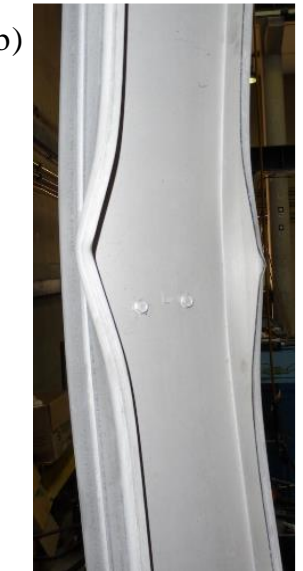

Figure 2.22 Distortional buckling of compression members. a) (Ranawaka, 2006). b) Experimental test. 


\subsubsection{Global buckling}

Global buckling is a term used to describe a buckling mode where the member deforms without any distortions in its cross-sectional shape, this means that any given cross-section moves as a rigid body. Global buckling includes flexural (Euler), torsional and flexural torsional buckling for columns. Flexural buckling is the deflection originated by bending and commonly associated with long columns (Figure 2.23a). Torsional buckling is characteristic of doublysymmetric columns with very slender cross-sectional elements and occurs due to rotation of the cross-section about the longitudinal axis (about the shear centre axis of the member) (Figure 2.23b). Flexural-torsional buckling is the summation of individual flexural and torsional buckling modes, hence torsional and flexural deformations occur along the axis of the member. This type of buckling is particularly prevalent in long columns with only one axis of symmetry (Figure 2.23c). The half wavelength of the global buckling mode is the largest among the buckling modes. Finally, it is worth mentioning that sectional modes (local and distortional buckling) can interact with each other as well as with global buckling (Dubina et al, 2012).

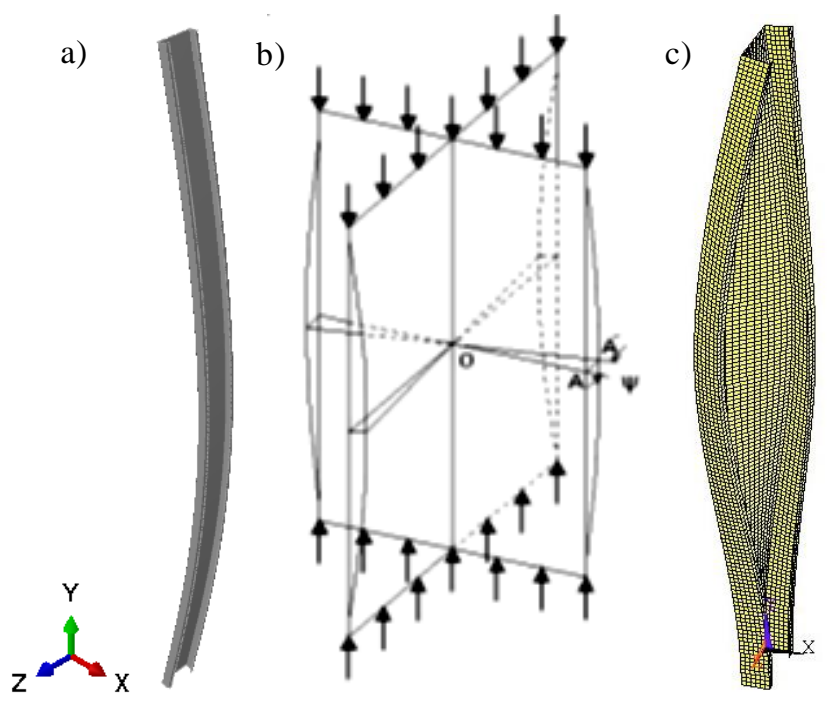

Figure 2.23 Global buckling modes. a) Flexural buckling. b) Torsional buckling. c) Flexuraltorsional buckling (Dubina et al, 2012).

\subsubsection{Previous research on cold-formed steel columns at ambient temperature}

There have been some significant developments in cold-formed steel structures over the past few decades, mainly due to improving technology of manufacture (higher quality steels, more complex section shapes, improved forming technology) and corrosion protection. This leads to greater competitiveness of this structural solution which has been translated into an increasing market share throughout the world. As a consequence, the demand for improved design 
procedures increases. In the past few decades researchers have been focused on the behaviour of cold-formed steel structures. Regarding the behaviour of CFS columns, research has been focused on open sections, such as plain and lipped channels, channels with simple and complex edge stiffeners, with and without holes, and angles. Regarding experimental research relevant studies were conducted in order to understand the behaviour of CFS columns with different cross-section shapes, different support conditions, namely pin-ended and fully fixed so that both lower and upper bounds for the strength of the tested columns could be determined, and different lengths in order to study different buckling modes. Based on the available experimental results several numerical studies using the Finite Element Method (FEM) were undertaken and after validation additional parametric studies. In the building construction industry built-up cross-sections are widely used since a built-up section can span more distance, present a higher buckling load and higher torsional stiffness. However, research on built-up CFS members is still scarce. Some of the most relevant studies conducted over the past few decades on single and built-up cross-sections are presented.

\subsubsection{Single section cold-formed steel columns}

Young and Rasmussen (Young and Rasmussen, 1998a) described an experimental study on compressed press-braked plain channels (2 cross-section geometries and different lengths ranging from $280 \mathrm{~mm}$ to $3500 \mathrm{~mm}$ ) considering pinned and fixed end-support conditions in order to define lower and upper bounds for the strength of the columns. In this investigation it was stated that for the fixed-end support condition local buckling does not induce flexural buckling, hence it was recommended that the applied load shall be assumed to act at the centroid of the effective cross-section. Based on this experimental research a finite element model was developed and validated (Young and Yan, 2002a). Using the validated finite element model, a parametric study was conducted in order to assess the influence of the cross-section geometry in the behaviour of compressed CFS plain channels. The flange width was kept constant at 80 $\mathrm{mm}$, while the width of the web varied from 100 to $200 \mathrm{~mm}$ and the thicknesses tested were 1.5 3.0 and $6 \mathrm{~mm}$. Experimental and numerical results obtained from the parametric study were compared with the available design standards, namely AISI (1996), AS/NZS 4600 (1996) and EC3 (1996) (see Section 2.4.3). It was found that the design methodologies are conservative for CFS plain channels.

Tests on compressed lipped channels between fixed ends were also conducted by Young and Rasmussen (1998b). The drawn conclusions were similar to the ones presented in the study performed on plain channels (Young and Rasmussen, 1998a). Regarding the end-supports it was stated that supports with rotational restraint above $3 \mathrm{EI}_{\mathrm{y}} / \mathrm{L}$ should be considered as fixed, 
since in practice columns have some degree of rotational restraint at the ends. Using the results provided in the experimental research conducted by Young and Rasmussen (1998b) a numerical investigation was conducted by Young and Yan (2002b). After validation of the finite element model an extensive parametric study was undertaken in order to assess the influence of the cross-section geometry in the behaviour of compressed lipped channel CFS columns. In the parametric study the flange width was $80 \mathrm{~mm}$, the lip length was $15 \mathrm{~mm}$, the width of the web ranged from 100 to $200 \mathrm{~mm}$ and the thicknesses tested were $1.5,3$ and $6 \mathrm{~mm}$. The tested lengths ranged from 500 to $3000 \mathrm{~mm}$. The ultimate strengths obtained in the parametric study were then compared with the ones obtained using the design standards AISI (1996), AS/NZS 4600 (1996) and EC3 (1996). It was observed that the design standards conservatively predicted the ultimate strength of fixed-ended CFS lipped channels. Some details of the experimental test setup, as well as the comparison between the observed failure modes in tests and in numerical simulations for the previously mentioned research works is presented in Figure 2.24.
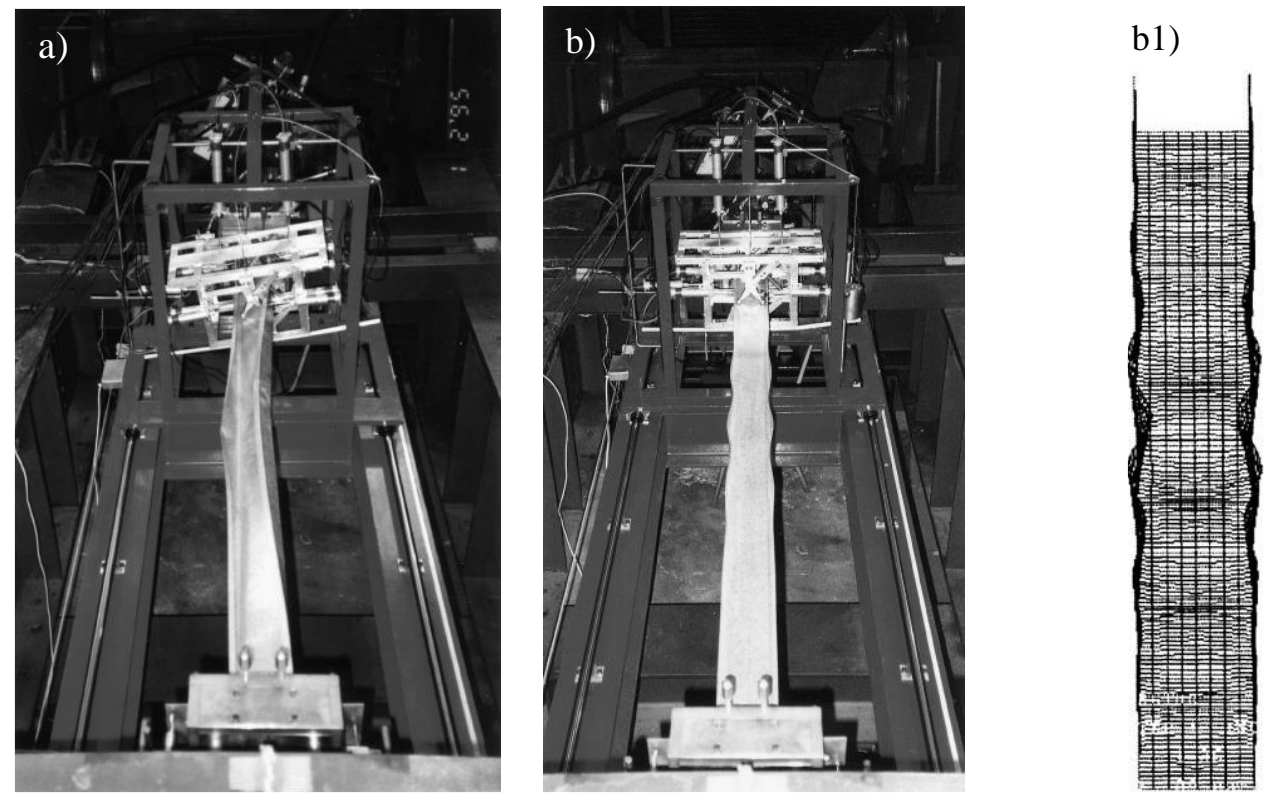

Figure 2.24 a) Failure of fixed-ended lipped channel. b) Failure of fixed-ended plain channel (Young and Rasmussen, 1999). b1) Deformed shape for the finite element model (Young and Yan, 2002a).

Kesti and Davies (1999) presented a study into the local and distortional buckling of short columns. The compression capacity of short columns with open cross-sections, namely $\mathrm{C}$, hat and rack upright, is determined by combining the effective width of plane elements due to local buckling and the effective stiffener thickness due to distortional buckling. The applicability of Eurocode 3 (EC3) (1996) to predict the compression capacity of short fixed-ended columns 
with open cross-sections was assessed. Different methods, namely presented in the AS/NZS 4600 (1996), EC3 (1996) and Generalized Beam Theory (GBT) were used to determine the elastic distortional buckling stress in compression. It was found that the influence of boundary conditions is significant for short columns. For instance, for a column length of $500 \mathrm{~mm}$ the elastic distortional buckling stress of a fixed-ended column is 2.3 times higher than of the pinended column. Regarding the applicability of EC3 (1996) it was found that the method gives conservative compression resistance values.

Popovic et al. (1999, 2001) and Dhanalakshmi and Shanmugam (2001) presented an experimental research work on compression stub CFS equal angle columns. Fixed-ended and pin-ended conditions as well as the influence of eccentric loading were assessed in these studies. The comparison between the experimental results and the predictions provided by AS/NZS 4600 (1996) and AS 4100 (1996) showed that the design standards are conservative. It was found that the additional bending moment about the minor axis due to an eccentricity of L/1000, recommended by the AS/NZS 4600 (1996) for the design of concentrically loaded angles, does not need to be considered. In the research presented by Dhanalakshmi and Shanmugam (2001) a finite element model was also developed and validated against the experimental results and based on this model a parametric study was also undertaken. Tests showed that eccentric load lead to a significant reduction in the ultimate strength and that with increasing slenderness ratios the influence of eccentric loading in the ultimate strength decreases (Dhanalakshmi and Shanmugam 2001). Figure 2.25 shows the final deformed shapes of the tested specimens in both investigations as well as the finite element model developed in the study conducted by Dhanalakshmi and Shanmugam (2001).
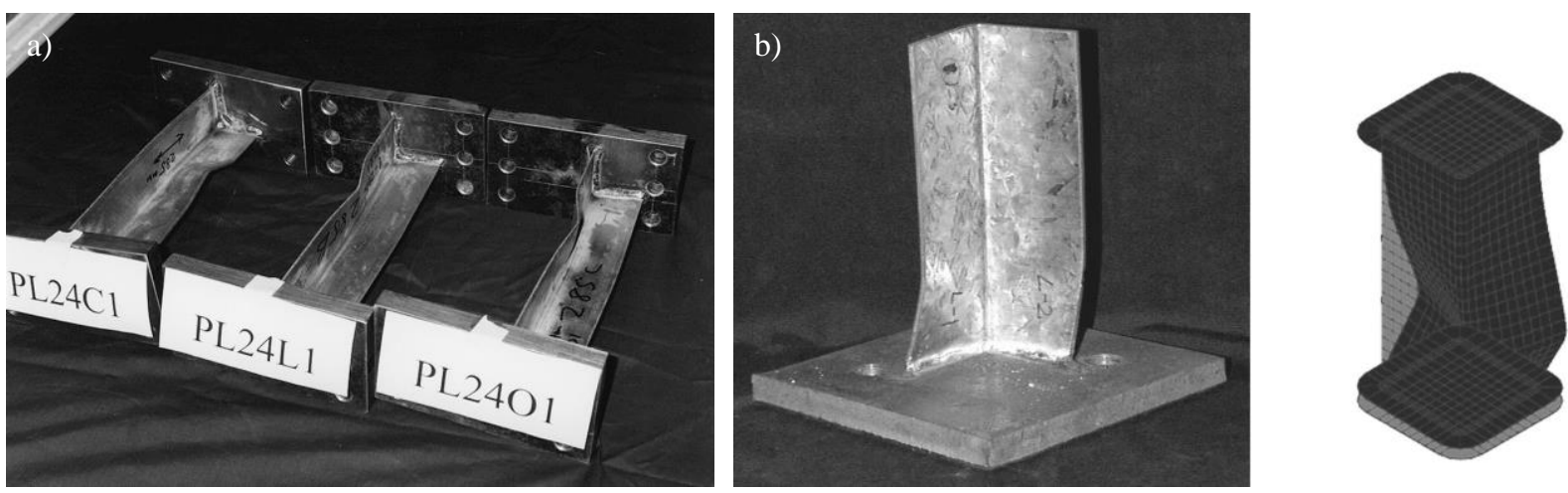

Figure 2.25 a) Local buckling of short columns (Popovic et al, 2001). b) Failure mode for both experimental and numerical analysis (Dhanalakshmi and Shanmugam 2001).

Young (2004a) also presented an experimental study on the behaviour of CFS unstiffened angle columns compressed between fixed ends. Different thicknesses and different lengths were 
tested and the tests conducted by Popovic et al (1999) were also considered and compared with the AS/NZS 4600 (1996) and AISI (1996). It was found that generally the design standards are very conservative, hence a modification proposal to the AS/NZS 4600 (1996) for the design of plane angle columns was presented (Equation 2.15).

$$
\begin{array}{ll}
F_{n}=\left(0.5 \lambda_{c}^{2}\right) F_{y} & \lambda_{c} \leq 1.4 \\
F_{n}=\left(0.5 / \lambda_{c}^{2}\right) F_{y} & \lambda_{c}<1.4
\end{array}
$$

Based on this study (Young, 2004a), Ellobody and Young (2005) developed and validated a finite element model to conduct additional parametric studies outside the bounds of the experimental research. Hence the influence of cross-section geometry on the strength of angle columns was studied. Different width-to-thickness ratios $(b / t)$ were tested, ranging from 85 to 5. Comparing the results of the parametric study with the design rules, AISI (1996) and AS/NZS 4600 (1996) it was found that they were conservative for angle columns with $b / t$ ratios above 25 and unconservative for angle columns with $b / t$ ratios under 15. Comparing the parametric results with the design proposal presented by Young (2004a) a good agreement was found.

An experimental (Young and Chen, 2008a) and numerical (Young and Ellobody, 2007) investigation on the behaviour of compressed CFS non-symmetric lipped channel angle sections between fixed ends was performed. The specimens used in the experimental campaign (Young and Chen, 2008) were brake-pressed from G450 and G550 structural steel, with 1.0, 1.5 and $1.9 \mathrm{~mm}$. Different lengths were tested, namely 250, 625, 1000, 1500, 2000, 2500 and $3000 \mathrm{~mm}$. Regarding the observed failure modes it was found that local buckling was predominant for short columns, whereas for intermediate and long columns the predominant buckling modes were the interaction between local and flexural-torsional and pure flexuraltorsional buckling, respectively. The finite element model developed by Young and Ellobody (2007) was validated against experimental results (Young and Chen, 2008a), and based on this validated model a parametric study was undertaken. In the parametric study different thicknesses were tested, namely $0.5,0.7,0.85,3$ and $5 \mathrm{~mm}$. The overall widths of both flanges was kept constant at 84 and $54 \mathrm{~mm}$, respectively, and the length of the lips was kept constant at $17 \mathrm{~mm}$. Comparing the results of the parametric study with the strengths predicted by the North American Specification (NAS, 2001) and with the strengths predicted using the proposed design rules presented by Young (2004a) it was observed that the NAS (2001) predictions are unconservative whereas the proposed design rules conservatively predicted the column strengths. 
Yang and Hancock (2004) conducted a series of compression tests on lipped channel columns with web and flange stiffeners to investigate local and distortional buckling and the interaction between them. Brake-pressed lipped channels with $0.42 \mathrm{~mm}$ thickness fabricated with G550 structural steel and with lengths ranging from 360 (stub columns to study local buckling) to $2000 \mathrm{~mm}(800,1300,2000 \mathrm{~mm}$, to study distortional buckling and the interaction between distortional and local buckling) were tested considering fixed ends. From the tests it was observed that the stub columns presented very high post local buckling strength. Also, it was observed that for intermediate length columns the interaction between distortional and local buckling reduced the ultimate strength. Regarding the type of failure modes three different modes were identified for stub columns, namely two flanges moving outward, two flanges moving inward and finally one moving outward while the other moved inward. For long columns two types of failure modes were identified, one with two flanges moving inward and other with the two flanges moving outward. Apparently the columns with flanges moving outward presented higher ultimate loads. Test results were also compared with the design methods presented in the North American Specification (NAS, 2001) and AS/NZS 4600 (1996). For both standards unconservative results were found and this fact was due to the inadequate consideration of the interaction between local and distortional buckling in these standards.

Yan and Young (2002) reported an experimental investigation on compressed CFS channels with complex stiffeners between fixed-ended columns and with different lengths, ranging from 500 to $3500 \mathrm{~mm}$. Lipped channels with return lips were brake-pressed from G450 structural steel with $1.5 \mathrm{~mm}$ and $1.9 \mathrm{~mm}$ thickness. In Figure 2.26 the tested cross-section and some results regarding a series of columns tested in this investigation, namely with $1.9 \mathrm{~mm}$ thickness, with a flange width of $80 \mathrm{~mm}$ and three lengths $(500,1500$ and $3000 \mathrm{~mm})$. Clearly it was observed that local buckling was the predominant failure mode for short columns, whereas for intermediate and long columns the predominant failure modes were the interaction between local and distortional buckling and the interaction between local and flexural-torsional buckling, respectively. Comparing the experimental results with the design strengths predicted by the Amercian Iron \& Steel Institute (AISI, 1996) and the AS/NZS (1996) it was found that the AISI (1996) predictions are unconservative whereas the AS/NZS 4600 (1996) predictions are conservative. 

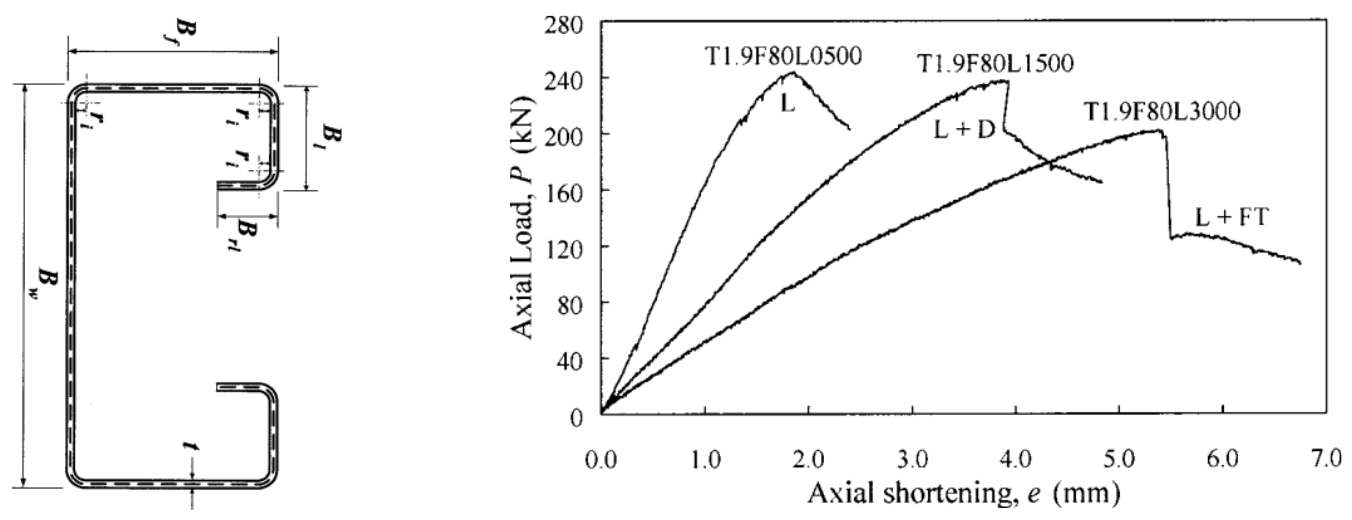

Figure 2.26 a) Configuration of the tested cross-section. b) Experimental results for three specimens with different lengths (Yan and Young (2002).

Based on the experimental results provided by the previously mentioned investigation (Yan and Young, 2002) numerical and parametric studies were undertaken (Yan and Young, 2004; Young and Yan, 2004). The finite element model was successfully calibrated against the experimental results. In the parametric study different sizes of the stiffeners were tested (Figure 2.26a). The centreline dimensions of the four sizes of complex stiffeners were 10 (return lip) $\times$ 20 (lip), $15 \times 30,20 \times 40$ and $25 \times 50 \mathrm{~mm}$, the tested thicknesses were 1 and $2 \mathrm{~mm}$ and column lengths ranging from 500 to $3500 \mathrm{~mm}$. It was found that the design strengths calculated from the AISI (1996) and AS/NZS 4600 (1996) are generally conservative for slender sections (1 $\mathrm{mm}$ thickness) with the flat flange width to thickness ratio of 57 and unconservative for sections with $2 \mathrm{~mm}$ and a flat flange width to thickness ratio of 28 .

Young (2004b) presented a study on the behaviour of channel columns with inclined edge stiffeners $\left(30^{\circ} \leq \theta \leq 150^{\circ}\right)$. Based on experimental tests (fixed-ended compression tests) a finite element model was developed and validated and a parametric study was conducted. Then determined strengths were compared with the ones obtained using the AISI (1996) and the AS/NZS 4600 (1996). Usually edge stiffeners are perpendicular to the flanges, however the available design rules can also be used for the design of inclined edge stiffeners but it seems that this extension is not entirely based on scientific facts, hence the importance of this study conducted by Young (2004). It was found that both AISI (1996) and AS/NZS 4600 (1996) conservatively predict the design strengths of CFS columns with inclined edge stiffeners.

Compression tests on lipped channels and lipped channels with intermediate stiffeners in the web and flanges were conducted by Kwon et al (2009) (Figure 2.27). The specimens were 0.6 and $0.8 \mathrm{~mm}$ thickness and were fabricated with the structural steel SGC570. Fix-end support condition was adopted in this investigation and a range of lengths was considered, namely 400, 
800,1000 and $1200 \mathrm{~mm}$. In order to study the interaction between local and overall buckling suitable cross-section geometries and lengths were adopted based on an elastic buckling analysis conducted using the elastic buckling analysis software Thin-Wall (Papangelis and Hancock, 1998) (Figure 2.28). It was observed that for stub columns local and distortional buckling occurred simultaneously, whereas for intermediate and long columns the interaction between local and global buckling was the predominant failure mode. Also it was stated that interaction between local and distortional buckling reduced significantly the ultimate strength of intermediate length columns. Consequently, a modification was proposed to the Direct Strength Method formula to determine the axial nominal axial strength.

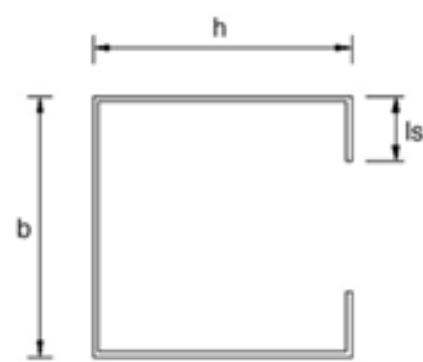

(a) Type A.

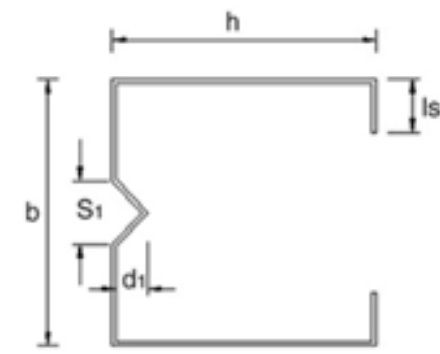

(b) Type B.

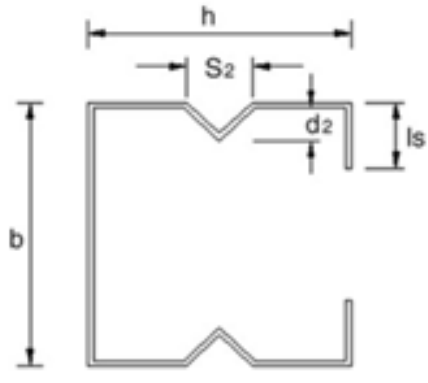

(c) Type C,

Figure 2.27 Cross-sections tested (Kwon et al, 2009).

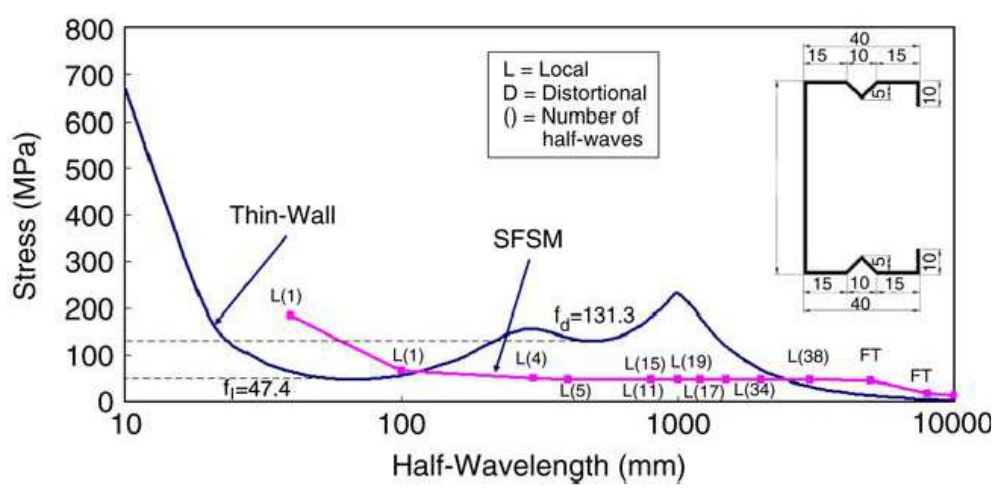

Figure 2.28 Buckling stress versus half-wavelength/column length curves for a lipped channel with intermediate stiffeners in the flanges (Kwon et al, 2009).

Also on lipped channel stub columns with intermediate stiffeners, an experimental and numerical investigation was conducted by Chen et al (2010). Specimens with $400 \mathrm{~mm}$ length and $2 \mathrm{~mm}$ thickness were brake-pressed. The observed failure mode for all tested specimens was distortional buckling. The experimental results were then compared with the Direct Strength Method (DSM) (see Section 2.4.3.1, page 2-37) considering the buckling stresses determined using the finite strip method and the finite element method. It was found that the 
design strengths calculated using the DSM, based on the buckling stresses obtained from the finite strip method, are very conservative, whereas the ones obtained using the DSM, based on the buckling stresses determined using the FEM, are in good agreement with the test results.

Camotim and Dinis (2011) and Dinis and Camotim (2011) presented numerical studies on the post-buckling behaviour of pinned channel columns undergoing local/distortional, distortional/global, local/distortional/ global buckling mode interaction. Geometries of the cross-sections and lengths were defined in order to ensure similar local, distortional and global buckling loads. The adopted magnitudes of initial geometric imperfections were $0.1 \mathrm{t}$ for local imperfection, $0.1 \mathrm{t}$ for distortional imperfection and L/1000 for global imperfection. From the GBT analysis it was found that the columns were affected by interaction between local, symmetric distortional and flexural-torsional-distortional (anti-symmetric modes), and distortional-flexural-torsional modes, respectively for the research presented by Camotim and Dinis (2011) and Dinis and Camotim (2011). The mentioned anti-symmetric distortional mode leads to a reduction on the post-critical strength of CFS columns. The imperfections leading to lower column strength are pure distortional for columns undergoing local/distortional buckling interaction and pure global for columns undergoing distortional/global or local/distortional/global interaction buckling mode.

An experimental research was conducted by Santos et al (2012) to assess the behaviour and ultimate strength of fixed-ended lipped channels undergoing local-distortional-global buckling mode interaction. The cross-section geometry and length of the tested specimens was determined after conducting buckling analyses using the code GBTUL (Bebiano et al, 2008a and 2008b) based on the Generalised Beam Theory (GBT) in order ensure similar local, distortional and global buckling loads. It was observed the occurrence of local-distortionalglobal buckling in the tests and that the interaction between distortional and global buckling was the predominant failure mode.

\subsubsection{Open and closed built-up cross-sections}

The behaviour of built-up I CFS sections and the accuracy of the design provisions presented in the North American Specification for the Design of Cold-Formed Steel Structural members (AISI, 2001) was assessed by means of experimental tests (Stone and LaBoube, 2005). The tested specimens consisted on two lipped channels (C) fastened back-to-back on the web. Different cross-section geometries were tested, as well as screw spacing $(304.8,609.6,762$, $914,1066.8)$. The length of the tested columns was $2.1 \mathrm{~m}$. In the compression tests the pinnedend support condition was adopted. The specimens experienced local buckling between the 
connections, however the predominant failure mode was the interaction between flexural and distortional buckling at mid-span (Figure 2.29). Comparing the results with the AISI specification it was found that the modified slenderness ratio (see Chapter 2.4.3.3) is applicable for built-up open sections with plate thickness of $0.89 \mathrm{~mm}$ whereas for thicker section the modified slenderness ratio is not applicable.

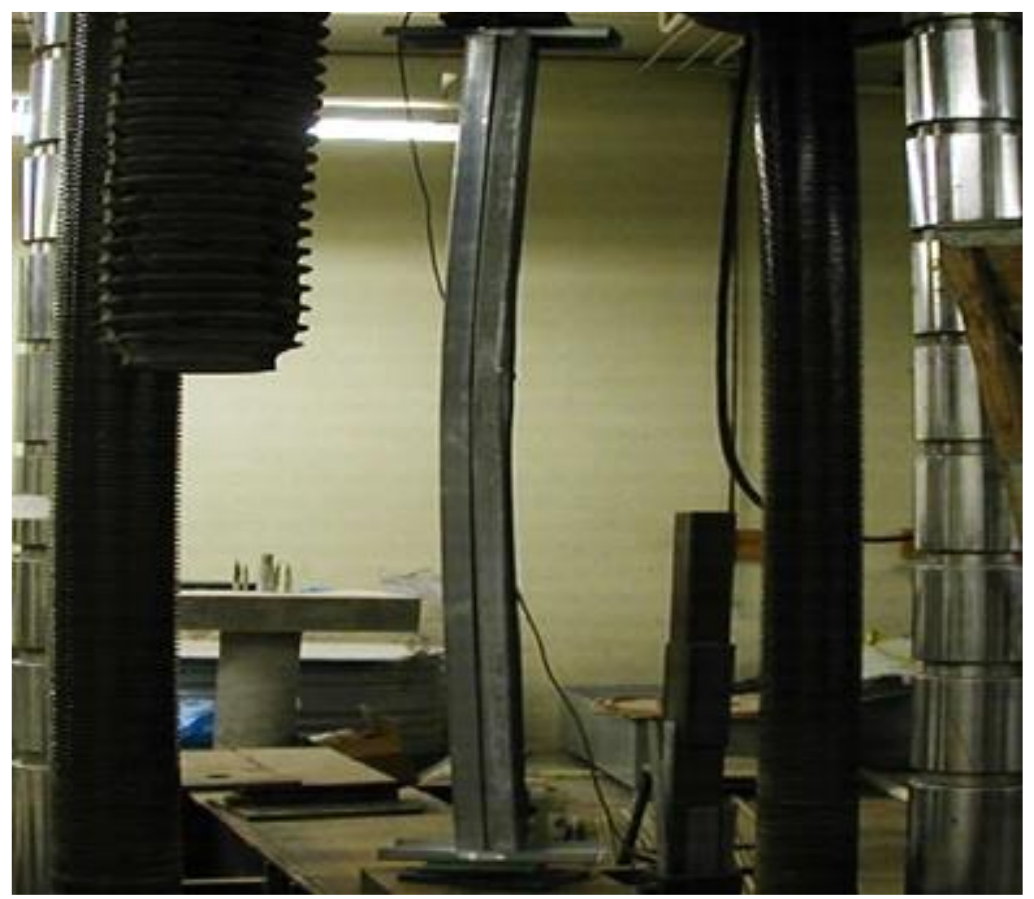

Figure 2.29 Failure observed for the open built-up I cross-section (Stone and LaBoube, 2005).

Young and Chen (2008b) reported an experimental research on compressed fixed-fixed CFS built-up closed sections with intermediate stiffeners. The sigma shaped single sections (plain channels with intermediate stiffeners) were fastened in the flanges using self-tapping screws. The single sections were fabricated from high strength steels, namely G450 and G550 with 1.5, $1.9 \mathrm{~mm}$ and $1.0 \mathrm{~mm}$ thickness, respectively. Different lengths were tested, ranging from 300 to $3000 \mathrm{~mm}$ and the screws spacing was approximately $100 \mathrm{~mm}$. Regarding the observed failure mode it was found that for short columns and thinner sections ( 1 and $1.5 \mathrm{~mm}$ ) the predominant failure mode was the interaction between local and distortional buckling, while for longer columns the predominant failure mode was the interaction between flexural and distortional buckling. For thicker sections $(1.9 \mathrm{~mm})$ distortional buckling was not observed. The applicability of the DSM to the design of built-up sections was assessed and it was found that generally it provides reliable results. 
Whittle and Ramseyer (2009) presented an experimental investigation on the behavior of compressed CFS built-up members, consisting on two lipped channels welded at the top and bottom to forma a closed box-section. Based on the experimental results it was intended to assess the accuracy of the AISI Specification, Section S1.2 (AISI, 2007) which that built-up members should be designed with a modified slenderness ratio (See Chapter 2.4.3.3) if shear forces are induced between the weld or screw connectors. Reyes and Guzmán (2011) also presented an experimental research on built-up CFS box sections and compared the experimental results with the ones determined using the modified slenderness ratio. In both investigations it was found that the modified slenderness ratio is too conservative and for that reason there is no need to consider it for thinner sections as the ones tested (1.5 to $2.54 \mathrm{~mm}$ ).

Zhang and Young (2012) reported an experimental study on compressed, between fixed-ends, CFS open built-up I cross-sections with intermediate web and edge stiffeners (sigma shaped cross-sections). Three thicknesses (0.48, 1.0 and $1.2 \mathrm{~mm})$ and six lengths $(300,800,1400,2000$, $2600,3200 \mathrm{~mm}$ ) were tested. In the experimental tests it was observed that for short columns the predominant failure mode was distortional buckling and that for intermediate and long columns the predominant failure mode was interaction between distortional and flexural buckling. The specimens with $0.48 \mathrm{~mm}$ thickness experienced as well local buckling and for longer columns (1400 to $3200 \mathrm{~mm}$ ) the interaction between local, distortional and flexural buckling was observed. For columns with $1.2 \mathrm{~mm}$ thickness and length above $2000 \mathrm{~mm}$ pure flexural buckling was observed.

Georgieva et al (2012b) and Georgieva et al (2012c) presented an experimental research on the behaviour of built-up CFS compression members. The tested columns were fabricated using two CFS Z-profiles (Figure 2.30). Two different cross-sections and two thicknesses were tested. The length of the columns was $4.45 \mathrm{~m}$. The failure mode observed was the interaction between flexural-torsional, distortional of one of the outer flanges and local buckling in the webs of the z-profiles. The experimental results were compared with design predictions EN 1993-1-1:2005 (2005), EN 1993-1-3 (2006) and NAS (2007), disregarding the fact that the studied members are out of the scope of this standards. Generally, it was found that all standards provide conservative results. Based on the experimental results a numerical study using the finite element method (FEM) was conducted. Two of the critical aspects mentioned in the paper are the initial imperfections and stiffness of connections between the two single Z-profiles.

Georgieva et al (2012a) assessed the applicability of the Direct Strength Method (DSM) to predict the axial compression capacity of innovative cross-section shapes using single CFS profiles (Figure 2.31). The built-up cross-sections were analysed using the software CUFSM 
(Li and Schafer, 2010), based on the finite strip method (FSM), in order to determine critical loads in local, distortional and overall buckling. It is worth to mention that the connections between the single profiles were modelled as rigid constraints. The predictions based on the DSM method presented good agreement with the experimental results.

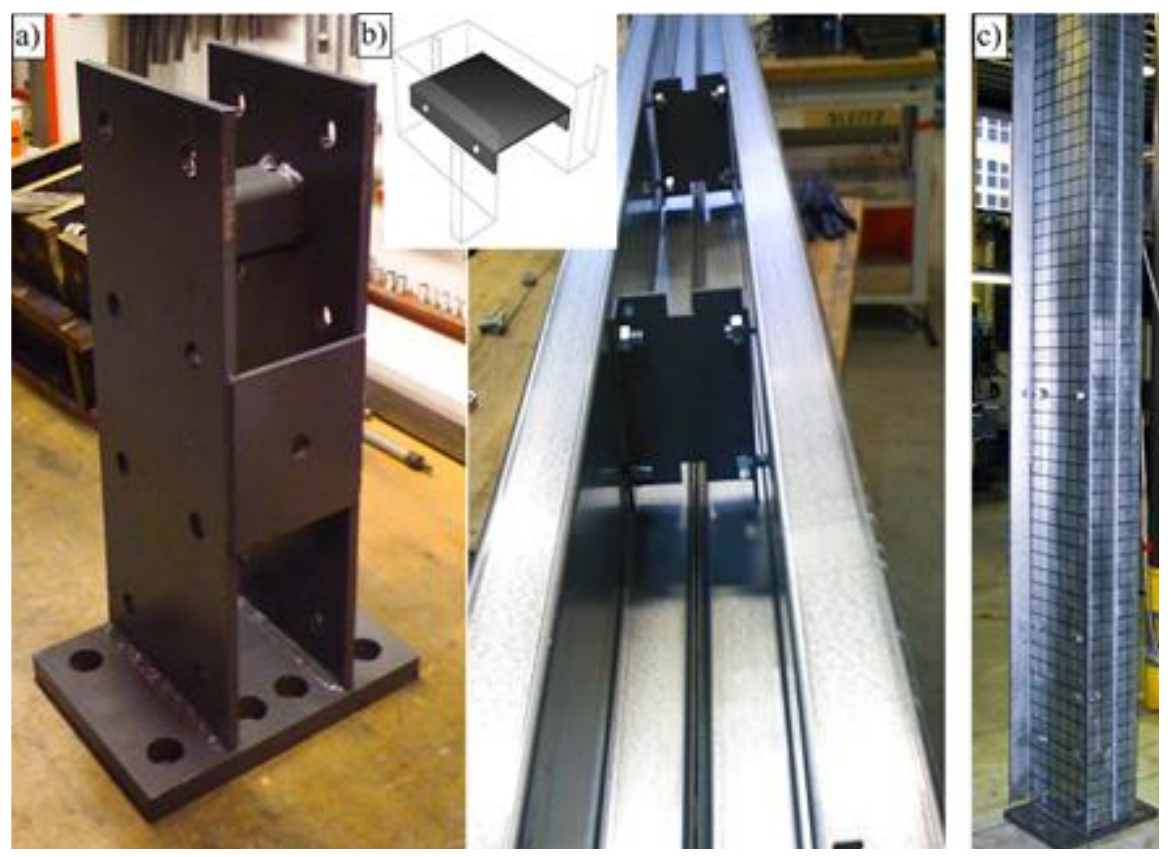

Figure 2.30 Connection pieces: a) column base. B) intermediate spacers. C) Built-up column (Georgieva et al, 2012b).

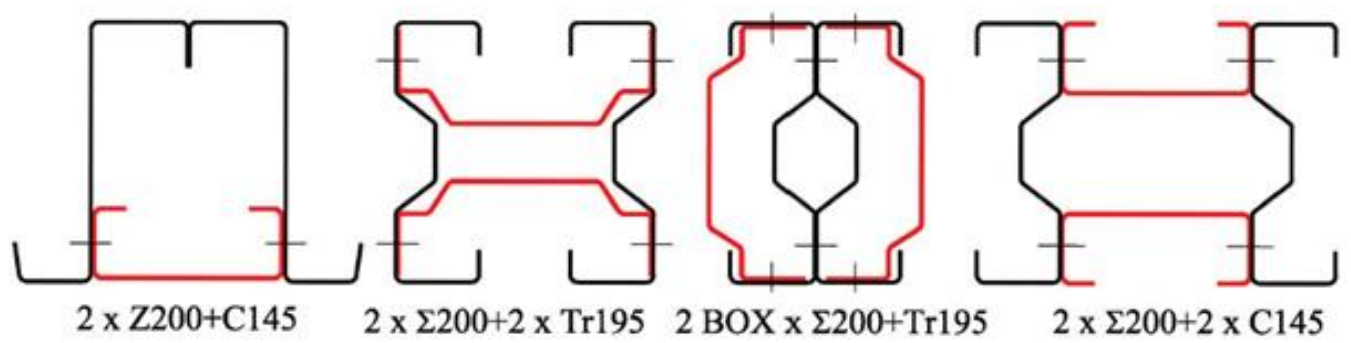

Figure 2.31 Built-up innovative cross-sections tested (Georgieva et al 2012a).

Li et al (2014) reported a series of experimental tests on CFS columns with built-up box and open (I) cross-sections subjected to axial compression. The single lipped channels (C) with web stiffeners were fabricated with structural steel G550. The thickness was $1 \mathrm{~mm}$. To form the built-up sections, the single profiles were connected at the flanges using self-drilling screws spaced from 300 or $600 \mathrm{~mm}$. Based on the experimental results a finite element model was developed and validated. Regarding the influence of spacing of the fasteners it was found that for the larger spacing $(600 \mathrm{~mm})$ a decrease on the buckling load of the column was not observed. 
Also the position of fasteners may improve the distortional buckling capacity of built-up sections since the flanges of the single sections are restrained. Finally, a new design method is proposed to assess the compression strength of built-up box and I section columns.

\subsubsection{Design rules at ambient temperature}

\subsubsection{General}

As previously mentioned cold-formed steel have some peculiar structural stability problems which are not found in thicker hot rolled steel sections. One of the first contributions for a better understanding of the local buckling mode was provided by von Karman et al in 1932 (1932). Analysing the behaviour of thin plates subjected to uniform compression it was observed that after buckling the stress diagram becomes non-uniform with stress concentration near the edges (parabolic stress pattern) as the load increases. To analyse the elastic post-buckling behaviour of the plate the "effective width" concept was introduced by von Karman et al in 1932 (1932). This method assumes uniform stress distribution over the effective width instead of nonuniform stress distribution over the entire width of the plate. Hence, the total load is carried by the effective width as illustrated in Figure 2.32.
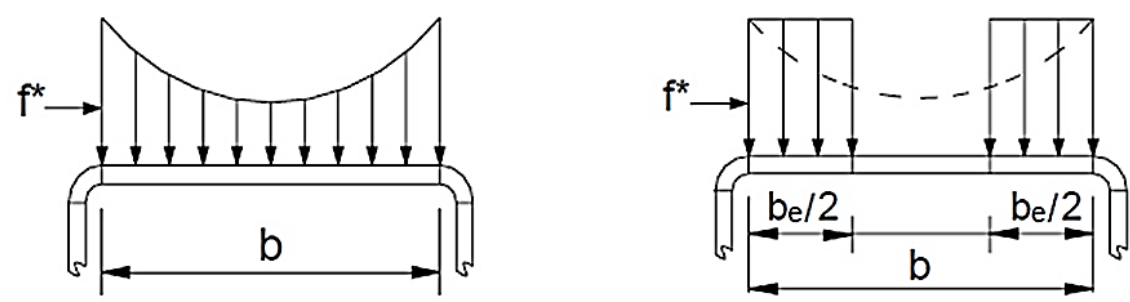

Figure 2.32. a) Non-uniform stress distribution (real situation). b) Uniform stress distribution over the effective width.

Von Karman (1932) proposed an equation (Equation 2.16) to determine the effective width of a plate element based on the design stress $\left(\sigma_{e}\right)$ and critical elastic buckling stress $\left(\sigma_{c r}\right)$ of the complete plate.

$$
\frac{b_{e f f}}{b}=\sqrt{\frac{\sigma_{c r}}{\sigma_{e}}}
$$

Where the elastic buckling stress is determined as follows (Equation 2.17): 


$$
\sigma_{c r}=\frac{k_{\sigma} \pi^{2} E}{12\left(1-v^{2}\right)(b / t)^{2}}
$$

In the limit $\sigma_{e}=f_{y}$ (minimum effective width), where $f_{y}$ is yield strength of the material. The effective width is determined as follows (Equation 2.18), and $\rho$ is the reduction factor of the plate.

$$
b_{\text {eff }}=\rho . b \quad \text { where } \quad \rho=\frac{b_{\text {eff }}}{b}=\frac{1}{\bar{\lambda}_{p}} \leq 1
$$

Later and based on experimental data Winter (1947) proposed some modifications to the formula presented by von Karman, to find the effective width of stiffened elements based on the plate slenderness as follows (Equation 2.19):

$$
\begin{array}{cc}
\frac{b_{\text {eff }}}{b}=1 & \bar{\lambda}_{p} \leq 0.673 \\
\frac{b_{\text {eff }}}{b}=\frac{1}{\bar{\lambda}_{p}}\left(1-\frac{0.22}{\bar{\lambda}_{p}}\right) \leq 1 & \bar{\lambda}_{p}>0.673
\end{array}
$$

Where the plate slenderness is $\bar{\lambda}_{p}=\sqrt{f_{y} / \sigma_{c r}}$. Nowadays the great majority of the available design codes use the Equations 2.19 to determine effective width of stiffened and unstiffened elements. However, this method presents some limitations, since it requires tedious and time consuming calculations to determine the effective width and becomes even more complicated for sections with complex shapes and additional edge and intermediate stiffeners.

In order to address the inherent limitations of the Effective Width Method (EWM), Schafer (Schafer and Peköz, 1998b; Schafer, 2001; Schafer, 2008) developed and presented a new design method entitled Direct Strength Method (DSM). The Direct Strength Method (DSM) is based on the member elastic stability in contrast to the Effective Width Method that is based on the effective width concept. Hence, this method considers the gross section instead of the effective section and thus the effective width calculations are not necessary. This method allows the interaction of local buckling with flexural or flexural-torsional buckling. In the DSM analysis adequate software (for instance, CUFSM, Thin-Wall) must be used in order to determine the elastic buckling loads. Specifically, for columns this includes local, distortional and global buckling loads. 
For the determination of the elastic buckling loads several tools can be used, namely by finite element analysis (FEA) software (e.g. ABAQUS) or by other simpler software based on the Finite Strip Method (FSM) or on the Generalised Beam Theory (GBT). Currently the most commonly used methods for elastic buckling prediction are the finite strip method FSM and the constrained finite strip method (cFSM) (Ádány and Schafer, 2006a and 2006b). There are some examples of software using the semi-analytical FSM such as CUFSM (Schafer, 2012) and ThinWall (Papangelis and Hancock, 2000). In the past few years some additional approaches for the determination of the elastic buckling loads, such as GBT (Silvestre and Camotim, 2002a, 2002b) and the correspondent software GBTUL (Bebiano et al, 2008a and 2008b), the effective area method (EAM) (Batista, 2009), the effective section method (ESM) (Batista, 2010) and the erosion of critical bifurcation load (ECBL) (Ungureanu and Dubina, 2004), were introduced.

Finite Strip Method is widely used and it is applicable to members with simply supported ends under longitudinal stress. The application of the FSM to a member provides the "signature curve", which is a buckling plot that can be used to find the elastic buckling load, buckling modes and buckling half-wavelength (Figure 2.33) (Li and Schafer, 2010). The FSM is a variant of the more common finite element method and in this method the member is discretized in longitudinal strips, and for each strip the elastic stiffness matrix is formulated based on the plane stress assumption and Kirchhoff thin plate theory (Li and Schafer, 2010). Since the FSM cannot individually identify and calculate the pure buckling modes the constrained finite strip method (cFSM) was proposed (Ádány and Schafer, 2006a, 2006b). The new method allows the decomposition of the stability buckling modes of a single-branched, open cross-section, into pure buckling modes (local, distortional and global) (Ádány and Schafer, 2006a, 2006b). However, the solutions (elastic buckling loads) provided by the cFSM are slightly different from the ones provided by the FSM and cannot be used for cross-sections models with corners. This brings some problems to the application of the cFSM, since the currently available DSM is calibrated to the conventional FSM. Li and Schafer (2010) conducted a series of parametric studies and found that the critical half-wavelengths determined using the cFSM models may be used to automatically identify local and distortional buckling in conventional FSM models. Since the critical half-wavelengths found in the cFSM models can be used in FSM models with rounded corners it is suggested that both FSM and CFSM analysis are run and only the critical half-wavelength values correspondent to the cFSM model are taken. Bear in mind that this procedure was only tested for lipped channels (Li and Schafer, 2010) (Figure 2.34).

The Generalised Beam Theory (GBT) (Silvestre and Camotim, 2002a, 2002b) is able to perform elastic buckling analysis with any type of end-support conditions and is able to characterize the 
buckling modes automatically, hence the buckling strengths associated with each one of the pure buckling modes (local, distortional and global) are directly provided. Due to this advantage the cFSM was developed taking into consideration the assumptions adopted in the GBT method which allow the identification and calculation of the pure buckling modes (Ádány and Schafer, 2006a).

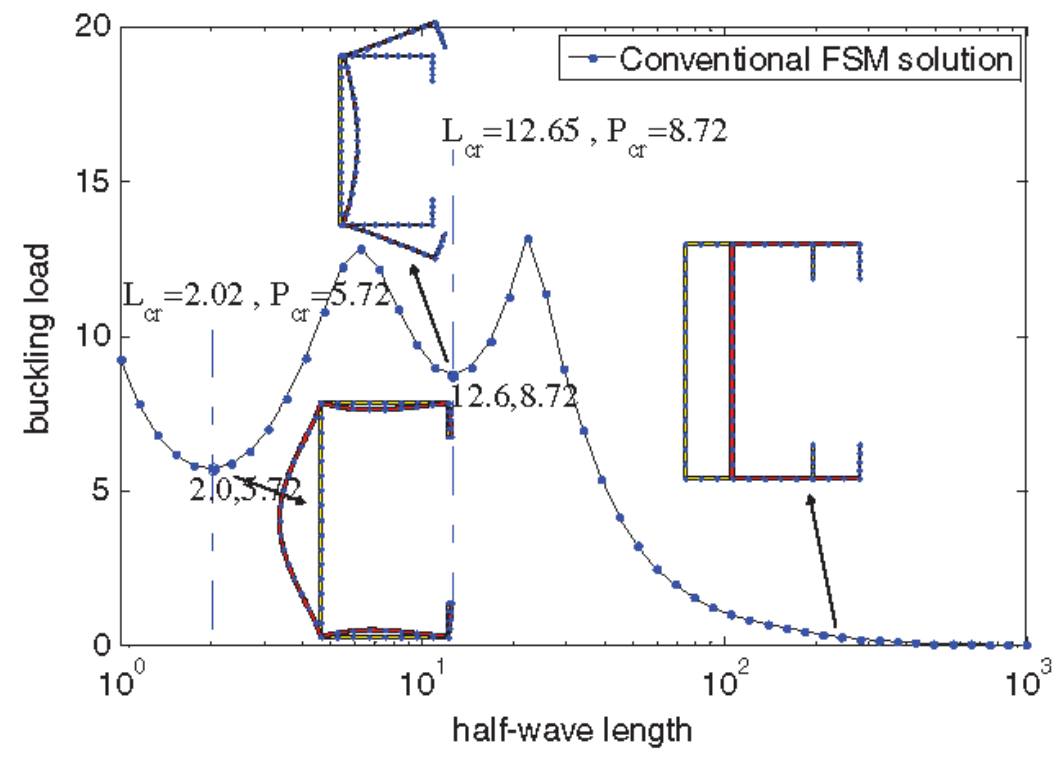

Figure 2.33 The cross-section stability "signature curve" from an FSM solution (Li and Schafer, 2010).

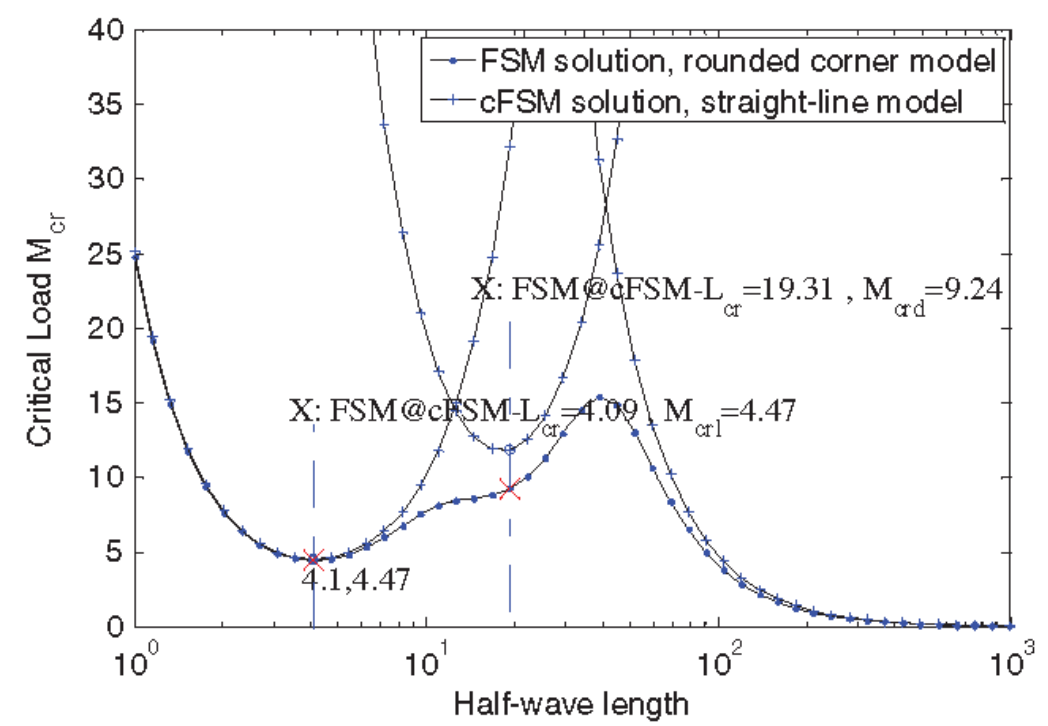

Figure 2.34 Signature curve with proposed methodology (FSM and cFSM) (Li and Schafer, 2010). 


\subsubsection{Design according to EN 1993-1-3:2006}

In the design process, before the determination of the resistance of the structural member, it is necessary to assess first the cross-sectional behaviour. According to the EN 1993-1-1 (2005) the cross-sectional resistance and the rotation capacity are limited by the effects of local buckling. Four behavioural classes were defined depending on material yield strength, widthto-thickness ratios of individual compression parts and the loading arrangement. Cold-formed steel cross-sections are traditionally Class 4 cross-sections, this means that local buckling will occur in the elastic range, before the attainment of yield stress in one or more parts of the crosssection. The design provisions for verification of local, distortional and global (flexural and flexural torsional) buckling strengths for CFS columns are presented in EN 1993-1-1 (2005), EN 1993-1-3 (2006) and EN 1993-1-5 (2006). The effective areas for Class 4 cross-sections fabricated with CFS are determined according to EN 1993-1-3 (2006). According to EN 19931-5 (2006) the effective area of a flat compression element is determined as follows (Equation 2.20):

$$
A_{c, e f f}=\rho A_{c}
$$

Where $\rho$ is the reduction factor and $A_{c}$ is the gross area of the compression element. The reduction factor, $\rho$, used to determine the effective width $\left(b_{\text {eff }}\right)$ shall be based on the compressive stress in the element, $\sigma_{c o m, E d}$. In Tables 2.2 and 2.3 the methodology for determination of the effective widths is specified for internal and outstand compression elements without stiffeners.

Assuming $\sigma_{c o m, E d}=f_{y b} / \gamma_{M 0}$ the reduction factor is obtained as follows (Equation 2.21 and 2.22) (EN 1993-1-5 (2006)):

- Internal compression elements;

$$
\begin{array}{cc}
\rho=1 & \text { for } \bar{\lambda}_{p} \leq 0.5+\sqrt{0.085-0.55 \psi} \\
\rho=\frac{\bar{\lambda}_{p}-0.055(3+\psi)}{\bar{\lambda}_{p}^{2}}<1.0 & \text { for } \bar{\lambda}_{p}>0.5+\sqrt{0.085-0.55 \psi}
\end{array}
$$

- Outstand compression elements (flange type);

$$
\rho=1 \quad \text { for } \bar{\lambda}_{p} \leq 0.748
$$




$$
\rho=\frac{\bar{\lambda}_{p}-0.188}{\bar{\lambda}_{p}^{2}}<1.0 \quad \text { for } \bar{\lambda}_{p}>0.748
$$

where, $\psi$ is the stress ratio and the plate slenderness, $\bar{\lambda}_{p}$, is given by (Equation 2.23 ):

$$
\bar{\lambda}_{p}=\frac{b / t}{28.4 \varepsilon \sqrt{k_{\sigma}}}
$$

If $\sigma_{c o m, E d}<f_{y b} / \gamma_{M 0}$ the reduction factor $\rho$ should be determined using the reduced plate thickness $\bar{\lambda}_{p, \text { red }}$ given by (Equation 2.24):

$$
\bar{\lambda}_{p, r e d}=\bar{\lambda}_{p} \cdot \sqrt{\frac{\sigma_{c o m, E d}}{f_{y b} / \gamma_{M 0}}}
$$

Hence, Equations 2.21 and 2.22 can be replaced by the following (Equations 2.25 and 2.26):

- For internal compression elements;

$$
\begin{array}{cr}
\rho=1 & \text { for } \bar{\lambda}_{p} \leq 0.5+\sqrt{0.085-0.55 \psi} \\
\rho=\frac{\bar{\lambda}_{p}-0.055(3+\psi) / \bar{\lambda}_{p, \text { red }}}{\bar{\lambda}_{p, \text { red }}}+0.18 \frac{\left(\bar{\lambda}_{p}-\bar{\lambda}_{p, \text { red }}\right)}{\left(\bar{\lambda}_{p}-0.6\right)}<1.0 \\
\text { for } \bar{\lambda}_{p}>0.5+\sqrt{0.085-0.55 \psi}
\end{array}
$$

- For outstand compression elements;

$$
\begin{array}{cr}
\rho=1 & \text { for } \bar{\lambda}_{p} \leq 0.748 \\
\rho=\frac{1-0.188 / \bar{\lambda}_{p, \text { red }}}{\bar{\lambda}_{p, \text { red }}}+0.18 \frac{\left(\bar{\lambda}_{p}-\bar{\lambda}_{p, \text { red }}\right)}{\left(\bar{\lambda}_{p}-0.6\right)}<1.0 & \text { for } \bar{\lambda}_{p}>0.748
\end{array}
$$


Table 2.2 Effective widths of internal compression elements (EN 1993-1-5:2006).

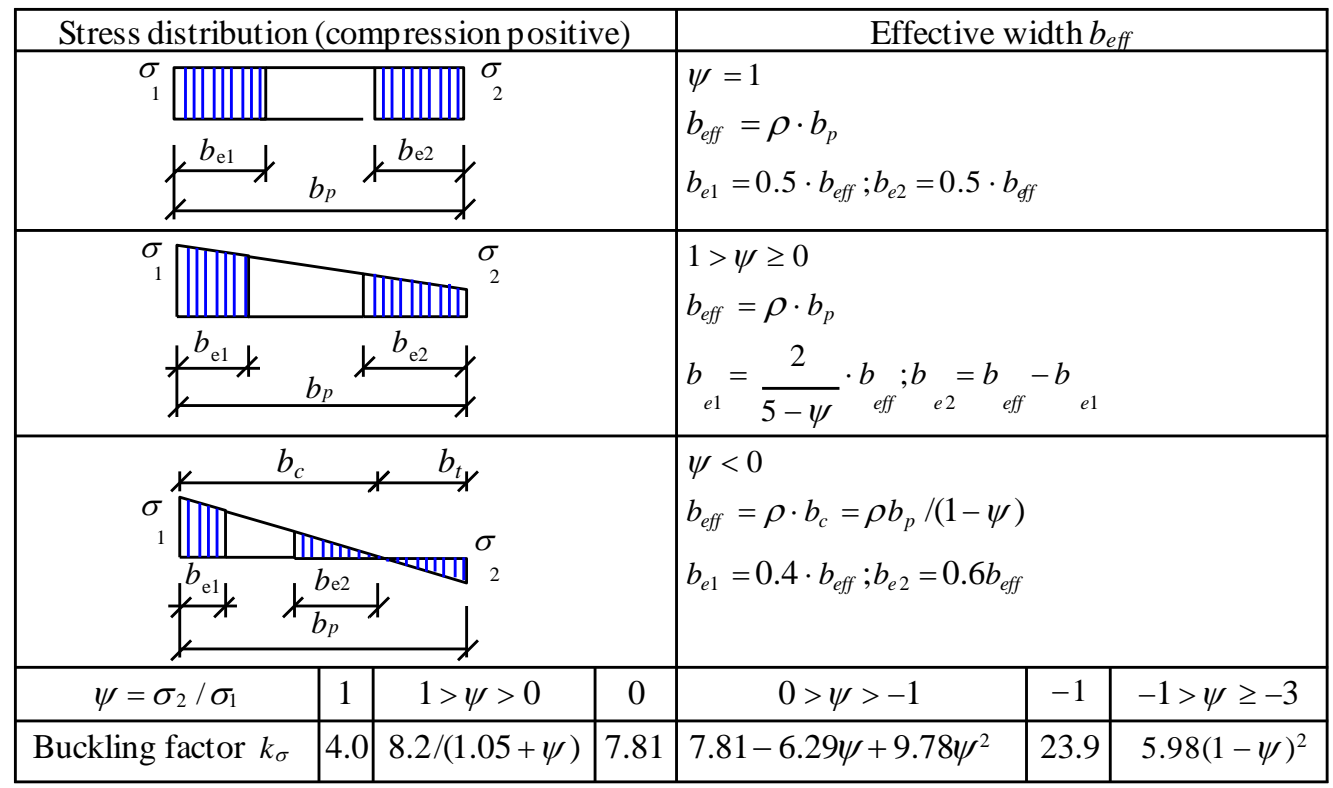

Table 2.3 Effective widths of outstand compression elements (EN 1993-1-5:2006).

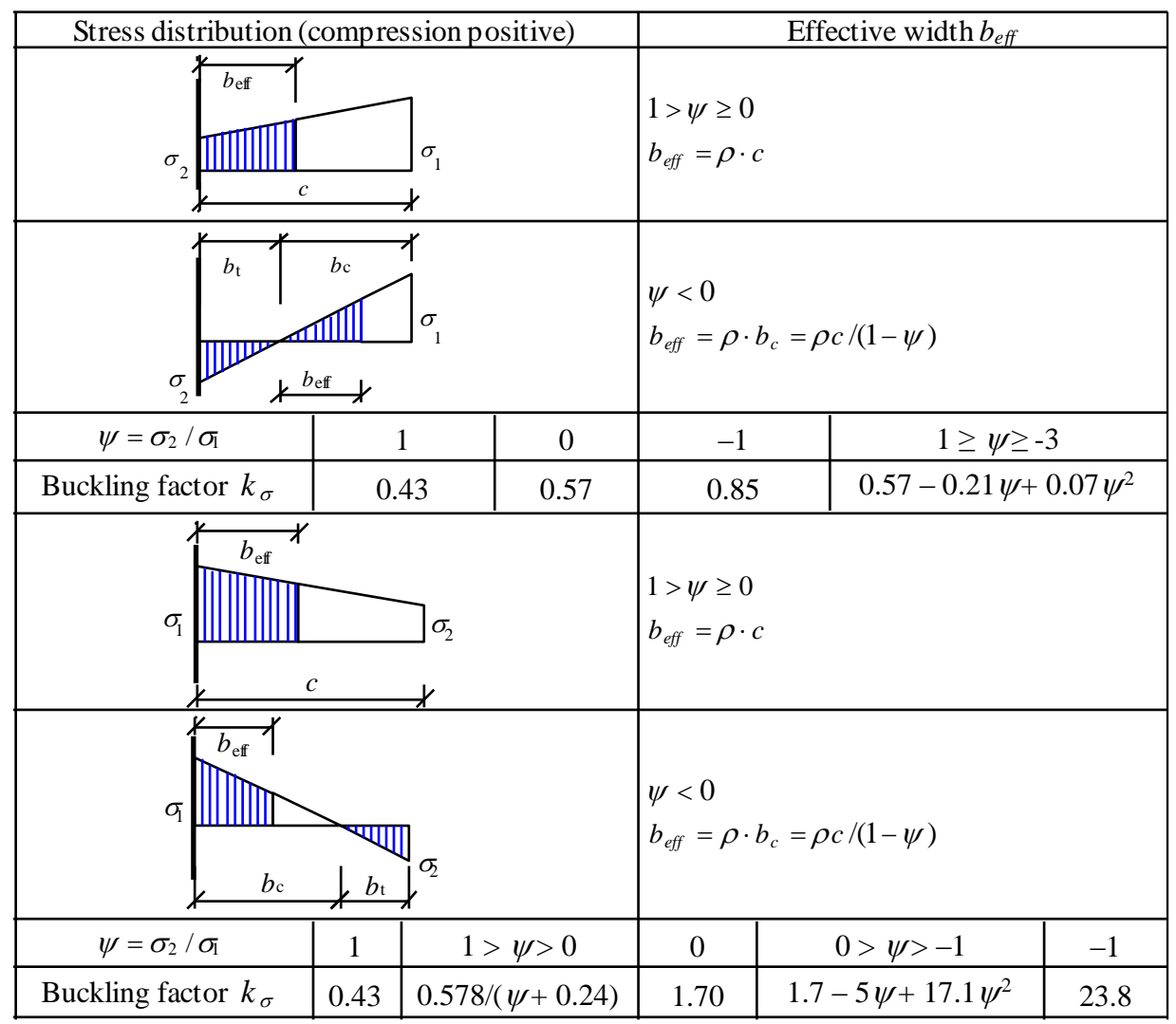


In the case of plane elements with edge stiffeners it shall be assumed that the stiffeners behave as a compression member with partial restraint, represented by a linear spring with stiffness that depends on the boundary conditions and flexural stiffness of the adjacent plane elements. This approach accounts for distortional buckling. The cross-section of an edge stiffener comprises the effective portions of the stiffener and the effective portion of the plane element (Dubina $e t$ $a l, 2012$ ) according to Figure 2.35. First the effective section for local buckling (assuming infinite spring stiffness) must be determined and the compressive stress in the element $\sigma_{c o m, E d}=f_{y b} / \gamma_{M 0}$. The initial values of the effective width $c_{e f f}$ in Figure 2.35 are determined as follows, for a single edge fold stiffener (Equation 2.27):

$$
c_{e f f}=\rho b_{p, c}
$$

But on this case, the buckling factor, $k_{\sigma}$, is determined as given in Equation 2.28:

$$
\begin{array}{cc}
k_{\sigma}=0.5 & \text { if } b_{p, c} / b_{p} \leq 0.35 \\
k_{\sigma}=0.5+\sqrt[3]{\left(b_{p, c} / b_{p}-0.35\right)^{2}} & \text { if } 0.35<b_{p, c} / b_{p} \leq 0.6
\end{array}
$$

where, $b_{p, c}$ is the width of the edge stiffener and $b_{p}$ is the flange width for a lipped channel (Figure 2.35).
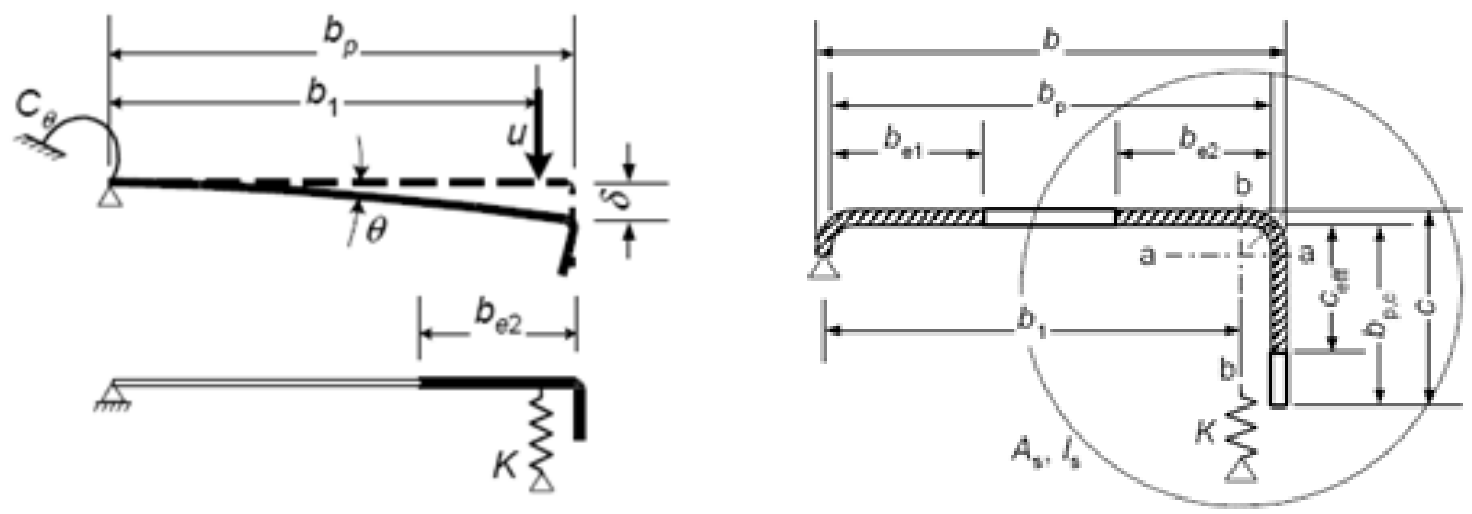

Figure 2.35 Edge stiffener of a lipped channel section (EN 1993-1-3, 2006).

The elastic critical buckling stress, $\sigma_{c r, s}$ for an edge stiffener should be determined as given in Equation 2.29. 


$$
\sigma_{c r, s}=\frac{2 \sqrt{K E I_{s}}}{A_{s}}
$$

where:
$K \quad$ is the linear spring stiffness;
$I_{s} \quad$ is the second moment area of the effective stiffener section about its centroidal axis a-a (Figure 2.33);
As is the cross-sectional area of the effective stiffener section.

The stiffness of the spring may be obtained by means of a unit load analysis (unit load per unit length, $K=u / \delta$ ) as presented in Figure 2.33. In the case of edge stiffeners of lipped channels, the stiffness of the linear spring is determined as follows (Equation 2.30):

$$
K=\frac{E t^{3}}{4\left(1-v^{2}\right)} \frac{1}{b_{1}^{2} h_{w}+b_{1}^{3}+0.5 b_{1} b_{2} h_{w} k_{f}}
$$

where:
$b_{1}$ is the distance from the web-to-flange junction to the gravity centre of the effective area of the edge stiffener (Figure 2.33);
$b_{2} \quad$ is the distance from the web-to-flange junction to the gravity centre of the effective area of the edge stiffener;
$h_{w} \quad$ is the web depth;
$k_{f}=A_{s 2} / A_{s 1} \quad$ if flange 2 is in compression, where $A_{s 1}$ and $A_{s 2}$ are the effective area of the edge stiffener of flange 1 and 2 ;
$k_{f}=1 \quad$ for symmetric section in compression.

Then the flexural buckling reduction factor $\chi_{d}$ (reduction factor for the distortional buckling) of the edge stiffener is calculated from the Equation 2.31.

$$
\begin{array}{cc}
\chi_{d}=1 & \text { for } \bar{\lambda}_{d} \leq 0.65 \\
\chi_{d}=1.47-0.723 \bar{\lambda}_{d} & \text { for } 0.65<\bar{\lambda}_{d} \leq 1.38 \\
\chi_{d}=0.66 / \bar{\lambda}_{d} & \text { for } \bar{\lambda}_{d} \geq 1.38
\end{array}
$$


where $\bar{\lambda}_{d}=\sqrt{f_{y b} / \sigma_{c r, s}}$. The reduction factor for flexural buckling may be refined iteratively if $\chi_{d}<1$, using modified values of $\rho$ obtained by assuming $\sigma_{c o m, E d}=\chi_{d} f_{y b} / \gamma_{M 0}$ for each iteration. Finally, the reduced area for the effective stiffener section is calculated from (Equation 2.32):

$$
A_{s, \text { red }}=\chi_{d} A_{s} \frac{f_{y b} / \gamma_{M o}}{\sigma_{c o m, E d}} \quad \text { but } A_{s, \text { red }} \leq A_{s}
$$

The reduced area is achieved by means of a reduction in thickness of the effective stiffener sections, $t_{\text {red }}=t A_{\text {red }} / A_{s}$.

Finally, the design buckling resistance of a compression member with class 4 cross-section is determined as follows (Equation 2.33):

$$
N_{b, R d}=\frac{\chi A_{e f f} f_{y}}{\gamma_{M 1}}
$$

in which $\chi$ is defined as follows (Equation 2.34):

$$
\chi=\frac{1}{\phi+\sqrt{\phi^{2}-\bar{\lambda}^{2}}} \quad \text { but } \chi \leq 1
$$

where

$$
\begin{aligned}
& \phi=0.5\left[1+\alpha(\bar{\lambda}-0.2)+\bar{\lambda}^{2}\right] \text { and } \bar{\lambda}=\sqrt{\frac{A_{e f f} f_{y}}{N_{c r}}} \text { for class } 4 \text { cross-sections. } \\
& \alpha \quad \text { is the imperfection factor, corresponding to the appropriate buckling }
\end{aligned}
$$

Regarding the design of built-up cross-sections the EN 1993-1-3:2006 (2006) simply predicts that the buckling resistance of a closed built-up cross-section should be determined using the buckling curve $b$ in association with the basic yield strength, $f_{y b}$, or curve c in association with the average yield strength, $f_{y a}$ provided that $A_{e f f}=A_{g}$. 


\subsubsection{Design of cold-formed steel columns according to the DSM established in Appendix of AISI S100}

Considering the DSM it is necessary to undertake previous analysis in order to determine the elastic buckling loads. Specifically, for columns this includes local $\left(P_{n l}\right)$, distortional $\left(P_{n d}\right)$ and global buckling loads $\left(P_{n e}\right)$. The axial strength of the columns will be the minimum of $P_{n e}, P_{n l}$, and $P_{n d}$ (AISI S100-7, 2007).

The axial strength of a column, $P_{n e}$, for flexural, torsional or flexural-torsional buckling is determined as follows (Equation 2.35):

$$
\begin{array}{ll}
\text { For } \lambda_{c} \leq 1.5 & P_{n e}=\left(0.658^{\lambda_{c}^{2}}\right) P_{y} \\
\text { For } \lambda_{c}>1.5 & P_{n e}=\left(\frac{0.877}{\lambda_{c}^{2}}\right) P_{y}
\end{array}
$$

where

$$
\begin{aligned}
& \lambda_{c}=\sqrt{P_{y} / P_{c r e}} \\
& P_{y}=A_{g} F_{y} \\
& P_{c r e}=\text { minimum of the critical elastic column buckling load in flexural, torsional, or } \\
& \text { flexural-torsional buckling (CUFSM). }
\end{aligned}
$$

The axial strength, $P_{n l}$, for local buckling is determined as follows (Equation 2.36):

$$
\begin{gathered}
\text { For } \lambda_{l} \leq 0.776 \\
P_{n l}=P_{n e} \\
\text { For } \lambda_{l}>0.776 \\
P_{n l}=\left[1-0.15\left(\frac{P_{c r l}}{P_{n e}}\right)^{0.4}\right]\left(\frac{P_{c r l}}{P_{n e}}\right)^{0.4} P_{n e}
\end{gathered}
$$

where

$$
\lambda_{l}=\sqrt{P_{n e} / P_{c r l}}
$$

$P_{n e}=$ axial strength of a column for flexural, torsional or flexural-torsional buckling;

$P_{c r l}=$ critical elastic local column buckling load determined by analysis (CUFSM). 
The axial strength, $P_{n d}$, for distortional buckling is determined as follows (Equation 2.37):

For $\lambda_{d} \leq 0.561$

$$
P_{n d}=P_{y}
$$

For $\lambda_{d}>0.561$

$$
P_{n d}=\left(1-0.25\left(\frac{P_{c r d}}{P_{y}}\right)^{0.6}\right)\left(\frac{P_{c r d}}{P_{y}}\right)^{0.6} P_{y}
$$

where

$$
\begin{aligned}
& \lambda_{d}=\sqrt{P_{y} / P_{c r d}} \\
& P_{y}=A_{g} F_{y} \\
& P_{c r d}=\text { critical elastic distortional column buckling load determined by analysis } \\
& \text { (CUFSM). }
\end{aligned}
$$

For doubly-symmetric sections, closed cross-sections, and any other sections that can be shown not to be subjected to torsional or flexural torsional buckling, the elastic flexural buckling stress, $F_{e}$ (Equation 2.38), shall be calculated as follows (Section C.4.1.1 - AISI S100-7, 2007):

$$
F_{e}=\frac{\pi^{2} E}{(K L / r)^{2}}
$$

where
E modulus of elasticity of steel;
$K \quad$ effective length factor;
$L \quad$ laterally unbraced length of member
$r \quad$ radius of gyration of full unreduced cross-section about axis of buckling.

For compression built-up members the axial strength is determined considering a modified slenderness ratio $(K L / r)_{m}$ if the buckling mode involves relative deformations that produce shear forces in the connectors between individual shapes (Equation 2.39):

$$
\left(\frac{K L}{r}\right)_{m}=\sqrt{\left(\frac{K L}{r}\right)_{0}^{2}+\left(\frac{a}{r_{i}}\right)^{2}}
$$


where
$\left(\frac{K L}{r}\right)_{0}$
is the overall slenderness ratio of the entire section about the built-up
$a$
member axis;
$r_{i}$
intermediate fastener or spot weld spacing;
minimum radius of gyration of full unreduced cross-sectional area of an individual shape in a built-up member.

\subsection{Cold-Formed Steel Columns under Fire Conditions}

\subsubsection{General}

The demand for CFS structures has increased significantly, as it has been recognised to be used effectively as primary structural elements, especially for residential, commercial and industrial buildings. Since fire can occur in any kind of building, causing loss of lives and properties, the fire safety engineering has received greater attention in the past few decades. This science is multidisciplinary and a building only can be considered safe if some specific areas in the scope of fire safety engineering are correctly addressed in design. Some of these specific areas are detection and warning systems, evacuation, controlling of ignition and limiting fire propagation and finally structural protection. Fire safety design of building structures is a key subject in the field of fire safety engineering. The ability to guarantee the structural integrity of a building in fire for a certain period of time is critical to guarantee adequate evacuation times for people, as well as to guarantee minimum safe conditions for firefighters' intervention. Structural protection against fire can be assessed in terms of critical temperature that a structural element can withstand, in terms of time and finally in terms of structural element capacity design.

Hot-rolled steel structures have been extensively investigated in the past few decades and as a consequence of product development and research some design specifications were implemented, namely EN 1993-1-2:2005 (2005). However, so far, there is a lack of research on the structural behaviour of cold-formed steel structural elements under fire conditions. As a result, the design guidelines for such structural members at elevated temperatures are not accurate and precise enough to be used by the structural designers. Currently the methods presented in the EN 1993-1-2:2005 (2005) for hot-rolled steel members are also applicable to CFS members with class 4 cross-sections, establishing the same reduction factors for the yield strength of steel and limiting the critical temperature to $350^{\circ} \mathrm{C}$. Some studies carried out in recent years have shown that the reduction of the mechanical properties for CFS as a function of temperature have been different from those presented in the EN 1993-1-2:2005 (2005) (Outinen, 1999; Kankanamge and Mahendran, 2011; Ranawaka and Mahendran, 2009), and 
that limiting the temperatures to $350^{\circ} \mathrm{C}$ without considering the load ratio may be overly conservative (Ranawaka and Mahendran, 2009; Heva, 2009).

The great majority of experimental studies at elevated temperatures have been performed on CFS short columns in order to evaluate the local and distortional buckling phenomena individually and in combination (Ranawaka and Mahendran, 2006; Heva, 2009; Feng et al, 2003a; Feng et al, 2003b; Lee, 2004; Kaitila, 2002; Outinen and Myllymaki, 1995) using experimental and numerical analysis (Feng et al, 2003c; Feng et al, 2004; Ranawaka and Mahendran, 2006). Concerning built-up cross-sections commonly used in building construction the lack of research is more substantial even at ambient temperatures. Also the influence of the surrounding structure on the structural behaviour of CFS columns has been neglected. Some studies regarding the influence of axial and rotational restraint have been conducted for hot rolled steel sections (Ali et al, 1998; Ali and O'Connor, 2001; Franssen, 2000; Wang, 1997a; Wang, 1997b; Wang, 2004; Rodrigues et al, 2000; Correia et al, 2011; Correia et al, 2012). It is clear that if the axial elongation is restrained the axial compression force will increase in the column depending on the stiffness of the surrounding structure and the initial applied load level leading to a premature failure of the column under fire situation. Some of these investigations, conducted on hot-rolled steel columns, are briefly detailed.

\subsubsection{Research on hot-rolled steel columns under fire conditions with restraint to thermal elongation}

Ali et al. (1998) presented an experimental study on the behaviour of steel columns in fire with axial restraint to thermal elongation. Three parameters were investigated, namely slenderness ratio $(\lambda=49,75,98)$, degree of axial restraint $\left(\alpha_{k}=K_{\text {structure }} / K_{\text {column }(T)}=0,0.1,0.2,0.3\right)$ and loading ratio $\left(\alpha_{L}=0,0.2,0.4,0.6\right)$. Generally, for all tested specimens it was found that imposing some level of restraint to thermal elongation to the steel columns lead to a reduction in the fire resistance. Also it was observed that increasing the level of restraint lead to an increase of the generated axial restraining forces and consequently to a reduction in the failure temperature.

Following this study, also the influence of rotational restraint on the failure of columns under fire conditions was assessed (Ali and O'Connor, 2001). Different load levels were tested and to all tested columns the same level of axial restraint was adopted $\left(\alpha_{k}=0.29\right.$, imposed axial stiffness of $57 \mathrm{kN} / \mathrm{mm}$ ). Two levels of rotational restraint were tested in this investigation, namely 0.18 and 0.93 . These rotational restraint levels were defined as follows (Equation 2.40): 


$$
\rho=\frac{\rho_{s}}{\rho_{s}+\rho_{c}}
$$

where
$\rho \quad$ degree of rotational restraint;
$\rho_{s} \quad$ rotational stiffness of the surrounding structure;
$\rho_{c} \quad$ rotational stiffness of the column.

As expected increasing the loading level lead to a reduction in failure temperature. Regarding the influence of increasing rotational restraint, it was found that the rotational restraint did not affect significantly the generated restraining force, however the failure temperatures were greatly increased.

Franssen (2000) presented a study on the influence of restraint to thermal elongation to a column inserted in a moment resisting frame. Assuming a localised fire, only one column of the moment resisting frame may be affected, hence the effects of restraint to thermal elongation may not be as severe as usually are for individual columns or for an isostatic system. The column in fire and subjected to some level of restraint to thermal elongation imposed by the surrounding structure will fail earlier due to the additional axial forces generated. However, if the column subjected to fire fails, the remaining columns of the moment resisting frame (not affected by fire, for instance due to compartmentation) are able to support the increase of the load, hence the failure of the entire structure is prevented. It was stated that in some situations the influence of restraint to thermal elongation may not be as severe as initially expected.

Wang and Davies (2003) reported an experimental research on the behaviour of non-sway loaded and rotational restrained steel columns in order to assess how bending moments in restrained columns change during a fire scenario and how this change could influence failure temperatures of the column. Each test consisted of a column and two beams connected to the web of the column, considering flexible or moment resisting beam-to-columns connections. Three load levels were investigated, namely 30, 50 and $70 \%$ of the column compressive strength at ambient temperature. It was observed that failure temperatures of the tested columns were mainly dependent on the applied load with reduced influence of the type of beam-to-column connection used.

\subsubsection{Research on cold-formed steel columns at elevated temperatures}

Ranby (1998) using FEM analysis assessed the load bearing resistance of thin walled coldformed steel sections in case of fire. It was showed that initial deflections have the same relative 
influence on the load bearing resistance at both ambient and fire conditions. Also it was stated that the basic methodologies presented in the EN 1993-1-3 (1996) can be directly used in fire design by simply applying the adequate reduced mechanical properties as a function of temperature. Also it was found that the critical temperature was above $350^{\circ} \mathrm{C}$.

In order to assess the physical behaviour and failure modes of lipped and unlipped short channel columns, with and without perforations, at elevated temperatures, an experimental investigation was conducted (Feng et al, 2003a). All fire tests were conducted under the steady state condition at different temperature levels, namely $250,400,550$ and $700^{\circ} \mathrm{C}$. During the heating process the specimen could freely expand and after the desired temperature was reached the axial compression load was applied until failure. The predominant failure mode observed was the interaction between local and distortional buckling. Also it was observed that depending on the initial imperfections, the failure mode of nominally identical columns can be different, however the failure loads were very close. Based on this experimental research Feng et al (2003b) a finite element model was developed and calibrated and the experimental results were compared with some design methods at ambient temperature, such as the one presented in the EN 19931-3 (1993). The mechanical properties provided by Outinen (1999) were tested, as well as the ones presented in the EN 1993-1-2:2001 (2001), and it was found that results are more accurate using the material model provided by Outinen (1999). Also it was showed that ambient temperature design formulations can be extended to elevated temperatures, provided the reduced yield strength based on the $0.2 \%$ proof stress and reduced elastic modulus are used.

Feng et al (2004) presented a numerical study, using the finite element method and the finite element software ABAQUS, on the influence of imperfections on the behaviour of thin-walled tubular short and long steel columns at elevated temperatures. For initial local imperfections the imperfection values of $h / 200, t$ (thickness of the cross-section), $0.1 t$ and $0.5 t$ were used whereas for global imperfections the values used were $L / 1000$ and $L / 500$. Three stress-strain relationships were used, namely the one proposed by Outinen (2000), the one presented in the EN 1993-1-2:2001 (2001) and an elastic perfectly plastic stress-strain relationship. It was found that the initial imperfection has a significant influence on the buckling load of a CFS column under uniform heating and also that simulation results are very sensitive to the stress-strain models used as input. Hence additional experimental studies are recommended to assess accurately the stress-strain relationship at both ambient and elevated temperatures.

Chen and Young (2007b) reported an investigation on the behaviour of CFS lipped channel columns at elevated temperatures using the finite element model and the finite element software ABAQUS. The material model was based on the stress-strain curve equations for CFS at 
elevated temperatures by Chen and Young (2007a). The numerical model was validated against the experimental tests conducted by Young and Rasmussen (1998) and Feng et al (2003a) at both ambient and elevated temperatures. Different cross-sections and different lengths were tested in the parametric study. The results were then compared with the design strengths obtained using the EWM and the DSM in the North American Specification (2001) and in the supplement to the North American Specification (2004), and it was found that using the adequate reduced material properties the design provisions give conservative results.

Ranawaka and Mahendran $(2009,2010)$ presented a detailed experimental and numerical investigation on the distortional buckling behaviour of cold-formed steel compression members at elevated temperatures. The experimental tests (Ranawaka and Mahendran, 2009) were conducted on two types of cross-sections with different thicknesses $(0.6,0.8$ and $0.95 \mathrm{~mm})$ and at different temperature levels, ranging from 20 to $800^{\circ} \mathrm{C}$. Also two types of structural steel were investigated, namely G250 and G550 (low and high strength steels). Test specimens were specifically designed to fail by pure distortional buckling, using the FSM and Thin-Wall software. All experimental tests were undertaken considering the steady state test method, hence each specimen was heated up to the desired temperature with free thermal expansion (no load on the specimens during the heating phase). After reaching the desired temperature level the compression test was performed (Figure 2.36).

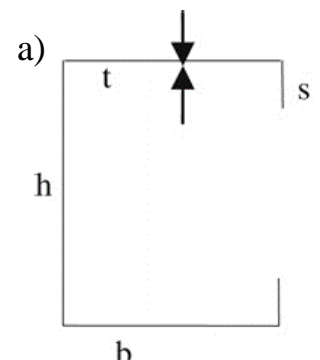

Type A

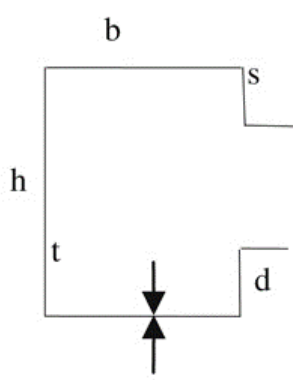

Type B

c)

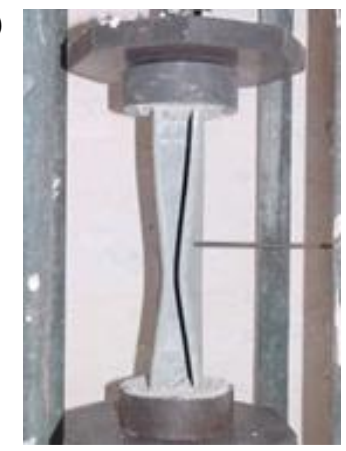

b)

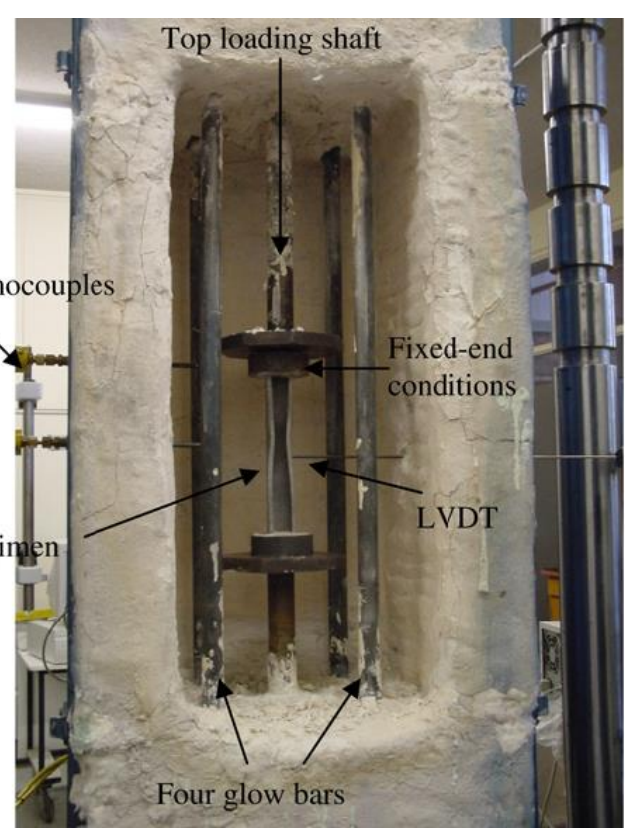

Figure 2.36 a) Cross-sections tested. b) Experimental test set-up. c) Final deformed shape of a Type A specimen at $650^{\circ} \mathrm{C}$. (Ranawaka and Mahendran, 2009). 
In the tests at elevated temperatures three failure modes were observed. In the first the columns failed by both flanges moving inwards, in the second by both flanges moving outwards and the third by one flange moving inwards and the other moving outward. Despite the different failure modes, the failure loads were about the same. Hence, it was stated that imperfections may influence the type of distortional buckling. The obtained ultimate loads were compared with the available design provisions presented in the AS/NZS 4600 and with the Direct Strength Method (DSM). The results showed that at ambient temperatures the design provisions are accurate and that at elevated temperatures the results are reasonably accurate provided that the adequate reduced mechanical properties are used.

Based on the experimental results Ranawaka and Mahendran (2010) developed and validated a finite element model able to reproduce the behaviour of CFS compression members subjected to distortional buckling at elevated temperatures. The geometric imperfections used were the ones measured in the specimens, and the residual stresses were included in the model. Regarding the residual stresses, it was observed that it had very small influence on the ultimate load $(<1 \%)$. For both types of steel (G250 and G550) tensile coupon tests were conducted to assess the reduction of mechanical properties with temperature increase (Ranawaka and Mahendran, 2009). The stress-strain relationship adopted in the numerical model was based on the experimental results using the Ramberg-Osgood material model. Also the influence of initial geometric imperfections was assessed, and was observed that the ultimate load decreases rapidly for small geometric imperfections. Finally, is also reinforced the idea that accurate mechanical properties at elevated temperatures are fundamental to accurately characterize the behaviour of compressed CFS members.

Shahbazian and Wang $(2011,2012)$ reported an investigation on the applicability of the Direct Strength Method (DSM) for determination of local and distortional buckling capacity of CFS columns with uniform and non-uniform elevated temperatures. The results obtained from the DSM calculations were compared with the results obtained from the finite element model developed and validated against the experimental tests conducted by Feng et al (2003). Using the calibrated model extensive parametric studies were undertaken. Regarding local buckling it was observed that the DSM equations in the AISI specification are applicable to columns under local buckling with uniform temperature distribution in the cross-section. For columns with non-uniform temperature in the cross-section new predictive equations were proposed (Shahbazian and Wang, 2011). The same conclusions were drawn for columns under distortional buckling and with uniform or non-uniform temperature distribution in the crosssection (Shahbazian and Wang, 2012). Hence, for non-uniform situation some modifications were proposed for both local (Equation 2.38) and distortional buckling (Equation 2.39). 


$$
\begin{array}{cc}
P_{n l}=P_{n e} & \text { For } \lambda_{l} \leq 0.776 \\
P_{n l}=\left(1-0.22\left(\frac{P_{c r l}}{P_{n e}}\right)^{0.75}\right)\left(\frac{P_{c r l}}{P_{n e}}\right)^{0.75} P_{n e} & \text { For } \lambda_{l}>0.776 \\
P_{n d}=P_{y} & \text { For } \lambda_{l} \leq 0.561 \\
P_{n d}=0.65\left(1-0.14\left(\frac{P_{c r d}}{P_{y}}\right)^{0.7}\right)\left(\frac{P_{c r d}}{P_{y}}\right)^{0.7} P_{y} & \text { For } \lambda_{l}>0.561
\end{array}
$$

\subsubsection{Design rules according to the EN 1993-1-2:2005}

EN 1993-1-2:2005 (2005) provides design provisions for steel compression elements, specific for class 1, 2 and 3 sections. For members with class 4 cross-section the area must be replaced by the effective area and the section modulus is replaced by the effective section modulus (determined according to EN 1993-1-3:2006 and EN 1993-1-5:2006) in the equations for compression members. Also, the EN 1993-1-2:2005 (2005) provides yield stress reduction factor for class 4 cross-sections, however, there is no difference between the reduction factors for hot-rolled and cold-formed steels. Additionally, no reduction factors for the elastic modulus of cold-formed steels are provided.

The design buckling resistance, $N_{b, f i, t, R d}$, of a compression member is determined as follows (Equation 2.40):

$$
N_{b, f i, t, R d}=\chi_{f i} A k_{y, \theta} f_{y} / \gamma_{M, f i}
$$

where

$\chi_{f i}$ is the reduction factor for flexural buckling in a fire design situation (Equation 2.40a);

$k_{y, \theta} \quad$ is the reduction factor for the yield strength of steel at the temperature $\theta_{a}$;

$\gamma_{M, f i}$ partial safety factor for fire situation.

The value of $\chi_{f i}$ should be taken as the lower of the values $\chi_{f i, y}$ and $\chi_{f i, z}$ determined as follows: 


$$
\chi_{f i}=\frac{1}{\phi_{\theta}+\sqrt{\phi_{\theta}^{2}+\bar{\lambda}_{\theta}^{2}}}
$$

where

$$
\phi_{\theta}=\frac{1}{2}\left[1+\alpha \bar{\lambda}_{\theta}+\bar{\lambda}_{\theta}^{2}\right]
$$

The imperfection factor, $\alpha$, was proposed by Franssen et al. (1995) (Equation 2.40c):

$$
\alpha=0.65 \sqrt{235 / f_{y}}
$$

The non-dimensional slenderness $\bar{\lambda}_{\theta}$ for the temperature $\theta_{a}$ is determined as follows:

$$
\bar{\lambda}_{\theta}=\bar{\lambda}\left[k_{y, \theta} / k_{E, \theta}\right]^{0.5}
$$

where

$k_{E, \theta} \quad$ is the reduction factor for the modulus of elasticity at the steel temperature $\theta_{a} ;$ is the non-dimensional slenderness at ambient temperature defined as:

$$
\bar{\lambda} \quad \bar{\lambda}=\frac{\lambda}{\lambda_{1}}=\frac{l_{f i} / i}{\pi \sqrt{E / f_{y}}} ;
$$

Where
$l_{f i} \quad$ is the buckling length of the column in fire situation;
$i \quad$ is the minimum radius of gyration;
$\mathrm{E} \quad$ is the elastic modulus at ambient temperature;
$f_{y} \quad$ is the yield strength at ambient temperature.

\subsection{Finite Element Analysis (ABAQUS)}

Finite Element Analysis (FEA) is the practical application of the Finite Element Method (FEM). Nowadays it is extensively used method for multiphysics problems. It is extensively used in the analysis of structures, part of structures and even isolated structural elements. It is also used to perform heat transfer analysis. There is several commercial software available, however in the scope of this investigation Abaqus v.6.12 (2012) was used. Other software packages are 
very well known and widely used in the field of structural fire engineering, such as SAFIR (2005), VULCAN (2010), ANSYS (2015), etc. Abaqus v.6.12 is able to perform Geometrically and Materially Nonlinear Analysis with Imperfections (GMNIA), which is a structural method designed to determine the strength capacity of a structure, part of a structure, or isolated structural element, taking into account both plasticity and buckling failure modes (Marques et $a l, 2013)$. In research FEA is extremely important since it is relatively inexpensive and time efficient when compared with more traditional full scale tests. Nevertheless, it is worth to mention that the FEA does not exclude the need to perform at least some experimental tests that will be used to compare and validate the finite element model developed. Hence, it is standard practice to perform some experimental tests that will be used for validation of the developed finite element model, and then the validated model is used for extensive parametric studies outside the bounds of the experimental tests. Several of the previously mentioned studies used the FEA to assess the behaviour of CFS columns at both ambient and elevated temperatures.

The FEA consists of three major stages, namely pre-processing, analysis and finally postprocessing. In the pre-processing stage the model is created, which includes definition of geometry, creating a suitable finite element mesh, input and assign appropriate material properties and applying the existent boundary conditions in the form of restraints or loads. In the analysis stage the numerical problem created is solved and finally in the post-processing stage the results are evaluated (Figure 2.37).

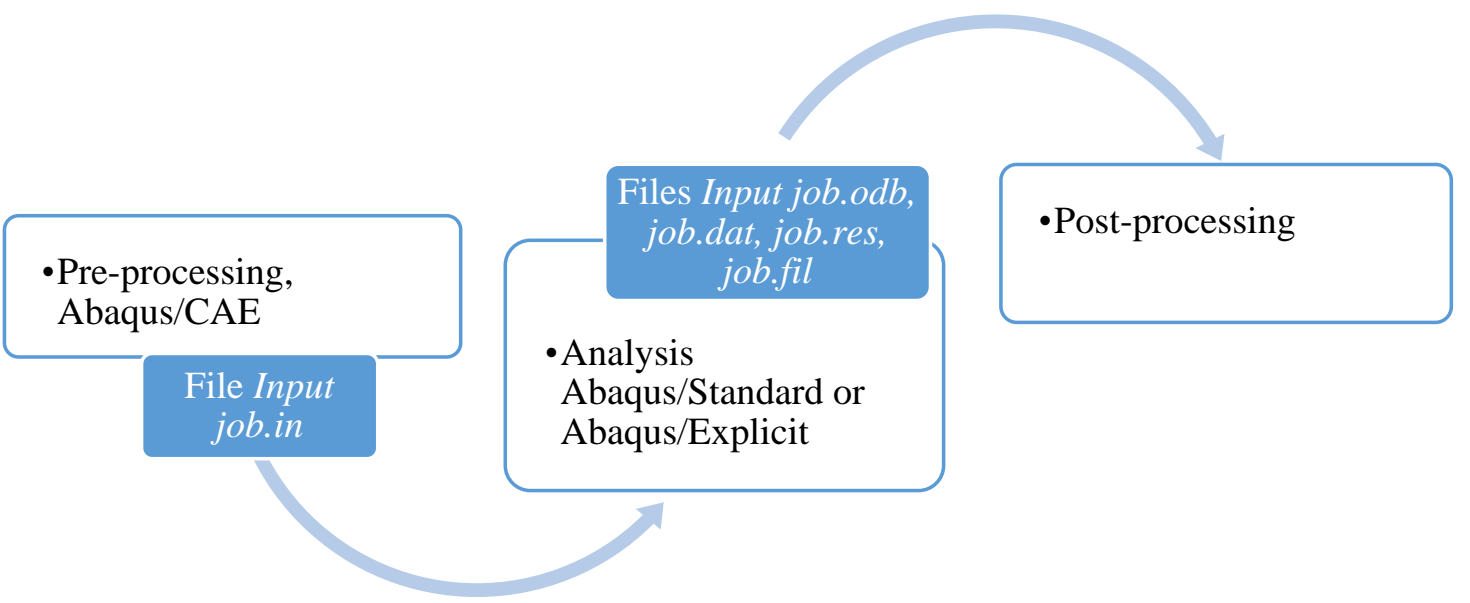

Figure 2.37 Main stages of a FEA.

The key issues with the FEA are related with the input data, such as initial geometric imperfections, residual stresses, material modelling (at ambient and elevated temperatures), boundary conditions, type of finite element used, element discretization and solution controls 
in collapse (Schafer and Moen, 2010). All the mentioned parameters have significant influence in the accuracy of the developed numerical model.

Since this method is extremely powerful it is commonly used to assess the behaviour of CFS single, open built-up and closed built-up columns at both ambient and fire conditions. In the scope of this investigation the structural behaviour of single, open built-up and closed built-up CFS columns at both ambient and fire conditions with restraint to thermal elongation will be assessed using the finite element analysis and the software Abaqus. The developed model and the correspondent validation are thoroughly detailed in Chapter 6.

\subsection{Final Remarks}

Extensive literature review was presented in the previous sections, allowing to obtain the necessary basic knowledge to undertake the proposed research on the field of columns fabricated with cold-formed steel profiles at both ambient and fire conditions. A literature overview was performed on the following topics: material characterization (mechanical and thermal properties) and residual stresses, imperfections, behaviour and buckling load of coldformed steel columns at ambient temperature, design methodologies, behaviour of cold-formed steel columns under fire conditions, behaviour of steel columns subjected to fire with restraint to thermal elongation and finally on finite element modelling.

Accurate evaluation of the mechanical and thermal properties of cold-formed steel is fundamental for the accuracy of the design methodologies at ambient and especially under fire situation. From the reported studies it is clear that the available design provisions in the EN 1993-1-2 (2005) are not applicable for cold-formed steels, however, there is some significant scatter among the available results in the literature. Hence, the assessment of the mechanical properties of the $\mathrm{S} 280 \mathrm{GD}+\mathrm{Z}$ steel in this research is extremely important to assess the accuracy of the proposed model in the EN 1993-1-2 (2005) and to establish some comparisons with the available data in the literature. Also the results of this experimental investigation will be used as input in the development of the numeric models. The topic of thermal properties of steel at both ambient and elevated temperatures has very few studies, at least among the civil engineering community. Hence, taking advantage of the existent equipment at the University of Coimbra the thermal properties of the $S 280 \mathrm{GD}+\mathrm{Z}$ steel were assessed in the scope of this investigation. Once again the experimental results will be used as input in the numerical models developed to reproduce the behaviour of CFS columns. 
Regarding the behaviour of CFS columns at ambient temperature the available research is extensive, both in terms of experimental tests and numerical investigations, translated in the available standards, namely the EN 1993-1-3 (2006) and the AISI S100-7 (2007), and in the available design methods, namely the Effective Width Method and the Direct Strength Method. Despite some existing limitations both methods are generally able to predict the strength of single CFS columns. However, regarding the commonly used open and closed built-up crosssections the research at both ambient and elevated temperatures is still very scarce. Also the available design standards only present small recommendations for the design of this type of elements. This is a specific subject that must be addressed since this type of elements is currently being used in the building construction industry without adequate scientific support.

The fire behaviour is another relevant area in the field of CFS structures that is not adequately addressed. Some important studies using experimental and numerical analysis have been presented, however so far there are no specific or standardised guidelines for the design of CFS columns subjected to fire. Also, so far, the great majority of studies were undertaken to study individually the different buckling phenomena traditionally associated with thin-walled structural elements. Moreover, the conducted investigations do not take into account the influence of the surrounding structure to the overall behaviour of the CFS column. This type of study has only been undertaken for hot-rolled steels. Since there is a significant gap in research on this matter the biggest subject of this research is the fire behaviour of unprotected CFS columns with single, open built-up and closed built-up cross-sections. Since there are few experimental investigations detailed in the literature on this subject the core of the research presented in this work is related to the conducted experimental analysis, from material characterization to behaviour of the structural element. Based on the obtained experimental results finite element models were developed and validated and using these validated models extensive parametric studies may be conducted in near future. 


\section{MATERIAL CHARACTERIZATION}

\subsection{Introduction}

Probably one the most important aspects for an accurate structural fire design is the evolution of mechanical properties such as yield strength and modulus of elasticity with increasing temperature. The deterioration of mechanical properties with increasing temperature results in a significant loss of buckling load of cold-formed steel structural elements.

The currently available design codes such as BS 5950 Part 8 (1990) and the EN 1993-1-2 (2005) provide reduction factors for mechanical properties of cold-formed steels at elevated temperatures but with some limitations. For instance, the BS 5950 Part 8 (1990) only provides reduction factors for yield strengths corresponding to $0.5 \%, 1.5 \%$ and $2.0 \%$ strain levels whereas the EN 1993-1-2 (2005) provides the same reduction factors used for class 4 hot-rolled steels.

Some relevant studies have been conducted on the mechanical properties of cold-formed steels at elevated temperatures for low and high strength cold-formed steels and with different thicknesses (Lee et al., 2003; Outinen, 1999; Ranawaka and Mahendran, 2009; Kankanamge and Mahendran, 2011; Makelainen et al., 1998; Chen and Young, 2007; Chen and Young, 2006; Mecozzi and Zhao, 2005). In the great majority of these studies it was found that the yield strength and modulus of elasticity reduction factors available in the current design codes are not suitable for steels used in the cold-formed steel building construction industry (Lee et al., 2003; Outinen, 1999; Ranawaka and Mahendran, 2009; Kankanamge and Mahendran, 2011; Makelainen et al., 1998; Chen and Young, 2007a; Chen and Young, 2006; Mecozzi and Zhao, 2005). Analysing the available results in the literature it seems that the reduction factors for the modulus of elasticity show little dependence on steel grade (Ranawaka and Mahendran, 2009; Kankanamge and Mahendran, 2011) whereas the yield strength reduction factors are more dependent on steel grade. However, the reduction factors obtained and the proposed equations still differ most likely due to the test method, strain rate, heating rate and material grade. Two test methods are widely used, namely steady state and transient state test method. Steady-state is more common and probably easier to conduct whereas the transient-state test method is considered more realistic as it simulates a structural member under static loading subjected to fire (Outinen and Makelainen, 2002). In this experimental investigation (Pinheiro, 2015), the steady-state method was used. Steady-state tests are easier to conduct guaranteeing accurate 
data acquisition. Consequently, possible errors may be reduced during the execution of the tensile tests and in the determination of the mechanical properties, since the stress-strain curves are plotted directly from the recorded data (Lee et al., 2003; Outinen, 1999; Ranawaka and Mahendran, 2009; Chen and Young, 2007; Mecozzi and Zhao, 2005). Moreover, some researchers showed that the difference between the steady-state and transient-state methodologies was not very significant (Lee et al., 2003; Outinen, 1999; Outinen et al., 2000). For instance, in the study conducted by Outinen (Outinen, 1999; Outinen et al., 2000) on the S350GD $+Z$ steel the difference between both methodologies was on average about $5 \%$ in the temperature range from 300 to $600^{\circ} \mathrm{C}$ and almost identical for higher temperatures (Lee et al., 2003).

The creep effect is time dependent and influenced by the temperature exposure and applied load. The time dependent inelastic deformation starts to occur when the temperature of the material exceeds 30\%-40\% of the absolute melting temperature (Ottosen and Ristinmaa, 2005; Cowan and Khandelwal, 2014). According to some authors, the amount of creep may be considered limited since steady state tests usually lasted less than one hour (Lee et al., 2003; Ranawaka and Mahendran, 2009; Kankanamge and Mahendran, 2011; Kesawan at al., 2015). Despite the eventual reduced influence of creep in tensile tests, this does not mean that in real applications creep should not be considered. However, to include explicitly creep strain in calculations is difficult and in most applications it is simpler to use stress-strain relationships that include some amount of creep that might be expected (Lu and Makelainen, 2003; Barsom and Rolfe, 1999; Lennon et al., 2007). It is currently accepted that within the time-scale of the accidental fires creep may not be explicitly included, provided that the elevated stress-strain model presented in the EN 1993-1-2:2005 (2005) is used (Lu and Makelainen, 2003; Lennon et al., 2007). Some authors have already published some studies on the creep effect on steels at high temperatures (Brnic et al., 2014; Brnic et al., 2010; Brnic et al., 2011; Cowan and Khandelwal, 2014; Brnic et al., 2009; Brnic et al., 2013). Generally, it was found that for temperatures of $400^{\circ} \mathrm{C}$, for a time exposure of 120 minutes and for a stress level of $0.9 \mathrm{f}_{\mathrm{y}, 20}$ all tested steels present good creep resistance. For instance, for the S275JR (Brnic et al., 2013) and ASTM A992 (Cowan and Khandelwal, 2014) steel the creep strain was about $0.5 \%$ and $0.25 \%$ respectively. At $500^{\circ} \mathrm{C}$, for the ASTM A992 steel (Cowan and Khandelwal, 2014), for a stress level of $0.9 f_{y, 20}$ and for a time exposure of 120 minutes creep strain is still reduced, around $0.3 \%$. For temperatures above $600^{\circ} \mathrm{C}$ it is clear that creep effect may become more significant depending on the steel tested and will increase with temperature increase.

Additionally, the assessment of thermal properties such as thermal conductivity, thermal elongation and specific heat capacity is extremely important for a better understanding of the 
behaviour of cold-formed steel structural elements in case of fire. Thermal properties are crucial to understand heat transfer phenomena and thermal deformations. However, so far, there is a lack of research in the field of thermal properties of cold-formed steels at ambient and elevated temperatures. For instance, some design codes such as AISC (2010) and AS 4100-1998 (1998) simply ignore that thermal expansion coefficient is temperature dependent. Some authors have already showed that the predicted thermal elongation in the EN 1993-1-2 (2005) is conservative (Chen and Young, 2007; Outinen et al., 2000). Other thermal properties such as thermal conductivity and specific heat may be determined using the Transient Plane Source method (He, 2005; Zhang et al., 2014; Kodur and Shakya; 2013; Gustavsson et al., 1994). This equipment is widely used to measure thermal conductivity of bulk and slab specimens such as the ones that were tested in the scope of this research. Among the existing transient techniques to determine thermal conductivity such as hot wire and laser flash, the hot disk technique is probably the faster and more accurate thermal conductivity technique (He, 2005).

With this experimental study it was intended to evaluate and compare mechanical and thermal properties of the $\mathrm{S} 280 \mathrm{GD}+\mathrm{Z}$ steel at both ambient and elevated temperatures with the provisions presented in the available design standards and with the available results in the literature. Also the obtained results will be used as input in the developed numerical models using the finite element method.

\subsection{Experimental Investigation}

\subsubsection{Mechanical properties of the S280GD+Z steel at ambient and elevated temperatures}

\subsubsection{Experimental test set-up}

Tensile coupon tests were conducted at both ambient and elevated temperatures, ranging from $20^{\circ} \mathrm{C}$ to $800^{\circ} \mathrm{C}\left(100\right.$ to $800^{\circ} \mathrm{C}$ in steps of $100^{\circ} \mathrm{C}$ ), to determine the mechanical properties of the S280GD $+Z$ steel with $2.5 \mathrm{~mm}$ thickness. A universal testing machine Servosis model ME402/20 with $200 \mathrm{kN}$ capacity (1) was used to perform these tests. To measure the strains of the tensile test specimens, the high temperature extensometer Epsilon model HI3548 (2) (2011), with a gage length of $50.8 \mathrm{~mm}$ and travel of $25.4 \mathrm{~mm}$ in tension, was used (Figure 3.1 and Figure 3.2). In these tests, conical tip alumina rods were used since these type of rods are suitable for flat specimens. The high temperature extensometer is water-cooled using a constant temperature bath system Caron 2050 series (3). Also a data acquisition system TML model TDS602 (4) was used to monitor the temperatures of the furnace chamber and the specimen in test. The thermal action was applied using an electrical split tubular furnace (5) controlled by a 
Eurotherm controller (6) (Figure 3.2). A constant heating rate of $10^{\circ} \mathrm{C} / \mathrm{min}$ was used in the tests.

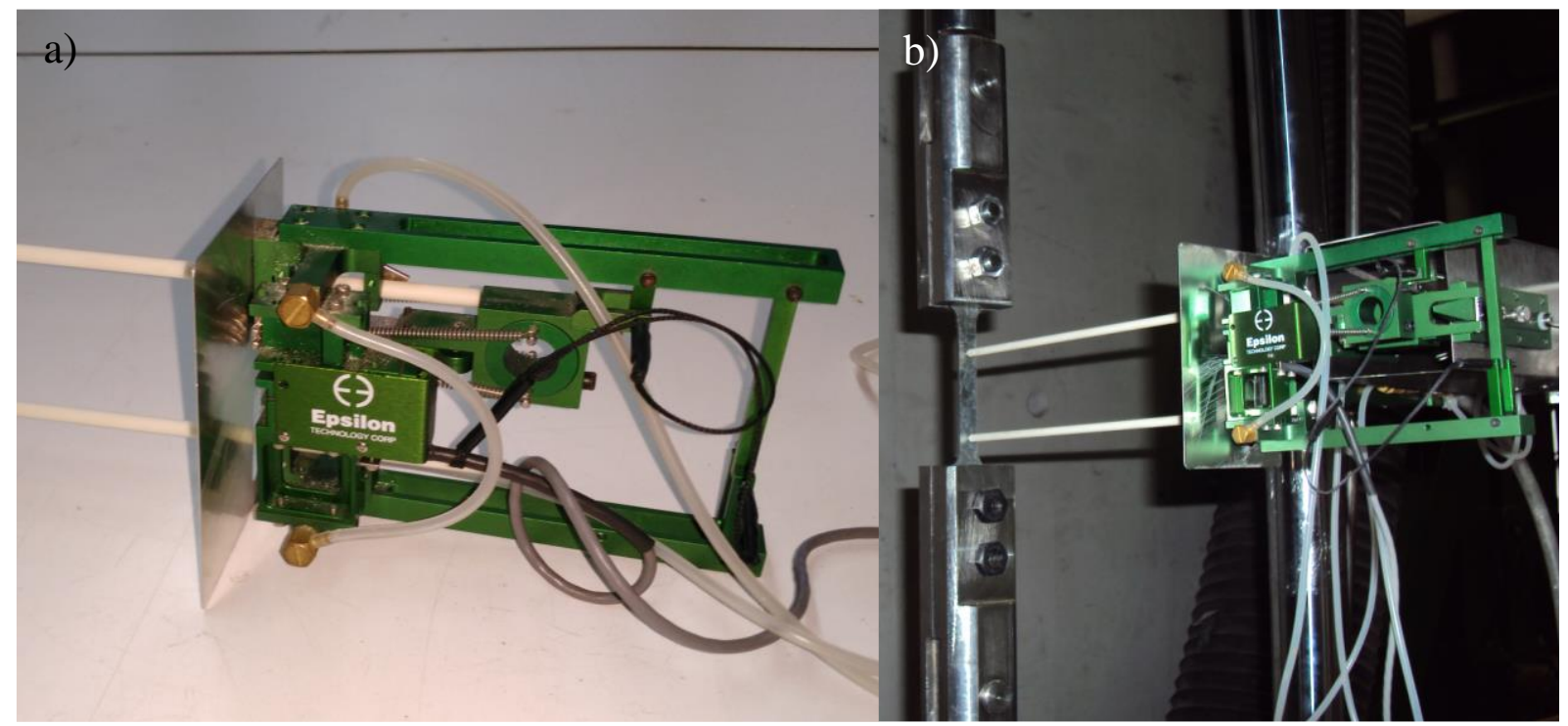

Figure 3.1 a) High temperature extensometer Epsilon HI 3548. b) Test set-up for ambient temperature testing.
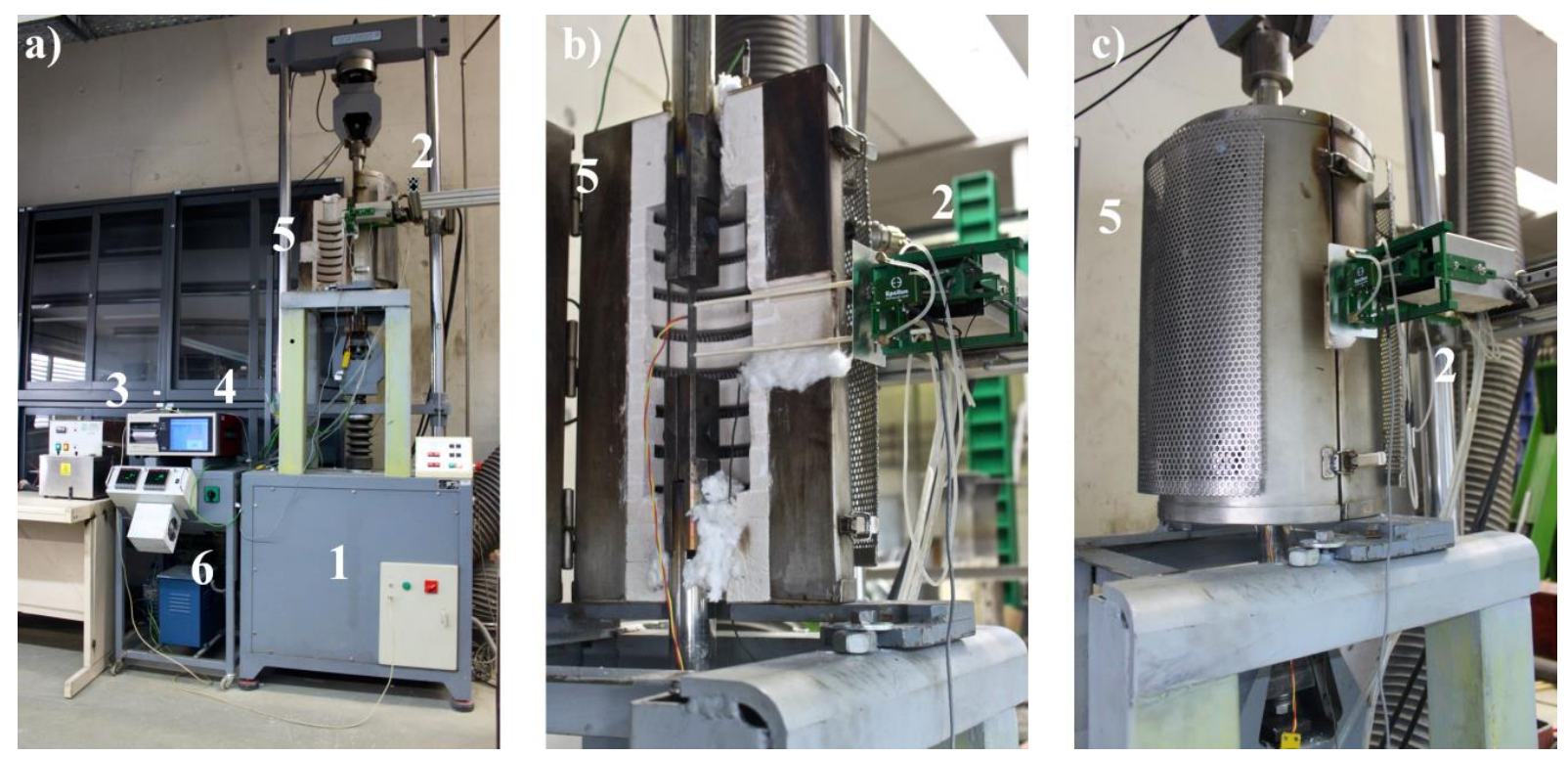

Figure 3.2 Test set-up. a) Global view. b) Detail of the high temperature extensometer. c) Electrical tubular split furnace and high temperature extensometer.

The high temperature extensometer HI3548 (2011) was previously calibrated, however, in the ambient temperature tests strain gauges FLA-6-11 were also used in order to compare the 
obtained results using both instruments assessing their accuracy. In Figure 3.9 it is possible to observe that the results obtained using both solutions are in very good agreement ensuring the accuracy of the high temperature extensometer. The tests were undertaken using the steady state test method. During the heating stage the specimen freely expanded and after stabilized at the desired temperature level and positioned the high temperature extensometer in the tensile coupon, the test was ready to start. The temperature of the specimen was monitored during the entire heating phase and tensile test using a thermocouple. The load was applied using displacement control with a rate of $0.15 \mathrm{~mm} / \mathrm{min}$ corresponding to a strain rate of $0.002 \mathrm{~min}^{-1}$ within the strain rate limit set to $0.003 \mathrm{~min}^{-1}$ according to the EN 10002-5 (1992).

\subsubsection{Test specimens}

Each test specimen was cut in the longitudinal direction of forming of the lipped channel S280GD $+Z$ profile according to the dimensions proposed in the EN 10002-1 (2001) (Figure 3.3). The specimens were extracted from the web of the lipped channel profiles.

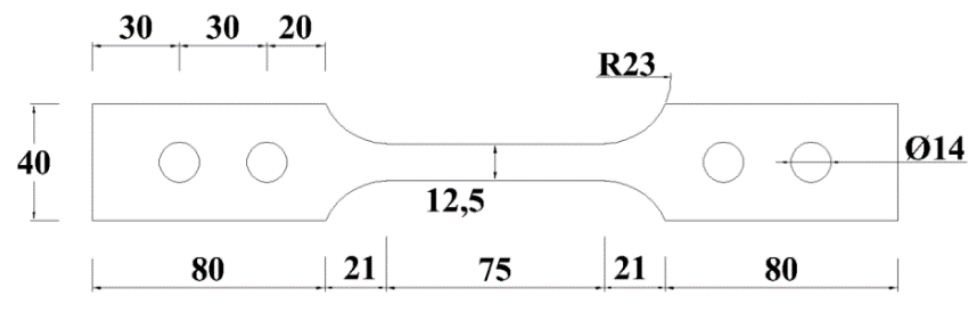

Figure 3.3 Dimensions of the tensile test specimens.

The chemical composition of the cold-formed steel is presented in Table 3.1.

Table 3.1 Chemical composition of the S280GD+Z steel provided by the manufacturer.

\begin{tabular}{l|c|c|c|c|c}
\hline & C [\%] & Mn [\%] & Si [\%] & S [\%] & P [\%] \\
\hline S280GD+Z & 0.06 & 0.43 & 0.026 & 0.007 & 0.012 \\
\hline
\end{tabular}

Two $14 \mathrm{~mm}$ holes were made at each end of the specimen to fix it in refractory steel pull rods. The use of two holes was decided in order to avoid localized crushing. After cut and machining, each specimen was measured using a micrometer at three different points of the gauge length region. These measured values were then used for the determination of the cross sectional area of the specimens. 


\subsubsection{Thermal properties of the S280GD+Z steel at ambient and elevated temperatures}

Thermal conductivity, specific heat and thermal elongation were experimentally determined in this investigation. Thermal elongation was assessed using the same test set-up of the coupon tests at elevated temperatures. A $100 \mathrm{~mm} \times 60 \mathrm{~mm}$ rectangular plate was cut and fixed in one end. The other end of the plate could freely expand with temperature increase. Thermal deformations were measured with the high temperature extensometer HI3548 (2011).

The transient plane source technique was used to determine the thermal conductivity, the diffusivity measurement and the specific heat capacity. It is a highly accurate technique that can be used in a wide range of material types, with a wide thermal conductivity range, with easy sample preparation and non-destructive (He, 2005; Zhang et al., 2014; Kodur; Gustavsson et al., 1994). The equipment used in this investigation was the TPS 2500S commercialized by Hot Disk AB (2015). Hot Disk AB is a company specialized in the development and fabrication of equipment for measuring and testing thermal conductivity, thermal diffusivity and specific heat capacity of almost any type of material (solids, liquids, powders, pastes and foams). Specifically, the TPS 2500S (Figure 3.4) is the high end product and can be used in different applications, from basic testing in isotropic materials (Isotropic module) to thin films, coatings or adhesive layers (Thin film module), high conducting sheets or slabs (Slab module), extremely light and low conducting materials (Low-density/Highly-insulating module) and anisotropic samples or layered samples (Anisotropic module) (Hot Disk AB, 2015). Different types of sensors can be used with this equipment, depending on the needs and testing conditions (Figure 3.4). The available types of sensors are: Kapton, Mica and Teflon sensors.
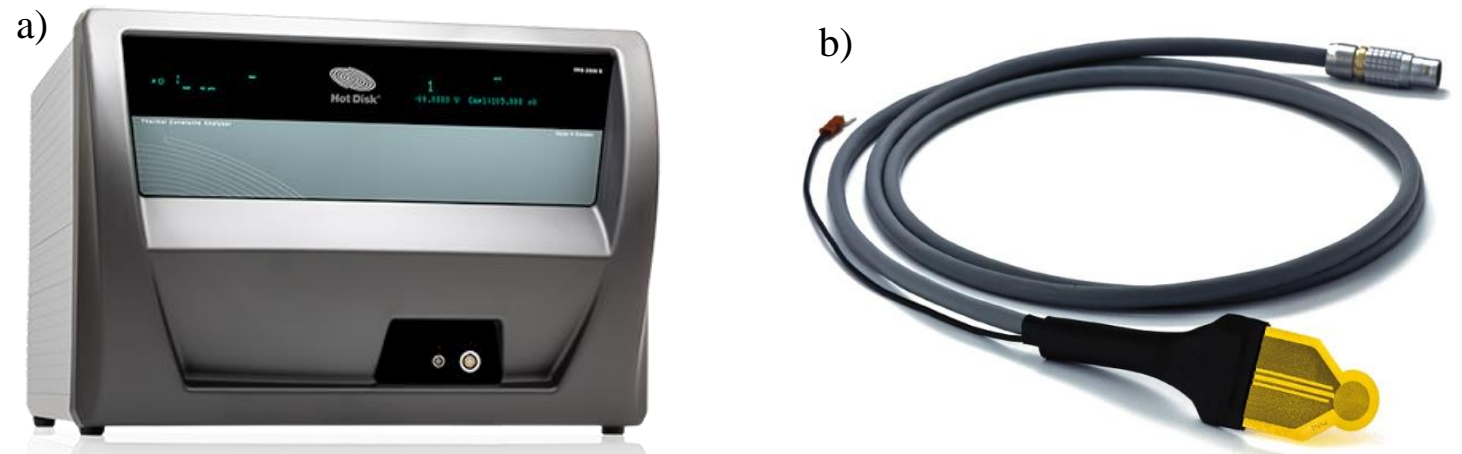

Figure 3.4 a) TPS 2500S equipment. b) Hot Disk Kapton sensor with cable (Hot Disk AB, 2015). 
The sensor is an insulated nickel double spiral which is used for both transient heating and precise temperature readings. The insulation to the nickel spiral is provided by two thin sheets of Kapton, Mica, or Teflon. In this experimental campaign Kapton and Mica sensors were used (Figure 3.5). The Hot Disk ref. 4922 (Hot Disk AB, 2015) Kapton and Mica sensors, with 14.6 $\mathrm{mm}$ radius, were used for ambient and high temperature tests ranging from 100 to $800{ }^{\circ} \mathrm{C}$, respectively.

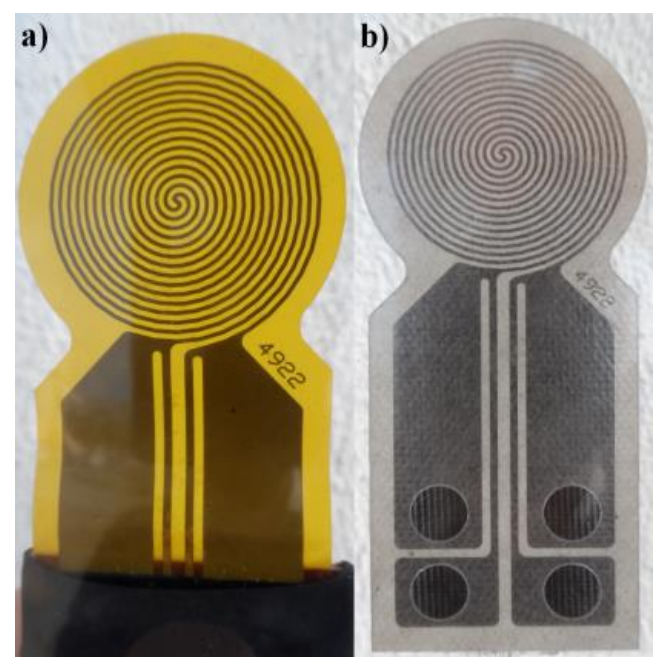

Figure 3.5 Hot disk sensors used in this investigation. a) Kapton sensor model 4922. b) Mica sensor model 4922.

To perform these tests two square sample pieces of S280GD+Z steel were used for each test (7 $\mathrm{cm} \times 7 \mathrm{~cm}$ ). The sensor was placed between the two sample pieces to be tested. Then a constant small electrical current was supplied to the sensor which is able to monitor the temperature increase through resistance measurement. The temperature increase due to the electrical current depends on the thermal transport properties of the two sample pieces surrounding the sensor. Thermal transport properties of the test samples are determined by monitoring the temperature increase over a short period of time (He, 2005; Zhang et al., 2014; Kodur; Gustavsson et al., 1994; Hot Disk AB, 2015) usually ranging from 5 to $20 \mathrm{~s}$ in the experimental tests undertaken. Due to the reduced thickness of the test samples $(2.5 \mathrm{~mm})$, the slab module of the software was used. Knowing the sample thickness this method only requires the measurement time and output power as input data. In Figure 3.6 the slab test layout for ambient temperatures using the Kapton insulation sensor is presented. The slab sample holder designed for use in combination with the slab software module was used as recommended in order to minimise heat losses to the sample holder. 


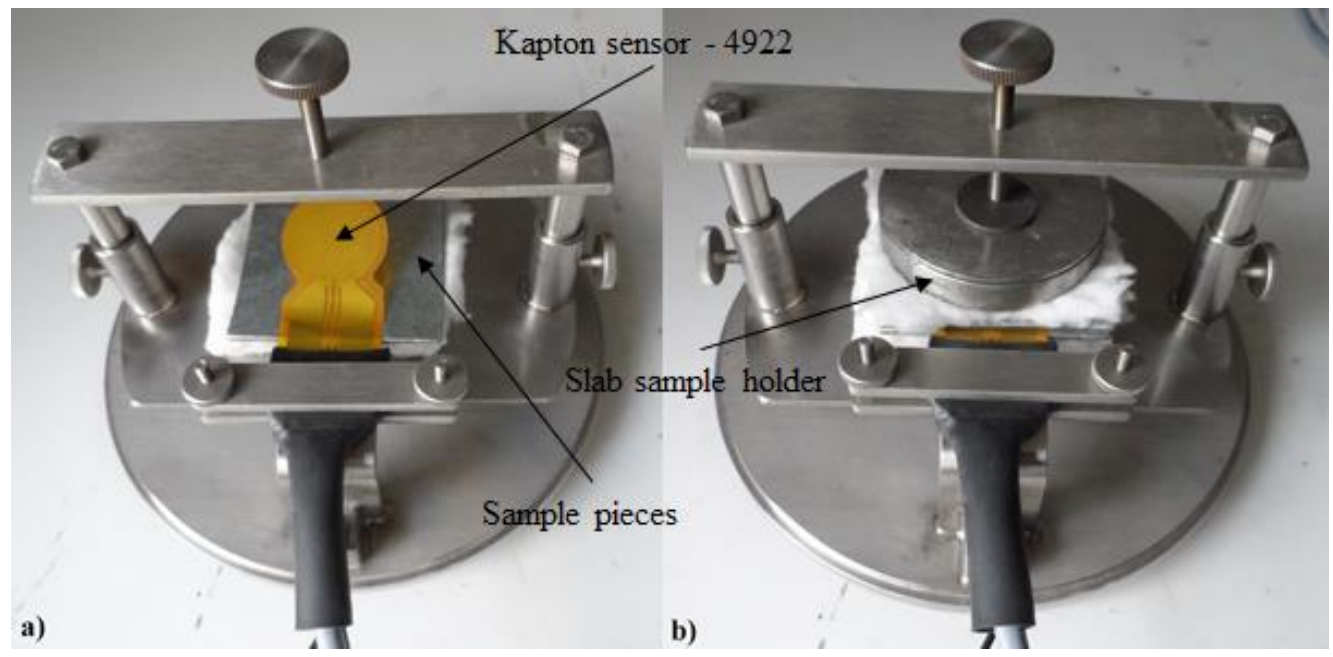

Figure 3.6 Ambient temperature sample support with slab sample holder setup.

For the high temperature tests a specific sample holder, as well as specific sensors (Mica sensors) and cables (Kanthal-Nikrothal 80 cable with ceramic rings) were used (Figure 3.7).
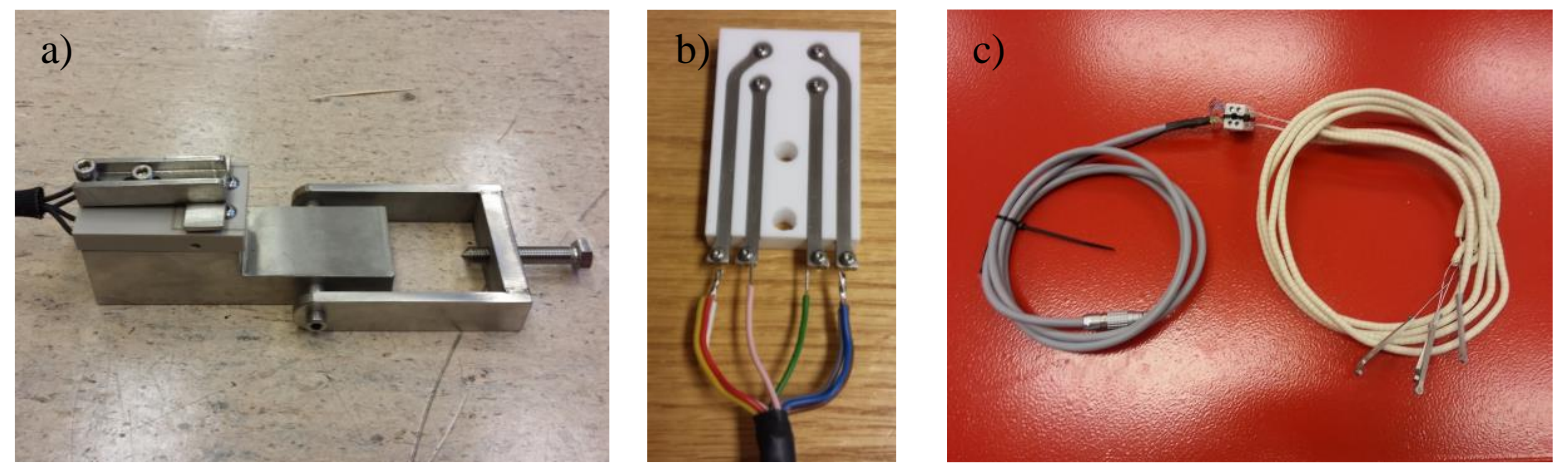

Figure 3.7 a) High temperature sensor holder. b) 4 wires Macor probe and connection interface. c) High temperature cable.

The high temperature test layout comprises a Carbolite furnace (1) with a Eurotherm controller, a laptop with the Hot Disk Thermal Constant Analyser software (2), the Transient Plane Source equipment model TPS 2500S (3) and a datalogger TML TDS 601 (4) used to monitor the gas and the cold-formed steel specimen temperatures. Figure 3.8 presents the test layout. 


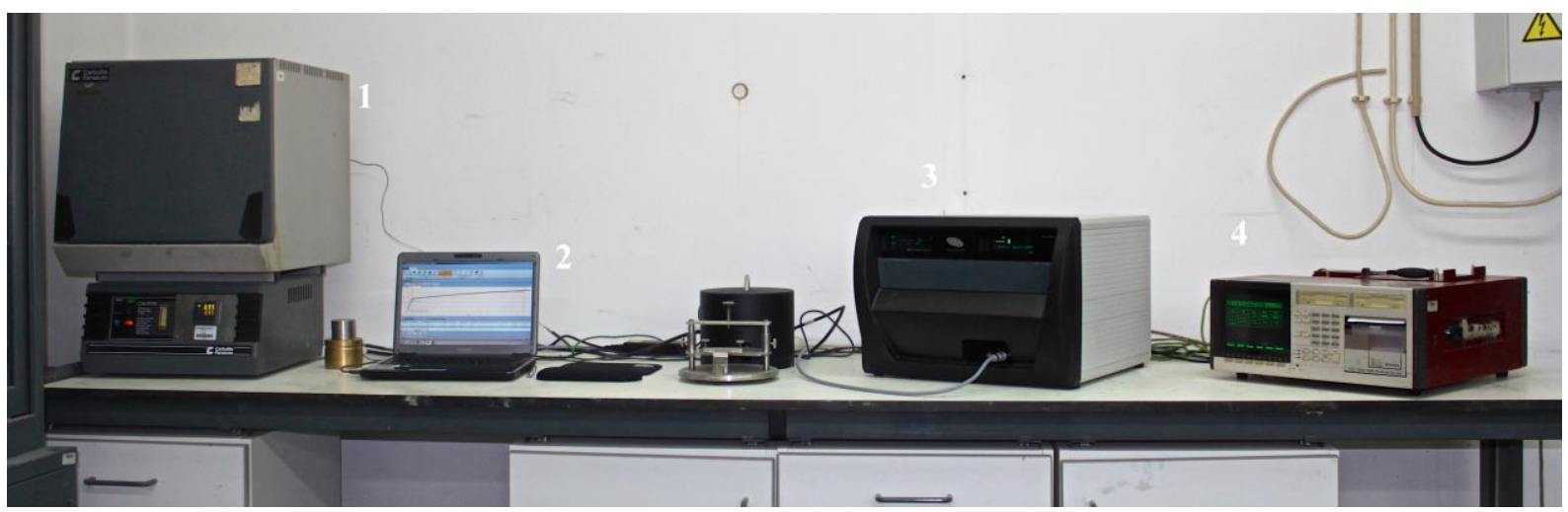

Figure 3.8 Thermal properties test layout.

As previously mentioned the special software module (thin slab module) was used since the tested specimens had reduced thickness $(2.5 \mathrm{~mm})$. The traditional analysis is based on the assumption that the sample dimensions are infinite and consequently that the sample boundaries do not influence the temperature increase measured by the used sensor. In this type of test, the outside lateral surfaces of the sample slabs must be insulated by a material (ceramic wool) with low thermal conductivity in order to reduce heat losses of these surfaces during measurement. Other parameter to take into account is the probing depth $(\Delta p[\mathrm{~mm}])$ which is defined as the distance from the sensor edge to the nearest free surface of the sample. It was found that if $\Delta p \geq$ $\sqrt{4 k t}$ the influence of the sample size on the results is negligible (He, 2005). For the thin slab module, the probing depth depends on the thermal diffusivity $\left(k\left[\mathrm{~mm}^{2} / \mathrm{s}\right]\right)$ and measuring time $(\mathrm{t}[\mathrm{s}])$ of the experiment.

\subsection{Experimental Results}

\subsubsection{Mechanical properties of the S280GD+Z steel at ambient and elevated temperatures}

\subsubsection{Stress-strain curves}

Based on the stress-strain curves obtained from experimental tests the yield strength was determined considering the $0.2 \%$ proof stress method at ambient and elevated temperatures. At elevated temperatures the $0.5 \%, 1.5 \%$ and $2.0 \%$ total strain method is also used by other researchers (Ranawaka and Mahendra, 2009; Kankanamge and Mahendran, 2011). In Figure 3.9 the results of an ambient temperature test are depicted. The stress-strain curves obtained with strain gauges and high temperature extensometer are presented as well as the method for determining the yield strength based on the $0.2 \%$ proof stress and $0.5 \%, 1.5 \%$ and $2.0 \%$ total 
strain method. The modulus of elasticity was determined from the stress-strain curve based on the tangent modulus of the initial elastic linear curve.

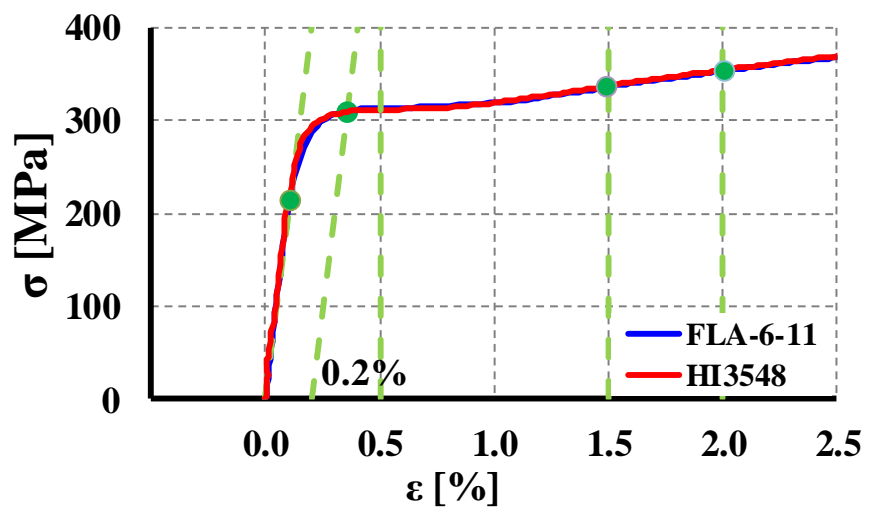

Figure 3.9 Comparison between the experimental results obtained with the high temperature extensometer Epsilon HI3548 and the extensometer FLA-6-11.

The obtained stress-strain curves at both ambient $\left(20^{\circ} \mathrm{C}\right)$ and elevated temperatures $(100,200$, $300,400,500,600,700$ and $800{ }^{\circ} \mathrm{C}$ ) are given in Figure 3.10. It is worth to mention that 3 repetitions were undertaken for each temperature level. The remaining test results are presented in Appendix A. The obtained curves for $20^{\circ} \mathrm{C}$ and $100{ }^{\circ} \mathrm{C}$ show a small yield plateau whereas for temperatures beyond $200{ }^{\circ} \mathrm{C}$ the stress-strain curves show a gradual yielding behaviour. In Table 3.2 the obtained mean results regarding the yield strength $\left(f_{y, \theta}\right)$, modulus of elasticity $\left(E_{\theta}\right)$, ultimate strength $\left(f_{u, \theta}\right)$ and proportional limit $\left(f_{p, \theta}\right)$ are presented. Detailed results for all valid experimental tests undertaken at both ambient and elevated temperatures, as well as the statistical analysis are presented in Appendix A. The observed failure modes for each tested specimen are presented in Figure 3.11.

Although the creep effect was not explicitly studied in the scope of this investigation it is worth noting that creep may have some influence on the obtained results for some temperature levels. No studies were found on the creep effect for the S280GD+Z steel. Using the available results in the literature it may be possible to provide an estimation (as a guidance) on the amount of creep in these experimental investigation. Assuming a similar behaviour between the S280GD+Z steel and the ASTM A992 (Cowan and Khandelwal, 2014) steel it can be stated that for 400 and $500^{\circ} \mathrm{C}$, for a stress level of $0.9 f_{y, 20}$ and for a time exposure of about 30 minutes (approximate duration of the tensile tests conducted in this investigation) the creep strain is reduced, specifically around $0.3 \%$. For temperatures of $600^{\circ} \mathrm{C}$ and above, creep most likely will become significant for higher stress levels and time exposure of 30 minutes (Cowan and Khandelwal, 2014) (creep strain of about $2 \%$ at $700^{\circ} \mathrm{C}$ for a stress level of $0.9 f_{y, 20}$; creep strain 
of about $5 \%$ at $800^{\circ} \mathrm{C}$ for a stress level of $0.9 f_{y, 20}$ ). Bear in mind that creep behaviour may be unique for every type of steel hence these conclusions must be verified against experimental tests in order to accurately assess the influence of creep for temperatures above $500^{\circ} \mathrm{C}$, time exposure of 120 minutes and different stress levels above $0.5 f_{y, 20}$. Finally it is worth mentioning that the existent amount of creep is included in the experimental results and also implicit in the proposed formulations based on the stress-strain model presented in the EN 1993-1-2 (2005).

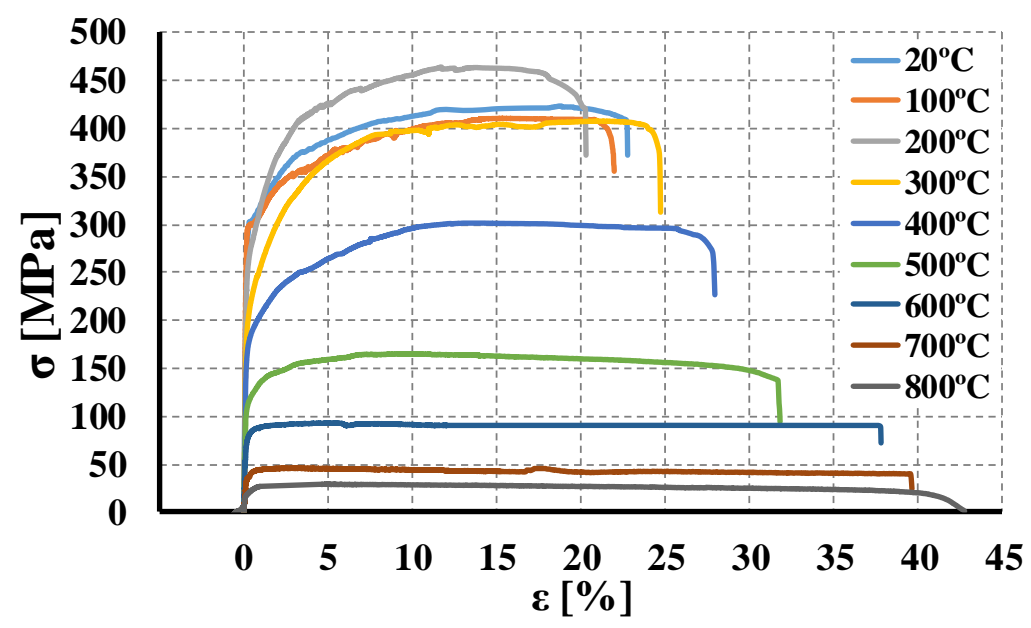

Figure 3.10 Stress-strain curves of the $\mathrm{S} 280 \mathrm{GD}+\mathrm{Z}$ steel; temperatures ranging from 20 to $800^{\circ} \mathrm{C}$.
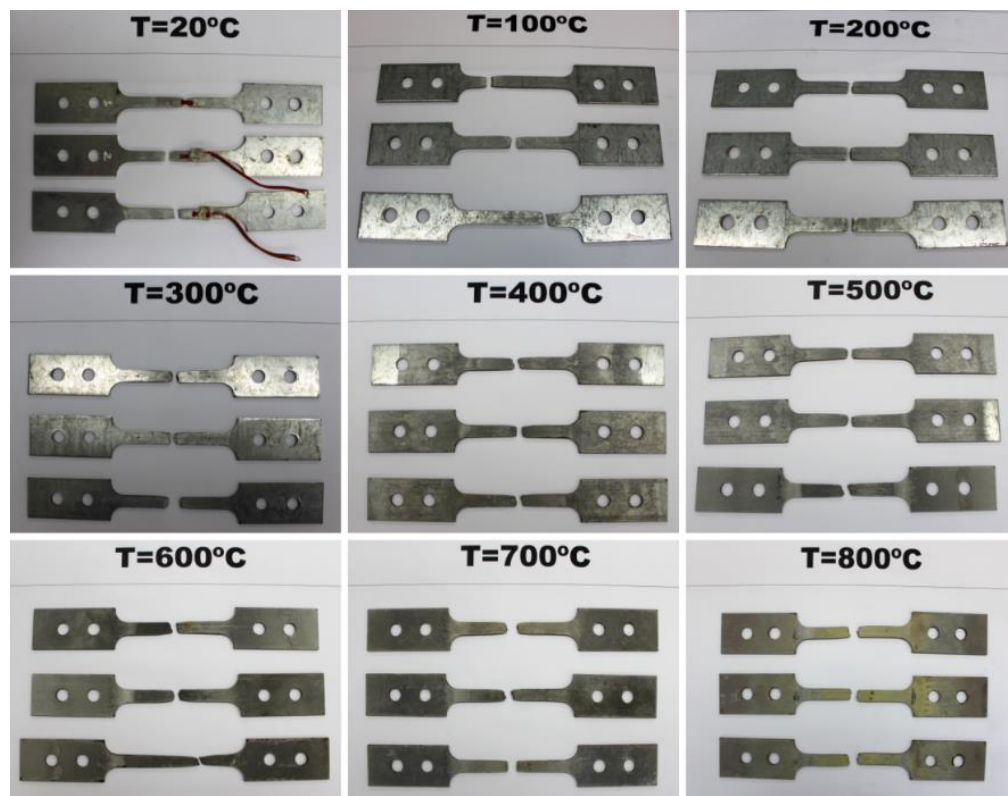

Figure 3.11 Failure modes observed in the tensile tests both at ambient and elevated temperatures ranging from 20 to $800^{\circ} \mathrm{C}$. 
Table 3.2 Mean values of the experimental results.

\begin{tabular}{c|c|c|c|c|c|c|c|c}
\hline Test & $\begin{array}{c}\text { Temperature } \\
{\left[{ }^{\mathbf{C}} \mathbf{C}\right]}\end{array}$ & $\begin{array}{c}\overline{\boldsymbol{E}}_{\boldsymbol{\theta}} \\
{[\mathbf{G P a}]}\end{array}$ & $\begin{array}{c}\overline{\boldsymbol{f}}_{\boldsymbol{y}, \mathbf{0 . 2} \%, \boldsymbol{\theta}}\left[\begin{array}{c}\overline{\boldsymbol{f}}_{\boldsymbol{y}, \mathbf{0 . 5} \%, \boldsymbol{\theta}} \\
{[\mathbf{M P a}]}\end{array}\right. \\
{[\mathbf{M P a}]}\end{array}$ & $\begin{array}{c}\overline{\boldsymbol{f}}_{\boldsymbol{y}, \mathbf{1 . 5} \%, \boldsymbol{\theta}} \\
{[\mathbf{M P a}]}\end{array}$ & $\begin{array}{c}\overline{\boldsymbol{f}}_{\boldsymbol{y}, \mathbf{2} \%, \boldsymbol{\theta}} \\
{[\mathbf{M P a}]}\end{array}$ & $\begin{array}{c}\overline{\boldsymbol{f}}_{\boldsymbol{u}, \boldsymbol{\theta}} \\
{[\mathbf{M P a}]}\end{array}$ & $\begin{array}{c}\overline{\boldsymbol{f}}_{\boldsymbol{p}, \boldsymbol{\theta}} \\
{[\mathbf{M P a}]}\end{array}$ \\
\hline CT_20 & 20 & 204.18 & 306.81 & 309.06 & 331.26 & 342.41 & 424.04 & 212.50 \\
\hline CT_100 & 100 & 200.11 & 295.24 & 297.17 & 325.29 & 342.53 & 415.49 & 209.25 \\
\hline CT_200 & 200 & 171.80 & 275.75 & 292.31 & 352.53 & 371.89 & 471.50 & 170.68 \\
\hline CT_300 & 300 & 143.59 & 223.57 & 237.61 & 284.66 & 298.18 & 397.41 & 136.37 \\
\hline CT_400 & 400 & 121.21 & 181.68 & 190.19 & 220.23 & 224.91 & 291.73 & 98.71 \\
\hline CT_500 & 500 & 84.54 & 113.67 & 122.49 & 142.73 & 146.44 & 165.85 & 80.25 \\
\hline CT_600 & 600 & 62.46 & 77.83 & 79.85 & 82.97 & 85.10 & 87.00 & 54.57 \\
\hline CT_700 & 700 & 22.21 & 36.34 & 40.32 & 49.10 & 49.83 & 50.48 & 19.26 \\
\hline CT_800 & 800 & 17.45 & 19.91 & 22.93 & 28.96 & 29.80 & 31.47 & 16.49 \\
\hline
\end{tabular}

\subsubsection{Mechanical properties reduction factors}

The reduction factors for each mechanical property $\left(k_{y, \theta}, k_{E, \theta}, k_{u, \theta}, k_{u, \theta^{*}}\right.$ and $\left.k_{p, \theta}\right)$ at elevated temperatures were determined as the ratio between the value obtained at elevated temperature to that obtained at ambient temperature except for the proportional limit which is determined in relation to the yield strength at ambient temperature. In Table 3.3 and 3.4 the yield strength, modulus of elasticity, ultimate strength and proportional limit reduction factors are presented.

The yield strength reduction factors $\left(k_{y, \theta}=f_{y, \theta} / f_{y, 20}\right)$ are presented for different strain values namely, $0.2 \%$ proof stress, $0.5 \%, 1.5 \%$ and $2 \%$ strain levels. In Figure $3.12 \mathrm{a}$ ) the reduction factors for yield strength are plotted. The reduction factors based on $0.5 \%$ total strain are relatively similar to the ones based on $0.2 \%$ proof stress. It seems that for yield strengths based on higher strain levels $(1.5 \%$ and $2.0 \%)$ the correspondent reduction factors were higher and also close to the reduction factors obtained for the ultimate strength for some temperature levels up to $500^{\circ} \mathrm{C}$. Regarding this observation, it is clear that the yield strength based on the $1.5 \%$ and $2.0 \%$ total strain should not be used in the design procedure.

The modulus of elasticity also decreases with increasing temperature. Table 3.4 presents the determined reduction factors for the modulus of elasticity at elevated temperature $\left(k_{E, \theta}=E_{\theta} / E_{20}\right)$, ultimate strength $\left(k_{u, \theta}=f_{u, \theta} / f_{u, 20}\right.$ and $\left.k_{u, \theta}{ }^{*}=f_{u, \theta} / f_{y, 20}\right)$ and proportional limit $\left(f_{p, \theta} / f_{y, 20}\right)$ for the $\mathrm{S} 280 \mathrm{GD}+\mathrm{Z}$ steel. In Figure 3.12b) the reduction factors for the modulus of elasticity at elevated temperatures are depicted. Between $100{ }^{\circ} \mathrm{C}$ and $700{ }^{\circ} \mathrm{C}$ the degradation of the reduction factors is almost linear. In Figure 3.12c) the obtained reduction factors for the ultimate strength $\left(f_{u, \theta} / f_{u, 20}\right)$ and for the proportional limit $\left(f_{p, \theta} / f_{y, 20}\right)$ are also presented. 
Table 3.3 Yield strength reduction factors for different strain values.

\begin{tabular}{c|c|c|c|c}
\hline \multicolumn{5}{|c}{ Yield strength reduction factors $-\boldsymbol{K}_{\boldsymbol{y}, \boldsymbol{\theta}}$} \\
\hline \multirow{2}{*}{$\mathbf{T}\left({ }^{\mathbf{}} \mathbf{C}\right)$} & \multicolumn{4}{|c}{$\boldsymbol{f}_{\boldsymbol{y}, \boldsymbol{\theta}} / \boldsymbol{f}_{\mathbf{y}, \mathbf{2 0}}$} \\
\cline { 2 - 5 } & $\mathbf{0 . 2 0 \%}$ & $\mathbf{0 . 5 0 \%}$ & $\mathbf{1 . 5 0 \%}$ & $\mathbf{2 . 0 0 \%}$ \\
\hline $\mathbf{2 0}$ & 1 & 1 & 1 & 1 \\
\hline $\mathbf{1 0 0}$ & 0.962 & 0.961 & 0.966 & 1.000 \\
\hline $\mathbf{2 0 0}$ & 0.898 & 0.945 & 1.047 & 1.086 \\
\hline $\mathbf{3 0 0}$ & 0.728 & 0.768 & 0.846 & 0.870 \\
\hline $\mathbf{4 0 0}$ & 0.592 & 0.615 & 0.654 & 0.656 \\
\hline $\mathbf{5 0 0}$ & 0.370 & 0.396 & 0.424 & 0.427 \\
\hline $\mathbf{6 0 0}$ & 0.253 & 0.258 & 0.246 & 0.248 \\
\hline $\mathbf{7 0 0}$ & 0.118 & 0.130 & 0.145 & 0.145 \\
\hline $\mathbf{8 0 0}$ & 0.064 & 0.074 & 0.086 & 0.087 \\
\hline
\end{tabular}

Table 3.4 Modulus of elasticity, ultimate strength and proportional limit reduction factors.

\begin{tabular}{|c|c|c|c|c|}
\hline \multicolumn{5}{|c|}{ Reduction factors - $K_{E, \theta} ; K_{u, \theta} ; K_{u, \theta}{ }^{*} ; K_{p, \theta}$} \\
\hline $\mathbf{T}\left({ }^{\circ} \mathrm{C}\right)$ & $E_{\theta} / E_{20}$ & $f_{u, \theta} / f_{u, 20}$ & $f_{u, \theta} / f_{y, 20}$ & $f_{p, \theta} / f_{y, 20}$ \\
\hline 20 & 1 & 1 & 1.382 & 0.692 \\
\hline 100 & 0.980 & 0.979 & 1.354 & 0.682 \\
\hline 200 & 0.841 & 1.111 & 1.537 & 0.556 \\
\hline 300 & 0.703 & 0.937 & 1.295 & 0.444 \\
\hline 400 & 0.593 & 0.687 & 0.951 & 0.321 \\
\hline 500 & 0.414 & 0.391 & 0.541 & 0.261 \\
\hline 600 & 0.305 & 0.205 & 0.284 & 0.177 \\
\hline 700 & 0.108 & 0.045 & 0.165 & 0.062 \\
\hline 800 & 0.085 & 0.038 & 0.103 & 0.053 \\
\hline
\end{tabular}

Regarding the ultimate strength, it was observed a singular behaviour at $200{ }^{\circ} \mathrm{C}$. As observed in the investigations performed by Ranawaka and Mahendran (2009) and Kankanamge and Mahendran (2011), for low strength steels the ultimate strength was higher at $200{ }^{\circ} \mathrm{C}(\approx 11 \%)$ than at ambient temperature $\left(20^{\circ} \mathrm{C}\right)$. This behaviour can be discussed in relation to the chemical composition of the steel tested. Increasing the temperature up to $200^{\circ} \mathrm{C}$ some chemical reactions and transformations in the steel base may occur leading to an increase of the ultimate strength (Ranawaka and Mahendran, 2009; Kankanamge and Mahendran, 2011). When the temperature grows beyond $200{ }^{\circ} \mathrm{C}$ it seems that these chemical reactions are retarded and as a consequence the ultimate strength reduces.

Regarding the ductility of the cold-formed steel tested, it was found that the ductility increased with increasing temperature from $200{ }^{\circ} \mathrm{C}$ to $800^{\circ} \mathrm{C}$. From Figure 3.10 it can be stated that the tested steel is ductile as expected since it has low levels of carbon. 

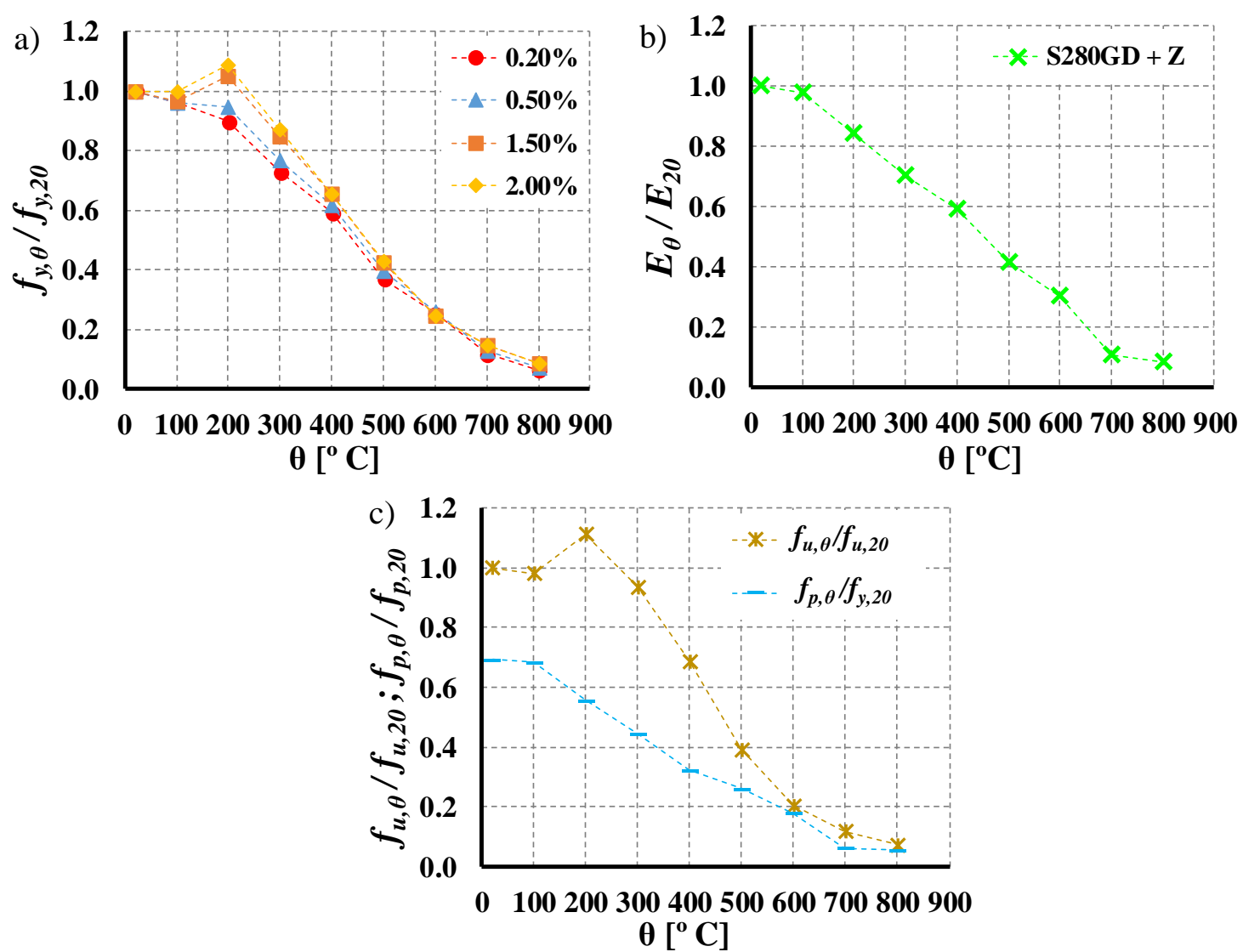

Figure 3.12 Reduction factors taken from the experimental tests. a) Yield strength. b) Elastic modulus. c) Ultimate strength and proportional limit.

\subsubsection{Comparison with available results in the literature}

Some researchers have already presented some relevant results regarding the reduction factors for mechanical properties of low and high strength steels used in cold-formed steel structures at elevated temperatures (Lee et al., 2003; Outinen, 1999; Ranawaka and Mahendran, 2009; Kankanamge and Mahendran, 2011; Makelainen et al., 1998; Chen and Young, 2007; Mecozzi and Zhao, 2005; Outinen and Makelainen, 2002). In this section those results will be compared with the ones obtained in this experimental research (Figure 3.13, 3.14 and 3.15). The results presented by Kankanamge and Mahendran (2011) for low strength steel G250 with $1.95 \mathrm{~mm}$ are in good agreement with the ones obtained in this investigation for the yield strength reduction factors. Regarding the modulus of elasticity, the reported values are also relatively similar but slightly unsafe for some temperature levels despite the difference in the steel grade and thicknesses used. It is worth to mention that the same displacement and heating rates were used in both investigations. Ranawaka and Mahendran (2009) results for the low strength steel G250 are conservative for the yield strength reduction factors. Regarding the modulus of 
elasticity, the results presented by Ranawaka and Mahendran (2009) are slightly higher for the low strength steel G250 when compared with the results reported in this investigation. The results provided by Outinen $(1999,2002)$ for the S350GD steel with $2.0 \mathrm{~mm}$ thickness are unconservative beyond $200^{\circ} \mathrm{C}$ both for yield strength and modulus of elasticity, when compared with the results presented in this investigation. Mecozzi and Zhao (2005) results are very overconservative for yield strength as well as for the modulus of elasticity reduction factors for temperatures above $200{ }^{\circ} \mathrm{C}$, when compared with the results presented in this investigation. The results presented by Ranawaka, Outinen, Mecozzi and Chen $(2009,1999,2002,2005)$ are not in agreement for the cold-formed steel considered in this study. Therefore, most equations available in the literature are not accurate to determine the reduction factors for yield strength and modulus of elasticity of the $\mathrm{S} 280 \mathrm{GD}+\mathrm{Z}$ steel tested at elevated temperatures. Generally, observing the results available in the literature high strength steels present higher reduction factors both for yield strength and elastic modulus. Also it is clear that the results provided by all researchers are more uniform for the modulus of elasticity than for the yield strength. It seems that the elastic modulus reduction factors are less dependent on the steel grade than the yield strength reduction factors, hence the influence of the steel grade on the reduction factors of the modulus of elasticity may be considered less important (Ranawaka and Mahendran, 2009; Kankanamge and Mahendran, 2011). The reduction factors for the ultimate strength determined in the different investigations are also depicted in Figure 3.15. The same behaviour was observed at about $200^{\circ} \mathrm{C}$ in some experimental investigations (Ranawaka and Mahendran, 2009; Kankanamge and Mahendran, 2011), predominantly for low strength steels. This behaviour was already explained.

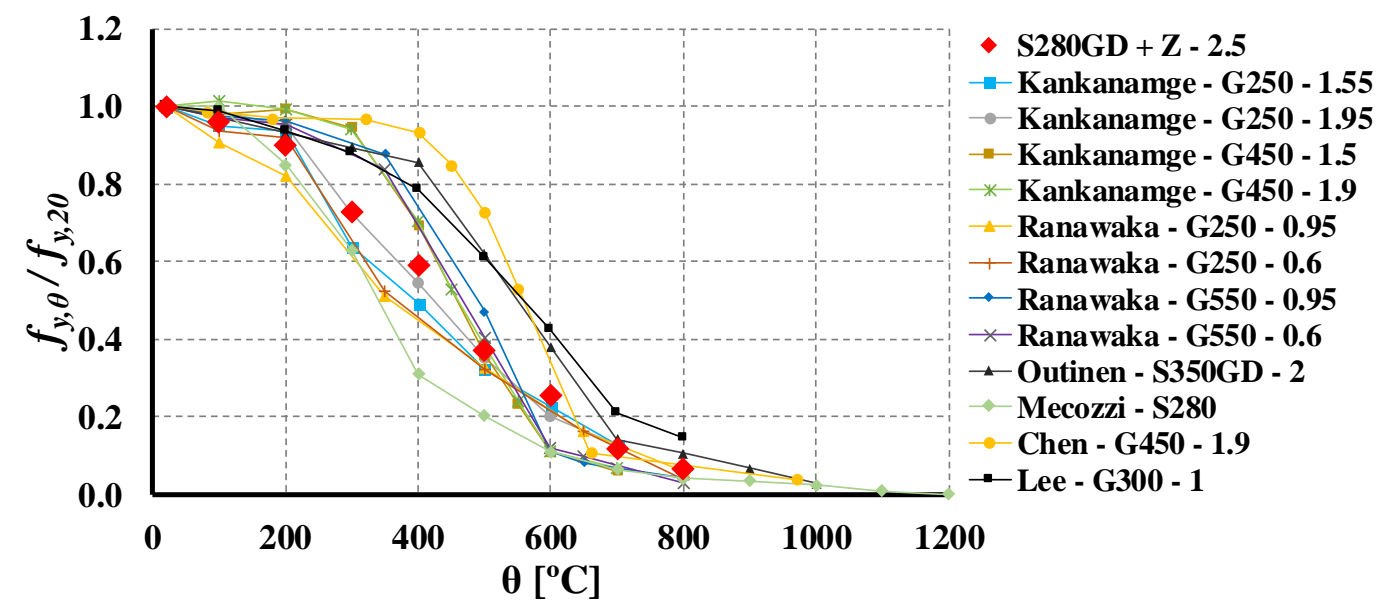

Figure 3.13 Comparison of the yield strength reduction factors at elevated temperatures determined in the scope of this investigation and with other authors. 


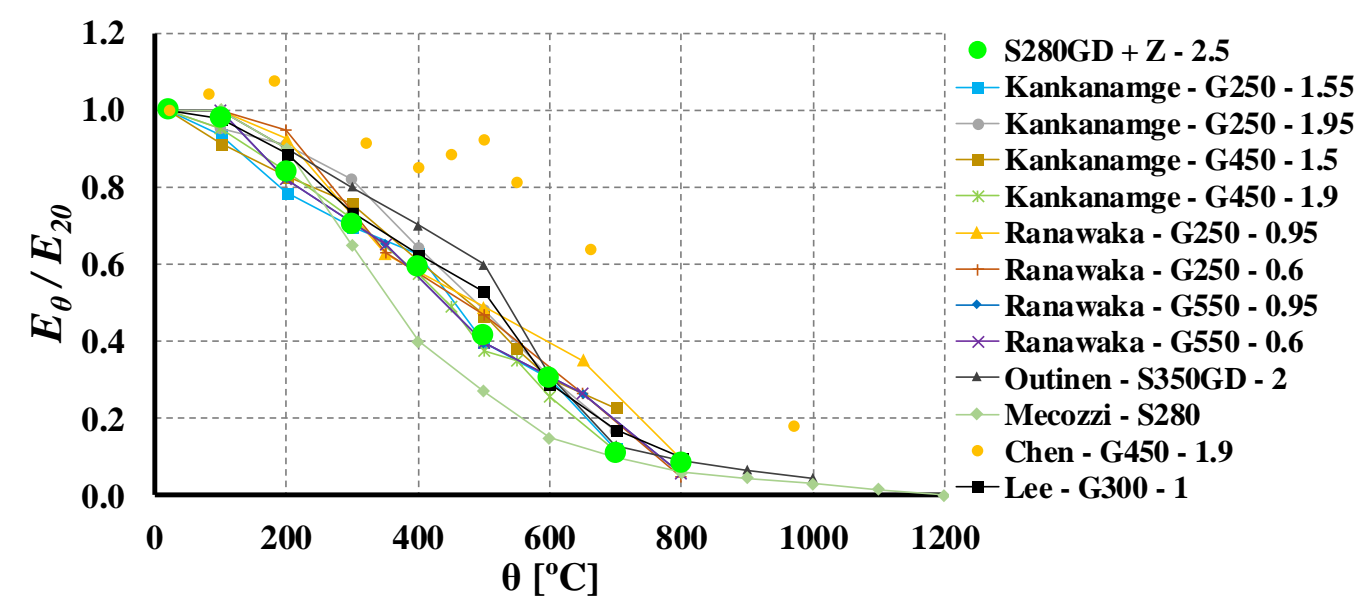

Figure 3.14 Comparison of the modulus of elasticity reduction factors at elevated temperatures determined in the scope of this investigation and with other authors.

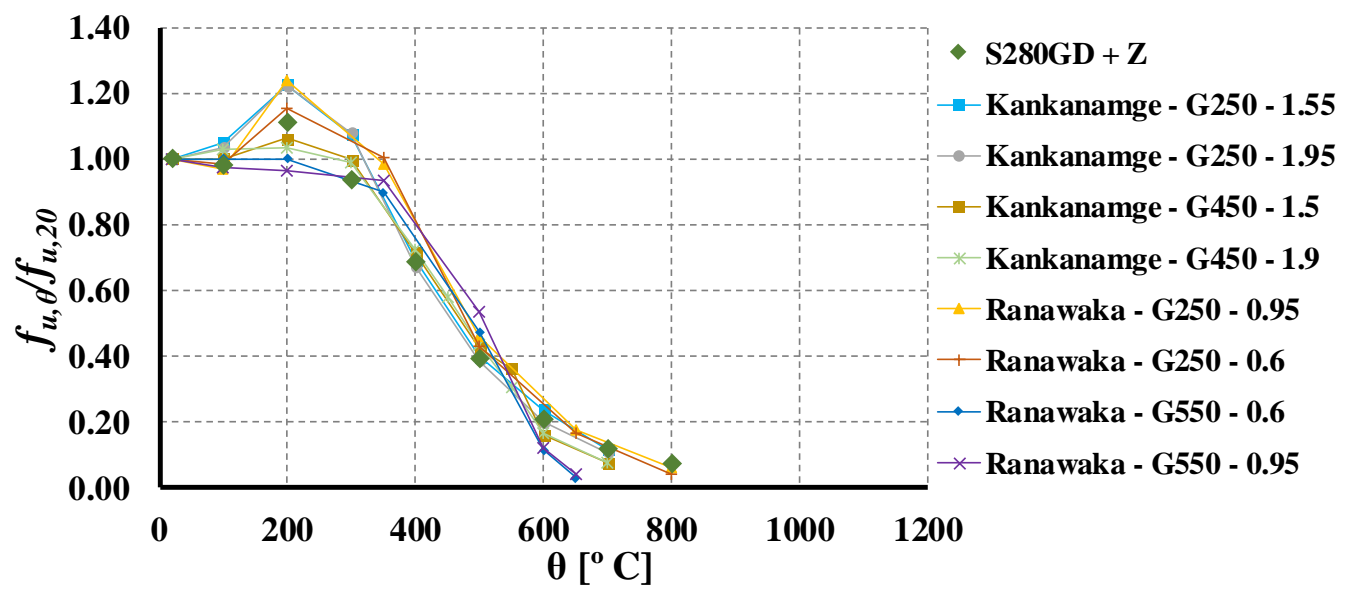

Figure 3.15 Comparison of the ultimate strength reduction factors at elevated temperatures determined in the scope of this investigation and with other authors.

\subsubsection{Comparison with available design standards}

The experimental results obtained in this research were compared with the current design standards, such as the EN 1993-1-2 (2005), BS 5950-Part 8 (1990) and AS 4100 (1998). The EN 1993-1-2 (2005) provides the same reduction factors used for class 4 hot-rolled steels for $0.2 \%$ proof stress whereas the BS 5950-Part 8 (1990) provide the reduction factors for steels used in cold-formed steel structures for yield strength at total strain levels of $0.5 \%, 1.5 \%$ and $2.0 \%$. Figures $3.16,3.17$ and 3.18 show the comparison between the reduction factors provided by the design standards against the reduction factors determined in this investigation for both yield strength and modulus of elasticity of a low strength cold-formed steel S280GD+Z. From the results it is clear that all design standards considered overestimate the reduction factors for 
yield strength. The EN 1993-1-2 (2005) overestimates the reduction factors beyond $200{ }^{\circ} \mathrm{C}$ and up to $600{ }^{\circ} \mathrm{C}$. For instance, for temperatures ranging from $200{ }^{\circ} \mathrm{C}$ up to $300{ }^{\circ} \mathrm{C}$ the prediction presented in the EN 1993-1-2 (2005) is about $8 \%$ higher than the reduction factor determined in the experimental tests, whereas for $500{ }^{\circ} \mathrm{C}$ the difference was about $30 \%$. Above $600{ }^{\circ} \mathrm{C}$ the results are in good agreement. The reduction factors provided by the BS 5950-Part 8 (1990) and AS 4100 (1998) are higher in the entire range of considered temperatures, suggesting that their reduction factors should be reviewed. Also reduction factors for different steel grades should be introduced since the influence of the steel grade on the determined reduction factors for yield strength has been proved by other researchers (Ranawaka and Mahendran, 2009; Kankanamge and Mahendran, 2011). Regarding the modulus of elasticity, the BS 5959-Part 8 (1990) does not include reduction factors. The reduction factors provided by the EN 1993-1-2 (2005) are unsafe up to $600{ }^{\circ} \mathrm{C}$ and the AS 4100 (1998) provides unsafe reduction factors beyond $100{ }^{\circ} \mathrm{C}$. The difference between the results from this experimental investigation and the predictions presented in the EN 1993-1-2 (2005) is quite relevant for temperatures ranging from $300{ }^{\circ} \mathrm{C}$ to $500{ }^{\circ} \mathrm{C}$ (average $\approx 20 \%$ ).
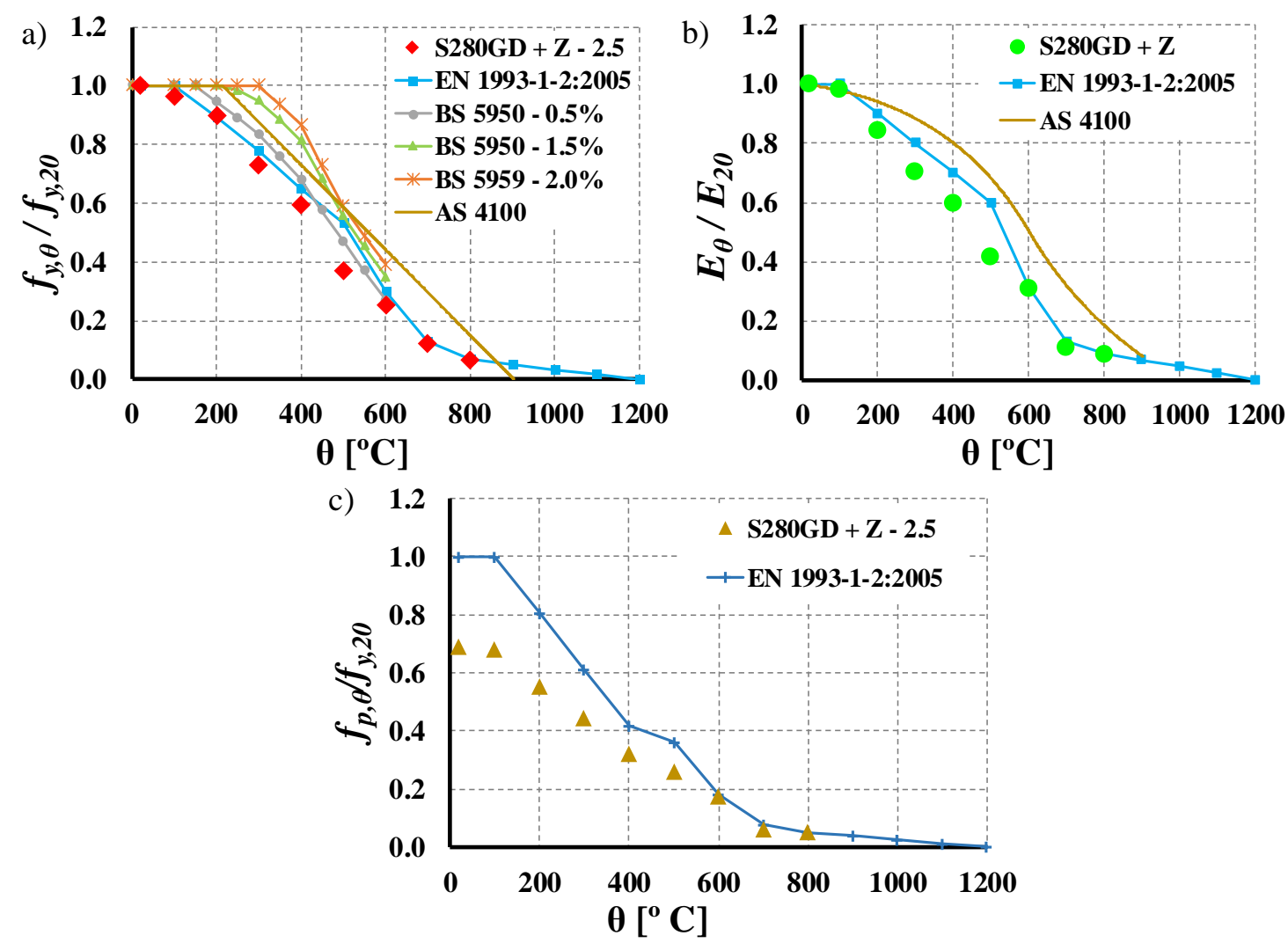

Figure 3.16 Comparison between the reduction factors determined in this research with the ones available in the current design standards. a) Yield strength reduction factors. b) Modulus of elasticity reduction factors. c) Proportional limit reduction factors. 
Comparing the proportional limit reduction factors determined in this experimental investigation with the ones provided in the EN 1993-1-2 (2005), it was found that the ones presented in the EN 1993-1-2 (2005) are unconservative for temperatures up to $500^{\circ} \mathrm{C}$. This observation was more or less expected since the reduction factors predicted in the EN 1993-12: (2005) were based on hot-rolled steels.

\subsubsection{Predictive equations for mechanical properties}

Based on the experimental results empirical equations were proposed to predict the evolution of the reduction factors for yield strength with temperature ranging from $20{ }^{\circ} \mathrm{C}$ to $800{ }^{\circ} \mathrm{C}$. The empirical equations (Equation 3.1) derived for the yield strength reduction factors were determined based on the $0.2 \%$ proof stress method. In Figure $3.17 \mathrm{a}$ ) it is possible to observe the good agreement between the proposed model and the experimental results. Additionally, already existent proposals that better fit the results of this investigation are presented.

$$
\begin{array}{cc}
\frac{f_{y, \theta}}{f_{y, 20}}=-5.5 \times 10^{-4} \theta+1.011 & 20{ }^{\circ} \mathrm{C} \leq \theta \leq 200{ }^{\circ} \mathrm{C} \\
\frac{f_{y, \theta}}{f_{y, 20}}=0.070876 \times\left(25.55-\theta^{0.482}\right) & 200{ }^{\circ} \mathrm{C}<\theta \leq 800{ }^{\circ} \mathrm{C}
\end{array}
$$

The predictive formulations proposed by Ranawaka and Kakanamge (2009, 2011) are conservative in comparison to the ones here presented. Nevertheless, the differences are not very relevant meaning that each model may be capable to accurately predict the reduction factors for yield strength of low strength cold-formed steels.

As for the yield strength, elevated temperatures lead to degradation of the modulus of elasticity. In order to predict the degradation of the modulus of elasticity with the temperature a new set of empirical equations (Equation 3.2) was developed for the $280 \mathrm{GD}+\mathrm{Z}$ steel. This equation is valid for low strength cold-formed steels. For instance, the equations proposed are similar to the ones provided by Ranawaka (2009) for the low strength steel G250. The proposed predictive equations depicted in Figure 3.17b) are in good agreement with the test results.

$$
\frac{E_{\theta}}{E_{20}}=-2.5 \times 10^{-4} \times \theta+1.005 \quad 20{ }^{\circ} \mathrm{C} \leq \theta \leq 100{ }^{\circ} \mathrm{C}
$$




$$
\begin{array}{ll}
\frac{E_{\theta}}{E_{20}}=-1.4 \times 10^{-3} \times \theta+1.118 & 100{ }^{\circ} \mathrm{C}<\theta \leq 700^{\circ} \mathrm{C} \\
\frac{E_{\theta}}{E_{20}}=-5.3 \times 10^{-4} \times \theta+0.509 & 700{ }^{\circ} \mathrm{C}<\theta \leq 800^{\circ} \mathrm{C}
\end{array}
$$
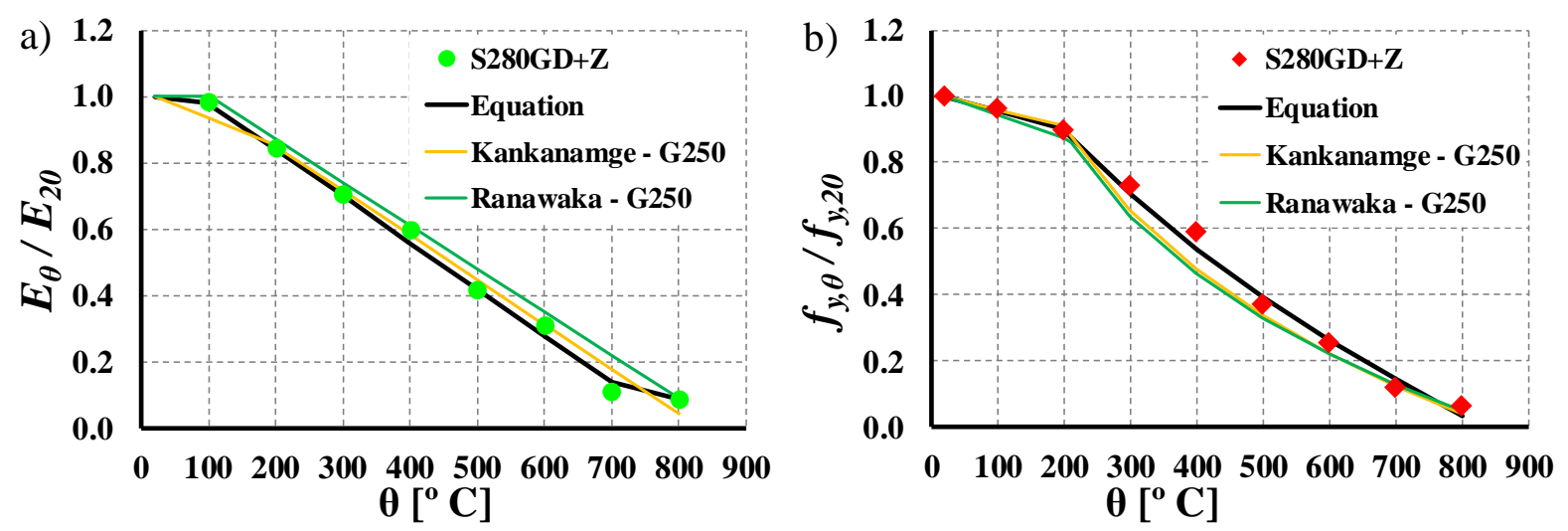

Figure 3.17 Comparison between proposed models and experimental results. a) Modulus of elasticity. b) Yield strength.

The previous proposal (Equation 3.2) may be slightly conservative. As an alternative a different model may be proposed (Equation 3.3). The proposal presented based on this investigation may be slightly conservative since it is possible to propose a model based only in two equations, which may be easier to use (Equation 3.3). The proposals presented in this research are very similar to the ones presented by Ranawaka and Kakanamge (2009; 2011).

$$
\begin{array}{ll}
\frac{E_{\theta}}{E_{20}}=-2.5 \times 10^{-4} \times \theta+1.005 & 20{ }^{\circ} \mathrm{C} \leq \theta \leq 100^{\circ} \mathrm{C} \\
\frac{E_{\theta}}{E_{20}}=-1.345 \times 10^{-3} \times \theta+1.109 & 100^{\circ} \mathrm{C}<\theta \leq 800^{\circ} \mathrm{C}
\end{array}
$$

\subsubsection{Stress-strain relationship to Ramberg-Osgood methodology}

There are two types of stress-strain curves, namely sharp yielding and gradual yielding. In this investigation sharp yielding stress-strain curves, with a small yield plateau, were obtained for lower temperatures $\left(20^{\circ} \mathrm{C}\right.$ and $100{ }^{\circ} \mathrm{C}$ ) and gradual yielding curves for higher temperatures $\left(200{ }^{\circ} \mathrm{C}, 300{ }^{\circ} \mathrm{C}, 400{ }^{\circ} \mathrm{C}, 500{ }^{\circ} \mathrm{C}, 600{ }^{\circ} \mathrm{C}, 700{ }^{\circ} \mathrm{C}\right.$ and $\left.800{ }^{\circ} \mathrm{C}\right)$. The stress-strain relationship of the tested steel coupons under steady state test was modelled considering the methodology 
presented by W. Ramberg and W. R. Osgood (1943) and then compared with the proposals presented by other researchers. The Ramberg-Osgood (1943) model was developed to describe stress-strain curves at ambient temperature. Based on this model Olawale and Plank (1988), Outinen (1999) and Lee et al. (2003) proposed stress-strain models at elevated temperatures respectively for hot-rolled steels, for S355 hot-rolled steel and for light gauge steel. The expression based on the Ramberg-Osgood model used in this investigation is as follows (Equation 4) (Gaggiano et al., 2004; MMPDS-01, 2003):

$$
\varepsilon_{\theta}=\frac{f}{E_{\theta}}+0.002 \times\left(\frac{f}{f_{y, \theta}}\right)^{n_{\theta}}
$$

The Ramberg-Osgood coefficient was determined based on the provisions presented in MMPDS-01 (2003):

$$
\begin{gathered}
\varepsilon_{u s, \theta}=100\left(\varepsilon_{r, \theta}-\frac{f_{u, \theta}}{E_{\theta}}\right) \\
n_{\theta}=\frac{\ln \left(\frac{\varepsilon_{u s, \theta}}{0.2}\right)}{\ln \left(\frac{f_{u, \theta}}{f_{y, \theta}}\right)}
\end{gathered}
$$

where $\varepsilon_{\theta}$ is the strain at temperature $\theta\left({ }^{\circ} \mathrm{C}\right), \varepsilon_{r, \theta}$ is the strain at rupture, $\varepsilon_{u s, \theta}$ is the plastic strain at maximum tension load, $f$ is the stress, $E_{\theta}$ is the modulus of elasticity, $f_{u, \theta}$ is ultimate strength and $f_{y, \theta}$, is the yield strength at temperature $\theta\left({ }^{\circ} \mathrm{C}\right)$. The $n_{\theta}$ parameter in this formulation is reciprocal to the strain hardening coefficient (MMPDS, 2003). The evolution of the parameter $n_{\theta}$ with temperature is presented in Table 3.5 for each temperature level. The stress-strain curves obtained using this formulation are compared with the experimental results obtained (Figure 3.18).

Table $3.5 n$ parameter determined for each temperature level.

\begin{tabular}{c|c|c|c|c|c|c|c|c|c}
\hline & \multicolumn{10}{|c}{ Temperature [ $\left.{ }^{\circ} \mathbf{C}\right]$} \\
\cline { 2 - 10 } & 20 & 100 & 200 & 300 & 400 & 500 & 600 & 700 & 800 \\
\hline $\mathbf{n}_{\boldsymbol{\theta}}$ & 14.602 & 13.724 & 8.586 & 8.353 & 10.411 & 13.397 & 47.024 & 16.113 & 11.701 \\
\hline
\end{tabular}

For low temperatures $\left(20^{\circ} \mathrm{C}\right.$ and $\left.100^{\circ} \mathrm{C}\right)$ the proposed equations are not suitable since the original equations were developed only for gradual yielding stress-strain curves and for lower 
temperatures sharp yielding stress-strain curves with a visible yield plateau were obtained. Between $200^{\circ} \mathrm{C}$ and $300^{\circ} \mathrm{C}$ the proposed equations fit reasonably well up to $1.6 \%$ strain however the equations may be considered slightly unsafe in the strain range of $0.2 \%$ to $1.3 \%$. For temperatures above $300^{\circ} \mathrm{C}$ the proposed equations are in very good agreement with stress-strain curves determined in the experimental tests.

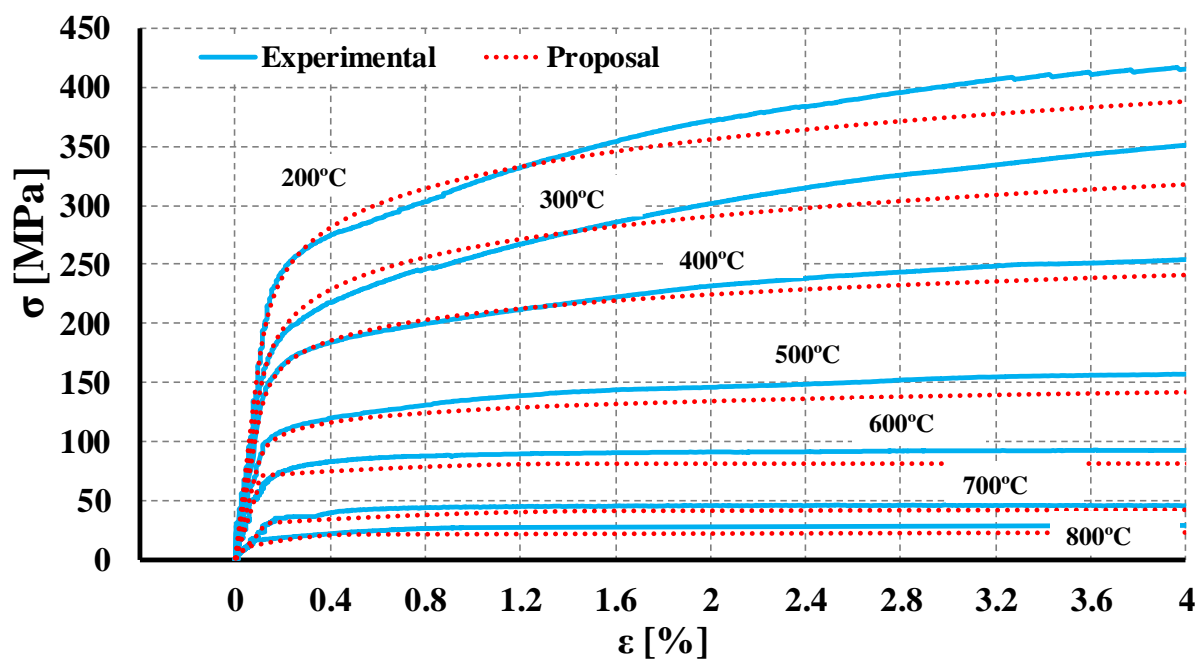

Figure 3.18 Comparison between experimental stress-strain curves and the proposed stressstrain model at elevated temperatures based on the Ramberg-Osgood model.

The proposed equations in this investigation for the stress-strain curves were also compared with the models proposed by Ranawaka (2009) (Equation 3.5), Chen and Young (2007) (Equation 3.6) and Kankanamge (2011) (Figure 3.19). The model proposed by Kankanamge (2011) is very similar to the one proposed by Ranawaka (2009). The only change is the $\beta$ parameter which has assumed the value 1.5 instead of 0.86 .

$$
\begin{gathered}
\varepsilon_{T}=\frac{f_{T}}{E_{T}}+\beta \times\left(\frac{f_{y, T}}{E_{T}}\right) \times\left(\frac{f_{T}}{f_{y, T}}\right)^{\eta_{T}} \\
\eta_{T}=0.000138 \times T^{2}-0.085468 T+19.212 \quad 350^{\circ} \mathrm{C} \leq \mathrm{T} \leq 800^{\circ} \mathrm{C} \\
\beta=0.86 \\
E_{T}=\left\{\begin{array}{c}
\frac{f_{T}}{E_{T}}+0.002 \times\left(\frac{f_{T}}{f_{y, T}}\right)^{n_{T}} \text { for } f_{T} \leq f_{y, T} \\
\frac{f_{T}-f_{y, T}}{E_{y, T}}+\varepsilon_{u, T} \times\left(\frac{f_{T}-f_{y, T}}{f_{u, T}-f_{y, T}}\right)^{m_{T}} \times \varepsilon_{y, T} \text { for } f_{T}>f_{y, T}
\end{array}\right.
\end{gathered}
$$


and

$$
\begin{gathered}
E_{y, T}=\frac{E_{T}}{1+0.002 \times n_{T} \times \frac{E_{T}}{f_{y, T}}} \\
n_{T}=20-0.6 \times \sqrt{T} \\
m_{T}=1+\frac{T}{350}
\end{gathered}
$$

Comparing all proposed models, it was found that above $500^{\circ} \mathrm{C}$ all models are in good agreement with the experimental results. Chen and Young (2007) model seem to be the less accurate. Kankanamge (2011) model is unsafe for lower levels of temperature $\left(300^{\circ} \mathrm{C}\right.$ and $400^{\circ} \mathrm{C}$ ), as well as the model proposed by Ranawaka (2009). Overall, the proposed model in this investigation is the one that fits best to the experimental stress-strain curves.

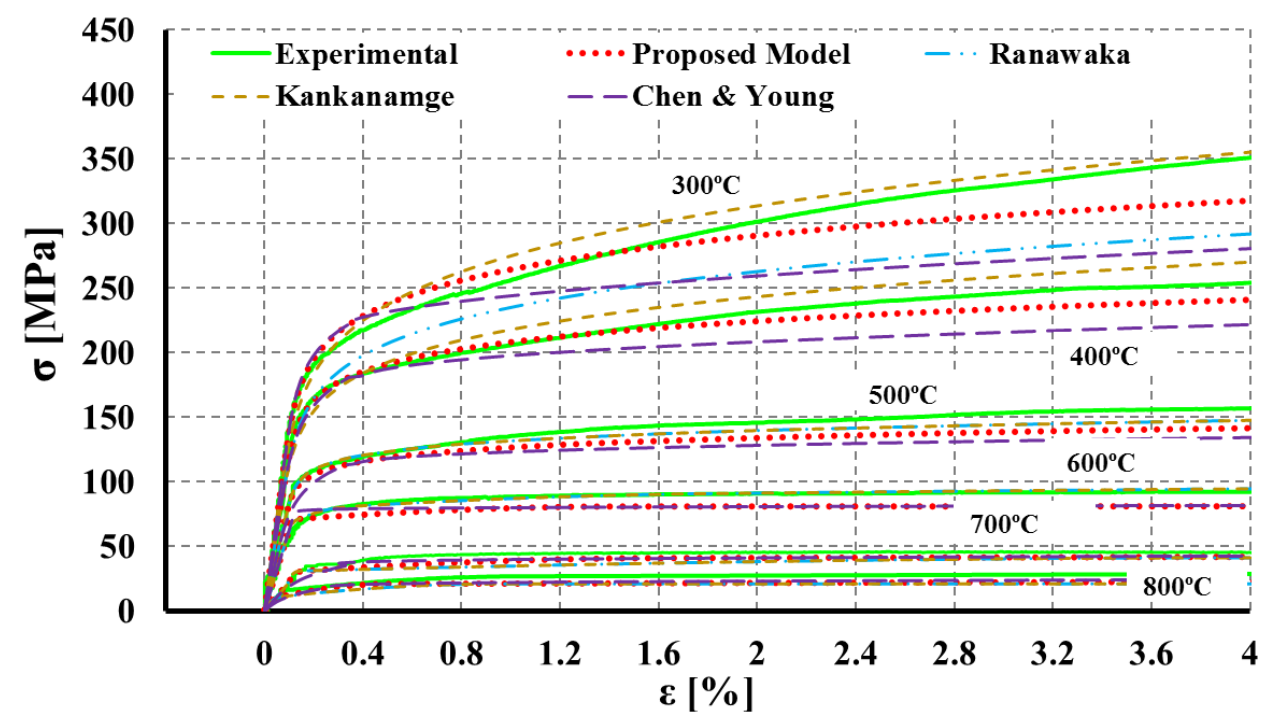

Figure 3.19 Comparison of the proposed stress-strain model with other predictive stress-strain models proposed by other authors.

\subsubsection{Stress-strain relationship of EN 1993-1-2}

The EN 1993-1-2 (2005) also proposes a stress-strain curve model which is based on hot-rolled steel, this could be the reason why it does not accurately reproduce the behavior of low strength cold-formed steel S280GD $+Z$ at elevated temperatures. The stress-strain model proposed by the EN 1993-1-2 (2005) is given by (Equation 3.7): 


$$
\sigma=\left\{\begin{array}{cl}
\varepsilon E_{a, \theta} & \text { for } \varepsilon \leq \varepsilon_{p, \theta} ; \\
f_{p, \theta}-c+\left(\frac{b}{a}\right)\left[a^{2}-\left(\varepsilon_{y, \theta}-\varepsilon\right)^{2}\right]^{0.5} & \text { for } \varepsilon_{p, \theta} \leq \varepsilon \leq \varepsilon_{y, \theta} \\
f_{y, \theta} & \text { for } \varepsilon_{y, \theta} \leq \varepsilon \leq \varepsilon_{t, \theta} \\
f_{y, \theta}\left[1-\frac{\left(\varepsilon-\varepsilon_{t, \theta}\right)}{\left(\varepsilon_{u, \theta}-\varepsilon_{t, \theta}\right)}\right] & \text { for } \varepsilon_{t, \theta} \leq \varepsilon \leq \varepsilon_{u, \theta} \\
0 & \text { for } \varepsilon=\varepsilon_{u, \theta}
\end{array}\right.
$$

and

$$
\begin{gathered}
\varepsilon_{p, \theta}=\frac{f_{p, \theta}}{E_{a, \theta}} ; \quad \varepsilon_{y, \theta}=0.02 ; \quad \varepsilon_{t, \theta}=0.15 ; \quad \varepsilon_{u, \theta}=0.20 ; \\
a^{2}=\left(\varepsilon_{y, \theta}-\varepsilon_{p, \theta}\right)\left(\varepsilon_{y, \theta}-\varepsilon_{p, \theta}+\frac{c}{E_{a, \theta}}\right) \\
b^{2}=c\left(\varepsilon_{y, \theta}-\varepsilon_{p, \theta}\right) E_{a, \theta}+c^{2} \\
c=\frac{\left(f_{y, \theta}-f_{p, \theta}\right)^{2}}{\left(\varepsilon_{y, \theta}-\varepsilon_{p, \theta}\right) E_{a, \theta}-2\left(f_{y, \theta}-f_{p, \theta}\right)}
\end{gathered}
$$

This model does not take into account the strain hardening of steel, but according to the EN 1993-1-2 (2005) the stress-strain model can be extended by the strain hardening model for temperatures below $400^{\circ} \mathrm{C}$ given in the Annex A (Equation 3.8):

$$
\sigma_{a}=\left\{\begin{array}{cl}
50\left(f_{u, \theta}-f_{y, \theta}\right) \varepsilon+2 f_{y, \theta}-f_{u, \theta} & \text { for } 0.02<\varepsilon<0.04 \\
f_{u, \theta} & \text { for } 0.04 \leq \varepsilon \leq 0.15 \\
f_{u, \theta}[1-20(\varepsilon-0.15)] & \text { for } 0.15<\varepsilon<0.2 \\
0 & \text { for } \varepsilon \geq 0.2
\end{array}\right.
$$

where the ultimate strength at elevated temperatures should be determined as follows:

$$
f_{u, \theta}=\left\{\begin{array}{cl}
1.25 f_{y, \theta} & \text { for } \theta_{a}<300^{\circ} C ; \\
f_{y, \theta}\left(2-0.0025 \theta_{a}\right) & \text { for } 300^{\circ} C \leq \theta_{a}<400^{\circ} C \\
f_{y, \theta} C & \text { for } \theta_{a} \geq 400^{\circ} C
\end{array}\right.
$$

The Eurocode models were compared with the experimental stress-strain curves as depicted in Figure 3.20a) and b) for both models presented in the EN 1993-1-2 (2005). As expected these models are not suitable to describe the stress-strain curves of cold-formed steels since these have been created for hot-rolled steels. Again, this shows that new stress-strain models should be developed specifically for steels used in cold-formed steel structures. 

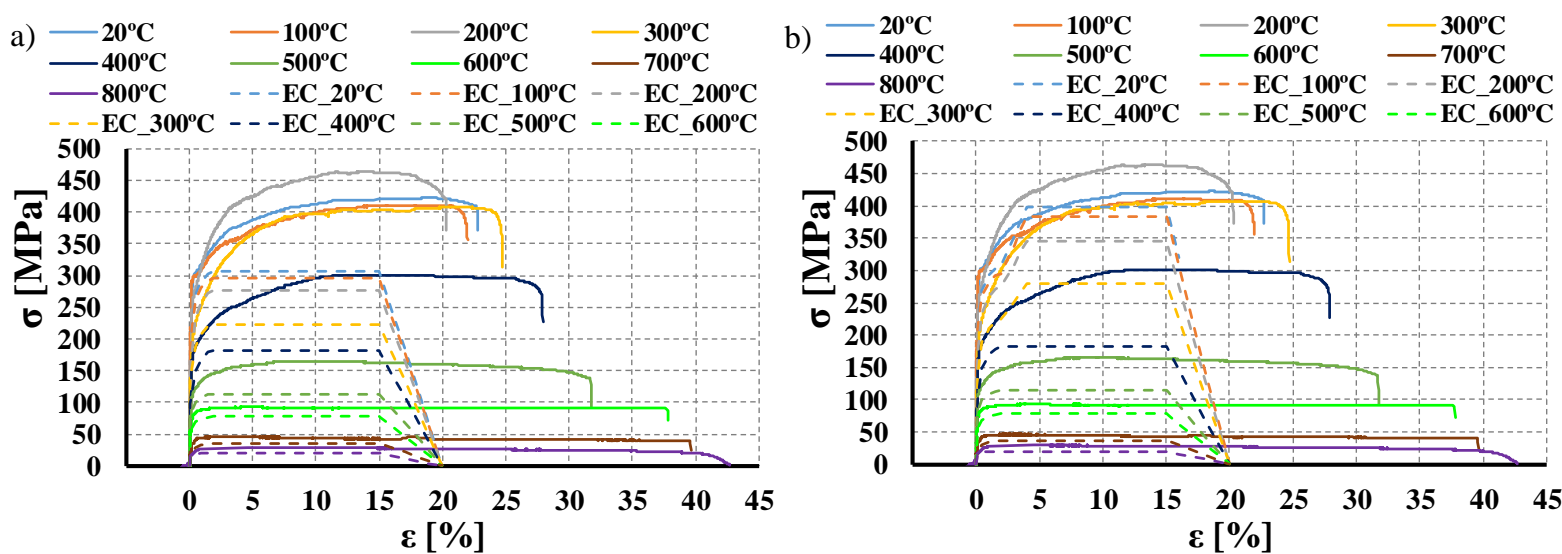

Figure 3.20 Comparison of stress-strain curves predicted by EN 1993-1-2:2005 model with test results. a) Stress-strain model without strain hardening. b) Stress-strain model with strain hardening.

Based on the stress-strain model presented in the EN 1993-1-2 (2005) for hot-rolled steels a modified predictive model is proposed for the S280GD+Z steel. Even though the material model presented in the EN 1993-1-2 (2005) is based on experimental results conducted using the transient state test method a new proposal, based on experimental tests conducted using the steady state test method is presented. Bear in mind that some authors state that since both types of tests (transient and steady state test methods) are usually conducted within an hour the amount of creep is limited (Ranawaka and Mahendran, 2009; Kankanamge and Mahendran, 2011). Moreover, in the study conducted by Outinen (Outinen, 1999; Outinen et al., 2000) on the $\mathrm{S} 350 \mathrm{GD}+\mathrm{Z}$ steel the difference between both methodologies was on average about $5 \%$ in the temperature range from 300 to $600^{\circ} \mathrm{C}$ and almost identical for higher temperatures. The available strain hardening model formulation was also used and modified in the new proposals. Two proposals are presented for the stress-strain relationship of low strength cold-formed steels at elevated temperatures based on the formulation presented in the EN 1993-1-2 (2005). The first proposed model is now thoroughly described (Equation 3.9):

- for $\varepsilon \leq \varepsilon_{p, \theta}$ and $20^{\circ} \mathrm{C} \leq \theta_{a} \leq 800^{\circ} \mathrm{C}$, where $\varepsilon_{p, \theta}=\frac{f_{p, \theta}}{E_{a, \theta}}$ :

$$
\sigma=\varepsilon \cdot E_{a, \theta}
$$

- for $\varepsilon_{p, \theta}<\varepsilon \leq \varepsilon_{y, \theta}^{*}$ and $20^{\circ} \mathrm{C} \leq \theta_{a} \leq 800^{\circ} \mathrm{C}$, where $\varepsilon_{y, \theta}=0.002$ and $\varepsilon_{y, \theta}^{*}=\frac{f_{y, \theta}}{E_{a, \theta}}+\varepsilon_{y, \theta}$ :

$$
\sigma=f_{p, \theta}-c+\frac{b}{a}\left[a^{2}-\left(\varepsilon_{y, \theta}^{*}-\varepsilon\right)^{2}\right]^{0.5}
$$


where:

$$
\begin{gathered}
a^{2}=\left(\varepsilon_{y, \theta}^{*}-\varepsilon_{p, \theta}\right) \times\left(\varepsilon_{y, \theta}^{*}-\varepsilon_{p, \theta}+\frac{c}{E_{a, \theta}}\right) \\
b^{2}=c \times\left(\varepsilon_{y, \theta}^{*}-\varepsilon_{p, \theta}\right) \times E_{a, \theta}+c^{2} \\
c=\frac{\left(f_{y, \theta}-f_{p, \theta}\right)^{2}}{\left(\varepsilon_{y, \theta}^{*}-\varepsilon_{p, \theta}\right) \times E_{a, \theta}-2\left(f_{y, \theta}-f_{p, \theta}\right)}
\end{gathered}
$$

- for $\varepsilon_{y, \theta}^{*}<\varepsilon \leq 0.1$ :

- $20^{\circ} \mathrm{C} \leq \theta_{a}<200^{\circ} \mathrm{C}$, the strain hardening is as follows.

$$
f_{u, \theta}=1.3 \times f_{y, \theta}
$$

- $200^{\circ} \mathrm{C} \leq \theta_{a}<400^{\circ} \mathrm{C}$, the strain hardening is as presented in Equation 3.9c.2. This behavior may be explained by the observed singularity in the behavior of low strength cold formed steels for temperatures between $200^{\circ} \mathrm{C}$ and $300^{\circ} \mathrm{C}$. At $200^{\circ} \mathrm{C}$ the ultimate strength was higher $(\approx 11 \%)$ than at ambient temperature $\left(20^{\circ} \mathrm{C}\right)$ and at $300^{\circ} \mathrm{C}$ the ultimate strength was close to the one obtained at ambient temperature. This behavior has been already explained.

$$
f_{u, \theta}=1.6 \times f_{y, \theta}
$$

- $400^{\circ} \mathrm{C} \leq \theta_{a}<600^{\circ} \mathrm{C}$, the strain hardening is as follows:

$$
f_{u, \theta}=f_{y, \theta}\left(2.8-0.003 \theta_{a}\right)
$$

For both temperature ranges $\left(20^{\circ} \mathrm{C} \leq \theta_{a}<300^{\circ} \mathrm{C}\right.$ and $\left.300^{\circ} \mathrm{C} \leq \theta_{a}<600^{\circ} \mathrm{C}\right)$ the stress is determined as follows:

$$
\begin{gathered}
\sigma=\frac{1}{\left(0.1-\varepsilon_{y, \theta}^{*}\right)} \times\left(f_{u, \theta}-f_{y, \theta}\right) \times \varepsilon+\left[1+\left(\frac{\varepsilon_{y, \theta}^{*}}{0.1-\varepsilon_{y, \theta}^{*}}\right)\right] \times f_{y, \theta} \\
-\left(\frac{\varepsilon_{y, \theta}^{*}}{0.1-\varepsilon_{y, \theta}^{*}}\right) \times f_{u, \theta}
\end{gathered}
$$

- $600^{\circ} \mathrm{C} \leq \theta_{a} \leq 800^{\circ} \mathrm{C}$, strain hardening is no longer observed, hence, $f_{u, \theta}=f_{y, \theta}$ and consequently $\sigma=f_{y, \theta}$. 
- for $0.1 \leq \varepsilon<\varepsilon_{t, \theta}$ and considering $\sigma=f_{u}$ :

- $20^{\circ} \mathrm{C} \leq \theta_{a} \leq 300^{\circ} \mathrm{C}$ the strain range is as follows $0.1 \leq \varepsilon \leq 0.18$;

- $300^{\circ} \mathrm{C}<\theta_{a} \leq 500^{\circ} \mathrm{C}$ the strain range is as follows $0.1 \leq \varepsilon \leq 0.2$;

- $500^{\circ} \mathrm{C}<\theta_{a} \leq 800^{\circ} \mathrm{C}$ the strain range is as follows $0.1 \leq \varepsilon \leq 0.3$.

- for $\varepsilon_{t, \theta} \leq \varepsilon<\varepsilon_{u, \theta}$ :

- $20^{\circ} \mathrm{C} \leq \theta_{a} \leq 300^{\circ} \mathrm{C}$ the strain range is as follows $0.18<\varepsilon \leq 0.2$ and the stress is determined according to Eq. (3.9d.1):

$$
\sigma=f_{u, \theta} \times\left[1-50 \times\left(\varepsilon-\varepsilon_{t, \theta}\right)\right], \text { where } \varepsilon_{t, \theta}=0.18
$$

- $300^{\circ} \mathrm{C}<\theta_{a} \leq 500^{\circ} \mathrm{C}$ the strain range is as follows $0.20<\varepsilon \leq 0.25$ and the stress is determined according to Eq. (3.9d.2):

$$
\sigma=f_{u, \theta} \times\left[1-20 \times\left(\varepsilon-\varepsilon_{t, \theta}\right)\right], \text { where } \varepsilon_{t, \theta}=0.2
$$

- $500^{\circ} \mathrm{C}<\theta_{a} \leq 800^{\circ} \mathrm{C}$ the strain range is as follows $0.30<\varepsilon \leq 0.35$ and the stress is determined according to Eq. (3.9d.3) and $f_{u, \theta}=f_{y, \theta}$ :

$$
\sigma=f_{u, \theta} \times\left[1-20 \times\left(\varepsilon-\varepsilon_{t, \theta}\right)\right], \text { where } \varepsilon_{t, \theta}=0.3
$$

In Figure 3.21 the proposed model is compared with the experimental results.

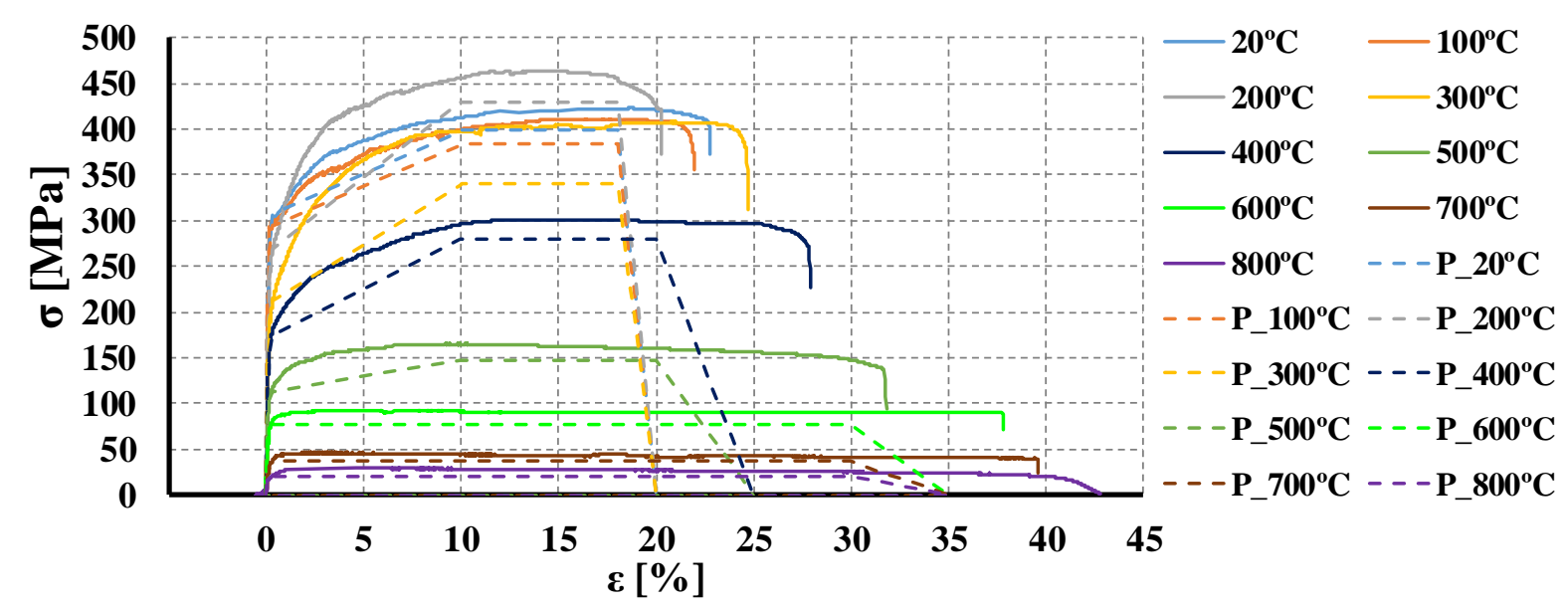

Figure 3.21 Comparison between the first modified proposed model based on the EN 1993-12 (2005) formulation with test results. Complete stress-strain curves. 
Alternatively, the second proposed model is now thoroughly described (Equation 3.10):

- for $\varepsilon \leq \varepsilon_{y, \theta}$ and $20^{\circ} \mathrm{C} \leq \theta_{a} \leq 800^{\circ} \mathrm{C}$, where $\varepsilon_{y, \theta}=\frac{f_{y, \theta}}{E_{a, \theta}}$ :

$$
\sigma=\varepsilon \cdot E_{a, \theta}
$$

- for $\varepsilon_{y, \theta}<\varepsilon \leq 0.1$ :

- $20^{\circ} \mathrm{C} \leq \theta_{a}<200^{\circ} \mathrm{C}$, the strain hardening is as follows.

$$
f_{u, \theta}=1.3 \times f_{y, \theta}
$$

- $200^{\circ} \mathrm{C} \leq \theta_{a}<400^{\circ} \mathrm{C}$, the strain hardening is as presented in Equation 10b.2. This behavior may be explained by the observed singularity in the behavior of low strength cold formed steels for temperatures between $200^{\circ} \mathrm{C}$ and $300^{\circ} \mathrm{C}$. At $200^{\circ} \mathrm{C}$ the ultimate strength was higher $(\approx 11 \%)$ than at ambient temperature $\left(20^{\circ} \mathrm{C}\right)$ and at $300^{\circ} \mathrm{C}$ the ultimate strength was close to the one obtained at ambient temperature. This behavior has been already explained.

$$
f_{u, \theta}=1.6 \times f_{y, \theta}
$$

- $400^{\circ} \mathrm{C} \leq \theta_{a}<600^{\circ} \mathrm{C}$, the strain hardening is as follows

$$
f_{u, \theta}=f_{y, \theta}\left(2.8-0.003 \theta_{a}\right)
$$

For both temperature ranges $\left(20^{\circ} \mathrm{C} \leq \theta_{a}<300^{\circ} \mathrm{C}\right.$ and $\left.300^{\circ} \mathrm{C} \leq \theta_{a}<600^{\circ} \mathrm{C}\right)$ the stress is determined as follows:

$$
\begin{gathered}
\sigma=\frac{1}{\left(0.1-\varepsilon_{y, \theta}\right)} \times\left(f_{u, \theta}-f_{y, \theta}\right) \times \varepsilon+\left[1+\left(\frac{\varepsilon_{y, \theta}}{0.1-\varepsilon_{y, \theta}}\right)\right] \times f_{y, \theta} \\
-\left(\frac{\varepsilon_{y, \theta}}{0.1-\varepsilon_{y, \theta}}\right) \times f_{u, \theta}
\end{gathered}
$$

- $600^{\circ} \mathrm{C} \leq \theta_{a} \leq 800^{\circ} \mathrm{C}$, strain hardening is no longer observed, hence, $f_{u, \theta}=f_{y, \theta}$ and consequently $\sigma=f_{y, \theta}$.

- for $0.1 \leq \varepsilon<\varepsilon_{t, \theta}$ and considering $\sigma=f_{u}$ : 
- $20^{\circ} \mathrm{C} \leq \theta_{a} \leq 300^{\circ} \mathrm{C}$ the strain range is as follows $0.1 \leq \varepsilon \leq 0.18$;

- $300^{\circ} \mathrm{C}<\theta_{a} \leq 500^{\circ} \mathrm{C}$ the strain range is as follows $0.1 \leq \varepsilon \leq 0.2$;

- $500^{\circ} \mathrm{C}<\theta_{a} \leq 800^{\circ} \mathrm{C}$ the strain range is as follows $0.1 \leq \varepsilon \leq 0.3$.

- for $\varepsilon_{t, \theta} \leq \varepsilon<\varepsilon_{u, \theta}$ :

- $20^{\circ} \mathrm{C} \leq \theta_{a} \leq 300^{\circ} \mathrm{C}$ the strain range is as follows $0.18<\varepsilon \leq 0.2$ and the stress is determined according to Eq. (3.10c.1):

$\sigma=f_{u, \theta} \times\left[1-50 \times\left(\varepsilon-\varepsilon_{t, \theta}\right)\right]$, where $\varepsilon_{t, \theta}=0.18$

- $300^{\circ} \mathrm{C}<\theta_{a} \leq 500^{\circ} \mathrm{C}$ the strain range is as follows $0.20<\varepsilon \leq 0.25$ and the stress is determined according to Eq. (3.10c.2):

$\sigma=f_{u, \theta} \times\left[1-20 \times\left(\varepsilon-\varepsilon_{t, \theta}\right)\right]$, where $\varepsilon_{t, \theta}=0.2$

- $500^{\circ} \mathrm{C}<\theta_{a} \leq 800^{\circ} \mathrm{C}$ the strain range is as follows $0.30<\varepsilon \leq 0.35$ and the stress is determined according to Eq. (3.10c.3) and $f_{u, \theta}=f_{y, \theta}$ :

$$
\sigma=f_{u, \theta} \times\left[1-20 \times\left(\varepsilon-\varepsilon_{t, \theta}\right)\right], \text { where } \varepsilon_{t, \theta}=0.3
$$

In Figure 3.22 the second modified predictive stress-strain curve model $\left(\mathrm{P}_{-} \theta\right)$ is compared with the experimental results obtained in the scope of this investigation for the S280GD+Z steel.

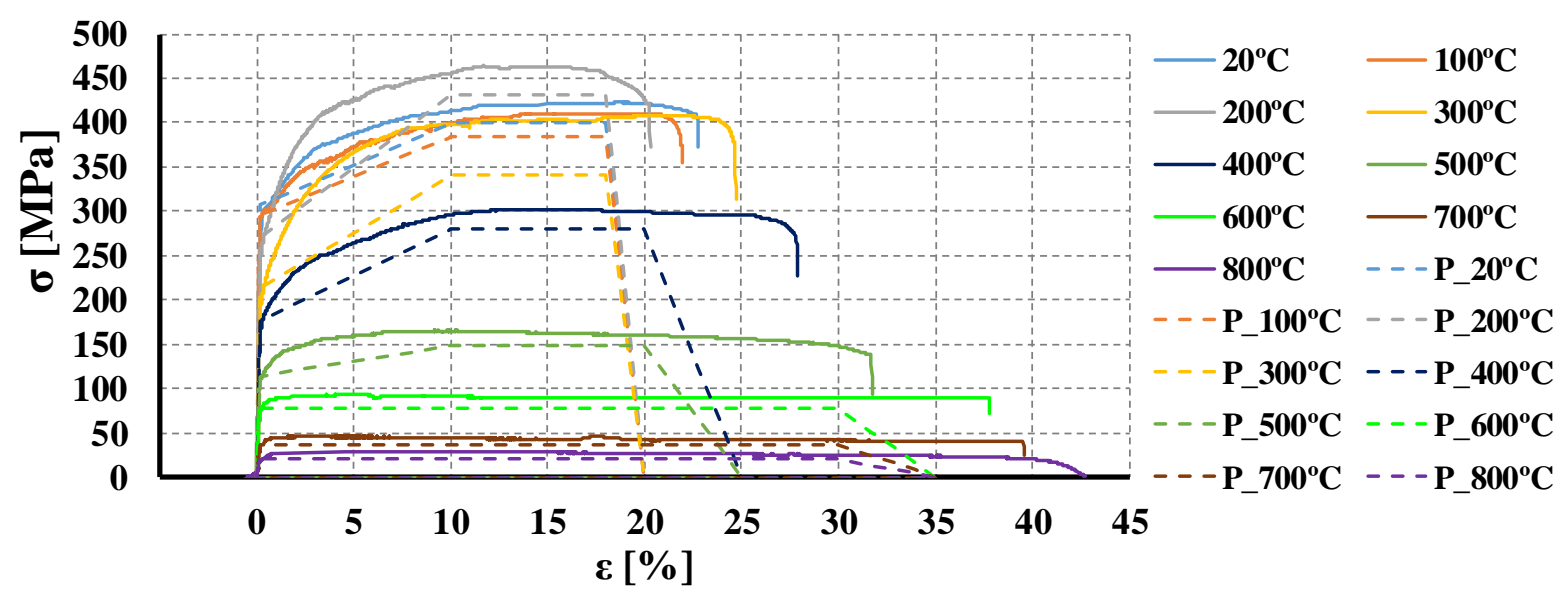

Figure 3.22 Comparison between the second modified proposed model based on the EN 19931-2 (2005) formulation with test results. Complete stress-strain curves. 
In Figure 3.23 a comparison between both proposed modified models is established. In order to provide a simpler model the mathematical formulation for strain range $\varepsilon_{p}<\varepsilon \leq \varepsilon_{y}$ was removed in the second proposal. It is clear that the proposed model P1 fits better the experimental results than the model $\mathrm{P} 2$, however its formulation is also a bit more complicated.

The comparison presented in Figures 3.21, 3.22 and 3.23 indicates that the proposed models conservatively predict the stress-strain curves for the $\mathrm{S} 280 \mathrm{GD}+\mathrm{Z}$ steel at elevated temperatures. At this point the model may be valid for the $S 280 \mathrm{GD}+Z$ steel and eventually for low strength steels up to $350 \mathrm{MPa}$. However further investigations must be conducted and the proposed model further developed. Nevertheless, this proposal for the S280GD+Z steel is a significant improvement against the current model.
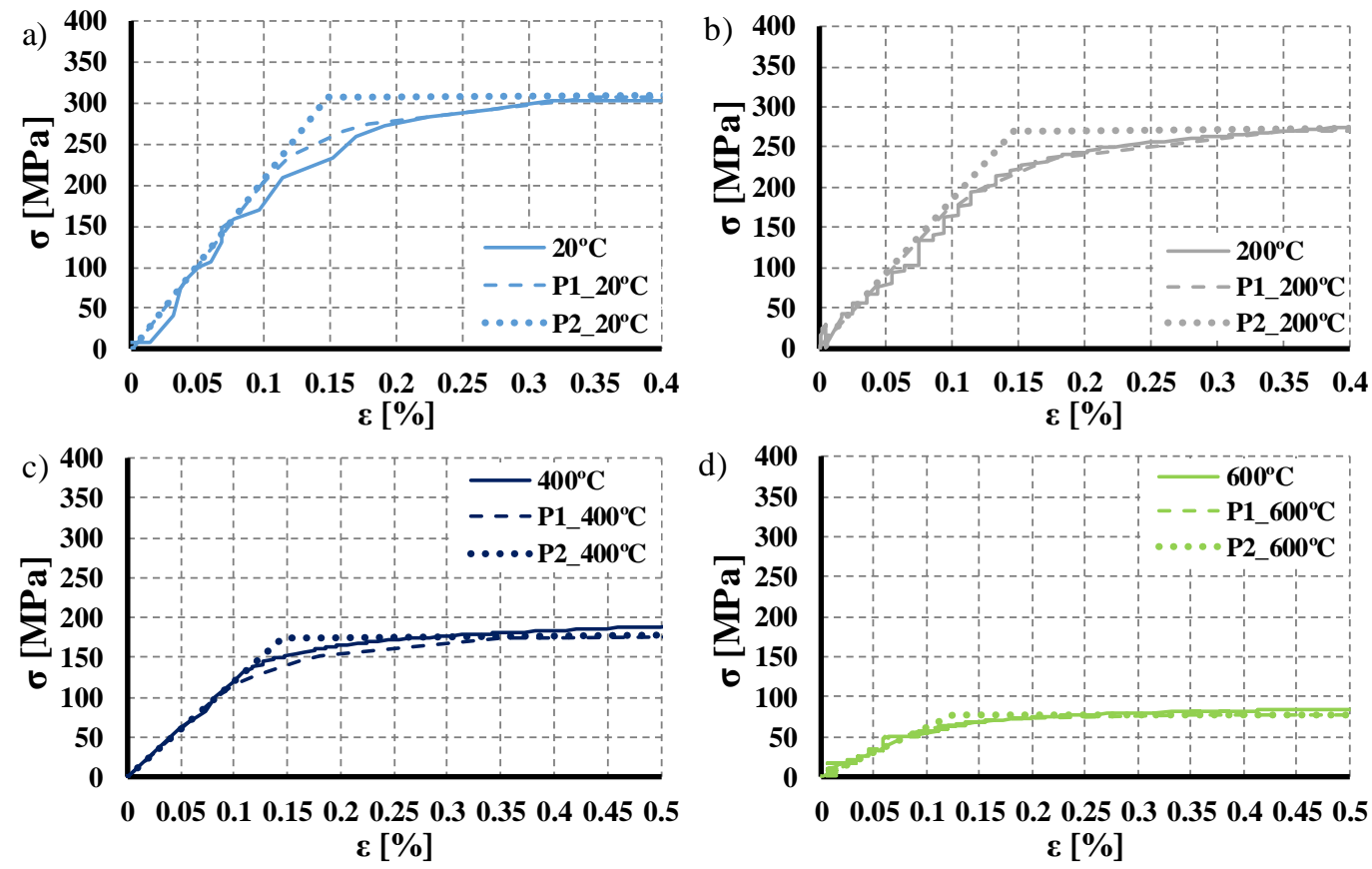

Figure 3.23 Comparison between both proposed models and the obtained results for different temperature levels (initial part of the stress-strain curves).

\subsubsection{Thermal properties of the $S 280 G D+Z$ steel}

\subsubsection{Thermal elongation}

The thermal elongation predicted in the available design standards (BS 5950-8, 1990); EN 1993-1-2, 2005) is conservative when compared with experimental results obtained in the scope of this investigation (Figure 3.24) and others previous studies (Outinen, 1999; Chen and Young, 
2007). The accurate assessment of this property is fundamental for structural fire design. For instance, while performing numerical analysis of cold-formed steel columns with restrained thermal elongation in case of fire, overestimated coefficient of thermal elongation will lead to overestimated generation of restraining forces and consequently to poor fitting between experimental and numerical results.

The determined thermal elongation was compared with the available design predictions presented in the EN 1993-1-2 (2005) and BS 5950-Part 8 (1990) (Figure 3.24). A new predictive equation based on the obtained results was proposed (Equation 3.11).

$$
\begin{array}{lr}
\frac{\Delta l}{l}=1.10235 \times 10^{-8} \times \theta^{2}+0.68575 \times 10^{-5} \times \theta- & 0.79712 \times 10^{-4} \\
\frac{\Delta l}{l}=1.1031 \times 10^{-2} & 20^{\circ} C \leq \theta \leq 740^{\circ} \mathrm{C} \\
\frac{\Delta l}{l}=2.16443 \times 10^{-5} \times \theta-8.04389 \times 10^{-3} & 740^{\circ} C<\theta \leq 890^{\circ} \mathrm{C} \\
& 890^{\circ} \mathrm{C}<\theta \leq 1000^{\circ} \mathrm{O} C
\end{array}
$$

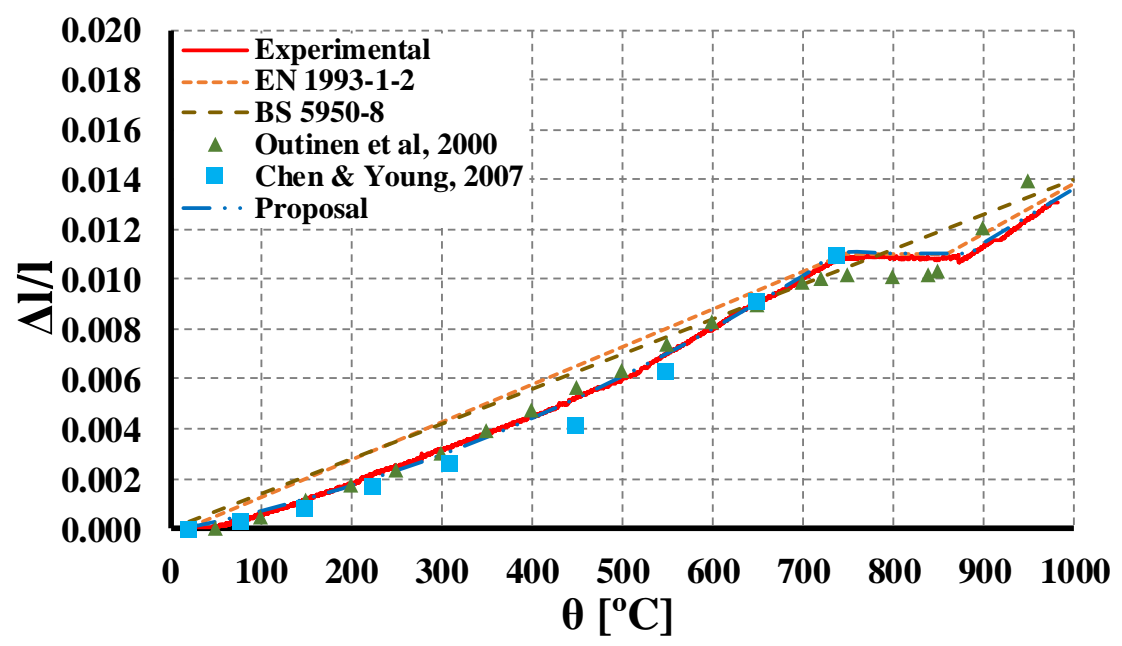

Figure 3.24 Thermal elongation as a function of temperature. Comparison between experimental results and proposed model with available design standards provisions and other proposed models.

\subsubsection{Thermal conductivity}

A suitable model for thermal conductivity for the $\mathrm{S} 280 \mathrm{GD}+\mathrm{Z}$ steel may be important to improve design methodologies at elevated temperatures. Rigorous thermal conductivity models will lead to an accurate prediction of the heat transfer phenomenon, hence the prediction of the ability of 
the S280GD+Z steel to transport heat energy from high to low temperature regions. With increasing temperature, the thermal conductivity decreases as it was observed in the experimental results. BS 5950-8 (1990) assumes that the thermal conductivity is constant with temperature increase $37.5 \mathrm{~W} / \mathrm{m}^{\circ} \mathrm{C}$. The proposed model for thermal conductivity presented in the EN 1993-1-2 (2005) was found unconservative for the S280GD+Z cold-formed steel tested. The difference between the two models was about $20 \%$ in the entire range of temperatures tested (Figure 3.25). The difference may be explained by the chemical composition of the steel tested as well as with the galvanized coating. Steels with higher levels of ferrite ( $\mathrm{Fe})$ and with lower levels of manganese $(\approx 0.3 \%)$ (Mn) tend to have values of thermal conductivity higher than $70 \mathrm{~W} \cdot \mathrm{m}^{-1} \cdot \mathrm{K}^{-1}$ (Peet et al., 2011). These results show that the EN 1993-1-2 (2005) should adopt different thermal conductivity models according to the chemical composition of the steel to be considered in design procedures, establishing lower and upper limits to the thermal conductivity.

The proposed thermal conductivity model for the $\mathrm{S} 280 \mathrm{GD}+\mathrm{Z}$ steel tested in this study is defined as follows (Equation 3.12):

$$
\lambda_{a}=-3.332 \times 10^{-2} \times \theta+65.206 \quad 20^{\circ} \mathrm{O} \leq \theta \leq 800^{\circ} \mathrm{C}
$$

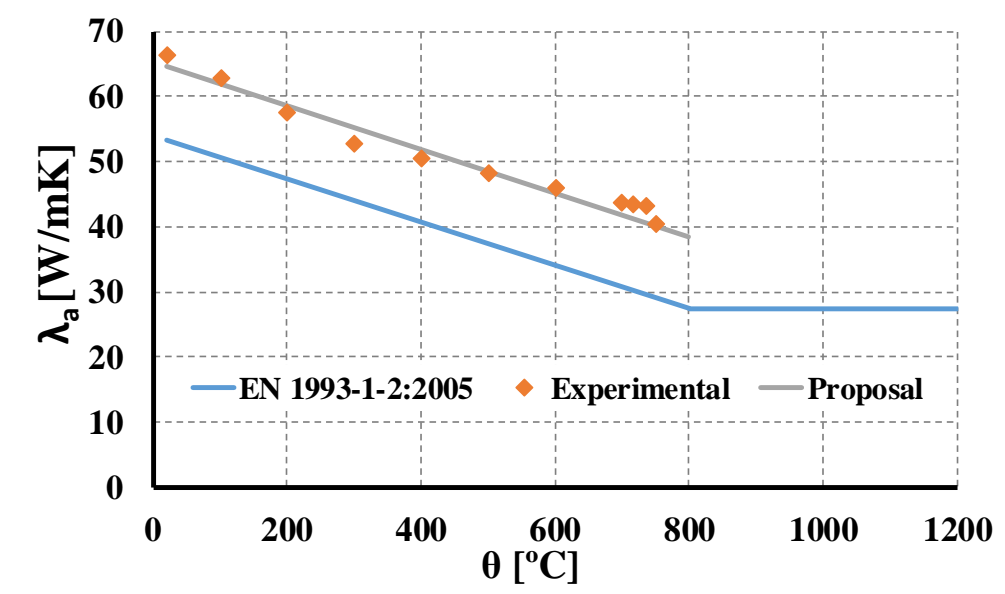

Figure 3.25 Thermal conductivity test results and new predictive model. Comparison with the EN 1993-1-2:2005 model.

\subsubsection{Specific heat}

The obtained experimental results for the specific heat $\left(c_{p}\right)$ were compared with the model available in the EN 1993-1-2 (2005) (Figure 20). Clearly the obtained results are in good 
agreement with the model available in the design standard hence can be used for structural fire design.

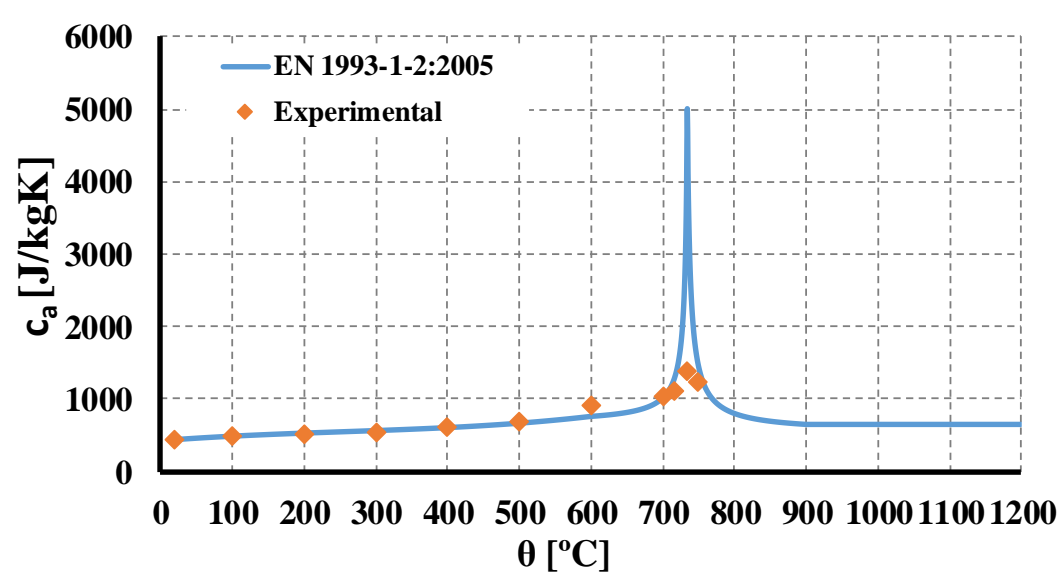

Figure 3.26 Specific heat experimental results and comparison with the model presented in the EN 1993-1-2 (2005).

\subsection{Final Remarks}

This chapter presented the conducted research, based on a detailed experimental study into the mechanical and thermal properties of the $\mathrm{S} 280 \mathrm{GD}+\mathrm{Z}$ steel with $2.5 \mathrm{~mm}$ thickness. Twentyseven tensile coupon tests were conducted at different temperatures, ranging from 20 to $800^{\circ} \mathrm{C}$. Peculiar behaviour was observed at about $200^{\circ} \mathrm{C}$, since a significant increase in the ultimate strength, when compared to that at ambient temperature, was observed. This may be due to chemical reactions and transformations taking place in the base at that temperature. This observation was described by Ranawaka and Mahendran (2009) and Kankanamge and Mahendran (2011). It was found that the available provisions in the current design standards are not accurate in terms of reduction factors for the mechanical properties. Moreover, the available stress-strain model presented in the EN 1993-1-2 (2005) do not predict accurately the stress-strain curves for the S280GD+Z steel. Hence, new predictive equations were presented in the scope of this investigation.

Regarding thermal properties, twenty-seven experimental tests were conducted to determine thermal conductivity, specific heat and thermal diffusivity of the S280GD+Z steel for temperatures ranging from 20 to $750^{\circ} \mathrm{C}$. Thermal elongation was also determined in the scope of this investigation. It was found that the thermal elongation predicted in the available design standards (BS 5950-8, 1990; EN 1993-1-2, 2005) is conservative when compared with experimental results obtained in the scope of this investigation and others previous studies 
(Outinen, 1999; Chen and Young, 2007). It was also found that the thermal conductivity of the S280GD+Z steel was about 20\% higher than the available model presented in the EN 1993-12 (2005). This may be due to the fact that the zinc coating was not removed and due to the chemical composition of the steel tested since steels with higher levels of ferrite $(\mathrm{Fe})$ and with lower levels of manganese $(\approx 0.3 \%)$ (Mn) tend to have values of thermal conductivity higher than $70 \mathrm{Wm}^{-1} \mathrm{~K}^{-1}$ (Peet et al., 2011). The specific heat is in good agreement with the available model presented in the EN 1993-1-2 (2005).

The determined material properties were extremely important in this investigation. All the reported data, in this chapter, was then used as input in the developed finite element model detailed in Chapter 5. 



\section{EXPERIMENTAL ANALYSIS ON COLD-FORMED STEEL COLUMNS}

\subsection{Introduction}

In this chapter the experimental investigation on cold-formed steel columns at both ambient and fire conditions is thoroughly detailed. Different test set-ups were used and the influence of different parameters was tested in order to accurately characterize the behavior of CFS columns at both ambient and fire conditions. The overview of the literature showed that experimental research on CFS columns is still scarce, especially on columns with open and closed built-up cross-sections under fire conditions with restraint to thermal elongation. The great majority of studies at elevated temperatures have been performed on short CFS columns (with only one profile, more commonly lipped and plain channels) in order to evaluate the local and distortional buckling phenomena individually and in combination (Ranawaka and Mahendran, 2009; Heva, 2009; Feng et al., 2003; Lee, 2004; Kaitila, 2002; Outinen and Myllymaki, 1995) using experimental and numerical analysis (Feng et al., 2003; Feng et al., 2004; Ranawaka and Mahendran, 2006). Concerning built-up cross-sections commonly used in building construction industry the lack of research is more substantial even at ambient temperatures (Stone and LaBoube, 2005; Georgieva et al., 2012). Also the influence of the surrounding structure on the structural behaviour of CFS columns has been neglected. Some studies regarding the influence of axial and rotational restraint have been conducted for hot rolled steel sections (Ali et al., 1998; Ali and O'Connor, 2001; Franssen, 2000; Wang, 1997a; Wang, 1997b; Wang, 2004; Rodrigues et al., 2000; Neves et al., 2002; Correia et al., 2011; Correia et al., 2012). Therefore, an extensive experimental study on CFS columns at both ambient and fire conditions was performed at the University of Coimbra. In the buckling tests the main objectives were to assess the buckling loads and failure modes of the tested columns as well as to compare the structural response of the different kinds of columns. In the fire tests it was intended to assess the behavior of different types of columns in fire considering the influence of restraint to thermal elongation in the overall behavior of the CFS columns. Twenty-four full scale buckling tests at ambient temperature and more than 96 fire tests with restraint to thermal elongation were performed in the scope of this investigation. The final goal of this extensive experimental research was to assess the behaviour of CFS columns and to provide relevant experimental data for validation of the developed finite element models that should be used for extensive parametric studies outside the bounds of the original experimental tests. 


\subsection{Test Specimens and Instrumentation}

All specimens were fabricated using CFS profiles provided by PERFISA, S.A. Commercially available profiles without perforations were used in the scope of this investigation. As previously mentioned, in this investigation single, open built-up and closed built-up crosssections were tested. All specimens consisted of one or more CFS profiles, namely plain channels (U) and lipped channel (C) profiles. All profiles were fabricated with S280GD+Z structural steel, hot dip galvanized with zinc on each side (zinc coating of $0.04 \mathrm{~mm}\left(275 \mathrm{~g} / \mathrm{m}^{2}\right.$ ), and with a yield strength of $280 \mathrm{MPa}$ and an ultimate tensile strength of $360 \mathrm{MPa}$, according to the EN 10147 (2002). The lipped channel profiles (C) cross-sections were $150 \mathrm{~mm}$ tall and 43 mm wide with $2.5 \mathrm{~mm}$ thickness, whereas the plain channel profiles (U) were $155 \mathrm{~mm}$ tall and $43 \mathrm{~mm}$ wide with $2.5 \mathrm{~mm}$ thickness. The inside bend radius and the length of the edge stiffeners of the lipped profiles was 2 and $15 \mathrm{~mm}$, respectively. With these dimensions it was possible to combine both types of single cross-sections to fabricate built-up cross-sections using selfdrilling screws Hilti S-6.3×19MD03Z. The open built-up cross-section was fabricated using two lipped channels positioned back-to-back (I) and fastened on the web using self-drilling screws along the length of the column. Two closed built-up cross-sections were fabricated. The first consisted on a lipped channel and a plain channel, positioned in such way to obtain a box shape (R cross-section). The plain channel and the lipped channel were fastened in the flanges using self-drilling screws along the length of the column. Finally, 2R cross-section consisted of two lipped channels (C) positioned back-to-back (I) and fastened in the web and two plain channels (U) positioned in such way to obtain a box shape (Figure 4.1). The length of all profiles was $2950 \mathrm{~mm}$ and the spacing of the fasteners along the length of the column was $725 \mathrm{~mm}$. The spacing adopted for the self-drilling screws was based on the observation of designed and built CFS structures using built-up members, in Portugal, by the company that provided the CFS profiles.

In both buckling tests and fire tests each cross-section tested was instrumented with strain gauges and thermocouples, respectively. In the buckling tests the strain gauges were placed in several points of the cross-section at mid-height of each CFS column tested (Figure 4.2). In the fire tests thermocouples were positioned in different points of the cross-section and in five different sections along the length of the column, in order to monitor the temperature in the tested cross-sections and along the length of the columns (Figure 4.3). 


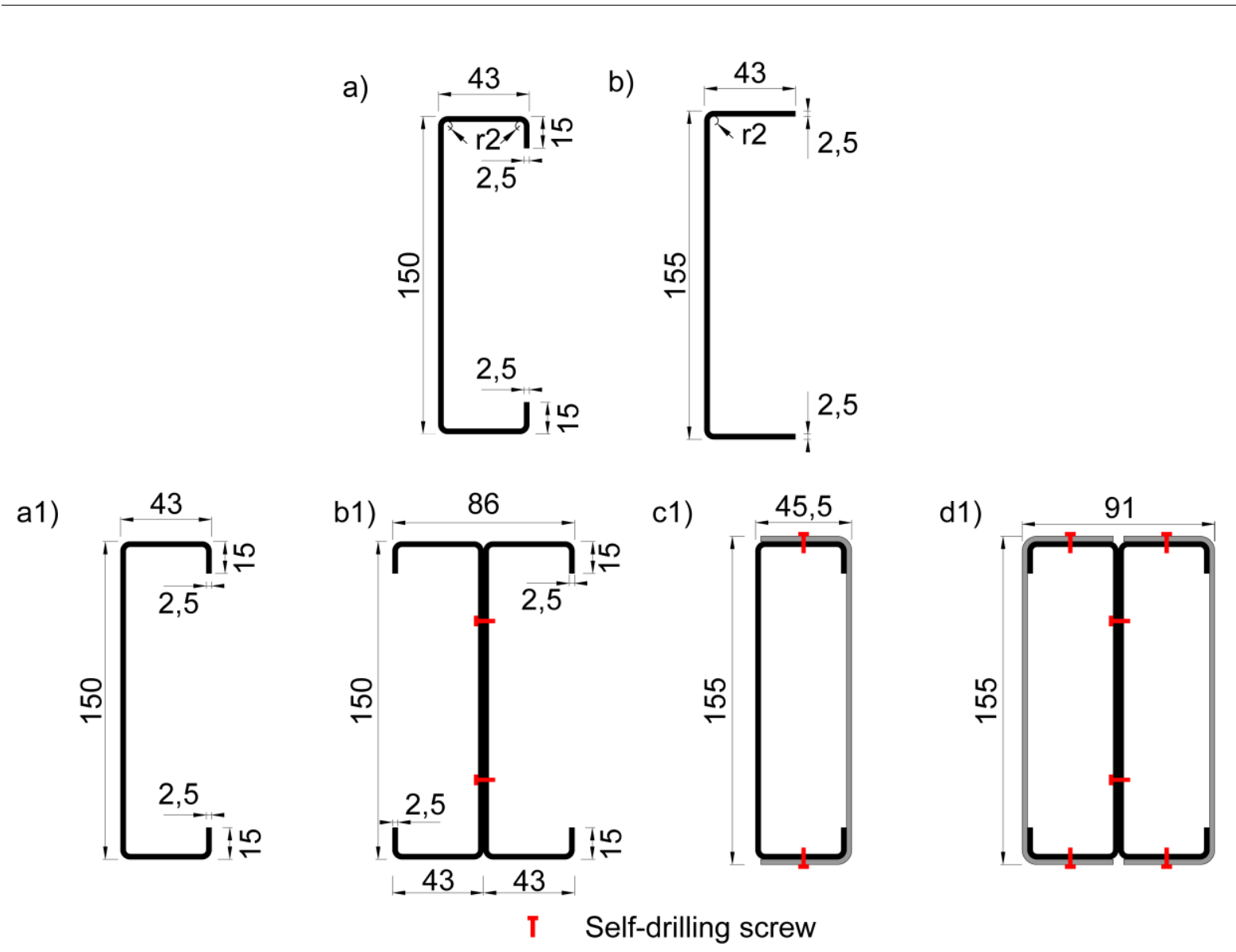

Figure 4.1 a) and b) Dimensions of the single profiles used. a1), b1), c1) and d1) Dimensions of the cross-sections tested in the scope of this investigation $(C, I, R, 2 R)$.
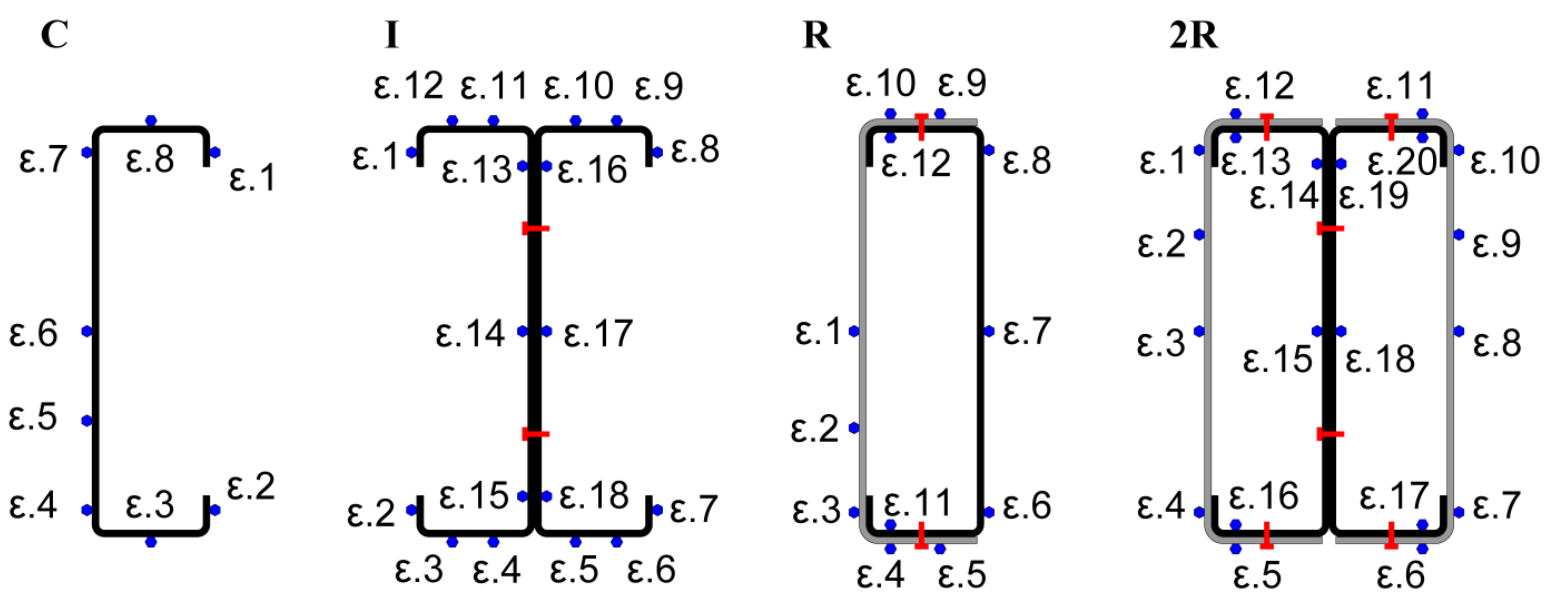

Figure 4.2 CFS cross-sections tested and positioning of the strain gauges used in buckling tests. 
a)

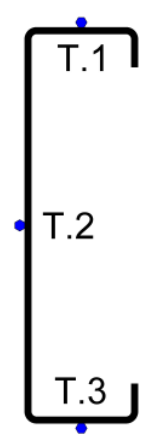

b)

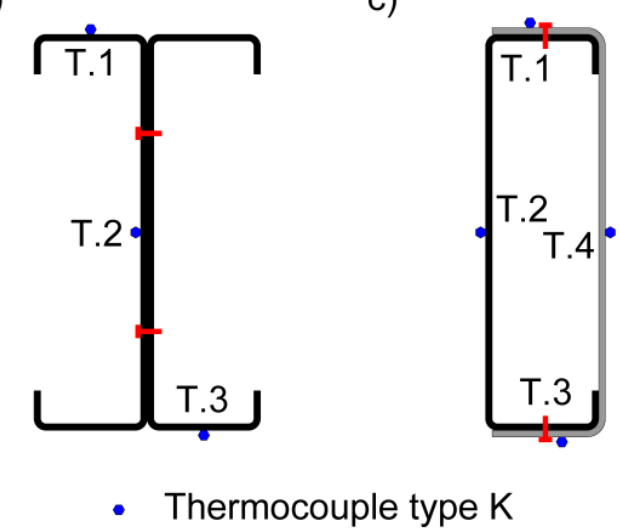

d)

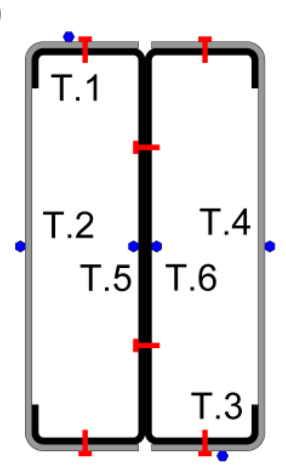

Figure 4.3 CFS cross-sections tested and positioning of the thermocouples used in the fire tests.

\subsection{Test Set-Up}

\subsubsection{Test set-up for buckling tests at ambient temperature}

The test set-up specifically designed for testing the buckling load of the CFS columns is thoroughly described in this section. With this experimental system it was attempted to simulate both pinned and fixed-end conditions in order to assess lower and upper bounds of the buckling load of the tested CFS columns. The experimental test set-up comprised a 2D reaction steel frame (1), a concrete footing (2), the designed end-support devices (3), load cell used to measure the applied load (4), the hydraulic jack (5) used to apply the load to the CFS column, the servo hydraulic central unit W+B NSPA700/DIG2000 (6) and the data acquisition system TML TDS530 (7) (Figure 4.4). The concrete footing was specifically designed and fabricated for this experimental campaign (Figure 4.5). To the concrete footing two steel plates were fixed. The hydraulic jack was connected to the top steel plate of the concrete footing (Figure 4.5). To the piston of the hydraulic jack a new set of steel plates were fixed, and to these steel plates the end-support devices were connected. Four threaded rods were used in order to aid levelling of the end-support devices. Additional steel plates were placed around the loading system in order to prevent any type of rotations during loading. The steel plates fixed to the hydraulic jack and connected to the end-support devices could slide through the threaded rods which were Teflon lined in order to reduce friction. In Figure 4.6 some details of the concrete footing are presented. 

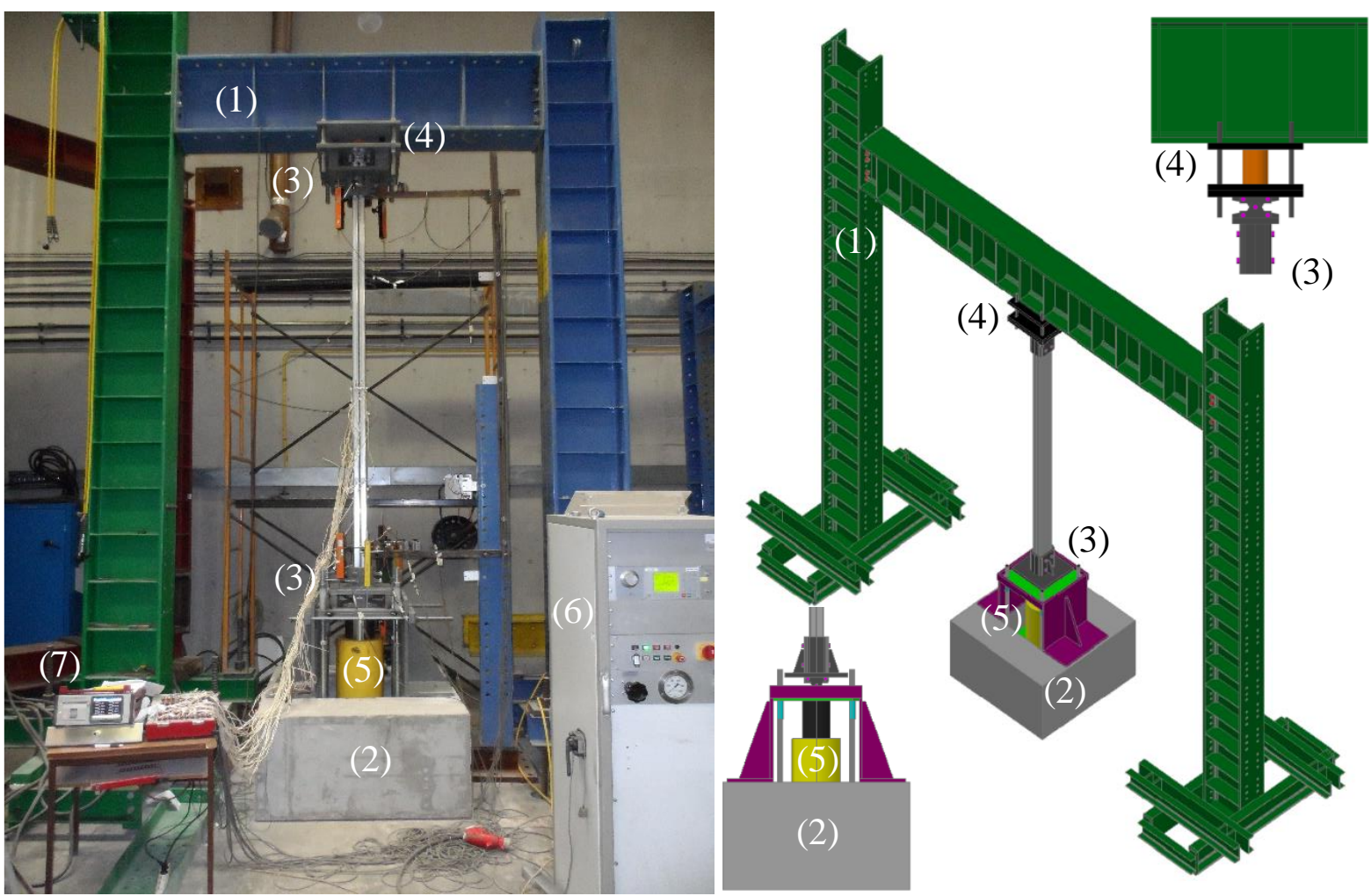

Figure 4.4 Experimental test set-up designed and built for the buckling tests. a) Test set-up. b) Schematic of the test set-up.

a)

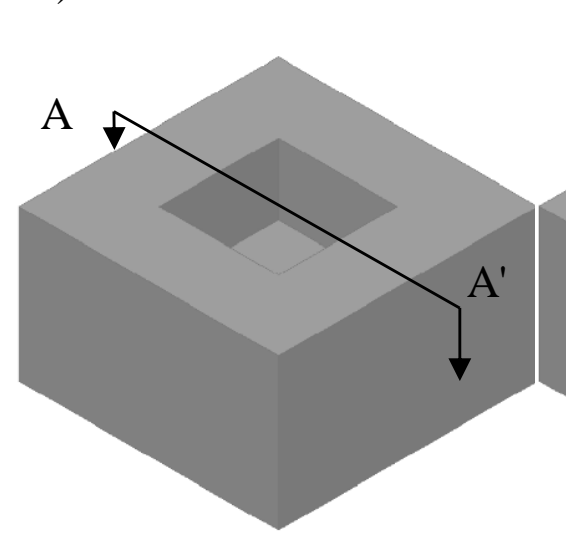

b)

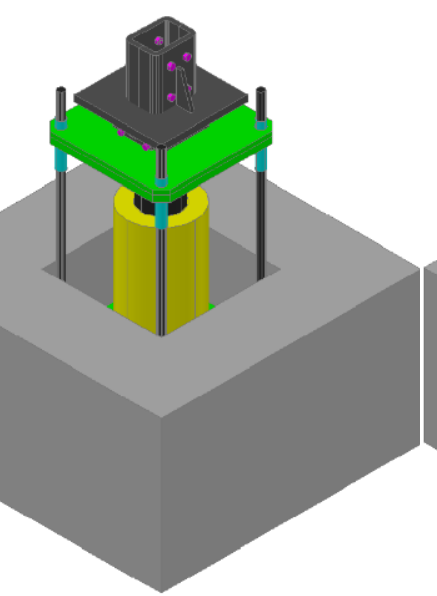

c)

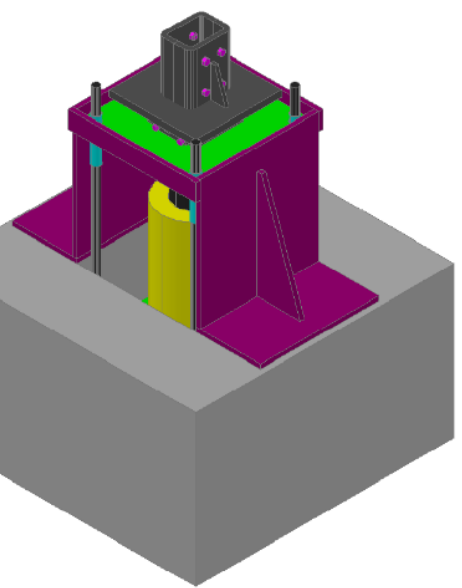

Figure 4.5 a) Concrete footing. b) Concrete footing with hydraulic jack and end-support device. c) Concrete footing with hydraulic jack, end-support device and steel plates used to prevent any type of rotation while applying the compressive load. 


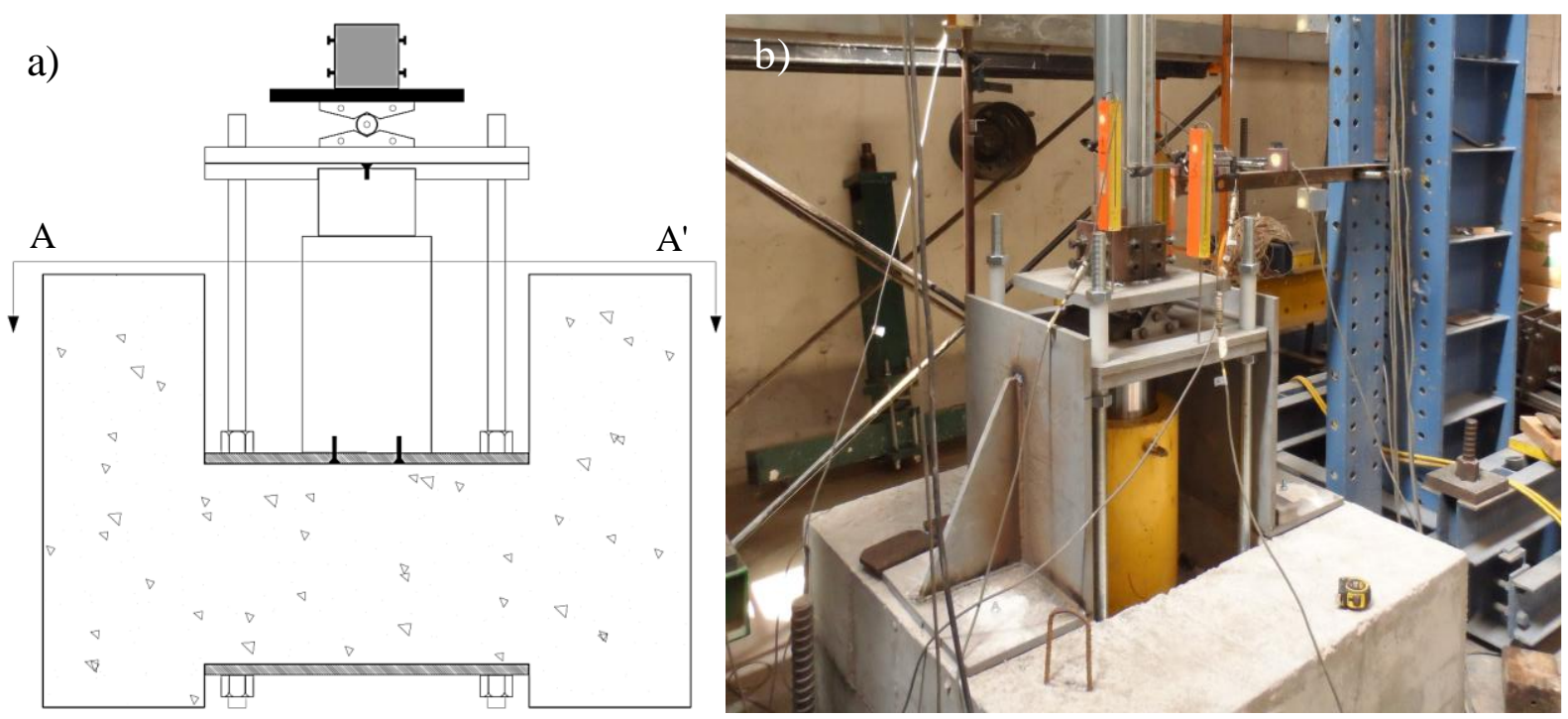

Figure 4.6 a) Concrete footing view cut. b) Concrete footing with hydraulic jack and endsupport device.

In an attempt to test both pinned and fixed-end support conditions a special end-support device was designed and built (Figure 4.7). It was intended that this end-support device could be used while testing all the studied cross-section shapes. It comprises a set of steel plates, a steel pin lubricated with copper grease and Teflon lined, a rectangular hollow section (RHS) welded to the top steel plate and to the end-support device. In the RHS four holes were drilled in each lateral plate and four nuts were welded at those holes. Using bolts, it was possible to adjust the free inner steel plates to each tested specimen in the scope of this investigation (Figure 4.7b)). Bear in mind that using this type of end-support devices lead to different lengths for pinned and fixed-ended columns. For pinned-ended columns the actual length was $3050 \mathrm{~mm}$ and for fixedend columns the actual length was $2850 \mathrm{~mm}$. These lengths were used in the calculations undertaken to determine the design buckling loads for the tested columns.

In all buckling tests axial load, axial displacements, lateral displacements, rotations of the endsupport devices and strains at several points of each cross-section, at mid-height of the column (Figure 4.2), were monitored and recorded. On the top and bottom end-support devices 4 linear variable displacement transducers (LVDT) were used to measure vertical displacements of the steel plate of the end-support device and consequently determine the rotations (Figure 4.8b) and c)). Lateral deflections were measured in both directions (about both minor and major axis) in three different points along the length of the column using linear wire transducer (LWT) as shown in Figure 4.8a). In Figure 4.8b) also a detail showing the load cell (TML CLC 1MN) is presented. 

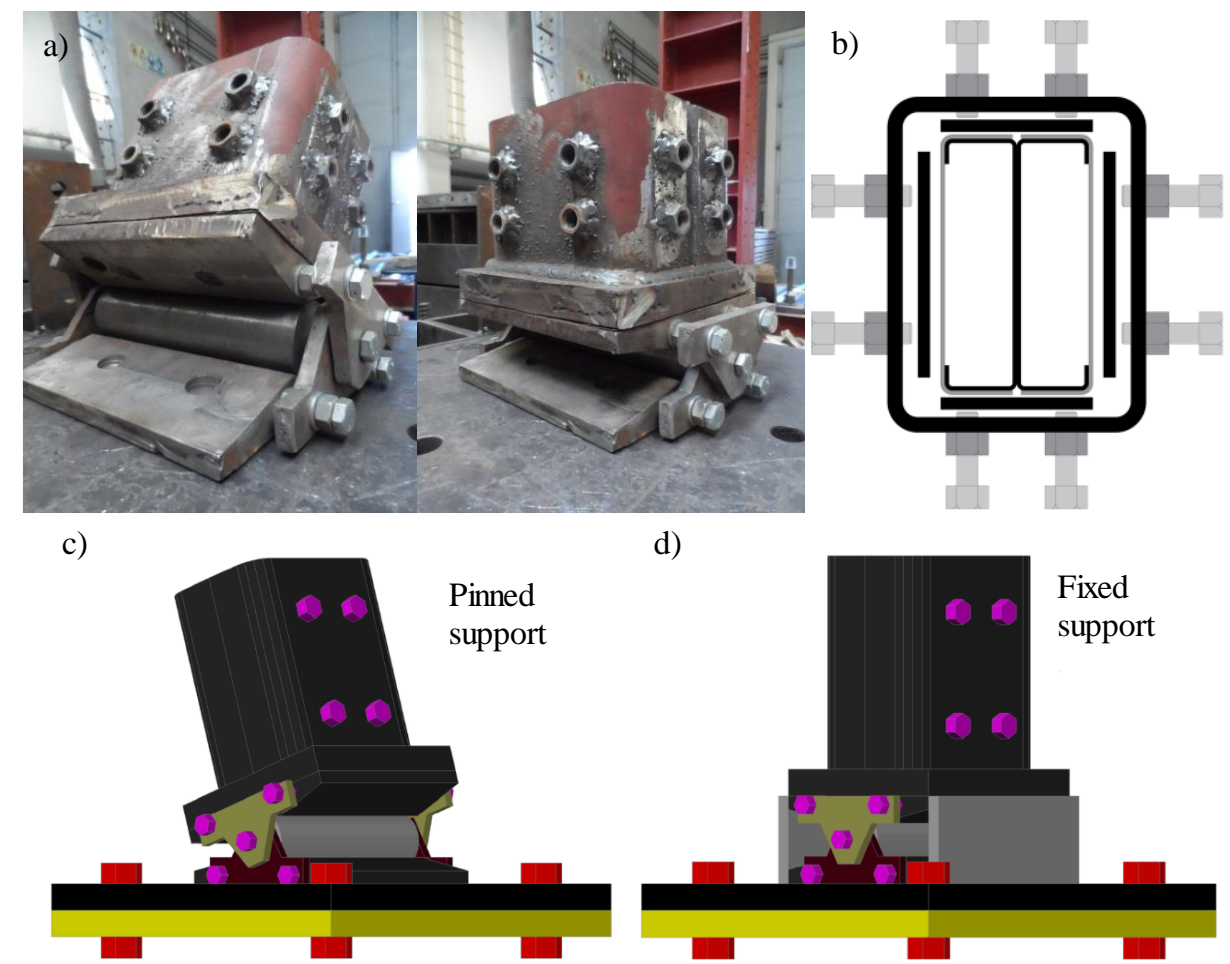

Figure 4.7 a) End-support devices. Schematics of the end-support device. b) Adjustable system adopted to fix each one of tested cross-sections. c) Pinned support. d) Fixed support.

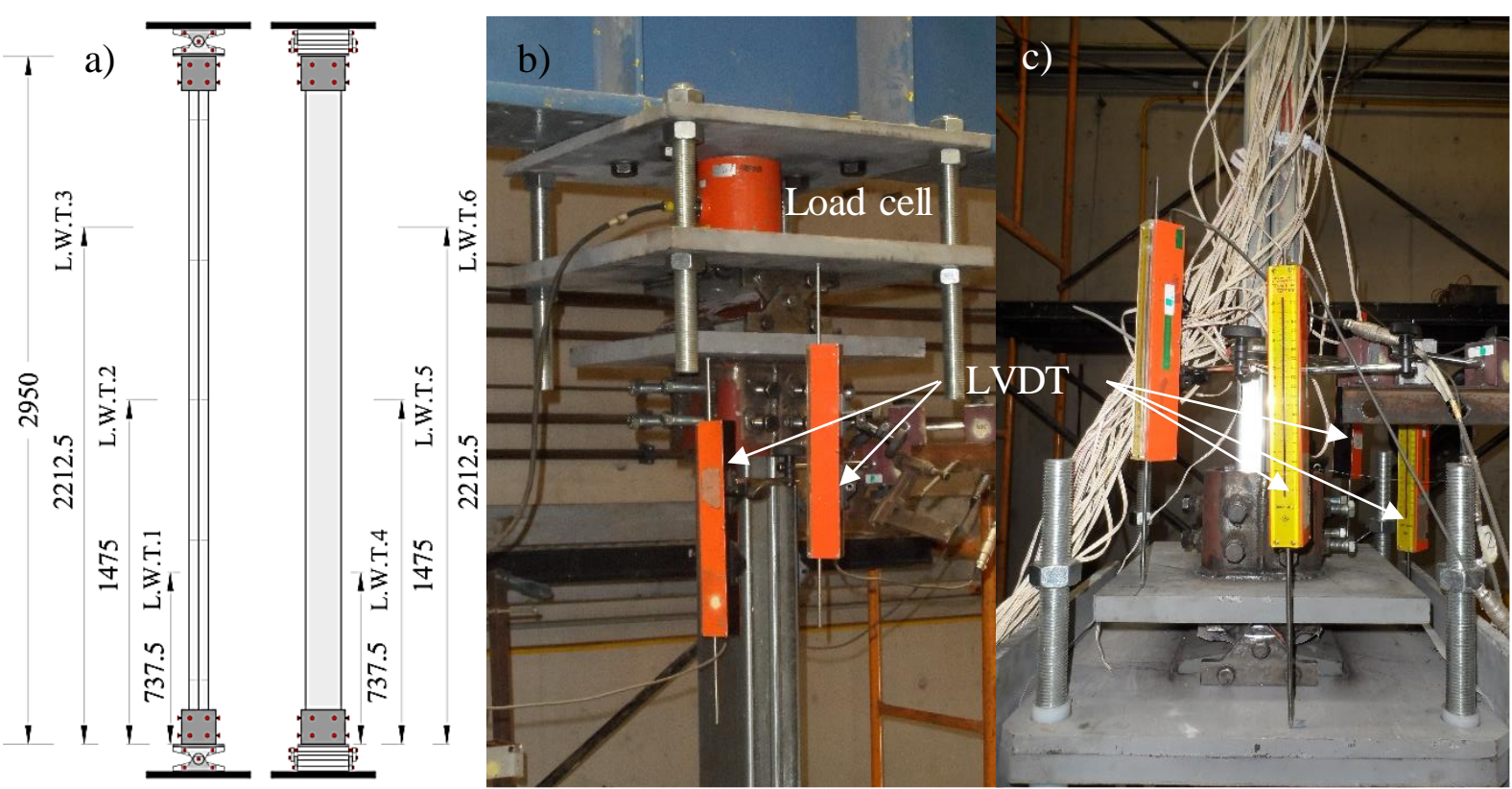

Figure 4.8 a) Positioning of the LWT along the length of the column in both directions. $b$ ) Load cell. c) Positioning of the LVDT in the end-support device. 


\subsubsection{Test set-up for fire tests with restraint to thermal elongation}

The experimental set-up comprised a two dimensional (2D) reaction steel frame ((1) in Figure 4.9a) and b)) and a three dimensional (3D) restraining steel frame adaptable for different levels of stiffness ((2) in Fig. 4.9a) and b)) in order to simulate the axial and rotational restraint imposed by the surrounding structure to a CFS column in fire. The $2 \mathrm{D}$ reaction frame was composed by two HEB 500 columns $6.6 \mathrm{~m}$ tall and by one HEB 600 beam $4.5 \mathrm{~m}$ long using M27 grade 8.8 bolts in the connections. The levels of stiffness of the surrounding structure adopted in the experimental tests were chosen after conducting numerical simulations considering a residential CFS building with two storeys and three bays. The height of the columns was $3.0 \mathrm{~m}$ and the length of the beams was $5.0 \mathrm{~m}$ spaced from $0.6 \mathrm{~m}$. Regarding the results obtained in these simulations it can be stated that $3 \mathrm{kN} / \mathrm{mm}$ of axial stiffness of the surrounding structure to the CFS column is a low value whereas $13 \mathrm{kN} / \mathrm{mm}$ is a medium/high value for the adopted experimental conditions (Laím, 2013). To achieve the desired levels of stiffness of the surrounding structure, in order to provide axial and rotational restraint (K1 to K4) to the thermal elongation of the CFS columns, two different 3D restraining frames were used in the experimental tests. The first one (RF.1) was composed by four columns HEA 200 and four beams HEA 200 while the second one (RF.2) was composed by four columns HEB 300 and four beams HEB 300 ( 2 on top and 2 on bottom) arranged orthogonally. This restraining system intended to reproduce the actual boundary conditions of a CFS column when inserted in a real building. In order to confirm the levels of stiffness, in addition to the numerical simulations, some experimental tests were carried out. Replacing the CFS column by a hydraulic jack a constant load was applied to the restraining frame and the respective vertical nodal (point of intersection of the top beams of the 3D restraining frame) displacement measured. The obtained values were also confirmed with the values of the restraining forces and axial displacements registered in the fire resistance tests of CFS columns.

The rotational stiffness of both restraining frames (RF.1 and RF.2) was determined through numerical simulations using the finite element software Abaqus (2012). This procedure was also used to confirm numerically the axial stiffness of the restraining frame (Appendix B). A geometrically nonlinear elastic analysis was performed. All hot rolled steel structural members of the restraining frames and the threaded rods used in the connections between the steel columns and the top beams of the restraining frames were modelled by using solid elements (C3D8R). The C3D8R element is defined as a 3D continuum hexahedral and a height-node brick element with reduced integration, hourglass control and first order interpolation. These finite elements have three degrees of freedom per node, corresponding to translations in the three directions. An elastic behaviour was assumed for the hot rolled steel members and threaded rods. The contact behaviour assumed between the steel elements was tie constraint for 
simplification. The finite element mesh was generated automatically with an approximate size of $20 \mathrm{~mm}$. The boundary conditions adopted were in agreement with those in the experimental system. In order to determine the axial and rotational stiffness of the restraining frames a rigid steel column, with $1 \mathrm{~m}$ long, was fixed in the intersection point between the top beams of the $3 \mathrm{D}$ restraining frames. The axial stiffness of the $3 \mathrm{D}$ restraining frame was determined by a vertical upward load applied on the bottom surface of the rigid steel column. The correspondent vertical deformation was measured at the intersection between the top beams of the $3 \mathrm{D}$ restraining frame. The rotational stiffness of the restraining frame, about the major and minor axis of the CFS column, was determined by applying a bending moment at the end connection between the rigid steel column and the top beams of the 3D restraining frame. The correspondent rotations were measured (Appendix B).

From calculations and for the restraining frame with axial stiffness of $3 \mathrm{kN} / \mathrm{mm}$ a rotational stiffness of $9253 \mathrm{kN} . \mathrm{m} / \mathrm{rad}$ and $2196 \mathrm{kN} . \mathrm{m} / \mathrm{rad}$ was obtained. For the restraining frame with an axial stiffness of $13 \mathrm{kN} / \mathrm{mm}$ a rotational stiffness of $37237 \mathrm{kN} . \mathrm{m} / \mathrm{rad}$ and $12620 \mathrm{kN} . \mathrm{m} / \mathrm{rad}$ was obtained, respectively about the minor and major axis of the CFS column. A global view of the experimental system is presented in Figure 4.10.

a)
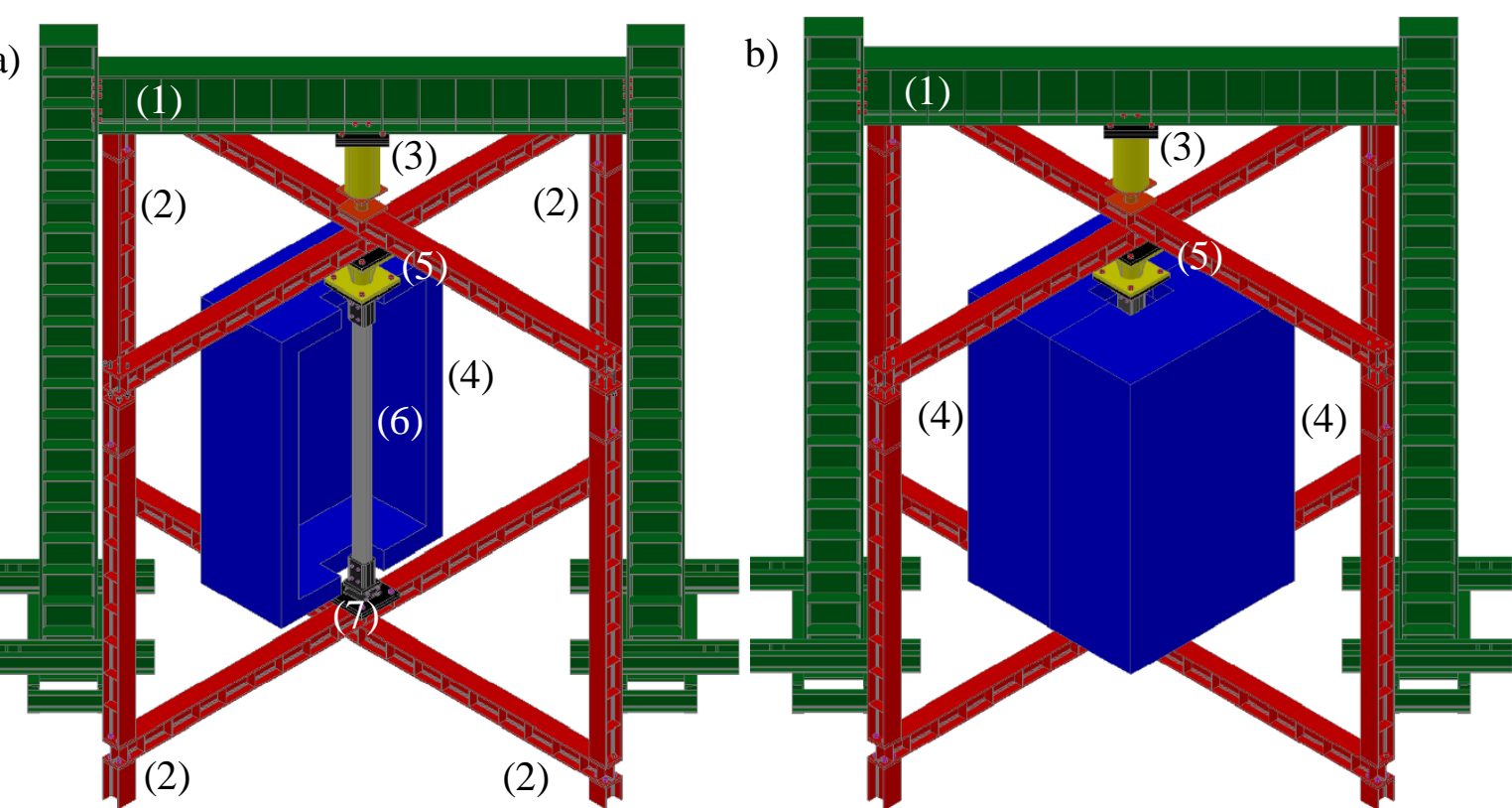

Figure 4.9 Schematic view of the experimental set-up. a) View with open furnace. b) View with closed furnace. 


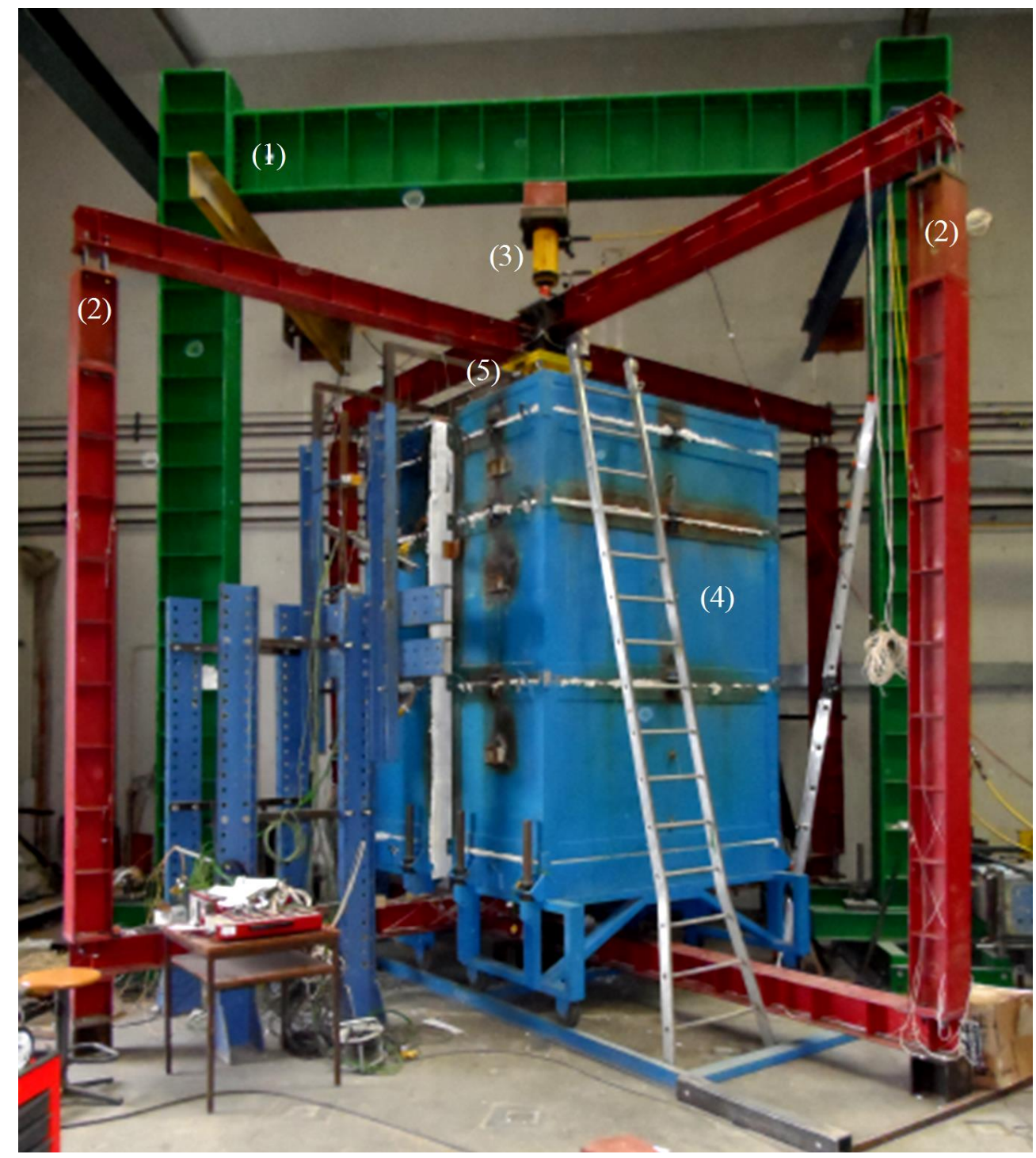

Figure 4.10 Global view of the experimental set-up.

The connections between the peripheral columns and the top beams of the restraining frame were made with threaded rods M24 or M27 grade 8.8 respectively for RF.1 and RF.2 restraining frames (Figure 4.12c)). A hydraulic jack, placed in the 2D reaction frame, was used to apply the serviceability load ((3) in Figure 4.9 a) and b)). This hydraulic jack has a maximum loading capacity in compression of $3 \mathrm{MN}$ and a maximum stroke length of $300 \mathrm{~mm}$ and was controlled by a servo hydraulic central unit W+B NSPA700/DIG2000. The thermal action was applied by a vertical modular electric furnace ((4) in Figure $4.9 \mathrm{~b})$ ) programmed to reproduce the standard fire curve ISO 834 (1999). This electric furnace was composed by three vertical modules that could open in a split system. Two of the vertical modules were $1 \mathrm{~m}$ (90 kVA each module) and 
the last one $0.5 \mathrm{~m}$ height $(45 \mathrm{kVA})$. When the two parts of the furnace were closed a chamber $(2.5 \mathrm{~m} \times 1.5 \mathrm{~m} \times 1.5 \mathrm{~m})$ was created around the test column.

The end-support devices used in the fire tests were the same used in the buckling tests. However, it is worth mentioning that due to space and equipment limitations the fire tests were conducted before the buckling tests. So there was a difference between the end-support devices when set to reproduce pinned-ended conditions. In the fire tests only Teflon (PTFE) was used in the steel pin in order to reduce friction, whereas in the buckling tests, as previously mentioned, copper grease and Teflon were used in the steel pin to reduce friction. It was expected that the friction could be reduced even more using both lubricant and Teflon.

To measure the restraining forces generated on the testing column during the heating process a special device was built ((5) in Figure 4.9a) and Figure 4.11), consisting of a hollow steel cylinder where a stiff steel cylinder Teflon (PTFE) lined slides through it (Figure 4.11). On the top of the stiff steel cylinder a $500 \mathrm{kN}$ load cell was placed and compressed against the top end plate of the hollow steel cylinder.

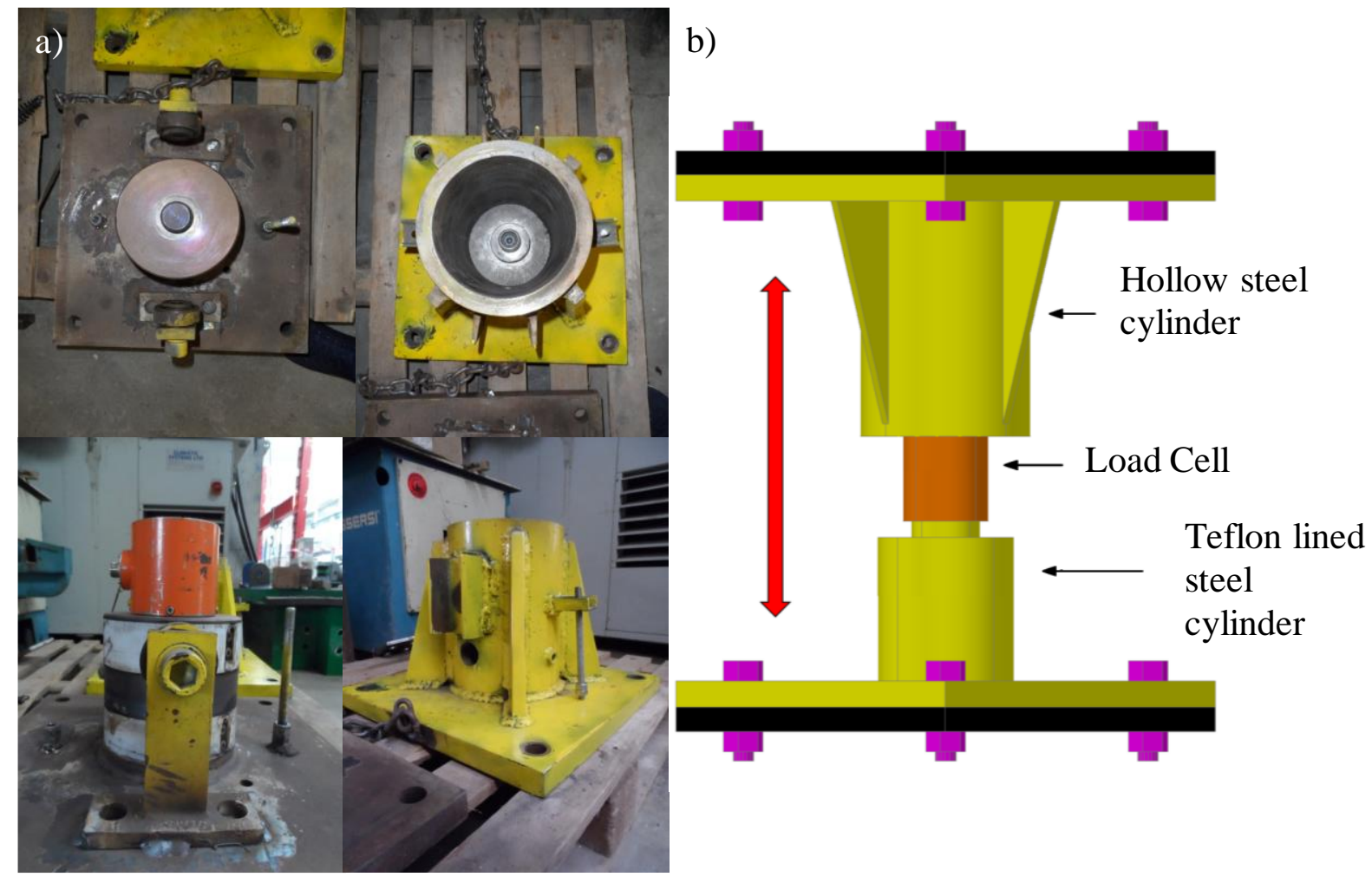

Figure 4.11 Device for measuring the restraining forces. 
In order to evaluate the temperature throughout the length of the column, type $\mathrm{K}$ thermocouples were welded on the flanges and web of each cross section (Figure 4.3) at five different sections (Figure 4.12).

The axial displacements of the testing columns were measured by 4 linear variable displacement transducers (LVDT) arranged orthogonally placed at both ends of the columns. Lateral displacements were also measured using 5 linear wire transducers (LWT) placed at specified positions throughout the length of the column about the major and minor axis (Figure 4.12).
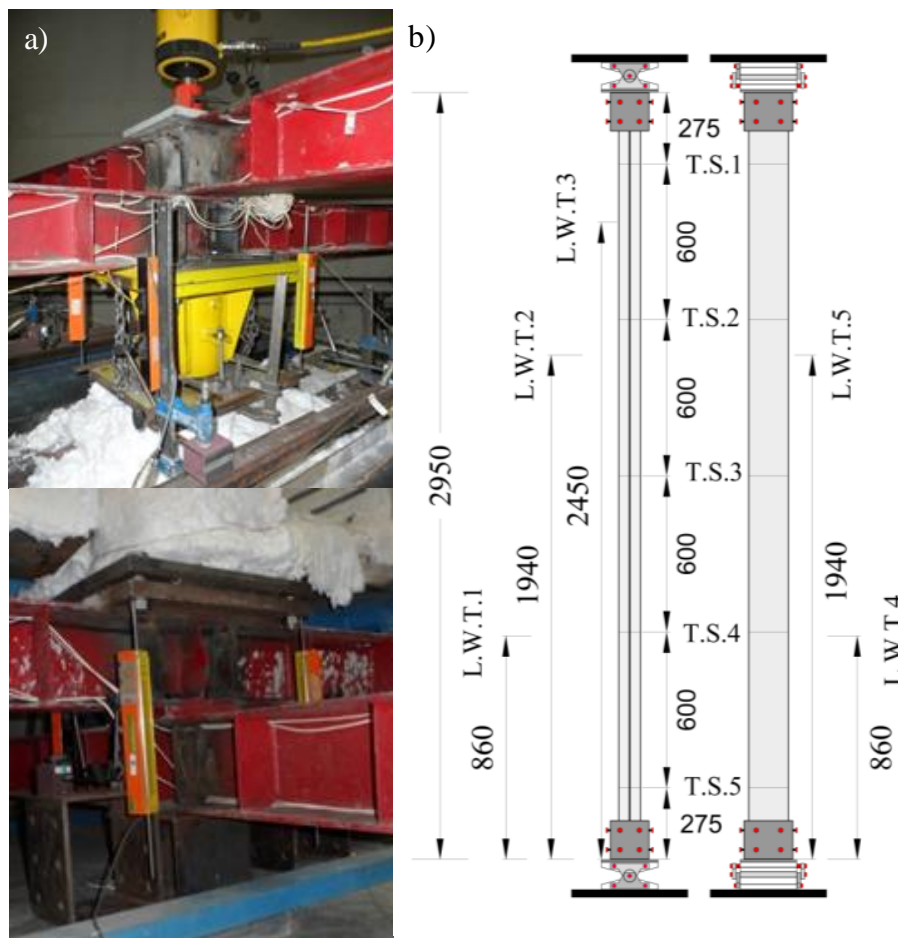

c)

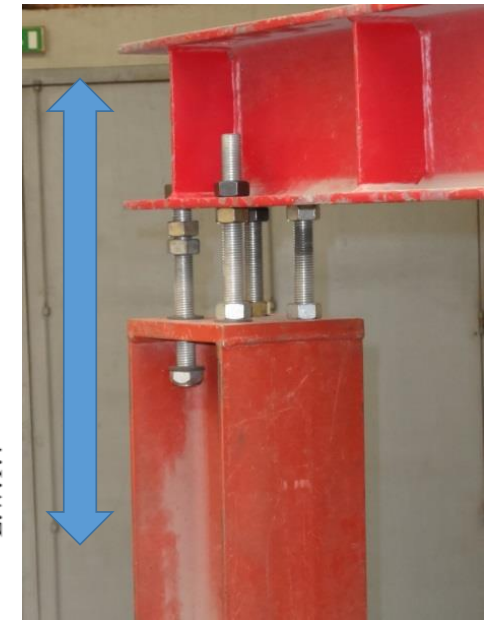

Figure 4.12 a) Positioning of LVDT on top and bottom of the CFS column. b) Location of thermocouples and LWT along the column length in both directions (about minor and major axis) c) Connections between peripheral columns and top beams.

\subsection{Test Plan}

\subsubsection{Buckling tests at ambient temperature}

The experimental campaign undertaken to assess the behavior, load-bearing capacity and failure modes of CFS columns consisted of 24 quasi-static compression tests. As previously mentioned four different cross-section shapes were tested, namely single lipped channels, open built-up I cross-section and two closed built-up cross-sections (R and 2R) (Figure 4.2). Also 
two end-support conditions were adopted using the designed devices. Hence it was attempted to simulate both pinned and fixed-end support conditions in order to assess lower and upper bounds to the strength of the tested columns and characterize the observed failure modes. For each cross-section and end-support condition, three repetitions were executed (total=24 tests). In Table 4.1 the test programme is detailed. In the Table 4.1, the reference C_PP_1, indicates the first test (1) of columns with lipped channel cross-section (C) and with pinned-end support condition (PP), while the reference 2R_FF_3 indicates the third (3) test of columns with closed built-up cross-section (2R) and with fixed end-support condition (FF).

Table 4.1 Test programme and predicted values for CFS columns.

\begin{tabular}{|c|c|c|c|c|c|}
\hline Test reference & $\bar{\lambda}$ & $\begin{array}{c}N_{b, R d} \\
{[\mathrm{kN}]}\end{array}$ & Load & End-support & Repetitions \\
\hline$\overline{\text { C_PP_i }}$ & 2.11 & 24.8 & \multirow{8}{*}{$\begin{array}{l}\text { Until } \\
\text { failure }\end{array}$} & \multirow{4}{*}{ Pinned } & 3 \\
\hline I_PP_i & 1.62 & 85.51 & & & 3 \\
\hline R_PP_i & 1.66 & 76.55 & & & 3 \\
\hline 2R_PP_i & 1.03 & 305.57 & & & 3 \\
\hline C_FF_i & 1.51 & 41.8 & & \multirow{4}{*}{ Fixed } & 3 \\
\hline I_FF_i & 0.9 & 187.7 & & & 3 \\
\hline R_FF_i & 0.94 & 168.27 & & & 3 \\
\hline 2R_FF_i & 0.59 & 443.42 & & & 3 \\
\hline
\end{tabular}

\subsubsection{Fire tests with restraint to thermal elongation}

The experimental campaign on CFS columns under fire situation with restraint to thermal elongation consisted of 96 fire tests. Several parameters were assessed in this extensive experimental investigation, namely the cross-section shape, the influence of end-support conditions, influence of initial service load applied to the column and the influence of the level of restraint to thermal elongation imposed by the surrounding structure to the CFS column. For each test condition three repetitions were conducted. For instance, lipped channel columns were tested considering two end-support conditions, two levels of service load and two levels of restraint to thermal elongation. Hence, 24 fire tests were undertaken for columns with lipped channel cross-section.

In Tables 4.2 and 4.3 the experimental campaign conducted is detailed. In the table, the reference C_PP_30LL_K1-2, indicates the second test (2) of the column with lipped channel (C) cross-section tested with $3 \mathrm{kN} / \mathrm{mm}$ of axial stiffness of the surrounding structure (K1) and a $30 \%$ load level (30LL) for the pin-ended support condition (PP), while the reference I_SR_50LL_K4-3 indicates the third test (3) of the column with built-up I (I) cross-section with 
$13 \mathrm{kN} / \mathrm{mm}$ of axial stiffness and 37237 and $12620 \mathrm{kN} . \mathrm{m} / \mathrm{rad}$ of rotational stiffness about the minor and major axis of the CFS column (K4), respectively and a 50\% load level (50LL) for the semi-rigid support condition (SR).

Also in Tables 4.2 and 4.3 the design $\left(N_{b, R d}\right)$ and applied loads $\left(P_{0}\right)$ are presented for the tested specimens. The applied load $\left(P_{0}\right)$ corresponded to 30 and $50 \%$ of the design buckling load at ambient temperature $\left(N_{b, R d}\right)$. The design buckling loads were determined according to the provisions presented in EN 1993-1-1 (2005), EN 1993-1-2 (2005) and EN 1993-1-5 (2006) (Appendix C). The non-dimensional slenderness was determined according the provisions presented in the EN 1993-1-1 (2005) for class 4 cross sections. The level of axial restraint imposed to a CFS column is defined as the ratio (Equation 4.1) between the axial stiffness of the surrounding structure to the CFS column $\left(K_{a, s}\right)$ and the axial stiffness of the column $\left(K_{a, c}\right)$ (Equation 4.2):

$$
\alpha_{K, 20^{\circ} \mathrm{C}}=\frac{K_{a, s}}{K_{a, c}}
$$

where

$$
k_{a, c}=\frac{A_{c} E_{c}}{L_{c}}
$$

The level of rotational restraint in each direction $\left(\rho_{i}\right)$ (Equation 4.3) on a CFS column is defined as the ratio between the rotational stiffness of the surrounding structure to the column $\left(K_{r, s}\right)$ and the sum of the flexural stiffness of the column (Equation 4.4) $\left(K_{r, c}\right)$ with the rotational stiffness of the surrounding structure $\left(K_{r, s}\right)$ in the intended direction:

$$
\rho_{i}=\frac{k_{r, s}}{k_{r, s}+k_{r, c}}
$$

where

$$
k_{r, c}=\frac{4 E_{c} I_{c}}{L_{c}}
$$

Regarding the Equation 4.3 when $\rho \approx 1$ the column is fully fixed and when $\rho=0$ the column is pin-ended. 
Table 4.2 Test programme and predicted values for CFS columns with single and open built-up cross-section.

\begin{tabular}{|c|c|c|c|c|c|c|c|c|c|c|c|c|c|}
\hline Test Reference & $\bar{\lambda}$ & $\begin{array}{l}\mathbf{N}_{\mathrm{cr}} \\
{[\mathrm{kN}]}\end{array}$ & $\begin{array}{c}\mathbf{N}_{b, R d} \\
{[k N]}\end{array}$ & $\begin{array}{c}\mathbf{P}_{0} \\
{[\mathrm{kN}]}\end{array}$ & $\begin{array}{c}\alpha_{k, 20 \circ C} \\
{[--]}\end{array}$ & $\begin{array}{c}\rho_{1,20} \mathrm{C} \\
{[--]} \\
\end{array}$ & $\begin{array}{c}\rho_{2,20 \circ C} \\
{[--]}\end{array}$ & $\begin{array}{c}\mathbf{K}_{\mathrm{a}, \mathbf{s}} \\
{[\mathrm{kN} / \mathbf{m m}]} \\
\end{array}$ & $\begin{array}{c}\mathbf{K}_{\mathbf{r}, \mathbf{s 1}} \\
{[\mathrm{kNm} / \mathbf{r a d}]}\end{array}$ & $\begin{array}{c}\mathbf{K}_{\mathbf{r}, \mathbf{s} 2} \\
{[\mathrm{kNm} / \mathrm{rad}]}\end{array}$ & $\begin{array}{c}\mathbf{K}_{\mathbf{a}, \mathbf{c}} \\
{[\mathrm{kN} / \mathbf{m m}]}\end{array}$ & $\begin{array}{c}\mathbf{K}_{\mathrm{r}, \mathrm{cl}} \\
{[\mathrm{kNm} / \mathrm{rad}]}\end{array}$ & $\begin{array}{c}\mathbf{K}_{\mathrm{r}, \mathrm{c} 2} \\
{[\mathrm{kNm} / \mathrm{rad}]}\end{array}$ \\
\hline C_PP_30LL_K1 & \multirow{4}{*}{2.11} & \multirow{4}{*}{32} & \multirow{4}{*}{24.8} & \multirow{2}{*}{$\begin{array}{c}7.4 \\
(30 \%)\end{array}$} & 0.067 & 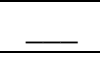 & $\underline{-}$ & \begin{tabular}{|l|}
3 \\
\end{tabular} & $\ldots$ & - & \multirow{4}{*}{44.8} & \multirow{4}{*}{31.4} & \multirow{4}{*}{436.8} \\
\hline C_PP_30LL_K2 & & & & & 0.290 & - & - & 13 & - & 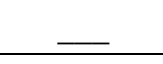 & & & \\
\hline C_PP_50LL_K1 & & & & \multirow{2}{*}{$\begin{array}{c}12.4 \\
(50 \%)\end{array}$} & 0.067 & + & + & 3 & 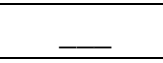 & - & & & \\
\hline C_PP_50LL_K2 & & & & & 0.290 & - & $\underline{-}$ & 13 & - & $\underline{-}$ & & & \\
\hline C_SR_30LL_K3 & \multirow{4}{*}{1.51} & \multirow{4}{*}{62} & \multirow{4}{*}{41.8} & \multirow{2}{*}{$\begin{array}{c}12.5 \\
(30 \%)\end{array}$} & 0.067 & 0.995 & 0.790 & 3 & 9253 & 2196 & \multirow{4}{*}{44.8} & \multirow{4}{*}{41.9} & \multirow{4}{*}{582.4} \\
\hline C_SR_30LL_K4 & & & & & 0.290 & 0.999 & 0.956 & 13 & 37237 & 12620 & & & \\
\hline C_SR_50LL_K3 & & & & \multirow{2}{*}{$\begin{array}{c}20.9 \\
(50 \%)\end{array}$} & 0.067 & 0.995 & 0.790 & 3 & 9253 & 2196 & & & \\
\hline C_SR_50LL_K4 & & & & & 0.290 & 0.999 & 0.956 & 13 & 37237 & 12620 & & & \\
\hline I_PP_30LL_K1 & \multirow{4}{*}{1.62} & \multirow{4}{*}{108} & \multirow{4}{*}{85.51} & \multirow{2}{*}{$\begin{array}{c}25.7 \\
(30 \%)\end{array}$} & 0.033 & 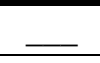 & $\overline{-}$ & 3 & $\ldots$ & 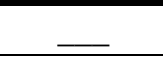 & \multirow{4}{*}{90.6} & \multirow{4}{*}{104.2} & \multirow{4}{*}{886.7} \\
\hline I_PP_30LL_K2 & & & & & 0.144 & - & - & 13 & 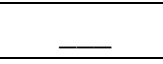 & - & & & \\
\hline I_PP_50LL_K1 & & & & \multirow{2}{*}{$\begin{array}{c}42.8 \\
(50 \%)\end{array}$} & 0.033 & - & - & 3 & $\ldots$ & - & & & \\
\hline I_PP_50LL_K2 & & & & & 0.144 & $\underline{-}$ & $\underline{-}$ & 13 & 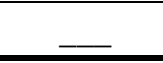 & $\underline{-}$ & & & \\
\hline I_SR_30LL_K3 & \multirow{4}{*}{0.9} & \multirow{4}{*}{350} & \multirow{4}{*}{187.7} & \multirow{2}{*}{$\begin{array}{c}56.3 \\
(30 \%)\end{array}$} & 0.033 & 0.985 & 0.650 & 3 & 9253 & 2196 & \multirow{4}{*}{90.6} & \multirow{4}{*}{138.9} & \multirow{4}{*}{1182.3} \\
\hline I_SR_30LL_K4 & & & & & 0.144 & 0.996 & 0.914 & 13 & 37237 & 12620 & & & \\
\hline I_SR_50LL_K3 & & & & \multirow{2}{*}{$\begin{array}{c}93.8 \\
(50 \%)\end{array}$} & 0.033 & 0.985 & 0.650 & 3 & 9253 & 2196 & & & \\
\hline I_SR_50LL_K4 & & & & & 0.144 & 0.996 & 0.914 & 13 & 37237 & 12620 & & & \\
\hline
\end{tabular}


Table 4.3 Test programme and predicted values for CFS columns with closed built-up cross-section.

\begin{tabular}{|c|c|c|c|c|c|c|c|c|c|c|c|c|c|}
\hline Test Reference & $\bar{\lambda}$ & $\begin{array}{l}\mathbf{N}_{\mathrm{cr}} \\
{[\mathrm{kN}]}\end{array}$ & $\begin{array}{c}\mathbf{N}_{\mathbf{b}, \mathbf{R d}} \\
{[\mathrm{kN}]}\end{array}$ & $\begin{array}{c}\mathbf{P}_{0} \\
{[\mathrm{kN}]}\end{array}$ & $\begin{array}{c}\boldsymbol{\alpha}_{\mathrm{k}, 20} \mathrm{oC}^{-} \\
{[--]}\end{array}$ & $\begin{array}{c}\boldsymbol{\rho}_{1,20} \mathrm{C} \\
{[--]}\end{array}$ & $\begin{array}{c}\rho_{2,20}{ }^{-C} \\
{[--]}\end{array}$ & $\begin{array}{c}\mathbf{K}_{\mathrm{a}, \mathbf{s}} \\
{[\mathrm{kN} / \mathbf{m m}]}\end{array}$ & $\begin{array}{c}\mathbf{K}_{\mathrm{r}, \mathrm{s} 1} \\
{[\mathrm{kNm} / \mathrm{rad}]}\end{array}$ & $\begin{array}{c}\mathbf{K}_{\mathrm{r}, \mathbf{s} 2} \\
{[\mathrm{kNm} / \mathrm{rad}]}\end{array}$ & $\begin{array}{c}\mathbf{K}_{\mathrm{a}, \mathrm{c}} \\
{[\mathrm{kN} / \mathbf{m m}]}\end{array}$ & $\begin{array}{c}\mathbf{K}_{\mathrm{r}, \mathrm{c1}} \\
{[\mathrm{kNm} / \mathrm{rad}]}\end{array}$ & $\begin{array}{c}\mathbf{K}_{\mathrm{r}, \mathrm{c} 2} \\
{[\mathrm{kNm} / \mathrm{rad}]}\end{array}$ \\
\hline R_PP_30LL_K1 & \multirow{4}{*}{1.66} & \multirow{4}{*}{96.0} & \multirow{4}{*}{76.55} & \multirow{2}{*}{$\begin{array}{l}22.96 \\
(30 \%)\end{array}$} & 0.034 & $\ldots$ & 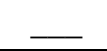 & 3 & 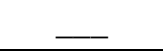 & - & \multirow{4}{*}{87.0} & \multirow{4}{*}{92.7} & \multirow{4}{*}{866.3} \\
\hline R_PP_30LL_K2 & & & & & 0.149 & $\ldots$ & 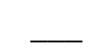 & 13 & 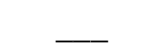 & $\ldots$ & & & \\
\hline R_PP_50LL_K1 & & & & \multirow{2}{*}{$\begin{array}{l}38.27 \\
(50 \%)\end{array}$} & 0.034 & $\ldots$ & 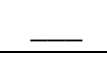 & 3 & - & 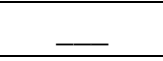 & & & \\
\hline R_PP_50LL_K2 & & & & & 0.149 & $\ldots$ & 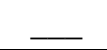 & 13 & 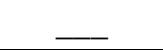 & $\ldots$ & & & \\
\hline R_SR_30LL_K3 & \multirow{4}{*}{0.94} & \multirow{4}{*}{301.1} & \multirow{4}{*}{168.27} & \multirow{2}{*}{$\begin{array}{l}50.48 \\
(30 \%)\end{array}$} & 0.034 & 0.987 & 0.655 & 3 & 9253 & 2196 & \multirow{4}{*}{87.0} & \multirow{4}{*}{123.6} & \multirow{4}{*}{1154.9} \\
\hline R_SR_30LL_K4 & & & & & 0.149 & 0.997 & 0.916 & 13 & 37237 & 12620 & & & \\
\hline R_SR_50LL_K3 & & & & \multirow{2}{*}{$\begin{array}{l}84.13 \\
(50 \%)\end{array}$} & 0.034 & 0.987 & 0.655 & 3 & 9253 & 2196 & & & \\
\hline R_SR_50LL_K4 & & & & & 0.149 & 0.997 & 0.916 & 13 & 37237 & 12620 & & & \\
\hline 2R_PP_30LL_K1 & \multirow{4}{*}{1.03} & \multirow{4}{*}{499} & \multirow{4}{*}{305.57} & \multirow{2}{*}{$\begin{array}{l}91.67 \\
(30 \%)\end{array}$} & 0.017 & $\underline{-}$ & 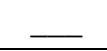 & 3 & - & $\ldots$ & \multirow{4}{*}{175.4} & \multirow{4}{*}{479.1} & \multirow{4}{*}{1740.1} \\
\hline 2R_PP_30LL_K2 & & & & & 0.074 & $\ldots$ & 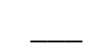 & 13 & 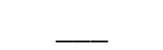 & $\ldots$ & & & \\
\hline 2R_PP_50LL_K1 & & & & \multirow{2}{*}{$\begin{array}{l}152.78 \\
(50 \%)\end{array}$} & 0.017 & 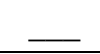 & - & 3 & 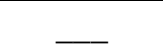 & $\ldots$ & & & \\
\hline 2R_PP_50LL_K2 & & & & & 0.074 & $\ldots$ & 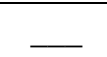 & 13 & $\ldots$ & $\ldots$ & & & \\
\hline 2R_SR_30LL_K3 & \multirow{4}{*}{0.59} & \multirow{4}{*}{1500} & \multirow{4}{*}{443.42} & \multirow{2}{*}{$\begin{array}{l}133.02 \\
(30 \%)\end{array}$} & 0.017 & 0.935 & 0.486 & 3 & 9253 & 2196 & \multirow{4}{*}{175.4} & \multirow{4}{*}{638.8} & \multirow{4}{*}{2320.1} \\
\hline 2R_SR_30LL_K4 & & & & & 0.074 & 0.983 & 0.845 & 13 & 37237 & 12620 & & & \\
\hline 2R_SR_50LL_K3 & & & & \multirow{2}{*}{$\begin{array}{l}221.71 \\
(50 \%)\end{array}$} & 0.017 & 0.935 & 0.486 & 3 & 9253 & 2196 & & & \\
\hline 2R_SR_50LL_K4 & & & & & 0.074 & 0.983 & 0.845 & 13 & 37237 & 12620 & & & \\
\hline
\end{tabular}




\subsection{Test Procedure}

\subsubsection{Buckling tests at ambient temperature}

Compression tests were conducted in order to assess the ultimate load-bearing capacity and characterize the observed failure modes of different types of CFS columns fabricated using CFS single profiles. These tests provided useful results to understand the behavior of CFS columns as well as to be used for validation of finite element models. The compressive load was applied under displacement control, which was controlled by a TML SDP-200D LVDT (Linear Variable Displacement Transducer), adopting an approximate rate of $0.01 \mathrm{~mm} / \mathrm{s}$, until the tested specimen failed and the unloading stage was reached, where the lateral deformation was too large. As previously mentioned, during each experimental test axial load, axial displacement, lateral deformations, rotations of the end-support devices and strains in different points of the cross-section at mid-height of the column were monitored and recorded using the data acquisition system TML TDS-530. In Figure 4.13 some details regarding the positioning of strain gauges in the tested cross-sections is presented (schematic of the strain gauges positioning see Figure 4.2).

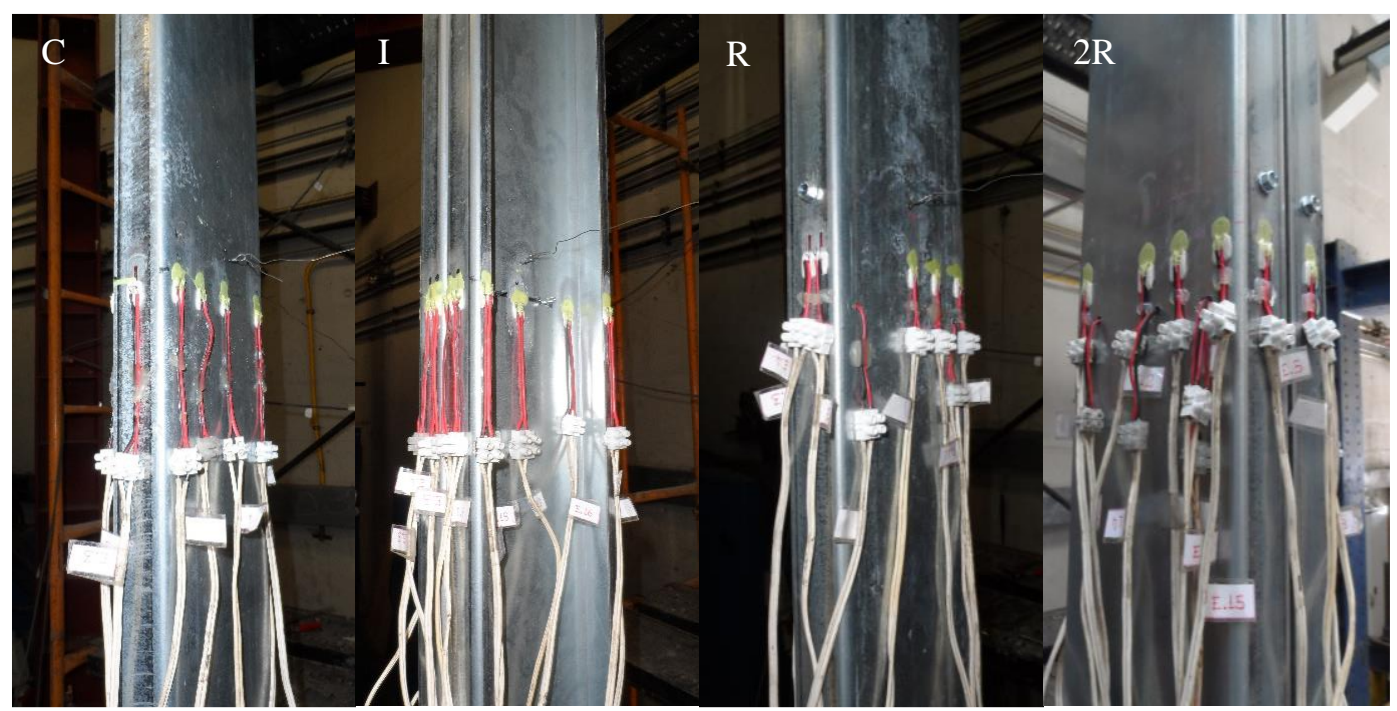

Figure 4.13 Positioning of the strain gauges in all cross-sections tested.

\subsubsection{Fire tests with restraint to thermal elongation}

In order to assess the behavior of CFS columns, with different cross-section shapes, under fire conditions and with restraint to thermal elongation imposed by the surrounding structure an 
extensive experimental campaign was conducted. As previously mentioned, different types of cross-section shapes, different levels of service load, different end-support conditions and different levels of restraint to thermal elongation were tested. In each experimental test the service load (30 and 50\% $N_{b, R d}$ ) (Appendix C) was applied to the CFS column using the hydraulic jack ((3) in Figure 4.9a) and b) and Figure 4.10) fixed to the reaction frame ((1) in Figure 4.9a) and b) and Figure 4.10) and with the nuts of the threaded rods loosened (Figure $4.12 \mathrm{c})$ ). This means that the orthogonal top beams of the restraining frame had free vertical movement, guaranteeing that the compressive load was totally transferred to the CFS column. The applied service load was controlled by a load cell placed between the top beams of the restraining frame and the hydraulic jack. Reaching the serviceability load, the nuts of the threaded rods of the peripheral columns to top beams connections were tightened (Figure 4.12c) and from that moment the restraining frame ((2) in Figure 4.9a) and b) and Figure 4.10) started to impose axial and rotational restraint to the CFS column being tested in fire ((6) in Figure 4.9a)). During the entire test the initial applied load was kept constant.

The thermal action was applied by a vertical modular electric furnace ((4) in Figure 4.9a) and b) and Figure 4.10) programmed to reproduce the standard fire curve ISO 834 (1999). With heating the CFS column started to expand due to thermal elongation of steel and since the column was restrained additional axial forces were generated. The generated restraining forces were measured by the load cell placed in the device presented in Figure 4.11. At the same time and with temperature increase the mechanical properties of the S280GD+Z steel degraded. After reaching a maximum the column cannot withstand additional axial stresses and the generated restraining forces started to decrease, reaching once again the initially applied serviceability load (failure criteria). At this moment it was assumed that the column failed. This instant was used to establish the values for critical times and temperature for each CFS column tested in the scope of this investigation.

This experimental investigation was attempted to simulate both pinned (about the minor axis) and semi-rigid end-support conditions. Finally, it is worth mentioning, once again, that in these fire tests, compressive service load, generated restraining forces, axial displacements, lateral deflections and temperatures in different points of the tested cross-sections and along the length of the column were monitored and recorded using the data acquisition system TML TDS-530. 


\subsection{Results and Discussion}

\subsubsection{Buckling tests at ambient temperature}

Figure 4.14 shows the obtained results in the experimental tests for both pinned and fixed-end support conditions. Load vs axial displacement curves are presented. As comparison, also the loads determined using the data provided by the strain gauges (SG) (considering the influence area of each one of the used strain gauges and assuming an elastic-perfectly plastic material behaviour as simplification) are presented as a function of the axial displacements. Comparing the loads monitored with the load cell with the loads determined using the strain gauges data (SG) a very good agreement was observed in the loading stage and in terms of the maximum axial load. It was observed that for the three tests conducted for each type of cross-section the loading stage, failure load and unloading stage was very similar. In this section only one test result of each cross-section tested is presented. In Appendix D all test results are presented. For all tests undertaken a small curvature is observed in the first part of the load vs axial displacement curves. This is due to small adjustments in the end-support devices that occur in the initial stage of the loading process. Hence, the actual axial displacement of the CFS column is slightly lower than the ones presented. However, the actual axial displacement can be easily estimated using the linear part of each test result.
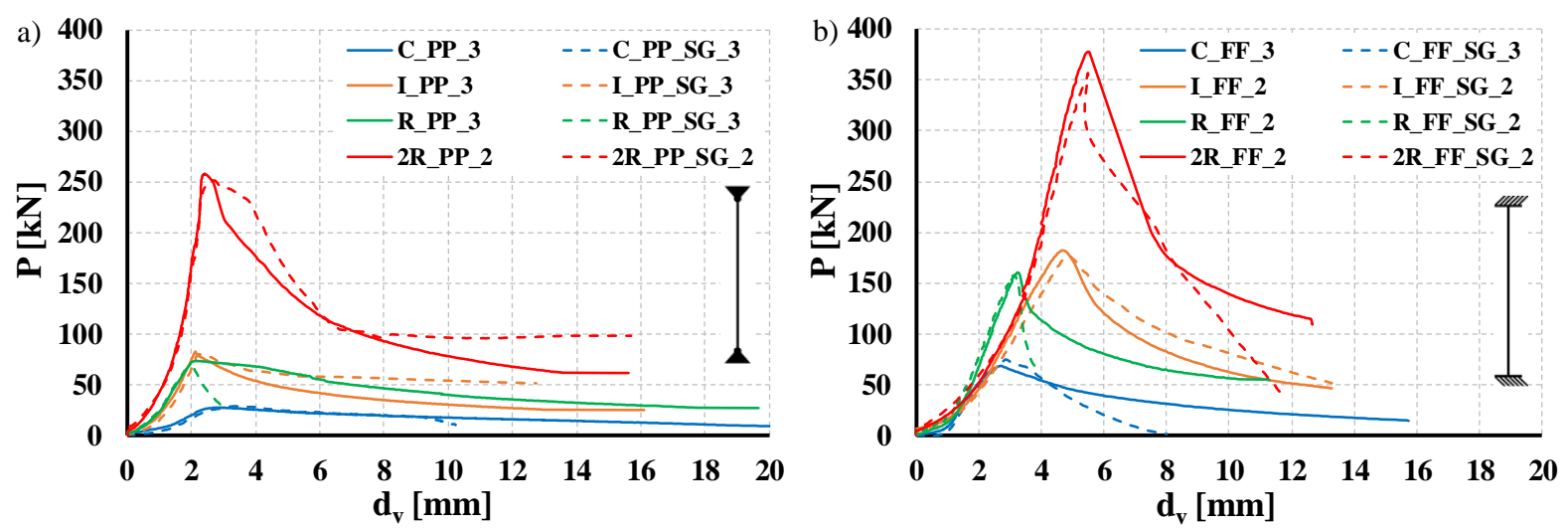

Figure 4.14 Load-axial displacement diagrams for the compression tests undertaken on CFS columns with C, I, R and 2R cross-sections. a) Results for the pinned-end support condition.

b) Results for the fixed-end support condition.

In Figure 4.15 the evolution of the relative axial compressive load $\left(P / P_{\max }\right)$ as a function of the lateral deflections about the minor axis of the CFS columns tested is presented. It was observed that for all tested columns the lateral deflections about the major axis were almost negligible $(<$ $5 \mathrm{~mm}$ ). Generally, it can be stated that for the same $P / P_{\max }$ the biggest lateral deformations were 
observed in the lipped channel (C) columns, as it was expected. In Table 4.4 and 4.5 the obtained results are detailed for all tested cross-sections and end-support conditions.
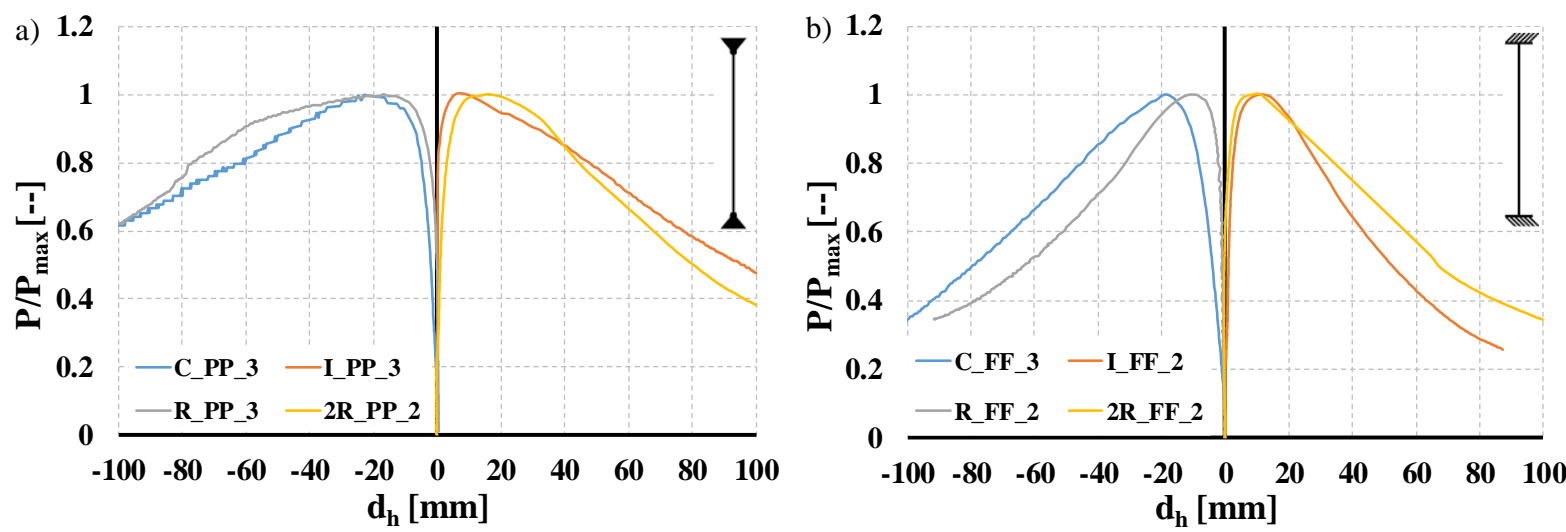

Figure 4.15 Evolution of the relative compressive loads $\left(P / P_{\max }\right)$ as a function of the lateral deflection at mid-height.

Table 4.4 Test results for pinned-end columns.

\begin{tabular}{|c|c|c|c|c|}
\hline \multirow{2}{*}{ Test } & \multicolumn{2}{|c|}{$\mathbf{P}_{\max }[\mathrm{kN}]$} & \multirow{2}{*}{$\frac{\mathbf{d}_{\mathbf{v}, \mathbf{P m a x}}}{[\mathrm{mm}]}$} & \multirow{2}{*}{$\begin{array}{c}\mathbf{d}_{\mathbf{h}, \mathbf{P m a x}} \\
{[\mathbf{m m}]}\end{array}$} \\
\hline & L.C. & SG & & \\
\hline C_PP-1 & 24.53 & -- & 2.68 & 29.3 \\
\hline C_PP-2 & 29.14 & 29.32 & 2.7 & 25.2 \\
\hline C_PP-3 & 27.04 & 28.64 & 2.95 & 23.1 \\
\hline$\mu$ & 26.90 & 28.98 & $2 . \overline{7} \overline{7}$ & 25.86 \\
\hline$\sigma$ & 1.88 & 0.34 & 0.12 & 2.57 \\
\hline C.V. & 7.00 & 1.17 & 4.42 & 9.95 \\
\hline I_PP-1 & 74.69 & -- & 2.63 & 25.82 \\
\hline I_PP-2 & 69.06 & 67.28 & 2.88 & 24.6 \\
\hline I_PP-3 & 83.18 & 85.22 & 2.13 & 17.1 \\
\hline$\mu$ & 75.64 & 76.25 & $2 . \overline{5}$ & 27.85 \\
\hline$\sigma$ & 5.80 & 8.97 & 0.31 & 2.37 \\
\hline C.V. & 7.67 & 11.76 & 2.13 & 8.54 \\
\hline R_PP-1 & 66.25 & -- & 2.63 & 28.68 \\
\hline R_PP-2 & 70.43 & 68.1 & 2.2 & 25.1 \\
\hline R_PP-3 & 73.37 & 72.9 & 2.17 & 16.8 \\
\hline$\mu$ & 70.01 & 70.5 & 2.33 & 23.52 \\
\hline$\sigma$ & 2.92 & 2.4 & 0.21 & 4.97 \\
\hline C.V. & 4.17 & 3.40 & 9.0 & 21.15 \\
\hline 2R_PP-1 & 268.79 & -- & 2.33 & 12.48 \\
\hline 2R_PP-2 & 257.7 & 251.7 & 2.40 & 16 \\
\hline 2R_PP-3 & 235.14 & 219.45 & 2.50 & 20.7 \\
\hline$\mu$ & 253.87 & 235.57 & $2 . \overline{4}$ & 16.39 \\
\hline$\sigma$ & 14.00 & 16.12 & 0.07 & 3.36 \\
\hline C.V. & 5.51 & 6.84 & 2.89 & 20.54 \\
\hline
\end{tabular}


Table 4.5 Test results for fixed-end columns.

\begin{tabular}{|c|c|c|c|c|}
\hline \multirow{2}{*}{ Test } & \multicolumn{2}{|c|}{$\mathbf{P}_{\max }[\mathbf{k N}]$} & \multirow{2}{*}{$\frac{d_{v, P m a x}}{[\mathrm{~mm}]}$} & \multirow{2}{*}{$\frac{d_{\mathbf{h}, \text { Pmax }}}{[\mathrm{mm}]}$} \\
\hline & L.C. & SG & & \\
\hline C_FF-1 & 65.62 & -- & 2.65 & 18.4 \\
\hline C_FF-2 & 64.35 & 74.03 & 2.67 & 20.8 \\
\hline C_FF-3 & 69.22 & 75.3 & 2.73 & 19 \\
\hline$\mu$ & 66.39 & 74.66 & $2 . \overline{68}$ & 19.40 \\
\hline$\sigma$ & 2.06 & 0.63 & 0.03 & 1.02 \\
\hline C.V. & 3.10 & 0.85 & 1.27 & 5.25 \\
\hline I_FF-1 & 198.75 & -- & 4.46 & 9.6 \\
\hline I_FF-2 & 182.07 & 175.23 & 4.71 & 12.2 \\
\hline I_FF-3 & 180.11 & 185.05 & 4.14 & 10.3 \\
\hline$\mu$ & 186.97 & 180.14 & $4 . \overline{43}$ & 10.7 \\
\hline$\sigma$ & 8.36 & 4.91 & 0.23 & 1.09 \\
\hline C.V. & 4.47 & 2.72 & 5.25 & 10.26 \\
\hline R_FF-1 & 148.03 & -- & 3.30 & 18 \\
\hline R_FF-2 & 160.78 & 157.87 & 3.26 & 11.2 \\
\hline R_FF-3 & 138.22 & 128.93 & 3.66 & 18.8 \\
\hline$\mu$ & 149.01 & 143.4 & $3 . \overline{4} 1$ & 16 \\
\hline$\sigma$ & 9.23 & 14.47 & 0.18 & 3.40 \\
\hline C.V. & 6.19 & 10.09 & 5.28 & 21.31 \\
\hline 2R_FF-1 & 388.67 & -- & 5.62 & 7.6 \\
\hline 2R_FF-2 & 377.88 & 355.92 & 5.51 & 8 \\
\hline 2R_FF-3 & 356.59 & 372.56 & 5.68 & 11.5 \\
\hline$\mu$ & 374.38 & 364.24 & 5.60 & 9.03 \\
\hline$\sigma$ & 13.328 & 8.32 & 0.07 & 1.75 \\
\hline C.V. & 3.560 & 2.284 & 1.25 & 19.39 \\
\hline
\end{tabular}

Observing the obtained results, it is clear the advantages of using built-up cross-sections in CFS building construction industry. For instance, the buckling load of columns with $2 \mathrm{R}$ crosssection was 9.4 times higher than the buckling load of columns with lipped channels, 3.35 times higher than the buckling load of columns with open built-up I cross-section and 3.62 times higher than the buckling load of columns with closed built-up R cross-section, for the pinnedend support condition. For fixed-end support condition, the buckling load of columns with $2 \mathrm{R}$ cross-section was 5.6 times higher than the buckling load of columns with lipped channels, 2.01 times higher than the buckling load of columns with open built-up I cross-section and 2.51 times higher than the buckling load of columns with closed built-up R cross-section, for the pinned-end support condition.

For the pinned-end support conditions the rotations of the end-support devices were determined using the monitored data during each test. In Figure 4.16 the determined rotations are presented 
for each cross-section tested. The rotations are due to flexural buckling of the CFS columns about the minor axis.
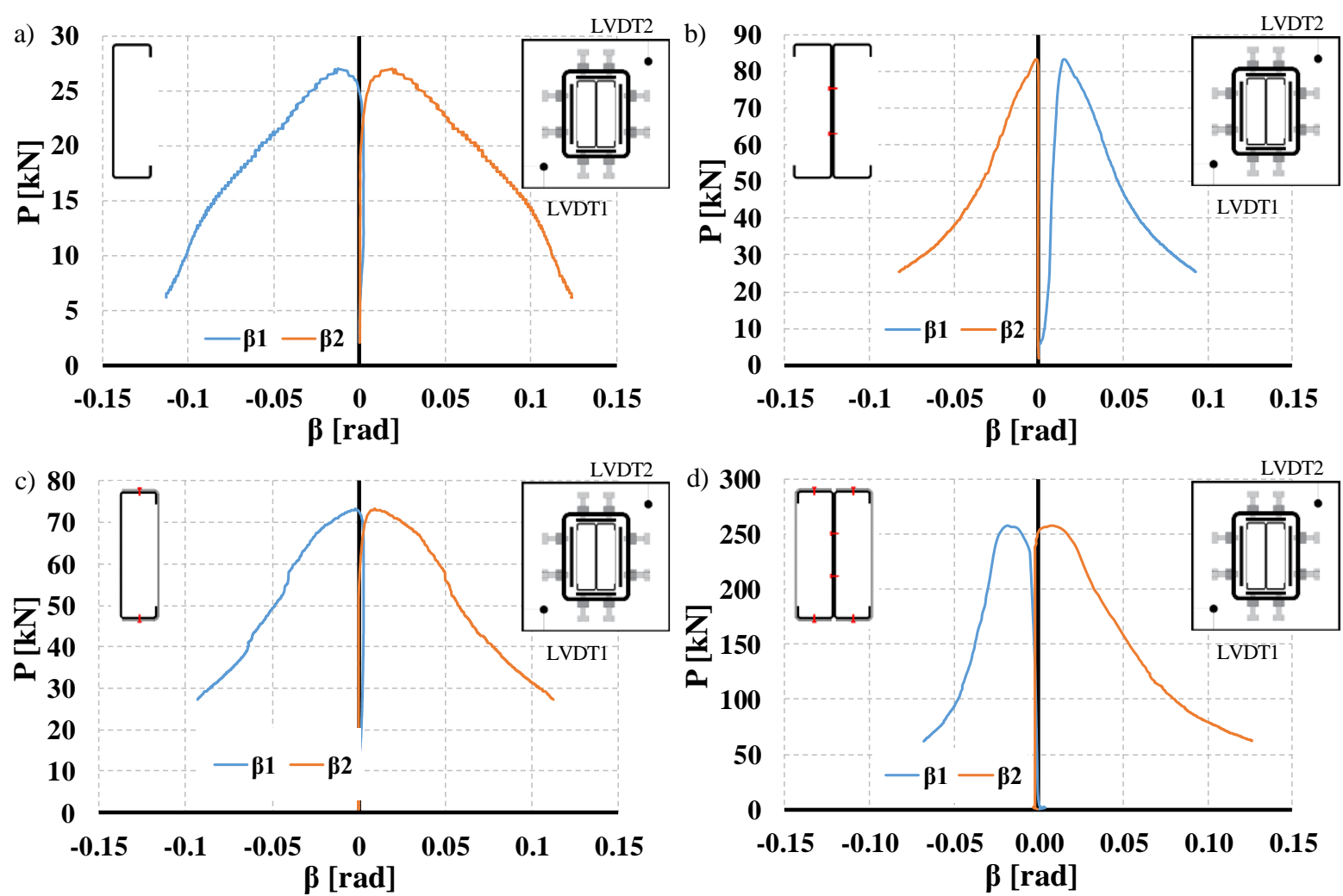

Figure 4.16 Rotations of the pinned-end support devices determined using the measurements obtained during each experimental test. a) C_PP_3.b) I_PP_3.c) R_PP_3.d) 2R_PP_2.

In Figure 4.17, 4.18, 4.19 and 4.20 lateral deflections about the minor axis along the length of the column, for each cross-section of the tested columns and for both end-support conditions, are presented. The lateral deflections along the length of the columns are presented as a function of the applied loading, namely $25 \% P_{\max }, 50 \% P_{\max }, 75 \% P_{\max }, P_{\max }$ (loading stage), $75 \% P_{\max }$, $50 \% P_{\max }$ and $25 \% P_{\max }$ (unloading stage). Observing the obtained curves, the influence of the end-support devices on the final deformed shape was clear. For the fixed-end support condition the points of inflection in the deformed shape are recognizable. However, it is worth mentioning that especially for the closed built-up cross-sections the inflection points in the deformed shape at maximum axial load $\left(\mathrm{P}_{\max }\right)$ are slightly subtler. This may be due, eventually, to a minor rotation in the end-support device, hence, it is not possible to guarantee for sure that the fully fixed condition was actually achieved in these experimental tests. Hence, it is possible that the upper bound of resistance for the tested columns was not determined in these tests. The same can be stated regarding the lower bound of resistance of the CFS columns, since the perfect pinned-end support was not achieved in these tests due to friction between the steel pin and 
steel plates of the end-support devices. In reality semi-rigid end-support conditions with low and high rotational stiffness were tested in the scope of this investigation. Based on the obtained experimental results finite element models were developed and validated (Chapter 5) and in the future extensive parametric studies should be undertaken.

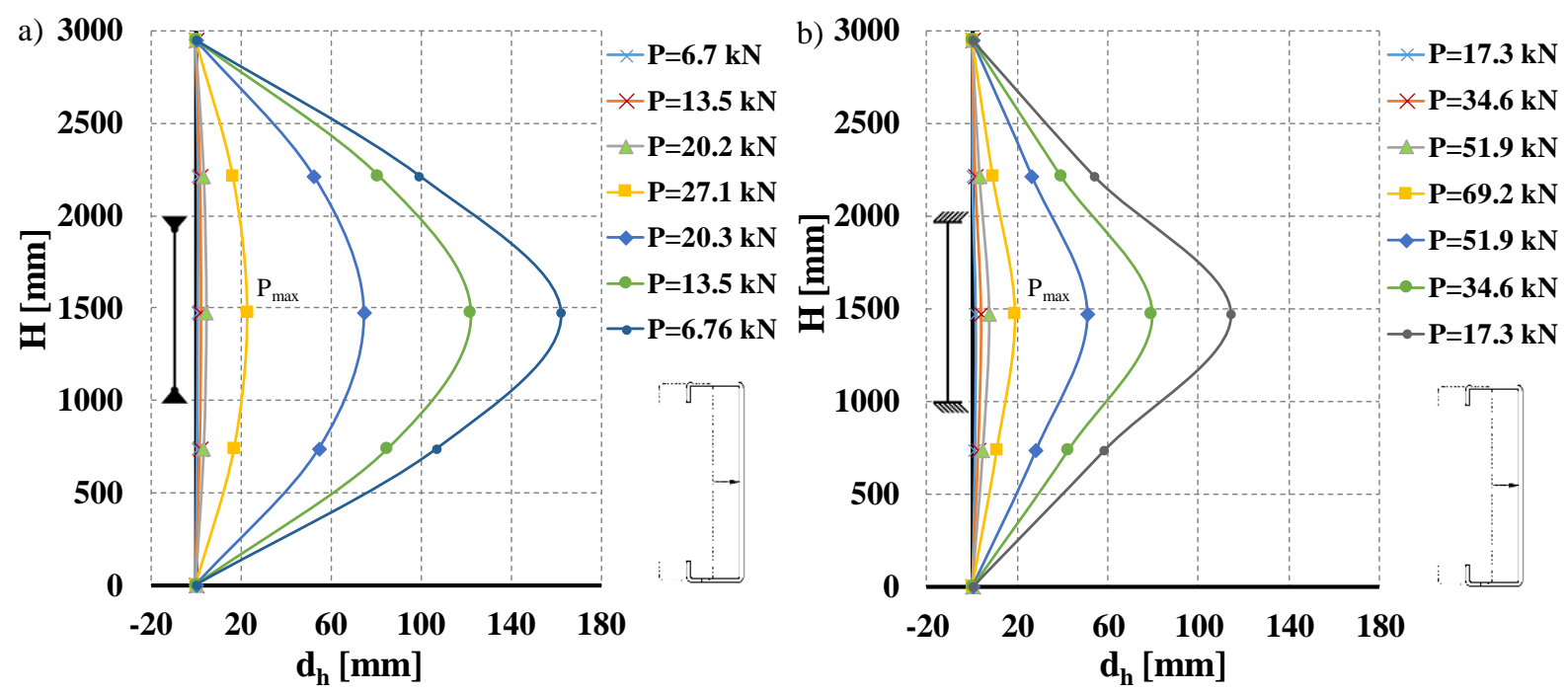

Figure 4.17 Evolution of lateral deflections about the minor axis along the column for the lipped channel (C) cross-section. a) Pinned-end. b) Fixed-end.
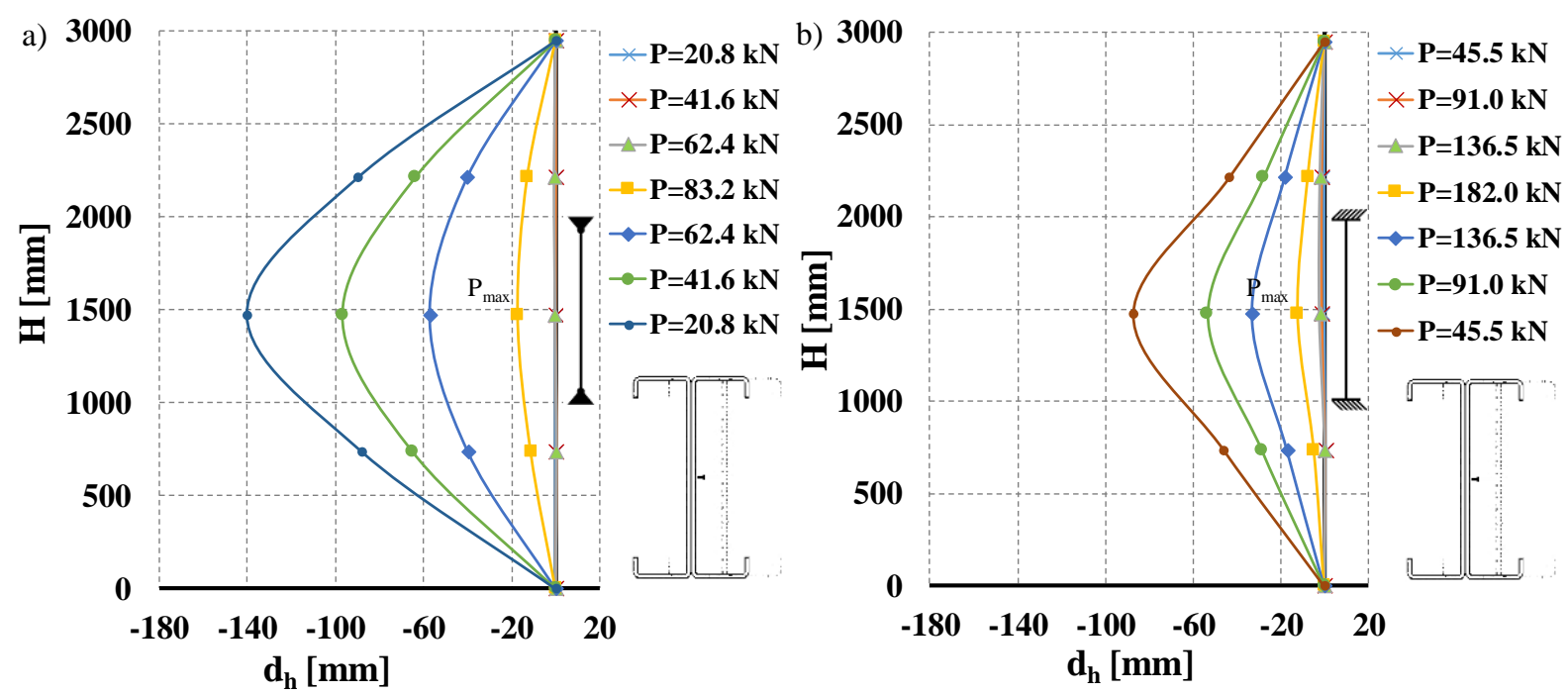

Figure 4.18 Evolution of lateral deflections about the minor axis along the column for the open built-up (I) cross-section. a) Pinned-end. b) Fixed-end. 

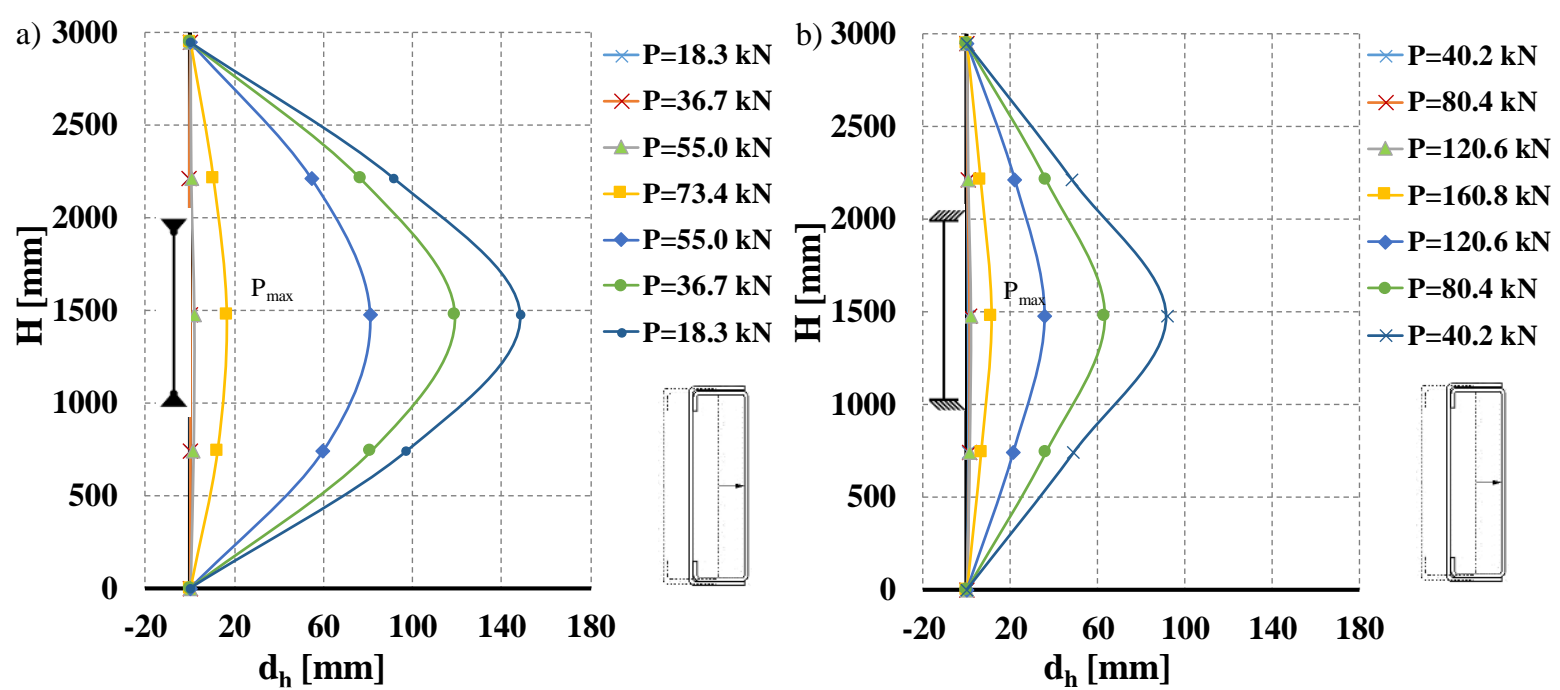

Figure 4.19 Evolution of lateral deflections about the minor axis along the column for the closed built-up (R) cross-section. a) Pinned-end. b) Fixed-end.
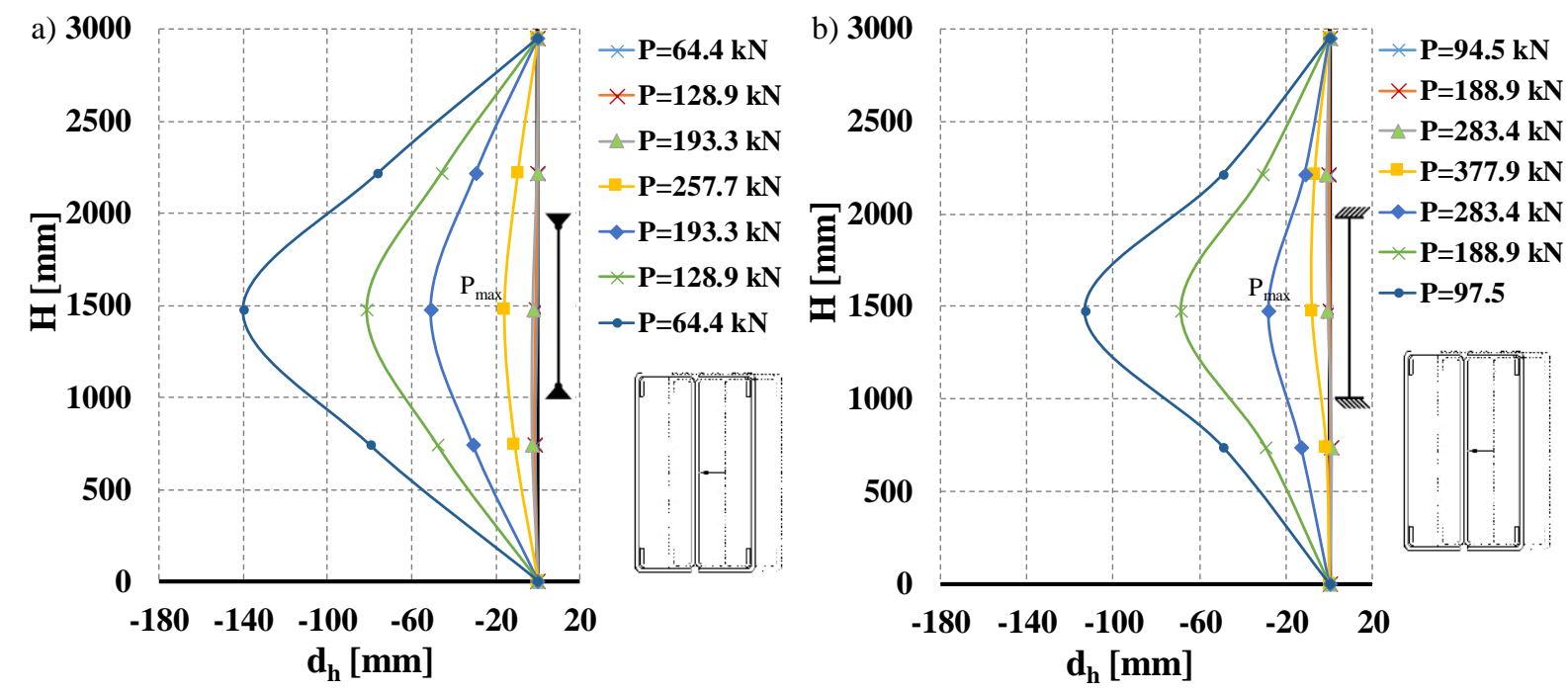

Figure 4.20 Evolution of lateral deflections about the minor axis along the column for the closed built-up (2R) cross-section. a) Pinned-end. b) Fixed-end.

Another important parameter assessed in this experimental study was the evolution of strains, during compressive loading, in the different tested cross-sections. The positioning of the strain gauges is presented in Figure 4.2. In Figure 4.14 the monitored axial load was compared with the axial load determined using data provided by the strain gauges. It is worth mentioning that in this step an elastic perfectly-plastic material behaviour $\left(f_{y}=306.8 \mathrm{MPa}\right)$ was assumed for simplification. It was observed a very good agreement between obtained axial loads using both measurement devices (Figure 4.14), namely load-cell and strain gauges, especially in the 
loading stage. In Figures 4.20 to 4.24 the obtained results for the strain evolution for the different tested cross-sections and end-support conditions are presented and detailed. It is clear that for all tested cross-sections and for all tested conditions (pinned-end and fixed-end) the readings of the strain gauges were very uniform during the loading stage of the compression tests. During the loading stage, for all tested cross-sections and tested conditions, the entire cross-section was subjected to compression stresses.
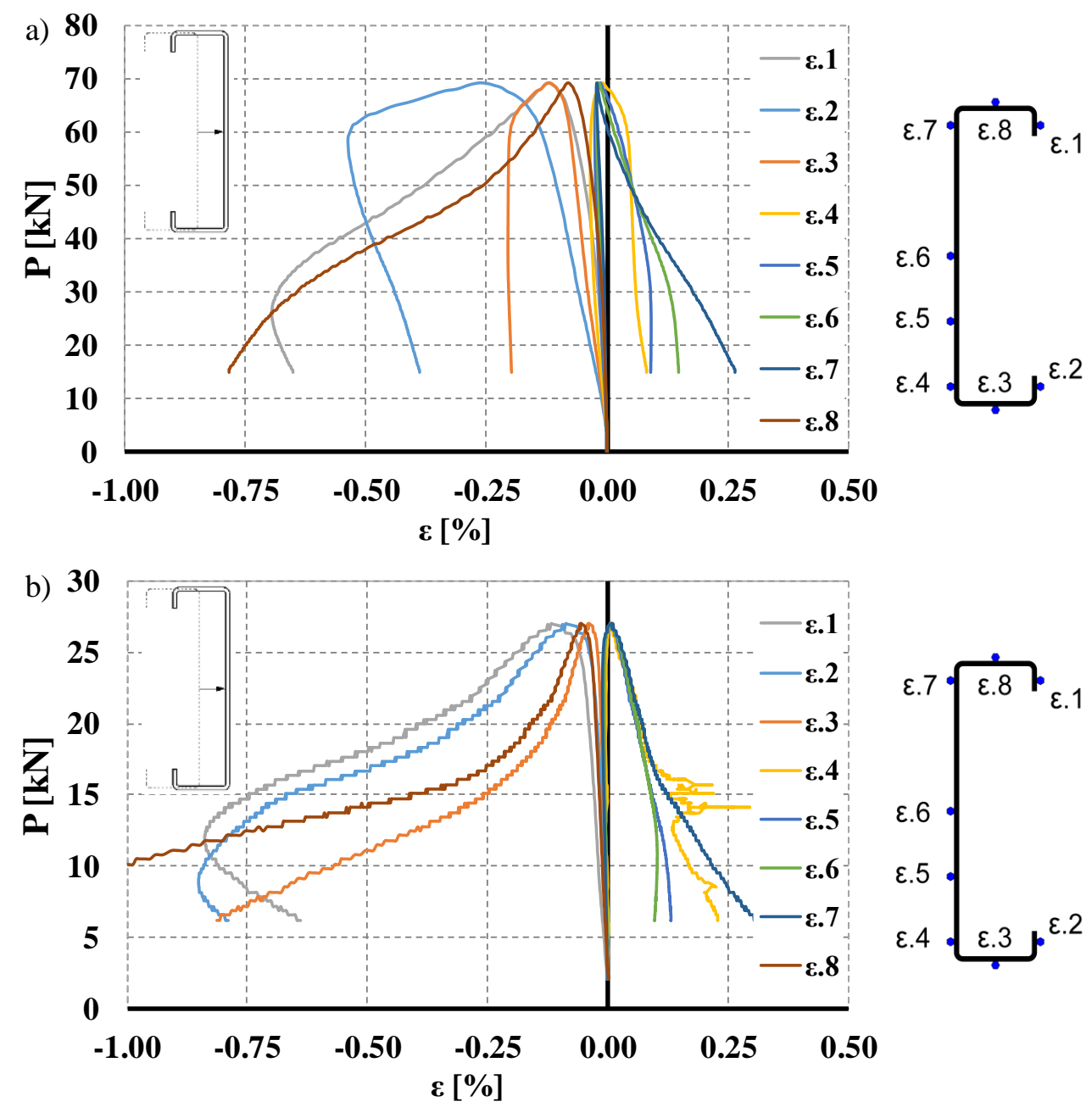

Figure 4.21 Load vs strain curves obtained from the strain gauges. a) C_FF_3. b) C_PP_3.

Observing the load vs strain curves it is clear that during the unloading stage of the tests part of the section was subjected to compressive stresses and the remaining part of the cross-section was subjected to tensile stresses. For the lipped channel cross-section and for both pinned and fixed-end support the lips moved in the direction of the web of the cross-section as presented

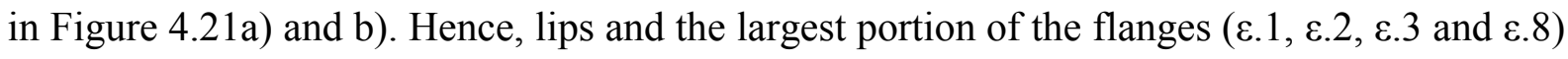
were subjected to compression stresses whereas the web $(\varepsilon .4, \varepsilon .5, \varepsilon .6, \varepsilon .7)$ was subjected to 
tensile stresses in the unloading stage. It is interesting to observe the behaviour of the strain gauges $\varepsilon .1$ and $\varepsilon .2$ for both fixed and pinned-end support. This peculiar behaviour may indicate the occurrence of a sectional buckling mode, namely distortional buckling near the position of the strain gauges.

Analysing the results for the open built-up I cross-section (Figure 4.22 a1), a2) and 4.22 a1)

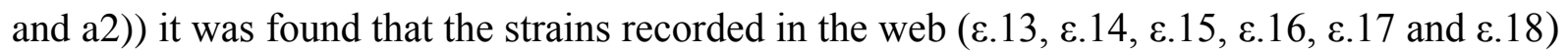
of the built-up open I cross-section are almost identical in the loading and unloading stage of the test. Hence, it is most likely that during the test no relevant sectional buckling modes occur in the web of the built-up open I cross-section. The same type of behaviour in the readings of the strains gauges observed in the lips of the lipped channel cross-section, was found for the lips subjected to compression stresses of open built-up cross-section $(\varepsilon .7, \varepsilon .8)$. Hence, this is probably due to the occurrence of distortional buckling near the position of the strain gauges.
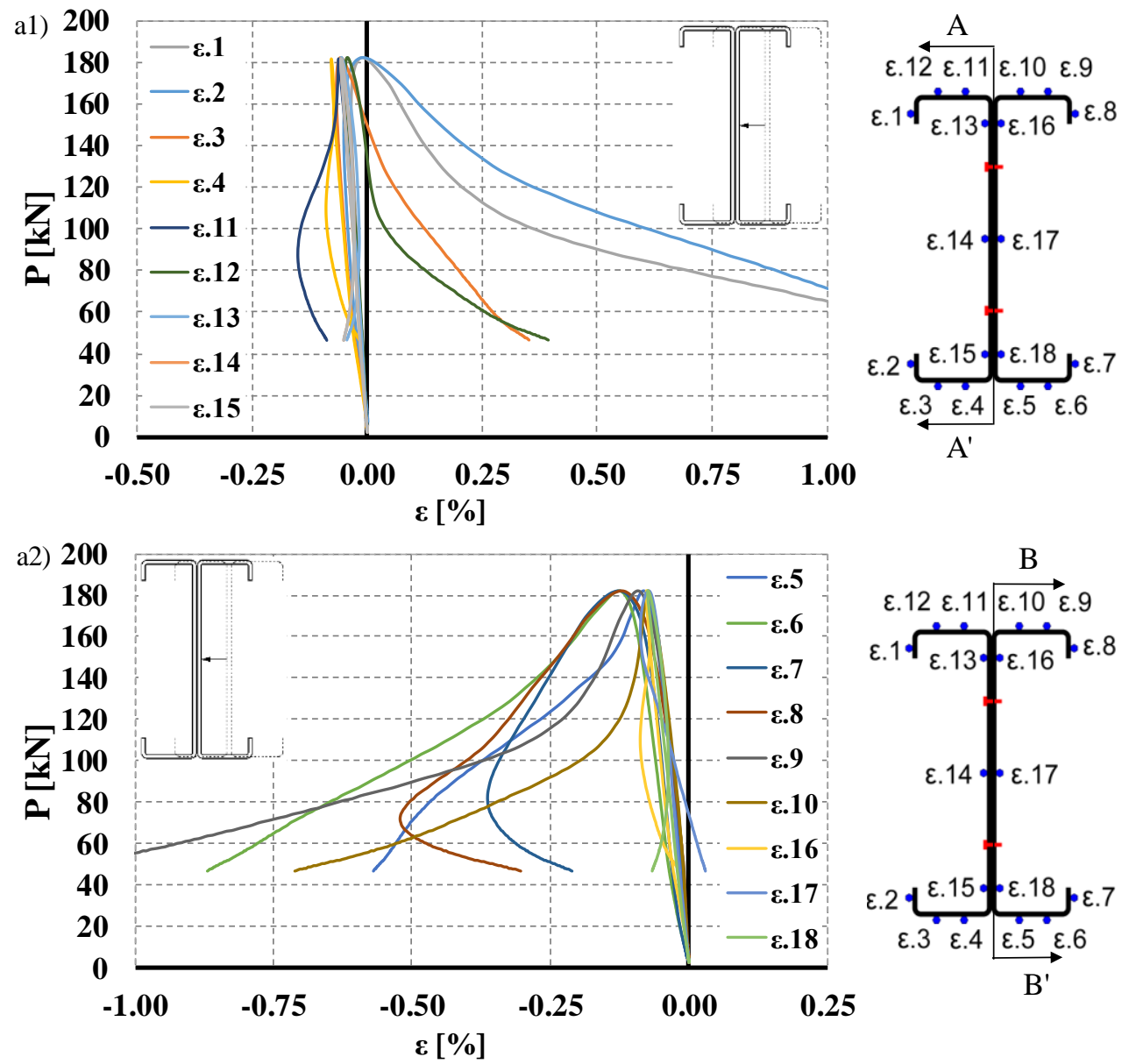

Figure 4.22 Load vs strain curves obtained from the strain gauges. a1) and a2) I_FF_2. 

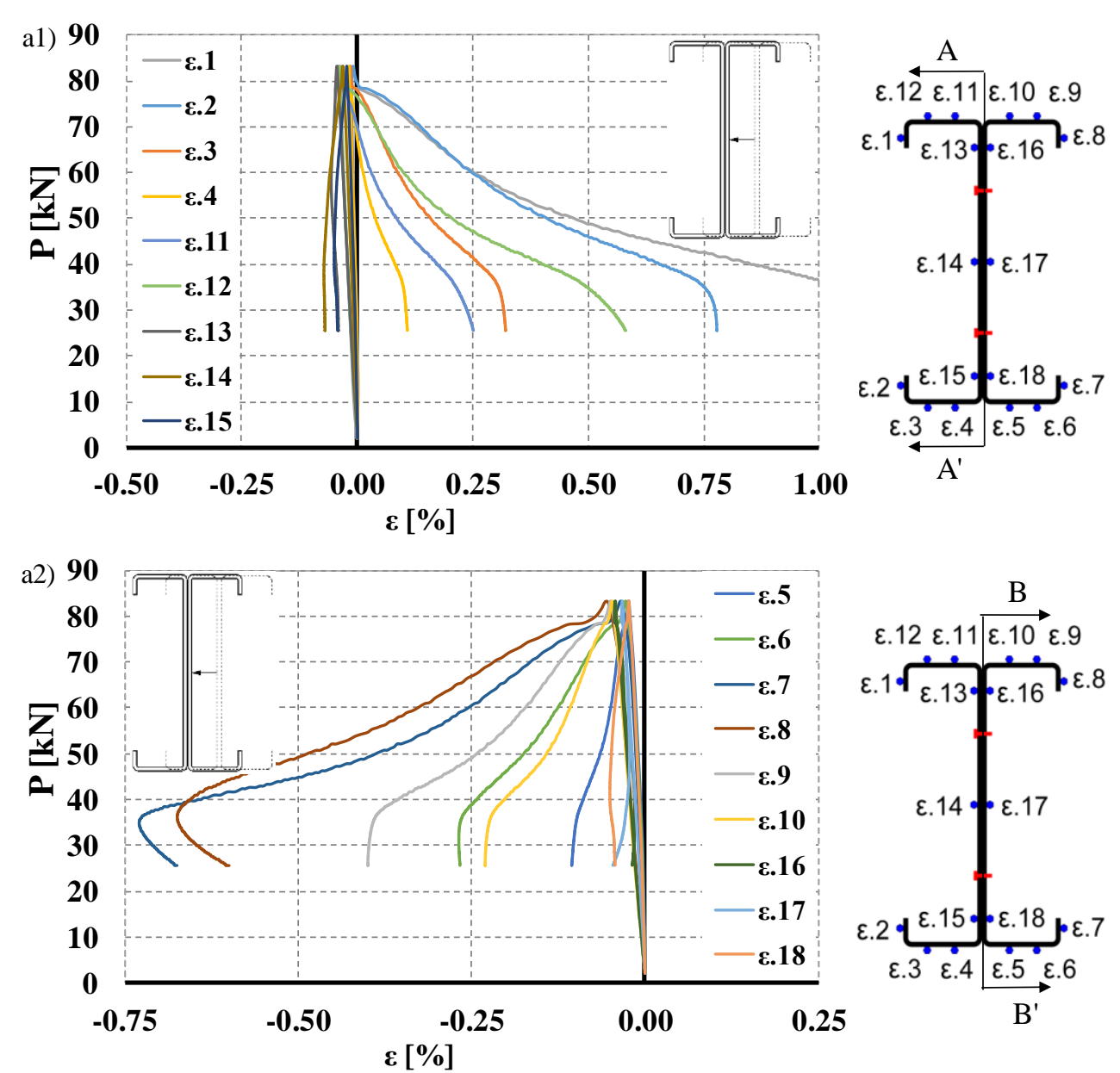

Figure 4.23 Load vs strain curves obtained from the strain gauges for the pinned-end column. a1) and a2) I_PP_3.

For the closed built-up R cross-section, it was observed that during the loading stage the entire cross-section was subjected to compressive stresses. In the unloading stage and for both pinned and fixed-end support some singularities in the readings of the strain gauges were found. For instance, in the unloading stage, for an axial load of approximately $100 \mathrm{kN}$ some type of interaction between sectional buckling modes (local and distortional) has occurred. This is very clear for $\varepsilon .1, \varepsilon .2$ and $\varepsilon .5$. This singularity in the readings of these strain gauges is most likely due to the occurrence of local buckling where the strain gauges were positioned. Further, since there is a sudden change from compressive strains to tensile strains, the strain gauges were positioned exactly on top of the wave originated in the web of the plain channel (U) due to local buckling. Regarding the lipped channel of the built-up closed column it seems that no particular sectional buckling has occurred. After reaching the buckling load the plain channel moved in the direction of the web of the lipped channel. Hence, lips and flanges of the lipped channel were subjected to compressive stresses whereas the web was subjected to tensile stresses. 

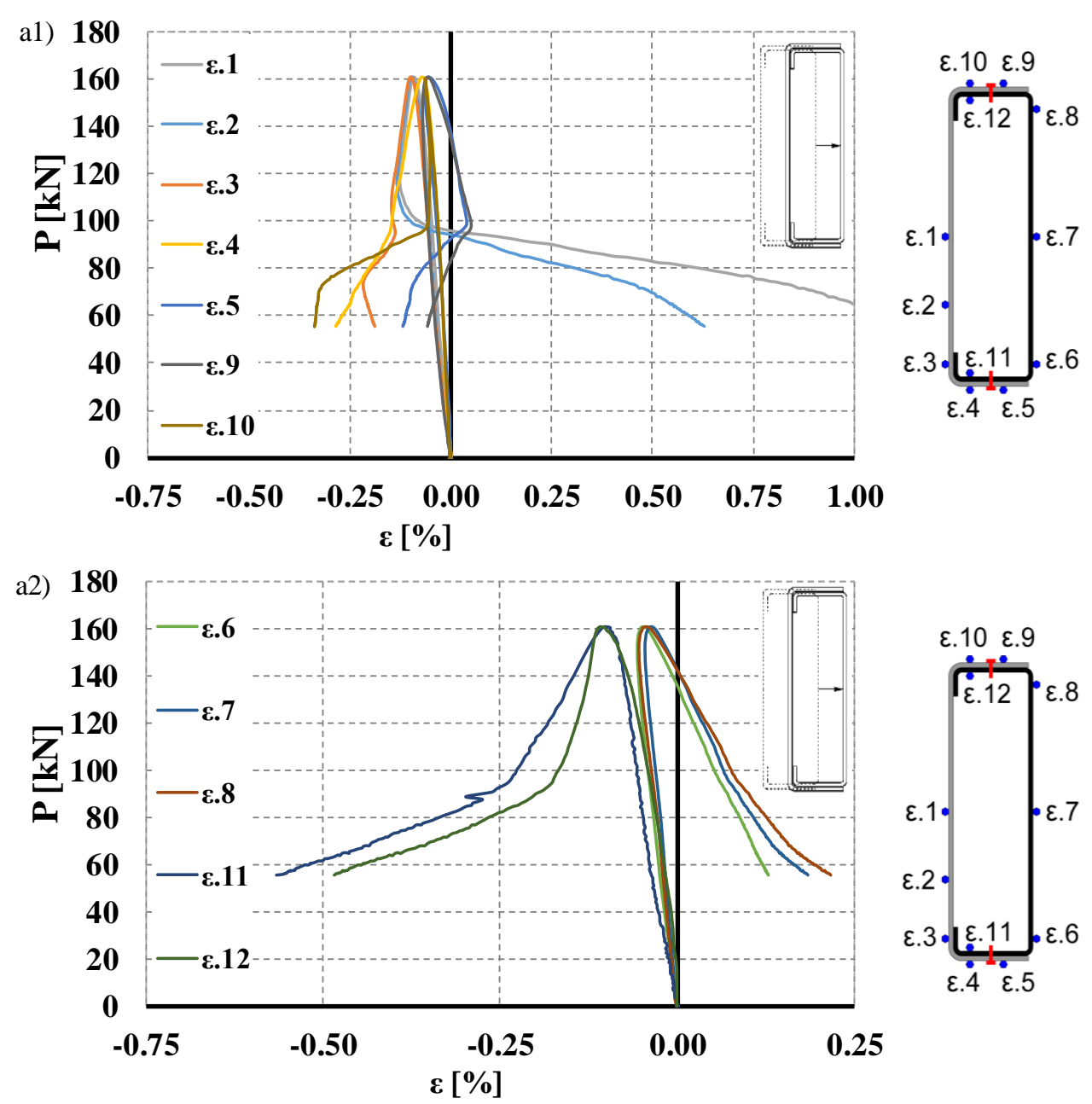

Figure 4.24 Load vs strain curves obtained from the strain gauges for the fixed-end column.

a1) and a2) R_FF_2.

For the pinned-end test the behaviour was relatively similar to the one observed for the fixedend support condition in terms of qualitative strain evolution throughout the experimental test. Once again during the loading stage the entire cross-section was subjected to compressive stresses. For the strain gauges positioned in the plain channel (U) once again some singularities were observed during the unloading stage, translated in sectional buckling modes (local and distortional buckling). 

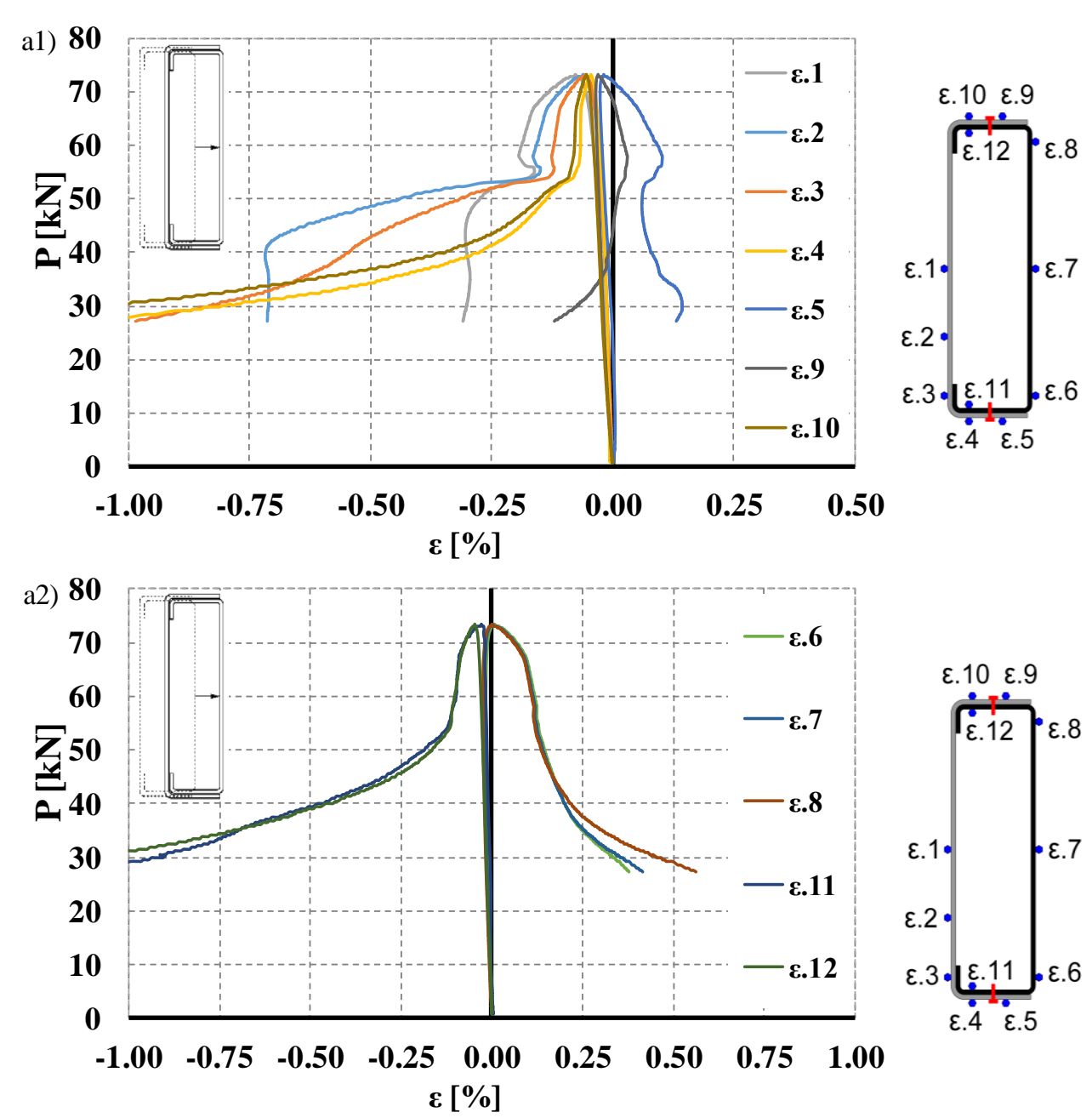

Figure 4.25 Load vs strain curves obtained from the strain gauges for the pinned-end column. a1) and a2) R_PP_3.

For the $2 \mathrm{R}$ cross-section and for both end-support conditions tested it was observed that during the loading stage all the cross-section was subjected to compressive stresses and that for the strain gauges positioned in the inner web of the closed built-up section presented a very similar behaviour in both loading and unloading stage. For the fixed-end condition some parts of the cross-section reached the yield plateau at the maximum axial load (Figure 4.26), namely $\varepsilon .6$, $\varepsilon .7, \varepsilon .10$, ع.11, ع.20 $\left(f_{y}=306.8 \mathrm{MPa}\right)$. For all strain gauges significant strain variations were recorded at maximum axial load. After the observed strain variation at maximum axial load a very sudden drop in axial load was observed.

For pinned-ended columns the behaviour was slightly different since no sudden strain variations were observed. In this case load decreased much more gradually than in fixed-ended situation. 

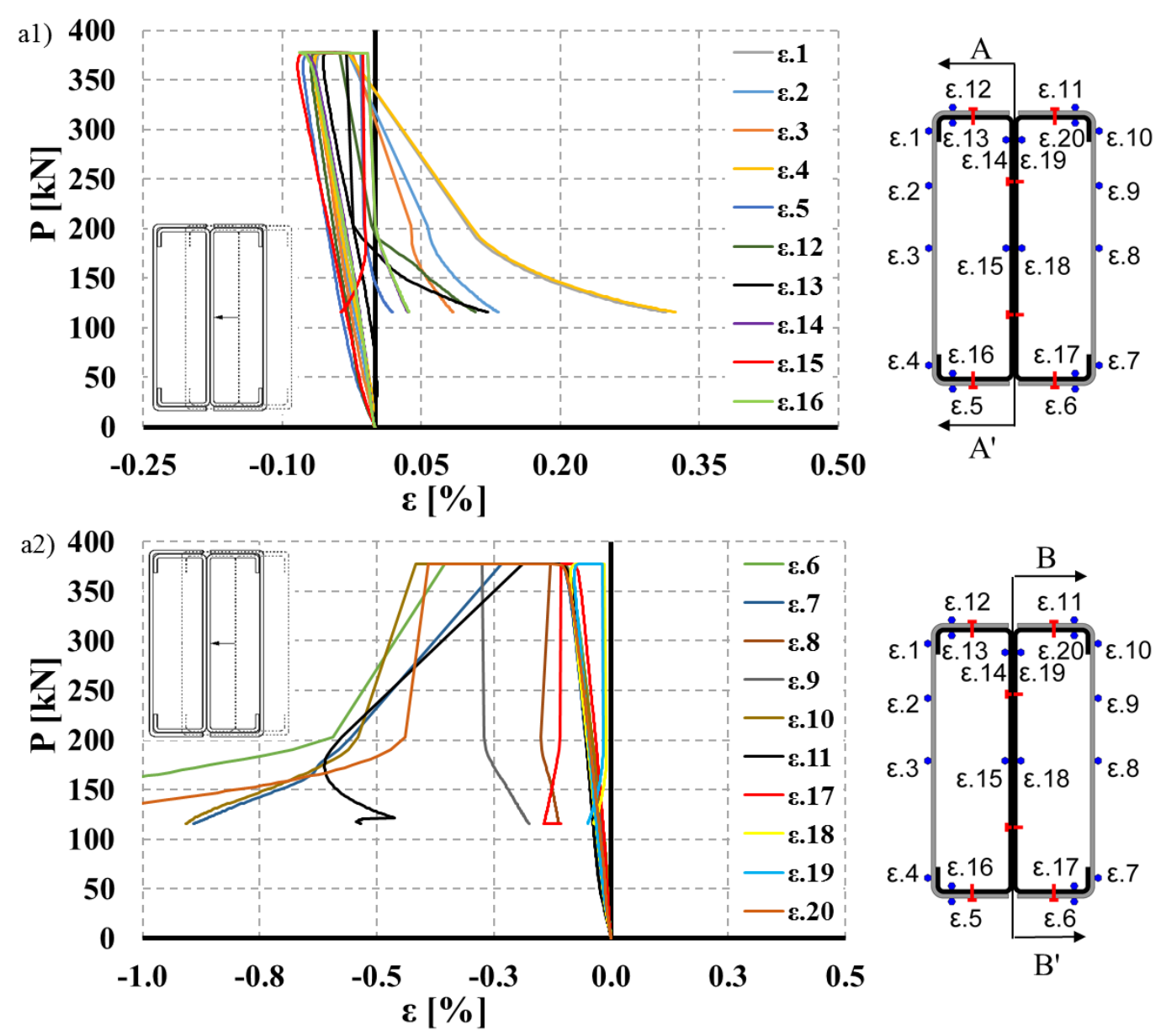

Figure 4.26 Load vs strain curves obtained from the strain gauges for the fixed-end column. a1) and a2) 2R_FF_2. 

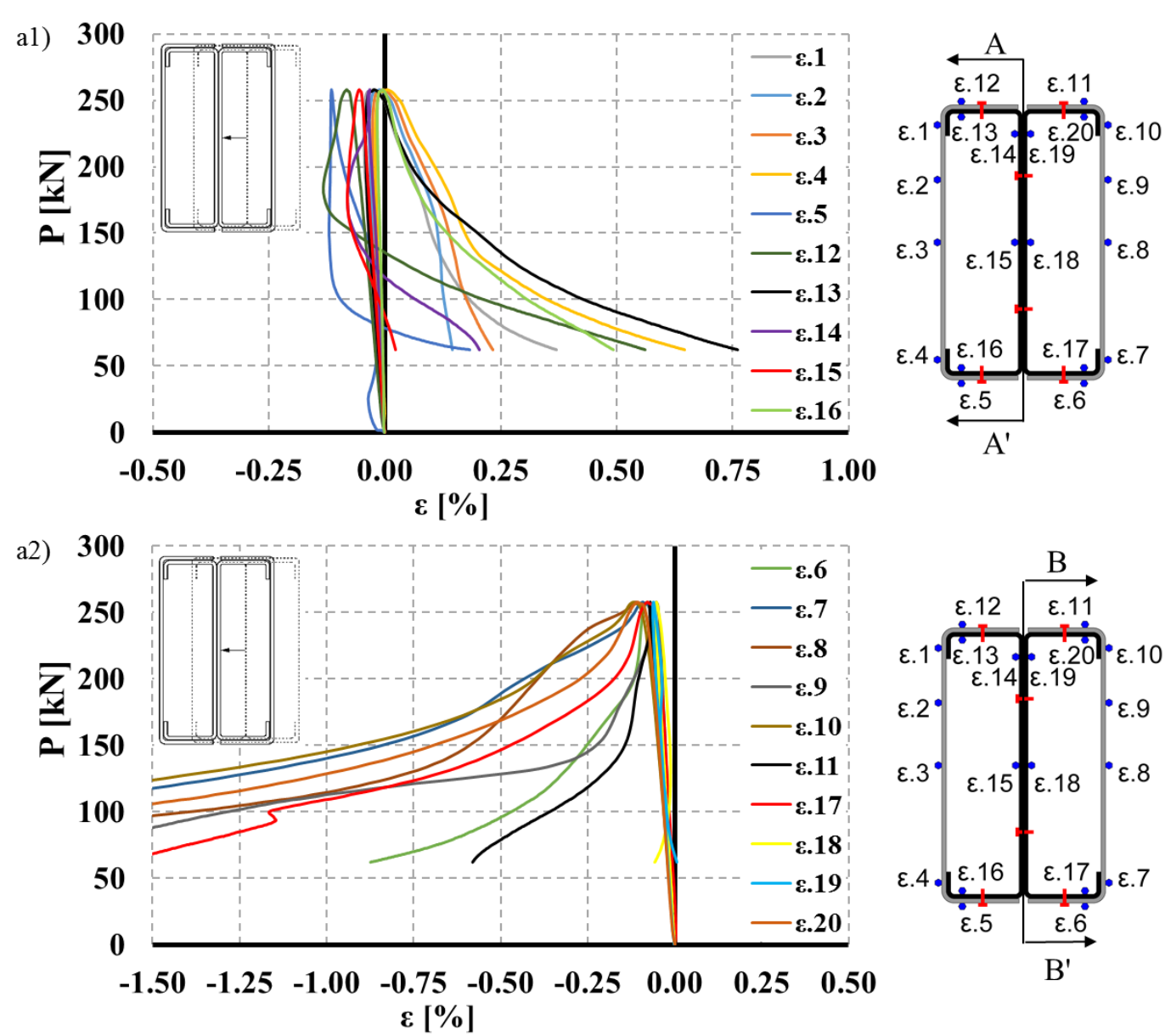

Figure 4.27 Load vs strain curves obtained from the strain gauges for the pinned-end column. a1) and a2) 2R_PP_2.

Finally, based on the obtained results the stress field at mid-height of the column and for the maximum axial load, for each cross-section and end-support condition, is presented in Figures $4.28,4.29,4.30$ and 4.31 .

Also it is worth mentioning that all the obtained experimental results may be affected by some additional imperfections, such as out of alignment of the columns while positioning in the test set-up leading to eccentric loading, non-verticality of the columns, out of level end-support devices and specifically for the pinned-end condition, friction between the steel plates and the steel pin. Nevertheless, in order to reduce the influence of friction in the test results Teflon and copper grease were used in the steel pin. 
a)

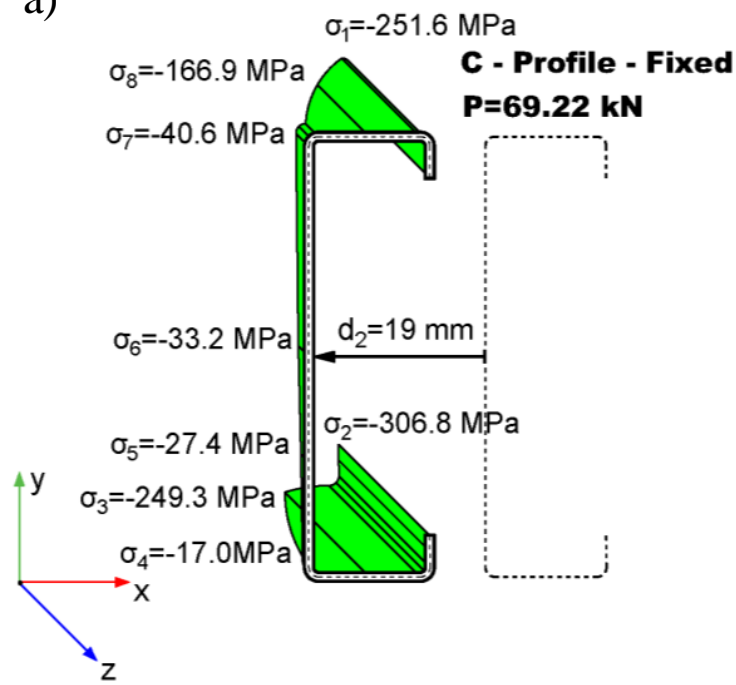

b)

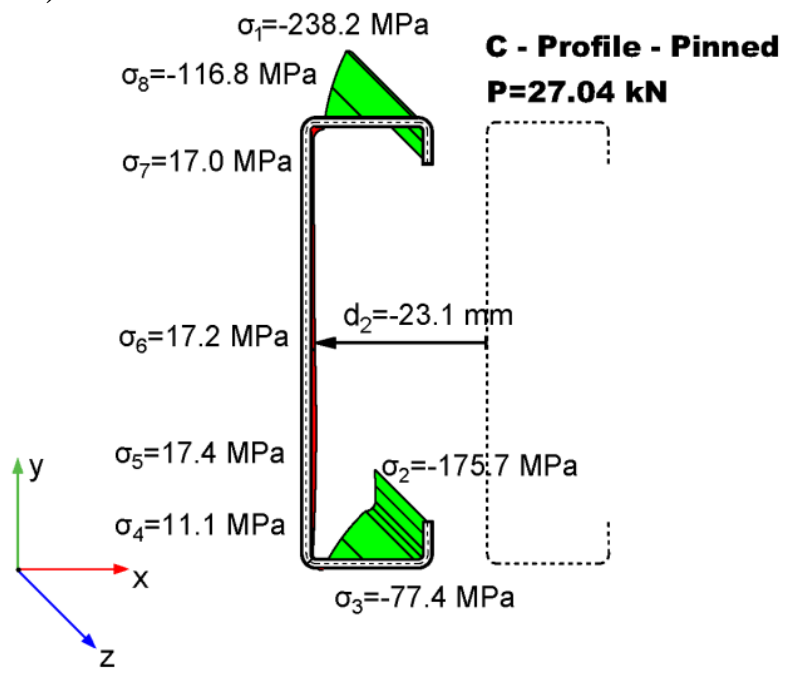

Figure 4.28 Stress field determined for the maximum axial load for lipped channel columns considering both pinned and fixed-end support conditions. a) Fixed-end. b) Pinned-end.

a) $\quad \sigma_{11}=-128.2 \mathrm{MPa} \sigma_{9}=-193.3 \mathrm{MPa}$

$\sigma_{12}=-87.1 \mathrm{MPa} \quad \sigma_{10}=-152.6 \mathrm{MPa}$

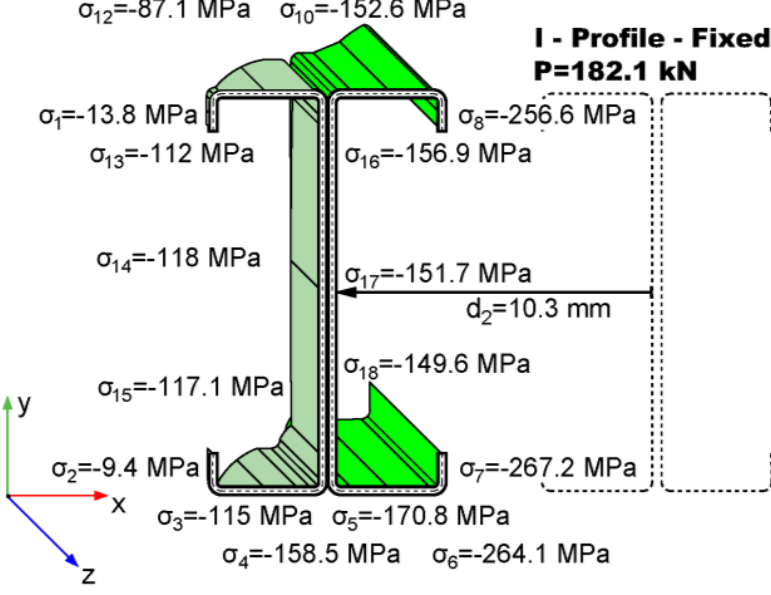

b) $\quad \sigma_{11}=-84.3 \mathrm{MPa} \quad \sigma_{9}=-103.3 \mathrm{MPa}$

$\sigma_{12}=-77.23 \mathrm{MPa} \quad \sigma_{10}=-98.2 \mathrm{MPa} \quad$ I - Profile - Pinned $\mathbf{P}=83.18 \mathrm{kN}$

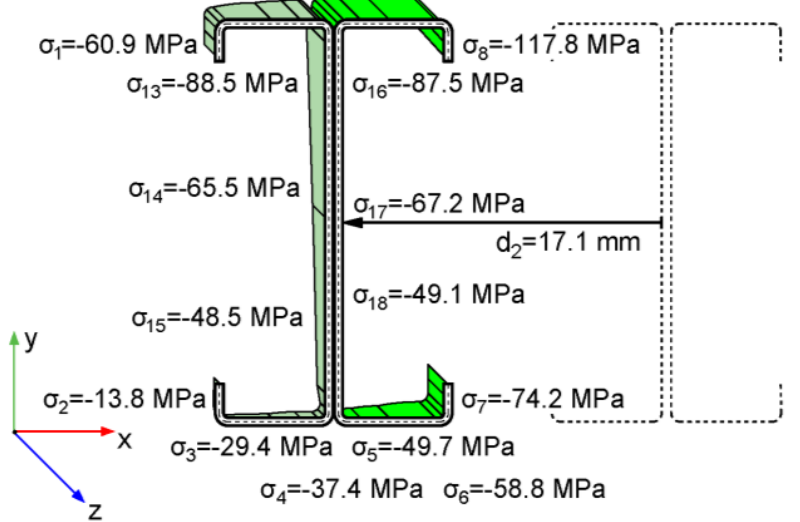

Figure 4.29 Stress field determined for the maximum axial load for open built-up I columns considering both pinned and fixed-end support conditions. a) Fixed-end. b) Pinned-end. 
a)

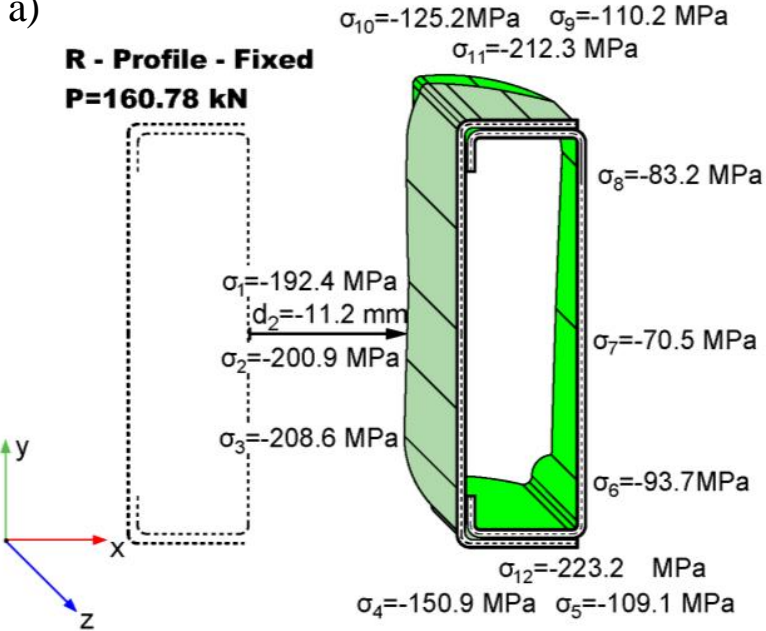

b)

R - Profile - Pinned $\sigma_{10}=-110.3 \mathrm{MPa} \quad \sigma_{9}=-64.4 \mathrm{MPa}$ $P=73.37 \mathrm{kN}$ $\sigma_{11}=-60.9 \mathrm{MPa}$

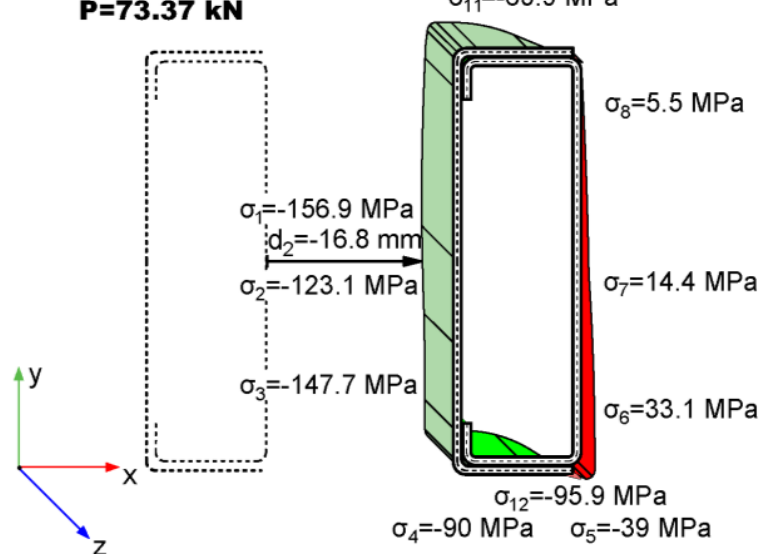

Figure 4.30 Stress field determined for the maximum axial load for closed built-up R columns considering both pinned and fixed-end support conditions. a) Fixed-end. b) Pinned-end.
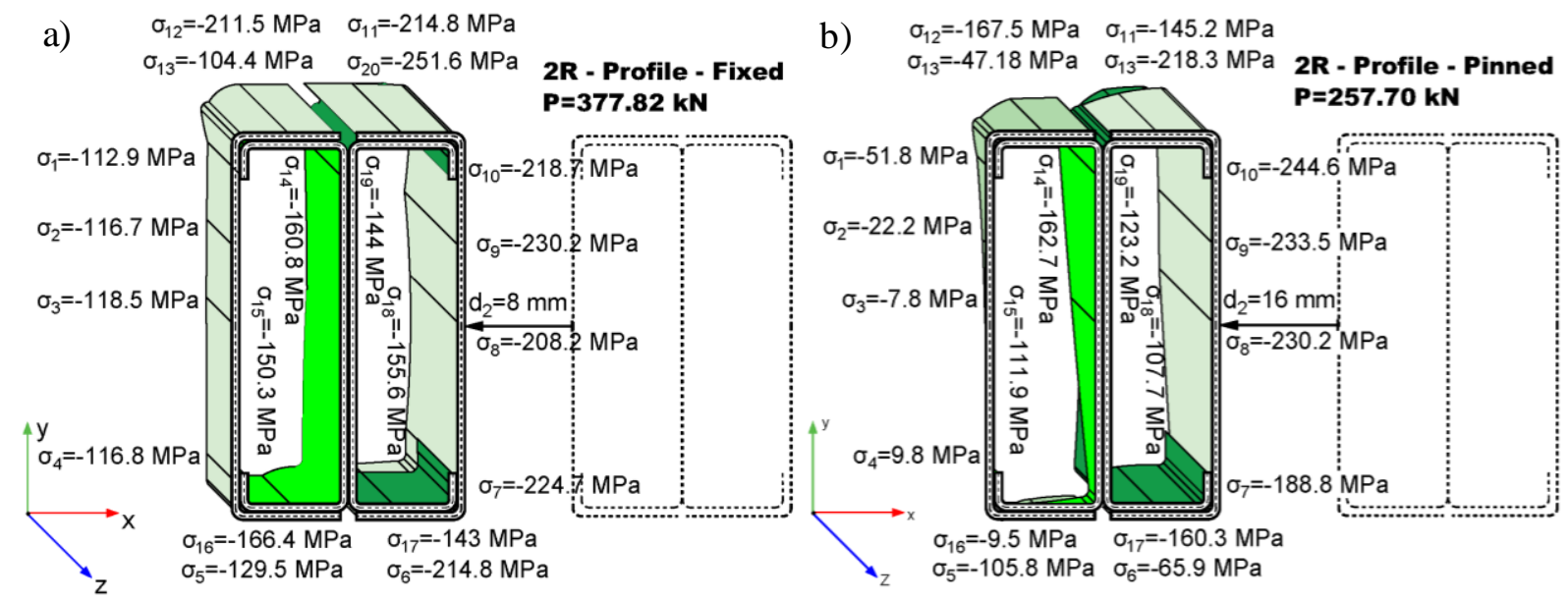

Figure 4.31 Stress field determined for the maximum axial load for closed built-up $2 \mathrm{R}$ columns considering both pinned and fixed-end support conditions. a) Fixed-end. b) Pinnedend.

\subsubsection{Fire tests with restraint to thermal elongation}

\subsubsection{Temperature evolution}

In the fire tests it was attempted to reproduce the ISO 834 (1999) standard fire curve, however due to the type of furnace used (electrical furnace), to the volume of the inside chamber (high initial thermal inertia) and eventually due to less effective insulation (heat loss near the endsupport devices) the actual thermal action obtained in these tests is different from the thermal 
action presented in the ISO 834 (1999), especially in the first few minutes. In Figure 4.32 the average furnace temperature evolution is presented for some test conditions (all cross-sections (i), pinned and fixed-end supports, all levels of restraint to thermal elongation tested and the $30 \%$ load level) and compared with the ISO 834 (1999) fire curve. The presented temperature evolution for the furnace is representative of remaining tests conducted.
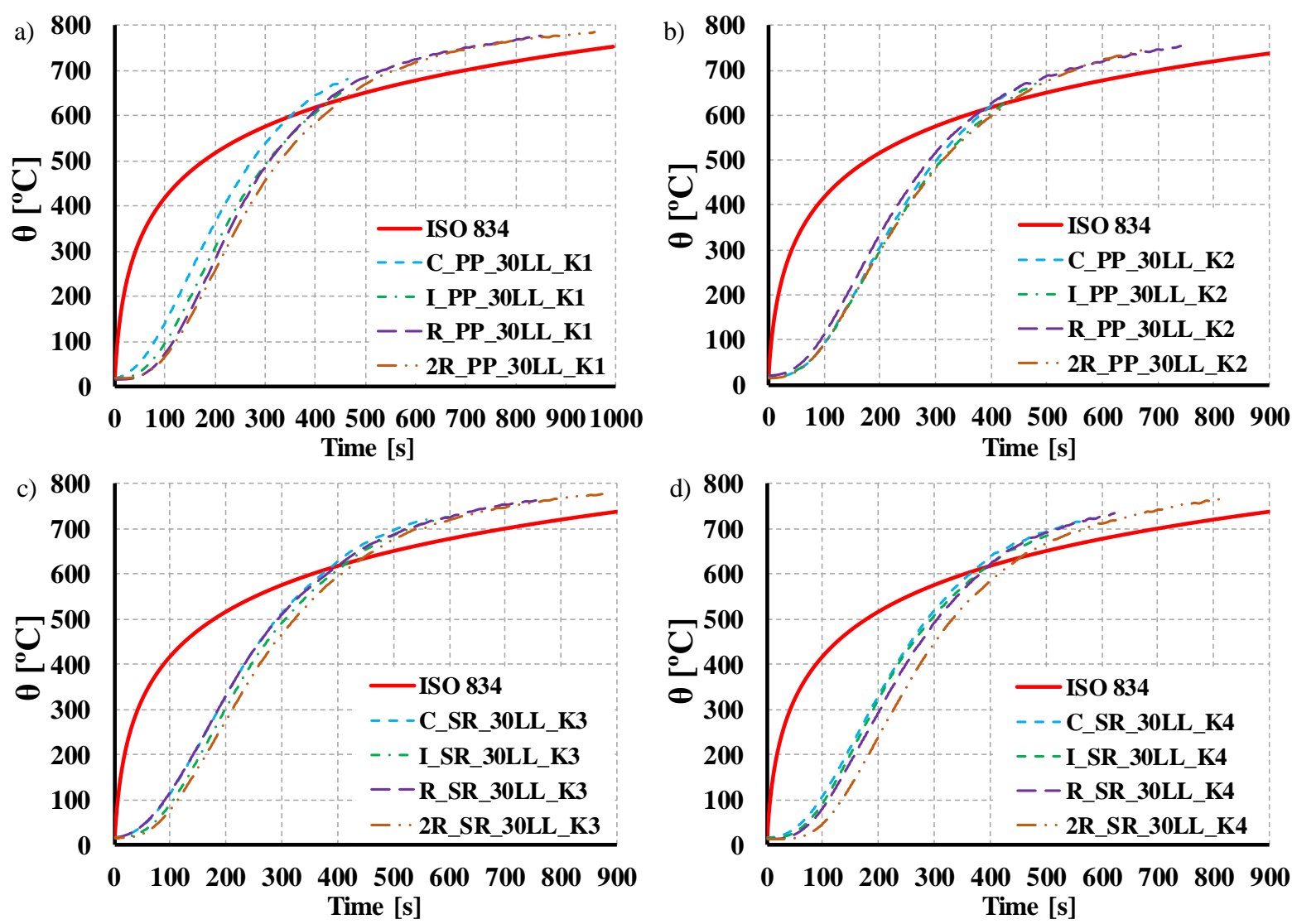

Figure 4.32 Comparison between temperature evolution of the furnace in the experimental

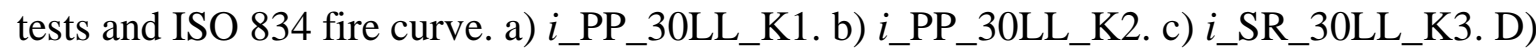

$$
\text { i_SR_30LL_K4. }
$$

Analyzing the results presented in Figure 4.32 it was observed a few differences in the thermal action in terms of time in the different tests, hence it might not be adequate to establish comparisons between the different test conditions in terms of time. In the future, based on the developed and validated numerical models, it will be possible to perform extensive parametric research which will allow comparisons between the simulated conditions in terms of time. In terms of temperature the agreement for all tested conditions is very good, hence comparisons may be established in terms of critical temperatures monitored in the tests. 
As previously mentioned all CFS columns were instrumented with thermocouples in several points of the cross-section and in different sections along the length of the column (5 sections). Using the measured temperatures from each thermocouple $\left(\mathrm{T}_{\mathrm{i}}\right)$, placed in each one of the five instrumented sections, the mean temperature $\left(\bar{\theta}_{s}\right)$ for each one of those sections was determined taking into account the influence areas defined by thermocouples spot-welded to the steel profiles. For the $2 \mathrm{R}$ cross-section the thermocouples placed on the web of the inner profiles were not taken into account in this calculation. Using the average temperatures in each section it was possible to determine the temperature distribution along the length of the column as a function of time and consequently to determine the mean temperature of the CFS column $\left(\bar{\theta}_{c}\right)$. This value represents the integral of the mean temperatures calculated in each cross-section. The evolution of temperatures for each type of cross-section tested (C, I, R, 2R), in the TS3 section (mid-height of the columns), as well as the ISO 834 (1999) standard fire, furnace mean temperature, the mean temperature in the TS3 cross-section $\left(\bar{\theta}_{S}\right)$ and mean temperature of the column $\left(\bar{\theta}_{c}\right)$ are presented in Figure 4.33, 4.34, 4.35 and 4.36. The presented evolution of temperatures in the TS3 cross section is representative of the different tests carried out. It was observed that the heating rate was similar to each identical column and non-dependent, as expected, on the load level, boundary conditions or levels of stiffness of the surrounding structure to the CFS column.

It was observed that the evolution of temperature in the thermocouples was almost uniform for the lipped channel (C) cross-section (T.1, T.2 and T.3) (Figure 4.33). For open built-up and closed built-up cross-sections the temperature evolution was not uniform in the cross-section. For the built-up I (2C) cross-section the thermocouple welded to the web (T.2) registered lower temperatures than the ones welded to the flanges (T.1 and T.3) (Figure 4.34). This fact can be explained by the greater thickness of the web $(5 \mathrm{~mm})$ and the thermal conductance between the two CFS profiles and also due to the position of the web in relation to the electrical resistances. This positioning may lead to the existence of some shadow zones (not directly exposed to radiation) in the web of the column. Regarding the evolution of temperature in both closed built-up cross-sections (Figure 4.35 and 4.36) it is clear that the thermocouples placed in the flanges registered (T.1 and T.3) lower temperatures than the ones placed in the web (T.2 and T.4) of the cross-sections. This is due to the bigger thickness $(5 \mathrm{~mm})$ in these areas and thermal conductance between the steel profiles. Also, for the closed built-up $2 \mathrm{R}$ cross-section the temperature difference between thermocouples placed in the web and in the flanges is even bigger than the difference observed for the $\mathrm{R}$ cross-section. Further, it was observed that the temperature measured in the web of the inner profiles $(\mathrm{C}+\mathrm{C}=\mathrm{I})$ was significantly lower than the temperatures monitored in the flanges and web of the plain channels ( $U$ profiles). This is due to the existence of confined air in the interior of the built-up closed cross-section which has low 
thermal conductivity and due to the fact that only the external profiles (U) were directly exposed to radiation.

Based on the obtained results depicted in Figures 4.33 to 4.36 it can be stated that the evolution of temperature depended on the cross-section shape since the temperature rate was different for all tested cross-sections, namely about $43^{\circ} \mathrm{C} /$ minute for $2 \mathrm{R}$, about $50^{\circ} \mathrm{C} /$ minute for $\mathrm{R}$, about $60^{\circ} \mathrm{C} /$ minute for I and about $76^{\circ} \mathrm{C} /$ minute for $\mathrm{C}$ cross-section. This can be easily observed in Figure 4.37. The rate of temperature increase is significantly more severe for single and open built-up I cross-sections than for closed built-up cross-sections. It is worth mentioning that the temperature rate was determined considering the initial temperature of $100{ }^{\circ} \mathrm{C}$ in order to reduce the influence of the initial thermal inertia of the furnace.

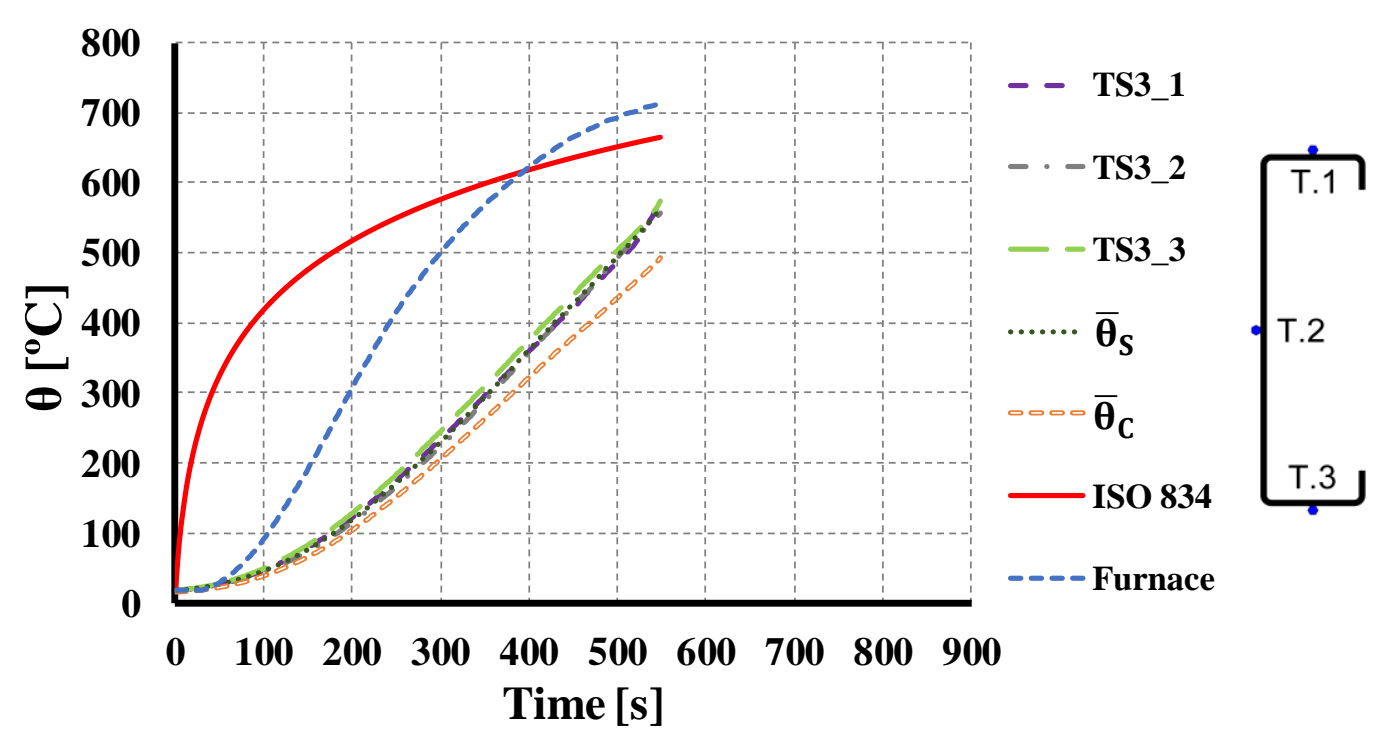

Figure 4.33 Evolution of temperature in the cross-section TS3 for the test column C_SR_30LL_K3-1 as a function of time. 


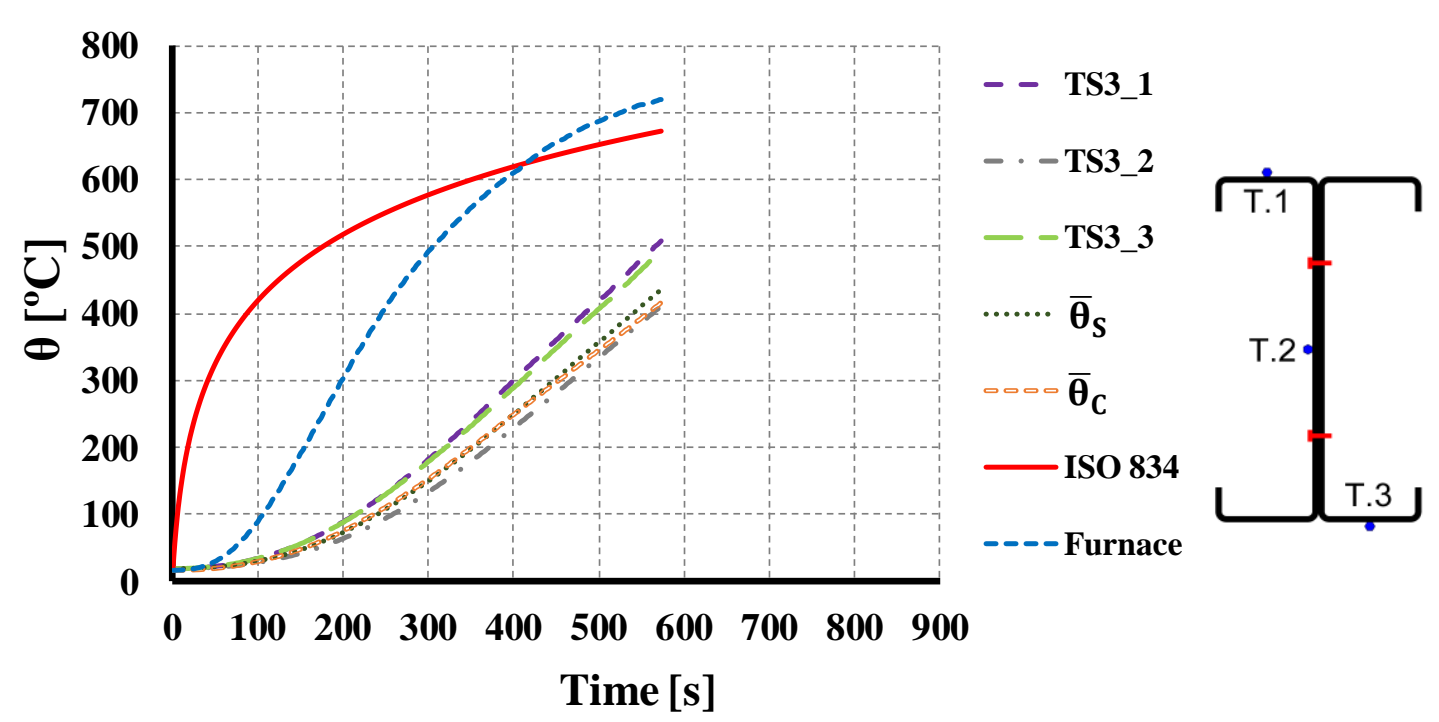

Figure 4.34 Evolution of temperature in the cross-section TS3 for the test column I_SR_30LL_K3-2 as a function of time.

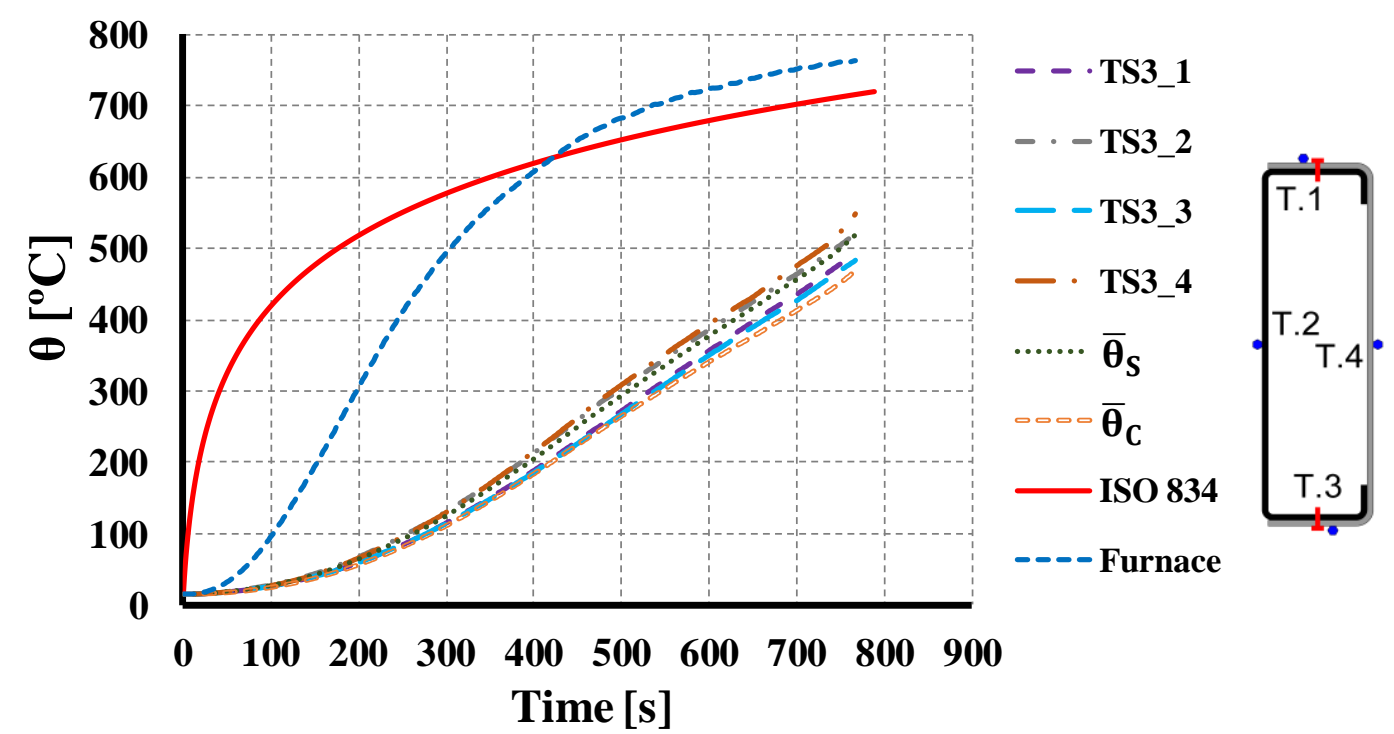

Figure 4.35 Evolution of temperature in the cross-section TS3 for the test column R_SR_30LL_K3-2 as a function of time. 


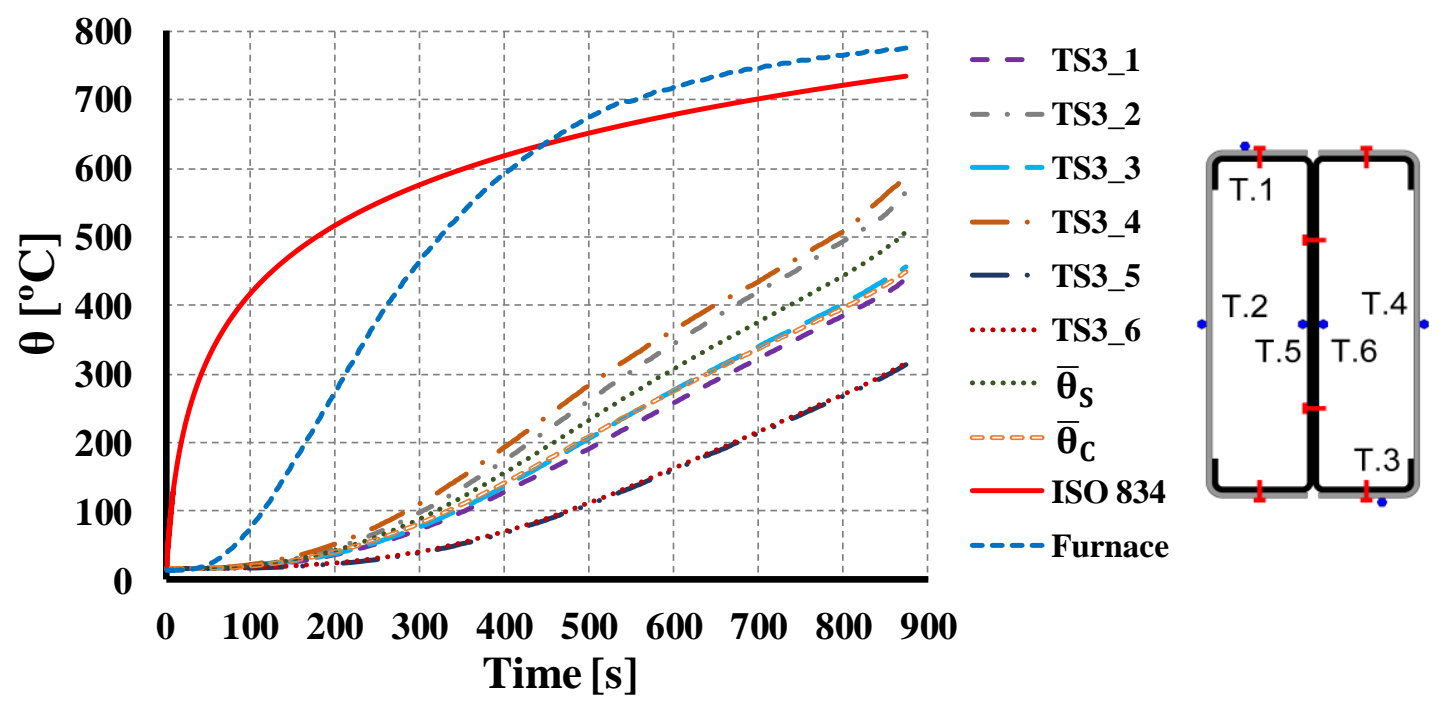

Figure 4.36 Evolution of temperature in the cross-section TS3 for the test column 2R_SR_30LL_K3-1 as a function of time.

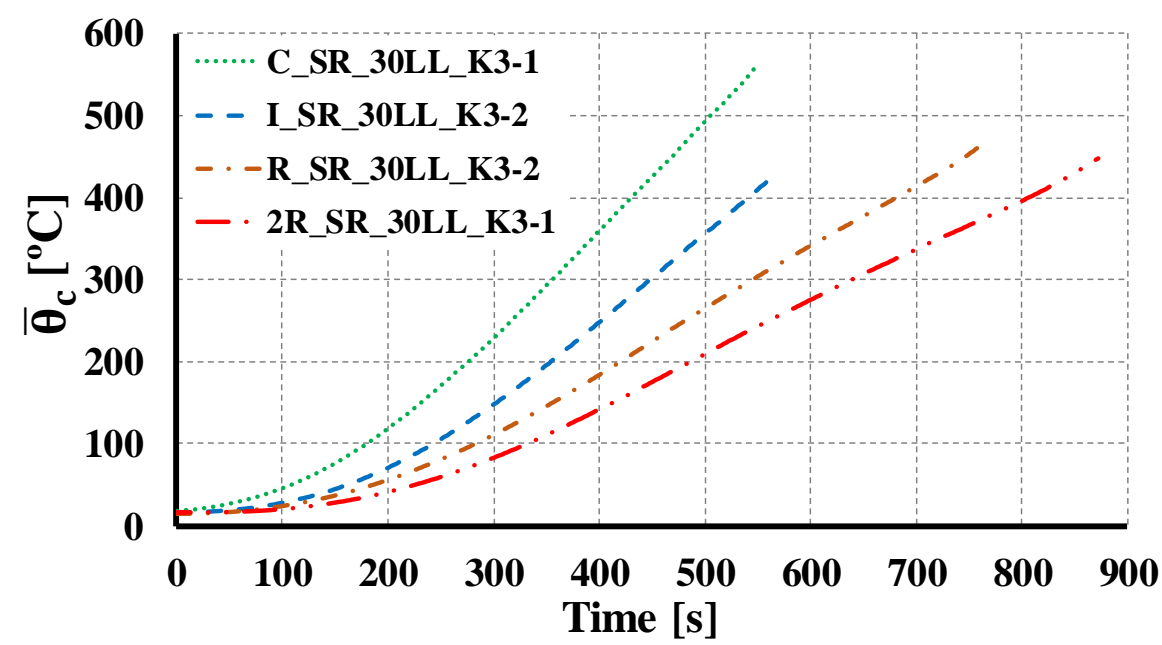

Figure 4.37 Evolution of the mean temperature of the column for all tested cross-sections as a function of time.

In Figure 4.38 a), b), c) and d) the evolution of temperature along the length of the column is depicted as a function of time. For each one of the 5 instrumented sections the mean temperature in that section $\left(\bar{\theta}_{S}\right)$ is plotted for different times. It is possible to observe a thermal gradient along the length of the column since the end-support devices were connected outside the furnace and protected with a $50 \mathrm{~mm}$ layer of ceramic wool and also due to heat losses through thermal conduction. In the three central sections the temperatures were quite uniform reducing significantly near the end support devices. 

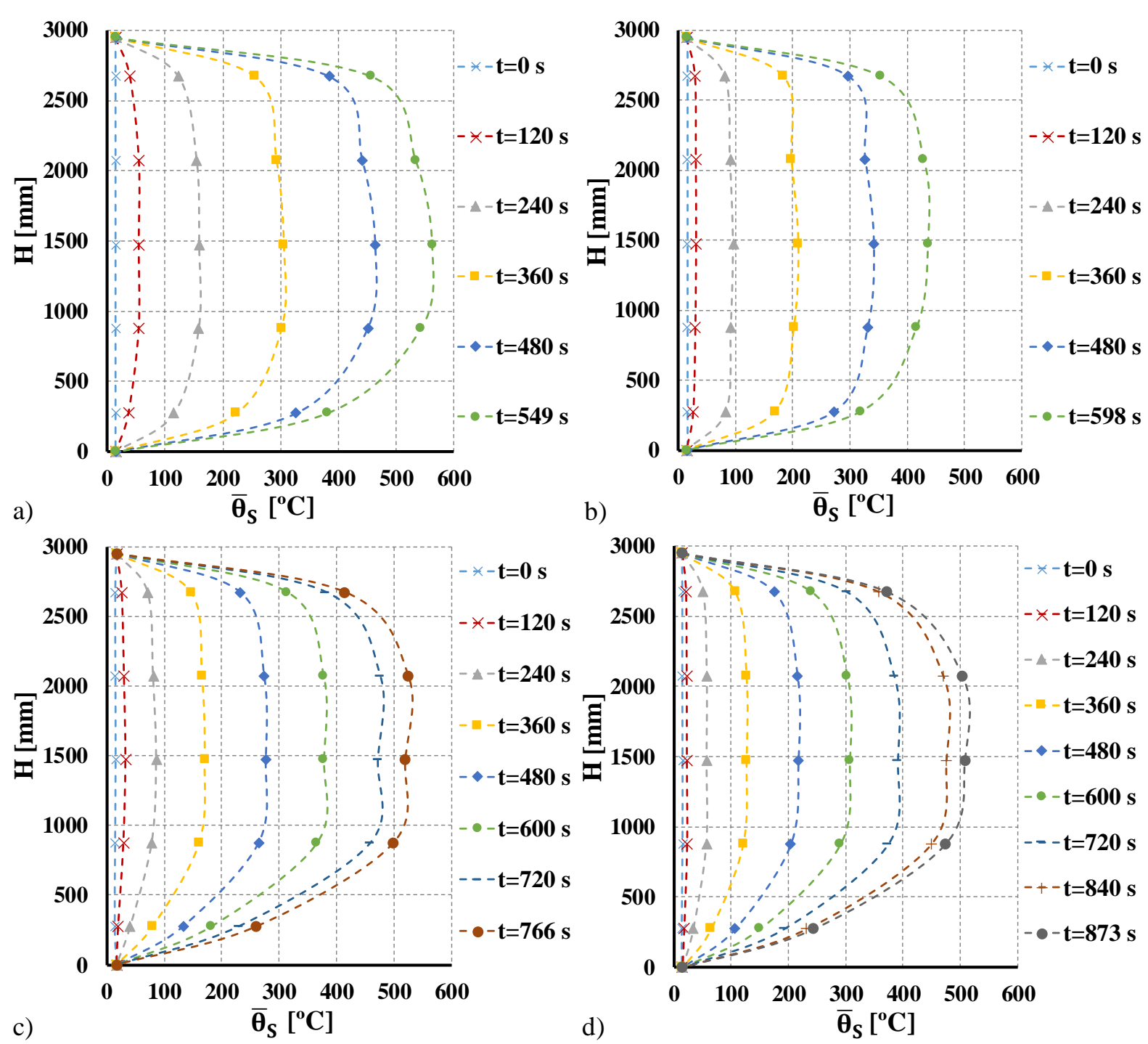

Figure 4.38 Evolution of temperature along the length of the column. a) C_SR_30LL_K3-1. b) I_SR_30LL_K3-2.c) R_SR_30LL_K3-2.d) 2R_SR_30LL_K3-1.

\subsubsection{Evolution of restraining forces}

The evolution of restraining forces is a non-dimensional $P / P_{0}$ ratio presented as a function of the mean temperature of the column $\left(\bar{\theta}_{C}\right)$ for the different cross-sections, end-support conditions, load levels and levels of axial and rotational restraint to thermal elongation. In these graphs it is possible to observe the expected behaviour of a column in a real structure as the effect of a surrounding structure was simulated with the 3D restraining frames. Each tested column was loaded with a compressive serviceability load (30 and 50\% $N_{b, R d}$ ) that was kept constant throughout the entire fire test. Due to the thermal action and since the column was axially restrained the axial compression force (restraining forces) on the column started to 
increase whereas the mechanical properties of CFS degraded with the temperature increase. After reaching a maximum $\left(P_{\max }\right)$ the restraining forces $(P)$ started to decrease reaching the initial service load applied $\left(P_{0}\right)$ to the CFS column. This point defines the critical time $\left(t_{c r}\right)$ and temperature $\left(\theta_{c r}\right)$ as the failure criteria in these experimental tests (see Tables 4.6-4.14).

Due to the large number of experimental results all the information is presented in two steps. First all the information regarding single and open built-up cross-sections ( $\mathrm{C}$ and $\mathrm{I}$ ) is presented and then all the information regarding closed built-up cross-sections ( $R$ and $2 \mathrm{R}$ ). In the end a comparison between all tested cross-sections and tested conditions will be established. In figure 4.39 the evolution of the restraining forces as a non-dimensional ratio is presented as a function of the mean temperature of the column for both single and open built-up I cross-sections.
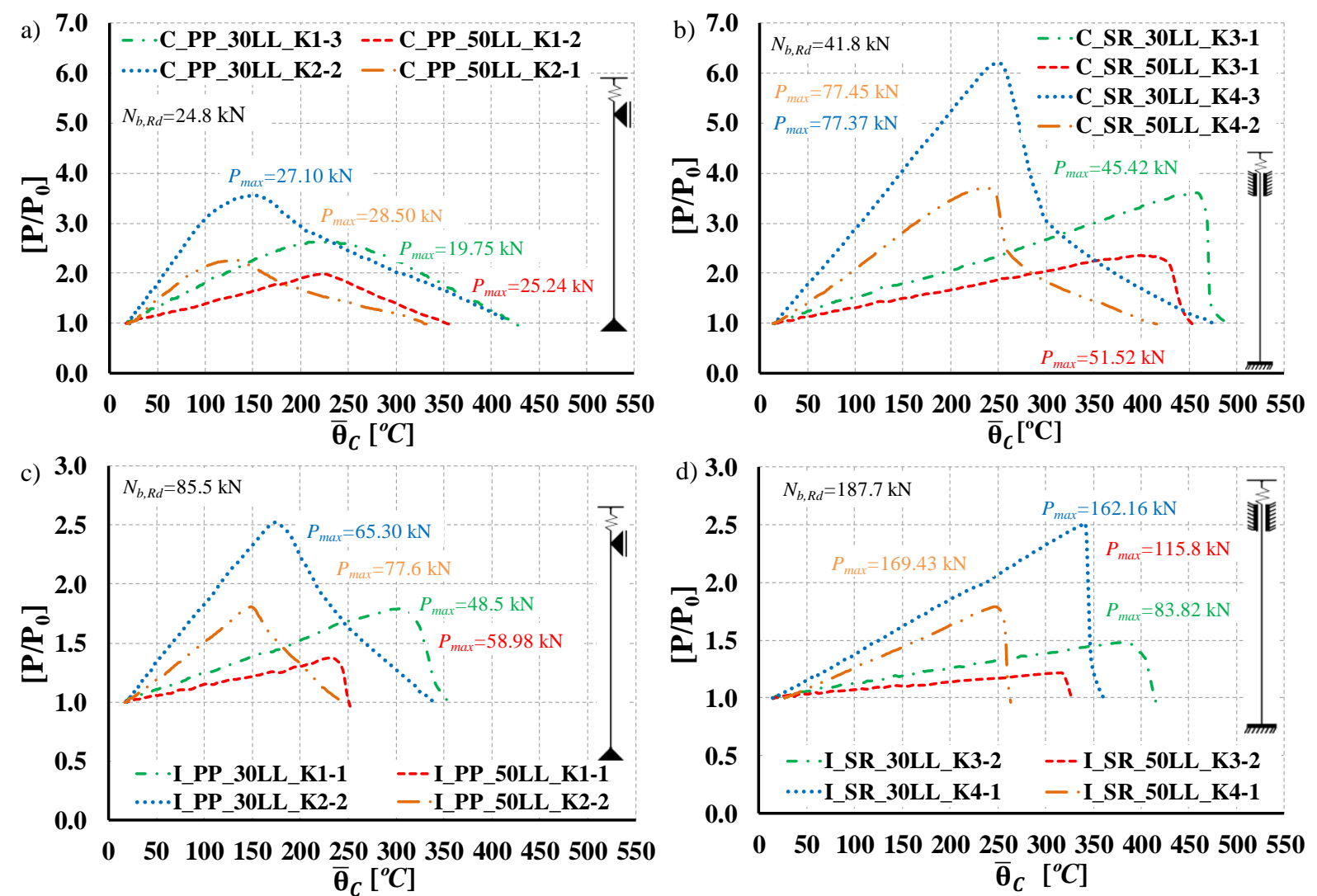

Figure 4.39 Non-dimensional restraining forces ratio for lipped and open built-up I columns. a) $\mathrm{C}$ cross-section with pinned-end support. b) $\mathrm{C}$ cross-section with semi-rigid end support. c) I cross-section with pinned-end support. d) I cross-section with semi-rigid end support.

Analysing the results for the lipped channel columns, considering pin-ended support conditions and the $\mathrm{K} 1$ level of stiffness of the surrounding structure to the CFS column ( $3 \mathrm{kN} / \mathrm{mm}$ of axial 
restraint), the $P_{\max } / P_{0}$ ratio is 2.616 and 1.987 times the value of the initial applied load respectively for the 30 and 50\% load level. (Figure 4.39 a)) (Table 4.6). Analysing the results obtained for the K2 level of stiffness of the surrounding structure to the CFS column $(13 \mathrm{kN} / \mathrm{mm}$ of axial restraint) it was found out that the mean $P_{\max } / P_{0}$ ratio increased up to 3.712 times for the $30 \%$ load level and up to 2.262 times the initial load for the $50 \%$ load level (Figure 4.39 a)) (Table 4.6) which are significantly higher than those obtained for the K1 level (Figure 4.39 a)). However, in terms of critical temperatures it was found out that despite the different levels of stiffness of the surrounding structure the final results were almost identical, for example the critical temperature for C_PP_30LL_K1-3 was $425.21{ }^{\circ} \mathrm{C}$ and for C_PP_30LL_K2-1 was $412.85^{\circ} \mathrm{C}$ (Table 4.6). In terms of critical times it was observed that for unprotected lipped channel columns in fire situation, considering the influence of restraint to thermal elongation, the times are very low, mainly due to high section factor and high thermal conductivity of steel.

Table 4.6 Results for the lipped channel (C) cross-section specimens - pin-ended support.

\begin{tabular}{|c|c|c|c|c|c|c|}
\hline Test Reference & $\begin{array}{l}\theta_{\text {peak }} \\
{\left[{ }^{\circ} \mathbf{C}\right]}\end{array}$ & $\begin{array}{c}t_{\text {peak }} \\
{[\mathrm{min}]}\end{array}$ & $\begin{array}{l}\boldsymbol{P}_{\max } \\
{[\mathbf{k N}]}\end{array}$ & $\begin{array}{c}\boldsymbol{P}_{\max } \boldsymbol{P}_{\mathbf{0}} \\
{[--]}\end{array}$ & $\begin{array}{c}\theta_{c r} \\
{\left[{ }^{\circ} \mathbf{C}\right]}\end{array}$ & $\begin{array}{c}t_{c r} \\
{[\mathrm{~min}]}\end{array}$ \\
\hline C_PP_30LL_K & 261.16 & 5.17 & 19.7 & 2.558 & 418.85 & 7.38 \\
\hline C_PP_30LL_K $K_{1-2}$ & 255.75 & 5.28 & 20.5 & 2.733 & 422.35 & 7.67 \\
\hline C_PP_30LL_K $K_{1}-3$ & 238.94 & 5.02 & 19.75 & 2.616 & 425.21 & 7.65 \\
\hline$\mu$ & $25 \overline{1.95}$ & 5.16 & 19.98 & 2.64 & $42 \overline{2} .14$ & $\overline{7.57}$ \\
\hline$\sigma$ & 9.46 & 0.11 & 0.37 & 0.07 & 2.60 & 0.13 \\
\hline $\mathrm{CV}[\%]$ & 3.76 & 2.07 & 1.83 & 2.76 & 0.62 & 1.75 \\
\hline C_PP_50LL_K $K_{1-1}$ & 211.83 & 5.02 & 24.00 & 1.920 & 338.76 & 6.75 \\
\hline C_PP_50LL_K $K_{1-2}$ & 225.36 & 5.07 & 25.24 & 1.987 & 351.10 & 6.95 \\
\hline C_PP_50LL_K $K_{1-3}$ & 216.42 & 4.87 & 25.29 & 1.976 & 346.71 & 6.85 \\
\hline$\mu$ & 217.87 & 4.99 & 24.84 & 1.96 & 345.52 & 6.85 \\
\hline$\sigma$ & 5.62 & 0.08 & 0.60 & 0.03 & 5.11 & 0.08 \\
\hline $\mathrm{CV}[\%]$ & 2.58 & 1.70 & 2.40 & 1.50 & 1.48 & 1.19 \\
\hline C_PP_30LL_K $K_{2}-1$ & 144.56 & 3.92 & 28.40 & 3.712 & 412.85 & 7.75 \\
\hline C_PP_30LL_K ${ }_{2-2}$ & 153.49 & 4.27 & 27.10 & 3.566 & 426.61 & 8.05 \\
\hline C_PP_30LL_K2-3 & 138.16 & 3.97 & 26.40 & 3.520 & 423.08 & 7.63 \\
\hline$\mu$ & 145.40 & 4.05 & 27.30 & 3.60 & 420.85 & 7.81 \\
\hline$\sigma$ & 6.29 & 0.15 & 0.83 & 0.08 & 5.84 & 0.18 \\
\hline $\mathrm{CV}[\%]$ & 4.32 & 3.81 & 3.04 & 2.27 & 1.39 & 2.26 \\
\hline C_PP_50LL_K $K_{2}-1$ & 134.47 & 4.15 & 28.50 & 2.262 & 332.33 & 6.85 \\
\hline C_PP_50LL_K ${ }_{2}-2$ & 123.81 & 3.88 & 28.90 & 2.312 & 338.31 & 6.82 \\
\hline C_PP_50LL_K & 126.22 & 3.95 & 29.30 & 2.260 & 344.95 & 7.13 \\
\hline$\mu$ & 128.17 & 3.99 & 28.90 & 2.28 & 338.53 & 6.93 \\
\hline$\sigma$ & 4.56 & 0.11 & 0.33 & 0.02 & 5.15 & 0.14 \\
\hline $\mathrm{CV}[\%]$ & 3.56 & 2.86 & 1.13 & 1.06 & 1.52 & 2.01 \\
\hline
\end{tabular}


For the semi-rigid end-support conditions (Figure 4.39 b)) the same conclusions can be drawn for the restraining forces, the higher the level of stiffness of the surrounding structure to the CFS column the higher the restraining forces are. For the highest level of stiffness of the surrounding structure $\mathrm{K} 4$ and for the $30 \%$ load level the $P_{\max } / P_{0}$ ratio increased up to 6.194 times the initial load while for the lower level of stiffness of the surrounding structure K3 that ratio increased just up to 3.605 times (Figure 4.39 b)) (Table 4.7). For 50\% load level the results in terms of the $P_{\max } / P_{0}$ ratio obtained were 2.473 and 3.702 respectively for C_SR_50LL_K31 and for C_SR_50LL_K4-2 (Figure 4.39 b)) (Table 4.7). The critical temperatures were reduced increasing the level of stiffness of the surrounding structure ( $\mathrm{K} 3$ and $\mathrm{K} 4$ ), specifically $490.36^{\circ} \mathrm{C}$ for C_SR_30LL_K3-1 and $475.47^{\circ} \mathrm{C}$ for C_SR_30LL_K4-3 and finally $453.23^{\circ} \mathrm{C}$ for C_SR_50LL_K3-1 and $415.6^{\circ} \mathrm{C}$ for C_SR_50LL_K4-2 (Table 4.7).

Table 4.7 Results for the lipped channel (C) cross-section specimens - semi-rigid end support.

\begin{tabular}{|c|c|c|c|c|c|c|}
\hline Test Reference & $\begin{array}{l}\theta_{\text {peak }} \\
{\left[{ }^{\circ} \mathbf{C}\right]}\end{array}$ & $\begin{array}{c}t_{\text {peak }} \\
{[\mathrm{min}]}\end{array}$ & $\begin{array}{l}P_{\max } \\
{[\mathrm{kN}]}\end{array}$ & $\begin{array}{c}\boldsymbol{P}_{\max } \boldsymbol{P}_{\boldsymbol{O}} \\
{[--]}\end{array}$ & $\begin{array}{l}\theta_{c r} \\
{\left[{ }^{\circ} \mathbf{C}\right]}\end{array}$ & $\begin{array}{c}t_{c r} \\
\text { [min] }\end{array}$ \\
\hline C_SR_30LL_K & 458.53 & 8.70 & 45.42 & 3.605 & 490.36 & 9.15 \\
\hline C_SR_30LL_K ${ }_{3}-2$ & 460.05 & 8.50 & 43.82 & 3.415 & 524.10 & 9.32 \\
\hline C_SR_30LL_K K $_{3}-3$ & 461.48 & 8.52 & 45.32 & 3.486 & 490.52 & 8.93 \\
\hline$\mu$ & 460.02 & 8.57 & 44.85 & 3.50 & 501.66 & 9.13 \\
\hline$\sigma$ & 1.20 & 0.09 & 0.73 & 0.08 & 15.87 & 0.16 \\
\hline $\mathrm{CV}[\%]$ & 0.26 & 1.05 & 1.63 & 2.24 & 3.16 & 1.75 \\
\hline C_SR_50LL_K ${ }_{3-1}$ & 400.16 & 7.65 & 51.52 & 2.473 & 453.23 & 8.38 \\
\hline C_SR_50LL_K ${ }_{3}-2$ & 406.88 & 7.52 & 50.84 & 2.260 & 458.43 & 8.27 \\
\hline C_SR_50LL_K ${ }_{3}-3$ & 418.75 & 8.12 & 53.17 & 2.532 & 449.11 & 8.57 \\
\hline$\mu$ & 408.60 & 7.76 & 51.84 & 2.42 & 453.59 & 8.41 \\
\hline$\sigma$ & 7.69 & 0.26 & 0.98 & 0.12 & 3.81 & 0.12 \\
\hline $\mathrm{CV}[\%]$ & 1.88 & 3.32 & 1.89 & 4.82 & 0.84 & 1.47 \\
\hline $\bar{C}$ C_SR_30LL_K ${ }_{4}-1$ & 255.01 & 6.30 & 79.70 & 6.330 & 494.55 & 9.78 \\
\hline C_SR_30LL_K ${ }_{4}-2$ & 244.54 & 6.20 & 76.30 & 6.109 & 465.98 & 9.53 \\
\hline C_SR_30LL_K 4 -3 & 250.26 & 6.20 & 77.37 & 6.194 & 475.47 & 9.50 \\
\hline$\mu$ & 249.94 & 6.23 & 77.79 & 6.21 & 478.67 & 9.60 \\
\hline$\sigma$ & 4.28 & 0.05 & 1.42 & 0.09 & 11.88 & 0.13 \\
\hline $\mathrm{CV}[\%]$ & 1.71 & 0.76 & 1.82 & 1.47 & 2.48 & 1.31 \\
\hline C_SR_50LL_K 4 -1 & 239.15 & 6.22 & 76.3 & 3.606 & 405.51 & 8.65 \\
\hline C_SR_50LL_K ${ }_{4}-2$ & 237.29 & 6.45 & 77.45 & 3.702 & 415.6 & 9.08 \\
\hline C_SR_50LL_K4-3 & 242.18 & 6.25 & 79.38 & 3.540 & 425.33 & 8.87 \\
\hline$\mu$ & 239.54 & 6.31 & $7 \overline{7} . \overline{7}$ & 3.62 & 415.48 & 8.87 \\
\hline$\sigma$ & 2.02 & 0.10 & 1.27 & 0.07 & 8.09 & 0.18 \\
\hline $\mathrm{CV}$ [\%] & 0.84 & 1.62 & 1.64 & 1.84 & 1.95 & 1.98 \\
\hline
\end{tabular}


Regarding the open built-up cross-section (I) (Figure $4.39 \mathrm{c}$ ) and d)) it was observed that for the pin-ended condition and for the K1 level of stiffness of the surrounding structure $(3 \mathrm{kN} / \mathrm{mm}$ of axial restraint) the $P_{\max } / P_{0}$ ratios were 1.790 and 1.376 respectively for the 30 (I_PP_30LL_K1-1) and 50\% (I_PP_50LL_K1-1) load level while for the K2 level of stiffness of the surrounding structure (13 kN/mm of axial restraint) were 2.521 (I_PP_30_K2-2) and 1.805 (I_PP_50LL_K2-2) (Figure 4.39 c)) (Table 4.8). In terms of critical temperatures, it was observed that despite the different levels of stiffness of the surrounding structure the temperatures were once again relatively similar, $354.71{ }^{\circ} \mathrm{C}$ and $339.38{ }^{\circ} \mathrm{C}$ respectively for the $\mathrm{K} 1$ and $\mathrm{K} 2$ level of stiffness (Figure $4.39 \mathrm{c}$ )) (Table 4.8). A reduction in critical temperature was observed of about $20^{\circ} \mathrm{C}$ was observed.

Table 4.8 Results for the built-up I (2C) cross-section specimens - pin-ended support.

\begin{tabular}{|c|c|c|c|c|c|c|}
\hline Test Reference & $\begin{array}{l}\theta_{\text {peak }} \\
{\left[{ }^{\circ} \mathbf{C}\right]}\end{array}$ & $\begin{array}{c}t_{\text {peak }} \\
{[\mathrm{min}]}\end{array}$ & $\begin{array}{l}P_{\max } \\
{[\mathrm{kN}]}\end{array}$ & $\begin{array}{c}\boldsymbol{P}_{\max } / \boldsymbol{P}_{0} \\
{[--]}\end{array}$ & $\begin{array}{c}\theta_{c r} \\
{\left[{ }^{\circ} \mathbf{C}\right]}\end{array}$ & $\begin{array}{c}t_{c r} \\
{[\mathbf{m i n}]}\end{array}$ \\
\hline I_PP_30LL_K $K_{1-1}$ & 306.24 & 7.2 & 48.5 & 1.790 & 354.71 & 7.88 \\
\hline I_PP_30LL_K ${ }_{1}-2$ & 296.89 & 7.25 & 46.28 & 1.787 & 342.81 & 8.05 \\
\hline I_PP_30LL_K ${ }_{1-3}$ & 308.78 & 6.98 & 49.13 & 1.868 & 348.64 & 7.65 \\
\hline$\mu$ & 303.97 & 7.14 & 47.97 & 1.82 & 348.72 & 7.86 \\
\hline$\sigma$ & 5.11 & 0.12 & 1.22 & 0.04 & 4.86 & 0.16 \\
\hline $\mathrm{CV}[\%]$ & 1.68 & 1.64 & 2.55 & 2.07 & 1.39 & 2.09 \\
\hline I_PP_50LL_K ${ }_{1-1}$ & 231.78 & 5.78 & 58.98 & 1.376 & 251.61 & 6.12 \\
\hline I_PP_50LL_K 1 -2 & 219.01 & 5.62 & 61.2 & 1.351 & 241.13 & 5.98 \\
\hline I_PP_50LL_K ${ }_{1}-3$ & 225.65 & 5.9 & 60.31 & 1.392 & 246.33 & 6.25 \\
\hline$\mu$ & 225.48 & $5.7 \overline{7}$ & 60.16 & 1.37 & 246.36 & 6.12 \\
\hline$\sigma$ & 5.21 & 0.11 & 0.91 & 0.02 & 4.28 & 0.11 \\
\hline $\mathrm{CV}[\%]$ & 2.31 & 1.99 & 1.52 & 1.23 & 1.74 & 1.80 \\
\hline I_PP_30LL_K ${ }_{2-1}$ & 171.85 & 5.58 & 67.70 & 2.584 & 336.56 & 8.08 \\
\hline I_PP_30LL_K ${ }_{2-2}$ & 174.01 & 5.38 & 65.30 & 2.521 & 339.38 & 7.78 \\
\hline I_PP_30LL_K $\mathrm{K}_{2-3}$ & 187.62 & 5.77 & 71.16 & 2.784 & 349.84 & 8.40 \\
\hline$\mu$ & 177.83 & $5 . \overline{58}$ & 68.05 & 2.63 & 341.93 & 8.09 \\
\hline$\sigma$ & 6.98 & 0.16 & 2.41 & 0.11 & 5.71 & 0.25 \\
\hline $\mathrm{CV}[\%]$ & 3.93 & 2.86 & 3.53 & 4.26 & 1.67 & 3.13 \\
\hline I_PP_50LL_K ${ }_{2-1}$ & 174.04 & 5.22 & 83.3 & 1.924 & 266.36 & 6.8 \\
\hline I_PP_50LL_K ${ }_{2-2}$ & 148.33 & 4.8 & 77.6 & 1.805 & 245.45 & 6.5 \\
\hline I PP $50 \mathrm{LL} \mathrm{K}_{2-3}$ & 166.58 & 5.02 & 81.1 & 1.886 & 244.17 & 6.3 \\
\hline$\mu$ & 162.98 & 5.01 & 80.67 & 1.87 & 251.99 & 6.53 \\
\hline$\sigma$ & 10.80 & 0.17 & 2.35 & 0.05 & 10.17 & 0.21 \\
\hline $\mathrm{CV}[\%]$ & 6.63 & 3.42 & 2.91 & 2.65 & 4.04 & 3.15 \\
\hline
\end{tabular}


For the semi-rigid end-support conditions the $P_{\max } / P_{0}$ ratios were 1.480 (I_SR_30LL_K3-2) and 1.219 (I_SR_50LL_K3-2) respectively for 30 and 50\% load level and K3 level of stiffness of the surrounding structure (Figure $4.39 \mathrm{~d}$ )) (Table 4.9). For the K4 level of stiffness the $P_{\max } / P_{0}$ ratios were 2.888 (I_SR_30LL_K4-2) and 1.784 (I_SR_50LL_K4-1) (Figure 4.39 d)) (Table 4.9). In terms of critical temperatures, it was observed that for the semi rigid end-support conditions increasing the level of stiffness of the surrounding structure may lead to lower critical temperatures of the CFS columns. For the $\mathrm{K} 3$ level of stiffness the mean critical temperatures obtained were $416.0^{\circ} \mathrm{C}$ (I_SR_30LL_K3-2) and $327.74{ }^{\circ} \mathrm{C}$ (I_SR_50LL_K3-2) for 30 and $50 \%$ load level whereas for the $\mathrm{K} 4$ level the mean critical temperatures were 353.86 ${ }^{\circ} \mathrm{C}$ (I_SR_30LL_K4-2) and $263.88^{\circ} \mathrm{C}$ (I_SR_50LL_K4-1) (Figure 4.39d)) (Table 4.9).

Table 4.9 Results for the built-up I (2C) cross-section specimens - semi-rigid ended support.

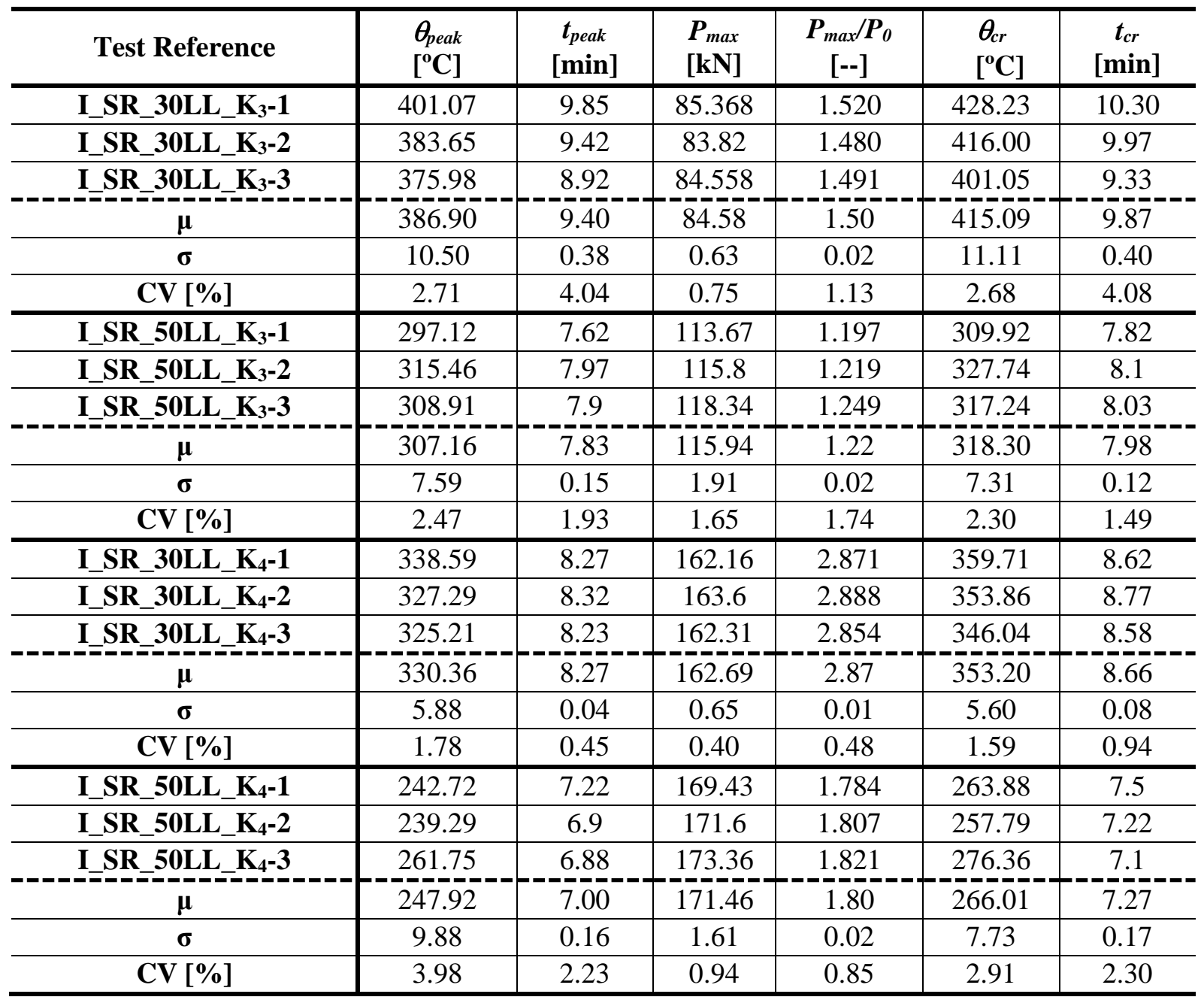


It was found out that the interaction between the stiffness of the surrounding structure (axial and rotational stiffness) and the initially applied load level significantly influenced the maximum load reached $\left(P_{\max }\right)$ during each experimental test. The higher the level of stiffness and initial applied load is, the higher the maximum load was for both cross-sections. In some cases it was found out that the maximum load reached actually exceeded the design buckling load at ambient temperature $\left(N_{b, R d}\right)$. For instance the obtained $P_{\max }$ values ranged approximately from $78 \%$ to $186 \%$ of the buckling load at ambient temperature $\left(N_{b, r d}\right)$ for the lipped channel columns. This may be due to the fact that the design buckling load for fixed lipped channel columns may be too conservative (according to the obtained results for the buckling tests), hence the initial service load used may not correspond exactly to 30 and $50 \%$ of the actual buckling load of fixed lipped channel columns. For the open built-up I cross-section considering the highest load level $\left(50 \% N_{b, R d}\right)$ and the highest level of restraint to thermal elongation the $P_{\max }$ recorded was very close to the design buckling load at ambient temperature (above $90 \%$ $N_{b, R d}$ for both pinned and fixed supports).

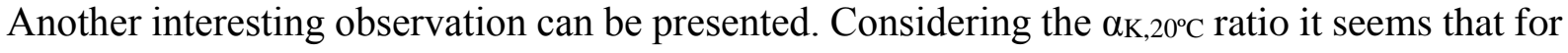
higher values of $\alpha \mathrm{K}, 20^{\circ} \mathrm{C}$ the reduction in critical temperature is smaller. For instance, considering

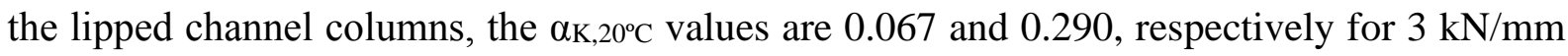
and $13 \mathrm{kN} / \mathrm{mm}$ of axial restraint to thermal elongation, it was observed that the reduction of critical temperature was small, on average, considering all test conditions, about $20{ }^{\circ} \mathrm{C}$. This may indicate that the imposed axial restraint of $3 \mathrm{kN} / \mathrm{mm}$ may be already high for the lipped channel columns. Assuming that the column could freely expand $\left(\alpha_{\left.\mathrm{K}, 20^{\circ} \mathrm{C}=0\right)}\right)$ the critical temperature should be higher than the one obtained in the experimental tests, and probably the big reduction in critical temperature due to increase of restraint to thermal elongation should be observed for values of $\alpha \mathrm{K}, 20^{\circ} \mathrm{C}$ between 0 and 0.067 . This type of interpretations can be investigated using the developed finite element model presented in Chapter 5 in future parametric studies.

For lower values of $\alpha \mathrm{K}, 20^{\circ} \mathrm{C}$ the reduction in critical temperatures are more significant. This can

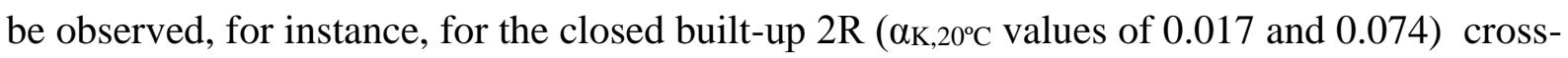
section, in Figure 4.40 and Tables 4.12 and 4.13. In figure 4.40 the evolution of the restraining forces as a non-dimensional ratio is presented as a function of the mean temperature of the column for both closed built-up $\mathrm{R}$ and $2 \mathrm{R}$ cross-sections. 

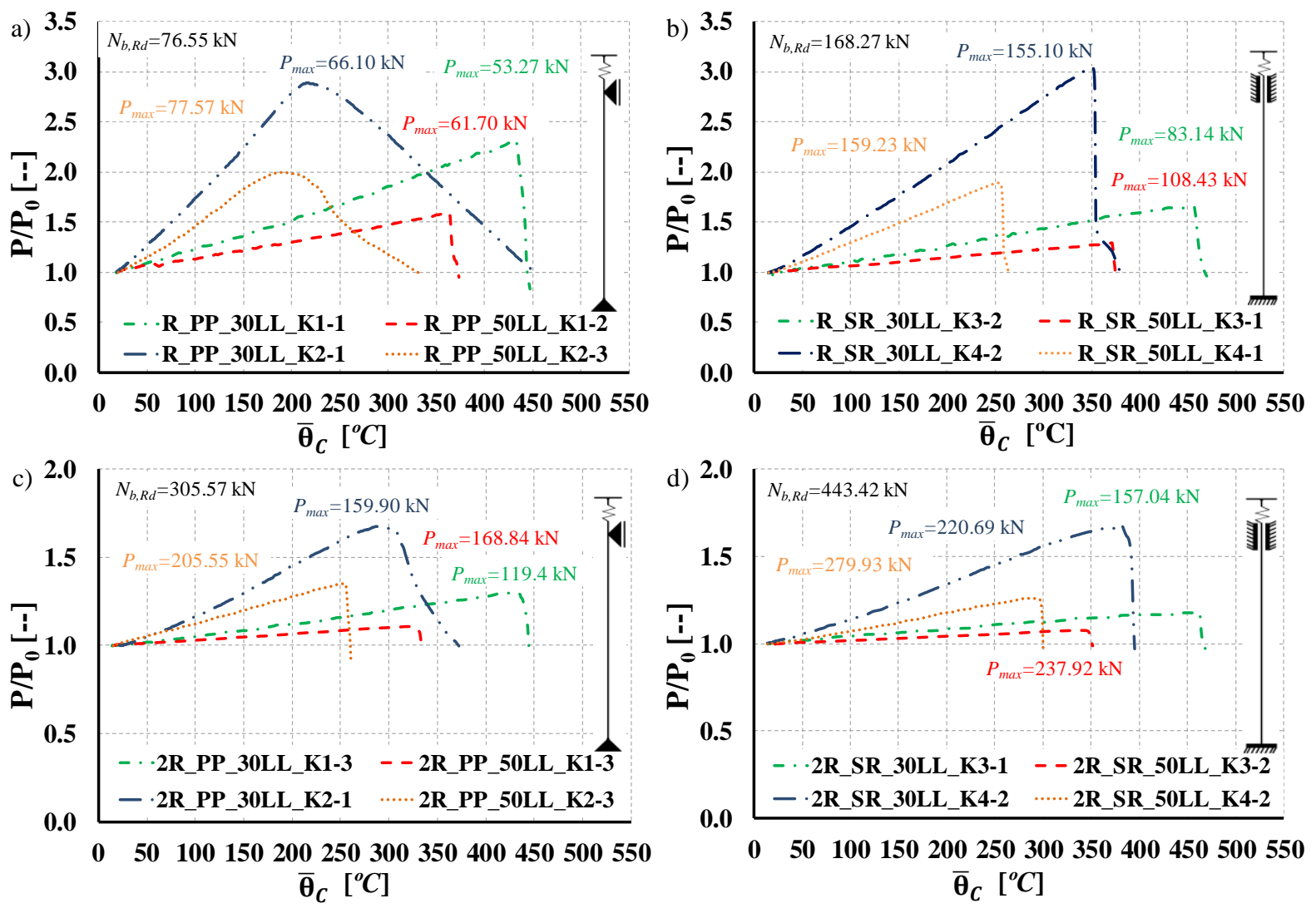

Figure 4.40 Non-dimensional restraining forces ratio for closed built-up CFS columns. a) R cross-section with pinned-end support. b) $\mathrm{R}$ cross-section with semi-rigid end support. c) $2 \mathrm{R}$ cross-section with pinned-end support. d) $2 \mathrm{R}$ cross-section with semi-rigid end support.

Observing the obtained results it is clear that increasing the initial service load ( 30 to $50 \% \mathrm{~N}_{\mathrm{b}, \mathrm{Rd}}$ ) critical temperatures and critical times decreased for both cross-sections. In Tables 4.10 to 4.14 more detailed results are presented. For instance, for the tests R_PP_30LL_K1 the obtained mean critical temperature $\left(\theta_{c r}\right)$ was $443.33^{\circ} \mathrm{C}$ whereas for the tests R_PP_50LL_K1 the obtained mean $\theta_{c r}$ was $372.67^{\circ} \mathrm{C}$. Analysing the results taking into consideration the imposed levels of restraint to thermal elongation to the CFS column it was observed that, generally, increasing the level of the imposed restraint to thermal elongation the critical times and temperatures decreased. Only for one test condition this evidence was not observed. For the R cross-section considering pin-end support condition and 30\% load level it was observed that the mean critical temperature was almost the same (R_PP_30LL_K1, $\theta_{c r}=443.33^{\circ} \mathrm{C}$ whereas for R_PP_30LL_K2, $\left.\theta_{c r}=436.26\right)$ despite the increase of the level of restraint to thermal elongation ( 3 to $13 \mathrm{kN} / \mathrm{mm}$ ) (Figure $4.40 \mathrm{a}$ )) and Table 4.10). However, considering the 50\% load level, increasing the level of restraint imposed to the CFS column lead to a reduction in the mean critical temperature (R_PP_50LL_K1, $\theta_{c r}=372.67^{\circ} \mathrm{C}$ whereas for R_PP_50LL_K2, $\theta_{c r}$ 
$=328.67^{\circ} \mathrm{C}$ ) (Figure $4.40 \mathrm{a}$ ) ) and Table 4.10 ). Hence the obtained results for the $30 \%$ load level and for both levels of restraint to thermal elongation may be somehow more influenced by friction in the end-support devices (translated into a reduction of the effective length). Another possible justification may be related to the fact that, for the higher imposed level of restraint, the maximum axial force is reached for much lower temperatures and the decrease of the generated restraining force was very slow up to the initial applied service load (Figure $4.40 \mathrm{a}$ )). Nevertheless, it is worth mentioning that for the pin-end condition all conducted tests may be more or less influenced by friction between the Teflon lined steel pin and the steel plates of the end-support devices, aggravated by the thermal expansion. Moreover, all tests results may be also affected by imperfections of the steel profiles, imperfections during the assembly of the built-up columns and even due to eccentricities in the applied loading.

For the closed built-up 2R cross-section for all tested conditions increasing the level of restraint to thermal elongation lead to a reduction in critical temperatures (Fig. $4.40 \mathrm{c}$ ) and d)). For instance, comparing the critical temperatures of the 2R_SR_30LL_K3 tests with the 2R_SR_30LL_K4 tests it was found that the critical temperature reduction was on average about $75^{\circ} \mathrm{C}$. For all tested situations it was observed that increasing the level of imposed restraint ( 3 to $13 \mathrm{kN} / \mathrm{mm}$ ) to thermal elongation to the CFS column the generated restraining forces increased faster and the maximum axial force (initial service load plus the generated restraining forces) was reached for much lower temperatures and times (Figure 4.40 and Tables 4.10 to 4.14). For example, for the R built-up cross-section considering a 50\% initial load level and pinned end-support condition, increasing the imposed level of restraint to thermal elongation ( 3 to $13 \mathrm{kN} / \mathrm{mm}$ ) lead on average to a reduction in the peak temperature ( $\theta_{\text {peak }}$ ) of $175.86^{\circ} \mathrm{C}$, whereas for the $2 \mathrm{R}$ cross-section and considering a $30 \%$ initial load level and semirigid end-support condition, increasing the level of restraint lead on average to a reduction of the peak temperature of $91.4^{\circ} \mathrm{C}$.

Regarding the obtained values for the $P / P_{0}$ ratio it was observed that for lower initial load level $(30 \% \mathrm{Nb}, \mathrm{Rd})$ the $P / P$ o ratio was higher for all tested situations, whereas for higher imposed levels of restraint to thermal elongation the $P / P_{0}$ ratio was bigger (Figure 4.40 and Tables 4.10 to 4.14). Analysing the results for the closed built-up R (Figure 4.40 a)) columns, considering pinend support conditions and the $\mathrm{K} 1$ level of stiffness of the surrounding structure to the CFS column ( $3 \mathrm{kN} / \mathrm{mm}$ of axial restraint), the mean $P_{\max } / P_{0}$ ratio was 2.27 and 1.62 times the value of the initial applied load respectively for the 30 and 50\% load level. (Figure 4.40 a)) (Table 4.10). For the K2 level of stiffness of the surrounding structure to the CFS column $(13 \mathrm{kN} / \mathrm{mm}$ of axial restraint) it was found that the mean $\mathrm{P}_{\max } / \mathrm{P}_{0}$ ratio increased up to 2.95 times for the $30 \%$ load level and up to 2.03 times the initial load for the $50 \%$ load level (Figure $4.40 \mathrm{~b}$ )) 
(Table 4.11) which are significantly higher than those obtained for the K1 level (Figure 4.40 b)). For the semi-rigid end-support condition and for K3 level of stiffness of the surrounding structure it was observed that the mean $P_{\max } / P_{0}$ ratios were 1.65 and 1.3 respectively for the 30 and 50\% load level (Figure 4.40 b)) (Table 4.12), whereas for the K4 level the mean $P_{\max } / P_{0}$ ratios were 3.0 and 1.89, respectively for the 30 and $50 \%$ load level (Figure $4.40 \mathrm{~b}$ )) (Table 4.12), which were once again significantly higher than those obtained for the lower level of stiffness of the surrounding structure. In Tables 4.11 and 4.12 all relevant results for the closed built-up R cross-section are presented.

Table 4.10 Results for the closed built-up R column with pinned-end supports.

\begin{tabular}{|c|c|c|c|c|c|c|c|c|}
\hline Test Reference & $\begin{array}{l}\theta_{\text {peak }} \\
{\left[{ }^{\circ} \mathrm{C}\right]} \\
\end{array}$ & $\begin{array}{c}\theta_{\text {peak }}^{\max } \\
{\left[{ }^{\circ} \mathrm{C}\right]}\end{array}$ & $\begin{array}{c}\boldsymbol{t}_{\text {peak }} \\
{[\mathrm{min}]}\end{array}$ & $\begin{array}{l}P_{\max } \\
{[\mathbf{k N}]}\end{array}$ & $\begin{array}{c}\boldsymbol{P}_{\max } / \boldsymbol{P}_{0} \\
{[--]}\end{array}$ & $\begin{array}{c}\theta_{c r} \\
{\left[{ }^{\circ} \mathbf{C}\right]} \\
\end{array}$ & $\begin{array}{c}\boldsymbol{\theta}_{\boldsymbol{c r}}^{\max } \\
{\left[{ }^{\circ} \mathbf{C}\right]} \\
\end{array}$ & $\begin{array}{c}t_{c r} \\
{[\mathbf{m i n}]}\end{array}$ \\
\hline R_PP_30LL_K1-1 & 432.9 & 476.4 & 13.12 & 53.271 & 2.319 & 447.2 & 489.90 & 13.42 \\
\hline R_PP_30LL_K1-2 & 419.1 & 466.7 & 12.5 & 49.514 & 2.162 & 435.4 & 466.70 & 12.8 \\
\hline R_PP_30LL_K1-3 & 443.8 & 496.2 & 13.5 & 53.659 & 2.343 & 447.4 & 500.90 & 13.58 \\
\hline$\mu$ & 431.93 & 479.77 & 13.04 & 52.15 & 2.27 & 443.33 & 485.83 & 13.27 \\
\hline$\sigma$ & 10.11 & 12.28 & 0.41 & 1.87 & 0.08 & 5.61 & 14.26 & 0.34 \\
\hline CV [\%] & 2.34 & 2.56 & 3.16 & 3.58 & 3.53 & 1.27 & 2.93 & 2.54 \\
\hline R_PP_50LL_K1-1 & 353.50 & 380.00 & 9.92 & 61.90 & 1.620 & 364.00 & 391.80 & 10.17 \\
\hline R_PP_50LL_K1-2 & 364.60 & 407.60 & 10.47 & 61.70 & 1.590 & 374.07 & 417.80 & 10.68 \\
\hline R_PP_50LL_K1-3 & 366.92 & 403.90 & 10.28 & 64.74 & 1.663 & 379.93 & 417.50 & 10.58 \\
\hline$\mu$ & 361.67 & 397.17 & 10.22 & 62.78 & 1.62 & 372.67 & 409.03 & 10.48 \\
\hline $\boldsymbol{\sigma}$ & 5.86 & 12.23 & 0.23 & 1.39 & 0.03 & 6.58 & 12.19 & 0.22 \\
\hline $\mathrm{CV}[\%]$ & 1.62 & 3.08 & 2.23 & 2.21 & 1.84 & 1.77 & 2.98 & 2.11 \\
\hline R_PP_30LL_K2-1 & 218.41 & 224.40 & 7.28 & 66.10 & 2.886 & 453.78 & 480.60 & 12.35 \\
\hline R_PP_30LL_K2-2 & 216.00 & 231.80 & 7.37 & 62.02 & 2.708 & 429.90 & 468.50 & 12.12 \\
\hline R_PP_30LL_K2-3 & 223.79 & 236.00 & 7.38 & 74.68 & 3.262 & 425.10 & 455.40 & 11.58 \\
\hline$\mu$ & 219.40 & 230.73 & 7.34 & 67.60 & 2.95 & 436.26 & 468.17 & 12.02 \\
\hline$\sigma$ & 3.26 & 4.80 & 0.04 & 5.28 & 0.23 & 12.54 & 10.29 & 0.32 \\
\hline CV [\%] & 1.48 & 2.08 & 0.61 & 7.80 & 7.82 & 2.88 & 2.20 & 2.69 \\
\hline R_PP_50LL_K2-1 & 182.70 & 196.00 & 5.88 & 79.74 & 2.082 & 342.40 & 362.40 & 9.28 \\
\hline R_PP_50LL_K2-2 & 184.05 & 203.80 & 6.23 & 76.83 & 1.995 & 312.01 & 346.20 & 9.13 \\
\hline R_PP_50LL_K2-3 & 190.70 & 204.70 & 6.12 & 77.57 & 1.999 & 331.60 & 362.00 & 9.17 \\
\hline$\mu$ & 185.82 & 201.50 & 6.08 & 78.05 & 2.03 & 328.67 & 356.87 & 9.19 \\
\hline$\sigma$ & 3.50 & 3.91 & 0.15 & 1.23 & 0.04 & 12.58 & 7.54 & 0.06 \\
\hline $\mathrm{CV}$ [\%] & 1.88 & 1.94 & 2.40 & 1.58 & 1.98 & 3.83 & 2.11 & 0.69 \\
\hline
\end{tabular}


Table 4.11 Results for the closed built-up R columns with semi-rigid end support.

\begin{tabular}{|c|c|c|c|c|c|c|c|c|}
\hline Test Reference & $\begin{array}{l}\theta_{\text {peak }} \\
{\left[{ }^{\circ} \mathbf{C}\right]}\end{array}$ & $\begin{array}{c}\theta_{\text {peak }}^{\max } \\
{\left[{ }^{\circ} \mathrm{C}\right]}\end{array}$ & $\begin{array}{c}t_{p e a k} \\
{[\mathrm{~min}]}\end{array}$ & $\begin{array}{l}\boldsymbol{P}_{\max } \\
{[\mathrm{kN}]}\end{array}$ & $\begin{array}{c}\boldsymbol{P}_{\max } / \boldsymbol{P}_{0} \\
{[--]}\end{array}$ & $\begin{array}{c}\theta_{c r} \\
{\left[{ }^{\circ} \mathbf{C}\right]}\end{array}$ & $\begin{array}{c}\theta_{c r}^{\max } \\
{\left[{ }^{\circ} \mathbf{C}\right]}\end{array}$ & $\begin{array}{c}t_{c r} \\
\text { [min] }\end{array}$ \\
\hline R_SR_30LL_K3-1 & 463.05 & 514.70 & 12.87 & 82.47 & 1.628 & 480.81 & 534.30 & 13.15 \\
\hline R_SR_30LL_K3-2 & 454.47 & 501.70 & 12.52 & 83.14 & 1.652 & 469.45 & 518.90 & 12.77 \\
\hline R_SR_30LL_K3-3 & 437.20 & 498.00 & 12.07 & 84.17 & 1.668 & 446.05 & 508.70 & 12.23 \\
\hline$\mu$ & 451.57 & 504.80 & 12.49 & 83.26 & 1.65 & 465.44 & 520.63 & 12.72 \\
\hline$\sigma$ & 10.75 & 7.16 & 0.33 & 0.70 & 0.02 & 14.47 & 10.52 & 0.38 \\
\hline $\mathrm{CV}[\%]$ & 2.38 & 1.42 & 2.62 & 0.84 & 1.00 & 3.11 & 2.02 & 2.97 \\
\hline R_SR_50LL_K3-1 & 371.41 & 407.4 & 10.12 & 108.43 & 1.289 & 375.51 & 412.3 & 10.2 \\
\hline R_SR_50LL_K3-2 & 370.6 & 416.9 & 10.47 & 108.59 & 1.286 & 384.4 & 433 & 10.77 \\
\hline R_SR_50LL_K3-3 & 378.3 & 419.1 & 10.35 & 111.36 & 1.325 & 386 & 426.6 & 10.48 \\
\hline$\mu$ & 373.44 & 414.47 & 10.31 & 109.46 & 1.30 & 381.97 & 423.97 & 10.48 \\
\hline$\sigma$ & 3.45 & 5.08 & 0.15 & 1.35 & 0.02 & 4.61 & 8.65 & 0.23 \\
\hline $\mathrm{CV}[\%]$ & 0.93 & 1.22 & 1.41 & 1.23 & 1.36 & 1.21 & 2.04 & 2.22 \\
\hline R_SR_30LL_K4-1 & 324.93 & 365.70 & 9.92 & 148.57 & 2.924 & 349.59 & 390.20 & 10.38 \\
\hline R_SR_30LL_K4-2 & 350.4 & 384.90 & 10.52 & 155.10 & 3.043 & 381.3 & 421.00 & 11.22 \\
\hline R_SR_30LL_K4-3 & 353.5 & 387.80 & 10.50 & 153.40 & 3.026 & 367.6 & 404.00 & 10.82 \\
\hline$\mu$ & 342.94 & 379.47 & 10.31 & 152.36 & 3.00 & 366.16 & 405.07 & 10.81 \\
\hline$\sigma$ & 12.80 & 9.81 & 0.28 & 2.77 & 0.05 & 12.99 & 12.60 & 0.34 \\
\hline $\mathrm{CV}[\%]$ & 3.73 & 2.58 & 2.70 & 1.82 & 1.75 & 3.55 & 3.11 & 3.17 \\
\hline R_SR_50LL_K4-1 & 268.27 & 290.6 & 8.7 & 159.23 & 1.893 & 277.4 & 301.6 & 8.9 \\
\hline R_SR_50LL_K4-2 & 255.01 & 289.4 & 8.4 & 157.18 & 1.867 & 264.01 & 299.3 & 8.58 \\
\hline R_SR_50LL_K4-3 & 264.18 & 299.7 & 8.37 & 161.1 & 1.911 & 269.83 & 306.8 & 8.5 \\
\hline$\mu$ & 262.49 & 293.23 & 8.49 & 159.17 & 1.89 & 270.41 & 302.57 & 8.66 \\
\hline$\sigma$ & 5.54 & 4.60 & 0.15 & 1.60 & 0.02 & 5.48 & 3.14 & 0.17 \\
\hline CV [\%] & 2.11 & 1.57 & 1.75 & 1.01 & 0.96 & 2.03 & 1.04 & 2.00 \\
\hline
\end{tabular}

Regarding the closed built-up 2R cross-section (Figure $4.40 \mathrm{c}$ ) and d)) it was observed that for the pin-ended condition and for the K1 level of stiffness of the surrounding structure $(3 \mathrm{kN} / \mathrm{mm}$ of axial restraint) the mean $\mathrm{P}_{\max } / \mathrm{P}_{0}$ ratios were 1.30 and 1.11 respectively for the 30 and $50 \%$ load level while for the $\mathrm{K} 2$ level of stiffness of the surrounding structure $(13 \mathrm{kN} / \mathrm{mm}$ of axial restraint) were 1.68 and 1.36 (Figure $4.40 \mathrm{c}$ )) (Table 4.12). For the semi-rigid end-support conditions the mean $\mathrm{P}_{\max } / \mathrm{P}_{0}$ ratios were 1.19 and 1.08 respectively for 30 and $50 \%$ load level and $\mathrm{K} 3$ level of stiffness of the surrounding structure (Figure $4.40 \mathrm{~d}$ )) (Table 4.13). For the K4 level of stiffness the mean $\mathrm{P}_{\max } / \mathrm{P}_{0}$ ratios were 1.67 and 1.26 (Figure $4.40 \mathrm{~d}$ )) (Table 4.13), respectively for 30 and 50\% load level. In Tables 4.12 and 4.13 all relevant results are presented.

Considering the ratio between $P_{\max }$ and $N_{b, R d}$ it is interesting to observe the differences between the tested cross-sections, specifically for the higher load level $\left(50 \% N_{b, R d}\right)$ and higher level of 
restraint to thermal elongation ( $\mathrm{K} 2$ and $\mathrm{K} 4)$. For the lipped channel columns, considering the $50 \%$ load level and K2 and K4 restraint level, the monitored load at elevated temperatures was higher than the design buckling load at ambient temperature $\left(\approx 1.1\right.$ times the $N_{b, R d}$ for pinnedend support condition; $\approx 1.8$ times the $N_{b, R d}$ for the fixed-end support condition). For the open built-up I and closed built-up R cross-sections the ratio between $P_{\max }$ and $N_{b, R d}$ ranged from 0.90 to approximately 1 , whereas for the closed built-up $2 \mathrm{R}$ cross-section the ratio was about 0.65 (50\% load level, K2 and K4 restraint levels). At this point it can be stated that the available design predictions may not be completely accurate and as a consequence the initial applied load may not correspond exactly to 30 or $50 \%$ of the buckling load of each tested type of column.

Table 4.12 Results for the closed built-up 2R columns with pin-ended supports.

\begin{tabular}{|c|c|c|c|c|c|c|c|c|}
\hline Test Reference & $\begin{array}{l}\theta_{\text {peak }} \\
{\left[{ }^{\circ} \mathbf{C}\right]}\end{array}$ & $\begin{array}{c}\theta_{\text {peak }}^{\max } \\
{\left[{ }^{\circ} \mathrm{C}\right]}\end{array}$ & $\begin{array}{c}t_{\text {peak }} \\
\text { [min] }\end{array}$ & $\begin{array}{l}P_{\max } \\
{[\mathbf{k N}]}\end{array}$ & $\begin{array}{c}\boldsymbol{P}_{\max } \boldsymbol{P}_{\mathbf{0}} \\
{[--]}\end{array}$ & $\begin{array}{c}\theta_{c r} \\
{\left[{ }^{\circ} \mathrm{C}\right]}\end{array}$ & $\begin{array}{c}\theta_{c r}^{\max } \\
{\left[{ }^{\circ} \mathrm{C}\right]}\end{array}$ & $\begin{array}{c}t_{c r} \\
{[\mathbf{m i n}]}\end{array}$ \\
\hline 2R_PP_30LL_K1-1 & 434.1 & 462.6 & 14.52 & 119.9 & 1.313 & 453.1 & 485.5 & 15.02 \\
\hline 2R_PP_30LL_K1-2 & 410.1 & 444.2 & 14.32 & 118.67 & 1.295 & 427.2 & 463.8 & 14.77 \\
\hline 2R_PP_30LL_K1-3 & 431.5 & 479 & 14.82 & 119.4 & 1.301 & 446.1 & 499 & 15.17 \\
\hline$\mu$ & $4 \overline{25} . \overline{3}$ & 461.93 & 14.55 & $1 \overline{9} .32$ & 1.30 & $4 \overline{4} . \overline{13}$ & 482.77 & 14.99 \\
\hline$\sigma$ & 10.75 & 14.21 & 0.21 & 0.51 & 0.01 & 10.94 & 14.50 & 0.16 \\
\hline $\mathrm{CV}[\%]$ & 2.53 & 3.08 & 1.41 & 0.42 & 0.57 & 2.47 & 3.00 & 1.10 \\
\hline 2R_PP_50LL_K1-1 & 345.5 & 383.6 & 11.78 & 169.8 & 1.117 & 351.5 & 390.3 & 11.95 \\
\hline 2R_PP_50LL_K1-2 & 294.5 & 312.4 & 10.1 & 167.4 & 1.099 & 309.3 & 328 & 10.47 \\
\hline 2R_PP_50LL_K1-3 & 322.8 & 358.1 & 11.18 & 168.84 & 1.106 & 334 & 370.8 & 11.48 \\
\hline$\mu$ & 320.93 & 351.37 & 11.02 & 168.68 & 1.11 & 331.60 & 363.03 & 11.30 \\
\hline$\sigma$ & 20.86 & 29.45 & 0.70 & 0.99 & 0.01 & 17.31 & 26.02 & 0.62 \\
\hline $\mathrm{CV}[\%]$ & 6.50 & 8.38 & 6.31 & 0.58 & 0.67 & 5.22 & 7.17 & 5.46 \\
\hline 2R_PP_30LL_K2-1 & 293.00 & 320.40 & $\overline{10.72}$ & 159.90 & 1.674 & 372.40 & 405.00 & 12.78 \\
\hline 2R_PP_30LL_K2-2 & 263.30 & 281.10 & 9.45 & 148.00 & 1.610 & 358.90 & 384.20 & 11.90 \\
\hline 2R_PP_30LL_K2-3 & 309.40 & 344.00 & 10.87 & 161.82 & 1.769 & 384.20 & 429.90 & 12.82 \\
\hline$\mu$ & 288.57 & 315.17 & 10.35 & 156.57 & 1.68 & 371.83 & 406.37 & 12.50 \\
\hline$\sigma$ & 19.08 & 25.94 & 0.64 & 6.11 & 0.07 & 10.34 & 18.68 & 0.42 \\
\hline $\mathrm{CV}[\%]$ & 6.61 & 8.23 & 6.16 & 3.90 & 3.88 & 2.78 & 4.60 & 3.40 \\
\hline 2R_PP_50LL_K2-1 & 224 & 243.30 & 8.77 & 206.8 & 1.353 & 241.1 & 262.50 & 9.17 \\
\hline 2R_PP_50LL_K2-2 & 253.2 & 272.30 & 9.22 & 213.19 & 1.388 & 264.5 & 284.40 & 9.52 \\
\hline 2R_PP_50LL_K2-3 & 253.4 & 287.00 & 9.42 & 205.55 & 1.348 & 260.3 & 294.70 & 9.6 \\
\hline$\mu$ & 243.53 & 267.53 & 9.14 & 208.51 & 1.36 & 255.30 & 280.53 & 9.43 \\
\hline$\sigma$ & 13.81 & 18.16 & 0.27 & 3.35 & 0.02 & 10.19 & 13.43 & 0.19 \\
\hline $\mathrm{CV}$ [\%] & 5.67 & 6.79 & 2.98 & 1.60 & 1.31 & 3.99 & 4.79 & 1.98 \\
\hline
\end{tabular}

The differences in the ratio between $P_{\max }$ and $N_{b, R d}$ may be explained at some extent by the eventual inaccuracy of the available design methods. However, the key parameter influencing 
these results is the ratio between the stiffness of the surrounding structure imposing axial restraint to thermal elongation of the CFS column and the axial stiffness of the column $\left(\alpha_{K, 20^{\circ} C}\right)$.

Table 4.13 Results for the closed built-up 2R columns with semi-rigid end supports.

\begin{tabular}{|c|c|c|c|c|c|c|c|c|}
\hline Test Reference & $\begin{array}{l}\theta_{\text {peak }} \\
{\left[{ }^{\circ} \mathbf{C}\right]}\end{array}$ & $\begin{array}{c}\theta_{\text {peak }}^{\text {max }} \\
{\left[{ }^{\circ} \mathrm{C}\right]}\end{array}$ & $\begin{array}{c}t_{\text {peak }} \\
{[\mathrm{min}]}\end{array}$ & $\begin{array}{l}\boldsymbol{P}_{\max } \\
{[\mathrm{kN}]}\end{array}$ & $\begin{array}{c}\boldsymbol{P}_{\max } / \boldsymbol{P}_{\boldsymbol{0}} \\
{[--]}\end{array}$ & $\begin{array}{c}\theta_{c r} \\
{\left[{ }^{\circ} \mathbf{C}\right]}\end{array}$ & $\begin{array}{c}\theta_{c r}^{\max } \\
{\left[{ }^{\circ} \mathbf{C}\right]}\end{array}$ & $\begin{array}{c}t_{c r} \\
{[\mathrm{~min}]}\end{array}$ \\
\hline 2R_SR_30LL_K3-1 & 462.3 & 530.8 & 15.37 & 157.04 & 1.180 & 470.5 & 542.2 & 15.53 \\
\hline 2R_SR_30LL_K3-2 & 441.9 & 499.4 & 14.25 & 158.45 & 1.189 & 448.4 & 507.9 & 14.55 \\
\hline 2R_SR_30LL_K3-3 & 482.1 & 535.3 & 15.63 & 159.48 & 1.197 & 488.7 & 541.3 & 15.75 \\
\hline$\mu$ & 462.1 & 521.8 & 15.08 & 158.32 & 1.19 & 469.2 & 530.47 & 15.28 \\
\hline $\boldsymbol{\sigma}$ & 16.41 & 15.97 & 0.60 & 1.00 & 0.01 & 16.48 & 15.96 & 0.52 \\
\hline CV [\%] & 3.55 & 3.06 & 3.97 & 0.63 & 0.58 & 3.51 & 3.01 & 3.41 \\
\hline 2R_SR_50LL_K3-1 & 304 & 340.9 & 10.37 & 234.95 & 1.067 & 322.7 & 361.7 & 10.97 \\
\hline 2R_SR_50LL_K3-2 & 340.7 & 355.1 & 11.45 & 237.92 & 1.078 & 351.4 & 366.9 & 11.73 \\
\hline 2R_SR_50LL_K3-3 & 352.7 & 371.9 & 11.82 & 238.19 & 1.08 & 363.4 & 383.8 & 12.13 \\
\hline$\mu$ & 332.4 & 355.9 & 11.21 & 237.02 & 1.08 & 345.8 & 370.80 & 11.61 \\
\hline$\sigma$ & 20.72 & 12.67 & 0.62 & 1.47 & 0.01 & 17.08 & 9.43 & 0.48 \\
\hline $\mathrm{CV}[\%]$ & 6.23 & 3.56 & 5.49 & 0.62 & 0.53 & 4.94 & 2.54 & 4.14 \\
\hline 2R_SR_30LL_K4-1 & 380.4 & 417.5 & 12.62 & 226.33 & 1.7 & 412.1 & 450.5 & 13.55 \\
\hline 2R_SR_30LL_K4-2 & 369.3 & 404.6 & 13.17 & 220.69 & 1.662 & 394.7 & 432.2 & 13.9 \\
\hline 2R_SR_30LL_K4-3 & 362.4 & 404 & 12.55 & 216.35 & 1.633 & 375.2 & 417.6 & 12.87 \\
\hline$\mu$ & 370.7 & 408.7 & 12.78 & 221.12 & 1.67 & 394.0 & 433.43 & 13.44 \\
\hline$\sigma$ & 7.41 & 6.23 & 0.28 & 4.09 & 0.03 & 15.07 & 13.46 & 0.43 \\
\hline CV [\%] & 2.00 & 1.52 & 2.17 & 1.85 & 1.65 & 3.83 & 3.11 & 3.18 \\
\hline 2R_SR_50LL_K4-1 & 263 & 292.8 & 9.5 & 274.51 & 1.237 & 273.6 & 305.3 & 9.77 \\
\hline 2R_SR_50LL_K4-2 & 284 & 324 & 10.02 & 279.93 & 1.261 & 301.6 & 344.1 & 10.47 \\
\hline 2R_SR_50LL_K4-3 & 299.8 & 332.7 & 10.6 & 287.71 & 1.296 & 314.1 & 349.4 & 11 \\
\hline$\mu$ & 282.2 & 316.5 & 10.04 & 280.72 & 1.26 & 296.4 & 332.93 & 10.41 \\
\hline$\sigma$ & 15.07 & 17.13 & 0.45 & 5.42 & 0.02 & 16.93 & 19.66 & 0.50 \\
\hline CV [\%] & 5.34 & 5.41 & 4.48 & 1.93 & 1.92 & 5.71 & 5.90 & 4.84 \\
\hline
\end{tabular}

In Figure 4.41 and Figure 4.42 for each one of defined tested conditions the behaviour of the different types of columns considered in this study is compared. At this point is difficult to establish accurate and conclusive comparisons between all tested cross-sections since all crosssections were submitted to the same levels of restraint, and consequently to different values of the ratio between axial stiffness of the surrounding structure to the CFS column and the axial stiffness of the column (Equation 4.1). The same can be said regarding the ratio between the rotational stiffness of the surrounding structure to the CFS column in each direction $\left(K_{r, s}\right)$ and the sum of the rotational stiffness of the column about the major and minor axis $\left(K_{r, c}\right)$ with the rotational stiffness of the surrounding structure in each direction $\left(K_{r, s}\right)$ (Equation 4.3). In order to draw conclusive comparisons, the use of numerical simulations is fundamental. After 
development and validation of finite element model using the finite element software ABAQUS a series of extensive parametric studies should be conducted. In these future parametric studies, the actual value of restraint to be imposed to the CFS column shall be determined for each type of cross-section as a function of pre-defined values of the non-dimensional ratio between axial stiffness of the surrounding structure to the CFS and the axial stiffness of the column (Equation 4.1) for all cross-section shapes considered. This means that the rate of generated restraining forces will be the same for all considered cross-section shapes.
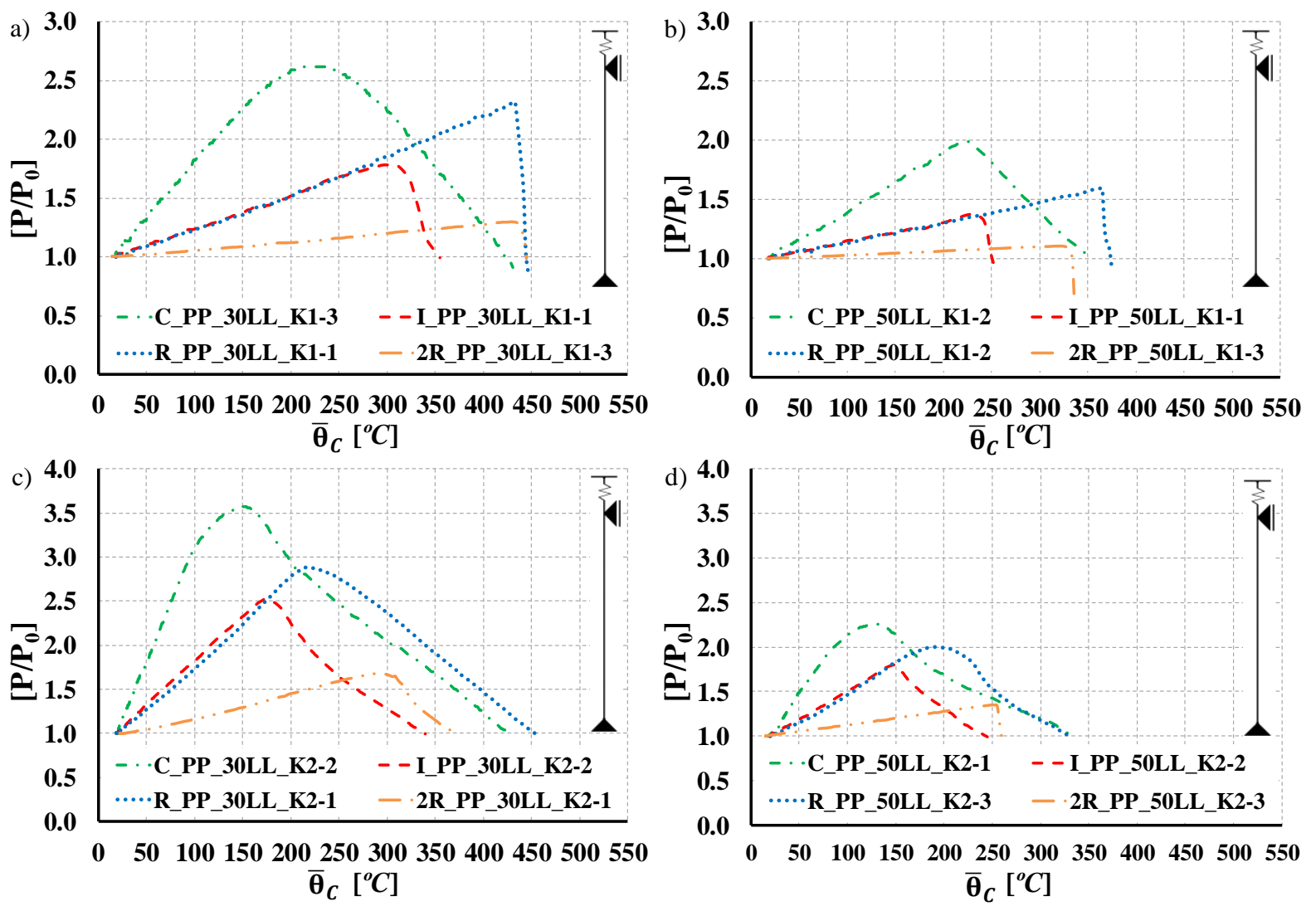

Figure 4.41 Evolution of the non-dimensional restraining forces ratio as a function of the mean temperature of the columns. a) $i \_P P \_30 L L \_K 1-j$. b) $i \_P P \_50 L L \_K 1-j$. c) $i \_$PP_30LL_K2-j. d) $i \_$PP_50LL_K2-j. ( $i$ is the type of cross-section; $j$ is the presented test result).

Analysing the Figures 4.41 and 4.42 is clear that the same levels of restraint used in the experimental investigation induce different rates in the generated restraining forces due to thermal elongation of the columns. Another conclusion that can be drawn for all presented results is that increasing the level of restraint to thermal elongation the failure of the columns is controlled by the generated restraining forces, whereas for lower levels of restraint 
temperature, and consequently degradation of mechanical properties, plays a more important role. Hence, higher levels of imposed restraint to thermal elongation will lead to higher values of generated restraining forces and eventually to lower values of critical temperature and time.
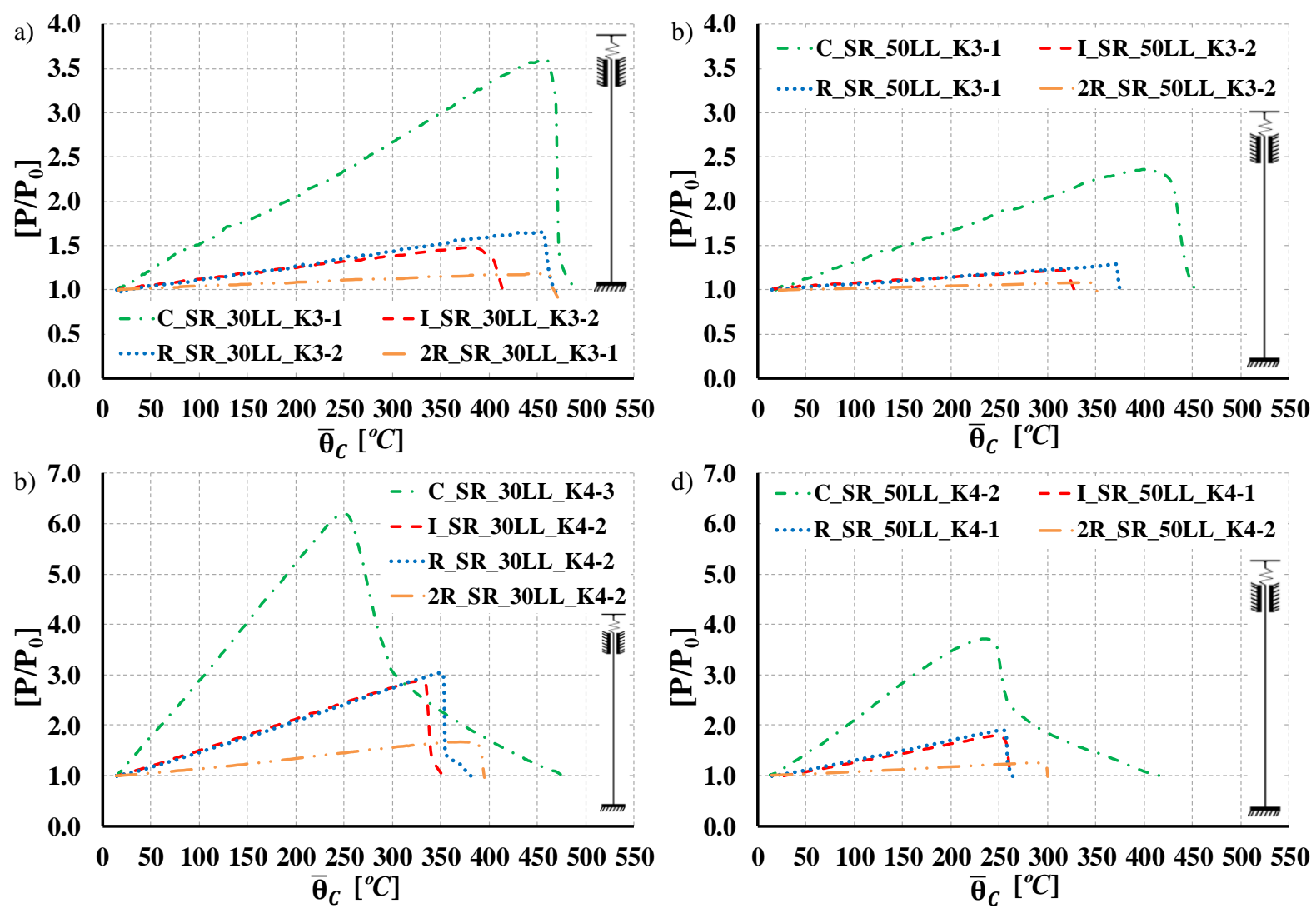

Figure 4.42 Evolution of the non-dimensional restraining forces ratio as a function of the mean temperature of the columns. a) $i \_S R \_30 L L \_K 3-j$. b) $\left.i \_S R \_50 L L \_K 3-j . c\right)$ $i \_$SR_30LL_K3-j.d) $i \_S R \_50 L L \_K 3-j$. ( $i$ is the type of cross-section; $j$ is the presented test result).

In terms of the post-critical behavior (instant from when the generated restraining forces started to decrease) it was observed that the lipped channel columns for the pin-ended support conditions presented a gradual decrease of restraining forces accompanied by a gradual increase of the lateral displacements whereas for the semi-rigid ended conditions the decrease of restraining forces was more sudden for the lower level of stiffness and in two stages for the higher level of stiffness of the surrounding structure. The post-critical behavior was characterized by a sudden drop followed by a gradual decrease in the restraining forces. Regarding the built-up I columns it was observed that the post-critical behavior presented a more or less gradual decrease of the restraining forces for the pin-ended whereas for the semi- 
rigid support conditions the post-critical behavior was characterized by a sudden decrease of the restraining forces followed by large lateral displacements. For the closed built-up R columns and for pinned condition the behavior of the column depended on the level of stiffness of the surrounding structure. For the lower level of imposed restraint, the behavior was characterized by a sudden drop followed by large lateral displacements whereas for higher levels of restraint to thermal elongation the behavior was characterized by a gradual decrease of restraining forces accompanied by a gradual increase of lateral displacements. For the semi-rigid end-support condition and for all tested load and restraint levels the behavior of the CFS columns was characterized by a sudden drop of the restraining forces followed by large lateral displacements. For the $2 \mathrm{R}$ cross-section only for one test condition (2R_PP_30LL_K1) a gradual decrease of the restraining forces was observed. For the remaining test conditions, a sudden drop of the restraining forces was observed. It is worth mentioning that for the pin-ended support conditions the obtained results may be affected by the friction in the hinge of the end-support device despite the use of Teflon (PTFE) between the steel pin and the steel plates. Due to friction, which is difficult to quantify, the fully pinned-end support condition is not guaranteed, hence the actual existent end-support condition may be classified as semi-rigid with a low rotational restraint. In Appendix D the observed influence of friction in the behavior of CFS columns is presented for a pinned-ended lipped channel column tested with and without Teflon. Teflon reduced friction, however, some friction still exists and as a consequence, the obtained results may not correspond to the lowest bound in terms of load-bearing capacity, critical temperatures and critical times of the CFS columns.

Finally, in order to clarify the behavior of CFS columns with restraint to thermal elongation in case of fire it is interesting to represent the observed critical temperatures $\left(\theta_{c r}\right)$ as a function of the ratio $\left(\alpha_{k}\right)$ between the axial stiffness of the surrounding structure to the CFS column $\left(\mathrm{K}_{\mathrm{a}, \mathrm{s}}\right)$ and the axial stiffness of the column $\left(K_{a, c}\right)$. The previously drawn conclusions regarding critical temperature $\left(\theta_{c r}\right)$ are clearly observed in Figure 4.43. Once again it is clear the influence of the interaction between the initial service load applied to the column and the level of restraint to thermal elongation. However, if only the influence of restraint to thermal elongation is considered it was found that for the pinned-end support condition the reduction in critical temperature was not very relevant for lipped channel and open built-up I columns. Once again friction may have significant influence on these results and its influence may not be exactly the same for all tested conditions. To completely clarify this situation future parametric studies using the developed and validated finite element model should be conducted, considering ideal support conditions, namely "perfectly" pinned and fully fixed. Regarding the semi-rigid end support condition, the reduction in critical temperature with the increase of restraint to thermal elongation is very clear for all tested cross-sections. Hence, in the scope of this research it can 
be stated that using the semi-rigid end support condition provided more reliable results to future numerical investigations.
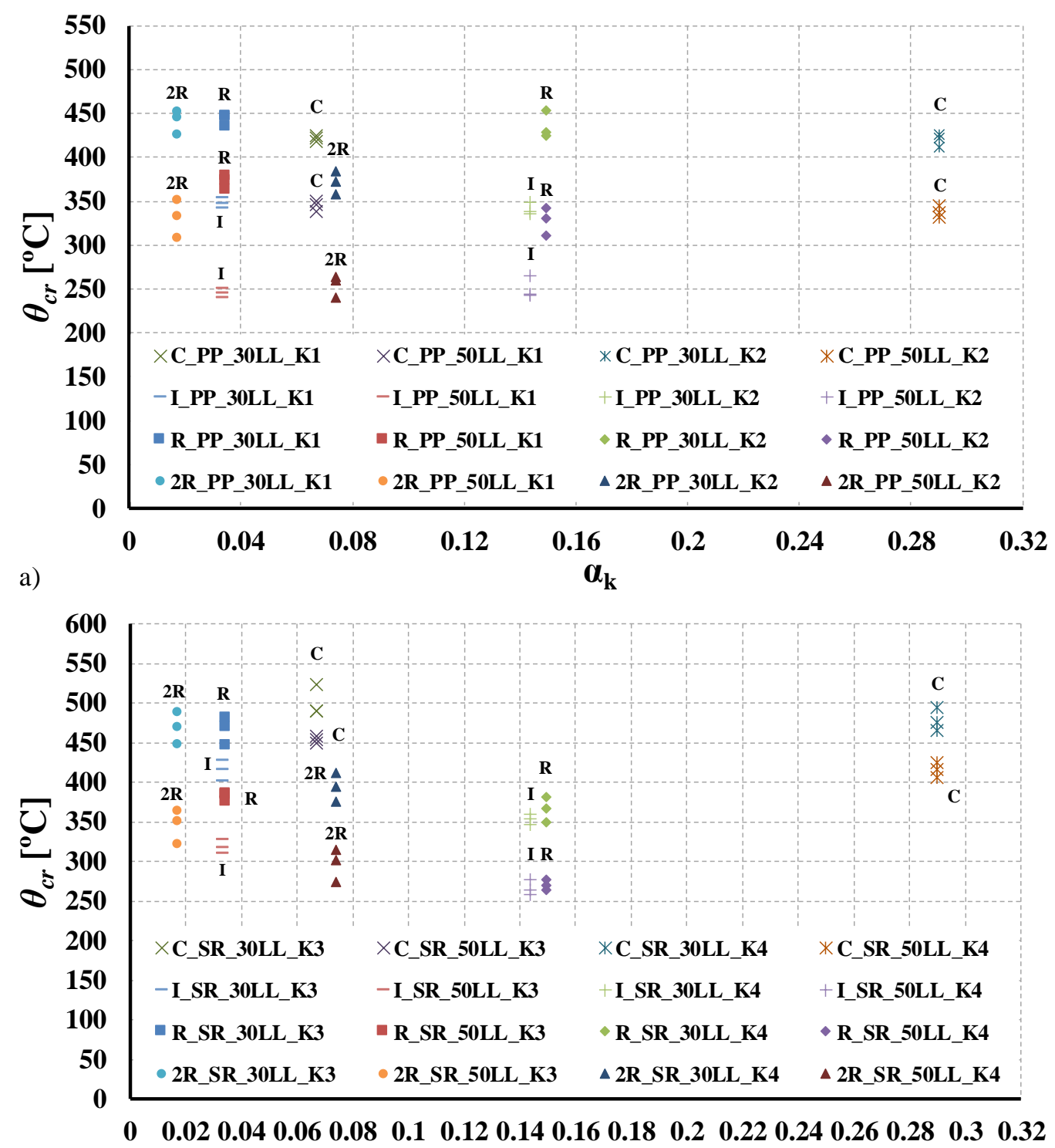

b)

$\boldsymbol{\alpha}_{\mathbf{k}}$

Figure 4.43 Variation of critical temperatures $\left(\theta_{c r}\right)$ as a function of the non-dimensional axial restraint ratio $\left(\alpha_{k}\right)$. a) Pinned-end support. b) Fixed-end support.

\subsubsection{Lateral and axial displacements}

Lateral deformations and axial displacements were monitored in every experimental test carried out in this investigation. In Figure 4.44 the evolution of lateral deflections about the minor axis 
is presented for all tested cross-sections and end-support conditions in order to show the global deformed shape. The presented results concern the $30 \%$ load level situation and are representative of the remaining test conditions.
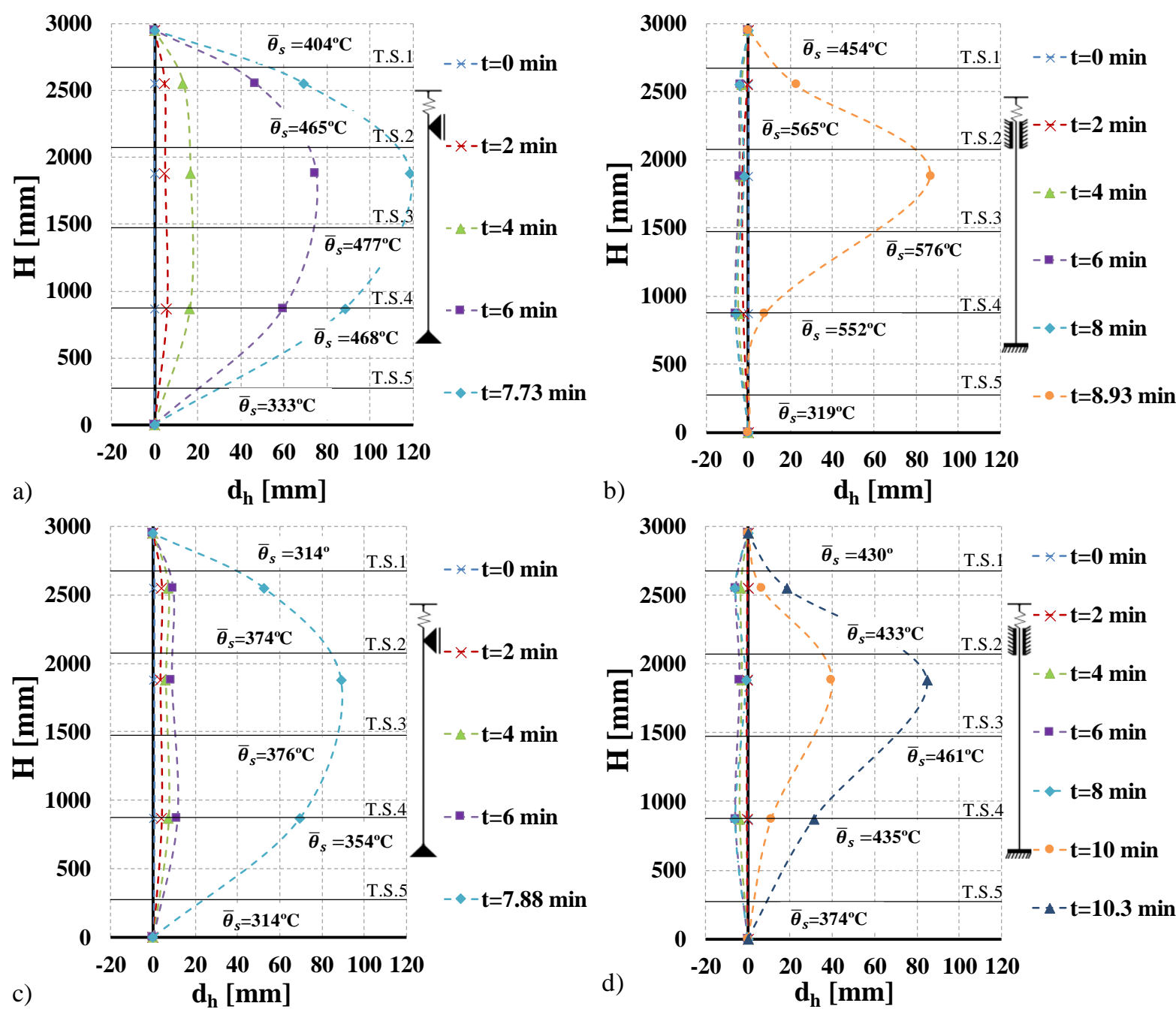

Figure 4.44 Lateral deformations about the minor axis for lipped channels and open built-up I cross-sections for both end-support conditions tested. a) C_PP_30LL_K1-3.b)

$$
\text { C_SR_30LL_K3-1.c) I_PP_30LL_K1-1.d) I_SR_30LL_K3-2. }
$$

Observing the obtained curves for the lateral displacements throughout the length of the column it was found in some cases a reversal in the direction of the lateral displacement during the fire test. For example, in Figure 4.44 b) and d) and Figure 4.45 a), b), c) and d) it is clear that initially the column started to buckle in one direction and then buckled in the opposite direction up to its final deformed shape. This may occur due to geometrical imperfections of the specimens and/or the restraining frame or even due to some small eccentricities of the applied loading. 
Also observing the obtained curves, it is clear the difference on the final deformed shape for both pin and semi-rigid ended support conditions. For the semi-rigid ended support conditions, the points of inflection in the deformed shape are clearly recognizable (Figure 4.44 and $4.45 \mathrm{~b}$ ) and d)). Also it is worth mentioning that for the pinned situation friction between the steel pin, acting as a hinge, and the steel plates may lead to a reduction of the effective length of the tested columns. Finally, it is worth mentioning that the lateral displacements about the major axis were not relevant for the presented experimental conditions.
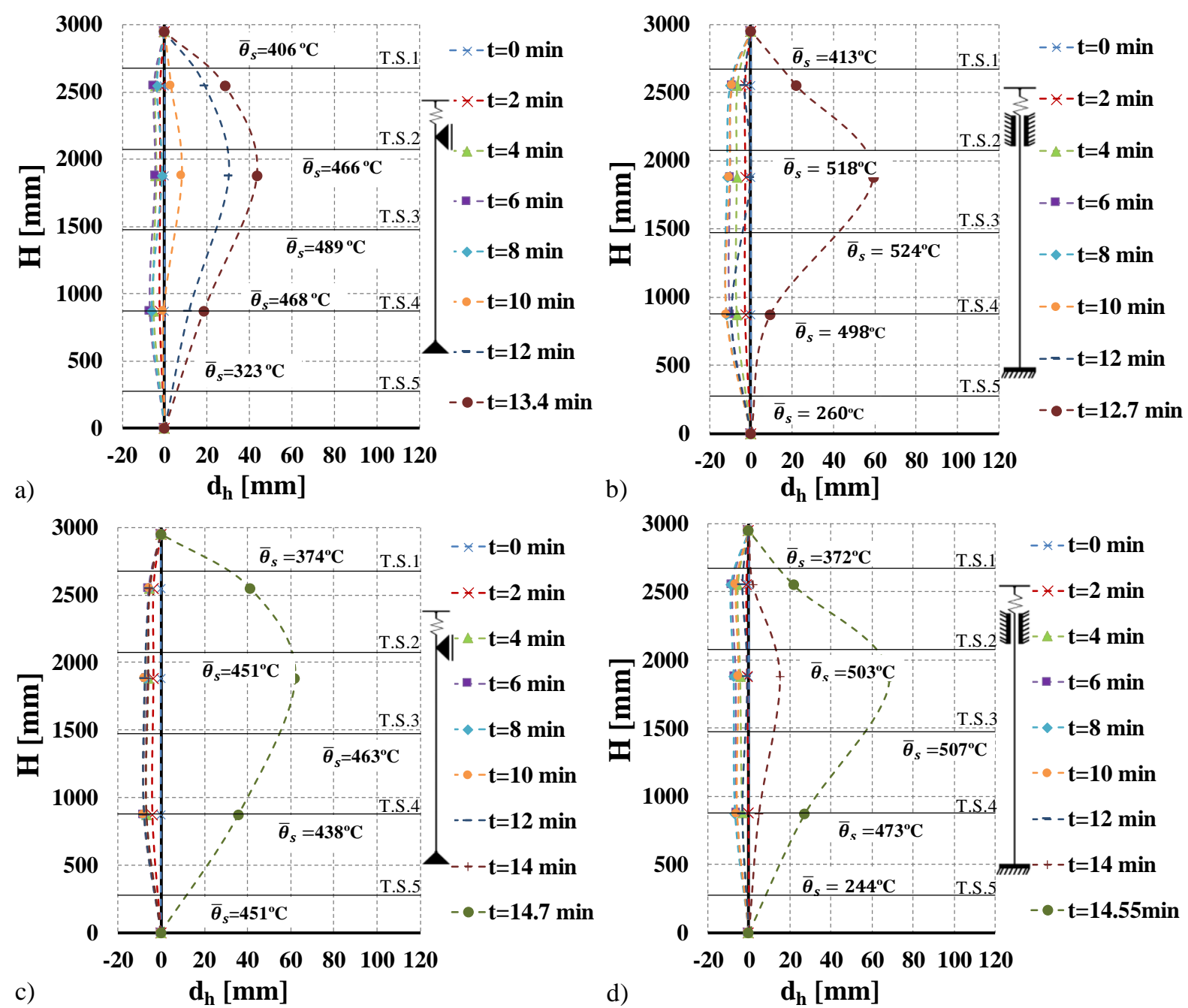

Figure 4.45 Lateral deformations about the minor axis for lipped channels and open built-up I cross-sections for both end-support conditions tested. a) R_PP_30LL_K1-1.b) R_SR_30LL_K3-2.c) 2R_PP_30LL_K1-3.d) 2R_SR_30LL_K3-1.

In terms of axial displacements and for both types of columns it was observed that lower load levels and lower levels of restraint to thermal elongation lead to higher axial displacements. In 
Figure 4.46 the obtained axial displacements as a function of the mean temperature of the column are presented. For example, to the C_PP_30LL_K $2-2$ specimen the maximum axial displacement was $3.72 \mathrm{~mm}$ whereas to the C_SR_30LL_K3-1 specimen the maximum axial displacement was $12.31 \mathrm{~mm}$.
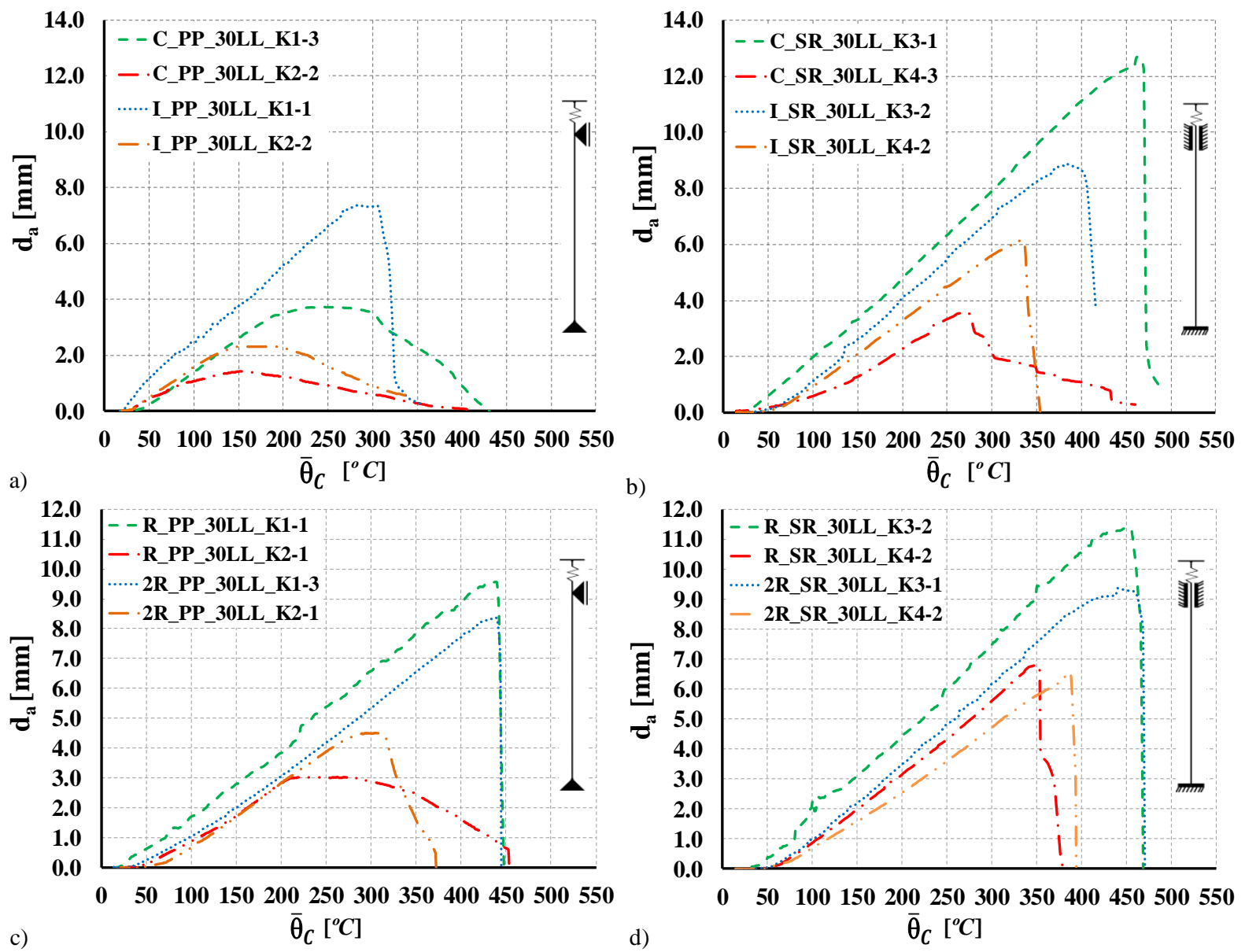

Figure 4.46 Axial displacements. a) Lipped and open built-up I cross-section columns with pinned-end supports. b) Lipped and open built-up I cross-section columns with semi-rigid end supports. c) Closed built-up R and 2R cross-section columns with pinned-end supports. d)

Closed built-up R and 2R cross-section columns with semi-rigid end supports.

\subsubsection{Failure modes}

\subsubsection{Buckling tests at ambient temperature}

Figure 4.47 to 4.54 illustrate the experimental failure modes observed for one specimen of each test condition adopted in the scope of this experimental research. Generally, it was observed 
that the predominant failure mode for almost all tested conditions was the flexural buckling about the minor axis of the CFS column in interaction with distortional buckling at mid height of the column (pinned and fixed-end support) and near the end-support devices (fixed-end support condition). In some specific cases additional buckling modes were also observed. A description of all observations regarding buckling modes is presented for all tested conditions.

For lipped channel column with pinned-end support the predominant failure mode was flexural buckling about the minor axis and distortional buckling. However, distortional buckling only occurred when a flexural deflection was already visible. Distortional buckling was observed at about mid-height of the column. It was observed that lips and flanges move outwards. Also the movement of lips and flanges on opposite sides was not horizontally aligned as Figure 4.47 a1) and a3) shows. This means that flexural-torsional buckling may have occurred in some pinned lipped channel columns. The final deformed shape can be described as almost a perfect arc.

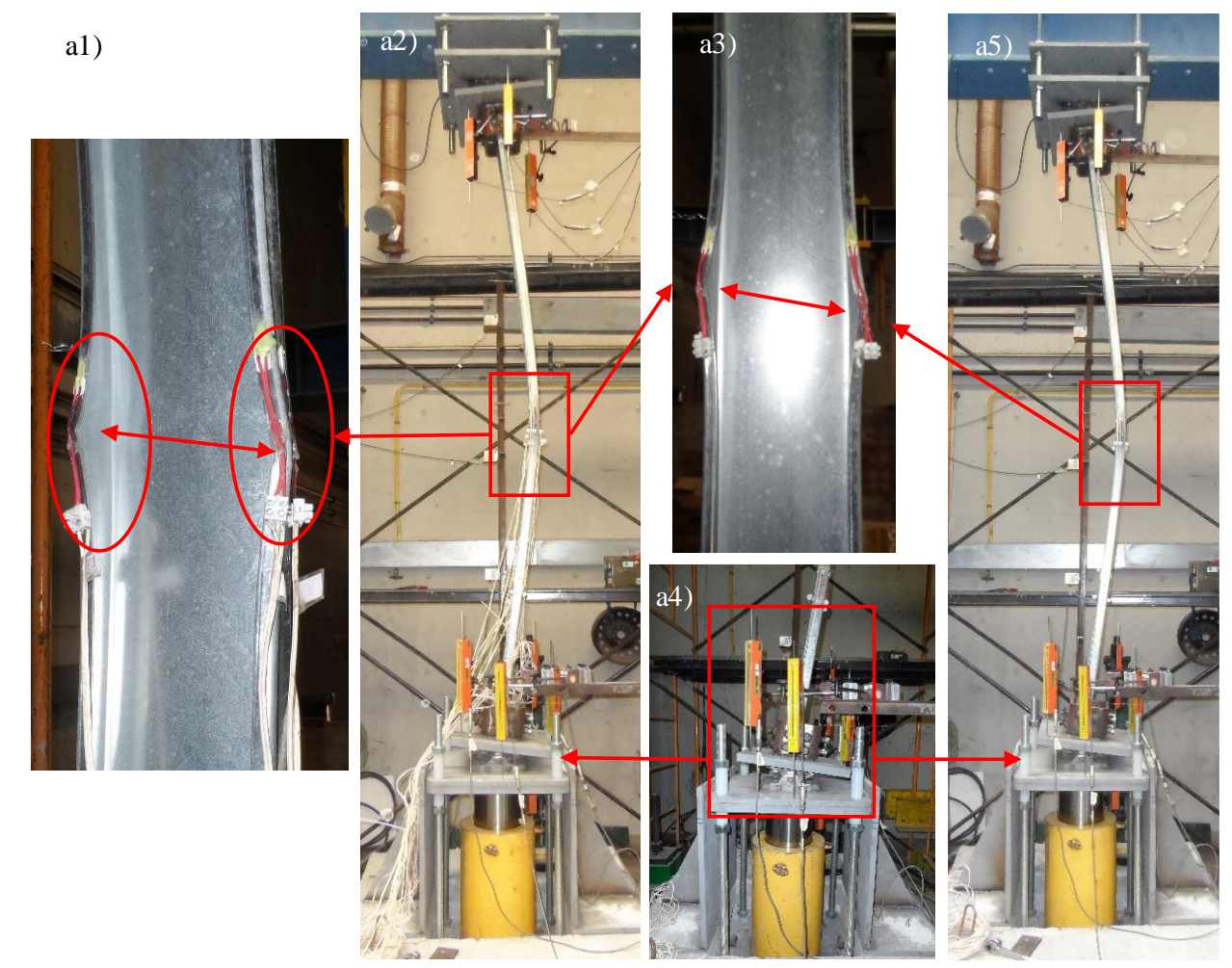

Figure 4.47 Failure modes of the test column C_PP_3 at ambient temperature. a1) and a3)

Distortional buckling. a2) and a5) Flexural buckling. a4) Rotation of the end-support.

For lipped channel columns with fixed-end supports the predominant failure mode was the interaction between flexural-torsional buckling (Figure 4.48 a1)) and distortional buckling at mid-height of the column (Figure $4.48 \mathrm{a} 3$ ) and a4)). Near the end-support devices some local 
crushing was observed in the lips and flanges of the columns. This can lead to buckling lengths bigger than $0.5 \mathrm{~L}$. In Figure $4.48 \mathrm{a}$ ) and a5) this observation is more or less evident.
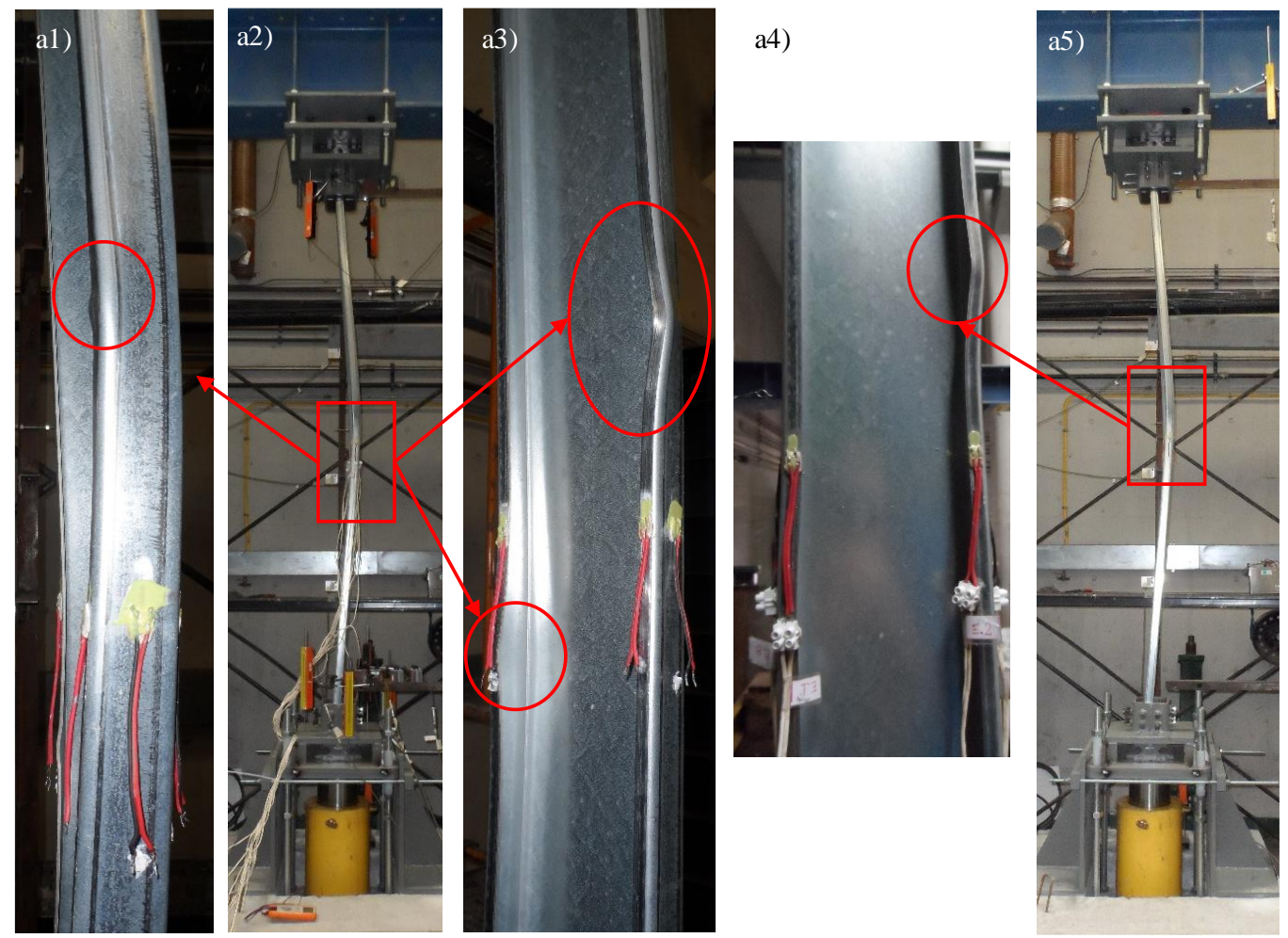

Figure 4.48 Failure modes of the test column C_FF_3 at ambient temperature. a1), a3) and a4) Distortional buckling. a2) and a5) Flexural buckling.

For columns with open built-up I cross-section and pinned-end supports (Figure 4.49) the predominant failure mode was the interaction between flexural buckling about the minor axis and distortional buckling at mid-height of the column. Just like it was observed for the lipped channel columns the distortional buckling was characterized by lips and flanges moving outwards and non-horizontal alignment between lips and flanges (Figure $4.49 \mathrm{a} 1$ ) and a3)). This may also indicate the existence of some eccentricities in loading.

For columns with open built-up cross-section and fixed-end supports (Figure 4.50) the predominant failure mode was the interaction between flexural buckling about the minor axis and distortional buckling at mid-height and near the end-support devices. The observed distortional buckling near the end-support devices is very subtle. Also localised crushing in lips and flanges was observed in the end-support devices. Distortional buckling was clearly visible only in one of the lipped channels used to fabricate the built-up cross-section and was characterized by lips and flanges moving outwards. Inflection points in the final deformed shape 
are a bit subtler due to the observed localised crushing in the end-support devices, hence the buckling length may be actually slightly bigger than $0.5 \mathrm{~L}$.

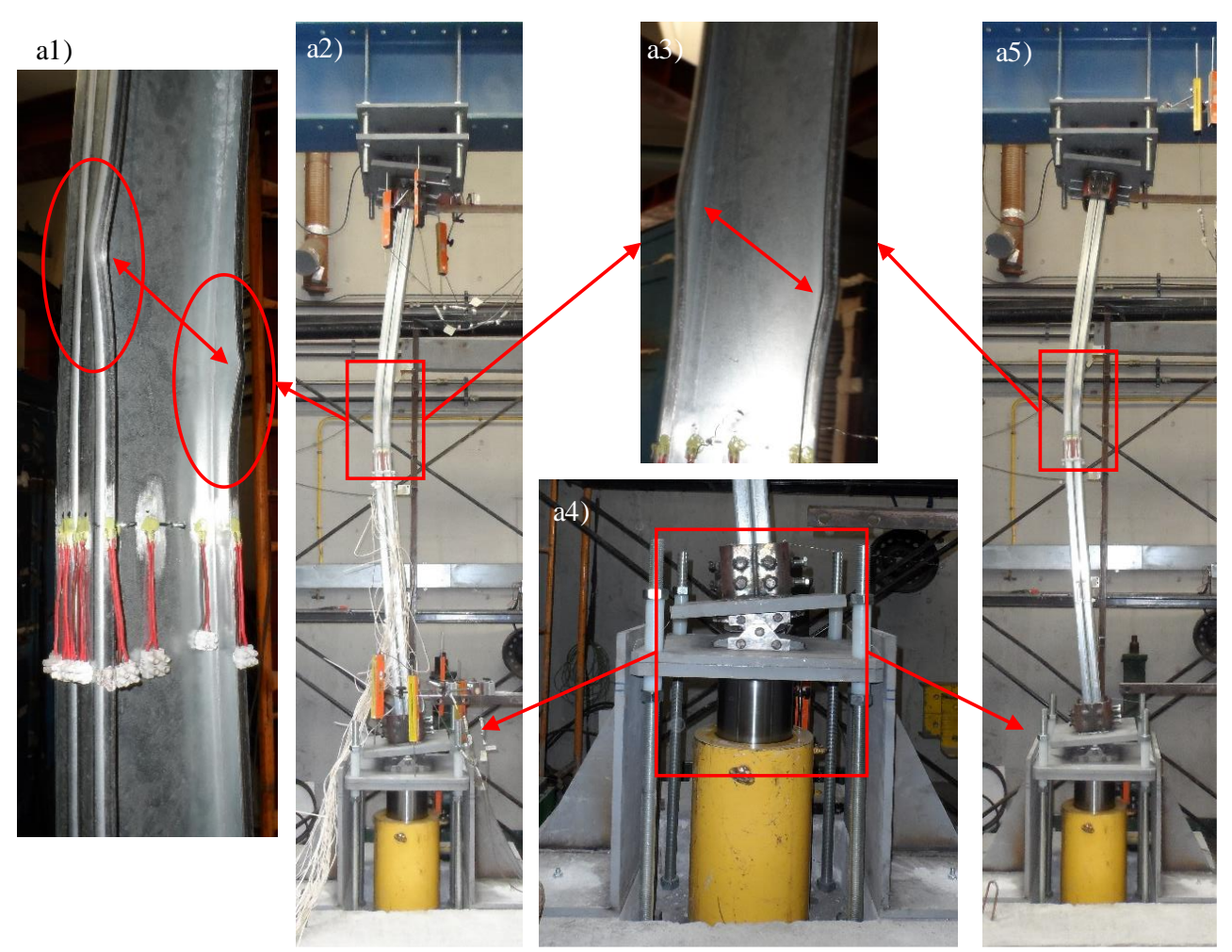

Figure 4.49 Failure modes of the test column I_PP_3 at ambient temperature. a1) and a3)

Distortional buckling. a2) and a5) Flexural buckling. a4) Rotation of the end-support.

For columns with closed built-up cross-section and with pinned (Figure 4.51) and fixed-end supports (Figure 4.52) the predominant failure mode was the interaction between flexural buckling about the minor axis and distortional and local buckling at mid-height of the column and in the case of fixed columns also near the end-support devices. Despite the use of fasteners in the flanges of the lipped and plain channels it is clear that the flanges of the plain channels moved outwards. Local buckling is way more visible for the fixed-end support. For the pinnedend support two waves were clearly identified, whereas for the fixed-end supports four waves were identified at mid-height of the column. For the columns with fixed-end support local crushing as well as distortional and local buckling was observed in the end support devices (Figure 4.52 a5). 


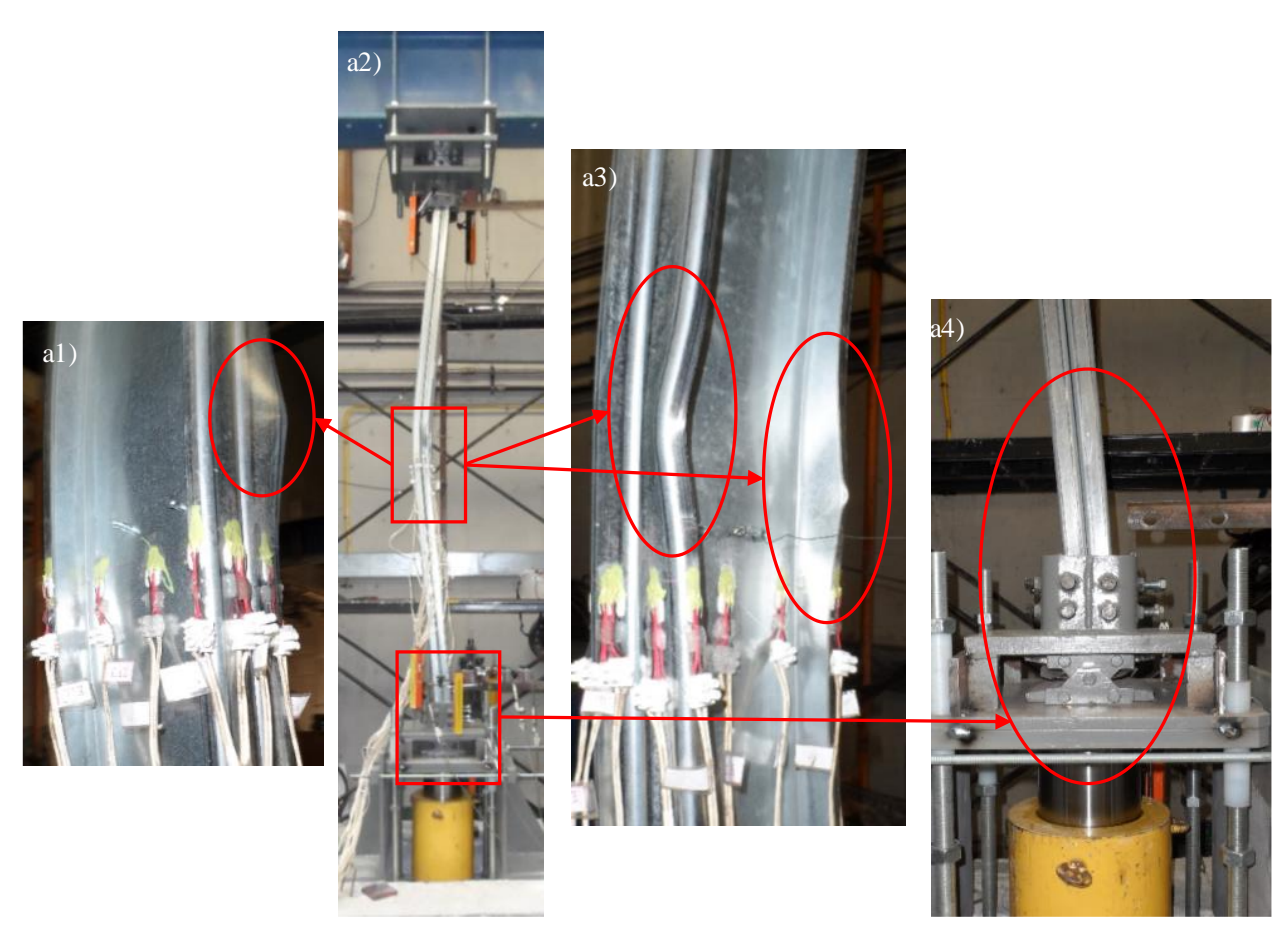

Figure 4.50 Failure modes of the test column I_FF_2 at ambient temperature. a1) and a3) Distortional buckling. a2) Flexural buckling. a4) End-support device.
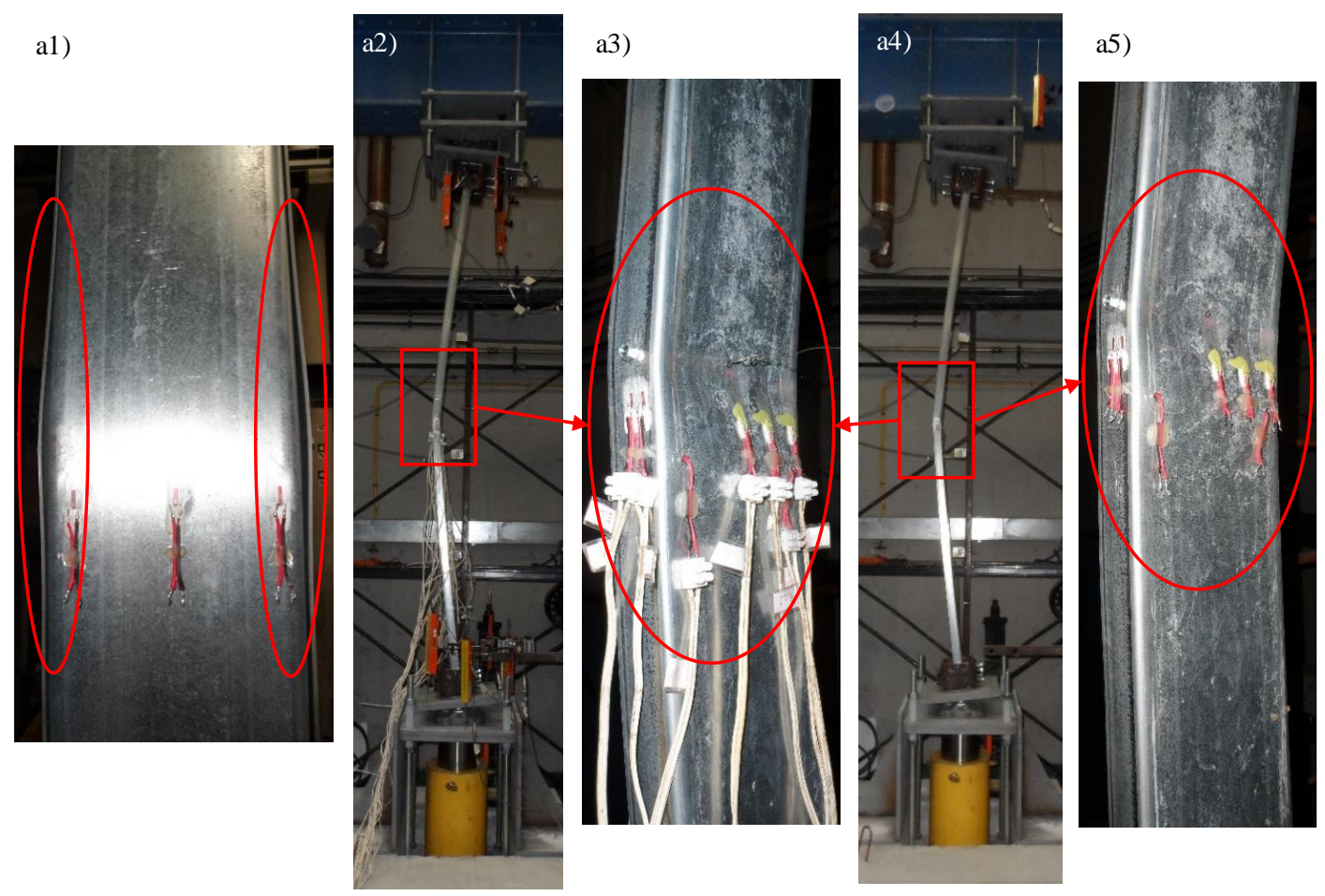

Figure 4.51 Failure modes of the test column R_PP_3 at ambient temperature. a1) Distortional buckling. a2) and a4) Flexural buckling. a3) and a5) Local buckling. 


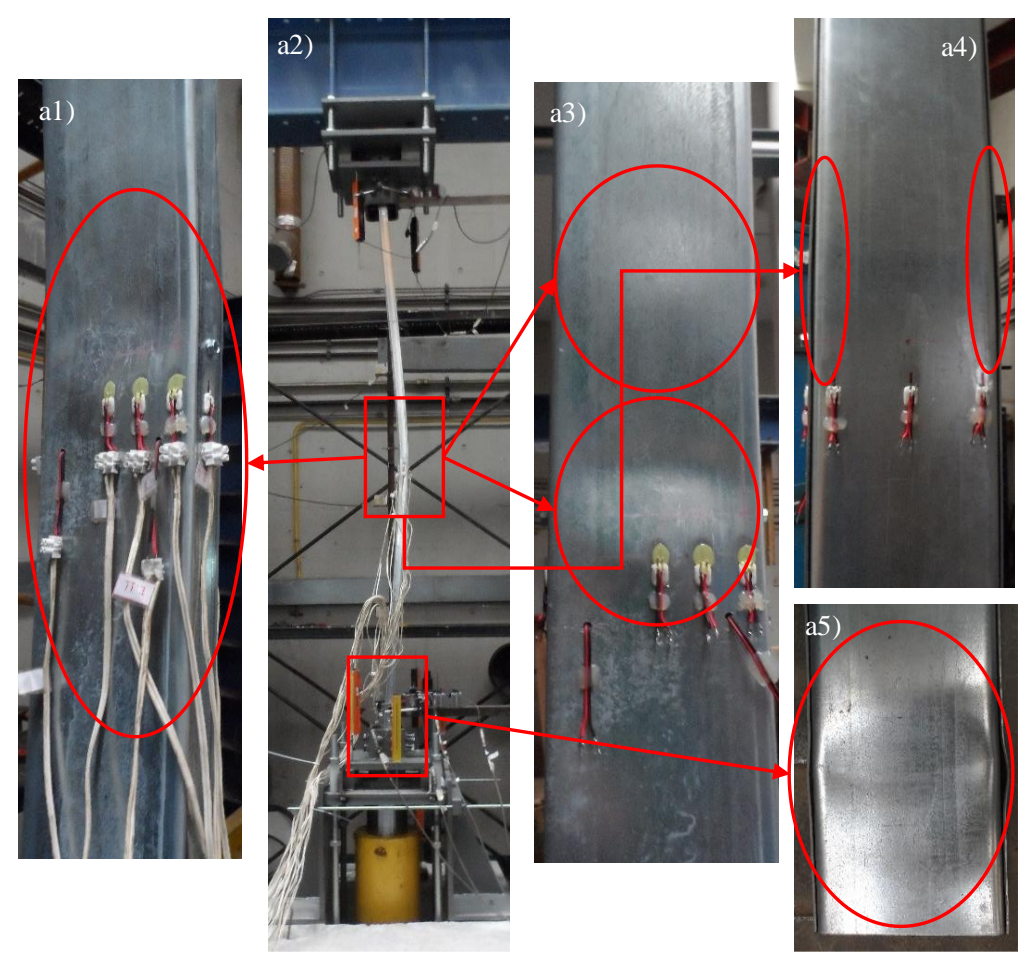

Figure 4.52 Failure modes of the test column R_FF_2 at ambient temperature. a1), a3) and a5) Local buckling. a2) Flexural buckling. a4) Distortional buckling.

For columns with closed built-up $2 \mathrm{R}$ cross-section and for both end-supports tested the predominant failure mode was the interaction between flexural buckling about the minor axis, distortional and local buckling at about mid height of the column. Distortional buckling for both end-support conditions was characterized by flanges of plain channels (U profiles) moving outwards, despite the use of fasteners in the flanges to connect lipped and plain channels together. At mid-height the distortional buckling was observed near the position of the fasteners (Figure 4.53 and 4.54). For both end-supports one plain channel presented distortional buckling, whereas the opposite plain channel presented local buckling at about mid-height of the column. For the fixed-end support condition local crushing was observed in the end-support device and near the end-support device distortional buckling in one of the plain channels (U) of the builtup cross-section was observed.

The observed failure modes in the buckling tests at ambient temperature will be also compared with the ones observed in the fire tests with restraint to thermal elongation. 

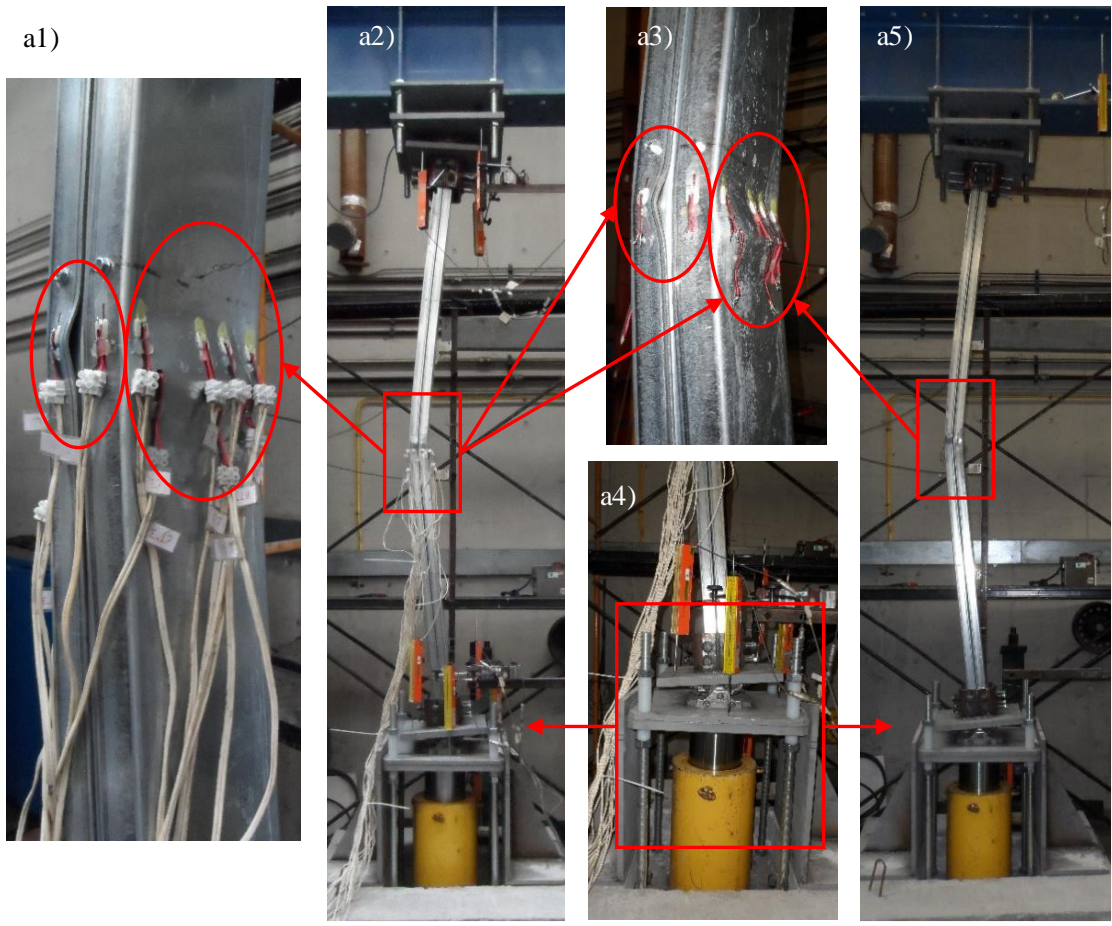

Figure 4.53 Failure modes of the test column 2R_PP_2 at ambient temperature. a1) and a3) Local and distortional buckling. a2) and a5) Flexural buckling. a4) Rotation of the support.

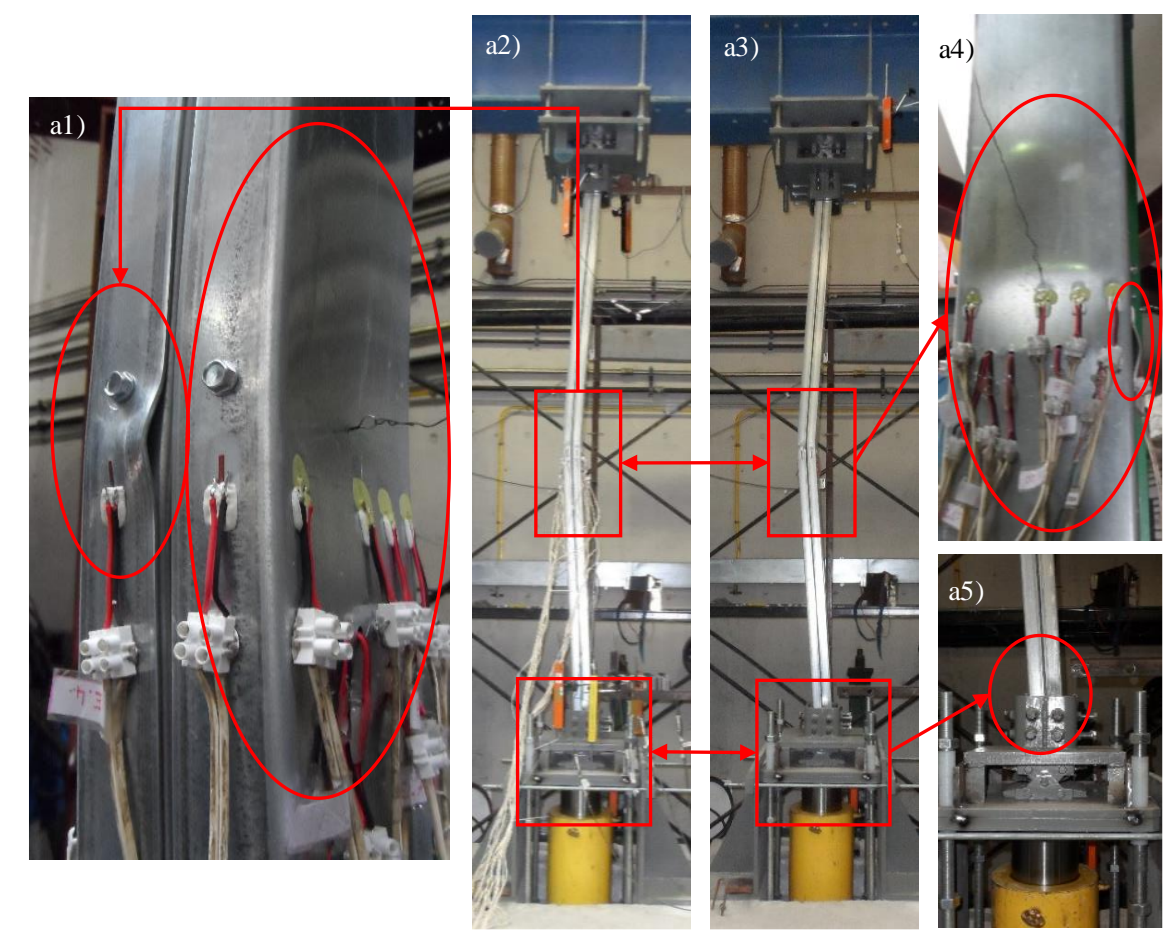

Figure 4.54 Failure modes of the test column 2R_FF_2 at ambient temperature. a1), a4) and a5) Local and distortional buckling. a2) and a3) Flexural buckling. 


\subsubsection{Fire tests with restraint to thermal elongation}

Since all tests were conducted inside the vertical modular electrical furnace only the final deformed shape could be observed. In Figure 4.55 to 4.62 the final deformed shapes for all cross-sections and end-support conditions are presented and described. It was observed that in the majority of the tests identical columns presented identical deformed shapes.

For pin-ended support conditions and for all tested cross-sections the predominant failure mode was global flexural buckling about the minor axis in interaction with distortional buckling (Figures 4.55, 4.57, 4.59 and 4.61). For the lipped channel columns two global buckling modes were observed, one moving in the direction of the lips of the cross section and another moving in the direction of the web of the lipped channel (Figure $4.55 \mathrm{a} 1$ ), a2), a3) and a4)). In the lipped channel cross-section when the column moved in the direction of the web the distortional buckling was more visible (Figure 4.55 b1) and b2)). Despite the different failure modes observed, the peak loads of identical columns were about the same. It seems that the existence of imperfections may influence the failure modes.

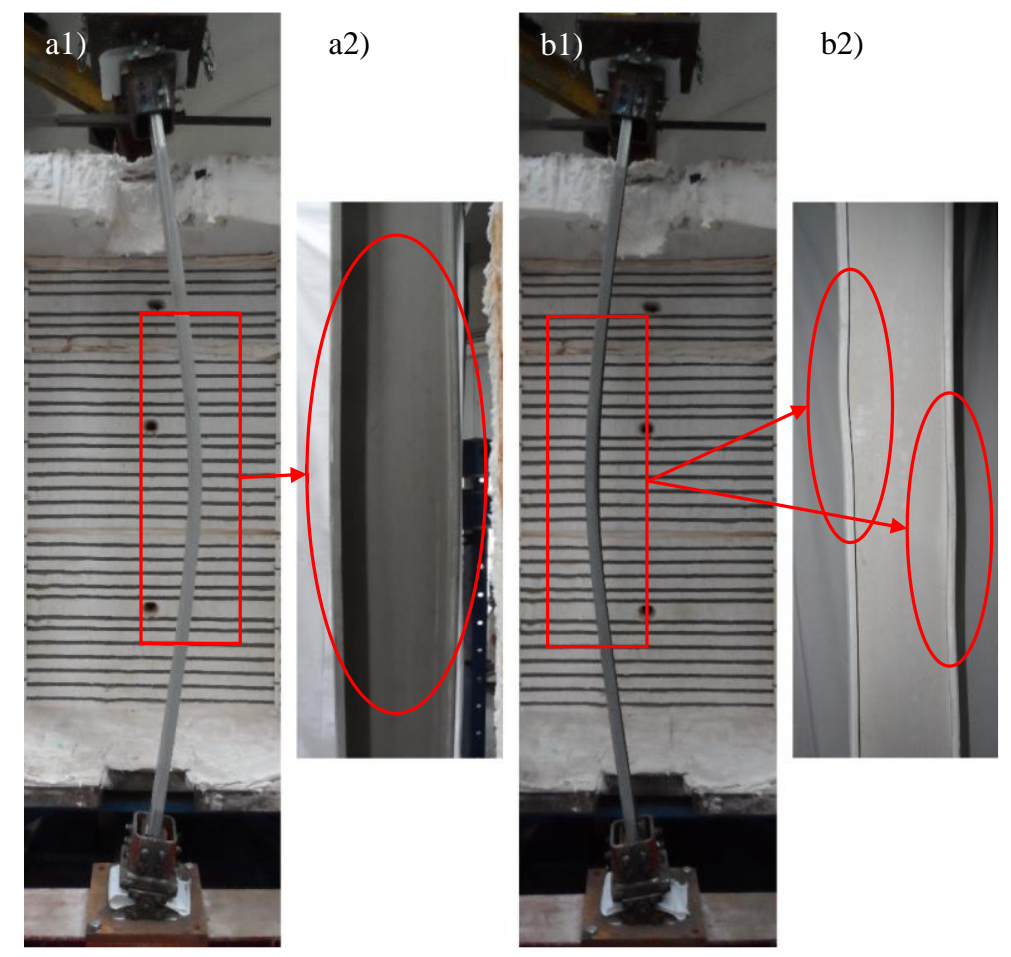

Figure 4.55 Failure modes for the specimen C_PP_50LL_K1-2 and C_PP_50LL_K1-3.a1) and b1) Flexural buckling. a2) and b2) Minor distortional buckling. 
Concerning the semi-rigid end-support conditions and the lipped channel columns it was observed that the predominant buckling mode was the interaction between global flexural buckling about the minor axis and distortional buckling (Figure 4.56 a1), a2) a3) and a4)). Distortional buckling was observed at about $400 \mathrm{~mm}$ from the end-support devices (Figure 4.56 a4) and at mid-height of the column (Figure 4.56 a1) and a3)). Local buckling was almost not observed in these experimental tests. Inflection points in the final deformed shape are clearly recognizable.

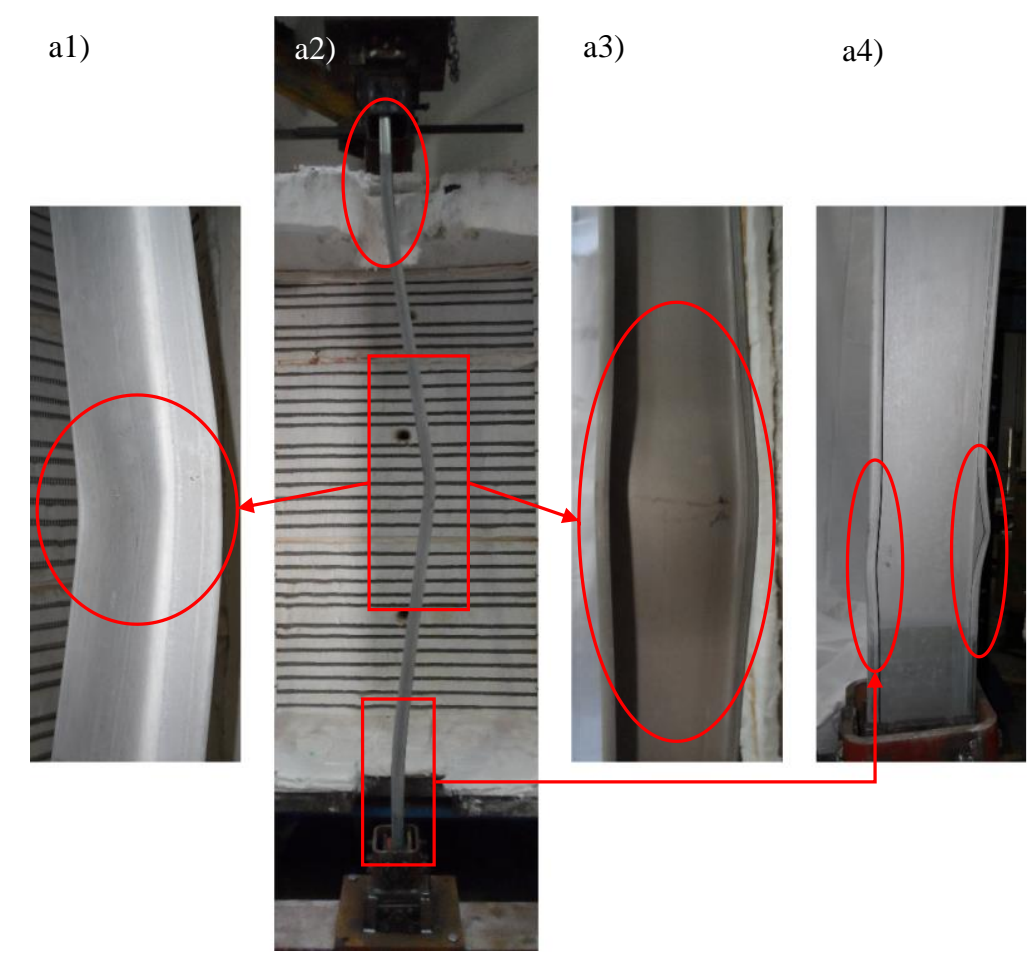

Figure 4.56 Failure modes for the specimen C_SR_50LL_K3-1. a1), a3) and a4) Distortional buckling. a2) Flexural buckling.

For columns with open built-up I cross-sections the predominant failure mode was the interaction between global flexural about the minor axis and distortional buckling at mid-height of the column in one of the lipped channel profiles (Figure 4.57 and 4.58). It should be pointed out that for the semi-rigid ended support condition the distortional buckling was by far more severe (Figure 4.58) than for the pin-ended conditions, playing a more important role in the failure of the column. In Figure 4.58 the inflection points in the final deformed shape are clearly recognizable. 


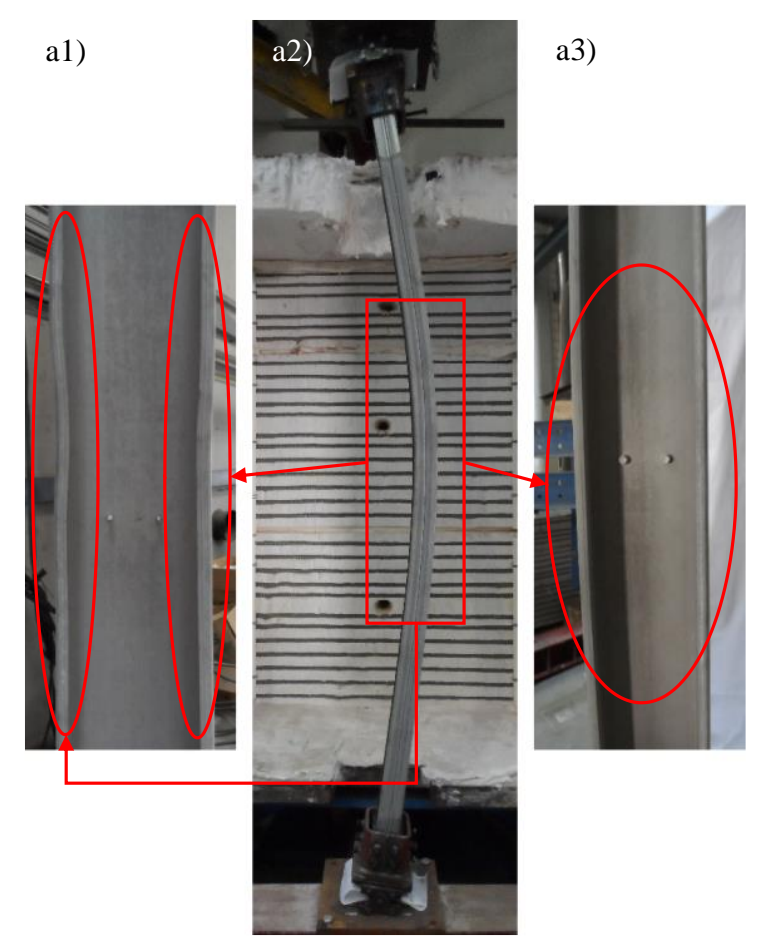

Figure 4.57 Failure modes for the specimen I_PP_50LL_K1-1. a1) and a3) Minor distortional buckling. a2) Flexural buckling

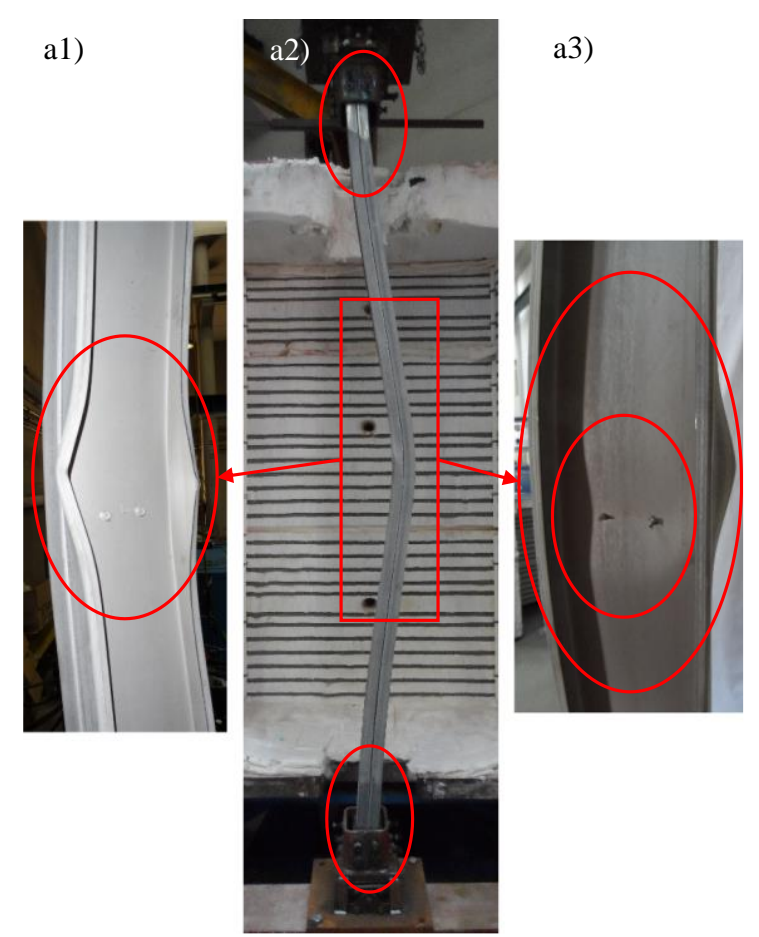

Figure 4.58 Failure modes for the specimen I_SR_50LL_K3-2.a1) and a3) Distortional buckling. a2) Flexural buckling. 
For columns with closed built-up R cross-section (Figure 4.59 and 4.60) the predominant failure mode was the interaction between flexural buckling about the minor axis, distortional and local buckling at about mid-height of the column, for both end-support conditions. For the semi-rigid end-support condition distortional and local buckling was also observed at $50 \mathrm{~cm}$ of the endsupport devices. Points of inflection in the final deformed shape for the semi-rigid end-support condition are clearly recognizable.

For columns with closed built-up 2R cross-section (Figure 4.61 and 4.62) the predominant failure mode was the interaction between flexural buckling about the minor axis, distortional and local buckling at about mid-height of the column. For the $2 \mathrm{R}$ cross-section local buckling is clearly recognizable in Figure $4.61 \mathrm{a} 1)$. It is worth mentioning that distortional and local buckling play a more relevant role in failure of columns with $2 \mathrm{R}$ cross-section. Once again, for the semi-rigid end-support condition and for the $2 \mathrm{R}$ cross-section distortional buckling was observed near the end support devices. Moreover, points of inflection in the final deformed shape are clearly recognizable (Figure 4.62).

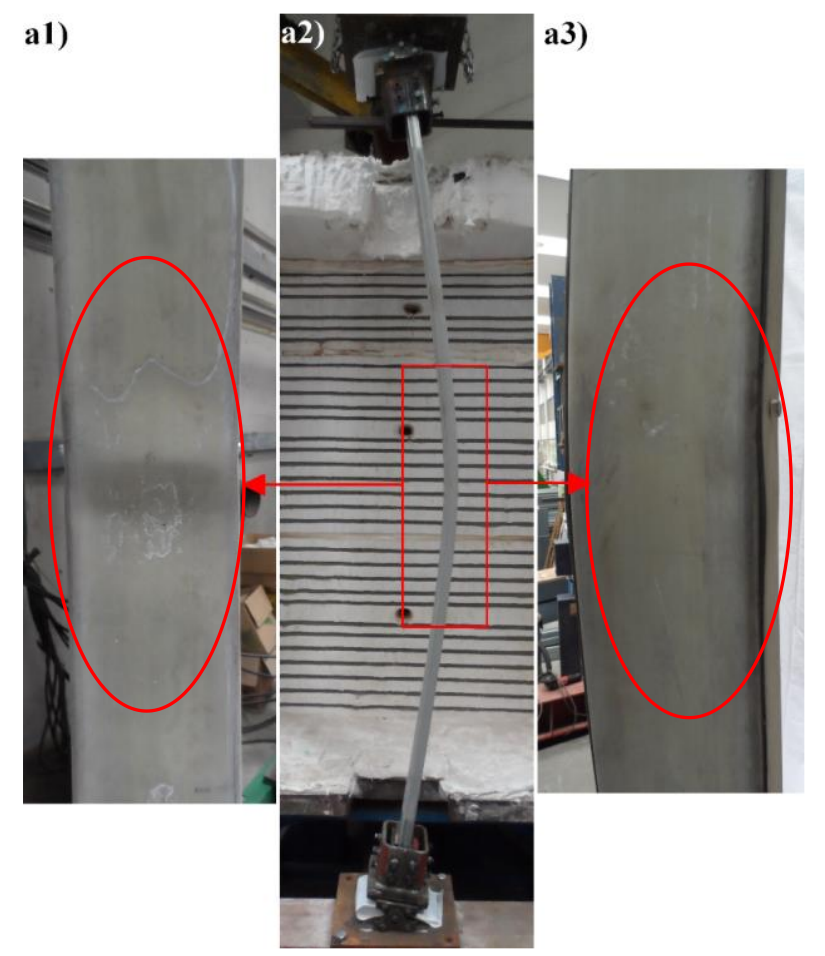

Figure 4.59 Failure modes for the specimen R_PP_30LL_K1-1. a1) Local buckling. a2)

Flexural buckling. a3) Distortional buckling. 


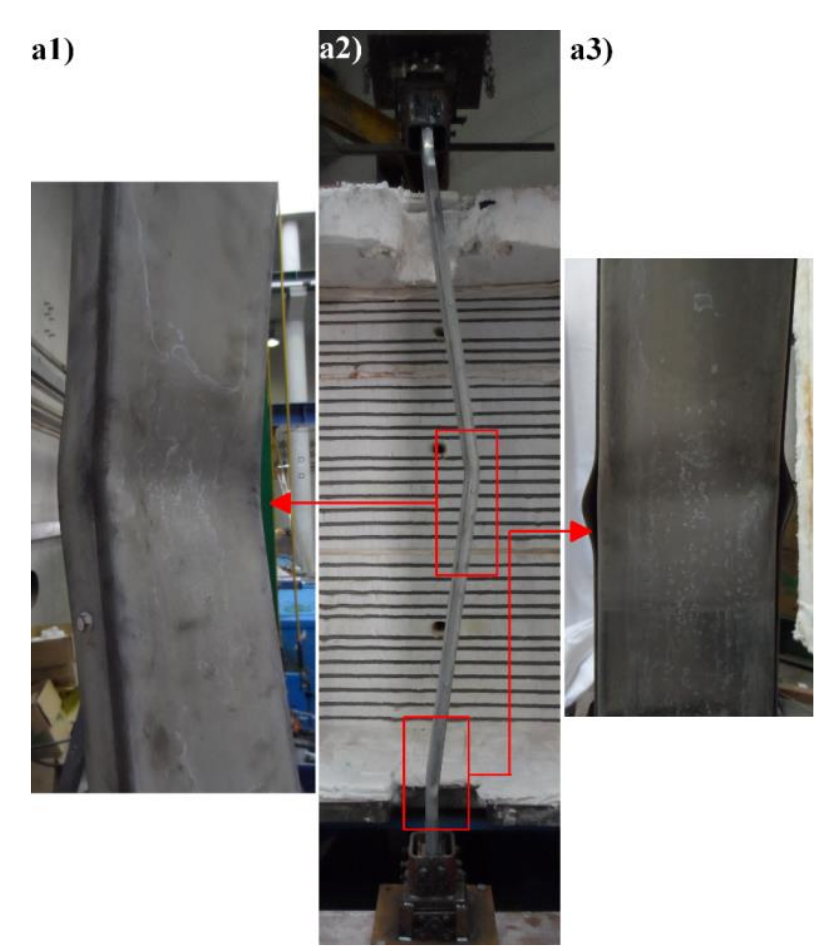

Figure 4.60 Failure modes for the specimen R_SR_30LL_K3-2. a1) and a3) Local and distortional buckling. a2) Flexural buckling.

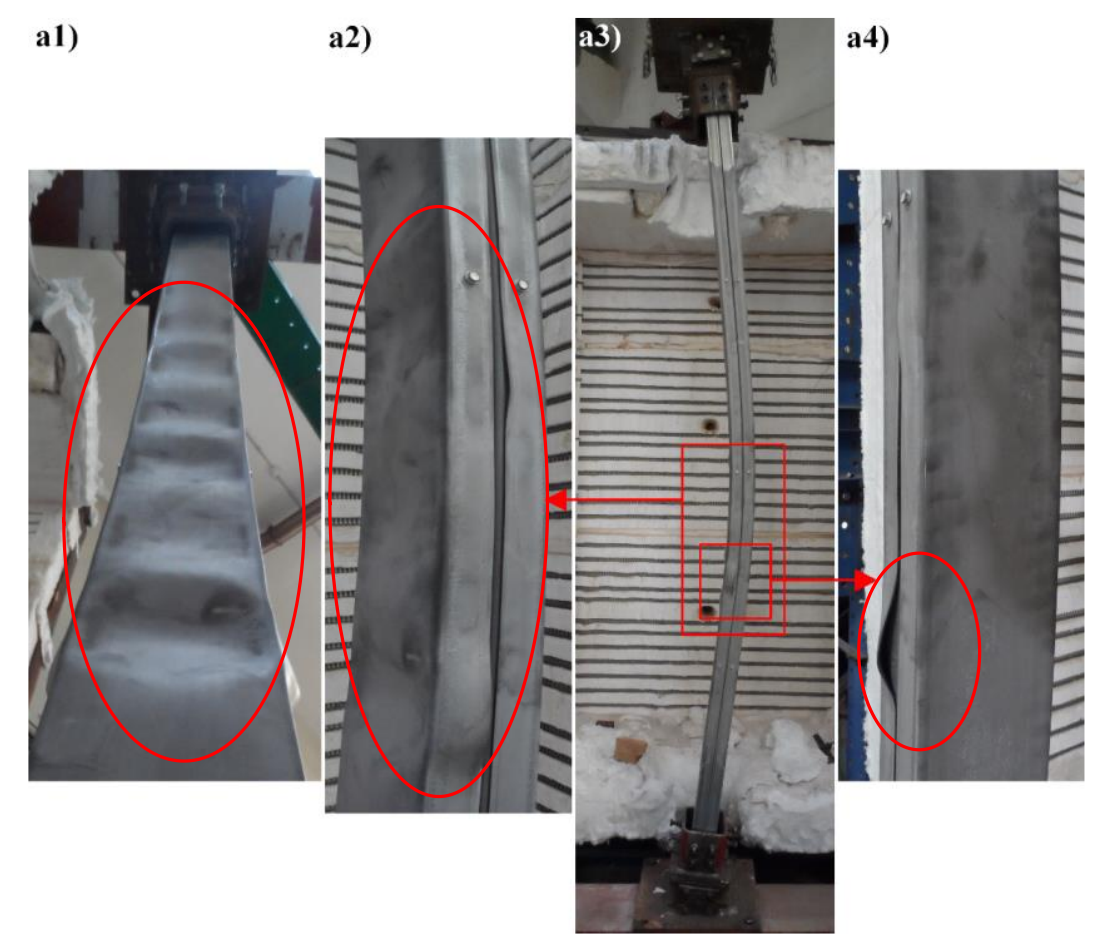

Figure 4.61 Failure modes for the specimen 2R_PP_30LL_K1-3.a1), a2) and a3) Local and distortional buckling. a3) Flexural buckling 


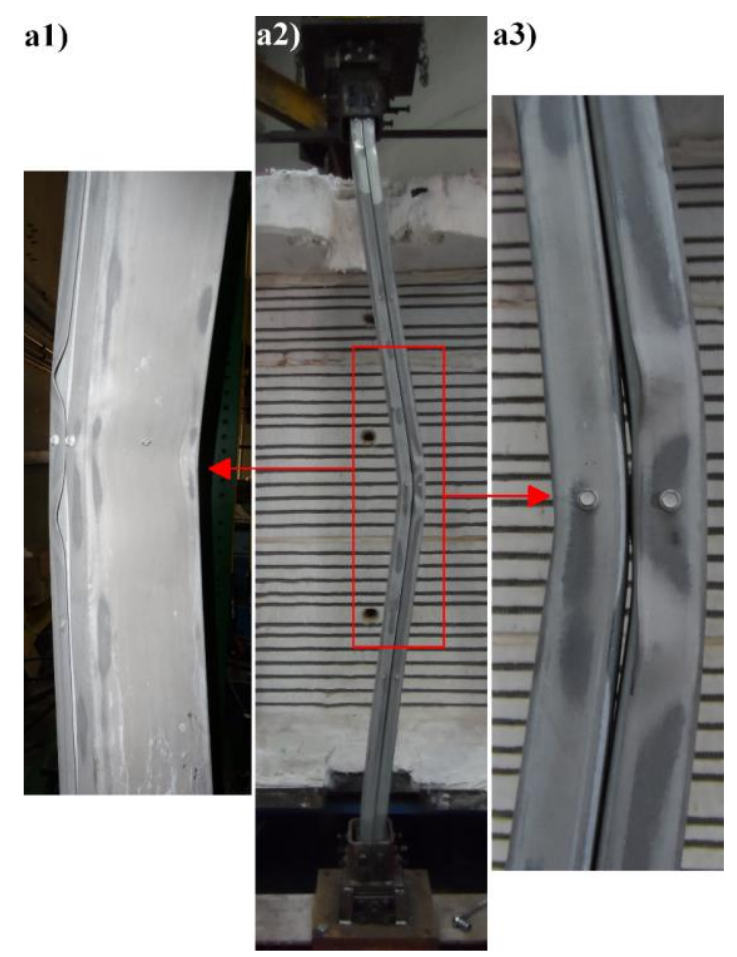

Figure 4.62 Failure modes for the specimen 2R_SR_30LL_K3-1. a1) and a3) Local and distortional buckling. a2) Flexural buckling.

\subsection{Final Remarks}

In this chapter a large experimental research on compressed single and built-up cold-formed steel columns at both ambient and fire conditions with restraint to thermal elongation was reported. Twenty-four buckling tests at ambient temperature were undertaken as reference tests. In those tests four cross-section shapes were studied, as well as two end-support conditions, namely pinned-end and fixed-end support. The buckling load of each type of column was assessed for both end-support conditions in order to establish lower and upper limits. During each test buckling load, axial and lateral displacements were monitored and recorded. Strains were also monitored at mid-height of the columns and in several points of the cross-section. Failure modes were observed and thoroughly characterized for each cross-section tested. These tests may be considered as reference tests for the larger campaign of tests under fire conditions, however due to lack of experimental results available in the literature regarding columns with built-up cross-sections the results can also be used as reference for the development and validation of finite element models. As a consequence, this results are very important and may be the basis for future numerical parametric investigations with the ultimate goal of improving/presenting available/new design guidelines for CFS columns with built-up cross- 
sections. In the scope of this thesis a finite element model was developed and validated using the available experimental results (Chapter 5).

As mentioned, the buckling load of each CFS column was assessed for both end-support conditions. It is worth mentioning that columns described as pinned did not represent exactly columns with perfect pinned ends due to friction between the steel pin and the steel plated of the end-support devices. Nevertheless, it can be stated that the use of copper grease simultaneously with Teflon in the steel pin may have reduced friction even more. Bear in mind that in the fire tests only Teflon was used to reduce friction in the end-support devices. Similar comment may be presented regarding fixed columns. It may be difficult to guarantee that rotations were totally prevented in the experimental tests. Hence, the actual boundary conditions may be described as semi-rigid with very low (pinned) and very high (fixed) values of rotational stiffness. These observations are coherent with the observed final deformed shapes. For instance, for the fixed-end support condition observing the final deformed shape of some columns it can be stated that the actual buckling length may be slightly higher than $0.5 \mathrm{~L}$.

The predominant failure mode was the flexural buckling about the minor axis of the CFS column with interaction with distortional buckling. Then, depending on the type of crosssection tested and the type of end-support adopted other buckling phenomena were observed. For instance, in some lipped channel columns with fixed-ends the interaction between flexuraltorsional buckling and distortional buckling was observed. For closed built-up cross-sections local buckling was clearly visible, whereas for single and open built-up cross-sections local buckling was less prevalent or inexistent for the pinned-end support condition. For the fixed end-support condition distortional buckling may have played a more relevant role in failure of the CFS columns.

Ninety-six fire tests on CFS columns with restraint to thermal elongation were reported in this chapter. Just like in the buckling tests at ambient temperature, four cross-section shapes and two end support conditions were tested. Additionally, the influence of restraint to thermal elongation in the overall behaviour of CFS columns was assessed. To assess the influence of restraint to thermal elongation two restraining frames were used to impose different levels of restraint to thermal elongation to the CFS columns in simulated fire conditions.

Analysing all the obtained results some general considerations may be presented. For isolated columns under fire conditions subjected to a low level of restraint to thermal elongation their failure is clearly controlled by temperature increase and by consequent degradation of mechanical properties of the S280GD+Z steel. The additional restraining forces are generated 
gradually during the heating phase. However, if an isolated column under fire conditions is subjected to high or very high levels of restraint to thermal elongation its failure may be controlled by the severity of the generated restraining forces during the heating phase. Higher levels of restraint will lead to higher rates of the generated restraining forces and as a consequence buckling load of the columns under these conditions may be reached for lower temperatures.

Also the influence of the initial applied load was relevant and obvious. Higher values of initial applied service load may lead to lower critical temperature and times. To fully understand the behaviour of isolated CFS columns under fire conditions with restraint to thermal elongation the ratio between the axial stiffness of the surrounding structure to the CFS columns and the axial stiffness of the CFS column $\left(\alpha_{K, 20^{\circ} C}\right)$ is a key parameter to be considered. It is worth mentioning that for all tested cross-sections and tested conditions a low fire resistance was observed.

Regarding the observed failure modes for the tested columns and tested end-support conditions a brief comparison should be established between the buckling tests at ambient temperature and fire tests with restraint to thermal elongation. For pinned-end columns the failure modes and final deformed shapes are very similar for both types of tests performed in this research. However, for fixed-ended (semi-rigid) columns some differences were observed specifically for the final deformed shape. Comparing the deformed shapes obtained in both types of tests it was clear that points of inflection were clearly recognizable in the deformed shapes of fire tests whereas for buckling tests at ambient temperature this observation was not so clear. For fire tests the buckling length was about $0.5 \mathrm{~L}$ whereas for buckling tests the buckling length was slightly higher. This may occur due to the reduction of rigidity of the CFS columns under simulated fire conditions, originated by the degradation of the modulus of elasticity with increasing temperature.

All the available data, obtained in the scope of this research study, was used to provide input data as well as to provide results to validate the developed finite element model. Based on the developed and validated model future extensive parametric studies on CFS columns with single and built-up cross-sections at both ambient and simulated fire conditions with axial and rotational restraint to thermal elongation should be conducted. 


\section{NUMERICAL MODELLING OF COLD-FORMED STEEL COLUMNS}

\subsection{Introduction}

Abaqus (Abaqus Analysis - User's Manual, 2012) is a commercial finite element analysis (FEA) software with a graphical user interface called $C A E$. It is one of the most used applications for solving routine and sophisticated engineering problems in a wide range of industries. It is also extensively used by the academic community throughout the world due to the wide range of capabilities, namely numerous options for material models, analysis and solution techniques and also multiphysics capabilities. Hence, it can simulate engineering problems from different fields such as heat transfer, mass diffusion, thermal management of electrical components (coupled thermal-electrical analyses), acoustics, soil-mechanics structural analysis and piezoelectric analysis. The Abaqus product suite comprises three main software products more commonly used, namely Abaqus/CAE, Abaqus/Standard and Abaqus/Explicit. Abaqus/CAE (Complete Abaqus Environment) is the complete solution for finite element modelling, hence it is possible to create, edit, monitor, diagnose and visualize advanced Abaqus analyses. Abaqus is a powerful finite element analysis software capable of modelling structures, parts of structures or isolated structural elements with material and geometric nonlinear behaviour.

Finite element analysis has become widely popular among researchers. Problem solving based on computer simulations is very efficient and less time and resources consuming than the more traditional experimental tests. Computer simulations are especially useful for conducting large parametric studies based on developed finite element models previously validated using some more traditional experimental tests. Computer simulations do not replace experimental tests but may reduce the number of experiments needed to conduct in any kind of research. In this particular research the large experimental campaign conducted was used to provide data to the developed finite element model and also to gather relevant experimental results used to validate the same developed model. The developed models should be used in the future for extensive parametric studies with the ultimate goal of developing/improving new/available design guidelines for both single and built-up columns and for both ambient and fire conditions with restraint to thermal elongation. 


\subsection{Structural Analysis}

\subsubsection{Selection of finite element type}

Shell elements are commonly used to model thin-walled structural elements. Several types of shell elements are available in the finite element simulation with Abaqus, namely S4, S4R, S4R5, S8R5 and S9R5. The first letter in the element name indicates the type of element $(S-$ shell element) and the first number indicates the number of nodes in the element, whereas $R$ stands for reduced integration with hourglass control and the last number indicates the degrees of freedom at each node. Hence, the S4R element (Figure 5.1) is a four node general-purpose shell, quadrilateral and stress/displacement shell element with reduced integration, a large strain formulation, hourglass control and a first order interpolation. The S4R finite element type was used to model the cold-formed steel profiles.
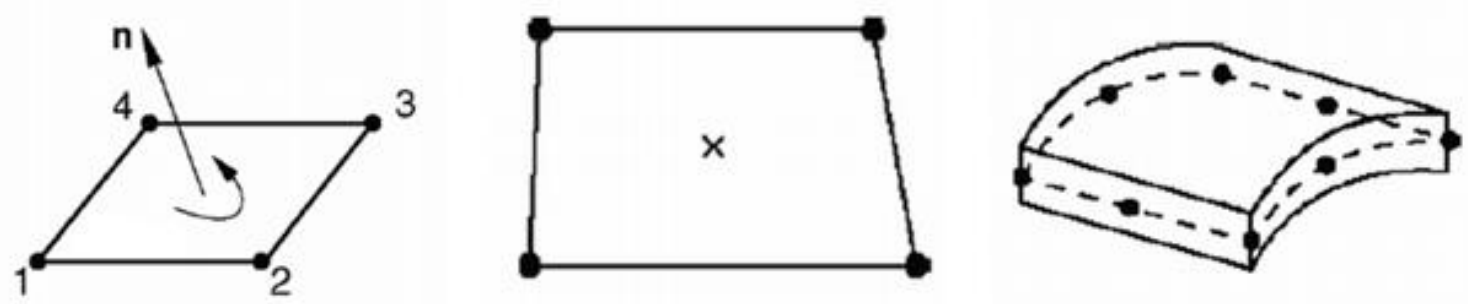

Figure 5.1 Schematic representation of the S4R element (Abaqus Analysis - User's manual, 2012.)

Regarding the self-drilling screws, the finite element chosen was the C3D8R. The C3D8R element (Figure 5.2) is defined as a three-dimensional, continuum hexahedral and an eight-node brick element with reduced integration, hourglass control and first-order interpolation.

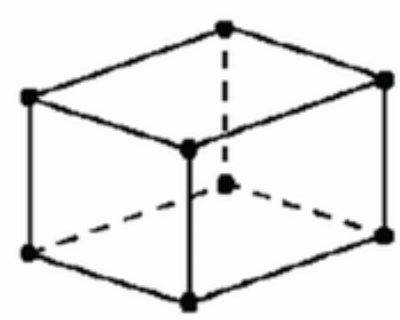

Figure 5.2 Schematic representation of the C3D8R element (Abaqus Analysis - User's Manual, 2012). 


\subsubsection{Material modelling}

Accurate numerical simulations depend on the accuracy of the input data. Hence all mechanical and thermal properties of the structural steel S280GD $+Z$ were determined experimentally as previously described in Chapter 3. The obtained results were used as input in the developed finite element models. It was the objective of this research to develop finite element analyses of cold-formed steel columns with different types of cross-sections at both ambient and fire conditions with restrain to thermal elongation. Using experimental tests, yield strength, elastic modulus and the stress-strain curves were determined at both ambient and elevated temperatures up to $800^{\circ} \mathrm{C}$. Based on the obtained stress-strain curves, stress-strain equations were developed based on Ramberg and Osgood model and already presented in Chapter 3 (Figure 3.18 and Equations (3.4), (3.4a), (3.4b) and Table 3.4 where the $n$ parameter is defined). The elastic modulus determined in the experimental research at ambient temperature was 204.18 GPa and the yield strength at ambient temperature was $306.81 \mathrm{MPa}$. In this research a strain hardening model was used. In Figure 5.3 the stress-strain curves used in the finite element model is presented. For the Poisson's ratio it was assumed that it remains unchanged with temperature increase. The adopted value was 0.3 .

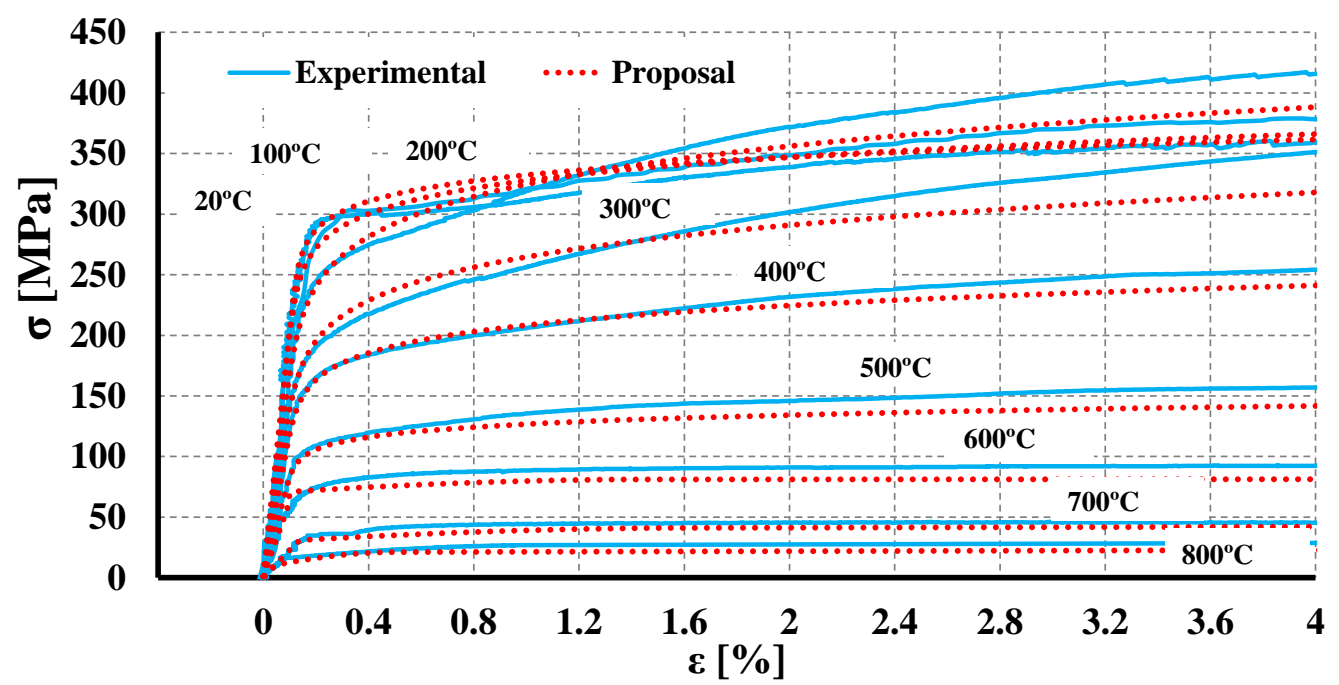

Figure 5.3 Experimental stress-strain curves and proposal based on the Ramberg-Osgood model.

Stress-strain curves as input data were converted to true stress and logarithmic plastic strain from the following equations:

$$
\sigma_{\text {true }}=\sigma_{\text {nom }}\left(1+\varepsilon_{\text {nom }}\right)
$$




$$
\varepsilon_{l n}^{p l}=\ln \left(1+\varepsilon_{\text {nom }}\right)-\frac{\sigma_{\text {true }}}{E}
$$

Where:

$$
\begin{array}{ll}
E & \text { modulus of elasticity; } \\
\sigma_{\text {nom }} & \text { nominal stress; } \\
\sigma_{\text {true }} & \text { true stress; } \\
\varepsilon_{\text {nom }} & \text { nominal strain; } \\
\varepsilon_{l n}^{p l} & \text { logarithmic plastic strain. }
\end{array}
$$

In Figure 5.4 the true stress vs true strain curves are plotted for the plastic range.

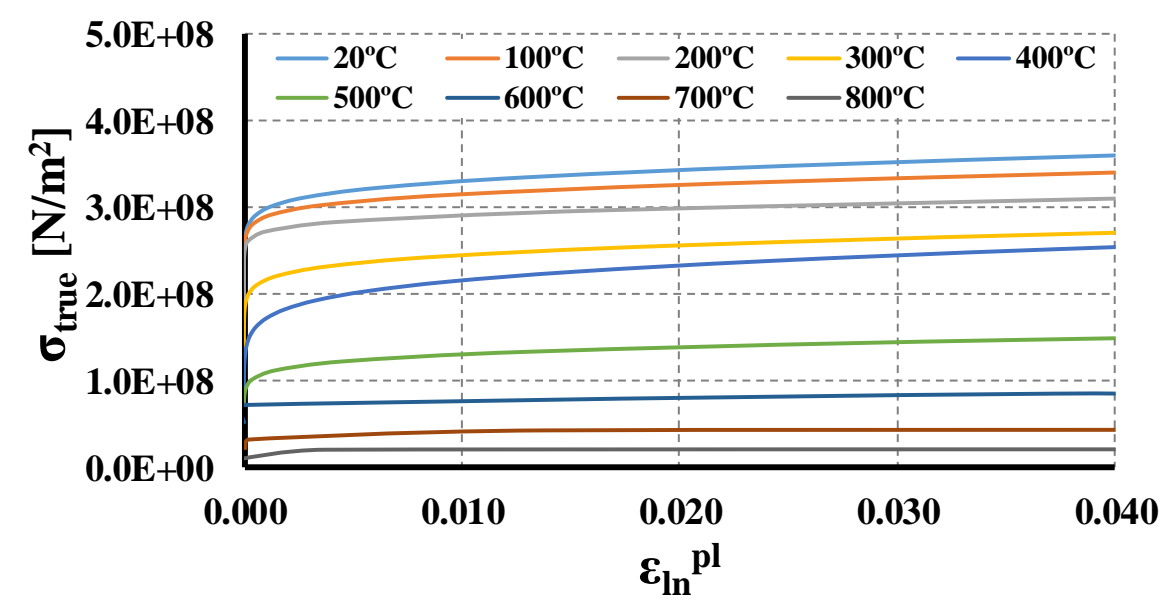

Figure 5.4 True stress and logarithmic plastic strain curves used as input in the finite element model.

The thermal properties determined in the experimental campaign were used as input in the numerical model, namely thermal elongation (Figure 3.24 and Equation 3.11), thermal conductivity (Figure 3.25 and Equation 3.12) and the specific heat used was the one presented in the EN 1993-1-2:2005 (2005) (Figure 3.26), since the experimental results and the presented model were very similar. In Appendix $G$ the accuracy of the material properties presented in the EN 1993-1-2:2005 is assessed. Hence, a comparison between FEA results using the determined material properties and the material properties presented in the EN 1993-1-2:2005 (2005), as input in the finite element model, is established.

Residual stresses were already discussed in Chapter 2 of this thesis, however at this point residual stresses were not included in the developed finite element model. Nevertheless, in the future it is intended to investigate the influence of residual stresses on the estimation of the ultimate failure load of columns. Ranawaka and Mahendran (2010) investigated the influence 
of residual stresses and performed several numerical simulations with and without residual stresses and concluded that the influence of residual stresses on the ultimate load was less than $1 \%$.

\subsubsection{Finite element mesh}

The accuracy of the finite element model is governed by the mesh size. Hence a sufficiently fine mesh shall be used. However, computational resources are limited and it is often necessary to assess the adequate mesh size in order to obtain accurate results with adequate computational times. The influence of the mesh size was investigated in order to decide the most suitable finite element mesh size. Different mesh sizes were tested, namely $5 \mathrm{~mm} \times 5 \mathrm{~mm}, 10 \mathrm{~mm} \times 10 \mathrm{~mm}$ and $20 \mathrm{~mm} \times 20 \mathrm{~mm}$. In Figure 5.6 the obtained results for some of the conducted simulations, considering different mesh sizes, are presented for the estimation of the buckling load of lipped channel columns at ambient temperature. Analysing the obtained results, the same mesh size was used for all tested cross-section, namely $5 \mathrm{~mm} \times 5 \mathrm{~mm}$. In Figure 5.5 the finite element mesh used for all tested cross-sections is depicted.

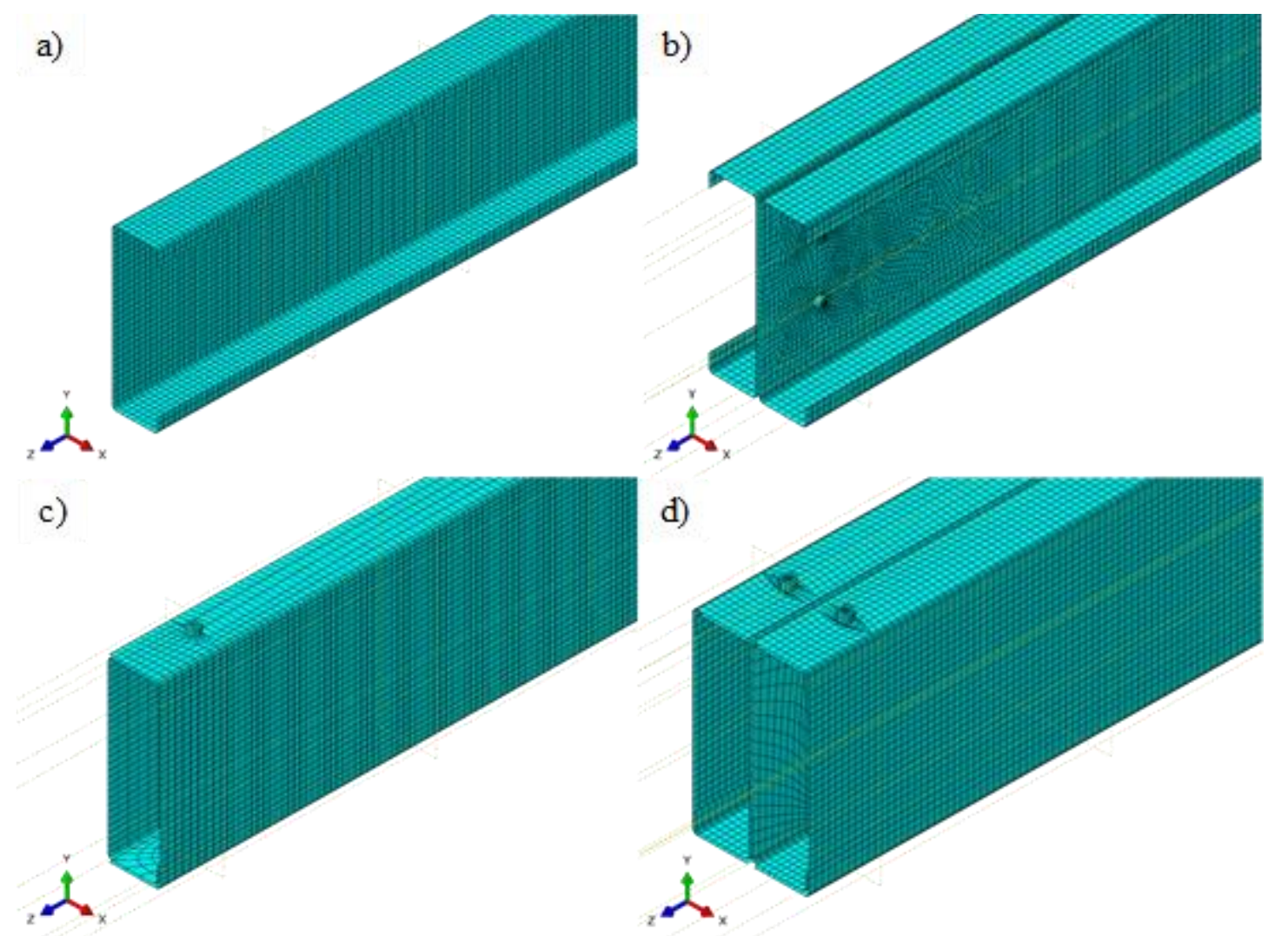

Figure 5.5 Detail of the finite element mesh used in all CFS columns. a) C. b) I. c) R. d) 2R. 


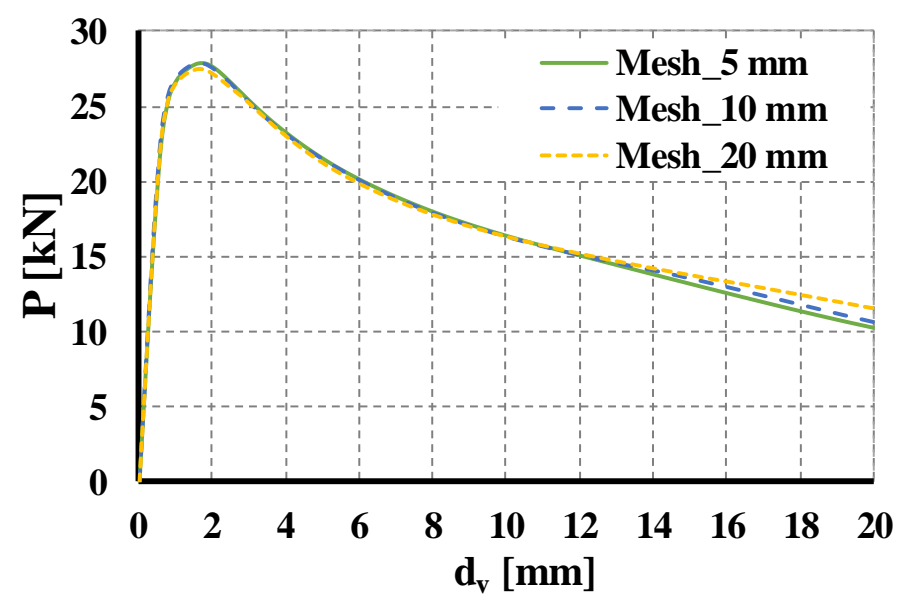

Figure 5.6 Predicted load for the lipped channel column at ambient temperature using different mesh sizes.

\subsubsection{Loading, boundary conditions and contact conditions}

In order to reproduce accurately the behaviour of CFS columns observed in the experimental tests appropriate boundary, loading and contact conditions, for single and built-up crosssections, must be defined in the finite element model. Hence, in the validation process of the finite element model was attempted to simulate the actual boundary conditions observed in the experimental tests. In the future, while conducting parametric studies ideal boundary conditions (perfect pinned ends and fully fixed ends) will be considered in order to define lower and upper bounds to the buckling load of CFS columns, as well as to critical times, temperatures and generated restraining forces. As previously mentioned in Chapter 4 for both ambient and fire tests, friction between the steel pin and steel plates of the end-support device for the pinnedend support condition may have influenced the overall behaviour of CFS columns. This friction may have prevented the columns from developing totally free rotation and consequently causing some increase in stiffness which may lead to overestimated values of buckling load or generated restraining forces. To address this issue, and using the capabilities of the software Abaqus, hinges, acting as rotational springs, were considered in the boundary conditions of the finite element model. These hinges were positioned in $\mathrm{Z}$ direction (in order to prevent totally free rotation about the minor axis of the columns) simulating a rotational stiffness (Figure 5.7). A linear behaviour was adopted for these hinges and the adopted values of rotational stiffness defined for each situation intended to simulate the friction observed in the experimental tests. Also, for the fixed-end columns in the buckling tests this boundary condition can be used since it was observed that a fully fixed condition was not achieved using the designed end support devices. In this case a high value of rotational stiffness must be used. In this study and for each one of the tested cross-sections the value of $4 E I / L[\mathrm{~N} . \mathrm{m} / \mathrm{rad}]$ was used to simulate the actual 
fixed-end condition. To simulate friction a small value of rotational restraint could be used whereas to simulate the experimental fixed-end support a very high value of rotational restraint was used for the defined hinges. To simulate friction different values of rotational stiffness were introduced in the finite element model, depending on the type of column to be simulated. The values ranged from $2 \%$ of $3 \mathrm{EI} / \mathrm{L}$ ( $\mathrm{C}$ columns) up to $20 \%$ of $3 \mathrm{EI} / \mathrm{L}$ ( $2 \mathrm{R}$ columns). Also, the experimental results may be affected by eccentricities in loading which may have led to lower ultimate buckling load. Hence, eccentricities may also be used in the numerical models in order to obtain good agreement with the experimental tests and in future parametric studies for sensitivity studies. In this particular study eccentricities were not used in the calibration process.

To reproduce the behaviour of CFS columns under simulated fire conditions with restraint to thermal elongation the surrounding structures used in the experimental tests were replaced by linear springs ( 3 and $13 \mathrm{kN} / \mathrm{mm}$ ) connected to the centroid of the column to be simulated (Figure 5.7).

To simulate ambient temperature tests displacement loading control was used in the numerical simulations. A node was created in the geometric centroid of each cross-section tested and then was connected to the perimeter of the section to create multi-purpose constraints. Using this methodology, the originated force (due to the defined axial displacement) can be equally distributed along the perimeter of the cross-section. Then an axial displacement was defined in the Y direction (Figure 5.7). For the fire tests load control was used to apply the serviceability load defined for each column. The axial compression load was defined as a concentrated nodal force at the top end of the column. That node was then connected to the perimeter of the crosssection and consequently the applied load was equally distributed along the perimeter of the cross-section.

Open and closed built-up cross-sections investigated in the scope of this research comprised two or more profiles connected by self-drilling screws in different points in the cross-section and along the length of the column. Therefore, contact between the CFS profiles and between profiles and self-drilling screws must be carefully modelled. In modelling it was assumed a tangential friction coefficient of 0.2 for the contact behaviour in tangential direction and a hard contact (full transmission of compressive forces and no transmission of tensile forces) for the contact behaviour in normal direction between the profile surfaces (Laím, 2013). The surfaceto-surface contact was used considering the finite-sliding tracking method to model the interaction between the surface of the individual profiles. For the contact between CFS profiles and self-drilling screws a rough and hard contact was also used. 
In Figure 5.7 a representation of the finite element model developed in this investigation is presented. The models showed in Figure 5.7 correspond to the ones used in the simulation of fire tests with restraint to thermal elongation.

a)

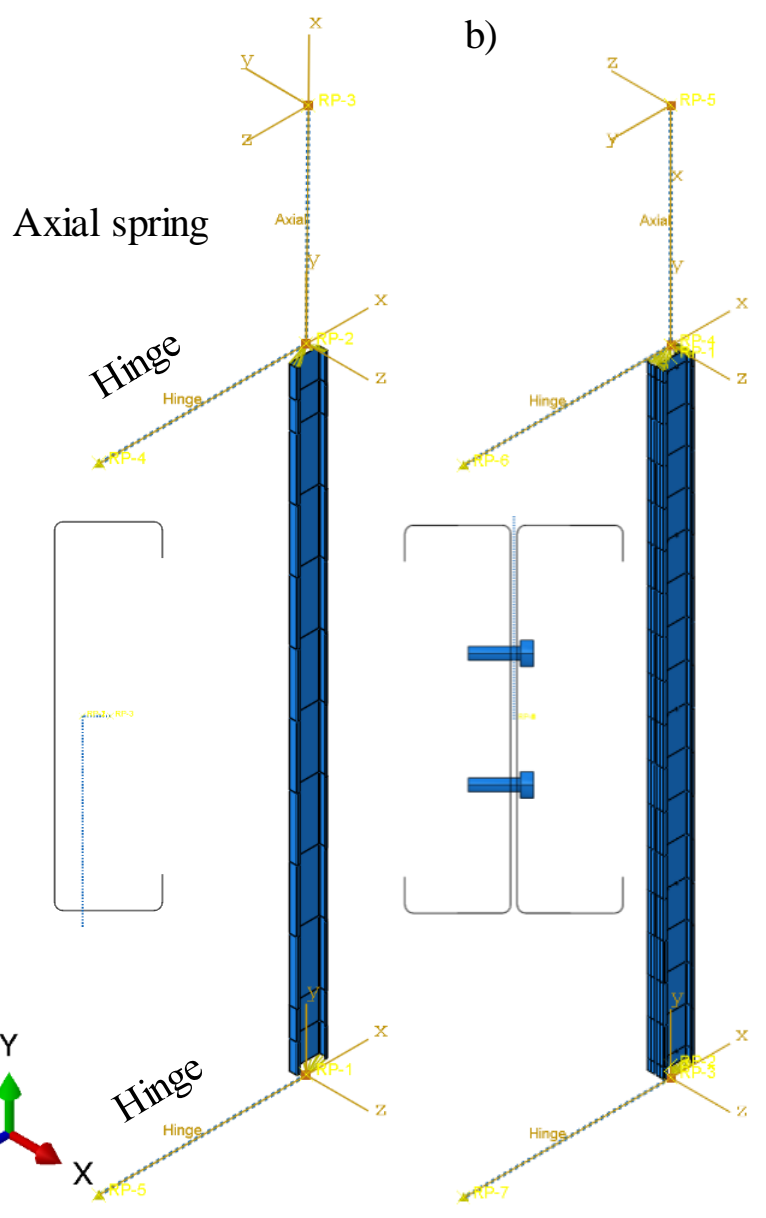

c)

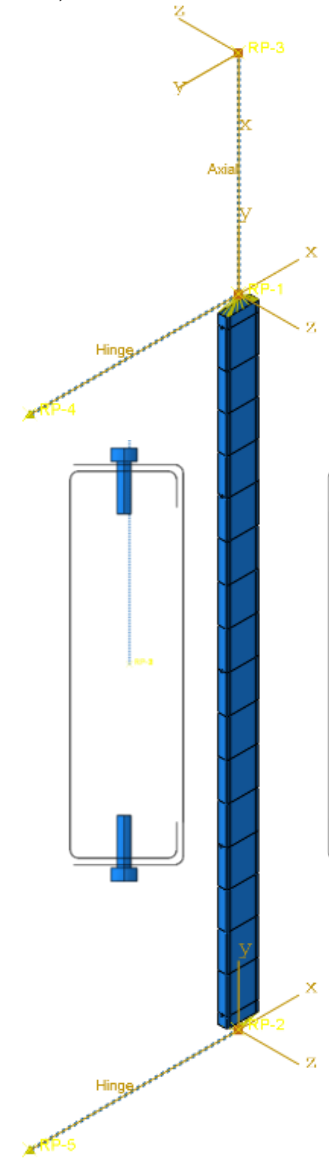

d)

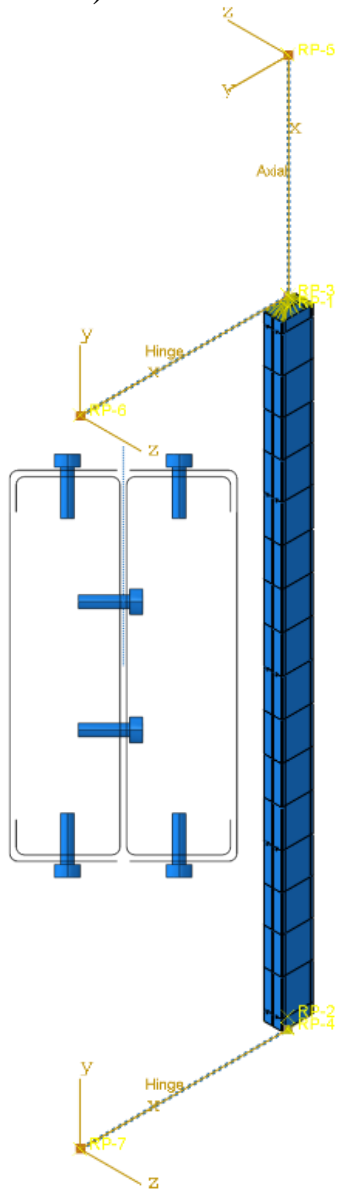

Figure 5.7 Finite element model developed for each type of columns tested. Model for CFS columns under simulated fire conditions with restraint to thermal elongation. a) Lipped channel column. b) Column with open built-up I cross-section. c) Column with closed builtup R cross-section. d) Column with closed built-up 2R cross-section.

The finite element used to simulate buckling tests were identically to the ones developed for fire tests. However, it is worth mentioning that for the buckling tests the axial spring was not used.

Concerning the fire tests the monitored temperatures in the cross-section and along the length of the column were used as input in the validation process of the finite element model. Influence areas were defined for each thermocouple and depending on the position of a specified 
thermocouple its temperature evolution as a function of time was allocated to the defined influence area. This will reproduce the non-uniform temperature distribution observed in the experimental tests. In the future, while performing parametric studies, uniform temperature distribution will be assumed along the length of the column and the ISO 834 (1999) standard fire curve will be used as thermal action. And to determine the temperature distribution in the cross-section due to ISO 834 fire curve a 2D heat transfer analysis shall be conducted based on a validated 2D finite element heat transfer analysis. Using ABAQUS capabilities and the available experimental results a heat transfer analysis was conducted. The developed model is thoroughly described in Chapter 5.5.

\subsubsection{Analysis method}

The finite element 3D models were created for all tested cross-sections and for all types of tests conducted, namely buckling tests at ambient temperature and fire tests with restraint to thermal elongation. Two different types of analysis were conducted by using the developed finite element model, namely elastic buckling analyses, to determine critical buckling loads and the associated buckling modes, and then a nonlinear static analysis. The buckling modes are then used to input the geometric imperfections in the nonlinear analysis. Despite the first buckling mode may be the critical mode, interaction between different buckling modes was observed in the experimental tests, therefore a combination of two or three buckling modes was considered. Also it is worth mentioning that in some situations the initial geometric imperfections were applied in the opposite direction, in order to accurately reproduce the behaviour observed in the experimental tests. For all tested columns the adopted maximum value for global imperfections was $L / 1000$, for distortional imperfections a value of $t$, and finally for local imperfections $h / 200$.

Finally, a structural analysis was undertaken in order to simulate the behaviour of CFS columns at ambient and fire conditions with restraint to thermal elongation, with different types of crosssections, different end-support conditions and different levels of restraint to thermal elongation. In all structural simulations the nonlinear geometric parameter $(* \mathrm{NLGEOM}=\mathrm{ON})$ was active in order to deal with the geometric nonlinear analysis. 


\subsection{Validation of Finite Element Model}

\subsubsection{Validation of the finite element model for buckling tests of CFS columns at ambient temperature}

The validation of the finite element model developed to reproduce the behaviour of cold-formed steel columns under compressive loading until failure at ambient temperature consisted of comparing the available experimental results with the ones obtained in the numerical simulations. For that purpose, axial compression load versus axial shortening curves were compared for both experimental and numerical simulations. For each tested cross-section and for each end-support condition tested the comparison is established. In Figures 5.8 and 5.9 the comparison between axial compression load versus axial shortening for all tested conditions is presented. In Tables 5.1 to 5.4 the results and difference between experimental and numerical simulations is also presented. As previously mentioned, in the development of the finite element models was assumed that friction in the end-support devices may significantly influence the behaviour of CFS columns. Hence, a hinge was modelled to reproduce the rotational restraint that may be caused by friction, preventing a totally free rotation of the columns. However, during the validation process, for pinned-ended columns, it was found that it was not needed to use rotational restraint in the modelled hinge in order to simulate friction. This may be due to the fact that before the experimental campaign on CFS columns at ambient temperature it was attempted to improve the end-support devices (use of copper grease and Teflon in the steel pin). It is worth mentioning that due to space and equipment limitations in the Laboratory of the Department of Civil Engineering of the University of Coimbra, the buckling tests were undertaken after the fire tests with restraint to thermal elongation, hence the pinned-ended support may present less friction than it was observed in the fire tests (steel pin lined with Teflon). Also, it can be mentioned the possibility that loading eccentricities are more significant in these experimental tests, and thus playing a more relevant role than the actual friction in the end-supports. It was observed that the assumptions considered in the finite element model led to very good agreement between experimental and numerical results. For all tested conditions a small translation in the axial shortening axis was assumed, for the obtained results of the finite element analysis, since the initial part of the experimental curves was not linear, due to adjustments in the end-support devices in the initial stage of loading. Generally, it can be stated that the agreement between experimental and numerical results is very good, especially in terms of estimated ultimate load and the load evolution during the loading stage. 

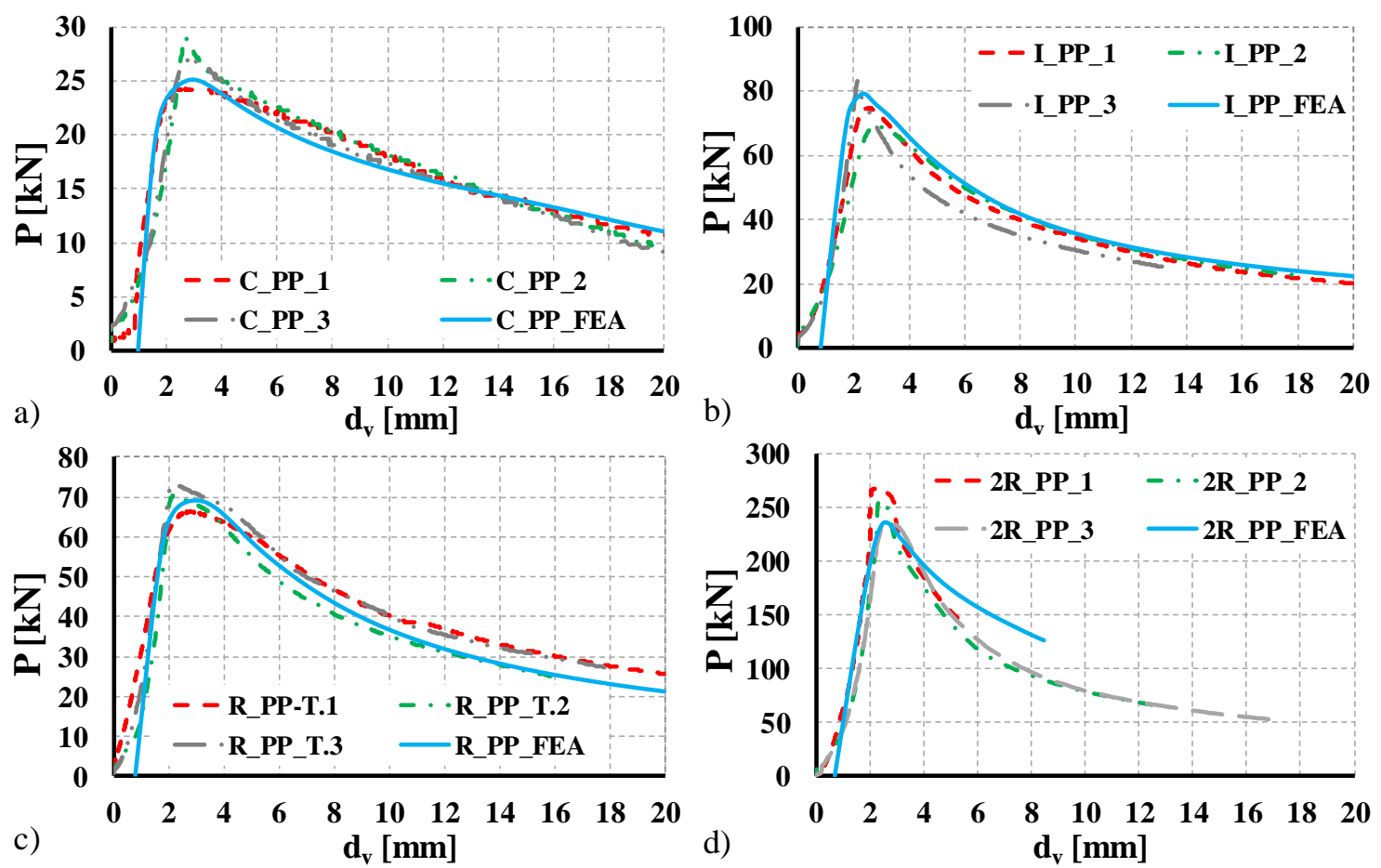

Figure 5.8 Comparison of FEA and experimental axial load versus axial shortening curves for all tested cross-sections and pinned-ended supports. a) C. b) I. c) R. d) 2 R cross-section.
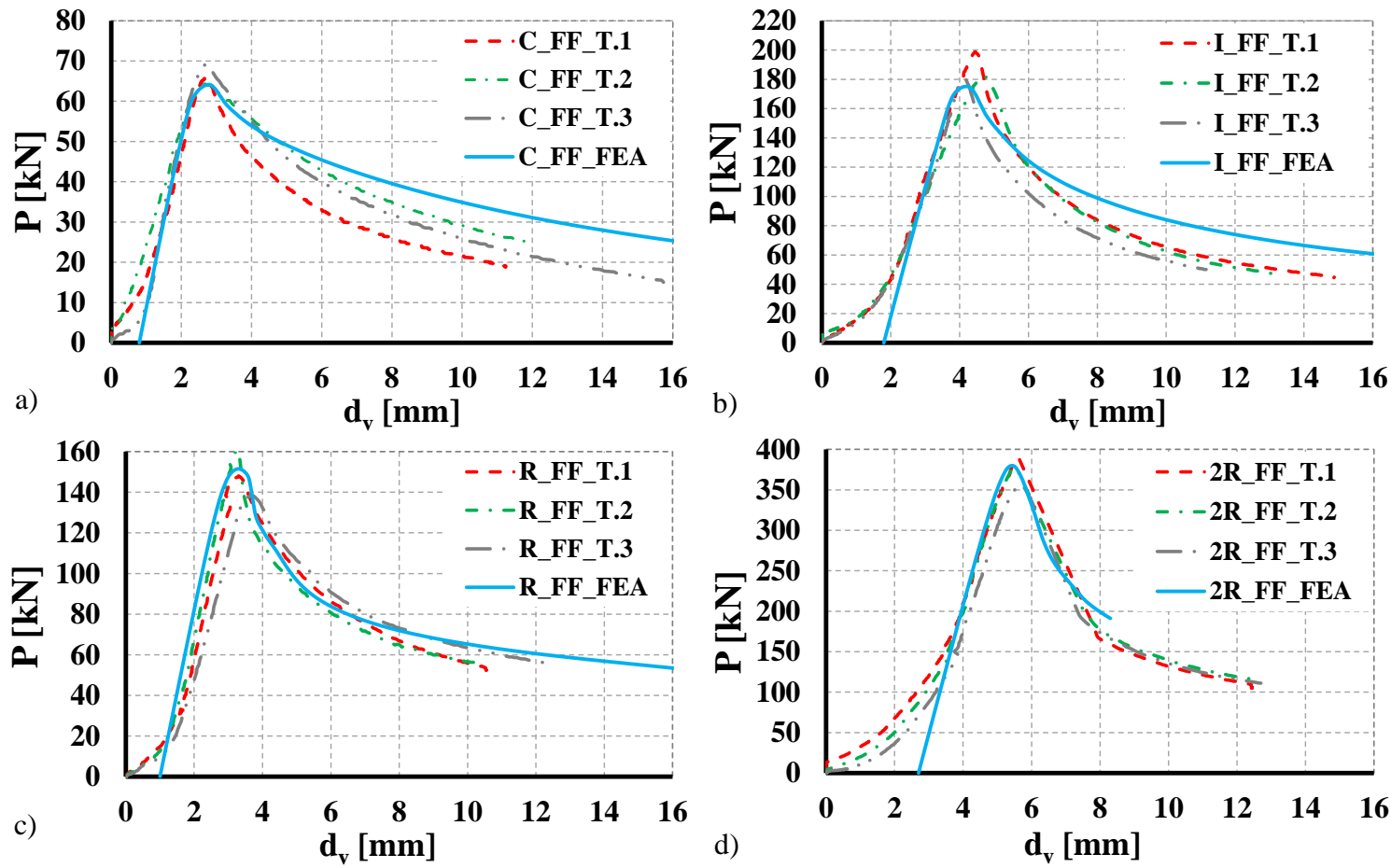

Figure 5.9 Comparison of FEA and experimental axial load versus axial shortening curves for all tested cross-sections and fixed-ended supports. a) C. b) I. c) R. d) 2R cross-section. 
In Tables 5.1 to 5.4 the test results are compared with the FEA results and with the design predictions. Once again the good agreement between experimental and FEA results is observed. Comparing both experimental and FEA results with the design buckling load $\left(N_{b, R d}\right)$ determined according the EN 1993-1-3 (2006) some relevant differences were observed. For instance, it seems that the design buckling load is too conservative for fixed-ended lipped channel columns. Also it was observed that increasing the number of profiles it seems that the design buckling predictions become unconservative, as it can be observed for columns with $2 \mathrm{R}$ cross-section. However, the presented comparisons must be analyzed carefully, since the obtained experimental results do not represent ideal end-support conditions (perfect pinned and fully fixed) and FEA results are based on assumptions adopted to accurately reproduce the boundary conditions observed in the experimental tests. Hence it is fundamental to conduct parametric studies, in the near future, using the developed and validated finite element model considering ideal end-support conditions, in order to provide estimations for lower and upper bounds of the ultimate strength of columns, and also to assess the accuracy of the available design methodologies.

Table 5.1 Experimental and numerical load-bearing capacity of lipped channel CFS columns.

\begin{tabular}{|c|c|c|c|c|c|c|}
\hline Test & $\begin{array}{c}\text { Experimental } \\
\mathbf{P}_{\max }[\mathrm{kN}] \\
\end{array}$ & $\begin{array}{c}\text { Numerical } \\
\mathbf{P}_{\mathrm{FEA}}[\mathrm{kN}]\end{array}$ & $\begin{array}{l}\mathbf{N}_{\mathbf{b}, \mathbf{R d}} \\
{[\mathbf{k N}]}\end{array}$ & $\mathbf{P}_{\mathrm{FEA}} / \mathbf{P}_{\max }$ & $\mathbf{P}_{\max } / \mathbf{N}_{\mathrm{b}, \mathbf{R d}}$ & $\mathbf{P}_{\mathrm{FEA}} / \mathbf{N}_{\mathrm{b}, \mathbf{R d}}$ \\
\hline C_PP-1 & 24.53 & \multirow{3}{*}{25.15} & \multirow{3}{*}{24.8} & 1.03 & 0.99 & \multirow{3}{*}{1.01} \\
\hline C_PP-2 & 29.14 & & & 0.86 & 1.18 & \\
\hline C_PP-3 & 27.04 & & & 0.93 & 1.09 & \\
\hline$\mu$ & 26.90 & 25.15 & 24.8 & 0.94 & 1.06 & 1.01 \\
\hline C_FF-1 & 65.62 & \multirow{3}{*}{64.14} & \multirow{3}{*}{41.8} & 0.98 & 1.57 & \multirow{3}{*}{1.53} \\
\hline C_FF-2 & 64.35 & & & 1.00 & 1.54 & \\
\hline C_FF-3 & 69.22 & & & 0.93 & 1.66 & \\
\hline$\mu$ & 66.40 & 64.14 & 41.8 & 0.97 & 1.59 & 1.53 \\
\hline
\end{tabular}

Table 5.2 Experimental and numerical load-bearing capacity of open built-up I CFS columns.

\begin{tabular}{|c|c|c|c|c|c|c|}
\hline Test & $\begin{array}{c}\text { Experimental } \\
\mathbf{P}_{\max }[\mathrm{kN}] \\
\end{array}$ & $\begin{array}{c}\text { Numerical } \\
\mathbf{P}_{\mathrm{FEA}}[\mathrm{kN}] \\
\end{array}$ & $\begin{array}{l}\mathbf{N}_{\mathrm{b}, \mathrm{Rd}} \\
{[\mathrm{kN}]}\end{array}$ & $\mathbf{P}_{\mathrm{FEA}} / \mathbf{P}_{\max }$ & $\mathbf{P}_{\max } / \mathbf{N}_{\mathbf{b}, \mathbf{R d}}$ & $\mathbf{P}_{\mathrm{FEA}} / \mathbf{N}_{\mathrm{b}, \mathbf{R d}}$ \\
\hline I_PP-1 & 74.69 & \multirow{3}{*}{78.97} & \multirow{3}{*}{85.51} & 1.06 & 0.87 & \multirow{3}{*}{0.92} \\
\hline I_PP-2 & 69.06 & & & 1.14 & 0.81 & \\
\hline I_PP-3 & 83.18 & & & 0.95 & 0.97 & \\
\hline$\mu$ & 75.64 & 78.97 & 85.51 & 1.05 & 0.88 & 0.92 \\
\hline I_FF-1 & 198.75 & \multirow{3}{*}{174.49} & \multirow{3}{*}{187.7} & 0.88 & 1.06 & \multirow{3}{*}{0.93} \\
\hline I_FF-2 & 182.07 & & & 0.96 & 0.97 & \\
\hline I_FF-3 & 180.11 & & & 0.97 & 0.96 & \\
\hline$\mu$ & 186.98 & 174.49 & 187.7 & 0.94 & 0.99 & 0.93 \\
\hline
\end{tabular}


Table 5.3 Experimental and numerical load-bearing capacity of closed built-up R CFS columns.

\begin{tabular}{|c|c|c|c|c|c|c|}
\hline Test & $\begin{array}{c}\text { Experimental } \\
\mathbf{P}_{\max }[\mathrm{kN}] \\
\end{array}$ & $\begin{array}{c}\text { Numerical } \\
\mathbf{P}_{\mathrm{FEA}}[\mathrm{kN}]\end{array}$ & $\begin{array}{l}\mathbf{N}_{\mathbf{b}, \mathbf{R d}} \\
{[\mathbf{k N}]}\end{array}$ & $\mathbf{P}_{\text {fEA }} / \mathbf{P}_{\max }$ & $\mathbf{P}_{\max } / \mathbf{N}_{\mathbf{b}, \mathbf{R d}}$ & $\mathbf{P}_{\mathrm{FEA}} / \mathbf{N}_{\mathrm{b}, \mathbf{R d}}$ \\
\hline R_PP-1 & 66.25 & \multirow{3}{*}{67.73} & \multirow{3}{*}{76.55} & 1.02 & 0.87 & \multirow{3}{*}{0.88} \\
\hline R_PP-2 & 70.43 & & & 0.96 & 0.92 & \\
\hline R_PP-3 & 73.37 & & & 0.92 & 0.96 & \\
\hline$\mu$ & 70.02 & 67.73 & 76.55 & 0.97 & 0.92 & 0.88 \\
\hline$\overline{R \_F F-1}$ & 148.03 & \multirow{3}{*}{151.01} & \multirow{3}{*}{168.27} & 1.02 & 0.88 & \multirow{3}{*}{0.90} \\
\hline R_FF-2 & 160.78 & & & 0.94 & 0.96 & \\
\hline R_FF-3 & 138.22 & & & 1.09 & 0.82 & \\
\hline$\mu$ & 149.01 & 151.01 & 168.27 & 1.02 & 0.87 & 0.90 \\
\hline
\end{tabular}

Table 5.4 Experimental and FEA load-bearing capacity of closed built-up 2R CFS columns.

\begin{tabular}{|c|c|c|c|c|c|c|}
\hline Test & $\begin{array}{c}\text { Experimental } \\
\mathbf{P}_{\max }[\mathrm{kN}]\end{array}$ & $\begin{array}{c}\text { Numerical } \\
\mathbf{P}_{\mathrm{FEA}}[\mathrm{kN}]\end{array}$ & $\begin{array}{l}\mathbf{N}_{\mathbf{b}, \mathbf{R d}} \\
{[\mathbf{k N}]}\end{array}$ & $\mathbf{P}_{\mathrm{FEA}} / \mathbf{P}_{\text {max }}$ & $\mathbf{P}_{\max } / \mathbf{N}_{\mathrm{b}, \mathbf{R d}}$ & $\mathbf{P}_{\mathbf{F E A}} / \mathbf{N}_{\mathbf{b}, \mathbf{R d}}$ \\
\hline 2R_PP-1 & 268.79 & \multirow{3}{*}{235.1} & \multirow{3}{*}{305.57} & 0.87 & 0.88 & \multirow{3}{*}{0.77} \\
\hline 2R_PP-2 & 257.7 & & & 0.91 & 0.84 & \\
\hline 2R_PP-3 & 235.14 & & & 0.99 & 0.77 & \\
\hline$\mu$ & 253.88 & 235.1 & 305.57 & 0.93 & 0.83 & 0.77 \\
\hline 2R_FF-1 & 388.67 & \multirow{3}{*}{379.07} & \multirow{3}{*}{443.42} & 0.98 & 0.88 & \multirow{3}{*}{0.85} \\
\hline 2R_FF-2 & 377.88 & & & 1.00 & 0.85 & \\
\hline 2R_FF-3 & 356.59 & & & 1.06 & 0.80 & \\
\hline$\mu$ & 374.38 & 379.07 & 443.42 & 1.01 & 0.84 & 0.85 \\
\hline
\end{tabular}

\subsubsection{Validation of the finite element model for fire tests with restraint to thermal elongation}

The validation of the finite element model developed to reproduce the behaviour of CFS columns under fire condition with restraint to thermal elongation consisted of comparing the evolution of the non-dimensional ratio between the generated restraining forces during fire tests with the initial applied service load $\left(P / P_{0}\right)$ as a function of the mean temperature of the CFS column $\left(\bar{\theta}_{C}\right)$. In Figures 5.10 to 5.17 the comparison between experimental and FEA results is presented in order to show the accuracy of the finite element model. In Figure 5.10a) the difference between a simulation with (orange curve) and without (green curve) rotational restraint (provided by the hinge feature in the model) is presented to show the influence of the hinge in the finite element model. In Tables 5.5 to 5.12 the results are further detailed and compared in terms of maximum axial load and critical temperature. Generally, it can be stated that the developed finite element model is able to accurately reproduce the behaviour of CFS columns under simulated fire conditions with restraint to thermal elongation. 

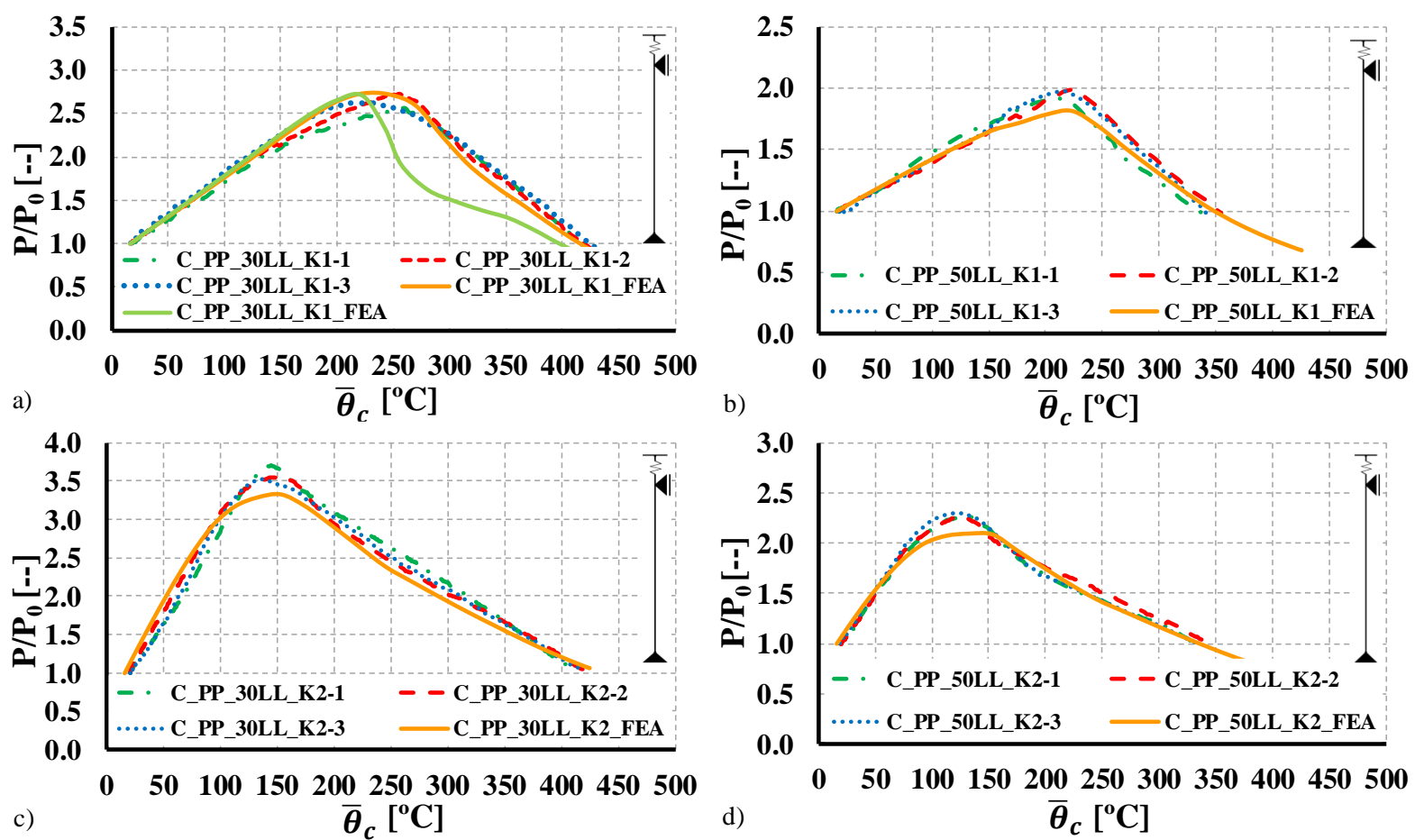

Figure 5.10 Comparison between experimental and FEA results for pinned-ended C columns.
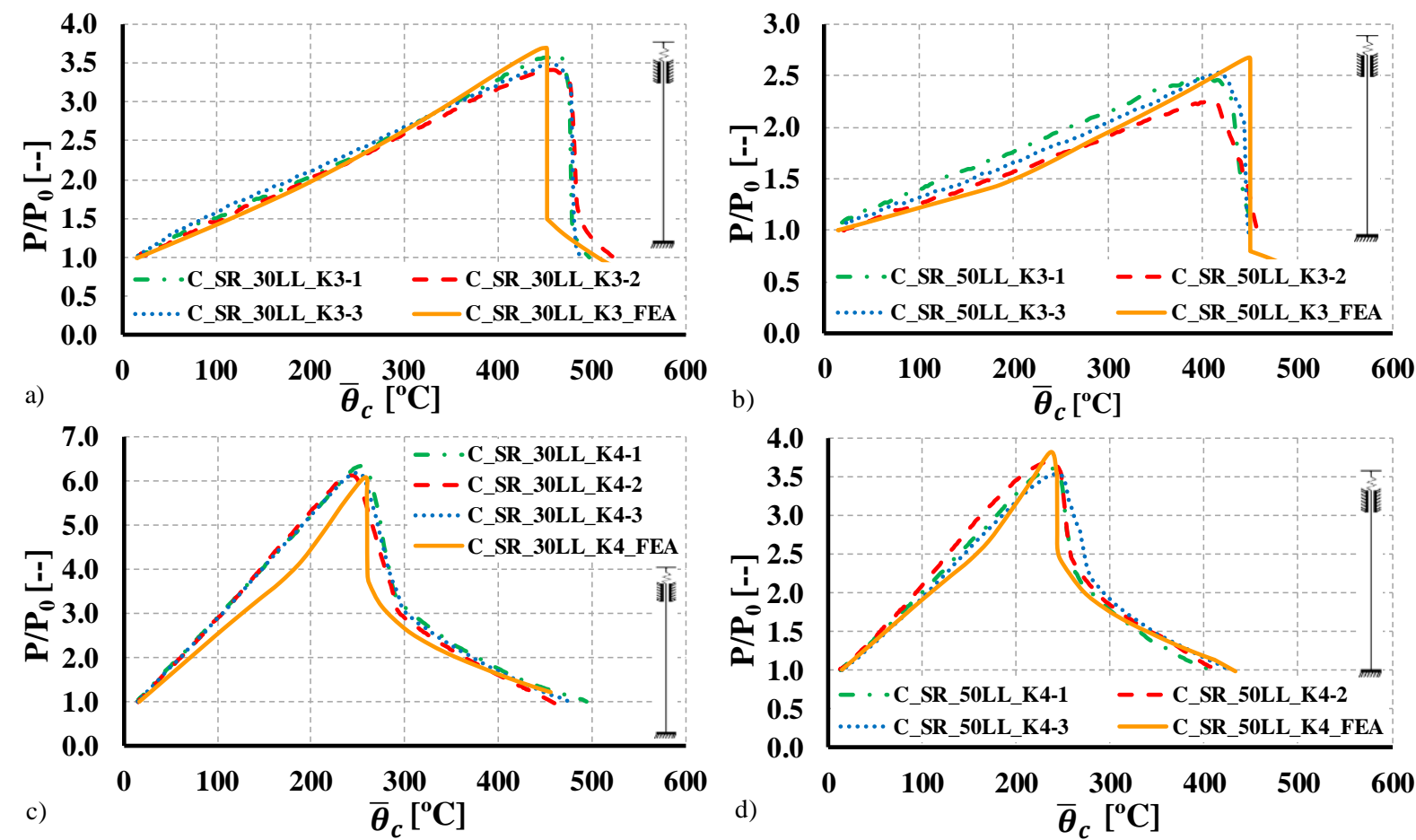

Figure 5.11 Comparison between experimental and FEA results for semi-rigid lipped channel columns. 

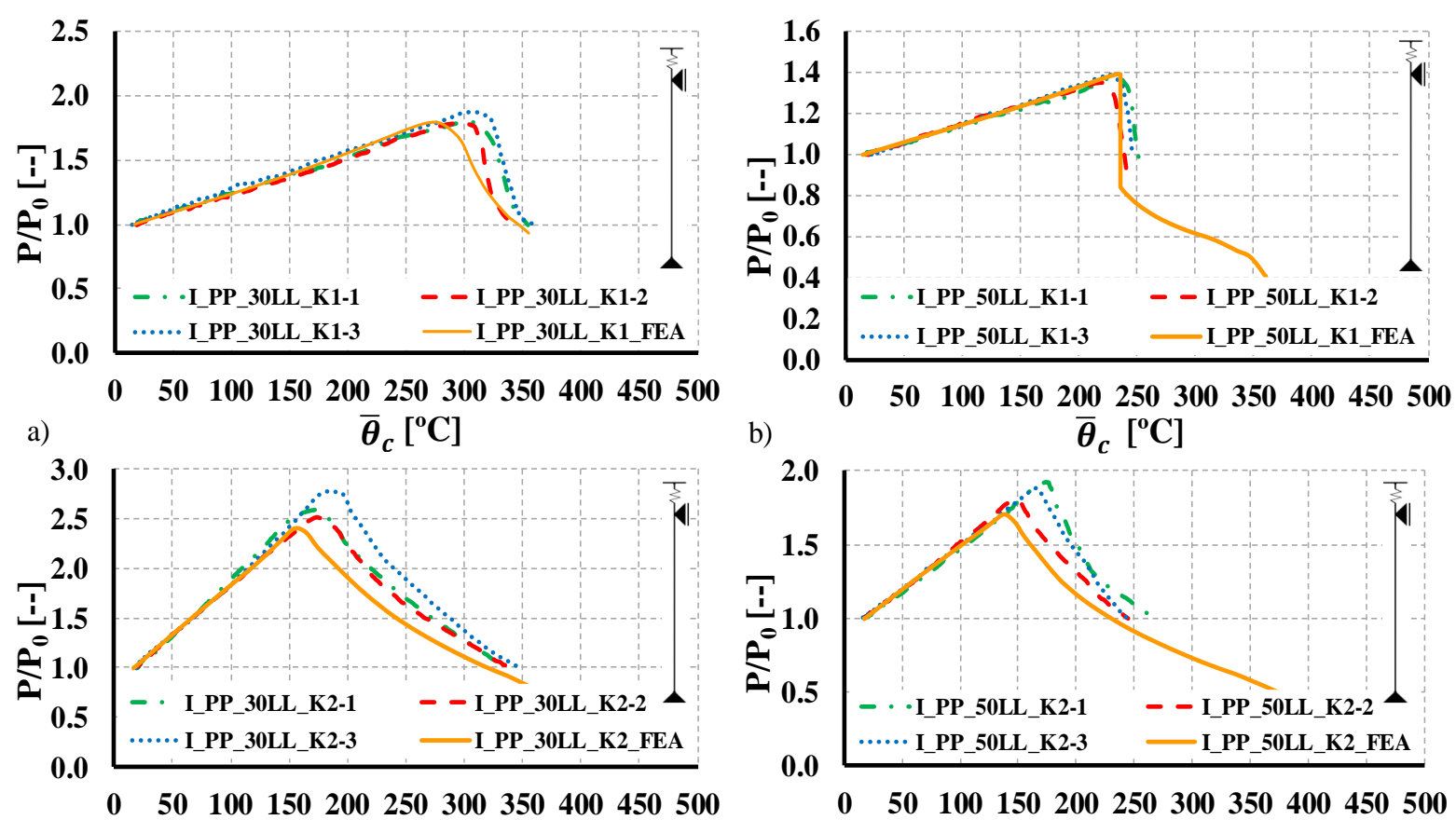

b) $\overline{\boldsymbol{\theta}}_{\boldsymbol{c}}\left[{ }^{\mathrm{O}} \mathrm{C}\right]$

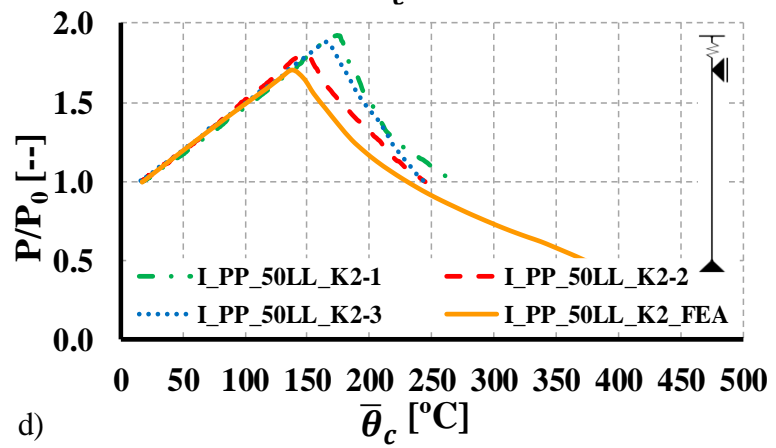
c) $\overline{\boldsymbol{\theta}}_{\boldsymbol{c}}\left[{ }^{\mathrm{O}} \mathrm{C}\right]$

d)

Figure 5.12 Comparison between experimental and FEA results for pinned-ended columns with open built-up I cross-section.
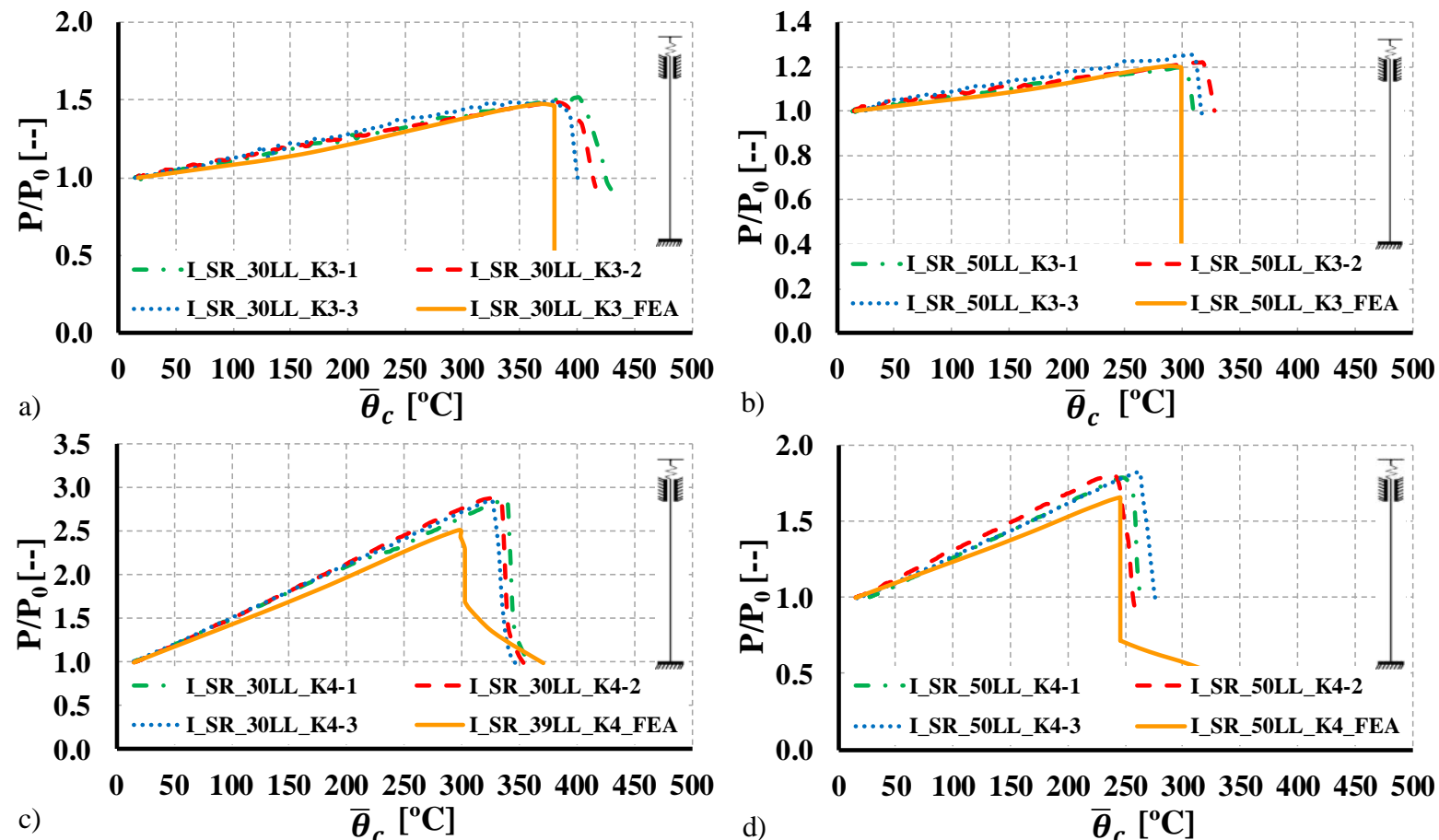
c) $\overline{\boldsymbol{\theta}}_{\boldsymbol{c}}\left[{ }^{\circ} \mathrm{C}\right]$

d) $\overline{\boldsymbol{\theta}}_{c}\left[{ }^{\circ} \mathbf{C}\right]$

Figure 5.13 Comparison between experimental and FEA results for semi-rigid columns with open built-up I cross-section. 

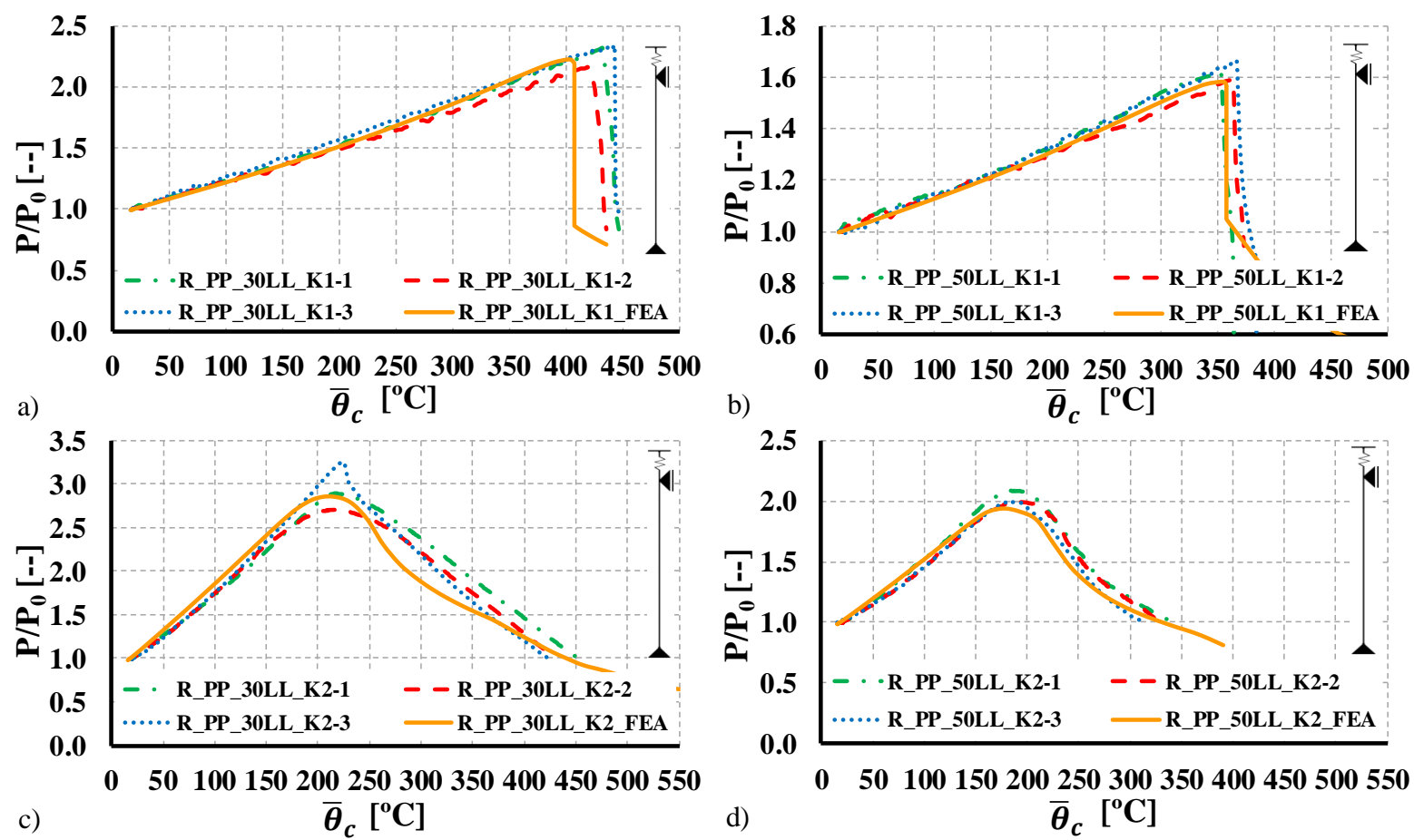

Figure 5.14 Comparison between experimental and FEA results for pinned-ended columns with closed built-up R cross-section.
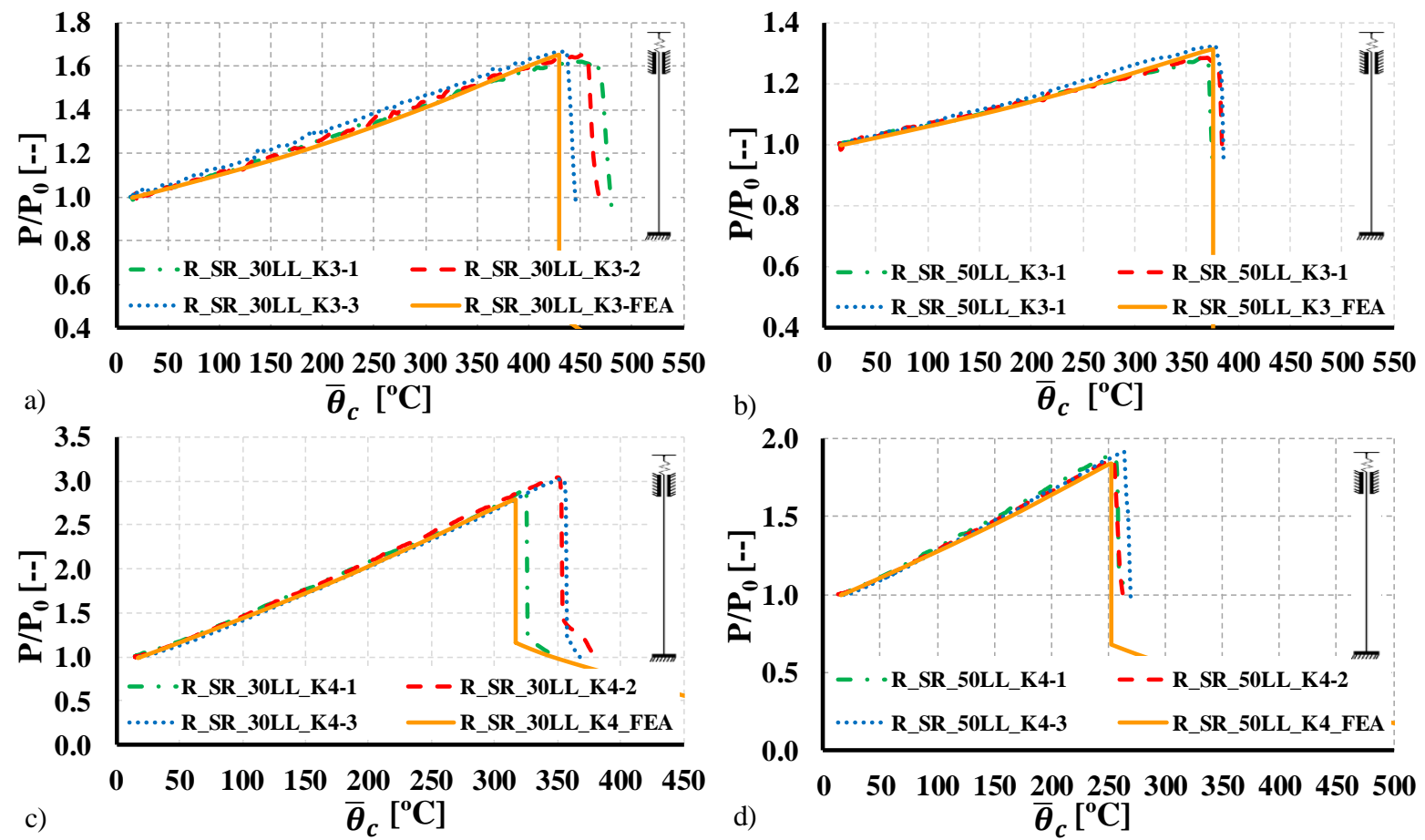

Figure 5.15 Comparison between experimental and FEA results for semi-rigid columns with closed built-up R cross-section. 

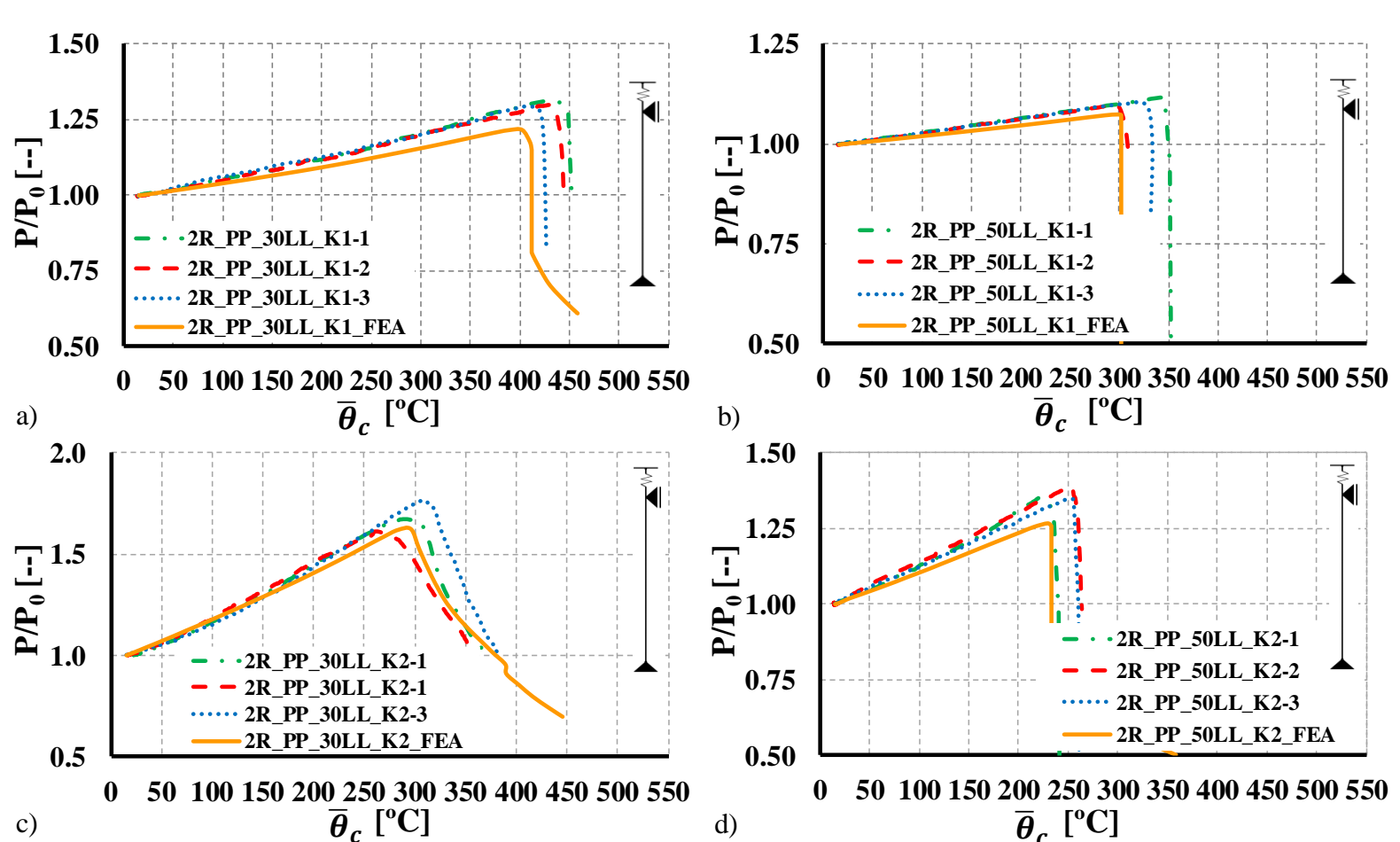

Figure 5.16 Comparison between experimental and FEA results for pinned-ended columns with closed built-up 2R cross-section.
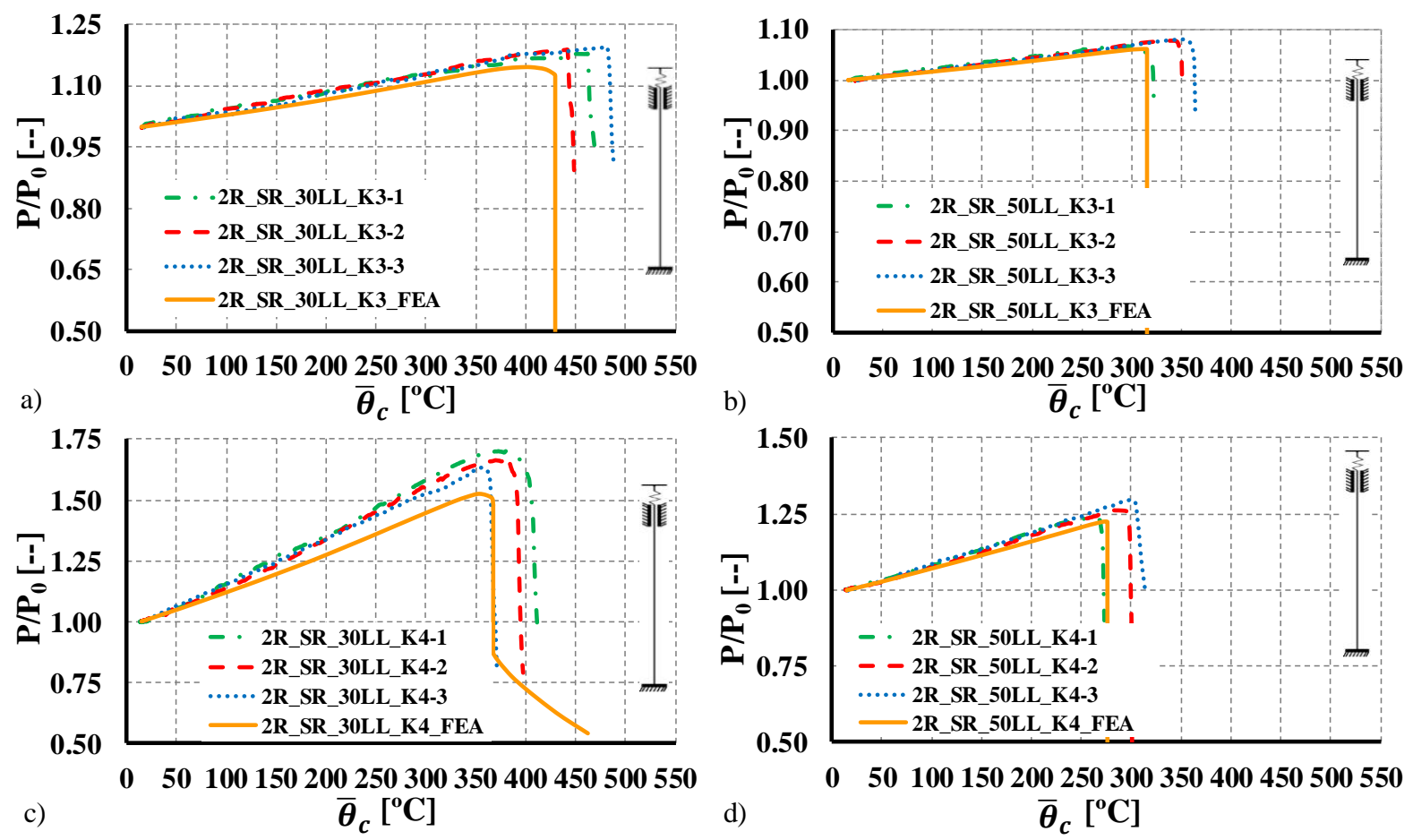

Figure 5.17 Comparison between experimental and FEA results for semi-rigid columns with closed built-up 2R cross-section. 
5. NUMERICAL MODELLING OF COLD-FORMED STEEL COLUMNS

Table 5.5 Experimental and FEA critical temperature and maximum axial load for pinnedended lipped channel columns.

\begin{tabular}{|c|c|c|c|c|c|c|}
\hline Test Reference & $\begin{array}{c}\theta_{\text {cr }} \\
{\left[{ }^{\circ} \mathbf{C}\right]}\end{array}$ & $\begin{array}{l}\mathbf{P}_{\max } \\
{[\mathbf{k N}]}\end{array}$ & $\begin{array}{c}\theta_{\text {cr,FEA }} \\
{\left[{ }^{\circ} \mathbf{C}\right]} \\
\end{array}$ & $\begin{array}{c}\mathbf{P}_{\max , \mathrm{FEA}} \\
{[\mathrm{kN}]}\end{array}$ & $\theta_{\mathrm{cr}} / \boldsymbol{\theta}_{\mathrm{cr}, \mathrm{FEA}}$ & $\mathbf{P}_{\max } / \mathbf{P}_{\text {max }, \mathrm{FEA}}$ \\
\hline C_PP_30LL_K1-1 & 418.85 & 19.7 & \multirow{3}{*}{428.16} & \multirow{3}{*}{20.34} & 0.98 & 0.97 \\
\hline C_PP_30LL_K1-2 & 422.35 & 20.5 & & & 0.99 & 1.01 \\
\hline C_PP_30LL_K1-3 & 425.21 & 19.75 & & & 0.99 & 0.97 \\
\hline$\mu$ & 422.14 & 19.98 & 428.16 & 20.34 & 0.99 & 0.98 \\
\hline C_PP_50LL_K1-1 & 338.76 & 24.00 & \multirow{3}{*}{369.23} & \multirow{3}{*}{22.49} & 0.92 & 1.07 \\
\hline C_PP_50LL_K1-2 & 351.10 & 25.24 & & & 0.95 & 1.12 \\
\hline C_PP_50LL_K1-3 & 346.71 & 25.29 & & & 0.94 & 1.12 \\
\hline$\mu$ & 345.52 & 24.84 & 369.23 & 22.49 & 0.94 & 1.10 \\
\hline C_PP_30LL_K2-1 & 412.85 & 28.40 & \multirow{3}{*}{426.42} & \multirow{3}{*}{24.74} & 0.97 & 1.15 \\
\hline C_PP_30LL_K2-2 & 426.61 & 27.10 & & & 1.00 & 1.10 \\
\hline C_PP $=30 L L=K 2-3$ & 423.08 & 26.40 & & & 0.99 & 1.07 \\
\hline$\mu$ & 420.8467 & 27.3 & 426.42 & 24.74 & 0.99 & 1.10 \\
\hline C_PP_50LL_K2-1 & 332.33 & 28.50 & \multirow{3}{*}{342.55} & \multirow{3}{*}{25.92} & 0.97 & 1.07 \\
\hline C_PP_50LL_K2-2 & 338.31 & 28.90 & & & 0.99 & 1.12 \\
\hline C_PP 50LL_K2-3 & 344.95 & 29.30 & & & 1.01 & 1.12 \\
\hline$\mu$ & 338.53 & 28.9 & 342.55 & 25.92 & 0.99 & 1.10 \\
\hline
\end{tabular}

Table 5.6 Experimental and FEA critical temperature and maximum axial load for semi-rigid lipped channel columns.

\begin{tabular}{|c|c|c|c|c|c|c|}
\hline Test Reference & $\begin{array}{c}\boldsymbol{\theta}_{\mathrm{cr}} \\
{\left[{ }^{\circ} \mathbf{C}\right]} \\
\end{array}$ & $\begin{array}{l}\mathbf{P}_{\max } \\
{[\mathbf{k N}]}\end{array}$ & $\begin{array}{c}\boldsymbol{\theta}_{\text {cr,FEA }} \\
{\left[{ }^{\circ} \mathbf{C}\right]} \\
\end{array}$ & $\begin{array}{c}\mathbf{P}_{\max , \mathrm{FEA}} \\
{[\mathrm{kN}]}\end{array}$ & $\boldsymbol{\theta}_{\mathrm{cr}} / \boldsymbol{\theta}_{\mathrm{cr}, \mathrm{FEA}}$ & $\mathbf{P}_{\text {max }} / \mathbf{P}_{\text {max }, \text { FEA }}$ \\
\hline C_SR_30LL_K3-1 & 490.36 & 45.42 & \multirow{3}{*}{515.43} & \multirow{3}{*}{45.99} & 0.95 & 0.99 \\
\hline C_SR_30LL_K3-2 & 524.10 & 43.82 & & & 1.02 & 0.95 \\
\hline C_SR_30LL_K3-3 & 490.52 & 45.32 & & & 0.95 & 0.99 \\
\hline$\mu$ & 501.66 & 44.85 & 515.43 & 45.99 & 0.97 & 0.98 \\
\hline C_SR_50LL_K3-1 & 453.23 & 51.52 & \multirow{3}{*}{449.91} & \multirow{3}{*}{55.93} & 1.01 & 0.92 \\
\hline C_SR_50LL_K3-2 & 458.43 & 50.84 & & & 1.02 & 0.91 \\
\hline C_SR_50LL_K3-3 & 449.11 & 53.17 & & & 1.00 & 0.95 \\
\hline$\mu$ & 453.59 & 51.84 & 449.91 & 55.93 & 1.01 & 0.93 \\
\hline C_SR_30LL_K4-1 & 494.55 & 79.70 & \multirow{3}{*}{480.2} & \multirow{3}{*}{76.22} & 1.03 & 1.05 \\
\hline C_SR_30LL_K4-2 & 465.98 & 76.30 & & & 0.97 & 1.00 \\
\hline C_SR_30LL_K4-3 & 475.47 & 77.37 & & & 0.99 & 1.02 \\
\hline$\mu$ & 478.667 & 77.79 & 480.2 & 76.22 & 1.00 & 1.02 \\
\hline C_SR_50LL_K4-1 & 405.51 & 76.30 & \multirow{3}{*}{433.2} & \multirow{3}{*}{79.42} & 0.94 & 1.07 \\
\hline C_SR_50LL_K4-2 & 415.60 & 77.45 & & & 0.96 & 1.12 \\
\hline C_SR_50LL_K4-3 & 425.33 & 79.38 & & & 0.98 & 1.12 \\
\hline$\mu$ & 415.48 & $7 \overline{7} . \overline{1}$ & 433.2 & 79.42 & 0.96 & 1.10 \\
\hline
\end{tabular}


Table 5.7 Experimental and FEA critical temperature and maximum axial load for pinnedended columns with open built-up I cross-section.

\begin{tabular}{|c|c|c|c|c|c|c|}
\hline Test Reference & $\begin{array}{c}\boldsymbol{\theta}_{\mathrm{cr}} \\
{\left[{ }^{\circ} \mathbf{C}\right]}\end{array}$ & $\begin{array}{l}P_{\max } \\
{[\mathbf{k N}]}\end{array}$ & $\begin{array}{c}\boldsymbol{\theta}_{\mathrm{cr}, \mathrm{FEA}} \\
{\left[{ }^{\circ} \mathbf{C}\right]} \\
\end{array}$ & $\begin{array}{c}\mathbf{P}_{\max , \mathrm{FEA}} \\
{[\mathrm{kN}]}\end{array}$ & $\boldsymbol{\theta}_{\mathrm{cr}} / \boldsymbol{\theta}_{\mathrm{cr}, \mathrm{FEA}}$ & $\mathbf{P}_{\max } / \mathbf{P}_{\text {max }, \mathrm{FEA}}$ \\
\hline I_PP_30LL_K1-1 & 354.71 & 48.5 & \multirow{3}{*}{346.83} & \multirow{3}{*}{46.02} & 1.02 & 1.05 \\
\hline I_PP_30LL_K1-2 & 342.81 & 46.28 & & & 0.99 & 1.01 \\
\hline I_PP_30LL_K1-3 & 348.64 & 49.13 & & & 1.01 & 1.07 \\
\hline$\mu$ & 348.72 & 47.97 & 346.83 & 46.02 & 1.01 & 1.04 \\
\hline I_PP_50LL_K1-1 & 251.61 & 58.98 & \multirow{3}{*}{235.64} & \multirow{3}{*}{59.58} & 1.07 & 0.99 \\
\hline I_PP_50LL_K1-2 & 241.13 & 61.20 & & & 1.02 & 1.03 \\
\hline I_PP_50LL_K1-3 & 246.33 & 60.31 & & & 1.05 & 1.01 \\
\hline$\mu$ & 246.36 & 60.16 & 235.64 & 59.58 & 1.05 & 1.01 \\
\hline I_PP_30LL_K2-1 & 336.56 & 67.70 & \multirow{3}{*}{327.37} & \multirow{3}{*}{61.55} & 1.03 & 1.10 \\
\hline I_PP_30LL_K2-2 & 339.38 & 65.30 & & & 1.04 & 1.06 \\
\hline I_PP_30LL_K2-3 & 349.84 & 71.16 & & & 1.07 & 1.16 \\
\hline$\mu$ & 341.93 & 68.05 & 327.37 & 61.55 & 1.04 & 1.11 \\
\hline I_PP_50LL_K2-1 & 266.36 & 83.30 & \multirow{3}{*}{237.79} & \multirow{3}{*}{73} & 1.12 & 1.07 \\
\hline I_PP_50LL_K2-2 & 245.45 & 77.60 & & & 1.03 & 1.12 \\
\hline I_PP_50LL_K2-3 & 244.17 & 81.10 & & & 1.03 & 1.12 \\
\hline$\mu$ & 251.99 & 80.67 & 237.79 & 73.00 & 1.06 & 1.10 \\
\hline
\end{tabular}

Table 5.8 Experimental and FEA critical temperature and maximum axial load for semi-rigid columns with open built-up I cross-section.

\begin{tabular}{|c|c|c|c|c|c|c|}
\hline Test Reference & $\begin{array}{c}\boldsymbol{\theta}_{\mathrm{cr}} \\
{\left[{ }^{\circ} \mathbf{C}\right]}\end{array}$ & $\begin{array}{l}\mathbf{P}_{\max } \\
{[\mathrm{kN}]}\end{array}$ & $\begin{array}{c}\boldsymbol{\theta}_{\mathrm{cr}, \mathrm{FEA}} \\
{\left[{ }^{\circ} \mathbf{C}\right]}\end{array}$ & $\begin{array}{c}\mathbf{P}_{\max , \mathrm{FEA}} \\
{[\mathrm{kN}]}\end{array}$ & $\boldsymbol{\theta}_{\mathrm{cr}} / \boldsymbol{\theta}_{\mathrm{cr}, \mathrm{FEA}}$ & $\mathbf{P}_{\text {max }} / \mathbf{P}_{\text {max }, \mathrm{FEA}}$ \\
\hline I_SR_30LL_K3-1 & 428.23 & 85.368 & \multirow{3}{*}{380.4} & \multirow{3}{*}{84.09} & 1.13 & 1.02 \\
\hline I_SR_30LL_K3-2 & 416.00 & 83.82 & & & 1.09 & 1.00 \\
\hline I_SR_30LL_K3-3 & 401.05 & 84.558 & & & 1.05 & 1.01 \\
\hline$\mu$ & 415.09 & 84.58 & 380.40 & 84.09 & 1.09 & 1.01 \\
\hline I_SR_50LL_K3-1 & 309.92 & 113.67 & \multirow{3}{*}{314.4} & \multirow{3}{*}{117.43} & 0.99 & 0.97 \\
\hline I_SR_50LL_K3-2 & 327.74 & 115.80 & & & 1.04 & 0.99 \\
\hline I_SR_50LL_K3-3 & 317.24 & 118.34 & & & 1.01 & 1.01 \\
\hline$\mu$ & 318.30 & 115.94 & 314.40 & 117.43 & 1.01 & 0.99 \\
\hline I_SR_30LL_K4-1 & 359.71 & 162.16 & \multirow{3}{*}{370.42} & \multirow{3}{*}{143.1} & 0.97 & 1.13 \\
\hline I_SR_30LL_K4-2 & 353.86 & 163.60 & & & 0.96 & 1.14 \\
\hline I_SR_30LL_K4-3 & 346.04 & 162.31 & & & 0.93 & 1.13 \\
\hline$\mu$ & 353.20 & 162.69 & 370.42 & 143.10 & 0.95 & 1.14 \\
\hline I_SR_50LL_K4-1 & 263.88 & 169.43 & \multirow{3}{*}{245.53} & \multirow{3}{*}{154.4} & 1.07 & 1.07 \\
\hline I_SR_50LL_K4-2 & 257.79 & 171.60 & & & 1.05 & 1.12 \\
\hline I_SR_50LL_K4-3 & 276.36 & 173.36 & & & 1.13 & 1.12 \\
\hline$\mu$ & 266.01 & 171.46 & 245.53 & 154.40 & 1.08 & 1.10 \\
\hline
\end{tabular}


5. NUMERICAL MODELLING OF COLD-FORMED STEEL COLUMNS

Table 5.9 Experimental and FEA critical temperature and maximum axial load for pinnedended columns with closed built-up R cross-section.

\begin{tabular}{|c|c|c|c|c|c|c|}
\hline Test Reference & $\begin{array}{c}\theta_{\mathrm{cr}} \\
{\left[{ }^{\circ} \mathbf{C}\right]}\end{array}$ & $\begin{array}{l}\mathbf{P}_{\max } \\
{[\mathbf{k N}]}\end{array}$ & $\begin{array}{c}\boldsymbol{\theta}_{\mathrm{cr}, \mathrm{FEA}} \\
{\left[{ }^{\circ} \mathbf{C}\right]} \\
\end{array}$ & $\begin{array}{c}\mathbf{P}_{\max , \mathrm{FEA}} \\
{[\mathrm{kN}]}\end{array}$ & $\boldsymbol{\theta}_{\mathrm{cr}} / \boldsymbol{\theta}_{\mathrm{cr}, \mathrm{FEA}}$ & $\mathbf{P}_{\text {max }} / \mathbf{P}_{\text {max }, \text { FEA }}$ \\
\hline R_PP_30LL_K1-1 & 447.2 & 53.271 & \multirow{3}{*}{408.02} & \multirow{3}{*}{51.26} & 1.10 & 1.04 \\
\hline R_PP_30LL_K1-2 & 435.4 & 49.514 & & & 1.07 & 0.97 \\
\hline R_PP_30LL_K1-3 & 447.4 & 53.659 & & & 1.10 & 1.05 \\
\hline$\mu$ & 443.33 & 52.15 & 408.02 & 51.26 & 1.09 & 1.02 \\
\hline R_PP_50LL_K1-1 & 364.00 & 61.90 & \multirow{3}{*}{368.25} & \multirow{3}{*}{60.57} & 0.99 & 1.02 \\
\hline R_PP_50LL_K1-2 & 374.07 & 61.70 & & & 1.02 & 1.02 \\
\hline R_PP_50LL_K1-3 & 379.93 & 64.74 & & & 1.03 & 1.07 \\
\hline$\mu$ & 372.67 & 62.78 & 368.25 & 60.57 & 1.01 & 1.04 \\
\hline R_PP_30LL_K2-1 & 453.78 & 66.10 & \multirow{3}{*}{456.83} & \multirow{3}{*}{65.53} & 0.99 & 1.01 \\
\hline R_PP_30LL_K2-2 & 429.90 & 62.02 & & & 0.94 & 0.95 \\
\hline R_PP_30LL_K2-3 & 425.10 & 74.68 & & & 0.93 & 1.14 \\
\hline$\mu$ & 436.26 & 67.6007 & 456.83 & 65.53 & 0.95 & 1.03 \\
\hline R_PP_50LL_K2-1 & 342.40 & 79.74 & \multirow{3}{*}{348.3} & \multirow{3}{*}{74.18} & 0.98 & 1.07 \\
\hline R_PP_50LL_K2-2 & 312.01 & 76.83 & & & 0.90 & 1.12 \\
\hline R_PP_50LL_K2-3 & 331.60 & 77.57 & & & 0.95 & 1.12 \\
\hline$\mu$ & 328.67 & 78.0467 & 348.3 & 74.18 & 0.94 & 1.10 \\
\hline
\end{tabular}

Table 5.10 Experimental and FEA critical temperature and maximum axial load for semi-rigid columns with closed built-up R cross-section.

\begin{tabular}{|c|c|c|c|c|c|c|}
\hline Test Reference & $\begin{array}{c}\boldsymbol{\theta}_{\mathrm{cr}} \\
{\left[{ }^{\circ} \mathbf{C}\right]}\end{array}$ & $\begin{array}{l}\mathbf{P}_{\max } \\
{[\mathbf{k N}]}\end{array}$ & $\begin{array}{c}\theta_{\mathrm{cr}, \mathrm{FEA}} \\
{\left[{ }^{\circ} \mathrm{C}\right]}\end{array}$ & $\begin{array}{c}\mathbf{P}_{\max , \mathrm{FEA}} \\
{[\mathrm{kN}]}\end{array}$ & $\theta_{\mathrm{cr}} / \boldsymbol{\theta}_{\mathrm{cr}, \mathrm{FEA}}$ & $\mathbf{P}_{\max } / \mathbf{P}_{\max , \mathrm{FEA}}$ \\
\hline R_SR_30LL_K3-1 & 480.81 & 82.47 & \multirow{3}{*}{429.19} & \multirow{3}{*}{83.48} & 1.12 & 0.99 \\
\hline R_SR_30LL_K3-2 & 469.45 & 83.14 & & & 1.09 & 1.00 \\
\hline R_SR_30LL_K3-3 & 446.05 & 84.17 & & & 1.04 & 1.01 \\
\hline$\mu$ & 465.44 & 83.26 & 429.19 & 83.48 & 1.08 & 1.00 \\
\hline R_SR_50LL_K3-1 & 375.51 & 108.43 & \multirow{3}{*}{376.12} & \multirow{3}{*}{110.51} & 1.00 & 0.98 \\
\hline R_SR_50LL_K3-2 & 384.40 & 108.59 & & & 1.02 & 0.98 \\
\hline R_SR_50LL_K3-3 & 386.00 & 111.36 & & & 1.03 & 1.01 \\
\hline$\mu$ & 381.97 & 109.46 & 376.12 & 110.51 & 1.02 & 0.99 \\
\hline R_SR_30LL_K4-1 & 349.59 & 148.57 & \multirow{3}{*}{354.15} & \multirow{3}{*}{140.99} & 0.99 & 1.05 \\
\hline R_SR_30LL_K4-2 & 381.30 & 155.10 & & & 1.08 & 1.10 \\
\hline R_SR_30LL_K4-3 & 367.60 & 153.40 & & & 1.04 & 1.09 \\
\hline$\mu$ & 366.163 & 152.357 & 354.15 & 140.99 & 1.03 & 1.08 \\
\hline R_SR_50LL_K4-1 & 277.40 & 159.23 & \multirow{3}{*}{252.53} & \multirow{3}{*}{154.77} & 1.10 & 1.07 \\
\hline R_SR_50LL_K4-2 & 264.01 & 157.18 & & & 1.05 & 1.12 \\
\hline R_SR_50LL_K4-3 & 269.83 & 161.10 & & & 1.07 & 1.12 \\
\hline$\mu$ & 270.413 & 159.17 & 252.53 & 154.77 & 1.07 & 1.10 \\
\hline
\end{tabular}


Table 5.11 Experimental and FEA critical temperature and maximum axial load for pinnedended columns with closed built-up $2 \mathrm{R}$ cross-section.

\begin{tabular}{|c|c|c|c|c|c|c|}
\hline Test Reference & $\begin{array}{c}\boldsymbol{\theta}_{\mathrm{cr}} \\
{\left[{ }^{\circ} \mathbf{C}\right]}\end{array}$ & $\begin{array}{l}\mathbf{P}_{\max } \\
{[\mathrm{kN}]}\end{array}$ & $\begin{array}{c}\boldsymbol{\theta}_{\mathrm{cr}, \mathrm{FEA}} \\
{\left[{ }^{\circ} \mathbf{C}\right]} \\
\end{array}$ & $\begin{array}{c}\mathbf{P}_{\max , \mathrm{FEA}} \\
{[\mathrm{kN}]}\end{array}$ & $\boldsymbol{\theta}_{\mathrm{cr}} / \boldsymbol{\theta}_{\mathrm{cr}, \mathrm{FEA}}$ & $\mathbf{P}_{\text {max }} / \mathbf{P}_{\text {max }, \mathrm{FEA}}$ \\
\hline 2R_PP_30LL_K1-1 & 453.1 & 119.9 & \multirow{3}{*}{412.42} & \multirow{3}{*}{111.71} & 1.10 & 1.07 \\
\hline 2R_PP_30LL_K1-2 & 427.2 & 118.67 & & & 1.04 & 1.06 \\
\hline 2R_PP_30LL_K1-3 & 446.1 & 119.4 & & & 1.08 & 1.07 \\
\hline$\mu$ & 442.13 & 119.32 & 412.42 & 111.71 & 1.07 & 1.07 \\
\hline 2R_PP_50LL_K1-1 & 351.50 & 169.80 & \multirow{3}{*}{302.22} & \multirow{3}{*}{163.71} & 1.16 & 1.04 \\
\hline 2R_PP_50LL_K1-2 & 309.30 & 167.40 & & & 1.02 & 1.02 \\
\hline 2R_PP_50LL_K1-3 & 334.00 & 168.84 & & & 1.11 & 1.03 \\
\hline$\mu$ & 331.60 & 168.68 & 302.22 & 163.71 & 1.10 & 1.03 \\
\hline 2R_PP_30LL_K2-1 & 372.40 & 159.90 & \multirow{3}{*}{389.13} & \multirow{3}{*}{148.63} & 0.96 & 1.08 \\
\hline 2R_PP_30LL_K2-2 & 358.90 & 148.00 & & & 0.92 & 1.00 \\
\hline 2R_PP_30LL_K2-3 & 384.20 & 161.82 & & & 0.99 & 1.09 \\
\hline$\mu$ & 371.833 & 156.573 & 389.13 & 148.63 & 0.96 & 1.05 \\
\hline 2R_PP_50LL_K2-1 & 241.10 & 206.80 & \multirow{3}{*}{232.98} & \multirow{3}{*}{193.52} & 1.03 & 1.07 \\
\hline 2R_PP_50LL_K2-2 & 264.50 & 213.19 & & & 1.14 & 1.12 \\
\hline 2R_PP_50LL_K2-3 & 260.30 & 205.55 & & & 1.12 & 1.12 \\
\hline$\mu$ & 255.3 & 208.513 & 232.98 & 193.52 & 1.10 & 1.10 \\
\hline
\end{tabular}

Table 5.12 Experimental and FEA critical temperature and maximum axial load for semi-rigid columns with closed built-up R cross-section.

\begin{tabular}{|c|c|c|c|c|c|c|}
\hline Test Reference & $\begin{array}{c}\boldsymbol{\theta}_{\mathrm{cr}} \\
{\left[{ }^{\circ} \mathbf{C}\right]}\end{array}$ & $\begin{array}{l}\mathbf{P}_{\max } \\
{[\mathbf{k N}]}\end{array}$ & $\begin{array}{c}\theta_{\mathrm{cr}, \mathrm{FEA}} \\
{\left[{ }^{\circ} \mathrm{C}\right]} \\
\end{array}$ & $\begin{array}{c}\mathbf{P}_{\max , \mathrm{FEA}} \\
{[\mathrm{kN}]}\end{array}$ & $\theta_{\mathrm{cr}} / \theta_{\mathrm{cr}, \mathrm{FEA}}$ & $\mathbf{P}_{\max } / \mathbf{P}_{\max , \mathrm{FEA}}$ \\
\hline 2R_SR_30LL_K3-1 & 470.50 & 157.04 & \multirow{3}{*}{429.28} & \multirow{3}{*}{152.43} & 1.10 & 1.03 \\
\hline 2R_SR_30LL_K3-2 & 448.40 & 158.45 & & & 1.04 & 1.04 \\
\hline 2R_SR_30LL_K3-3 & 488.70 & 159.48 & & & 1.14 & 1.05 \\
\hline$\mu$ & 469.20 & 158.32 & 429.28 & 152.43 & 1.09 & 1.04 \\
\hline 2R_SR_50LL_K3-1 & 322.70 & 234.95 & \multirow{3}{*}{315.57} & \multirow{3}{*}{233.49} & 1.02 & 1.01 \\
\hline 2R_SR_50LL_K3-2 & 351.40 & 237.92 & & & 1.11 & 1.02 \\
\hline 2R_SR_50LL_K3-3 & 363.40 & 238.19 & & & 1.15 & 1.02 \\
\hline$\mu$ & 345.83 & 237.02 & 315.57 & 233.49 & 1.10 & 1.02 \\
\hline 2R_SR_30LL_K4-1 & 412.10 & 226.33 & \multirow{3}{*}{367.95} & \multirow{3}{*}{202.54} & 1.12 & 1.12 \\
\hline 2R_SR_30LL_K4-2 & 394.70 & 220.69 & & & 1.07 & 1.09 \\
\hline 2R_SR_30LL_K4-3 & 375.20 & 216.35 & & & 1.02 & 1.07 \\
\hline$\mu$ & 394 & 221.123 & 367.95 & 202.54 & 1.07 & 1.09 \\
\hline 2R_SR_50LL_K4-1 & 273.60 & 274.51 & \multirow{3}{*}{276.22} & \multirow{3}{*}{270.88} & 0.99 & 1.07 \\
\hline 2R_SR_50LL_K4-2 & 301.60 & 279.93 & & & 1.09 & 1.12 \\
\hline 2R_SR_50LL_K4-3 & 314.10 & 287.72 & & & 1.14 & 1.12 \\
\hline$\mu$ & 296.433 & 280.718 & 276.22 & 270.88 & 1.07 & 1.10 \\
\hline
\end{tabular}




\subsection{Validation of the Finite Element Models in Terms of the Observed Final Deformed Shape}

\subsubsection{Buckling tests}

In this part the obtained failure modes in the numerical simulations are compared with the ones observed in the experimental tests. This comparison is established in order to further validate the developed finite element model to reproduce the behaviour of CFS columns at ambient temperature. In Figures 5.18 to 5.21 experimental and FEA failure modes observed are compared. Flexural buckling about the minor axis of the CFS columns, as well as local and distortional buckling modes were clearly identified in the finite element models. Hence, once again, it can be stated that the developed finite element model is able to accurately reproduce the failure modes observed in the buckling tests. In Figures 5.18 to 5.21 only the pinned-end support condition is presented. For the remaining test conditions the agreement between experimental and FEA failure modes is also very good.
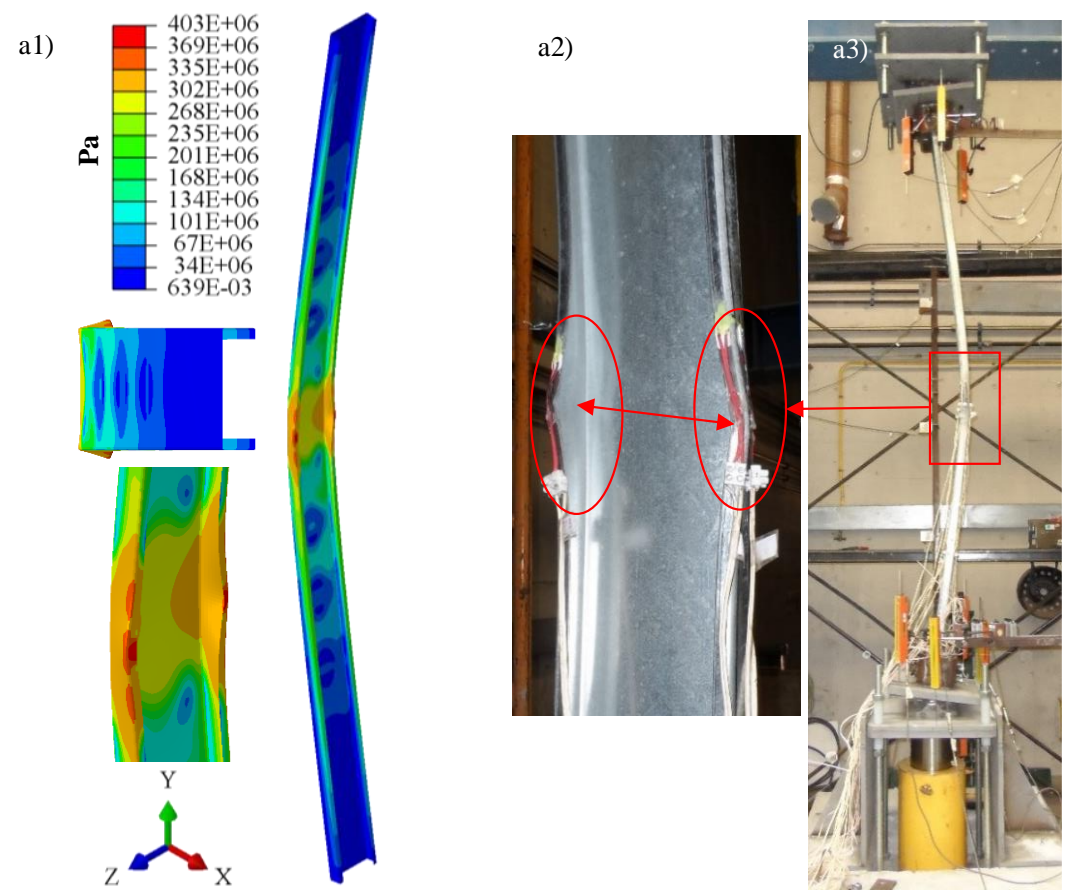

Figure 5.18 FEA (a) and experimental (b) failure modes for pinned-ended lipped channel columns. 

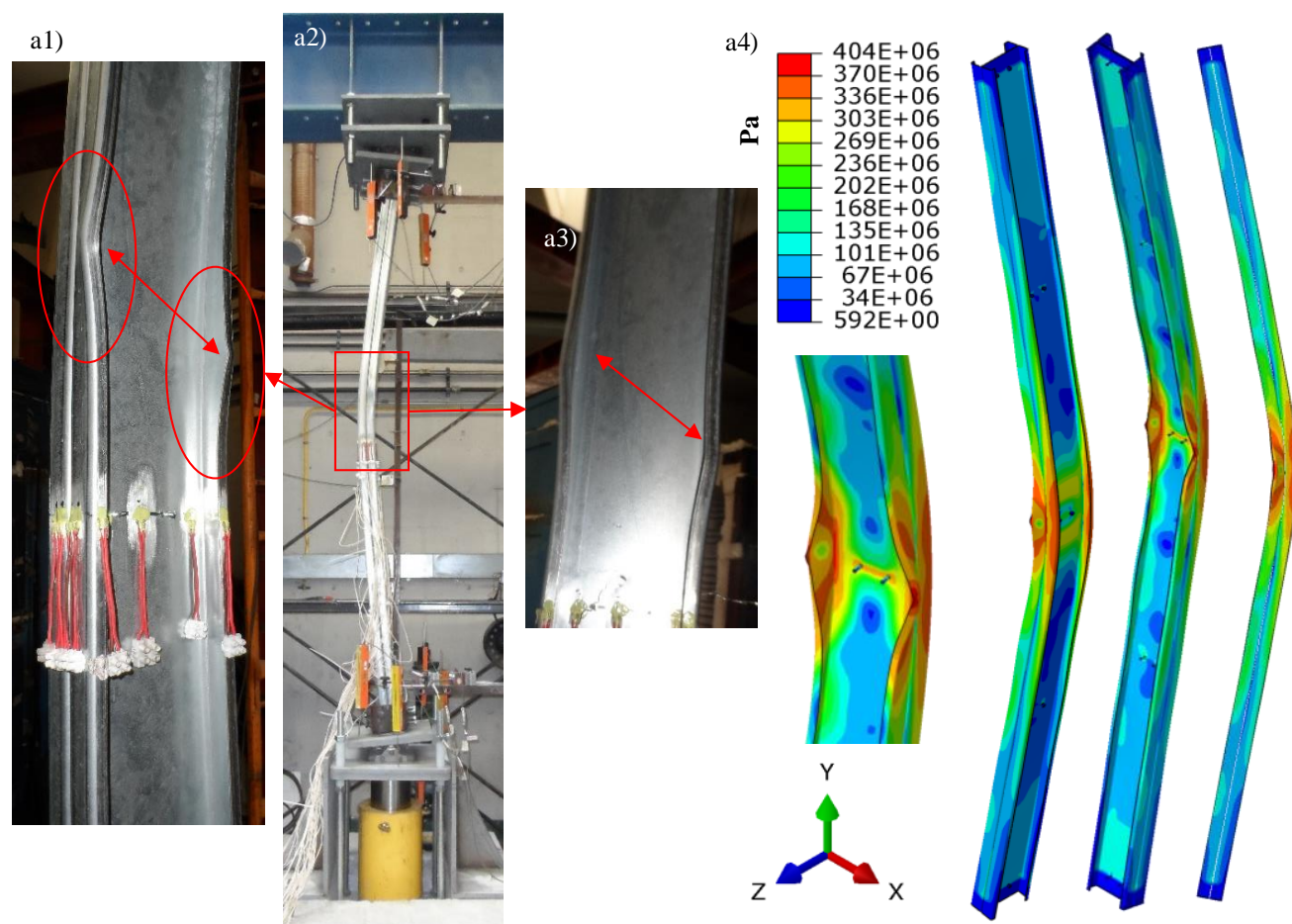

Figure 5.19 FEA (a) and experimental (b) failure modes for pinned-ended columns with open built-up I cross-section.
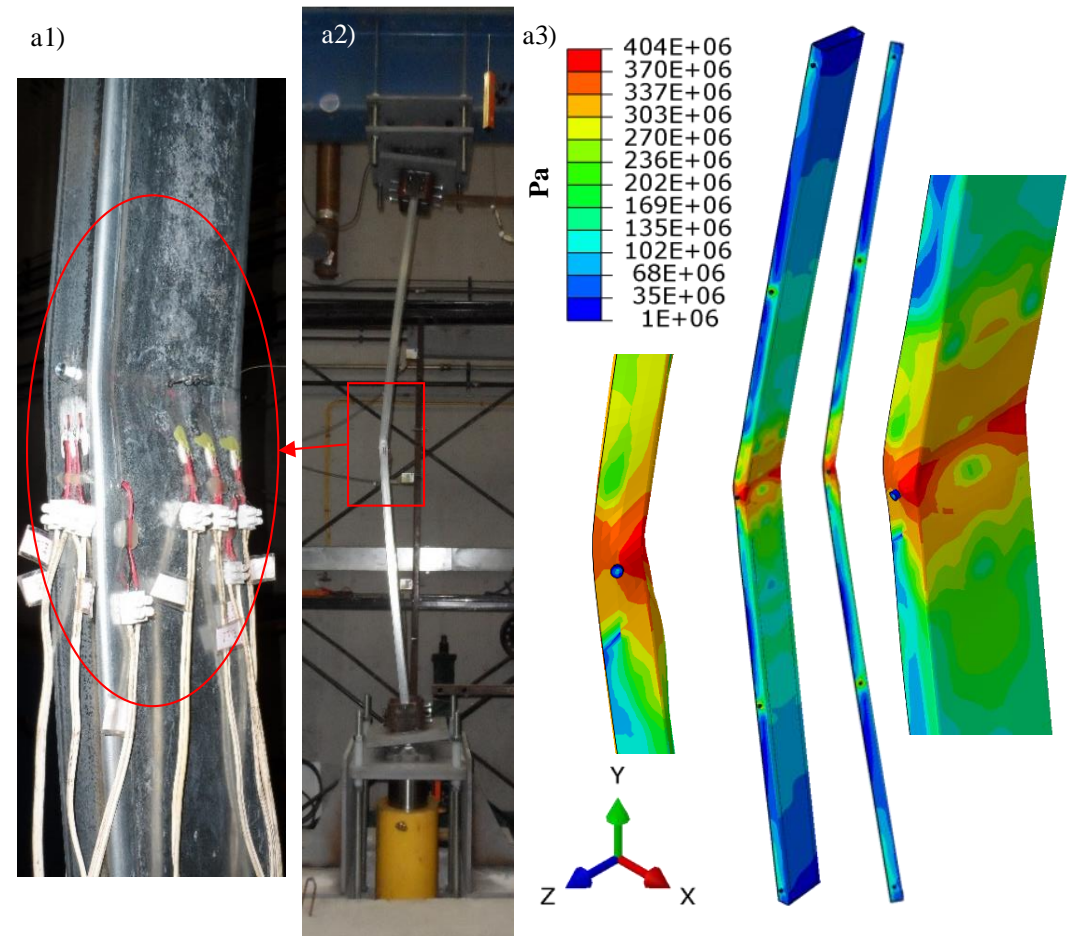

Figure 5.20 FEA (a) and experimental (b) failure modes for pinned-ended columns with closed built-up R cross-section. 

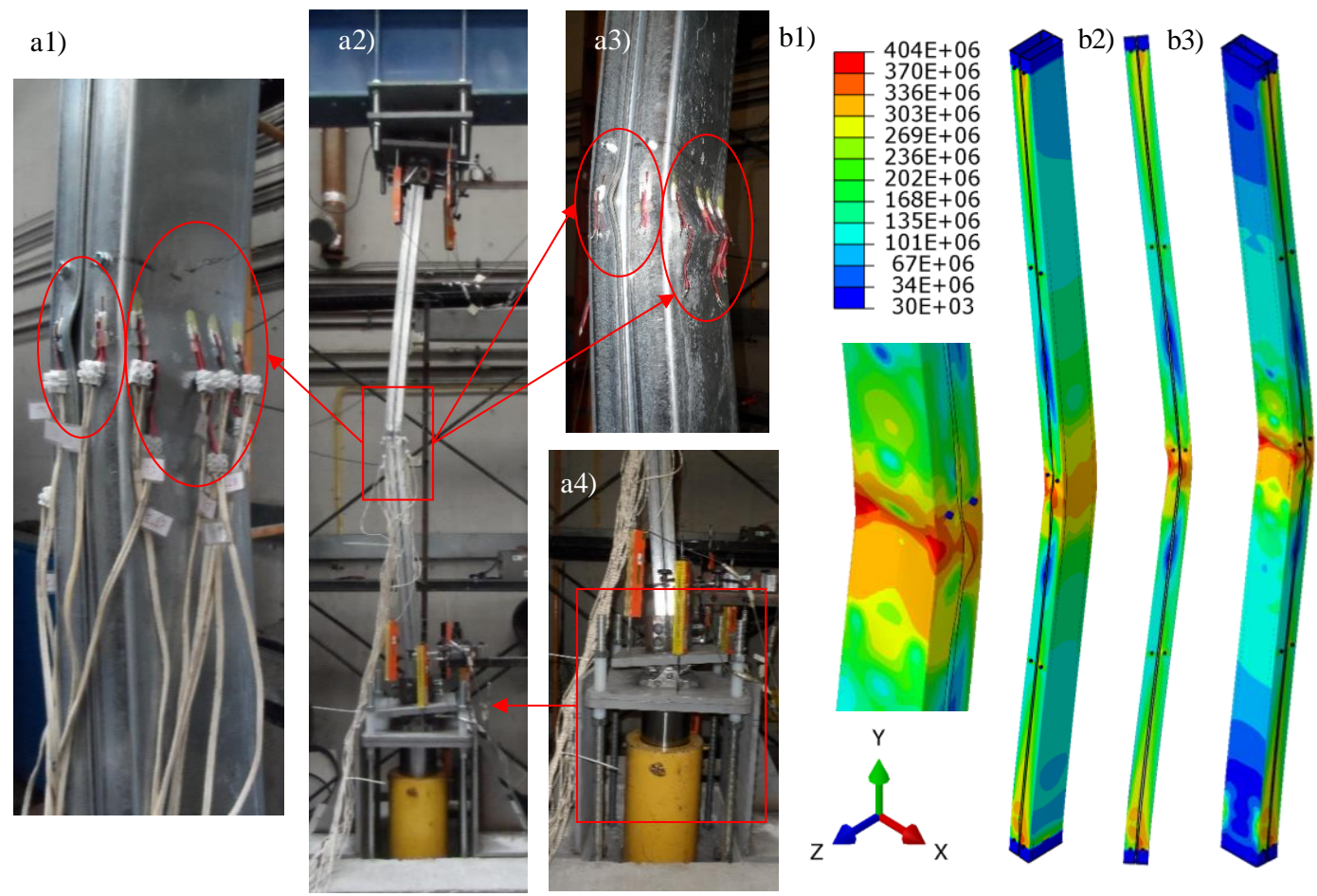

Figure 5.21 FEA (a) and experimental (b) failure modes for pinned-ended columns with closed built-up 2R cross-section.

\subsubsection{Fire tests with restraint to thermal elongation}

In this the obtained failure modes observed in the fire tests with restraint to thermal elongation are compared with the ones observed in the numerical simulations. This comparison is established in order to further validate the developed finite element model to reproduce the behaviour of CFS columns under simulated fire conditions with restraint to thermal elongation. In Figures 5.22 to 5.25 experimental and FEA failure modes observed are compared. Flexural buckling about the minor axis of the CFS columns, as well as local and distortional buckling modes were clearly identified in the finite element models. Hence, once again, it can be stated that the developed finite element model is able to accurately reproduce the failure modes observed in fire tests. In Figures 5.22 to 5.25 only the semi-rigid end-support condition is presented. For the remaining test conditions the agreement between experimental and FEA failure modes is also very good. 


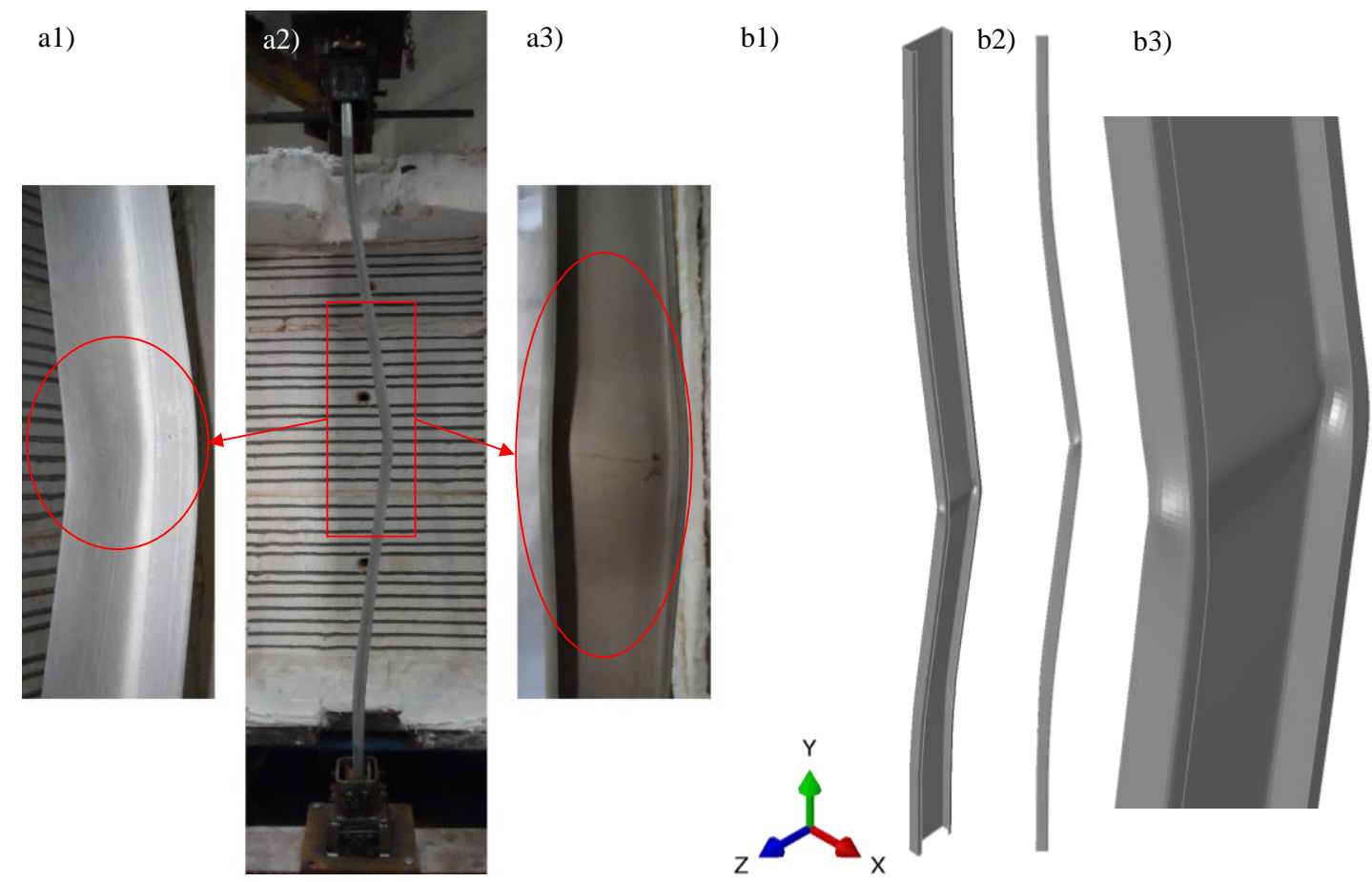

Figure 5.22 FEA (a) and experimental (b) failure modes for semi-rigid lipped channel columns.

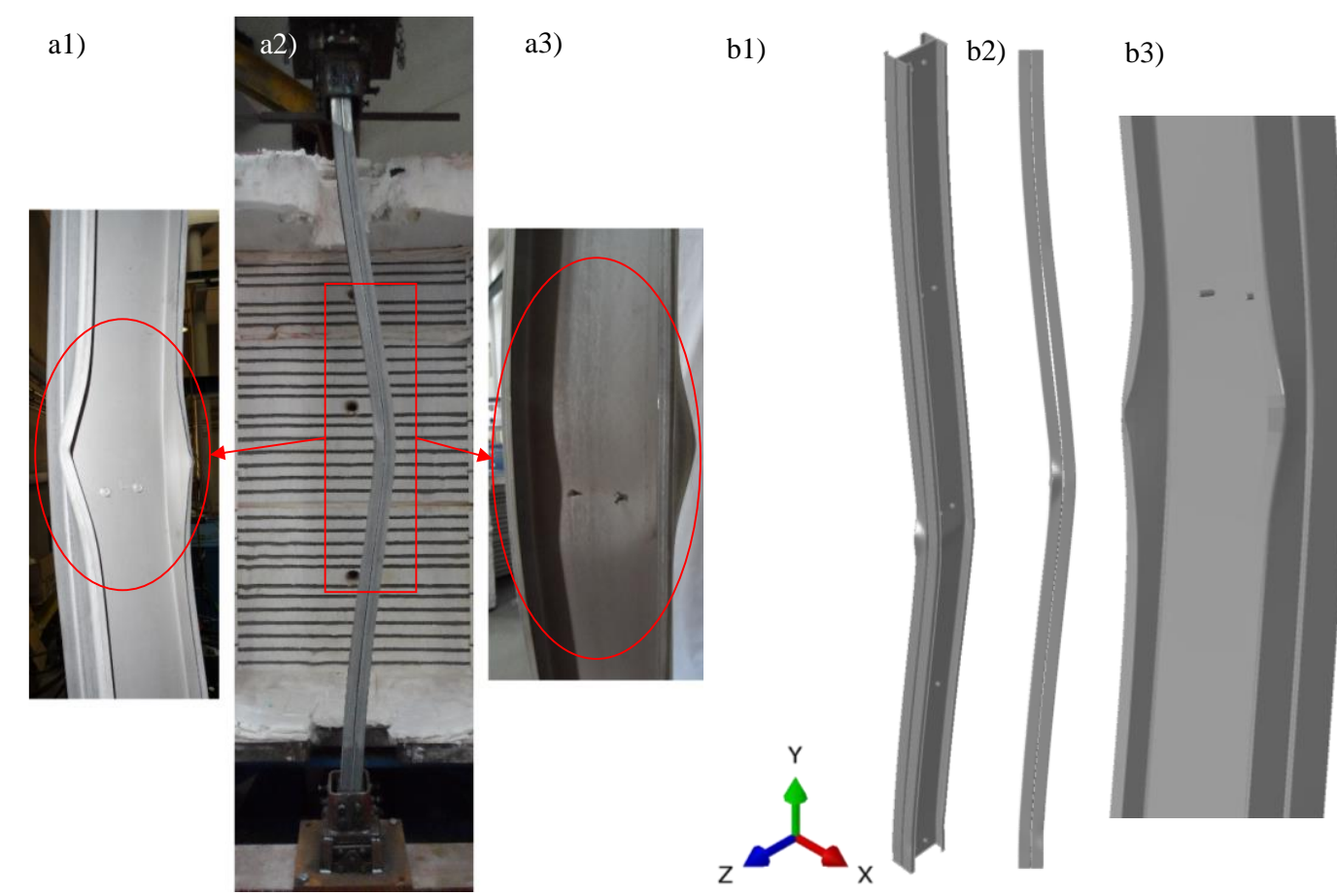

Figure 5.23 FEA (a) and experimental (b) failure modes for semi-rigid columns with open built-up I cross-section. 
5. NUMERICAL MODELLING OF COLD-FORMED STEEL COLUMNS

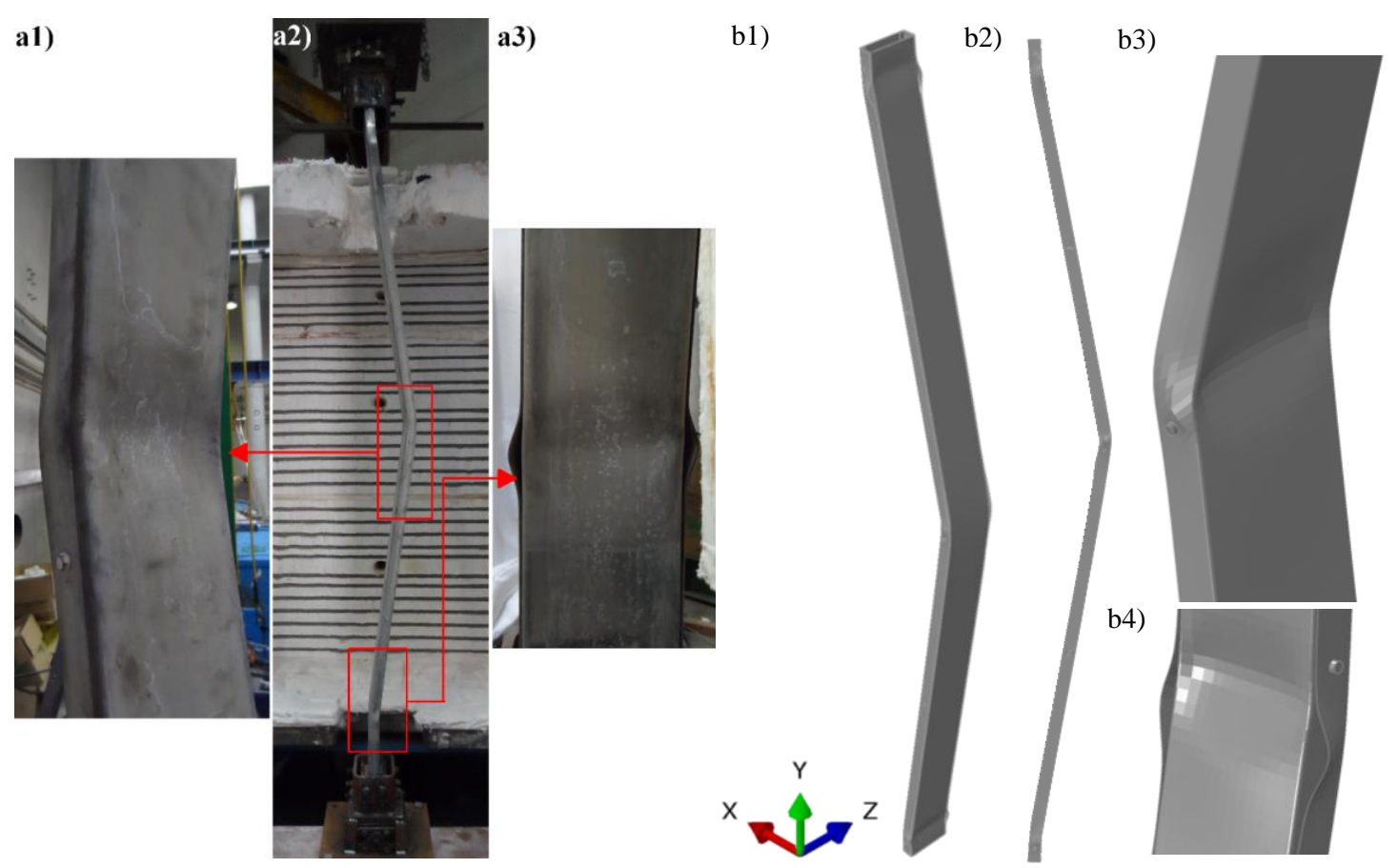

Figure 5.24 FEA (a) and experimental (b) failure modes for semi-rigid columns with closed built-up R cross-section.
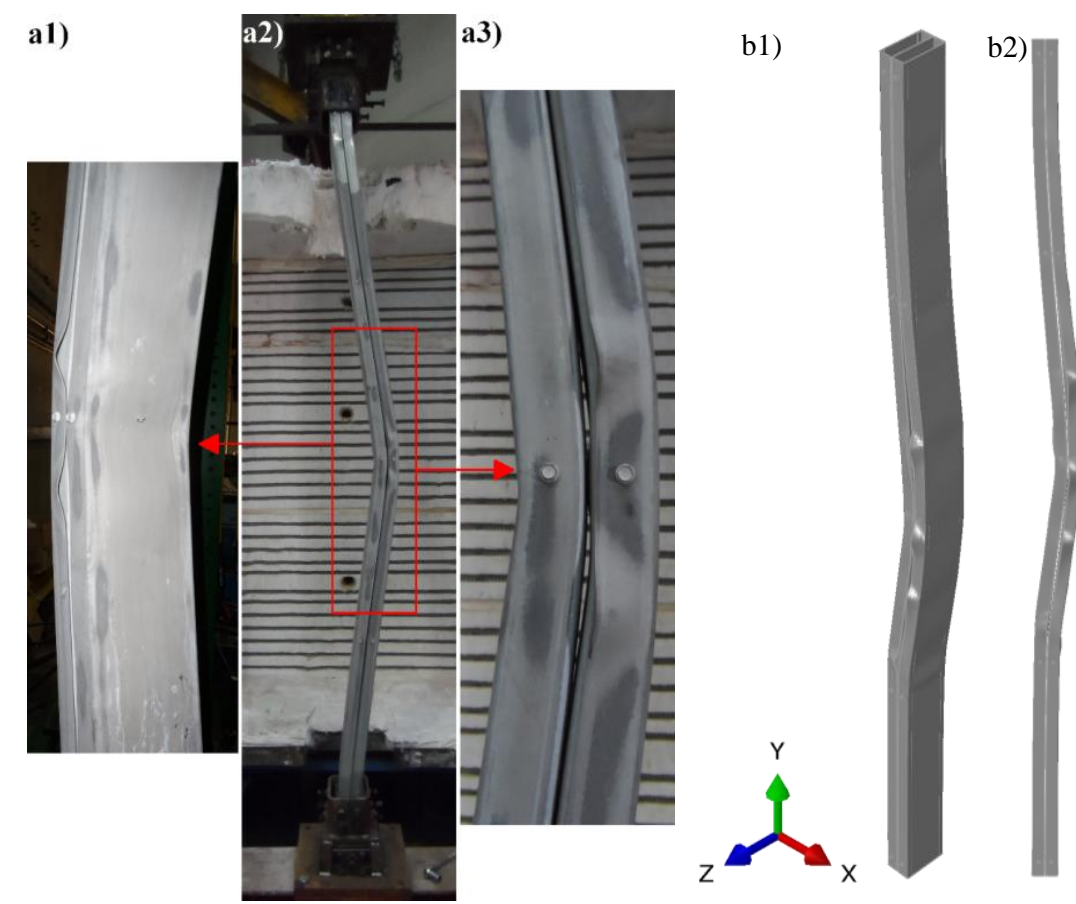

b3)

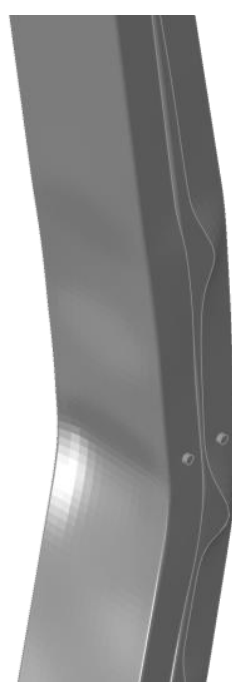

Figure 5.25 FEA (a) and experimental (b) failure modes for semi-rigid columns with closed built-up $2 \mathrm{R}$ cross-section. 


\subsection{Heat Transfer Analysis}

In this chapter the thermal model developed using the heat transfer analysis capabilities of ABAQUS is thoroughly detailed. It is intended, in this section, to determine the appropriate modelling parameters to define adequate thermal boundary conditions and material thermal properties (see Chapter 3) in order to simulate fire resistance tests of CFS single and built-up columns. The goal is to accurately reproduce temperature evolution/distribution in the TS3 cross-section (higher recorded temperatures) and then use the provisions presented in EN 19911-2 (2002) to determine the temperature distribution in the cross-section when the ISO 834 standard fire curve is the used thermal action. Bear in mind that in the experimental tests nonuniform temperature distribution was observed along the length of the column. Using the provisions in the EN 1991-1-2 (2002) and the ISO 834 standard fire curve a uniform temperature distribution along the length of the column will be adopted for future parametric studies.

In the finite element model, the fire action was modelled using two types of surface, namely film condition and radiant to ambient. These two types of surface correspond to heat transfer by convection and heat transfer by radiation, respectively. For calibration purposes the thermal action was the one recorded in the chamber of the furnace (gas furnace temperature). A 2D numerical model was developed to estimate temperature distribution in each cross-section tested, adopting a 4-node linear heat transfer or mass diffusion quadrilateral element, DC2D4. For all tested cross-sections the adopted convective heat transfer coefficient $\left(\alpha_{c}\right)$ was $16 \mathrm{~W} / \mathrm{m}^{2} \mathrm{~K}$ (lower than $25 \mathrm{~W} / \mathrm{m}^{2} \mathrm{~K}$ used for ISO 834 fire curve) for the fire test curves and the radiative heat flux was calculated using a steel (cold-formed steel with zinc coating) emissivity of 0.24 and 0.7 for the furnace electrical resistances $(\varepsilon=0.168)$. The Stefan-Boltzmann constant was $5.67 \times 10^{-8} \mathrm{~W} / \mathrm{m}^{2} \mathrm{~K}^{4}$. For future parametric studies the thermal action to be used is the ISO 834 standard fire curve and the coefficient of heat transfer by convection to be used is $25 \mathrm{~W} / \mathrm{m}^{2} \mathrm{~K}$ according to the EN 1991-1-2 (2002). The temperature distribution obtained from these numerical simulations will be used as input in the finite element structural models, considering a uniform temperature along the length of the column.

For the $\mathrm{R}$ and $2 \mathrm{R}$ cross-sections an additional consideration was adopted due to the existence of a volume with confined air, which as a low thermal conductivity. In this validation process air was assumed as a solid material, neglecting the heat transfer by convection in the cavity created. Hence, the thermal resistances to heat conduction developed at steel-to-steel and steelto-air interfaces were modelled considering thermal contact conductance coefficients of 200 and $10 \mathrm{~W} / \mathrm{m}^{2} \mathrm{~K}$, respectively. Moreover, thermal properties of air with temperature increase 
were also used as input in the model, namely thermal conductivity and specific heat of air (Figure 5.26). Finally, the specific weight was assumed constant and equal to $1.16 \mathrm{~kg} / \mathrm{m}^{3}$.
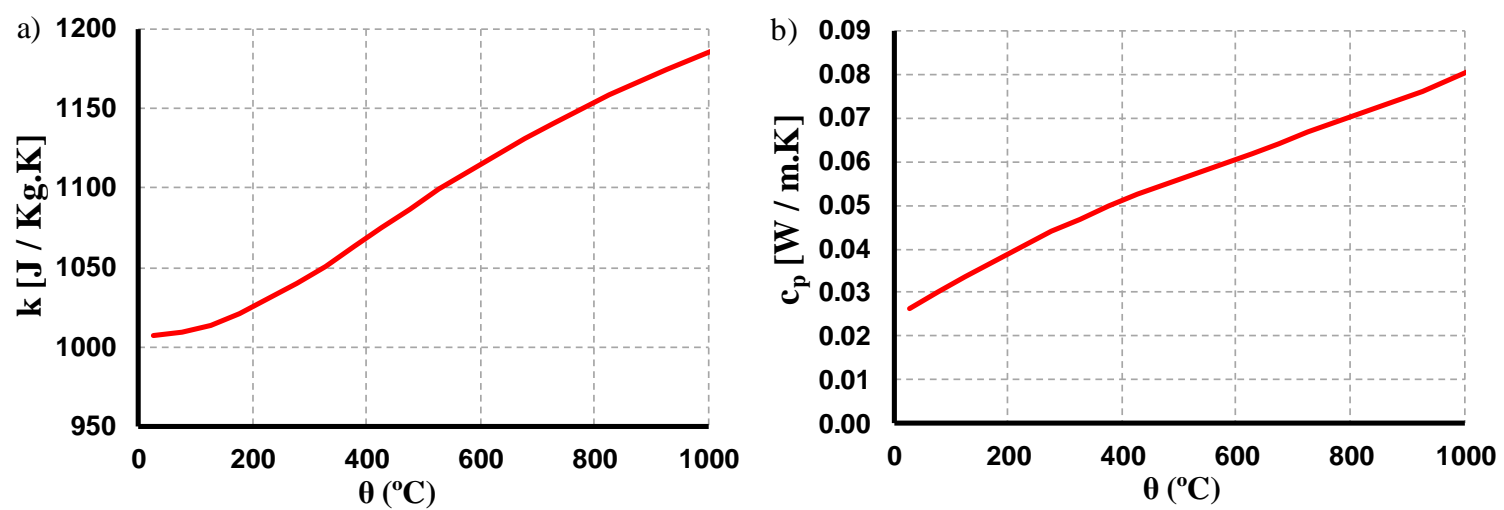

Figure 5.26 Thermal properties of air at one atmospheric pressure as a function of temperature. a) Thermal conductivity. b) Specific heat.

In Figures 5.27 to 5.30 a comparison between the experimental temperature evolution and the temperature evolution obtained in the numerical simulations is presented for all cross-sections. Generally, a very good agreement between experimental and numerical results, in terms of temperature evolution, was observed for all cross-sections. In Figure 5.31 the temperature evolution observed in the experimental tests and considering the ISO 834 (1999) fire curve as thermal action and the coefficient of heat transfer by convection equal to $25 \mathrm{~W} / \mathrm{m}^{2} \mathrm{~K}$, according to the EN 1991-1-2 (2002), is presented. As expected the temperature evolution is way more severe than the one observed in the experimental tests.

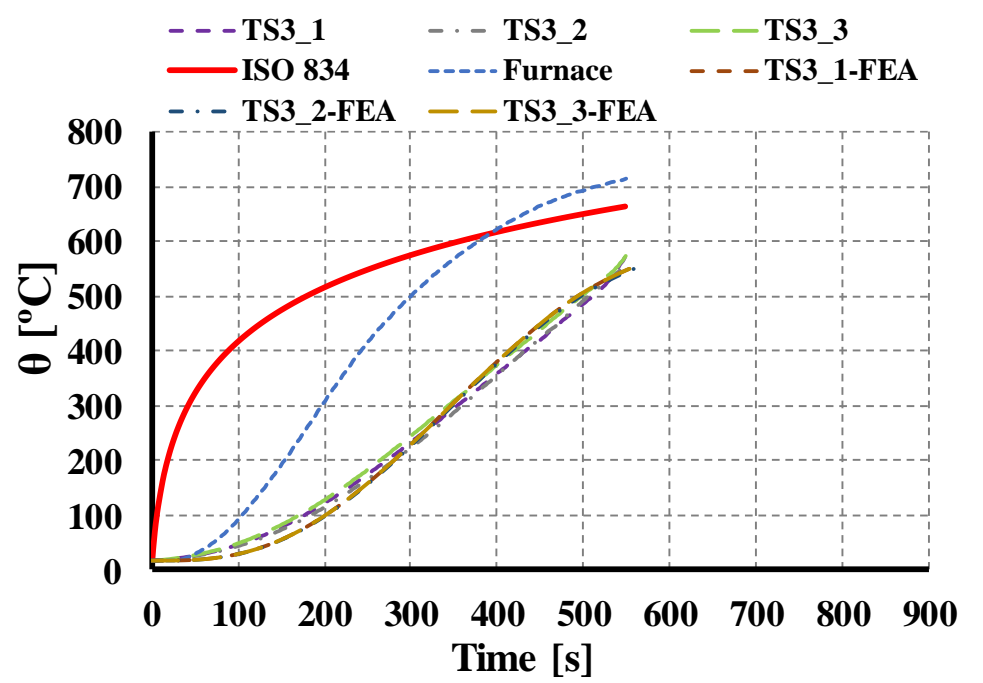

T.1

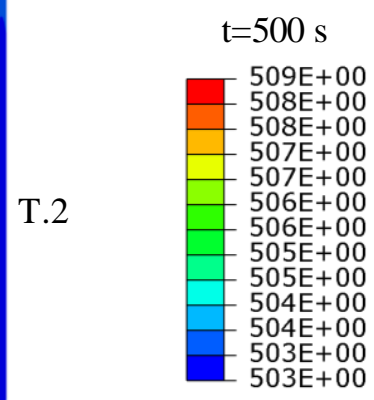

Figure 5.27 Comparison between test and FEA temperature evolution. C_SR_30LL_K3-1. 


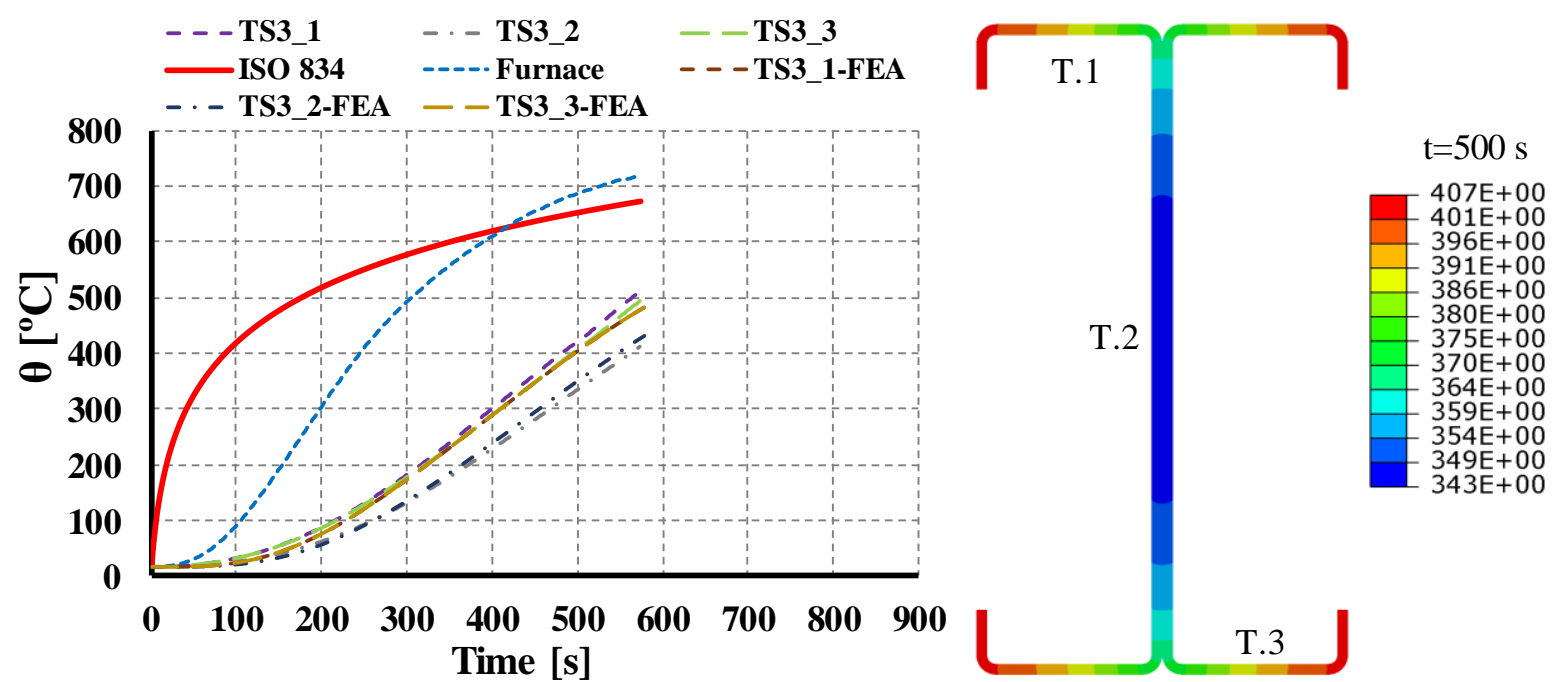

Figure 5.28 Comparison between test and FEA temperature evolution. I_SR_30LL_K3-2.
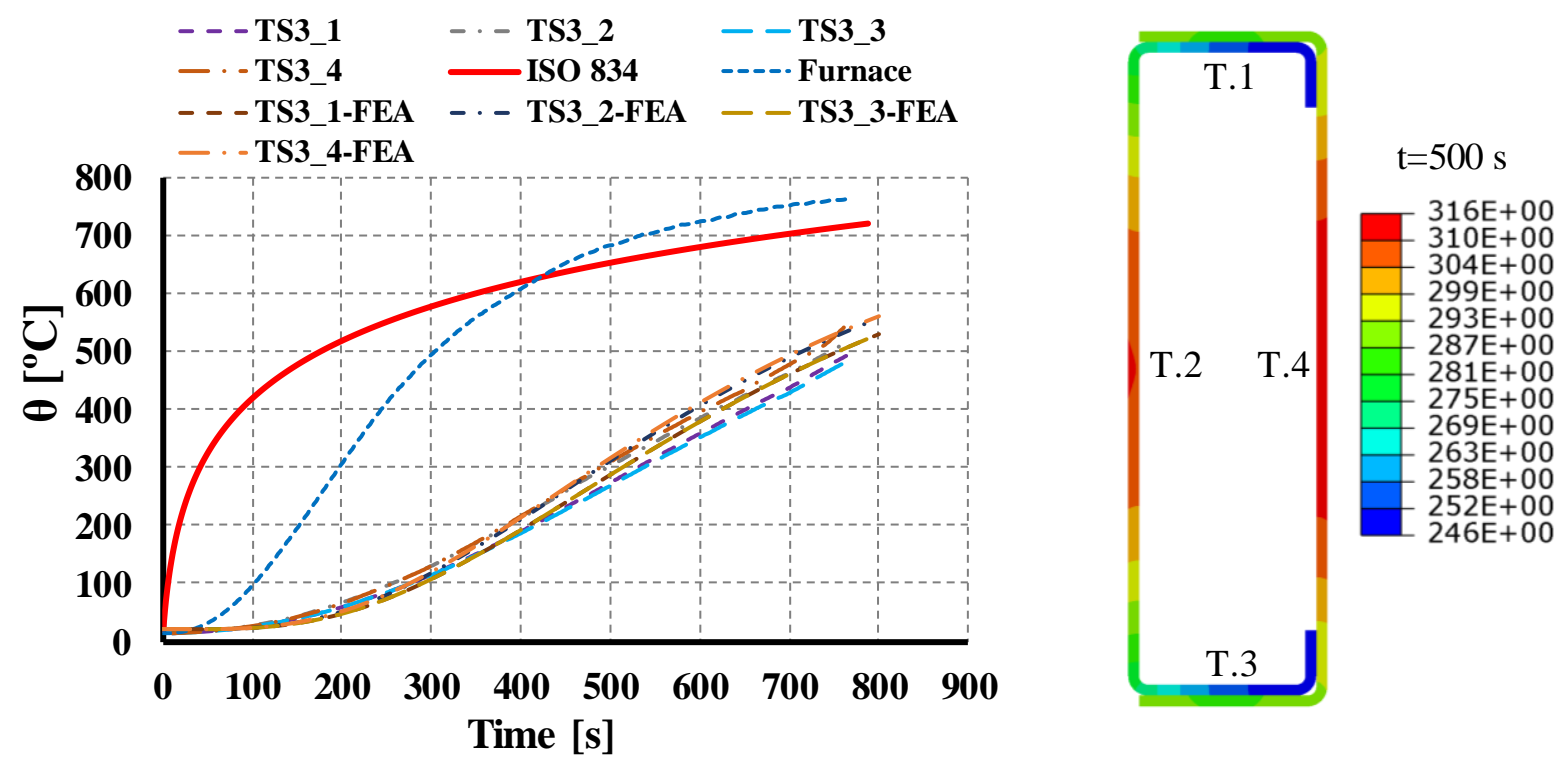

Figure 5.29 Comparison between test and FEA temperature evolution. R_SR_30LL_K3-2. 

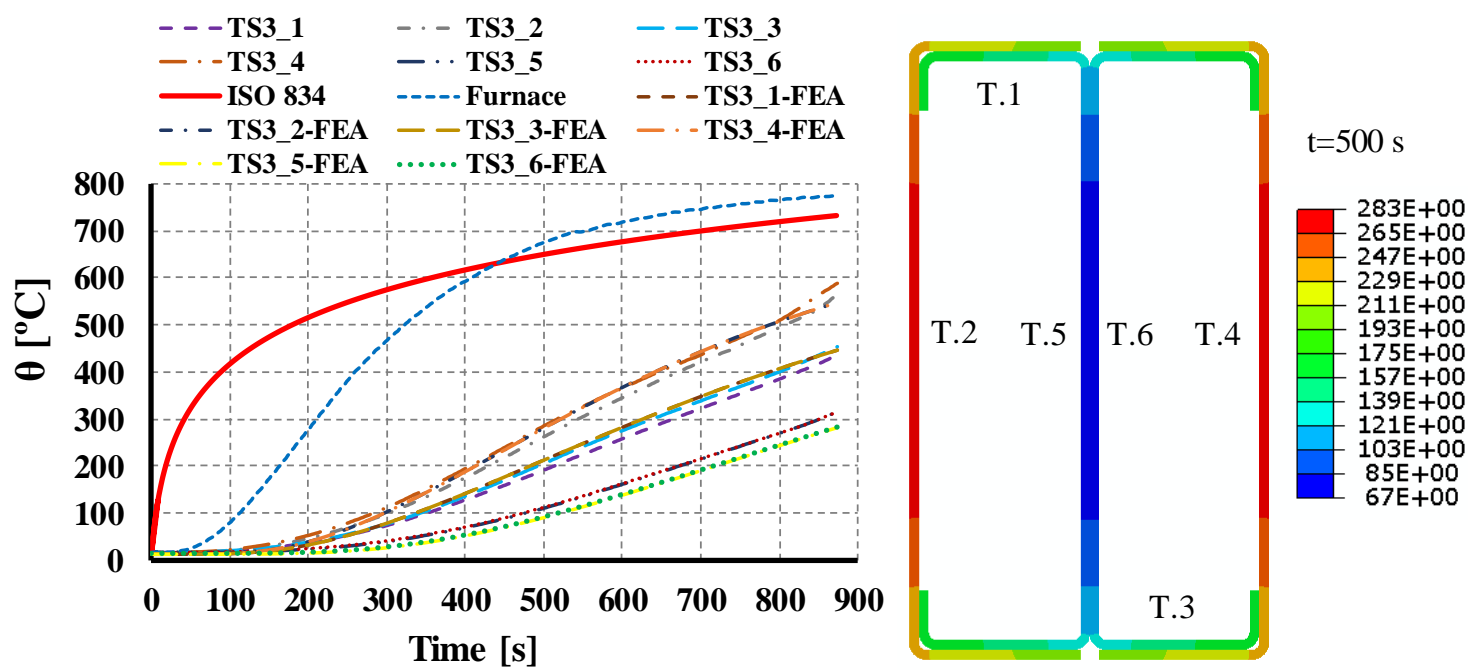

Figure 5.30 Comparison between test and FEA temperature evolution. 2R_SR_30LL_K3-1.

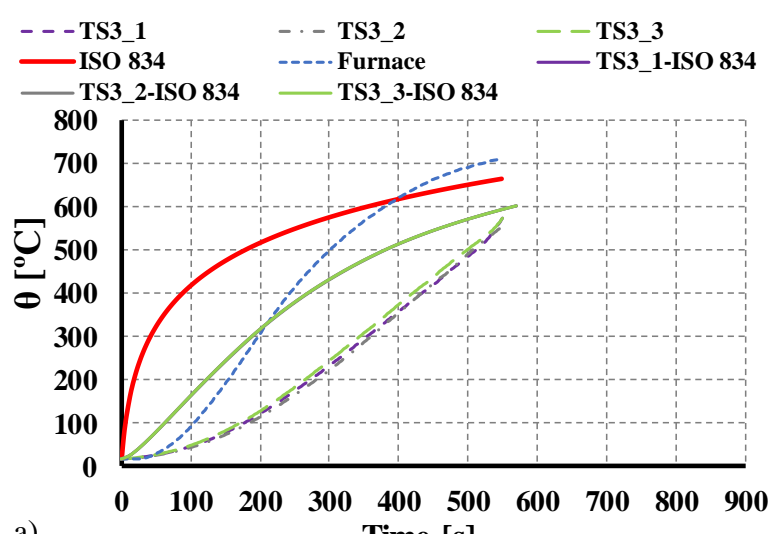

a)

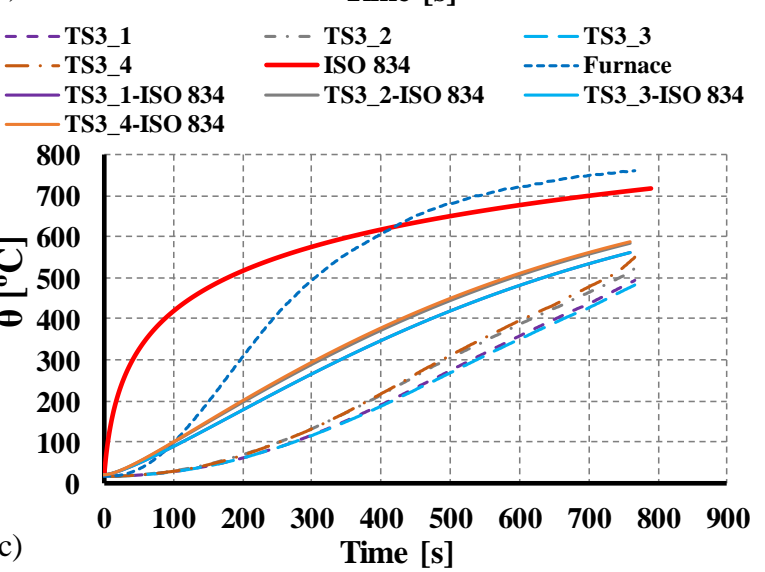

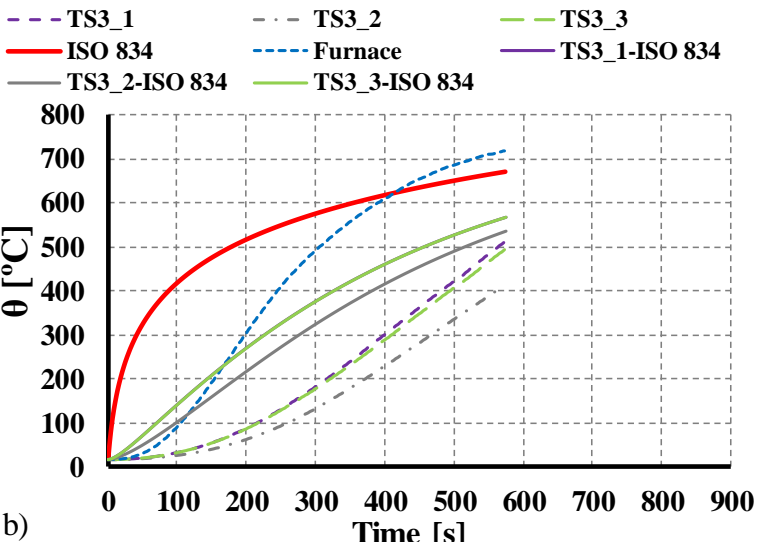

- - - TS3_1 - - TS3_2 - TS - TS3_3

$-\cdot-$ TS3_4 $\quad-\cdots$ TS3_5 $\quad \cdots \cdots \cdots \cdot$ TS3_6

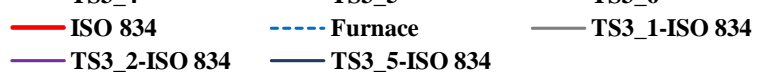

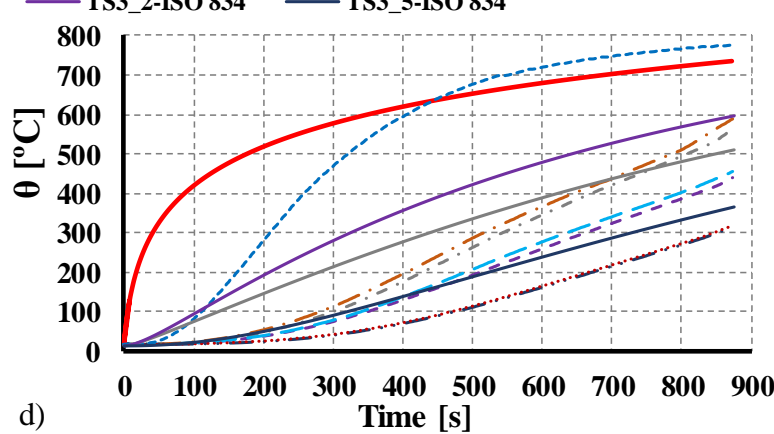

Figure 5.31 Comparison between test and FEA temperature evolution using the provisions presented in the EN 1991-1-2:2002 (2002) (ISO 834 as thermal action and coefficient of heat transfer by convection equal to $25 \mathrm{~W} / \mathrm{m}^{2} \mathrm{~K}$. a) C. b) I. c) R. d) $2 \mathrm{R}$ cross-section. 


\subsection{Final Remarks}

In this chapter the finite element models developed in order to reproduce the behavior of CFS columns at both ambient and simulated fire conditions with restraint to thermal elongation was thoroughly described. All assumptions were presented and described. At this point, and in the scope of this thesis the development and validation of the finite element model is presented. For the buckling tests the finite element model was validated in terms axial load versus endshortening of the columns as well as in terms of the observed failure modes. Comparing the obtained buckling load and the observed failure modes it was found a good agreement between experimental tests and FEA. Hence, the developed finite element model was able to reproduce the experimental conditions as consequently the behavior of CFS columns under compressive loading.

For the fire tests with restraint to thermal elongation the same conclusions can be drawn. Once again a good agreement between experimental and FEA was observed in terms of the maximum generated restraining forces, critical temperature and failure modes. Also it was found that rotational restraint has a positive effect on the fire resistance of CFS columns. The introduction of a hinge with a specific value of rotational stiffness leads to higher critical times and temperatures with low impact on the generated restraining forces.

Based on the presented analysis it is clear that the developed finite element model should be used for future parametric studies, in order to study the influence of several parameters outside the bounds of the experimental tests. For instance, at ambient temperature it is possible to study the influence of the cross-section geometry (height of the cross-section, width of the crosssection, thicknesses) and the influence of slenderness in the overall behaviour of the CFS columns. To study the fire resistance of CFS columns based on the developed finite element model, additional levels of axial and rotational restraint to thermal elongation. Columns with no restraint to thermal elongation (columns can freely expand) and columns fully restrained should be simulated using the available finite element model.

Finally, in the scope of this validation process the recommended value for global imperfection was $L / 1000$, for distortional imperfection was $t$ and for local imperfections $h / 200$. 



\section{CONCLUSIONS AND FUTURE WORK}

This thesis has described an extensive investigation on the behaviour of cold-formed steel columns with different types of cross-section shapes (from material characterization to behaviour of the structural element) at both ambient and fire conditions with restraint to thermal elongation. The presented investigation is mainly focused on experimental tests. Finite element analysis was undertaken to develop and validate the finite element model. Both investigations (experimental and numerical) were undertaken to improve the knowledge on the behaviour of cold-formed steel columns at both ambient temperature and simulated fire conditions. With the large experimental campaign undertaken was possible to gather significant data regarding material properties and behaviour of CFS columns at both ambient and simulated fire conditions.

In the first stage, the $\mathrm{S} 280 \mathrm{GD}+\mathrm{Z}$ steel was thoroughly characterized at both ambient and elevated temperatures. Mechanical and thermal properties were assessed in the experimental tests, namely modulus of elasticity, yield strength, thermal conductivity, relative thermal elongation and specific heat. In the second stage of this experimental research, the buckling load of different types of CFS columns was assessed and the observed failure modes characterized. In the third stage, the fire resistance of CFS columns with restraint to thermal elongation was assessed. In the last stage of this research work a finite element model capable of accurately reproduce the behaviour of CFS columns at both ambient and fire conditions with restraint to thermal elongation was developed and validated.

Generally, the most valuable outcomes of this research are as follows:

- All material properties determined and the correspondent proposals, namely the presented stress-strain relationship for the S280GD+Z steel, as well as the established comparisons with the available design standards;

- Assessment of the buckling load of CFS columns with single, open built-up and closed built-up cross-sections, commonly used in the building construction industry, as well as the observed failure modes;

- Assessment of the behaviour of CFS columns with different cross-section shapes, different end-support conditions, different applied load levels and different levels of imposed restraint to thermal elongation under simulated fire conditions;

- Development and validation of a finite element model able to accurately reproduce the behaviour of CFS columns at both ambient and simulated fire conditions with restraint 
to thermal elongation. The developed model should be used in the near future to conduct extensive parametric studies outside the bounds of the experimental tests. Different cross-section shape dimensions, length, and different levels of restraint to thermal elongation in case of fire should be tested in the parametric studies. The final goal would be the development/improvement of new/available design methods for the design of cold-formed steel single and built-up columns at both ambient and fire conditions with restraint to thermal elongation.

To sum up the presented results in this research can and will be used for other studies. Relevant data was gathered and reported in this thesis, from material characterization to the behaviour of a structural element at both ambient and elevated temperatures.

\subsection{Mechanical and Thermal Properties of the S280GD+Z Steel}

An experimental investigation on the mechanical and thermal properties of the S280GD+Z at elevated temperatures was presented and compared with other investigations and with the available predictions in design standards on this subject.

In the experimental campaign for the assessment of mechanical properties of the S280GD+Z steel with $2.5 \mathrm{~mm}$ thickness, tensile coupon tests were conducted at different temperatures, ranging from $20^{\circ} \mathrm{C}$ to $800^{\circ} \mathrm{C}$, using the steady state test method. Based on the stress-strain curves obtained for each temperature level, the yield strength, modulus of elasticity, proportional limit and ultimate strength were determined and the reduction factors for each property were obtained for each value of temperature. These results were then compared with the ones presented by other authors and with the design standards predictions such as EN 1993-1-2:2005 (2005), BS 5950-8 (1990) and AS 4100 (1998). It was found that for low strength cold formed steels such as the S280GD+Z, the yield strength predicted in the design standards are unconservative in the entire range of temperatures tested. The same conclusion can be observed for the modulus of elasticity and proportional limit. However, the BS 5950-8 (1990) does not provide any reduction factors for the modulus of elasticity and proportional limit whereas the AS 4100 (1998) does not present reduction factors for the proportional limit. Nevertheless, it is worth to mention that the reduction factors predicted in the EN 1993-1-2:2005 (2005) are the ones that best fit to the provided experimental results. In the scope of the research study a new set of predictive equations was presented for the yield strength, modulus of elasticity and proportional limit reduction factors. 
Creep may have significant influence for temperatures above $600^{\circ} \mathrm{C}$ and for higher stress levels hence specific creep tests must be conducted for the $\mathrm{S} 280 \mathrm{GD}+\mathrm{Z}$ steel at elevated temperatures (above $400^{\circ} \mathrm{C}$ ) in order to accurately assess the influence of creep and eventually understand how creep may affect the fire safety design of structures. In this experimental investigation the existent amount of creep is included in the experimental results as well as in the proposed formulation for stress-strain curves at elevated temperatures.

Also new predictive equations for stress-strain curves based on the Ramberg-Osgood model were proposed and compared with other proposals from other researchers. It was found that the proposal presented by Ranawaka and Mahendran (2009) also predict reasonably well the stressstrain curves for the $\mathrm{S} 280 \mathrm{GD}+\mathrm{Z}$ steel tested. The stress-strain curve model proposed by the EN 1993-1-2:2005 (2005) does not represent the behavior of low strength cold-formed steel at elevated temperatures even when the strain hardening model (Annex A, EN 1993-1-2:2005 (2005)) is included in the original formulation. Hence a new improved formulation of the stressstrain model is presented for the $\mathrm{S} 280 \mathrm{GD}+\mathrm{Z}$ steel. This new formulation conservatively predicts the stress-strain curves of the $\mathrm{S} 280 \mathrm{GD}+\mathrm{Z}$ and may be valid for low strength steels (up to 350 $\mathrm{MPa})$.

Regarding thermal properties of the $\mathrm{S} 280 \mathrm{GD}+\mathrm{Z}$ steel, it was found that the predictions available in the EN 1993-1-2:2005 (2005) are overconservative for the thermal elongation and thermal conductivity whereas for the specific heat the proposed model in the design standard fits well with the obtained results. An improved predictive equation was proposed for thermal elongation and thermal conductivity of the steel tested. Finally, it is recommended additional thermal properties tests in different types of steel in order to establish upper and lower limits for thermal conductivity as a function of the chemical composition of each steel.

\subsection{Experimental Analysis of Cold-Formed Steel Columns}

\subsubsection{Buckling tests}

An experimental investigation of the behaviour of CFS columns at ambient temperature was undertaken. Twenty-four buckling tests were conducted in this investigation. Four different cross-section shapes were tested, namely single lipped channel, open built-up I cross-section and closed built-up R and 2R cross-sections. The open built-up I columns consisted of two lipped channels fastened back-to-back on the web. The closed built-up R columns consisted of one lipped channel and one plain channel profile fastened in the flanges in order to build a closed section. The closed built-up 2R columns consisted of two lipped channels and 2 plain 
channels. The two lipped channels were fastened back-to-back (I) on the web and the plain channels were fastened to the lipped channels on the flanges. In the compressive tests two endsupport conditions were considered, namely pinned-end and fixed-end supports.

In these tests the advantages of using built-up members was clear, since the increase in the buckling load was significant. For instance, it was found that the buckling load of a column with closed built-up 2R cross-section was 9.4 times higher than the buckling load of columns with lipped channels, 3.35 times higher than the buckling load of columns with open built-up I cross-section and 3.62 times higher than the buckling load of columns with closed built-up R cross-section, for the pinned-end support condition. For fixed-end support condition, the buckling load of columns with $2 \mathrm{R}$ cross-section was 5.6 times higher than the buckling load of columns with lipped channels, 2.01 times higher than the buckling load of columns with open built-up I cross-section and 2.51 times higher than the buckling load of columns with closed built-up R cross-section, for the pinned-end support condition.

The obtained results were then compared with the design buckling loads determined according the EN 1993-1-2:2006 (2006). For pinned-ended lipped channel columns the design predictions are in good agreement with the experimental results, however for the fixed-ended columns the predictions may be conservative. This may be due to eccentricities in loading in the experimental tests. For built-up columns generally was found that design predictions are unsafe. However, it is worth to mention that at this stage it may not be adequate to draw firm conclusions, since the obtained results in the experimental tests may not represent exactly the lower and upper bounds of the buckling load of the tested columns. The end-supports used may not represent exactly a perfect pinned condition neither a fully fixed condition. Nevertheless, the obtained results are extremely important to validate the developed finite element model.

As some recommendations of good practice it is suggested that special care should be addressed to the assembly process of CFS columns with built-up cross-section. The assembly process may be influenced by human error, and as consequence the profiles may be misaligned leading to eccentricities in loading and consequently to lower buckling load of the CFS column. This problem may be critical for columns, since the load is applied at both ends of the member and the load should be equally distributed by all individual profiles. Hence, it is recommended that after assembly the ends of the cold-formed steel built-up member should be cut in order to guarantee a perfect alignment between all the individual profiles used in the built-up crosssection. Still on this matter, it may be possible to conduct some sensitivity simulations on the influence of misalignment of individual profiles on the overall behaviour of a cold-formed steel built-up column. 


\subsubsection{Fire tests with restraint to thermal elongation}

In this experimental research ninety-six fire resistance tests with restraint to thermal elongation were undertaken on CFS columns with different cross-sections, namely, C, I, R and 2R. In order to assess and understand the behaviour of CFS columns under simulated fire conditions several parameters were tested, namely, cross-section-shape, end-support conditions, load level initially applied and finally different levels of restraint to thermal elongation imposed to the tested columns. For each tests condition defined three repetitions were conducted.

The main conclusion of this investigation was that the interaction between the initial applied load and the imposed level of restraint to thermal elongation may significantly influence the overall behaviour of isolated CFS columns under fire conditions. If a column could freely expand when subjected to fire, no additional forces would be generated. However, when some level of restraint exists additional forces are generated, which may lead to premature collapse and consequently to lower critical times and temperatures. Load, as expected, is another important parameter in the fire behaviour of CFS columns. Columns subjected to higher service loads present lower critical times and temperature.

Generally, it was found that increasing the restraint to thermal elongation lead to a reduction in critical times and temperatures. However, and exclusively for the pinned-end support condition and for $\mathrm{C}$, I and R columns the reduction in critical temperature is small. However, with the increase of the level of restraint to thermal elongation ( 3 to $13 \mathrm{kN} / \mathrm{mm}$ ) the maximum generated restraining forces occurs to much lower mean temperatures of the column. This evidence was observed in all tests conducted with higher levels of restraint. Hence the load level and level of imposed restraint may lead to an increase in the generated restraining forces and as a consequence to a premature collapse. For pinned-ended columns with $2 \mathrm{R}$ cross-section increasing the level of restraint to thermal elongation lead to significant reduction in the critical temperature (about $70^{\circ} \mathrm{C}$ ). For the semi-rigid end-support condition increasing the level of restraint lead to a reduction in critical times and temperatures for all tested cross-sections. However, it is worth to mention that once again the lipped channel columns presented the smallest reduction, and columns with closed built-up cross-section presented the highest reduction in critical temperature. It seems that increasing the level of restraint to thermal elongation the failure of closed built-up cold-formed steel columns may be controlled by the generated axial restraining forces whereas for lower levels of restraint the failure is controlled by the temperature increase.

Probably one of the most important parameters that influence the behaviour of cold-formed steel columns under fire conditions is the ratio between the axial stiffness of the surrounding 


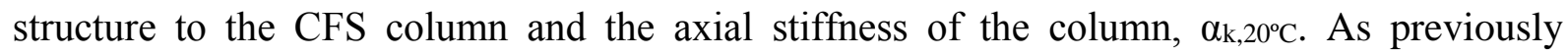

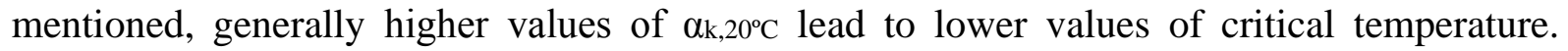

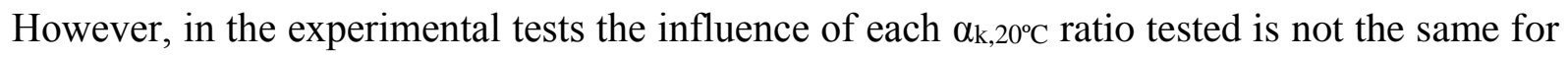
each type of CFS column tested. Hence, as a conclusion and as a suggestion for future research work the values of restraint to thermal elongation to be tested in numerical simulations for each

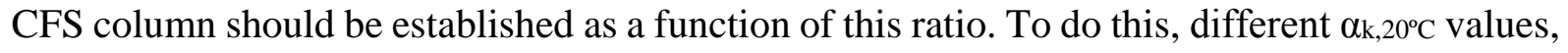
ranging from 0 (column can freely expand) to 1 (column fully restrained) should be tested.

In terms of evolution of temperatures in the cross-section it was observed that it depends greatly on the cross-section shape. The observed temperature increase rate was different for all tested cross-sections, namely about $43^{\circ} \mathrm{C} /$ minute for $2 \mathrm{R}$, about $50^{\circ} \mathrm{C} /$ minute for $\mathrm{R}$, about $60^{\circ} \mathrm{C} /$ minute for I and about $76^{\circ} \mathrm{C} /$ minute for $\mathrm{C}$ cross-section. The closed built-up cross-sections presented the lower temperature increase rate, mainly due to the existence of confined air in the interior of the built-up cross-sections, which has low thermal conductivity and also due to the fact that only external profiles (in the case of the $2 \mathrm{R}$ cross-section) were directly exposed to radiation.

Regarding the observed failure modes some slight differences were observed in function of the adopted boundary conditions. For instance, for the pin-end support condition the predominant failure mode was the global buckling about the minor axis for all cross-sections. However distortional and local buckling was also observed, especially for the $2 \mathrm{R}$ cross-section, but it seems that these buckling modes only become noticeable for large lateral deformations. Regarding the semi-rigid end-support condition the interaction between global flexural buckling about the minor axis and distortional buckling was clear for all types of columns. Distortional buckling was clearly observed at mid-height of the CFS columns and at about 40 $\mathrm{cm}$ from the end-support devices.

After analysing the behaviour of CFS columns at both ambient and elevated temperatures additional conclusions/recommendations may be introduced. Specifically, for the closed builtup cross-sections and for both ambient temperature and fire tests was observed that the lipped channels used in those sections did not present relevant sectional buckling modes. In the tests it seems that from the moment the plain channels failed the built-up cold-formed steel column collapsed. Hence, it can be suggested that additional studies should be undertaken in order to assess the behaviour of built-up columns using only plain channels (U). This may result in a major economic advantage without significant reduction in the structural response of the builtup member under compressive loading. 
Finally, and regarding the developed test set-up it is worth to mention that the pinned-end conditions brings additional complications to the execution of the experimental tests, especially at elevated temperatures. Friction may significantly influence the overall behaviour of a CFS column.

To conclude, it is worth mentioning the low fire resistance presented by all tested columns under all tested conditions.

\subsection{Finite Element Analysis}

Based on the obtained experimental results a finite element model, using the software ABAQUS, was developed in order to accurately reproduce the observed experimental behaviour of CFS columns at both ambient and fire conditions with restraint to thermal elongation. The developed finite element model was thoroughly described.

Comparing the results obtained in the FEA with the experimental results, generally, a good agreement was observed, proving that the FEA is a very reliable tool to simulate the behaviour of CFS columns at both ambient and elevated temperatures with restraint to thermal elongation. The comparison was established in terms of maximum axial restraining forces generated, critical temperature and observed failure modes.

In the validation process of the finite element model it was found that rotational restraint has a positive effect on critical temperature. For pinned-ended columns a hinge, acting as a rotational spring, with linear behaviour, imposing additional rotational restraint about the minor axis, was adopted in the finite element model. This hinge, acting as rotational spring, allows testing of semi-rigid end-support conditions with different levels of rotational restraint.

Also it was found that the used mechanical and thermal properties, determined in the scope of this research, as input in the finite element model are more accurate than the ones available in the EN 1993-1-2:2005 (2005). Using the determined properties, the agreement between experimental and FEA was significantly better than when the mechanical and thermal properties presented in the EN 1993-1-2:2005 (2005) were used as input. Nevertheless, the developed finite element model may still be improved. For instance, the material model, based on the EN 1993-1-2 (2005) formulation, proposed in this research should also be tested in the finite element model 
Finally, the developed finite element model should be extensively used in the future to conduct large parametric studies that will provide sufficient data to develop/improve new/available design methodologies.

\subsection{Future Research Work}

Full understanding of the behaviour of cold-formed steel columns with single and built-up cross-sections still needs additional investigation at both ambient and elevated temperature. For instance, additional studies on built-up members should be undertaken in order to accurately assess its behaviour and the possible interaction between individual buckling modes, such as flexural, distortional and local buckling. The study of the interaction between different buckling modes still represents a relevant challenge on the field of cold-formed steel structural elements.

Also the characterization of material properties, especially at elevated temperatures, still needs additional studies. Specifically, different steel grades and even different thicknesses should be tested (low and high strength steels) considering both steady state and transient state test methods, in order to accurately assess the degradation of the mechanical properties with temperature increase. Creep tests at elevated temperatures should also be conducted in order propose new predictive models that could be used in finite element models.

Following the reported research on this thesis it is fundamental to conduct extensive parametric studies using the developed and validated finite element model. Finite element analysis requires less time and resources. Hence, additional parameters, outside the bounds of the conducted experimental test, can be investigated, such as slenderness (variation of cross-section geometry, variation of the length of the column, variation of thickness of the cross-section), influence of the initial applied load level, and influence of axial and rotational restraint imposed to the coldformed steel columns under fire conditions. Using the results to be obtained in the parametric studies, comparisons must be established with the predictions of the currently available design standards. The ultimate goal is to improve/develop available/new design methodologies for cold-formed steel columns with single and built-up cross-sections at both ambient and elevated temperatures. To improve the knowledge on the behaviour of cold-formed steel columns additional experimental tests should be undertaken, especially considering other cross-section shapes, such as sigma $(\Sigma)$, zed $(Z)$, double lipped channel and also built-up sections composed by the mentioned single sections. Is also important, and recommended, to conduct numerical imperfection sensitivity analysis on CFS columns with single and built-up cross-sections. 
Another interesting topic to be addressed regarding the structural performance of cold-formed steel columns, with built-up cross-sections, is the spacing between fasteners along the length of the column. Experimental and numerical investigations should be conducted to assess the influence of connection spacing in the behaviour of CFS columns. Different spacing between fasteners should be tested, for instance ranging from 300 to $800 \mathrm{~mm}$.

Since all tested CFS columns showed low fire resistance it may be interesting to suggest some investigation on the behaviour of CFS columns with some type of protection. However, it is not intended to suggest additional studies on commonly used fire proof materials such as gypsum boards, since the aesthetics advantage of this type of structural solution is completely lost with the use of gypsum boards. The use of cementitious based fire proof materials seems an interesting solution that can be used, or alternatively the use of concrete as a fire protection material. Considering this suggestion two situations should be considered, namely, using concrete confined in a closed built-up cross-section (for instance cross-section $\mathrm{R}$ and $2 \mathrm{R}$ ) or using the cold-formed steel profiles as a replacement to the traditional reinforcement (protection against corrosion). In the first suggestion the aesthetics advantage is maintained. Hence, it is proposed the study of composite cold-formed steel columns at both ambient and fire conditions, considering concrete as a structural material or simply as a fire proof material. 



\section{REFERENCES}

AçoPotiguar@ (2015), http://acopotiguar.com.br, building construction company, Brazil, (Accessed: 5th August 2015).

Ádány, S., Schafer, B.W. (2006a), Buckling mode decomposition of single-branched open cross-section members via finite strip method: Derivation, Thin-Walled Structures, Vol. 44, pp. 563-584.

Ádány, S., Schafer, B.W. (2006b), Buckling mode decomposition of single-branched open cross-section members via finite strip method: Application and examples, Thin-walled Structures, Vol. 44, pp. 585-600.

AISC (2010), Specification for structural steel buildings, Appendix 4: Structural design for fire conditions. American Institute of Steel Construction, Chicago, IL.

AISI S100-1996: AISI specification for the design of cold-formed steel structures members. American Iron and Steel Institute, Washington (DC), USA; 1996.

Amada America@ (2015), http://www.amada.com, Sheet metal fabrication machines (Accessed: 22 ${ }^{\text {nd }}$ August 2015).

American Iron and Steel Institute, AISI (2001). North American Specification for the design of cold-formed steel structural members, Washington, DC, American Iron and Steel Institute, USA.

AISI S100-2007 (2007), American Iron and Steel Institute, AISI (2007), North American specification for the design of cold-formed steel structural members, Washington, DC, American Iron and Steel Institute, USA.

AISI S100-2012 (2012) North American Specification for the design of cold-formed steel structural members. American Iron and Steel Institute, Washington (DC), USA.

AISI S100-2004 - Appendix 1: Design of cold-formed steel structural members using the Direct Strength Method. In: 2004 supplement to the North American Specification for the design of cold-formed steel structures. American Iron and Steel Institute, Washington (DC), USA; 2004.

Ali, F.A., Shepherd, P., Randall, M., Simms, I., O’Connor, D., Burgess, I. (1998), The effect of axial restraint on the fire resistance of steel columns. Journal of Constructional Steel Research, Vol. 46, No. 177, pp. 305-306. 
Ali F. A., O’Connor D. (2001), Structural performance of rotationally restrained steel columns in fire. Fire Safety Journal, Vol. 36, pp. 679-691.

ANSYS ${ }^{\circledR}$ Academic Research (2015), Release 16.2, Help System, Ansys, Inc. (Accessed: $22^{\text {nd }}$ September 2015).

AS 4100 (1998): Steel structures. Australia standard, Sydney, Australia.

AS/NZS 4600 (1996): Cold formed steel structures. New Zealand standard, Australia.

Barsom, J.M. and Rolfe, S.T. (1999), Fatigue and Fracture Control in Structures: Application of fracture mechanics. American Society for Testing and Materials (ASTM), USA.

Batista, E.M. (2009), Local-global buckling interaction procedures for the design of coldformed columns: Effective width and direct method integrated approach, Thin-Walled Structures, Vol. 47, pp. 1218-1231.

Batista, E.M. (2010), Effective section method: A general direct method for the design of steel cold-formed members under local-global buckling interaction, Thin-Walled Structures, Vol. 48, pp. 345-356.

Bebiano, R., Pina, P., Silvestre, N., Camotim, D. (2008), http://www.civil.ist.utl.pt/gbt, GBTUL $1.0 \beta$ - Buckling and vibration analysis of thin-walled members, DECivil/IST, Technical University of Lisbon.

Bebiano, R., Silvestre, N., Camotim, D. (2008b). GBTUL - a code for the buckling analysis of cold-formed steel members. In: Laboube R., Yu, W.-W., editors. Proceedings of $19^{\text {th }}$ International Specialty Conference on Recent Research and Developments in Cold-Formed Steel Design and Construction (St. Louis, 14-15/10), pp. 61-79.

Brnic, J, et al. (2014) Comparison of material properties:Steel 20MnCr5 and similar steels. Journal of Constructional Steel Research, 95, pp. 81-89.

Brnic J., et al. (2009), Behavior of AISI 316L steel subjected to uniaxial state of stress at elevated temperatures. J. Mater. Sci. Technol., Vol 25 (02), pp. 175-180.

Brnic J., Canadija, M., Turkalj, G., Lanc, D. (2010) Behavior of S355JO steel subjected to uniaxial stress at lowered and elevated temperatures and creep. Bull Mater Sci, Vol 33, pp. 475489.

Brnic, J., Turkalj, G., Canadija, M., Lanc, D. (2011), AISI 316Ti (1.4571) steel-mechanical creep and fracture properties versus temperature. J. Constr Steel Res, Vol. 67(12), pp. 1948-52.

Brnic, J., Turkalij, G., Niu, J., Canadija, M., Lanc, D. (2013) Analysis of experimental data on the behavior of steel S275JR - Reliability of modern design. Materials and Design, Vol. 47, pp. 497-504. 
BS 5950-8 (1990), Structural Use of Steelwork in Building - Part 8: Code of Practice for Fire Resistance Design. British Standards Institution (BSI), London, UK.

Camotim, D., Dinis, B. (2011), Coupled instabilities with distortional buckling in cold-formed steel lipped channel columns, Thin-Walled Structures, Vol. 49, pp. 562-575.

Chajes, A., Britvec, S.J., Winter, G. (1963), Effect of cold-straining on structural sheet steels, Journal of the Structural Division, Proceedings of the American Society of Civil Engineers, Vol. 89, n. ST2, pp. 1-32.

Chen, J., He, Y., Jin, W.-L. (2010), Stub column tests of thin-walled complex section with intermediate stiffeners, Thin-Walled Structures, Vol. 48, pp. 423-429.

Chen, J., Young, B. (2006), Corner properties of cold-formed steel sections at elevated temperatures, Thin-Walled Structures, Vol. 44, pp. 216-223.

Chen, J., Young, B. (2007a), Experimental investigation of cold-formed steel material at elevated temperatures, Thin-Walled Structures, Vol. 45, pp. 96-110.

Chen, J., Young, B. (2007b), Cold-formed steel lipped channel columns at elevated temperatures, Engineering Structures, Vol. 29, pp. 2445-2456.

Correia, A. J. M. and Rodrigues, J. P. C. (2011), Fire Resistance of Partially Encased Steel Columns with Restrained Thermal Elongation, Journal of Constructional Steel Research, vol. 67, Issue 4, pp 593-601.

Correia, A. J. M. \& Rodrigues, J. P. C. (2012), Fire Resistance of Steel Columns with Restrained Thermal Elongation, Fire Safety Journal, vol. 50, May 2012, pp 1-11.

Cowan M., Khandelwal K. (2014), Modelling of high temperature creep in ASTM A992 structural steels. Engineering Structures, Vol. 80, 426-434.

Dassault Systèmes Simulia Corp., 2012, http://www.3ds.com/products/simulia/overview/, Abaqus Analysis - User's Manual, version 6.12-1, USA (Accessed: $14^{\text {th }}$ September 2015).

Dhanalakshmi, M. and Shanmugam, N.E. (2001), Design for Openings in Cold-Formed Steel Channel Stub Columns, Thin-Walled Structures, Vol. 39, Issue 12, pp. 961-981.

Dinis, P.B., Camotim, D. (2011), Post-buckling behaviour and strength of cold-formed steel lipped channel columns experiencing distortional/global interaction, Computers and Structures, Vol. 89, pp. 422-434.

Dreistern@ (2015), http://www.dreistern.com (Accessed: 11 ${ }^{\text {th }}$ July 2015). 
Dubina, D., Ungureanu, V. (2002), Effect of imperfections on numerical simulation of instability behaviour of cold-formed steel members, Thin-Walled Structures, Vol. 40, pp. 239262.

Dubina, D., Ungureanu, V., Landolfo, R. (2012), Design of cold-formed steel structures. Eurocode 3: Design of steel structures. Part 1-3: Design of cold-formed steel structures, ECCS - European Convention for Constructional Steelwork, Wiley-Blackwell, 676 p.

ECCS (1987). European recommendations for design of light gauge steel members, Publication P049, European Convention for Constructional Steelwork, Brussels.

Ellobody, E., Young, B. (2005), Behavior of Cold-Formed Steel Plain Angle Columns, Journal of Structural Engineering, Vol. 131, No. 3, pp. 457-466.

EN 1991-1-2 (2002), Eurocode 1: Actions on structures, Part 1-2: General actions - Actions on structures exposed to fire, European Committee for Standardisation, Brussels , $60 \mathrm{p}$.

EN 1993-1-1 (2004), Eurocode 3: Design of steel structures, Part 1-1: General rules and rules for buildings, European Committee for Standardisation, Brussels, $91 \mathrm{p}$.

EN 1993-1-2 (2005), Eurocode 3: Design of steel structures, Part 1-2: General rules, Structural fire design, European Committee for Standardisation, Brussels , 78 p.

EN 1993-1-3 (2006), Eurocode 3: Design of steel structures, Part 1-3: General rules, Supplementary rules for cold-formed members and sheeting, European Committee for Standardisation, Brussels, $125 \mathrm{p}$.

EN 1993-1-5 (2006), Eurocode 3: Design of steel structures, Part 1-5: Plated structural elements, European Committee for Standardisation, Brussels, 55 p.

EN 10002-1 (2001): Metallic materials-tensile Testing: I. Technique of test at ambient temperature, European Committee for Standardization (CEN), Brussels.

EN 10002-5 (1992): Metallic materials- tensile testing - Part 5: method of testing at elevated temperature, European Committee for Standardization (CEN), Brussels.

EN 10147 (2002), Specification for continuously hot-dip zinc coated structural steel sheet Technical delivery conditions, European Committee for Standardisation, CEN, Brussels

Epsilon (2011), http://www.epsilontech.com/ (Accessed: 21 ${ }^{\text {st }}$ June 2015).

Eurocode 3: (1996) CEN ENV 1993-1-3: Design of Steel Structures, General rulesSupplementary rules for Cold-Formed Thin Gauge Members and Sheeting, European Committee for Standardisation, CEN, Brussels. 
FAA AR MMPDS-01 (2003) - Metallic material properties development and standardization, US Department of Transportation, federal Aviation Administration, Washington DC.

Faggiano B., Matteis G.D., Landolfo R. and Mazzolani F.M. (2004), Behaviour of aluminium alloy structures under fire, Journal of Civil Engineering and Management, vol. X; 3, pp.183190.

Feng, M., Wang, Y.C., Davies, J.M. (2003a), Thermal performance of cold-formed thin-walled steel panel systems in fire, Fire Safety Journal, Vol. 38, pp. 365-394.

Feng M., Wang, Y.C., Davies, J.M. (2003b), Axial strength of cold-formed thin-walled steel channels under non-uniform temperatures in fire, Fire Safety Journal, Vol. 38, pp. 679-707

Feng, M., Wang, Y.C., Davies, J.M. (2003c), Structural behaviour of cold-formed thin-walled short steel channel columns at elevated temperatures. Part 2: Design calculations and numerical analysis, Thin-Walled Structures, Vol. 41, pp. 571-594.

Feng, M., Wang, Y.C. and Davies, J.M. (2004), A Numerical Imperfection Sensitivity Study of Cold-Formed Thin-Walled Tubular Steel Columns at Uniform Elevated Temperatures, ThinWalled Structures, Vol. 42, pp. 533-555.

Franssen J. M. (2000), Failure temperature of a system comprising a restrained column submitted to fire. Fire Safety Journal, Vol. 34, pp. 191-207.

Franssen, J.M., SAFIR: a thermal/structural program for modelling structures under fire, Eng J AM Inst Steel Construction, 42 (3), pp. 143-158.

Franssen, J.M., Schleich, J.B. and Cajot, L.G. (1995), A simple model for the fire resistance of axially loaded members according to Eurocode 3, Journal of Constructional Steel Research, Vol. 35, pp. 46-69.

Georgieva, I, Schueremans, L, Vandewalle, L., Pyl L. (2012a) Design of built-up cold-formed steel columns according to the direct strength method. Procedia Engineering, Vol. 40, pp. 119124.

Georgieva, I., Schueremans, L., Pyl, L., Vandewalle, L. (2012b), Experimental investigation of built-up double-Z members in bending and compression, Thin-Walled Structures, Vol. 53, pp. 48-57.

Georgieva, I., Schueremans, L., Pyl, L. (2012c), Composed columns from cold-formed steel Zprofiles: Experiments and code-based predictions of the overall compression capacity, Engineering Structures, Vol. 37, pp. 125-134. 
Gustavsson M, Karawacki E and Gustafsson SE. (1994), Thermal conductivity, thermal diffusivity, and specific heat of thin samples from transient measurements with hot disk sensors, Rev. Sci. Instrum.; Vol. 65, pp. 3856-3859.

He Y. (2005), Rapid thermal conductivity measurement with a hot disk sensor. Part 1. Theoretical considerations, Thermochimica Acta; 436, pp. 122-129.

Heva, D.Y.M.B. (2009), Behaviour and design of cold-formed steel compression members at elevated temperatures, $\mathrm{PhD}$ Thesis, Queensland University of Technology, Brisbane, Australia.

Hot Disk AB (2015), http://www.hotdisk.se/ (Accessed: $13^{\text {th }}$ September 2015)

Industrial Machinery Digest@ (2015), http://idustrialmachinerydigest.com, Monthly publication that serves the owners and managers of America's most diversified job shops (Accessed: 13 ${ }^{\text {th }}$ September 2015).

ISO 834-1 (1999): Fire resistance tests - elements of building construction, Part 1: general requirements, International Organization for Standardization ISO 834, Geneva, Switzerland.

Kaitila, O. (2002), Imperfection sensitivity analysis of lipped channel columns at high temperatures, Journal of Constructional Steel Research, Vol. 58, pp. 333-351.

Kankanamge, N.D., Mahendran, M. (2011), Mechanical properties of cold-formed steels at elevated temperatures, Thin-Walled Structures, Vol. 49, pp. 26-44.

Karren, K.W. and Winter, G. (1967), Effects of cold-forming on light-gauge steel members. Journal of Structural Division, ASCE Proceedings, 93, (ST1).

Karren K.W. (1967), Corner properties of cold-formed steel shapes. Journal of the Structural Division, ASCE 1967; 93(2), pp. 401-432.

Kesawan S., Jatheeshan V., Mahendran (2015) Elevated temperature mechanical properties of flange channel sections. Construction and Building Materials, Vol. 87, pp. 86-89.

Kesti, J. and Davies, J.M. (1999), Local and Distortional Buckling of Thin-Walled Short Columns, Thin-Walled Structures, Vol.34, No. 2, pp. 115-134.

Kwon, Y.B., Kim, B-S-, Hancock, G.J. (2009), Compression tests of high strength cold-formed steel channels with buckling interaction, Journal of Constructional Steel Research, Vol. 65, pp. 278-289.

Laím, L., (2013) Experimental and numerical analysis on the structural behavior of cold-formed steel beams subjected to fire, Dissertation for the degree of Doctor of Philosophy in Fire Safety Engineering, Department of Civil Engineering, Faculty of Science and Technology of the University of Coimbra. 
Lee, J.H. (2004), Local Buckling Behaviour and Design of Cold-Formed Steel Compression Members at Elevated Temperatures, Thesis submitted to the school of Civil Engineering Queensland University of Technology in partial fulfilment of requirements for the degree of Doctor of Philosophy, Australia.

Lee, J.H., Mahendran, M., Mäkeläinen, P. (2003), Prediction of mechanical properties of light gauge steels at elevated temperatures, Journal of Constructional Steel Research, Vol. 59, Issue 12, pp. 1517-1532.

Li, Y., Li, Y, Wang, S., Shen, Z. (2014), Ultimate load-carrying capacity of cold-formed thinwalled columns with built-up box and I section under axial compression, Thin-Walled Structures, Vol. 79, pp. 202-217.

Li, Z., Schafer B.W. (2010), Application of the finite strip method in cold-formed steel member design, Journal of Constructional Steel Research, Vol. 66, pp. 971-980.

Lennon, T., Moore, D.B., Wang, Y.C. and Bailey, C.G. (2007), Designers' guide to EN 19911-2, EN1992-1-2, EN 1993-1-2 and EN 1994-1-2. Handbook for the fire design of steel, composite and concrete structures to the Eurocodes, Thomas Telford.

Lu, Wei, Makelainen, P. (2003), Advanced steel structures: 1. Structural fire design; 2. Fatigue design. Helsinki University of Technology Laboratory of Steel Structures Publication 29.

LTH Steel Structure@ (2015), http://www.lthsteelstructures.com.

Makelainen, P. and Miller, K. (1983), Mechanical properties of cold-formed galvanized steel Z32 at elevated temperatures, Helsinki University of Technology, Finland.

Makelainen P, Outinen J and Kesti J. (1998), Fire design model for structural steel S420M based upon transient-state tensile test results, Journal of constructional steel research; 48, pp. 47-57.

Marques, L., Simões da Silva, L., Grainer, R., Rebelo, C., Taras, A. (2013), Development of a consistente design procedure for lateral-torsional buckling of tapered beams, Journal of Constructional Steel Research, Vol. 89, pp. 213-235.

Mecozzi, E., Zhao, B. (2005), Development of stress-strain relationships of cold-formed lightweight steel at elevated temperatures, Proceedings of Eurosteel 2005 - 4th European Conference on Steel and Composite Structures, Maastricht, Netherlands, pp. 5.1-41 - 5.1-49.

Moen, C.D., Igusa, T., Schafer, B.W. (2008), Prediction of residual stresses and strains in coldformed steel members, Thin-Walled Structures, Vol. 46, pp. 1274-1289.

Neves, I. C.; Valente, J. C. and Rodrigues, J. P. C. (2002); Thermal Restraint and Fire Resistance of Columns, Fire Safety Journal, vol. 37, Elsevier Science, UK, pp 753-771. 
North American Specification for the Design of Cold-Formed Steel Structural Members, NAS (2001), American Iron and Steel Institute.

Olawale A.O. and Plank R.J. (1988), The collapse analysis of steel columns in fire using a finite element strip method, International Journal of Numerical Methods in Engineering, Vol. 26: 2755-2764.

Ottosen N, Ristinmaa M. (2005), The mechanics of constitutive modeling. Amsterdam: Elsevier.

Outinen, J (1999), Mechanical properties of structural steels at elevated temperatures, Licentiate thesis, Helsinki University of Technology, Finland.

Outinen, J., Kaitila, O., Mäkeläinen, P. (2000), A study for the development of the design of steel structures in fire conditions, Proceedings of the 1st International Workshop of Structures in Fire, Copenhagen, Denmark, pp. 267-281.

Outinen, J., Mäkeläinen, P. (2002), Mechanical properties of structural steel at elevated temperatures and after cooling down, Proceedings of the 2nd International Workshop of Structures in Fire, Christchurch, New Zealand, pp. 273- 290.

Outinen MA and Myllymaki J. (1995), The local buckling of RHS members at elevated temperatures. VTT Research Notes 1672, Technical Research Centre of Finland, Espoo.

Papangelis, J.P., Hanckock, G.J., (1998), THIN-WALL (ver. 2.0). Sydney (Australia): Center for Advanced Structural Engineering, Dept. of Civil Engineering, University of Sydney.

Perfisa@ (2012), http://www.perfisa.net/ (Accessed: $8^{\text {th }}$ May 2015).

Peet MJ, Hasan HS and Bhadeshia HKDH. (2011) Prediction of thermal conductivity of steel, International journal of heat and mass transfer, 54: 2602-2608.

Pinheiro S. (2015), Experimental analysis of mechanical and thermal properties of the S280GD+Z steel, MSc thesis in civil engineering, university of Coimbra, (in Portuguese).

Popovich, D., Hancock, G.J. and Rasmussen, K.J.R. (1999), Axial Compression Tests on ColdFormed Angles Loaded parallel with a Leg, Journal of Structural Engineering, Vol. 127, Issue 6, pp. 600-607.

Popovich, D., Hancock, G.J. and Rasmussen, K.J.R. (2001), Compression Tests of ColdFormed Angles, Journal of Structural Engineering, Vol. 125, Issue 5, pp. 515-523.

Quach, W.M., Teng, J.G., Chung, K.F. (2004), Residual stresses in steel sheets due to coiling and uncoiling: a closed-form analytical solution, Engineering Structures, Vol. 26, pp. 12491259. 
Quach, W.M., Teng, J.G., Chung, K.F. (2006), Finite element predictions of residual stresses in press-braked thin-walled steel sections, Engineering Structures, Vol. 28, pp. 1609-1619.

Ramberg W, Osgood WR. (1943), Description of stress-strain curves by three parameters, NACA Technical Note 902.

Ranawaka, T., Mahendran, M. (2009), Experimental study of the mechanical properties of light gauge cold-formed steels at elevated temperatures, Fire Safety Journal, Vol. 44, pp. 219-229.

Ranawaka, T., Mahendran, M. (2009), Distortional buckling tests of cold-formed steel compression members at elevated temperatures, Journal of Constructional Steel Research, Vol. 65, pp. 249-259.

Ranawaka, T., Mahendran, M. (2010), Numerical modeling of light gauge cold-formed steel compression members subjected to distortional buckling at elevated temperatures, Thin-Walled Structures, Vol. 48, pp. 334-344.

Ranby, A. (1998), Structural fire design of thin-walled steel sections, Journal of Constructional Steel Research, Vol. 46, No. 1-3, pp. 303-304.

Reyes, G., Guzmán, A. (2011), Evaluation of the slenderness ratio in built-up cold-formed box sections, Journal of Constructional Steel Research, Vol. 67, pp. 929-935.

Rhodes, J. (1991), Design of cold formed steel members, New York: Elsevier Applied Science, $409 \mathrm{p}$.

Rodrigues, J. P. C.; Neves, I. C. and Valente, J. C. (2000), Experimental Research on the Fire Resistance of Steel Elements with Restrained Thermal Elongation, Fire Safety Journal, vol. 35, Elsevier Science, UK, pp 77 - 98.

Rondal, J., Dubina, D. (2005), Light gauge metal structures: recent advances, International centre for mechanical sciences (CISM), Udine, Italy, $259 \mathrm{p}$.

Santos, E.S., Batista, E.M., Camotim, D. (2012), Experimental investigation concerning lipped channel columns undergoing local-distortional-global buckling mode interaction, Thin-Walled Structures, Vol. 54, pp. 19-34.

Schafer, B.W. (2001), Direct strength prediction of thin-walled beams and columns, Research Report, John Hopkins University, USA.

Schafer; B.W. (2008), Review: The Direct Strength Method of cold-formed steel member design, Journal of Constructional Steel Research, Vol. 64, pp. 766-778.

Schafer, B.W. (2012), http://www.ce.jhu.edu/bschafer/cufsm/, CUFSM: elastic buckling analysis of thin-walled members by finite strip analysis, CUFSM, version 4.04 (Accessed: $29^{\text {th }}$ September 2015). 
Schafer, B.W., Peköz, T. (1998a), Computational modeling of cold-formed steel: characterizing geometric imperfections and residual stresses, Journal of Constructional Steel Research, Vol. 47, pp. 193-210.

Schafer, B.W., Peköz, T. (1998b), Direct strength prediction of cold-formed steel members using numerical elastic buckling solutions, In: Proceedings of the $14^{\text {th }}$ international specialty conference on cold-formed steel structures, St. Louis, Missouri, USA, pp. 69-76.

Shahbazian, A., Wang, Y.C. (2011), Application of the direct strength method to local buckling resistance of thin-walled steel members with non-uniform elevated temperatures under axial compression, Thin-Walled Structures, Vol. 49, pp. 1573-1583.

Shahbazian, A., Wang, Y.C. (2012), Direct strength method for calculating distortional buckling capacity of cold-formed steel columns with uniform and non-uniform elevated temperatures, Thin-Walled Structures, Vol. 53, pp. 188-199.

Shedquarters@ (2009), http://www.shedquarters.net.au. Domestic and commercial construction industry for over 20 years (Accessed: $21^{\text {st }}$ September 2015).

Sidey MP, Teague DP. (1988), Elevated temperature data for structural grades of galvanized steel, British steel (Welsh Laboratories) Report, UK.

Silvestre, N., Camotim, D. (2002a), First-order generalised beam theory for arbitrary orthotropic materials, Thin-Walled Structures, Vol. 40, Issue 9, pp. 755-789.

Silvestre, N., Camotim, D. (2002b), Second-order generalised beam theory for arbitrary orthotropic materials, Thin-Walled Structures, Vol. 40, Issue 9, pp. 791-820.

Sivakumaran, K.S. and Abdul-Rahman, N. (1998), A finite Element Analysis Model for the Behaviour of Cold-Formed Steel Members, Thin-Walled Structures, Vol. 31, Issue 4, pp. 305324.

Steel Construction Info@ (2010), http://www.steelconstruction.info, The free encyclopedia for UK steel construction information, UK (Accessed: $21^{\text {st }}$ September 2015).

Stone, T.A., LaBoube, R.A. (2005), Behavior of cold-formed steel built-up I-sections, ThinWalled Structures, Vol. 43, pp. 1805-1817.

SUEL, (2010), Vulcan - a software for structural fire engineering, Vulcan Solutions Ltd - a Company of Sheffield University Enterprises Limited, Shefield, UK.

Ungureanu, V., Dubina, D. (2004), Recent research advances on ECBL approach. Part I: Plastic-elastic interactive buckling of cold-formed steel sections, Thin-Walled Structures, Vol. 42, Issue 2, pp. 177-194. 
Vila Real, P.M.M., Cazeli, R., Simões DA Silva, L., Santiago, A., Piloto, P. (2004), The effect of residual stresses in the lateral-torsional buckling of steel I-beams at elevated temperature, Journal of Constructional Steel Research, Vol. 60, pp. 783-793.

Von Kármán, T., Sechler, E.E., Donnel, L.H. (1932), The strength of thin plates in compression, Transactions of the American Society of Mechanical Engineers, Vol. 54, pp. 53-57.

Wang Y. C. (1997a), The effects of frame continuity on the behavior of steel columns under fire conditions and fire resistant design proposals. Journal of Constructional Steel Research, vol. 41, n. ${ }^{\circ}$, pp.93-111.

Wang, Y. C. (1997b), Effects of structural continuity on the fire resistant design of steel columns in non-sway multi-storey frames. Fire Safety Journal, 28, 1997b, pp. 101-116.

Wang, Y. C. (2004), Postbuckling behavior of axially restrained and axially loaded steel columns under fire conditions. Journal of Structural Engineering, 130(3), pp. 371-380.

Wang, Y.C., Davies, J.M. (2003), Fire tests of non-sway loaded and rotationally restrained steel column assemblies, Journal of Constructional Steel Research, Vol. 59, pp. 359-383.

Wei, C., Jihong, Y. (2012), Mechanical properties of G550 cold-formed steel under transient and steady state conditions, Journal of Constructional Steel Research, Vol. 73, pp. 1-11.

Weng, C.C., Peköz, T. (1990), Residual stresses in cold-formed steel members, Journal of Structural Engineering, American Society of Civil Engineers (ASCE), Vol. 116, Issue 6, pp. 1611-1625.

Weng, C.C., White, R.N. (1990), Residual stresses in cold-bent thick steel plates, Journal of Structural Engineering, American Society of Civil Engineers (ASCE), Vol. 116, Issue 1, pp. 24-39.

Winter, G. (1947), Strength of Thin Steel Compression Flanges, Transactions of American Society of Civil Engineers (ASCE), Vol. 112, pp. 527-554

Whittle, J., Ramseyer, C. (2009), Buckling capacities of axially loaded, cold-formed, built-up C-channels, Thin-Walled Structures, Vol. 47, pp. 190-201.

Yan, J., Young, B., (2002), Column Tests of Cold-Formed Steel Channels with Complex Stiffeners, Journal of Structural Engineering, Vol. 128, Issue 6, pp. 737-745.

Yan, J. and Young, B. (2004), Numerical investigation of channel columns with complex stiffeners - part I: test verification, Thin-Walled Structures, Vol. 42, pp. 883-893.

Yang, D., Hancock, G.J. (2004), Compression tests of high strength steel channel columns with interaction between local and distortional buckling, Journal of structural Engineering, Vol. 130, No. 12, pp. 1954-1963. 
Young, B., Chen, J. (2008a), Column Tests of Cold-Formed Steel Non-Symmetric Lipped Angle Sections, Journal of Constructional Steel Research, Vol. 64, pp. 808-815.

Young, B., Chen, J. (2008b), Design of cold-formed steel built-up closed sections with intermediate stiffeners, Journal of Structural Engineering, Vol. 134, No. 5, pp. 727-737.

Young, B. Ellobody, E. (2007), Design of cold-formed steel unequal angle compression members, Thin-Walled Structures, Vol. 45, pp. 330-338.

Young B., Rasmussen, J.R. (1998a), Tests of Fixed-ended Plain Channel Columns, Journal of Structural Engineering, Vol. 124, Issue 2, pp. 131-139.

Young, B., Rasmussen, J.R. (1998b), Design of Lipped Channel Columns, Journal of Structural Engineering, Vol. 124, Iseu 2, pp. 140-148.

Young, B. (2004a), Tests and Design of Fixed-ended Cold-Formed Steel Plain Angle Columns, Journal of Structural Engineering, Vol. 130, pp. 1931-1940.

Young, B. (2004b), Design of channel columns with inclined edge stiffeners, Journal of Constructional Steel Research, Vol. 60, pp. 183-197.

Young, B., Yan, J. (2002), Finite element analysis and design of fixed-ended plain channel columns, Finite Elements in Analysis and Design, Vol. 38, pp. 549-566.

Young, B., Yan, J. (2004), Numerical investigation of channel columns with complex stiffeners - part II: parametric study and design, Thin-Walled Structures, Vol 42, pp. 895-909.

Yu, W.-W. and Laboube, R. (2010), Cold-Formed Steel Design - Fourth Edition, John Wiley \& Sons, Inc., USA, 767 p.

Zhang, J.-H., Young, B.(2012), Compression tests of cold-formed steel I-shaped open sections with edge and web stiffeners, Thin-Walled Structures, Vol. 52, pp. 1-11.

Zhang H, Li M, Fang W, Dan D, Li Z and Tao W. (2014), A numerical study on the theoretical accuracy of film thermal conductivity using transient plane source method, Applied thermal engineering; Vol. 72, pp. 62-69. 


\section{APPENDIX A MECHANICAL AND THERMAL PROPERTIES}

\section{A.1 Tensile Coupon Tests at both Ambient and Elevated Temperatures for the Structural Steel S280GD+Z}

\section{A.1.1 Stress-strain curves}

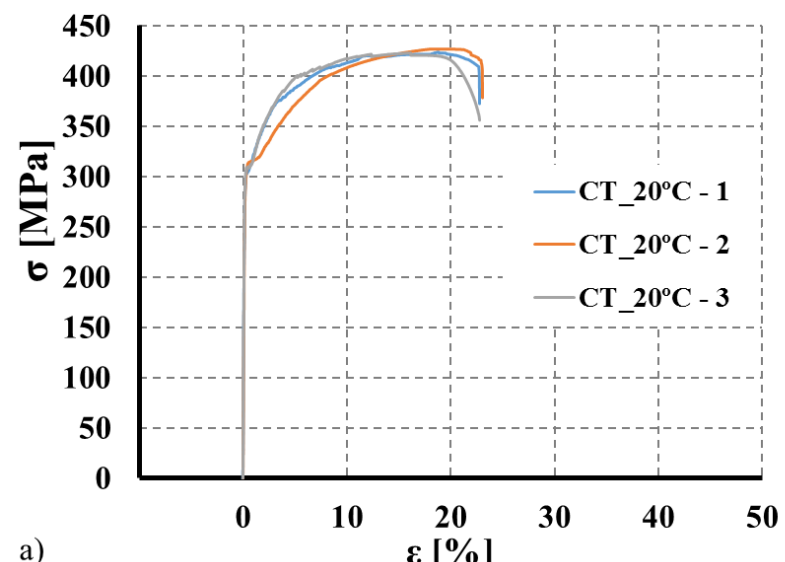

a)

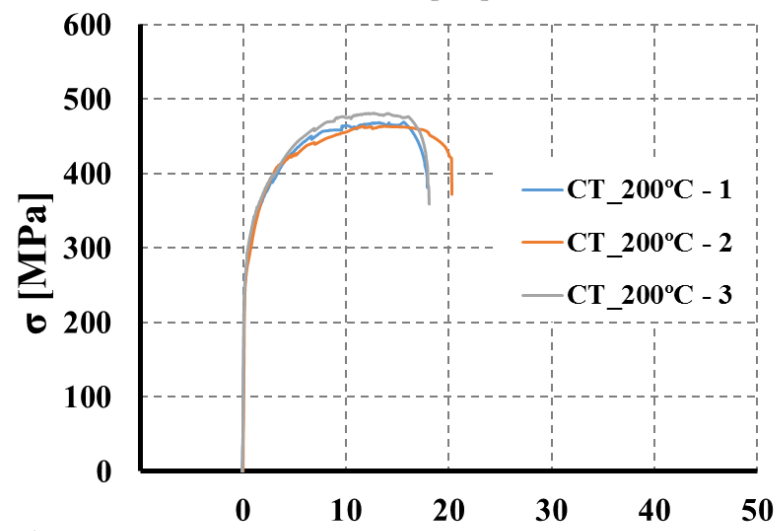

c)

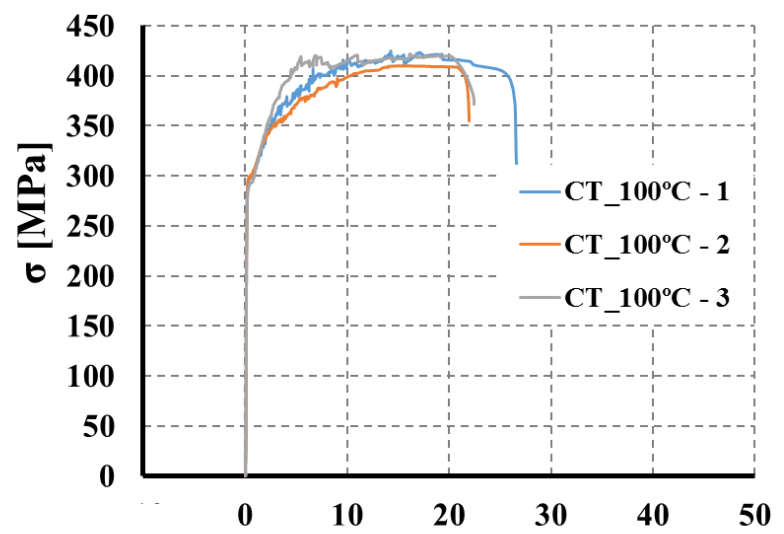

b)

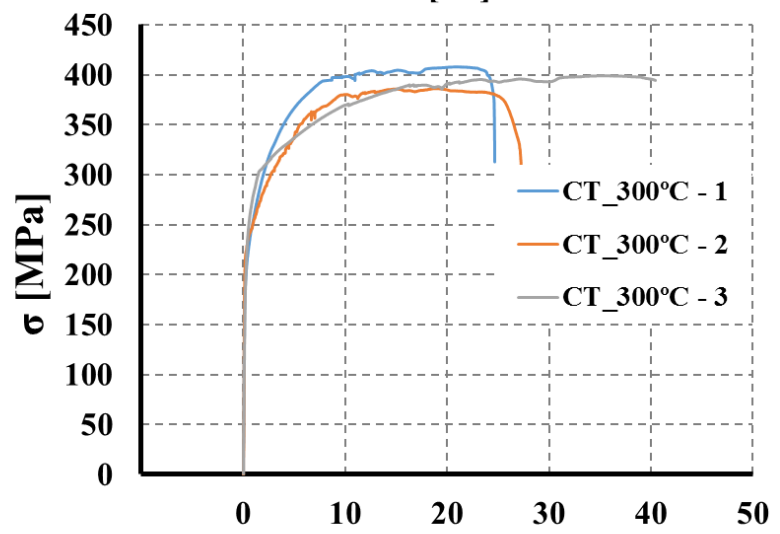

d)

Figure A.1 Stress vs strain curves for the structural steel S280GD+Z. a) $20^{\circ} \mathrm{C}$. b) $100^{\circ} \mathrm{C}$. c) $200^{\circ} \mathrm{C}$. d) $300^{\circ} \mathrm{C}$. 


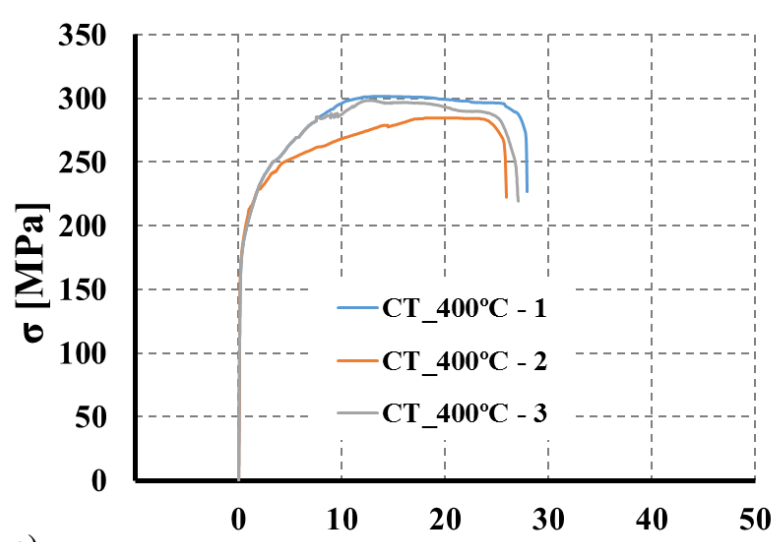

a)

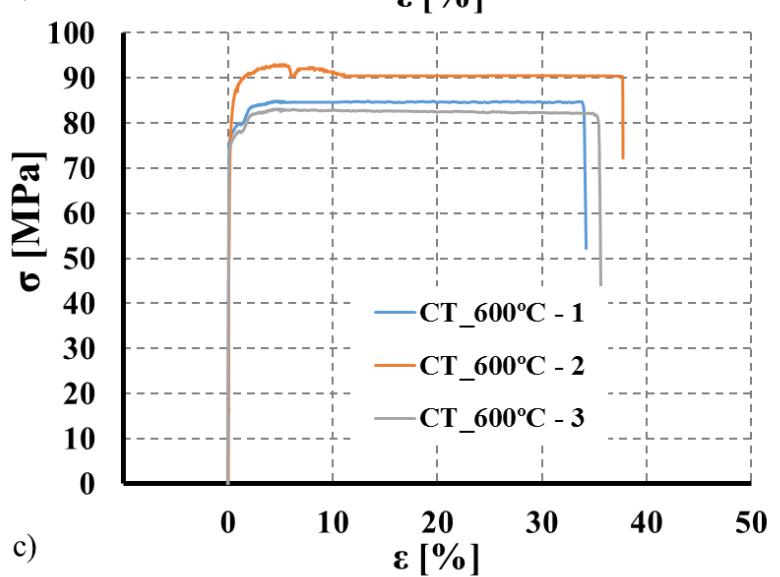

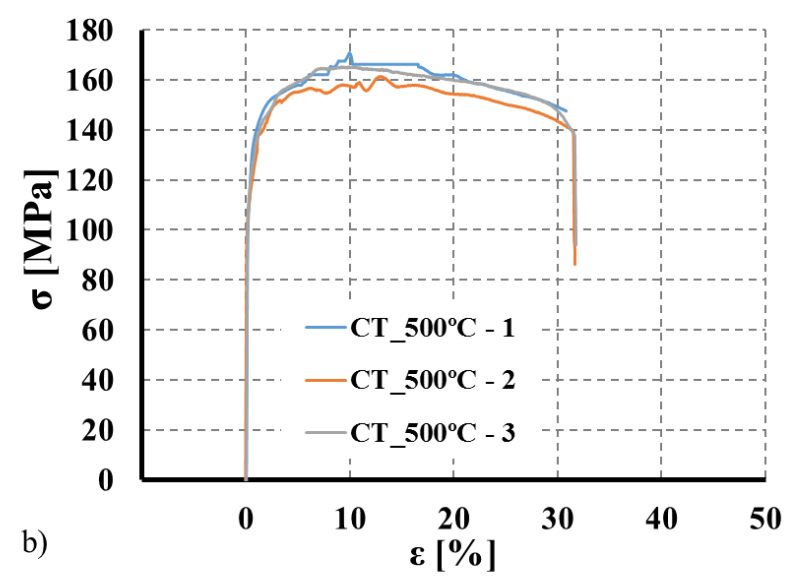

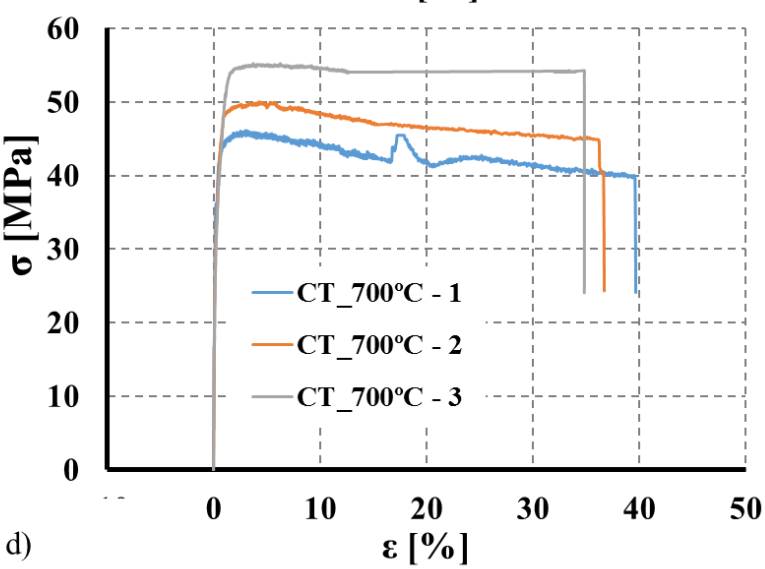

e)

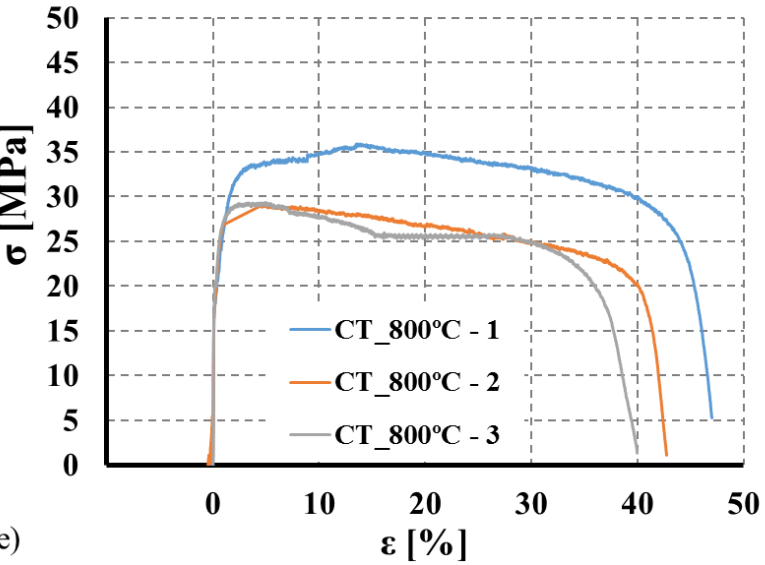

Figure A.2 Stress vs strain curves for the structural steel S280GD+Z. a) $400^{\circ} \mathrm{C}$. b) $500^{\circ} \mathrm{C}$. c) $600^{\circ} \mathrm{C}$.d) $700^{\circ} \mathrm{C}$.e) $800^{\circ} \mathrm{C}$. 
Table A.1 Experimental results.

\begin{tabular}{|c|c|c|c|c|c|c|c|c|c|c|c|c|c|}
\hline Test & $\begin{array}{c}\text { Temp. } \\
{\left[{ }^{\circ} \mathrm{C}\right]}\end{array}$ & $\begin{array}{c}E_{\theta} \\
{[\mathbf{G P a}]}\end{array}$ & $\bar{E}_{\theta}[\mathbf{G P a}]$ & $\sigma$ & $\begin{array}{c}f_{y, \theta} \\
{[\mathrm{MPa}]}\end{array}$ & $\begin{array}{c}\overline{\boldsymbol{f}}_{\boldsymbol{y}, \boldsymbol{\theta}} \\
{[\mathbf{M P a}]}\end{array}$ & $\sigma$ & $\begin{array}{c}f_{u, \theta} \\
{[\mathrm{MPa}]}\end{array}$ & $\begin{array}{c}\overline{\boldsymbol{f}}_{\boldsymbol{u}, \boldsymbol{\theta}} \\
{[\mathrm{MPa}]}\end{array}$ & $\sigma$ & $\begin{array}{c}f_{p, \theta} \\
{[\mathbf{M P a}]}\end{array}$ & $\begin{array}{c}\overline{\boldsymbol{f}}_{\boldsymbol{p}, \boldsymbol{\theta}} \\
{[\mathbf{M P a}]}\end{array}$ & $\sigma$ \\
\hline CT_20-1 & \multirow{3}{*}{20} & 205.23 & \multirow{3}{*}{204.18} & \multirow{3}{*}{0.94} & 302.54 & \multirow{3}{*}{306.81} & \multirow{3}{*}{3.70} & 422.47 & \multirow{3}{*}{424.04} & \multirow{3}{*}{2.25} & 208.28 & \multirow{3}{*}{212.50} & \multirow{3}{*}{5.15} \\
\hline CT_20-2 & & 203.90 & & & 308.92 & & & 426.61 & & & 210.97 & & \\
\hline CT_20-3 & & 203.42 & & & 308.98 & & & 423.04 & & & 218.24 & & \\
\hline CT_100-1 & \multirow{3}{*}{100} & 195.52 & \multirow{3}{*}{200.11} & \multirow{3}{*}{4.14} & 296.83 & \multirow{3}{*}{295.24} & \multirow{3}{*}{5.20} & 416.10 & \multirow{3}{*}{415.49} & \multirow{3}{*}{4.88} & 204.93 & \multirow{3}{*}{209.25} & \multirow{3}{*}{5.23} \\
\hline CT_100-2 & & 201.24 & & & 299.46 & & & 410.34 & & & 207.77 & & \\
\hline CT_100-3 & & 203.57 & & & 289.43 & & & 420.04 & & & 215.06 & & \\
\hline CT_200-1 & \multirow{3}{*}{200} & 176.90 & \multirow{3}{*}{171.80} & \multirow{3}{*}{4.48} & 275.71 & \multirow{3}{*}{275.75} & \multirow{3}{*}{3.05} & 469.11 & \multirow{3}{*}{471.50} & \multirow{3}{*}{8.95} & 170.91 & \multirow{3}{*}{170.68} & \multirow{3}{*}{2.26} \\
\hline CT_200-2 & & 168.48 & & & 272.73 & & & 463.98 & & & 172.81 & & \\
\hline CT_200-3 & & 170.03 & & & 278.82 & & & 481.40 & & & 168.31 & & \\
\hline$\overline{C T \_300-1}$ & \multirow{3}{*}{300} & 144.73 & & & 212.57 & & & 407.70 & & & 132.10 & & \\
\hline CT_300-2 & & 149.35 & 143.59 & 6.41 & 229.23 & 223.57 & 9.52 & 386.57 & 397.41 & $\begin{array}{c}10.3 \\
8\end{array}$ & 139.62 & 136.37 & 3.86 \\
\hline CT_300-3 & & 136.68 & & & 228.90 & & & 397.97 & & & 137.39 & & \\
\hline CT_400-1 & & 120.49 & & & 181.05 & & & 301.32 & & & 98.41 & & \\
\hline CT_400-2 & 400 & 120.34 & 121.21 & 1.39 & 183.55 & 181.68 & 1.64 & 284.86 & 291.73 & 8.56 & 99.32 & 98.71 & 0.53 \\
\hline CT_400-3 & & 122.81 & & & 180.45 & & & 289.01 & & & 98.39 & & \\
\hline CT_500-1 & & 84.07 & & & 113.64 & & & 170.97 & & & 78.50 & & \\
\hline CT_500-2 & 500 & 85.63 & 84.54 & 0.95 & 110.53 & 113.67 & 3.15 & 161.42 & 165.85 & 4.81 & 80.57 & 80.25 & 1.62 \\
\hline CT_500-3 & & 83.92 & & & 116.83 & & & 165.17 & & & 81.69 & & \\
\hline CT_600-1 & & 62.58 & & & 77.36 & & & 84.86 & & & 52.28 & & \\
\hline CT_600-2 & 600 & 62.71 & 62.46 & 0.32 & 80.62 & 77.83 & 2.58 & 92.91 & 87.00 & 5.18 & 53.53 & 54.57 & 2.95 \\
\hline CT_600-3 & & 62.10 & & & 75.52 & & & 83.24 & & & 57.90 & & \\
\hline$\overline{C T}$ CT00-1 & & 22.11 & & & 38.31 & & & 46.09 & & & 18.15 & & \\
\hline CT_700-2 & 700 & 22.58 & 22.21 & 0.33 & 36.28 & 36.34 & 1.95 & 50.05 & 50.48 & 4.61 & 20.25 & 19.26 & 1.06 \\
\hline CT_700-3 & & 21.95 & & & 34.42 & & & 55.29 & & & 19.38 & & \\
\hline CT_800-1 & & 17.47 & & & 19.45 & & & 35.89 & & & 16.81 & & \\
\hline CT_800-2 & 800 & 17.12 & 17.45 & 0.33 & 20.03 & 19.91 & 0.41 & 29.16 & 31.47 & 3.83 & 16.12 & 16.49 & 0.35 \\
\hline CT_800-3 & & 17.77 & & & 20.24 & & & 29.35 & & & 16.54 & & \\
\hline
\end{tabular}




\section{A.2 Thermal Properties of the Structural Steel S280GD+Z}

Table A.2 Experimental results.

\begin{tabular}{|c|c|c|c|c|c|c|c|c|c|c|}
\hline Test & $\begin{array}{c}\theta \\
{\left[{ }^{\circ} \mathrm{C}\right]}\end{array}$ & $\begin{array}{c}\lambda_{\mathrm{a}} \\
{[\mathrm{W} / \mathbf{m K}]}\end{array}$ & $\mu$ & $\sigma$ & $\begin{array}{c}\boldsymbol{\alpha} \\
{\left[\mathrm{mm}^{2} / \mathbf{s}\right]}\end{array}$ & $\mu$ & $\sigma$ & $\begin{array}{c}\mathrm{c}_{\mathrm{a}} \\
{[\mathrm{J} / \mathrm{kgK}]}\end{array}$ & $\mu$ & $\sigma$ \\
\hline TP_20-1 & \multirow{3}{*}{20} & 66.276 & \multirow{3}{*}{66.38} & \multirow{3}{*}{0.16} & 19.703 & \multirow{3}{*}{19.71} & \multirow{3}{*}{0.04} & 428.500 & \multirow{3}{*}{429.04} & \multirow{3}{*}{0.00} \\
\hline TP_20-2 & & 66.569 & & & 19.750 & & & 429.370 & & \\
\hline TP_20-3 & & 66.294 & & & 19.674 & & & 429.253 & & \\
\hline TP_100-1 & \multirow{3}{*}{100} & 62.716 & \multirow{3}{*}{62.84} & \multirow{3}{*}{0.36} & 17.016 & \multirow{3}{*}{16.70} & \multirow{3}{*}{0.46} & 469.503 & \multirow{3}{*}{479.51} & \multirow{3}{*}{0.09} \\
\hline TP_100-2 & & 63.251 & & & 16.912 & & & 476.448 & & \\
\hline TP_100-3 & & 62.560 & & & 16.179 & & & 492.568 & & \\
\hline TP_200-1 & \multirow{3}{*}{200} & 56.791 & \multirow{3}{*}{57.56} & \multirow{3}{*}{0.76} & 13.801 & \multirow{3}{*}{13.95} & \multirow{3}{*}{0.14} & 524.198 & \multirow{3}{*}{525.73} & \multirow{3}{*}{0.01} \\
\hline TP_200-2 & & 57.594 & & & 13.953 & & & 525.842 & & \\
\hline TP_200-3 & & 58.301 & & & 14.089 & & & 527.150 & & \\
\hline TP_300-1 & \multirow{3}{*}{300} & 53.253 & \multirow{3}{*}{52.68} & \multirow{3}{*}{1.56} & 12.712 & \multirow{3}{*}{12.18} & \multirow{3}{*}{0.79} & 533.675 & \multirow{3}{*}{551.85} & \multirow{3}{*}{0.17} \\
\hline TP_300-2 & & 53.863 & & & 12.563 & & & 546.189 & & \\
\hline TP_300-3 & & 50.914 & & & 11.266 & & & 575.691 & & \\
\hline TP_400-1 & \multirow{3}{*}{400} & 50.159 & \multirow{3}{*}{50.60} & & 10.284 & & & 621.327 & & \\
\hline TP_400-2 & & 50.640 & & 0.42 & 10.391 & 10.36 & 0.06 & 620.824 & 622.49 & 0.02 \\
\hline TP_400-3 & & 51.003 & & & 10.390 & & & 625.310 & & \\
\hline TP_500-1 & & 48.130 & & & 8.770 & & & 699.084 & & \\
\hline TP_500-2 & 500 & 47.248 & 48.26 & 1.09 & 8.528 & 8.77 & 0.24 & 705.741 & 700.99 & 0.03 \\
\hline TP_500-3 & & 49.415 & & & 9.016 & & & 698.159 & & \\
\hline TP_600-1 & & 45.902 & & & 6.205 & & & 942.318 & & \\
\hline TP_600-2 & 600 & 45.991 & 45.95 & 0.04 & 7.034 & 6.45 & 0.51 & 832.968 & 911.03 & 0.53 \\
\hline TP_600-3 & & 45.946 & & & 6.111 & & & 957.814 & & \\
\hline TP_700-1 & & 43.370 & & & 5.880 & & & 939.574 & & \\
\hline TP_700-2 & 700 & 43.772 & 43.71 & 0.32 & 5.392 & 5.43 & 0.43 & 1034.112 & 1030.26 & 0.70 \\
\hline TP_700-3 & & 43.995 & & & 5.017 & & & 1117.086 & & \\
\hline TP_750-1 & & 40.433 & & & 3.856 & & & 1335.690 & & \\
\hline TP_750-2 & 750 & 40.327 & 40.34 & 0.09 & 4.439 & 4.19 & 0.30 & 1157.251 & 1230.34 & 0.73 \\
\hline TP_750-3 & & 40.246 & & & 4.279 & & & 1198.081 & & \\
\hline
\end{tabular}




\section{APPENDIX B AXIAL AND ROTATIONAL STIFFNESS OF THE RESTRAINING FRAMES USED IN THE FIRE TESTS}

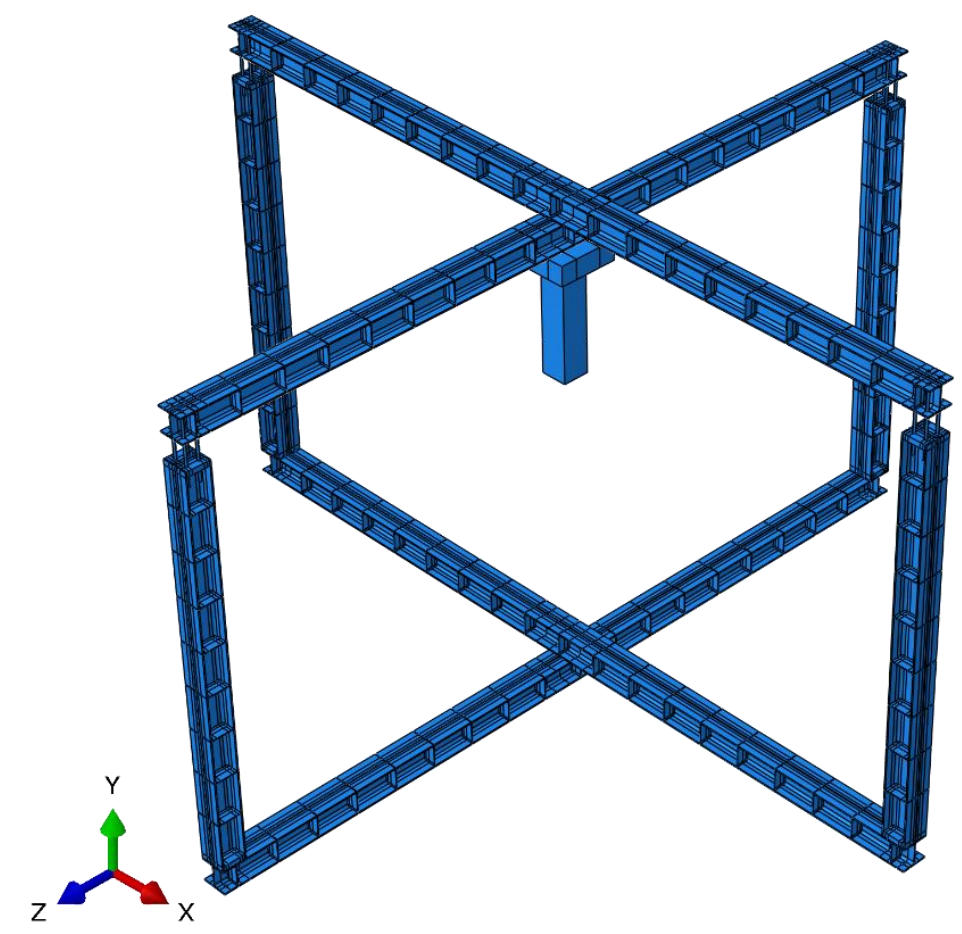

Figure B.1 3D restraining frame modelled using the finite element model and the finite element software ABAQUS.

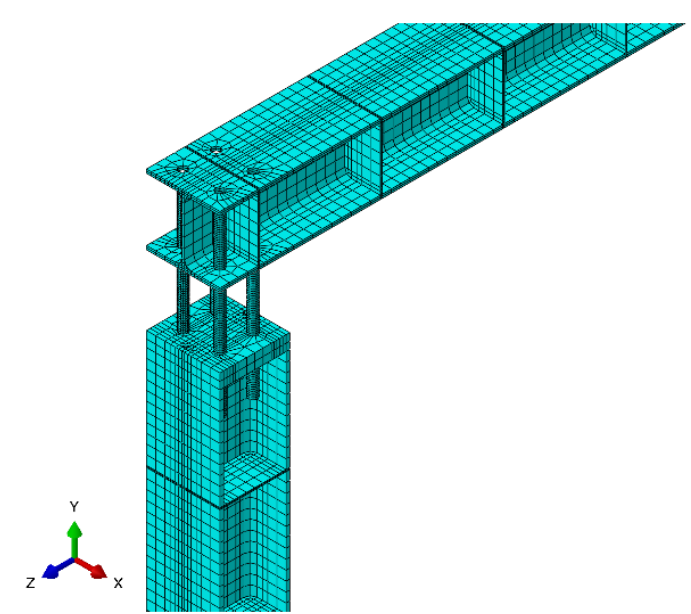

Figure B.2 Mesh detail of the developed finite element model. 
Visualization of the obtained results from the simulation conducted to determine the axial stiffness imposed by the 3D restraining frame to the CFS column.

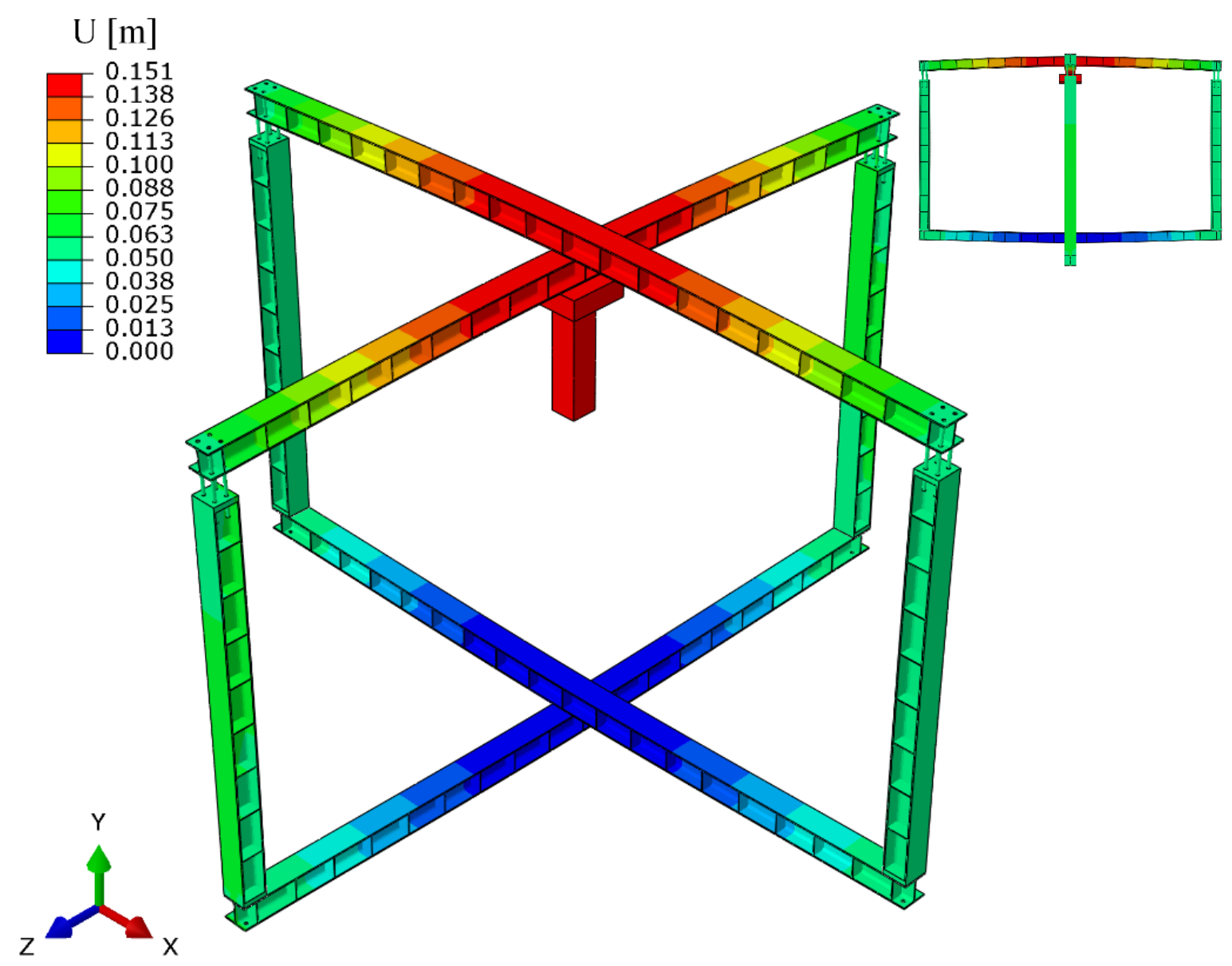

Figure B.3 Deformed shape of the 3D restraining frame due to a vertical force applied in the centre of the massive steel column. 
Visualization of the obtained results from the simulation conducted to determine the rotational stiffness imposed by the 3D restraining frame to the CFS column

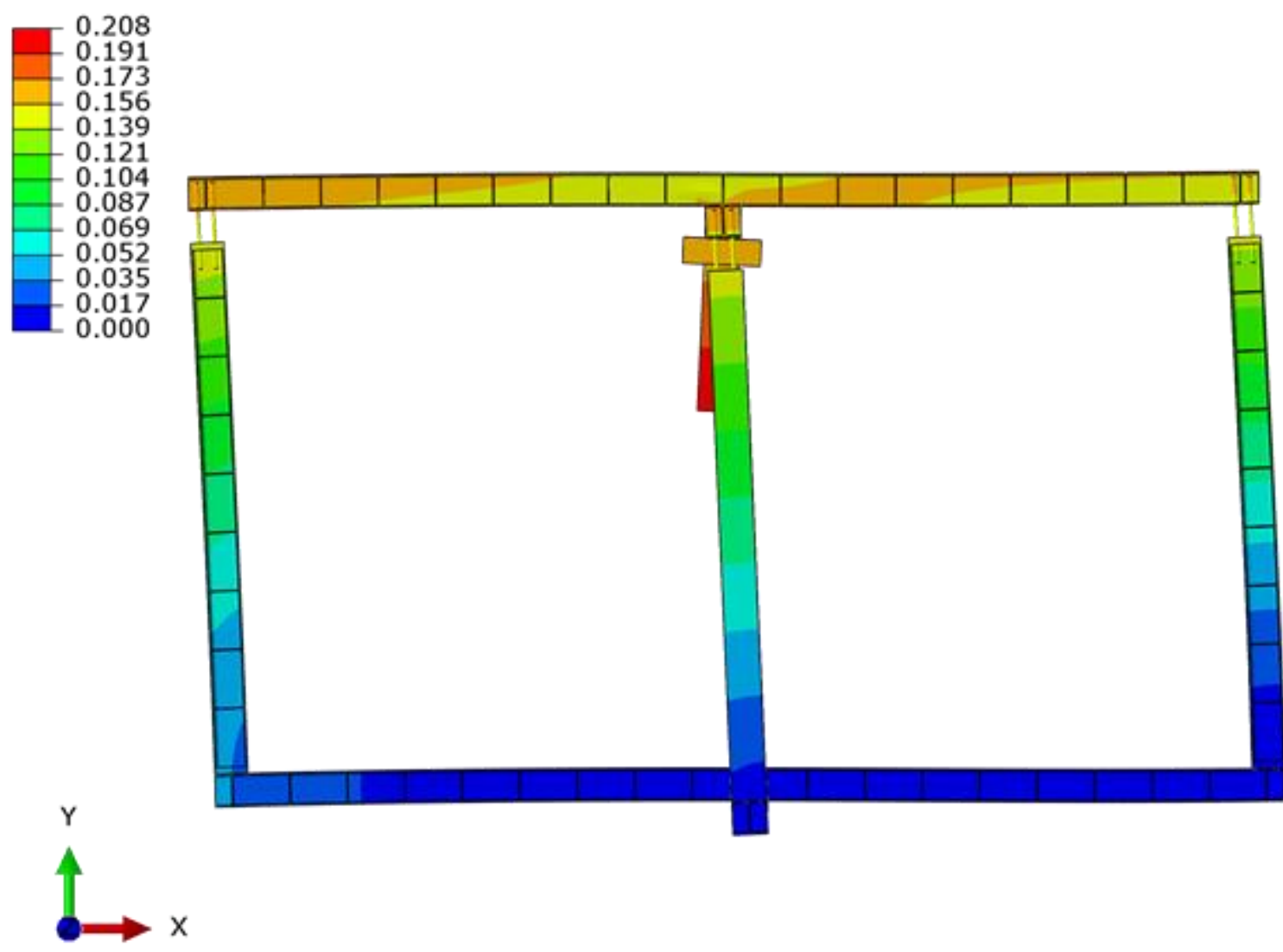

Figure B.4 Deformed shape of the 3D restraining frame due to a horizontal force applied in the massive steel column in order to induce rotation of the top beams.

Table B.1 Determined values for the imposed levels of axial and rotational restraint to the tested CFS columns.

\begin{tabular}{c|c|c|c}
\hline Restraining Frame & $\begin{array}{c}\mathbf{K}_{\mathbf{a}, \mathbf{s}} \\
{[\mathbf{k N} / \mathbf{m m}]}\end{array}$ & $\begin{array}{c}\mathbf{K}_{\mathbf{r}, \mathbf{s 1}} \\
{[\mathbf{k N m} / \mathbf{r a d}]}\end{array}$ & $\begin{array}{c}\mathbf{K}_{\mathbf{r}, \mathbf{s 2}} \\
{[\mathbf{k N m} / \mathbf{r a d}]}\end{array}$ \\
\hline RF.1 & 3 & 9253 & 2196 \\
\hline RF.2 & 13 & 37237 & 12620 \\
\hline
\end{tabular}





\section{APPENDIX C DETERMINATION OF THE DESIGN BUCKLING LOADS}

\section{C.1 U profile}

This example shows how was determined the effective section U according the EN 1993-13:2006 and EN 1993-1-5:2006.

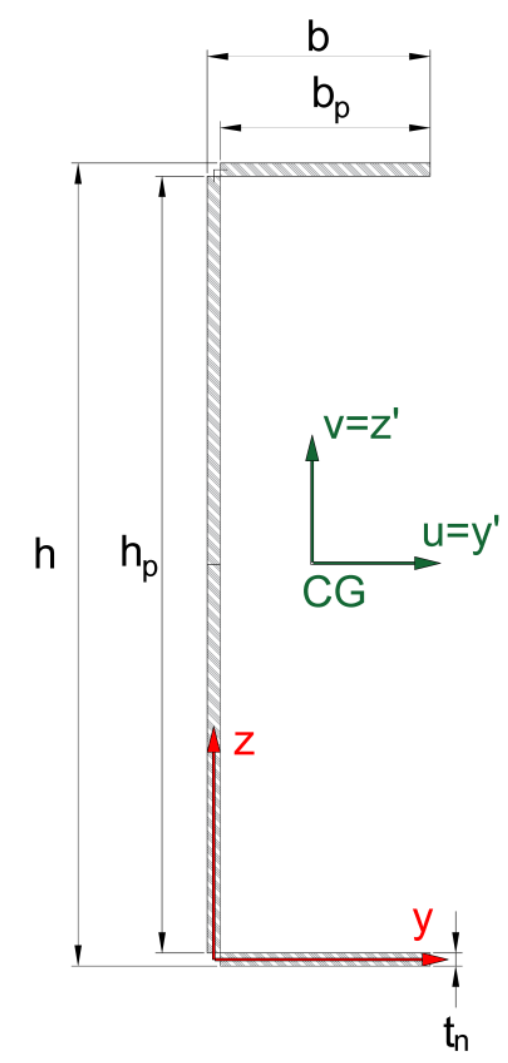

Figure C.1 U gross cross sectional scheme formed by flat plane elements.

\section{$>\mathrm{U}$ cross-sectional dimensions:}

Height of the section:

Width of the flange:

Nominal material thickness:

$$
\begin{gathered}
h=155 \mathrm{~mm} \\
b=43 \mathrm{~mm} \\
t_{n}=2.5 \mathrm{~mm}
\end{gathered}
$$


Material core thickness:

$$
\begin{gathered}
t_{\text {cor }}=t_{n}-t_{\text {zinc }} \\
t_{\text {zinc }}=0.04(\mathrm{~S} 280 \mathrm{GD}+\mathrm{Z})
\end{gathered}
$$$$
t_{c o r}=t=2.46 \mathrm{~mm}
$$

Height of the web flat plane

element:

Width of the flange flat plane

$$
\begin{gathered}
h_{p}=150.60 \mathrm{~mm} \\
b_{p}=40.80 \mathrm{~mm}
\end{gathered}
$$

element:

Column Height:

$\mathrm{L}=2950 / 3050 \mathrm{~mm}$

\section{$>$ Material properties:}

Modulus of elasticity:

Poisson ratio:

Shear modulus:

Nominal yield strength (S280GD + Z275):

Partial factor for resistance of crosssections (clause 2 of EN 1993-13:2006):

Partial factor for resistance of members (clause 2 of EN 1993-13:2006):

Elastic strain:

$$
\begin{gathered}
E=210000 \mathrm{~N} / \mathrm{mm}^{2} \\
v=0.3 \\
G=80769 \mathrm{~N} / \mathrm{mm}^{2} \\
f_{y b}=280 \mathrm{~N} / \mathrm{mm}^{2}
\end{gathered}
$$$$
G=E[2(1+v)]^{-1}
$$

$$
\gamma M 0=1.00
$$

$$
\gamma_{M 1}=1.00
$$

$$
\varepsilon=0.9161
$$

\section{$>$ Gross cross sectional properties:}

Cross-sectional area:

First moment of area with respect to y-axis:

First moment of area with respect to z-axis:

Gravity centre co-ordinate with respect to y-axis:

Gravity centre co-ordinate with respect to z-axis:

Second moment of area with respect to y-axis:

Second moment of area with respect to z-axis:

$$
\begin{gathered}
A=580.48 \mathrm{~mm}^{2} \\
S_{y}=44261.57 \mathrm{~mm}^{3} \\
S_{z}=4355.39 \mathrm{~mm}^{3}
\end{gathered}
$$

$Y_{G C}=S_{z} / A$

$$
Y_{G C}=7.50 \mathrm{~mm}
$$

$Z_{G C}=S_{x} / A$

$Z_{G C}=76.25 \mathrm{~mm}$

$I_{y}=5272614.66 \mathrm{~mm}^{4}$

$I_{z}=121482.75 \mathrm{~mm}^{4}$ 
Product moment of area with respect to $\mathrm{y}$ - and z-axis:

Second moment of area with respect to y'-axis:

Second moment of area with respect to z'-axis:

Product moment of area with respect to y'- and z'-axis:

Angle between y'-axis and u-axis (principal axis):

Second moment of area with respect to u-axis:

Second moment of area with respect to v-axis:

Radius of gyration with respect to u-axis:

Radius of gyration with respect to v-axis:

Shear centre co-ordinate with respect to u-axis:

Shear centre co-ordinate with respect to v-axis:

Polar radius of gyration about shear centre:

Torsional constant:

Warping constant:

Maximum co-ordinate with respect to u-axis:

Minimum co-ordinate with respect to u-axis:

Maximum co-ordinate with respect to $\mathrm{v}$-axis:

Minimum co-ordinate with respect to v-axis:

$$
I_{y z}=332098.56 \mathrm{~mm}^{4}
$$

$$
I_{y^{\prime}}=I_{y}-A \cdot Z_{C G}{ }^{2}
$$$$
I_{y^{\prime}}=1897662.02 \mathrm{~mm}^{4}
$$$$
I_{z}{ }^{\prime}=I_{z}-A \cdot Y_{C G}{ }^{2}
$$$$
I_{z^{\prime}}=88803.94 \mathrm{~mm}^{4}
$$

$$
I_{y^{\prime} z^{\prime}}=I_{y z}-\left(S_{y} . S_{z}\right) / A
$$

$$
I_{y^{\prime} z^{\prime}}=0
$$

$$
\alpha=0^{\circ}
$$

$$
\begin{gathered}
I_{u}=0.5\left[I_{y^{\prime}}+I_{z^{\prime}}+\sqrt{ }\left(\left(I_{z^{\prime}}-\right.\right.\right. \\
\left.\left.\left.I_{y^{\prime}}\right)^{2}+4 I_{y z^{\prime}}\right)\right] \\
I_{v}=0.5\left[I_{y^{\prime}}+I_{z^{\prime}}-\sqrt{ }\left(\left(I_{z^{\prime}}-\right.\right.\right. \\
\left.\left.\left.I_{y^{\prime}}\right)^{2}+4 I_{y z^{\prime}}\right)\right] \\
i_{u}=\sqrt{ }\left(I_{u} / A\right) \\
i_{v}=\sqrt{ }\left(I_{v} / A\right)
\end{gathered}
$$

$I_{u}=1897662.02 \mathrm{~mm}^{4}$

$$
I_{v}=88803.94 \mathrm{~mm}^{4}
$$$$
i_{u}=57.17 \mathrm{~mm}
$$$$
i_{v}=12.36 \mathrm{~mm}
$$

$$
U_{S C}=-20.84 \mathrm{~mm}
$$

$$
V_{S C}=0
$$

$$
\begin{aligned}
& i_{0}=\sqrt{ }\left(i_{u}{ }^{2}+i_{v}{ }^{2}+U_{S C}{ }^{2}+\right. \\
& V_{S C^{2}} \text { ) }
\end{aligned}
$$$$
i_{0}=62.10 \mathrm{~mm}
$$$$
I_{T}=1209.34 \mathrm{~mm}^{4}
$$$$
I_{W}=367018542.19 \mathrm{~mm}^{6}
$$$$
u_{\max }=34.25 \mathrm{~mm}
$$$$
u_{\text {min }}=-8.75 \mathrm{~mm}
$$$$
v_{\max }=77.5 \mathrm{~mm}
$$

$v_{\min }=-77.5 \mathrm{~mm}$ 
Effective cross sectional properties:

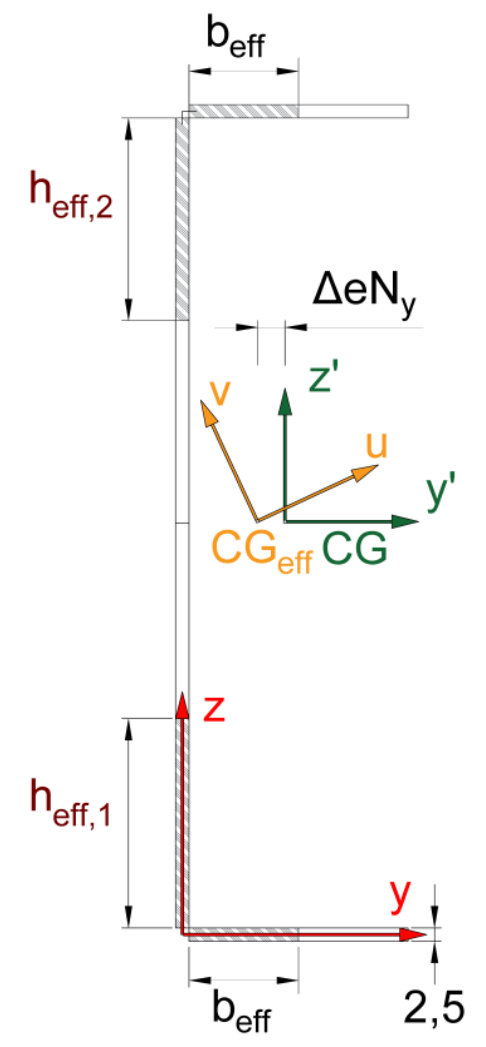

Figure C.2 Effective section scheme of the U cross-section.

\section{$1^{\text {st }}$ Iteration:}

\section{Flange (outstand compression element)}

Stress ratio in the plate:

Plate local buckling factor

(Table 4.2 of EN 1993-1-

5:2006):

Plate slenderness (clause 4.4 of EN 1993-1-5:2006):

Maximum design compressive stress in the plate:

Reduced plate slenderness (clause 4.4 of EN 1993-15:2006):

$$
\psi=\sigma_{\min } / \sigma_{\max }
$$

$\psi=1$

$$
k_{\sigma}=0.43
$$

$$
\bar{\lambda}_{p}=\frac{b_{p} / t}{28.4 \varepsilon \sqrt{k_{\sigma}}}
$$$$
\bar{\lambda}_{p}=0.957>0.748
$$

$$
\sigma_{\text {com, } E d}=280 \mathrm{~N} / \mathrm{mm}^{2}
$$

$$
\bar{\lambda}_{p, \text { red }}=\bar{\lambda}_{p} \sqrt{\frac{\sigma_{c o m, E d}}{f_{y b} / \gamma_{M 0}}}
$$

$$
\bar{\lambda}_{p, \text { red }}=0.957
$$


Reduction factor:

$$
\rho=\frac{\bar{\lambda}_{p}-0.188}{\bar{\lambda}_{p}{ }^{2}}
$$$$
b_{e f f}=\rho \cdot b_{p}
$$

Effective flange width (Table

4.2 of EN 1993-1-5:2006):

\section{Web (internal compression element)}

Stress ratio in the plate:

Plate local buckling factor

(Table 4.1 of EN 1993-1-

5:2006):

Plate slenderness (clause 4.4 of EN 1993-1-5:2006):

Maximum design compressive stress in the plate:

Reduced plate slenderness (clause 4.4 of EN 1993-15:2006):

Reduction factor:

Effective flange width (Table 4.1 of EN 1993-1-5:2006):

$$
\psi=\sigma_{\min } / \sigma_{\max }
$$

$$
\psi=1
$$$$
k_{\sigma}=4
$$

$$
\bar{\lambda}_{p}=\frac{h_{p} / t}{28.4 \varepsilon \sqrt{k_{\sigma}}}
$$

$\bar{\lambda}_{p}=1.158>0.673$

$$
\sigma_{c o m, E d}=280 \mathrm{~N} / \mathrm{mm}^{2}
$$

$$
\bar{\lambda}_{p, \text { red }}=\bar{\lambda}_{p} \sqrt{\frac{\sigma_{c o m, E d}}{f_{y b} / \gamma_{M 0}}}
$$$$
\rho=\frac{\bar{\lambda}_{p}-0.055(3+\psi)}{\bar{\lambda}_{p}^{2}}
$$

$$
h_{e f f}=\rho \cdot h_{p}
$$$$
h_{\text {eff }, 1}=0.5 \cdot h_{\text {eff }}
$$$$
h_{\text {eff }, 2}=0.5 . h_{\text {eff }}
$$

$$
\bar{\lambda}_{p, \text { red }}=1.158
$$

$$
\rho=0.700
$$

$h_{\text {eff }}=105.37 \mathrm{~mm}$

$h_{\text {,eff }, 1}=52.68 \mathrm{~mm}$

$h_{\text {,eff }, 2}=52.68 \mathrm{~mm}$ $\rho=0.84$

$b_{\text {,eff }}=34.27 \mathrm{~mm}$

\section{Effective sectional properties:}

Effective cross-sectional area:

$$
\begin{gathered}
A_{\text {eff }}=434.77 \mathrm{~mm}^{2} \\
Y_{\text {eff, }, G C}=7.128 \mathrm{~mm} \\
Z_{\text {eff, } G C}=76.25 \mathrm{~mm} \\
e N y=0.375 \mathrm{~mm} \\
e N z=0 \mathrm{~mm}
\end{gathered}
$$

Effective gravity centre co-ordinate with respect to z-axis:

Distance in y-direction from gravity centre to effective gravity centre

Distance in z-direction from gravity centre to effective gravity centre

Second moment of effective area with respect to y-axis:

Second moment of effective area with respect to z-axis:

Product moment of effective area with respect to $y-$ and $z-$ axis:

$$
\begin{gathered}
I_{\text {eff }, y}=4216378.17 \mathrm{~mm}^{4} \\
I_{\text {eff }, z}=72960.94 \mathrm{~mm}^{4} \\
I_{\text {eff, }, y z}=236310.89 \mathrm{~mm}^{4}
\end{gathered}
$$


Angle between $\mathrm{y}$-axis and $\mathrm{u}$-axis (principal axis):

Second moment of effective area with respect to u-axis:

Second moment of effective area with respect to v-axis:

Product moment of effective area with respect to $\mathrm{u}$ - and $\mathrm{v}$ axis:

Maximum co-ordinate with respect to u-axis:

Minimum co-ordinate with respect to u-axis:

Maximum co-ordinate with respect to v-axis:

Minimum co-ordinate with respect to v-axis:

$$
\begin{gathered}
\alpha=0^{\circ} \\
I_{\text {eff }, u}=1688590.29 \mathrm{~mm}^{4} \\
I_{\text {eff, }, v}=50869.35 \mathrm{~mm}^{4} \\
I_{\text {eff }, u v}=0 \mathrm{~mm}^{4} \\
u_{\max }=77.5 \mathrm{~mm} \\
u_{\min }=-77.5 \mathrm{~mm} \\
v_{\max }=8.378 \mathrm{~mm} \\
v_{\text {min }}=-28.09 \mathrm{~mm}
\end{gathered}
$$

\section{C.2 C profile}

This example shows how was determined the design value of resistant compression for the columns with section C according the EN 1993-1-1:2005, EN 1993-1-3:2006 and EN 1993-15:2006. For pinned-ended columns the actual length used in the calculations was $3050 \mathrm{~mm}$. For the semi-rigid end-support condition the actual length of the column used in the calculations was $2850 \mathrm{~mm}$.
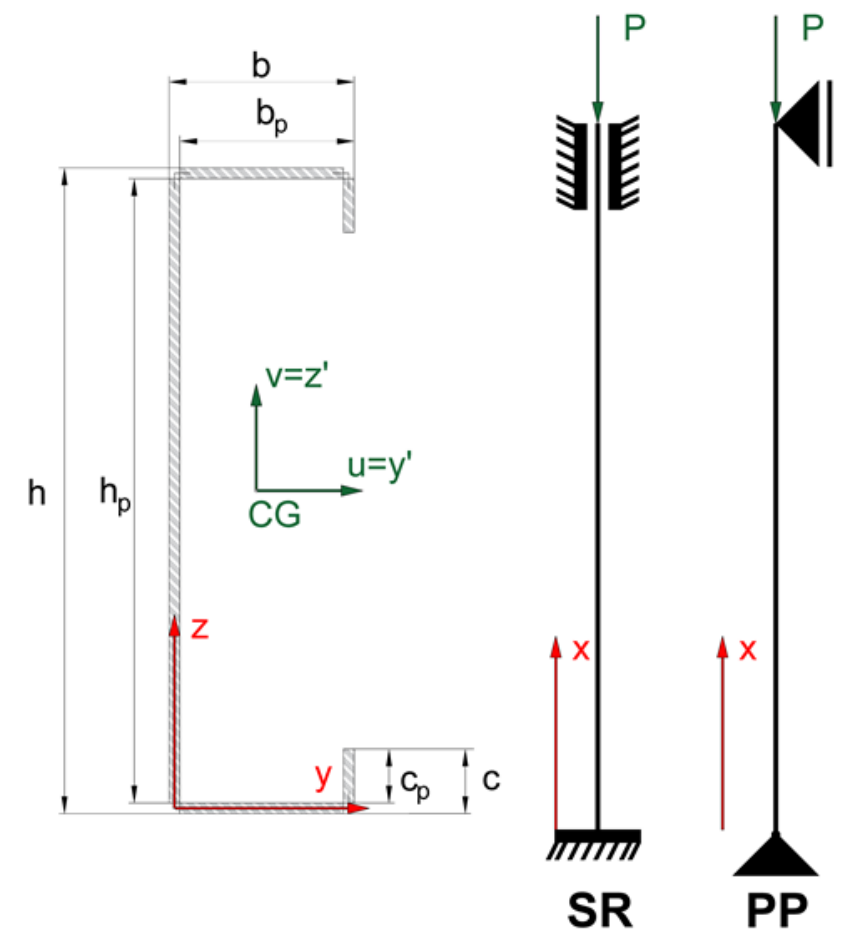

Figure C.3 Scheme of the lipped channel gross cross-section formed by flat plane elements and boundary conditions assumed. 


\section{$>$ C cross-sectional dimensions:}

Height of the section:

Width of the flange:

Width of the stiffener:

Nominal material thickness:

Material core thickness:

$$
\begin{gathered}
t_{c o r}=t_{n}-t_{\text {zinc }} \\
t_{\text {zinc }}=0.04(\mathrm{~S} 280 \mathrm{GD}+\mathrm{Z})
\end{gathered}
$$

Height of the web flat plane element:

Width of the flange flat plane element:

Width of the stiffener flat plane element:

Column Height:

$$
\begin{gathered}
h=150 \mathrm{~mm} \\
b=43 \mathrm{~mm} \\
\mathrm{c}=15 \mathrm{~mm} \\
t_{n}=2.5 \mathrm{~mm} \\
t_{c o r}=t=2.46 \mathrm{~mm} \\
h_{p}=145.60 \mathrm{~mm} \\
b_{p}=38.60 \mathrm{~mm} \\
\mathrm{cp}=12.80 \mathrm{~mm} \\
L=3050 / 2850 \mathrm{~mm}
\end{gathered}
$$

\section{$>$ Material properties:}

Modulus of elasticity:

$E=210000 \mathrm{~N} / \mathrm{mm}^{2}$

Poisson ratio:

$$
v=0.3
$$

Shear modulus:

$G=E[2(1+v)]^{-1}$

$G=80769 \mathrm{~N} / \mathrm{mm}^{2}$

Nominal yield strength (S280GD + Z275):

$f_{y b}=280 \mathrm{~N} / \mathrm{mm}^{2}$

Partial factor for resistance of crosssections (clause 2 of EN 1993-1-

3:2006):

$\gamma_{M 0}=1.00$

Partial factor for resistance of members (clause 2 of EN 1993-1-

3:2006):

Elastic strain:

$$
\varepsilon=\left(235 / f_{y b}\right)^{1 / 2}
$$

$$
\gamma_{M 1}=1.00
$$

$$
\varepsilon=0.9161
$$

\section{$>$ Gross cross sectional properties:}

Cross-sectional area:

First moment of area with respect to y-axis:

First moment of area with respect to z-axis:

$$
\begin{gathered}
A=611.53 \mathrm{~mm}^{2} \\
S_{y}=45112.96 \mathrm{~mm}^{3} \\
S_{z}=6412.12 \mathrm{~mm}^{3}
\end{gathered}
$$


Gravity centre co-ordinate with respect to y-axis:

Gravity centre co-ordinate with respect to Z-axis:

Second moment of area with respect to y-axis:

Second moment of area with respect to z-axis:

Product moment of area with respect to $\mathrm{y}$ - and $\mathrm{z}$-axis:

Second moment of area with respect to y'-axis:

Second moment of area with respect to $\mathrm{z}$ '-axis:

Product moment of area with respect to y'- and z'-axis:

Angle between y'-axis and u-axis (principal axis):

Second moment of area with respect to u-axis:

Second moment of area with respect to v-axis:

Radius of gyration with respect to u-axis:

Radius of gyration with respect to v-axis:

Shear centre co-ordinate with respect to u-axis:

Shear centre co-ordinate with respect to v-axis:

Polar radius of gyration about shear centre:

Torsional constant:

Warping constant:

Maximum co-ordinate with respect to u-axis:

Minimum co-ordinate with respect to u-axis:

Maximum co-ordinate with respect to v-axis:

$$
\begin{aligned}
& Y_{G C}=S_{z} / A \quad Y_{G C}=10.47 \mathrm{~mm} \\
& Z_{G C}=S_{x} / A \quad Z_{G C}=73.77 \mathrm{~mm} \\
& I_{y}=5275392.59 \mathrm{~mm}^{4} \\
& I_{z}=205701.29 \mathrm{~mm}^{4} \\
& I_{y z}=473022.03 \mathrm{~mm}^{4} \\
& I_{y^{\prime}}=I_{y}-A . Z_{C G^{2}}{ }^{2} \quad I_{y^{\prime}}=1947408.88 \mathrm{~mm}^{4} \\
& I_{z^{\prime}}=I_{z}-A . Y_{C G^{2}}{ }^{2} \quad I_{z^{\prime}}=138468.44 \mathrm{~mm}^{4} \\
& I_{y^{\prime} z^{\prime}}=I_{y z}-\left(S_{y} . S_{z}\right) / A \quad I_{y^{\prime} z^{\prime}}=0 \\
& \alpha=0^{\circ} \\
& I_{v}=\begin{array}{c}
0.5\left[I_{y^{\prime}}+I_{z^{\prime}}-\sqrt{ }\left(\left(I_{z^{\prime}}-\right.\right.\right. \\
\left.\left.\left.I_{y^{\prime}}\right)^{2}+4 I_{y z^{\prime}}, 2\right)\right]
\end{array} \quad I_{v}=138468.44 \mathrm{~mm}^{4} \\
& i_{u}=\sqrt{ }\left(I_{u} / A\right) \quad i_{u}=56.43 \mathrm{~mm} \\
& i_{v}=\sqrt{ }\left(I_{v} / A\right) \quad i_{v}=15.04 \mathrm{~mm} \\
& U_{S C}=-28.305 \mathrm{~mm} \\
& V_{S C}=0 \\
& i_{0}=\sqrt{ }\left(i_{u}^{2}+i_{v}^{2}+U_{S C^{2}}+\right. \\
& \left.V_{S C^{2}}\right) \\
& i_{0}=64.9 \mathrm{~mm} \\
& I_{T}=1233.56 \mathrm{~mm}^{4} \\
& I_{W}=618248537.53 \mathrm{~mm}^{6} \\
& u_{\max }=31.28 \mathrm{~mm} \\
& u_{\min }=-11.72 \mathrm{~mm} \\
& v_{\max }=75.0 \mathrm{~mm}
\end{aligned}
$$


Minimum co-ordinate with respect to $\mathrm{v}$-axis:

$v_{\min }=-75.0 \mathrm{~mm}$

\section{Effective cross sectional properties:}

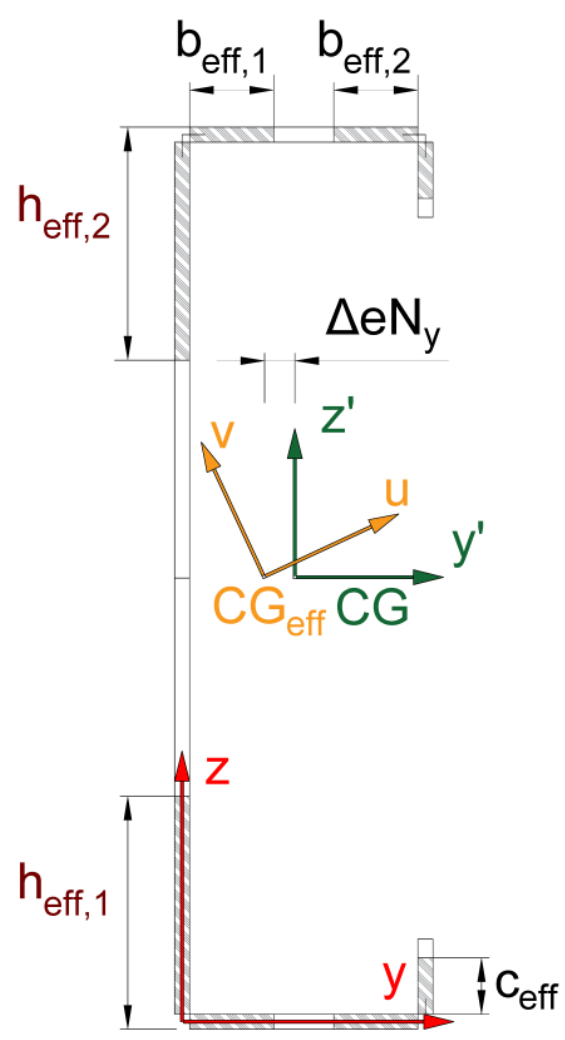

Figure C.4 Effective cross-section scheme of the lipped channel.

\section{$2^{\text {nd }}$ Iteration}

\section{Flange (outstand compression element):}

Stress ratio in the plate:

Plate local buckling factor

(Table 4.2 of EN 1993-1-

5:2006):

Plate slenderness (clause 4.4 of EN 1993-1-5:2006):

$$
\psi=\sigma_{\min } / \sigma_{\max }
$$

$$
\psi=1
$$$$
k_{\sigma}=4
$$

$$
\bar{\lambda}_{p}=\frac{b_{p} / t}{28.4 \varepsilon \sqrt{k_{\sigma}}}
$$

$\bar{\lambda}_{p}=0.302 \leq 0.673$ 
Maximum design compressive stress in the plate:

Reduced plate slenderness (clause 4.4 of EN 1993-15:2006):

Reduction factor:

Effective flange width (Table 4.2 of EN 1993-1-5:2006):

$$
\begin{gathered}
\bar{\lambda}_{p, r e d}=\bar{\lambda}_{p} \sqrt{\frac{\sigma_{c o m, E d}}{f_{y b} / \gamma_{M 0}}} \\
\rho=\frac{\bar{\lambda}_{p}-0.055(3+\psi)}{\bar{\lambda}_{p}^{2}} \\
b_{\text {eff }}=\rho \cdot b_{p} \\
b_{\text {eff }, 1}=0.5 \cdot b_{\text {eff }} \\
b_{\text {eff }, 2}=0.5 \cdot b_{\text {eff }}
\end{gathered}
$$

$\sigma_{c o m, E d}=280 \mathrm{~N} / \mathrm{mm}^{2}$

$$
\bar{\lambda}_{p, \text { red }}=0.302
$$

$$
\rho=1
$$

$b_{, e f f}=38.64 \mathrm{~mm}$

$b_{, e f f, l}=19.32 \mathrm{~mm}$

$b, e f f, 2=19.32 \mathrm{~mm}$

\section{Web (internal compression element):}

Stress ratio in the plate:

Plate local buckling factor

(Table 4.1 of EN 1993-1-

5:2006):

Plate slenderness (clause 4.4 of EN 1993-1-5:2006):

Maximum design

compressive stress in the

plate:

Reduced plate slenderness

(clause 4.4 of EN 1993-1-

5:2006):

Reduction factor:

Effective flange width (Table

4.1 of EN 1993-1-5:2006):

$$
\psi=\sigma_{\min } / \sigma_{\max }
$$

$$
\psi=1
$$$$
k_{\sigma}=4
$$

$$
\bar{\lambda}_{p}=\frac{h_{p} / t}{28.4 \varepsilon \sqrt{k_{\sigma}}}
$$

$\bar{\lambda}_{p}=1.137>0.673$

$$
\sigma_{c o m, E d}=280 \mathrm{~N} / \mathrm{mm}^{2}
$$

$$
\bar{\lambda}_{p, \text { red }}=\bar{\lambda}_{p} \sqrt{\frac{\sigma_{c o m, E d}}{f_{y b} / \gamma_{M 0}}}
$$

$$
\bar{\lambda}_{p, \text { red }}=1.137
$$

$$
\rho=\frac{\bar{\lambda}_{p}-0.055(3+\psi)}{\bar{\lambda}_{p}{ }^{2}}
$$

$$
\rho=1
$$

$$
\begin{gathered}
h_{\text {eff }}=\rho . h_{p} \\
h_{\text {eff }, 1}=0.5 . h_{\text {eff }} \\
h_{\text {eff }, 2}=0.5 \cdot h_{\text {eff }}
\end{gathered}
$$

\section{Stiffener (outstand compression element):}

Stress ratio in the plate:

$$
\psi=\sigma_{\min } / \sigma_{\max }
$$

$\psi=1$

Plate local buckling factor (clause 5.5.3.2(5) of EN 1993-1-3:2006):

$$
k_{\sigma}=0.5 \text { se } \frac{c_{p}}{b_{p}}=0.33 \leq 0.35 \quad k_{\sigma}=0.5
$$


Plate slenderness (clause 4.4 da EN 1993-1-5:2006):

Maximum design compressive stress in the plate:

Reduced plate slenderness (clause 4.4 of EN 1993-15:2006):

Reduction factor:

$$
\bar{\lambda}_{p}=\frac{h_{p} / t}{28.4 \varepsilon \sqrt{k_{\sigma}}}
$$$$
\bar{\lambda}_{p}=0.283<0.748
$$

$$
\sigma_{c o m, E d}=280 \mathrm{~N} / \mathrm{mm}^{2}
$$

$$
\bar{\lambda}_{p, \text { red }}=\bar{\lambda}_{p} \sqrt{\frac{\sigma_{\text {com,Ed }}}{f_{y b} / \gamma_{M 0}}}
$$$$
\bar{\lambda}_{p, \text { red }}=0.283
$$

$$
\rho=\frac{\bar{\lambda}_{p}-0.055(3+\psi)}{\bar{\lambda}_{p}^{2}}
$$

$$
\rho=1
$$

Effective flange width (Table 4.1 of EN 1993-1-5:2006):

$$
c_{\text {eff }}=\rho \cdot c_{p}
$$

$c$, eff $=12.82 \mathrm{~mm}$

Reduction factor $\chi_{d}$ for the distortional buckling resistance of the edge stiffener:

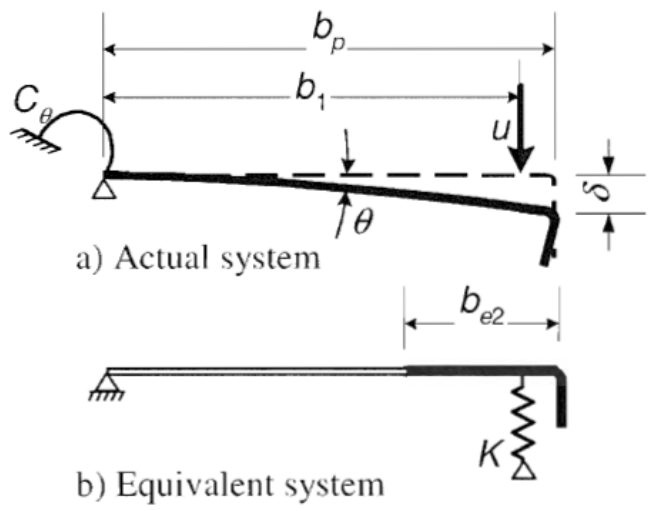

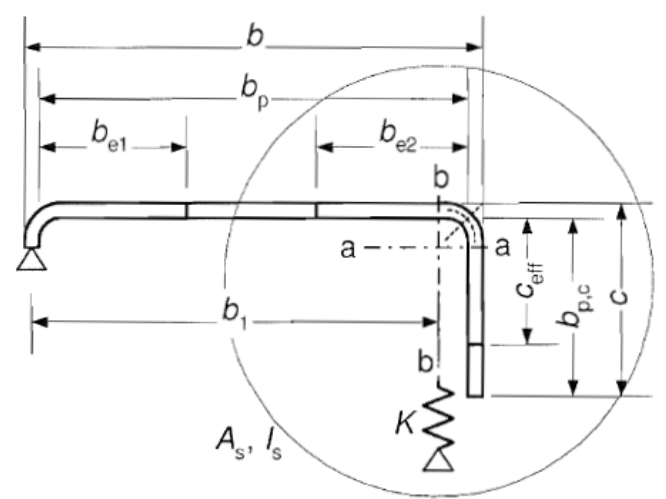

$b / t \leq 60$

a) single edge fold

Figure C.5 Reduction factor for the distortional buckling resistance of the edge stiffener.

Distance from the web-

to-flange junction to the centre of the effective $\mathrm{b}_{1}=19.32 \mathrm{~mm}$ area of the edge stiffener (including effective part $\mathrm{b}_{2}=19.32 \mathrm{~mm}$ $b_{1, \text { eff_ } 2}$ of the flange): 
Spring stiffness of the

edge stiffener per unit

length (clause 5.5.3.1 of

EN 1993-1-3:2006):

$$
=\frac{E t^{3}}{4\left(1-v^{2}\right)} \frac{1}{b_{1}{ }^{2} h_{w}+b_{1}{ }^{3}+0.5 b_{1} b_{2} h_{w} k_{f}} \quad K=2.91 \mathrm{~N} / \mathrm{mm}^{2}
$$

Second moment of

effective area of the edge

stiffener with respect to

$$
I_{s}=2164.27 \mathrm{~mm}^{4}
$$

a-axis:

Effective cross-sectional area

of the edge stiffener:

$$
A_{s}=79.08 \mathrm{~mm}^{2}
$$

Elastic critical buckling stress of the edge stiffener

(clause 5.5.3.2 of EN 1993-

$$
\sigma_{c r, s}=\frac{2 \sqrt{K E I_{s}}}{A_{s}} \quad \sigma_{c r, s}=761.67 \mathrm{Mpa}
$$

1-3:2006):

Edge stiffener slenderness

(clause 5.5.3.1 of EN 1993-

1-3:2006):

$$
\begin{aligned}
& \bar{\lambda}_{d}=\sqrt{\frac{f_{y b}}{\sigma_{c r, s}}} \quad \bar{\lambda}_{d}=0.61 \\
& \chi_{d}=\left\{\begin{array}{l}
1.0 \text { se } \bar{\lambda}_{d} \leq 0.65 \\
1.47-0.723 \bar{\lambda}_{d} \\
\text { se } 0.65 \leq \bar{\lambda}_{d}<1.38 \\
0.66 \text { se } \bar{\lambda}_{d} \geq 1.38
\end{array} \quad \chi_{d}=1\right.
\end{aligned}
$$

Reduction factor for the distortional buckling resistance of the edge stiffener (clause 5.5.3.1 of EN 1993-1-3:2006):

There is no need more iterations by clause 5.5.3.2(10), because the reduction factor $\chi_{d}$ is equal to 1 .

\section{Effective sectional properties:}

Effective cross-sectional area:

Effective gravity centre co-ordinate with respect to y-axis:

Effective gravity centre co-ordinate with respect to z-axis:

Distance in y-direction from gravity centre to effective gravity centre

Distance in z-direction from gravity centre to effective gravity centre

Second moment of effective area with respect to y-axis:

Second moment of effective area with respect to z-axis:

Product moment of effective area with respect to $y$ - and $\mathrm{z}-$ axis:

$$
\begin{gathered}
A_{\text {eff }}=507.25 \mathrm{~mm}^{2} \\
Y_{\text {eff, }, G C}=12.64 \mathrm{~mm} \\
Z_{\text {eff, }, G C}=73.75 \mathrm{~mm} \\
e N y=2.15 \mathrm{~mm} \\
e N z=0 \mathrm{~mm} \\
I_{\text {eff, } y}=4692301.54 \mathrm{~mm}^{4} \\
I_{\text {eff }, z}=205648.70 \mathrm{~mm}^{4} \\
I_{\text {eff, } y z}=473022.03 \mathrm{~mm}^{4}
\end{gathered}
$$


Angle between y-axis and u-axis (principal axis):

Second moment of effective area with respect to u-axis:

Second moment of effective area with respect to v-axis:

Product moment of effective area with respect to $\mathrm{u}$ - and vaxis:

Maximum co-ordinate with respect to u-axis:

Minimum co-ordinate with respect to u-axis:

Maximum co-ordinate with respect to $\mathrm{v}$-axis:

Minimum co-ordinate with respect to v-axis:

$$
\begin{gathered}
\alpha=0^{\circ} \\
I_{e f f, u}=1931794.69 \mathrm{~mm}^{4} \\
I_{\text {eff }, v}=124594.83 \mathrm{~mm}^{4} \\
I_{\text {eff }, u v}=0 \mathrm{~mm}^{4} \\
u_{\max }=29.13 \mathrm{~mm} \\
u_{\min }=-13.87 \mathrm{~mm} \\
v_{\max }=75 \mathrm{~mm} \\
v_{\min }=-75 \mathrm{~mm}
\end{gathered}
$$

\section{Design buckling load - Pinned (PP):}

Design resistance of a crosssection for compression:

Design buckling resistance for flexural buckling:

Design buckling resistance for torsional buckling:

Design buckling resistance for torsional-flexural buckling:

Where $\beta$ is:

Design buckling resistance:

Non-dimensional slenderness (clause 6.3.1.1 of EN 1993-11:2005):

Imperfection factor (Clause 6.3 of EN 1993-1-3:2006):

Capacity reduction factor:

$$
\begin{aligned}
& N_{c, R d}=\frac{A_{e f f} f_{y b}}{\gamma_{M 0}} \\
& N_{c, R d}=142.3 \mathrm{KN} \\
& N_{c r, F}=\frac{\pi^{2} E I}{L^{2}} \\
& N_{c r, T}=\frac{1}{i_{0}^{2}}\left(G I_{t}+\frac{\pi^{2} E I_{w}}{L_{T}^{2}}\right) \\
& N_{c r, T F} \\
& =\frac{N_{c r, F}}{2 \beta}\left[1+\frac{N_{c r, T}}{N_{c r, F}}\right. \\
& \left.-\sqrt{\left(1-\frac{N_{c r, T}}{N_{c r, F}}\right)^{2}+4\left(\frac{y_{o}}{i_{0}}\right)^{2} \frac{N_{c r, T}}{N_{c r, F}}}\right] \\
& \beta=1-\left(\frac{y_{0}}{i_{0}}\right)^{2} \\
& N_{c r}=\min \left(N_{c r, F} ; N_{c r, T} ; N_{c r, T F}\right) \\
& N_{c r}=32.8 \mathrm{KN} \\
& \beta=0.81 \\
& \bar{\lambda}=\frac{A_{e f f} f_{y}}{N_{c r}} \\
& \text { Curve b } \\
& \bar{\lambda}=2.107 \\
& \alpha=0.34 \\
& \Phi=0.5\left[1+\alpha(\bar{\lambda}-0.2)+\bar{\lambda}^{2}\right] \\
& \Phi=3.04
\end{aligned}
$$


Reduction factor for the

relevant buckling mode:

$$
\chi=\frac{1}{\Phi+\sqrt{\Phi^{2}-\bar{\lambda}^{2}}} \leq 1.0
$$

Design value of the buckling resistance:

Considering the variation of the centre of gravity:

$$
N_{b, R d}=\frac{\chi A_{e f f} f_{y}}{\gamma_{M 1}}
$$

\section{$>$ Design buckling load - Semi-rigid (SR):}

Design buckling resistance for flexural buckling:

Design buckling resistance for torsional buckling:

Design buckling resistance for torsional-flexural buckling:

Where $\beta$ is:

Design buckling resistance:

Non-dimensional slenderness (clause 6.3.1.1 of EN 1993-11:2005):

Imperfection factor (Clause 6.3 of EN 1993-1-3:2006):

Capacity reduction factor:

Reduction factor for the relevant buckling mode:

Design value of the buckling resistance:

Considering the variation of centre of gravity:

$$
\begin{array}{cc}
N_{c r, F}=\frac{\pi^{2} E I}{L^{2}} & N_{c r, F}=150.29 \mathrm{KN} \\
N_{c r, T}=\frac{1}{i_{0}{ }^{2}}\left(G I_{t}+\frac{\pi^{2} E I_{w}}{L_{T}{ }^{2}}\right) & N_{c r, T}=62.83 \mathrm{KN}
\end{array}
$$

$$
N_{c r, T F}
$$

$$
\begin{aligned}
& =\frac{N_{c r, F}\left[1+\frac{N_{c r, T}}{N_{c r, F}}\right.}{2 \beta} \\
& \left.-\sqrt{\left(1-\frac{N_{c r, T}}{N_{c r, F}}\right)^{2}+4\left(\frac{y_{o}}{i_{0}}\right)^{2} \frac{N_{c r, T}}{N_{c r, F}}}\right]
\end{aligned}
$$$$
N_{c r, T F}=61.27 \mathrm{KN}
$$

$$
\beta=1-\left(\frac{y_{0}}{i_{0}}\right)^{2}
$$

$$
\beta=0.81
$$

$$
N_{c r}=\min \left(N_{c r, F} ; N_{c r, T} ; N_{c r, T F}\right)
$$

$$
N_{c r}=61 K N
$$

$$
\bar{\lambda}=\frac{A_{e f f} f_{y}}{N_{c r}}
$$

$$
\bar{\lambda}=1.53
$$

Curve b

$$
\alpha=0.34
$$

$$
\begin{gathered}
\Phi=0.5\left[1+\alpha(\bar{\lambda}-0.2)+\bar{\lambda}^{2}\right] \\
\chi=\frac{1}{\Phi+\sqrt{\Phi^{2}-\bar{\lambda}^{2}}} \leq 1.0 \\
N_{b, R d}=\frac{\chi A_{e f f} f_{y}}{\gamma_{M 1}}
\end{gathered}
$$$$
\Phi=1.89
$$$$
\chi=0.33
$$$$
N_{b, R d}=47.28 \mathrm{KN}
$$$$
N_{E d}=\mathbf{N}_{\mathrm{b}, \mathbf{R d}}{ }^{*}
$$$$
N_{b, R d}=41.3 \mathrm{kN}
$$ 


\section{C.3 Open built-up I columns}

This example shows how was determined the design buckling resistance of compressed columns with open built-up I cross-section according the EN 1993-1-1:2005, EN 1993-13:2006 and EN 1993-1-5:2006. The open built-up I cross-section consists of two lipped channel profiles fastened back-to-back on the web using self-drilling screws. For pinned-ended columns the actual length used in the calculations was $3050 \mathrm{~mm}$. For the semi-rigid end-support condition the actual length of the column used in the calculations was $2850 \mathrm{~mm}$.
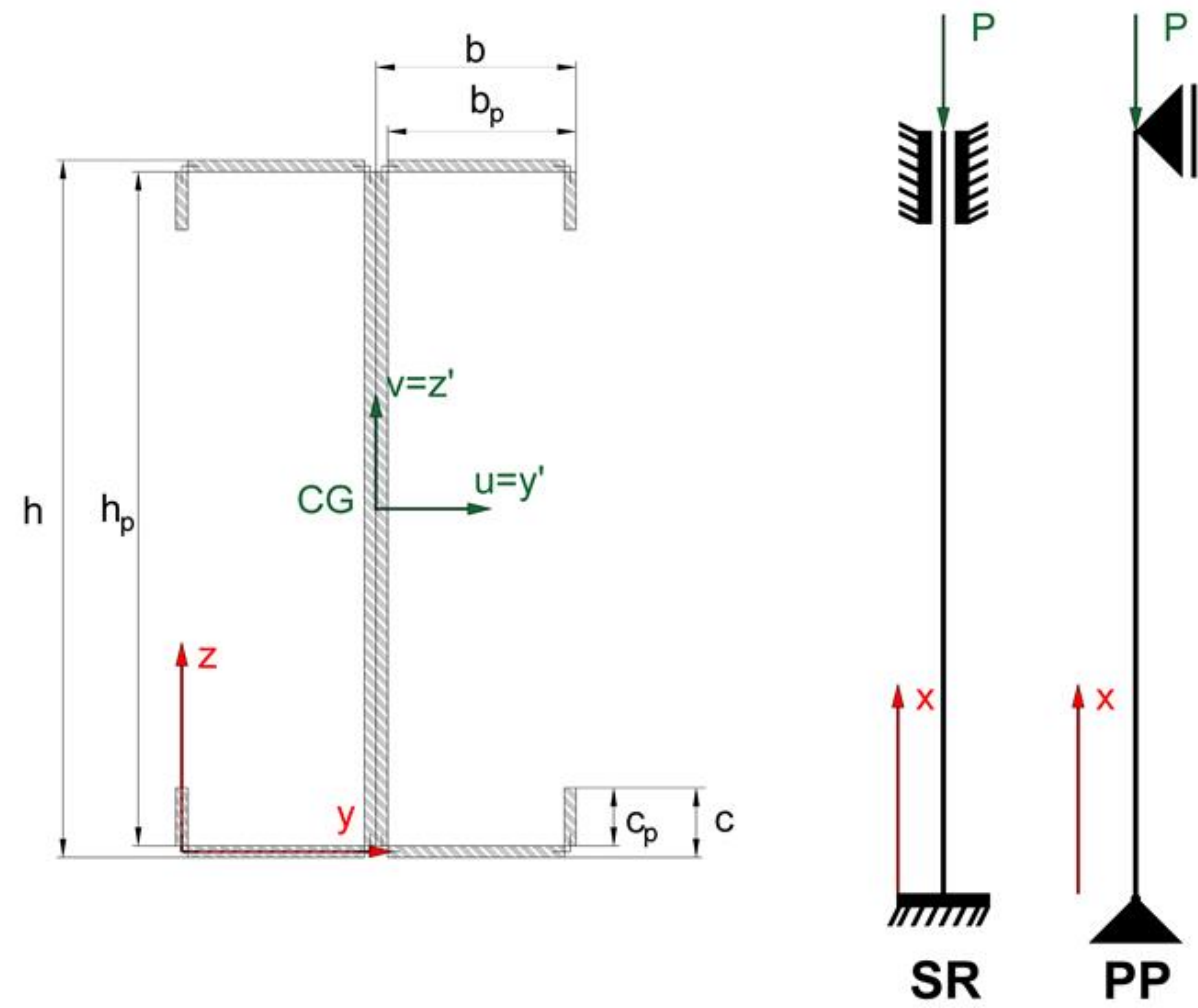

Figure C.6 Scheme of the open built-up I gross cross-section formed by flat plane elements and boundary conditions assumed.

\section{$>$ Material properties:}

Modulus of elasticity:

Poisson ratio:

Shear modulus:

Nominal yield strength (S280GD):

$$
E=210000 \mathrm{~N} / \mathrm{mm}^{2}
$$$$
v=0.3
$$

$$
\begin{gathered}
G=E[2(1+v)]^{-1} \quad G=80769 \mathrm{~N} / \mathrm{mm}^{2} \\
f_{y b}=280 \mathrm{~N} / \mathrm{mm}^{2}
\end{gathered}
$$


Partial factor for resistance of crosssections (clause 2 of EN 1993-1-3:2006):

Partial factor for resistance of members (clause 2 of EN 1993-1-3:2006):

Elastic strain:

$$
\begin{aligned}
\gamma_{M 0} & =1.00 \\
\gamma_{M 1} & =1.00 \\
\varepsilon=\left(235 / f_{y b}\right)^{1 / 2} & \varepsilon=0.9161
\end{aligned}
$$

\section{$>$ Gross cross sectional properties:}

Cross-sectional area:

First moment of area with respect to y-axis:

First moment of area with respect to z-axis:

Gravity centre co-ordinate with respect to y-axis:

Gravity centre co-ordinate with respect to Z-axis:

Second moment of area with respect to y-axis:

Second moment of area with respect to z-axis:

Product moment of area with respect to y- and z-axis:

Second moment of area with respect to y'-axis:

Second moment of area with respect to $z$ '-axis:

Product moment of area with respect to y'- and z'-axis:

Angle between y'-axis and u-axis (principal axis):

Second moment of area with respect to u-axis:

Second moment of area with respect to v-axis:

Radius of gyration with respect to u-axis:

Radius of gyration with respect to v-axis:

$$
\begin{aligned}
& A=1272.05 \mathrm{~mm}^{2} \\
& S_{y}=94648.24 \mathrm{~mm}^{3} \\
& S_{z}=53931.11 \mathrm{~mm}^{3} \\
& Y_{G C}=S_{z} / A \quad Y_{G C}=42.38 \mathrm{~mm} \\
& Z_{G C}=S_{x} / A \quad Z_{G C}=74.38 \mathrm{~mm} \\
& I_{y}=11192406.81 \mathrm{~mm}^{4} \\
& I_{z}=2773860.16 \mathrm{~mm}^{4} \\
& I_{y z}=4011665.97 \mathrm{~mm}^{4} \\
& I_{y^{\prime}}=I_{y}-A \cdot Z_{C G^{2}}{ }^{2} \quad I_{y^{\prime}}=4151944.34 \mathrm{~mm}^{4} \\
& I_{z^{\prime}}=I_{z}-A . Y_{C G^{2}}{ }^{2} \quad I_{z^{\prime}}=486520.71 \mathrm{~mm}^{4} \\
& I_{y^{\prime} z^{\prime}}=I_{y z}-\left(S_{y} . S_{z}\right) / A \quad I_{y^{\prime} z^{\prime}}=0 \\
& \alpha=0^{\circ} \\
& I_{u}=0.5\left[I_{y^{\prime}}+I_{z^{\prime}}+\sqrt{ }\left(\left(I_{z^{\prime}}-\right.\right.\right. \\
& \left.\left.\left.I_{y^{\prime}}\right)^{2}+4 I_{y z} z^{2}\right)\right] \\
& I_{u}=4151996.92 \mathrm{~mm}^{4} \\
& I_{v}=0.5\left[I_{y^{\prime}}+I_{z^{\prime}}-\sqrt{ }\left(\left(I_{z^{\prime}}-\right.\right.\right. \\
& \left.\left.\left.I_{y^{\prime}}\right)^{2}+4 I_{y z} z^{2}\right)\right] \\
& i_{u}=\sqrt{ }\left(I_{u} / A\right) \\
& i_{u}=57.12 \mathrm{~mm} \\
& i_{v}=\sqrt{ }\left(I_{v} / A\right) \\
& i_{v}=19.58 \mathrm{~mm}
\end{aligned}
$$


Shear centre co-ordinate with respect to u-axis:

$$
U_{S C}=0 \mathrm{~mm}
$$

Shear centre co-ordinate with respect to v-axis:

Polar radius of gyration about shear centre:

$$
\begin{aligned}
& V_{S C}=0 \mathrm{~mm} \\
& i_{0}=\sqrt{ }\left(i_{u}{ }^{2}+i_{v}{ }^{2}+U_{S C^{2}}+\right. \\
& V_{S C^{2}} \text { ) } \\
& i_{0}=60.39 \mathrm{~mm} \\
& I_{T}=6086.95 \mathrm{~mm}^{4} \\
& I_{W}=3085703631.28 \mathrm{~mm}^{6}
\end{aligned}
$$

Torsional constant:

Warping constant:

Maximum co-ordinate with respect to u-axis:

Minimum co-ordinate with respect to u-axis:

Maximum co-ordinate with respect to v-axis:

Minimum co-ordinate with respect to $\mathrm{v}$-axis:

\section{$>$ Effective cross sectional properties:}

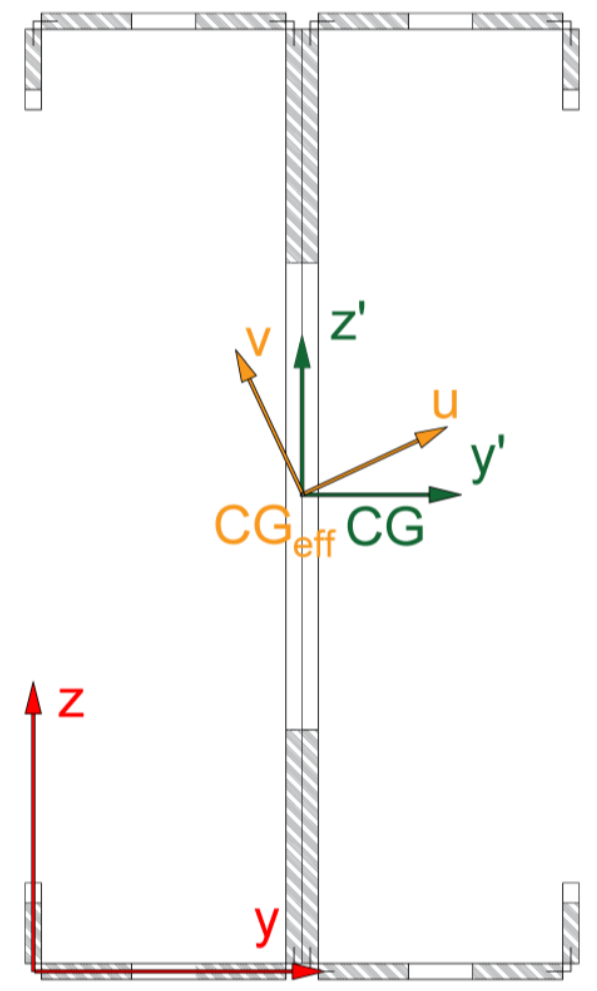

Figure C.7 Effective section scheme of the open built-up I cross-section. 


\section{Effective sectional properties:}

Effective cross-sectional area:

Effective gravity centre co-ordinate with respect to y-axis:

Effective gravity centre co-ordinate with respect to z-axis:
$A_{\text {eff }}=1014.52 \mathrm{~mm}^{2}$

$Y_{\text {eff }, G C}=41.75 \mathrm{~mm}$

$Z_{\text {eff, }, G C}=73.75 \mathrm{~mm}$

\section{Design buckling load - Pinned:}

Design resistance of a crosssection for compression:

Design buckling resistance for flexural buckling:

Design buckling resistance for torsional buckling:

Design buckling resistance for torsional-flexural buckling:

Where $\beta$ is:

Design buckling resistance:

Non-dimensional slenderness (clause 6.3.1.1 of EN 1993-11:2005):

Imperfection factor (Clause 6.3 of EN 1993-1-3:2005):

Capacity reduction factor:

Reduction factor for the relevant buckling mode:

$$
\begin{aligned}
N_{c, R d} & =\frac{A_{e f f} f_{y b}}{\gamma_{M 0}} & N_{c, R d} & =284.06 \mathrm{KN} \\
N_{c r, F} & =\frac{\pi^{2} E I}{L^{2}} & N_{c r, F} & =108.7 \mathrm{KN}
\end{aligned}
$$$$
N_{c r, T}=\frac{1}{i_{0}{ }^{2}}\left(G I_{t}+\frac{\pi^{2} E I_{w}}{L_{T}{ }^{2}}\right) \quad N_{c r, T}=323.35 \mathrm{KN}
$$

$N_{c r, T F}$

$$
\begin{aligned}
& =\frac{N_{c r, F}\left[1+\frac{N_{c r, T}}{2 \beta}\right.}{N_{c r, F}} \\
& \left.-\sqrt{\left(1-\frac{N_{c r, T}}{N_{c r, F}}\right)^{2}+4\left(\frac{y_{o}}{i_{0}}\right)^{2} \frac{N_{c r, T}}{N_{c r, F}}}\right]
\end{aligned}
$$$$
N_{c r, T F}=323.35 K N
$$$$
\beta=1-\left(\frac{y_{0}}{i_{0}}\right)^{2}
$$$$
\beta=1
$$

$$
N_{c r}=\min \left(N_{c r, F} ; N_{c r, T} ; N_{c r, T F}\right)
$$

$N_{c r}=108.7 \mathrm{KN}$

$$
\bar{\lambda}=\frac{A_{e f f} f_{y}}{N_{c r}}
$$

$$
\bar{\lambda}=1.622
$$

Curve b

$$
\alpha=0.34
$$

$$
\Phi=0.5\left[1+\alpha(\bar{\lambda}-0.2)+\bar{\lambda}^{2}\right]
$$$$
\Phi=2.057
$$

$$
\chi=0.301
$$


Design value of the buckling resistance:

$$
N_{b, R d}=\frac{\chi A_{e f f} f_{y}}{\gamma_{M 1}}
$$

$N_{b, R d}=85.51 K N$

\section{Design buckling load - Semi-rigid:}

Design buckling resistance for flexural buckling:

Design buckling resistance for torsional buckling:

Design buckling resistance for torsional-flexural buckling:

Where $\beta$ is:

Design buckling resistance:

Non-dimensional slenderness (clause 6.3.1.1 of EN 1993-11:2005):

Imperfection factor (Clause 6.3 of EN 1993-1-3:2005):

Capacity reduction factor:

Reduction factor for the relevant buckling mode: Design value of the buckling resistance:

$$
\begin{array}{cc}
N_{c r, F}=\frac{\pi^{2} E I}{L^{2}} & N_{c r, F}=498.1 K N \\
N_{c r, T}=\frac{1}{i_{0}{ }^{2}}\left(G I_{t}+\frac{\pi^{2} E I_{w}}{L_{T}{ }^{2}}\right) & N_{c r, T}=350.7 \mathrm{KN}
\end{array}
$$

$N_{c r, T F}$

$$
\begin{aligned}
& =\frac{N_{c r, F}\left[1+\frac{N_{c r, T}}{N_{c r, F}}\right.}{2 \beta} \\
& \left.-\sqrt{\left(1-\frac{N_{c r, T}}{N_{c r, F}}\right)^{2}+4\left(\frac{y_{o}}{i_{0}}\right)^{2} \frac{N_{c r, T}}{N_{c r, F}}}\right]
\end{aligned}
$$$$
N_{c r, T F}=305.7 \mathrm{KN}
$$

$$
\beta=1-\left(\frac{y_{0}}{i_{0}}\right)^{2}
$$

$$
\beta=1
$$

$$
N_{c r}=\min \left(N_{c r, F} ; N_{c r, T} ; N_{c r, T F}\right)
$$

$$
N_{c r}=350 \mathrm{KN}
$$

$$
\bar{\lambda}=\sqrt{\frac{A_{e f f} f_{y}}{N_{c r}}}
$$

$$
\bar{\lambda}=1.19
$$

Curve b

$$
\alpha=0.34
$$

$$
\begin{gathered}
\Phi=0.5\left[1+\alpha(\bar{\lambda}-0.2)+\bar{\lambda}^{2}\right] \\
\chi=\frac{1}{\Phi+\sqrt{\Phi^{2}-\bar{\lambda}^{2}}} \leq 1.0 \\
N_{b, R d}=\frac{\chi A_{e f f} f_{y}}{\gamma_{M 1}}
\end{gathered}
$$$$
\Phi=1.025
$$ 


\section{C.4 Closed built-up R columns}

This example shows how was determined the design buckling resistance of compressed columns with closed built-up R cross-section according the EN 1993-1-1:2005, EN 1993-13:2006 and EN 1993-1-5:2006. The closed built-up R cross-section consists of one lipped channel and one plain channel profile fastened in the flanges using self-drilling screws. For pinned-ended columns the actual length used in the calculations was $3050 \mathrm{~mm}$. For the semirigid end-support condition the actual length of the column used in the calculations was 2850 $\mathrm{mm}$.
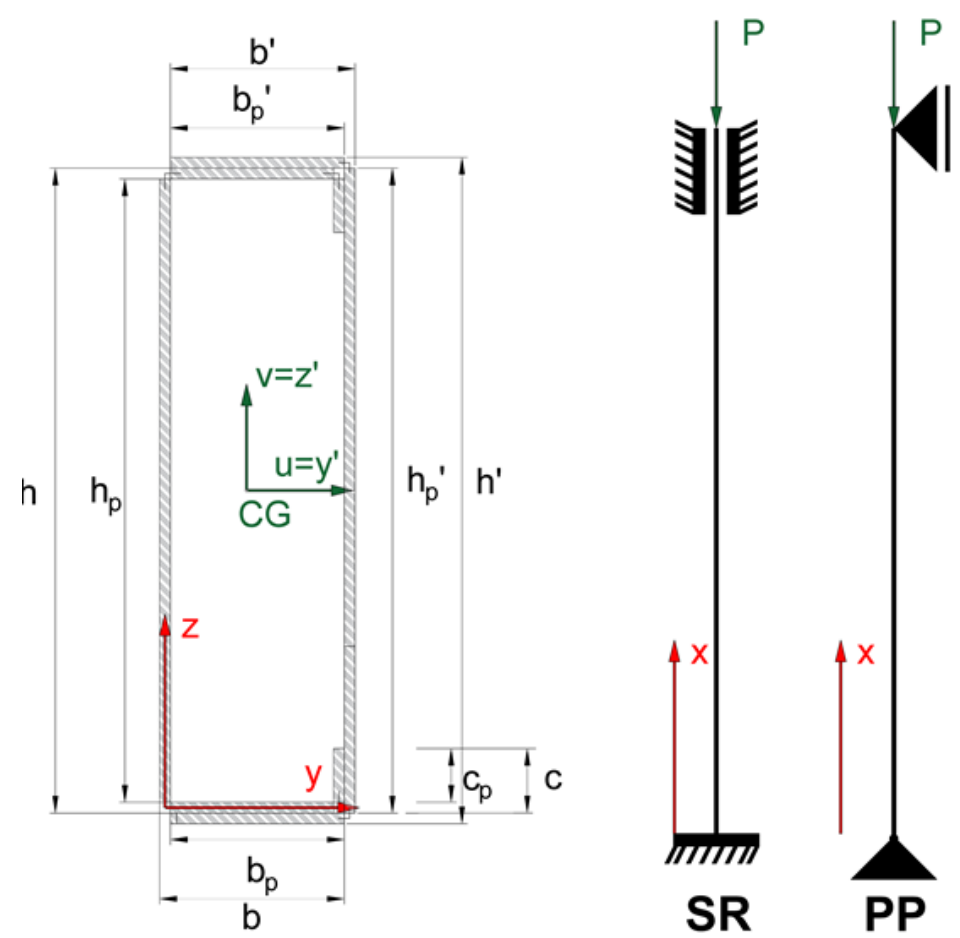

Figure C.8 Gross cross sectional scheme of the R cross-section formed by flat plane elements.

\section{$>$ Material properties:}

Modulus of elasticity:

Poisson ratio:

Shear modulus:

Nominal yield strength (S280GD + Z275):

$$
E=210000 \mathrm{~N} / \mathrm{mm}^{2}
$$$$
v=0.3
$$

$$
\begin{array}{r}
G=E[2(1+v)]^{-1} \quad G=80769 \mathrm{~N} / \mathrm{mm}^{2} \\
f_{y b}=280 \mathrm{~N} / \mathrm{mm}^{2}
\end{array}
$$


Partial factor for resistance of crosssections (clause 2 of EN 1993-1-

3:2006):

Partial factor for resistance of members (clause 2 of EN 1993-1-

3:2006):

Elastic strain:

$$
\gamma_{M 0}=1.00
$$

$$
\gamma_{M 1}=1.00
$$

$$
\varepsilon=\left(235 / f_{y b}\right)^{1 / 2}
$$

\section{Gross cross sectional properties:}

Cross-sectional area:

First moment of area with respect to y-axis:

First moment of area with respect to z-axis:

Gravity centre co-ordinate with respect to y-axis:

Gravity centre co-ordinate with respect to z-axis:

Second moment of area with respect to y-axis:

Second moment of area with respect to z-axis:

Product moment of area with respect to y-and z-axis:

Second moment of area with respect to y'-axis:

Second moment of area with respect to $\mathrm{z}$ '-axis:

Product moment of area with respect to y'- and z'-axis:

Angle between y'-axis and u-axis (principal axis):

Second moment of area with respect to u-axis:

Second moment of area with respect to v-axis:

Radius of gyration with respect to u-axis:

$$
\begin{aligned}
& A=1222.62 \mathrm{~mm}^{2} \\
& S_{y}=91696.5 \mathrm{~mm}^{3} \\
& S_{z}=27608.58 \mathrm{~mm}^{3} \\
& Y_{G C}=S_{z} / A \\
& Z_{G C}=S_{x} / A \\
& I_{y}=10933375.39 .55 \mathrm{~mm}^{4} \\
& I_{z}=1057366.73 \mathrm{~mm}^{4} \\
& I_{y z}=2070643.5 \mathrm{~mm}^{4} \\
& I_{y^{\prime}}=I_{y}-A . Z_{C G^{2}}{ }^{2} \quad I_{y^{\prime}}=4056137.89 \mathrm{~mm}^{4} \\
& I_{z^{\prime}}=I_{z}-A . Y_{C G^{2}}{ }^{2} \quad I_{z^{\prime}}=433923.89 \mathrm{~mm}^{4} \\
& I_{y^{\prime} z^{\prime}}=I_{y z}-\left(S_{y} . S_{z}\right) / A \quad I_{y^{\prime} z^{\prime}}=0 \\
& \alpha=0^{\circ} \\
& I_{u}=0.5\left[I_{y^{\prime}}+I_{z^{\prime}}+\sqrt{ }\left(\left(I_{z^{\prime}}-\right.\right.\right. \\
& \left.\left.\left.I_{y^{\prime}}\right)^{2}+4 I_{y z^{2}}{ }^{2}\right)\right] \\
& I_{u}=4056137.89 \mathrm{~mm}^{4} \\
& I_{v}=0.5\left[I_{y^{\prime}}+I_{z^{\prime}}-\sqrt{ }\left(\left(I_{z^{\prime}}-\right.\right.\right. \\
& \left.\left.\left.I_{y^{\prime}}\right)^{2}+4 I_{y z^{2}}{ }^{2}\right)\right] \\
& i_{u}=\sqrt{ }\left(I_{u} / A\right) \\
& I_{v}=433923.89 \mathrm{~mm}^{4} \\
& i_{u}=57.60 \mathrm{~mm}
\end{aligned}
$$


Radius of gyration with respect to v-axis:

Shear centre co-ordinate with respect to u-axis:

Shear centre co-ordinate with respect to v-axis:

Polar radius of gyration about shear centre:

Torsional constant:

Warping constant:

Maximum co-ordinate with respect to u-axis:

Minimum co-ordinate with respect to u-axis:

Maximum co-ordinate with respect to v-axis:

Minimum co-ordinate with respect to v-axis:

$$
i_{v}=\sqrt{ }\left(I_{v} / A\right) \quad i_{v}=18.84 \mathrm{~mm}
$$

$$
U_{S C}=-53.50 \mathrm{~mm}
$$

$$
V_{S C}=0 \mathrm{~mm}
$$

$$
\begin{aligned}
& i_{0}=\sqrt{ }\left(i_{u}{ }^{2}+i_{v}{ }^{2}+U S C^{2}+\right. \\
& V_{S C^{2}} \text { ) }
\end{aligned}
$$

$$
\begin{gathered}
i_{0}=80.84 \mathrm{~mm} \\
I_{T}=5771.17 \mathrm{~mm}^{4} \\
I_{W}=7295701211.59 \mathrm{~mm}^{6} \\
u_{\max }=-22.897 \mathrm{~mm} \\
u_{\min }=-23.811 \mathrm{~mm} \\
v_{\max }=77.46 \mathrm{~mm} \\
v_{\text {min }}=-77.46 \mathrm{~mm}
\end{gathered}
$$

\section{Effective cross sectional properties:}

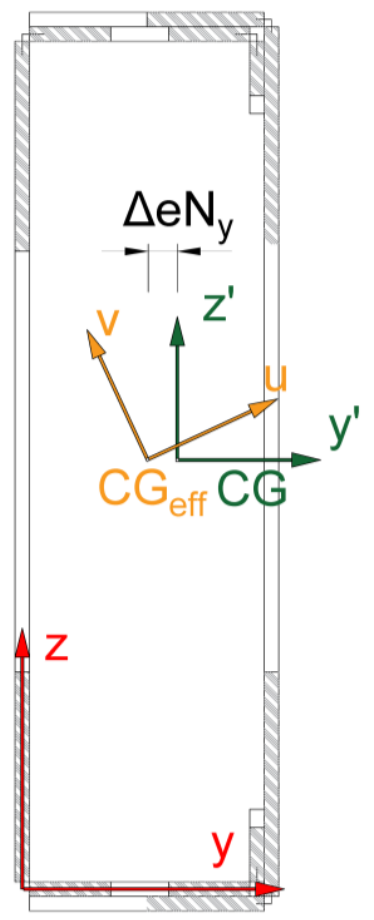

Figure C.9 Effective cross-section scheme for the closed built-up R cross-section. 


\section{Effective sectional properties:}

Effective cross-sectional area:

Effective gravity centre co-ordinate with respect to y-axis: Effective gravity centre co-ordinate with respect to z-axis:
$A_{\text {eff }}=942.03 \mathrm{~mm}^{2}$

$Y_{\text {eff }, G C}=24.36 \mathrm{~mm}$

$Z_{\text {eff, }, G C}=73.75 \mathrm{~mm}$

\section{Design buckling load - Pinned-end:}

Design resistance of a crosssection for compression:

Design buckling resistance for flexural buckling:

Design buckling resistance for torsional buckling:

Design buckling resistance for torsional-flexural buckling:

Where $\beta$ is:

Design buckling resistance:

Non-dimensional slenderness (clause 6.3.1.1 of EN 1993-11:2005):

Imperfection factor (Clause 6.3 of EN 1993-1-3:2006):

Capacity reduction factor:

Reduction factor for the relevant buckling mode:

$$
N_{c, R d}=\frac{A_{e f f} f_{y b}}{\gamma_{M 0}} \quad N_{c, R d}=263.77 \mathrm{KN}
$$$$
N_{c r, F}=\frac{\pi^{2} E I}{L^{2}}
$$$$
N_{c r, F}=96.68 K N
$$$$
N_{c r, T}=\frac{1}{i_{0}^{2}}\left(G I_{t}+\frac{\pi^{2} E I_{w}}{L_{T}{ }^{2}}\right)
$$$$
N_{c r, T}=320.04 K N
$$

$$
N_{c r, T F}
$$$$
=\frac{N_{c r, F}}{2 \beta}\left[1+\frac{N_{c r, T}}{N_{c r, F}}\right.
$$$$
\left.-\sqrt{\left(1-\frac{N_{c r, T}}{N_{c r, F}}\right)^{2}+4\left(\frac{y_{o}}{i_{0}}\right)^{2} \frac{N_{c r, T}}{N_{c r, F}}}\right]
$$

$$
N_{c r, T F}=269.76 K N
$$

$$
\beta=1-\left(\frac{y_{0}}{i_{0}}\right)^{2}
$$

$$
\beta=0.56
$$

$$
N_{c r}=\min \left(N_{c r, F} ; N_{c r, T} ; N_{c r, T F}\right)
$$

$$
N_{c r}=91.33 \mathrm{KN}
$$

$$
\bar{\lambda}=\frac{A_{e f f} f_{y}}{N_{c r}}
$$

$$
\bar{\lambda}=1.69
$$

Curve b

$\alpha=0.34$

$$
\begin{gathered}
\Phi=0.5\left[1+\alpha(\bar{\lambda}-0.2)+\bar{\lambda}^{2}\right] \\
\chi=\frac{1}{\Phi+\sqrt{\Phi^{2}-\bar{\lambda}^{2}}} \leq 1.0
\end{gathered}
$$

$\Phi=2.18$ 
Design value of the buckling resistance:

$$
N_{b, R d}=\frac{\chi A_{e f f} f_{y}}{\gamma_{M 1}}
$$

$$
N_{b, R d}=73.24 K N
$$

\section{> Design buckling load - Semi-rigid:}

Design buckling resistance for flexural buckling:

Design buckling resistance for torsional buckling:

Design buckling resistance
for torsional-flexural
buckling:

Where $\beta$ is:

Design buckling resistance:

Non-dimensional slenderness (clause 6.3.1.1 of EN 1993-11:2005):

Imperfection factor (Clause 6.3 of EN 1993-1-3:2006):

Capacity reduction factor:

Reduction factor for the relevant buckling mode:

Design value of the buckling resistance:

$$
N_{c r, F}=\frac{\pi^{2} E I}{L^{2}}
$$$$
N_{c r, F}=186.39 K N
$$

$$
N_{c r, T}=\frac{1}{i_{0}^{2}}\left(G I_{t}+\frac{\pi^{2} E I_{w}}{L_{T}^{2}}\right)
$$

$N_{c r, T}=448.84 K N$

$N_{c r, T F}$

$$
\begin{aligned}
& =\frac{N_{c r, F}\left[1+\frac{N_{c r, T}}{N_{c r, F}}\right.}{2 \beta} \\
& \left.-\sqrt{\left(1-\frac{N_{c r, T}}{N_{c r, F}}\right)^{2}+4\left(\frac{y_{o}}{i_{0}}\right)^{2} \frac{N_{c r, T}}{N_{c r, F}}}\right]
\end{aligned}
$$$$
N_{c r, T F}=448.13 \mathrm{KN}
$$

$$
\beta=1-\left(\frac{y_{0}}{i_{0}}\right)^{2}
$$

$$
\beta=1
$$

$$
N_{c r}=\min \left(N_{c r, F} ; N_{c r, T} ; N_{c r, T F}\right)
$$

$N_{c r}=186.39 K N$

$$
\bar{\lambda}=\sqrt{\frac{A_{e f f} f_{y}}{N_{c r}}}
$$

$$
\bar{\lambda}=0.936
$$

$$
\text { Curve b }
$$

$$
\alpha=0.34
$$

$$
\begin{gathered}
\Phi=0.5\left[1+\alpha(\bar{\lambda}-0.2)+\bar{\lambda}^{2}\right] \\
\chi=\frac{1}{\Phi+\sqrt{\Phi^{2}-\bar{\lambda}^{2}}} \leq 1.0
\end{gathered}
$$

$$
\Phi=1.34
$$

$$
\chi=0.63
$$

$$
N_{b, R d}=\frac{\chi A_{e f f} f_{y}}{\gamma_{M 1}}
$$




\section{C.5 Closed built-up 2R columns}

This example shows how was determined the design buckling resistance of compressed columns with closed built-up 2R cross-section according the EN 1993-1-1:2005, EN 1993-13:2006 and EN 1993-1-5:2006. The closed built-up R cross-section consists of two lipped channels fastened back-to-back on the web (I) and two plain channel profiles fastened on the flanges of each one of the lipped channels (I) using self-drilling screws. For pinned-ended columns the actual length used in the calculations was $3050 \mathrm{~mm}$. For the semi-rigid end-support condition the actual length of the column used in the calculations was $2850 \mathrm{~mm}$.
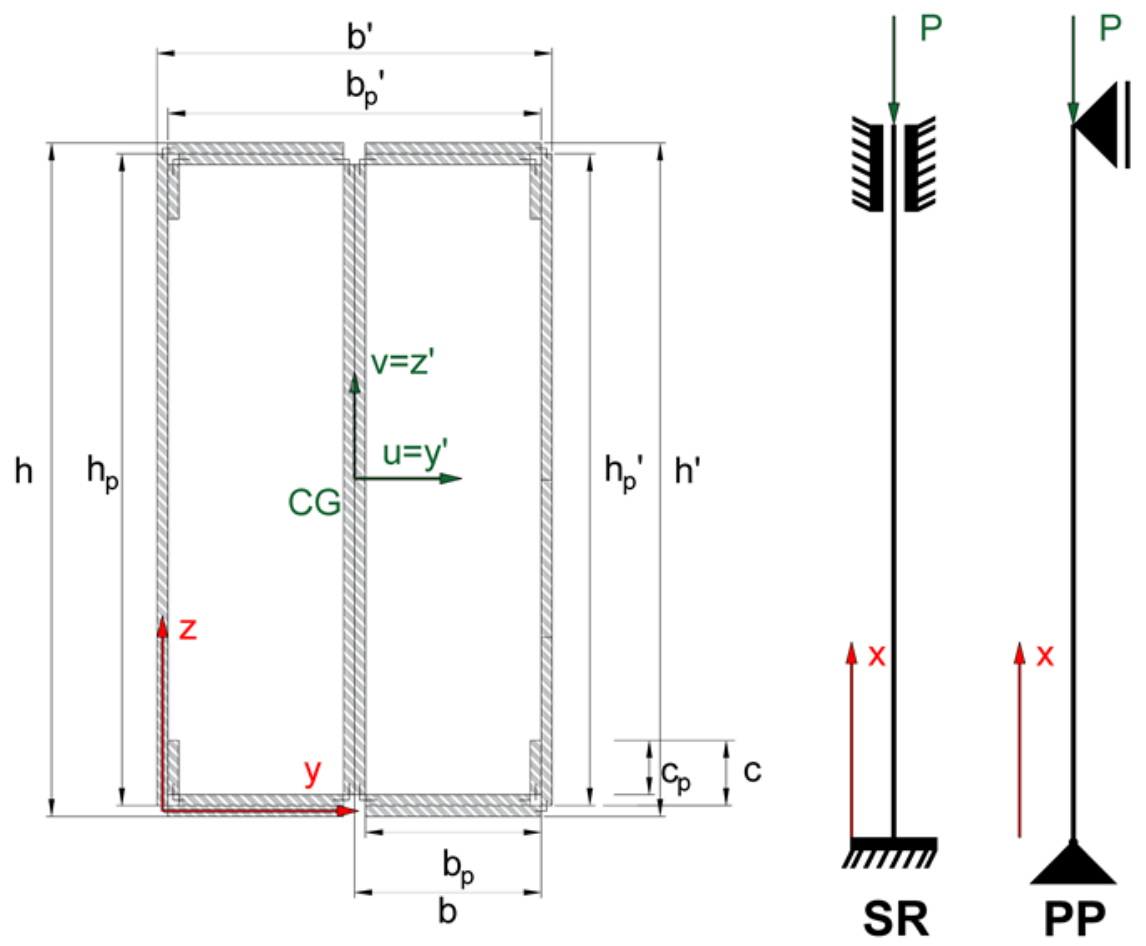

Figure C.10 Gross cross sectional scheme of the closed built-up 2R cross-section formed by flat plane elements.

\section{$>$ Material properties:}

Modulus of elasticity:

$$
\begin{gathered}
E=210000 \mathrm{~N} / \mathrm{mm}^{2} \\
v=0.3
\end{gathered}
$$

Poisson ratio: 
Shear modulus:

Nominal yield strength (S280GD + Z275):

Partial factor for resistance of crosssections (clause 2 of EN 1993-13:2006):

Partial factor for resistance of members (clause 2 of EN 1993-13:2006):

Elastic strain:

$$
\begin{gathered}
G=E[2(1+v)]^{-1} \quad G=80769 \mathrm{~N} / \mathrm{mm}^{2} \\
f_{y b}=280 \mathrm{~N} / \mathrm{mm}^{2} \\
\gamma_{M 0}=1.00 \\
\gamma_{M I}=1.00 \\
\varepsilon=\left(235 / f_{y b}\right)^{1 / 2} \\
\varepsilon=0.9161
\end{gathered}
$$

\section{Gross cross sectional properties:}

Cross-sectional area:

First moment of area with respect to y-axis:

First moment of area with respect to $\mathrm{z}$-axis:

Gravity centre co-ordinate with respect to y-axis:

Gravity centre co-ordinate with respect to Z-axis:

Second moment of area with respect to y-axis:

Second moment of area with respect to Z-axis:

Product moment of area with respect to $\mathrm{y}$ - and z-axis:

Second moment of area with respect to y'-axis:

Second moment of area with respect to z'-axis:

Product moment of area with respect to y'- and z'-axis:

Angle between y'-axis and u-axis (principal axis):

Second moment of area with respect to u-axis:

Second moment of area with respect to v-axis:

$$
\begin{aligned}
& A=2463.79 \mathrm{~mm}^{2} \\
& S_{y}=187814.58 \mathrm{~mm}^{3} \\
& S_{z}=108973.36 \mathrm{~mm}^{3} \\
& Y_{G C}=S_{z} / A \\
& Y_{G C}=44.23 \mathrm{~mm} \\
& Z_{G C}=S_{x} / A \\
& Z_{G C}=76.23 \mathrm{~mm} \\
& I_{y}=22465095.74 \mathrm{~mm}^{4} \\
& I_{z}=7063168.74 \mathrm{~mm}^{4} \\
& I_{y z}=8307039.30 \mathrm{~mm}^{4} \\
& I_{y^{\prime}}=I_{y}-A \cdot Z_{C G^{2}}{ }^{2} \quad I_{y^{\prime}}=8147989.60 \mathrm{~mm}^{4} \\
& I_{z^{\prime}}=I_{z}-A . Y_{C G}{ }^{2} \quad I_{z^{\prime}}=2243277.0 \mathrm{~mm}^{4} \\
& I_{y^{\prime} z^{\prime}}=I_{y z}-\left(S_{y} . S_{z}\right) / A \quad I_{y^{\prime} z^{\prime}}=0 \\
& \alpha=0^{\circ} \\
& I_{u}=0.5\left[I_{y^{\prime}}+I_{z^{\prime}}+\sqrt{ }\left(\left(I_{z^{\prime}}\right.\right.\right. \\
& \left.\left.\left.-I_{y^{\prime}}\right)^{2}+4 I_{y z} z^{2}\right)\right] \\
& I_{u}=8147989.6 \mathrm{~mm}^{4} \\
& I_{v}=0.5\left[I_{y^{\prime}}+I_{z^{\prime}}-\sqrt{ }\left(\left(I_{z^{\prime}}-\right.\right.\right. \\
& \left.\left.\left.I_{y}\right)^{2}+4 I_{y z},{ }^{2}\right)\right] \\
& I_{v}=2243277.0 \mathrm{~mm}^{4}
\end{aligned}
$$


Radius of gyration with respect to u-axis:

Radius of gyration with respect to v-axis:

Shear centre co-ordinate with respect to u-axis:

Shear centre co-ordinate with respect to v-axis:

Polar radius of gyration about shear centre:

Torsional constant:

Warping constant:

Maximum co-ordinate with respect to u-axis:

Minimum co-ordinate with respect to u-axis:

Maximum co-ordinate with respect to v-axis:

Minimum co-ordinate with respect to v-axis:

$$
\begin{array}{cc}
i_{u}=\sqrt{ }\left(I_{u} / A\right) & i_{u}=57.51 \mathrm{~mm} \\
i_{v}=\sqrt{ }\left(I_{v} / A\right) & i_{v}=30.17 \mathrm{~mm} \\
U_{S C} & =0 \mathrm{~mm} \\
V_{S C} & =0 \mathrm{~mm} \\
i_{0}=\sqrt{ }\left(i_{u}^{2}+i_{v}^{2}+U_{S C^{2}+}\right. & i_{0}=64.94 \mathrm{~mm} \\
\left.V_{S C^{2}}\right) & I_{T}=8423.8 \mathrm{~mm}^{4} \\
I_{W}= & 26302961553.3 \mathrm{~mm}^{6} \\
u_{\max } & =45.46 \mathrm{~mm} \\
u_{\min } & =-45.46 \mathrm{~mm} \\
v_{\max } & =77.46 \mathrm{~mm} \\
v_{\min } & =-77.46 \mathrm{~mm}
\end{array}
$$

\section{$>$ Effective cross sectional properties:}




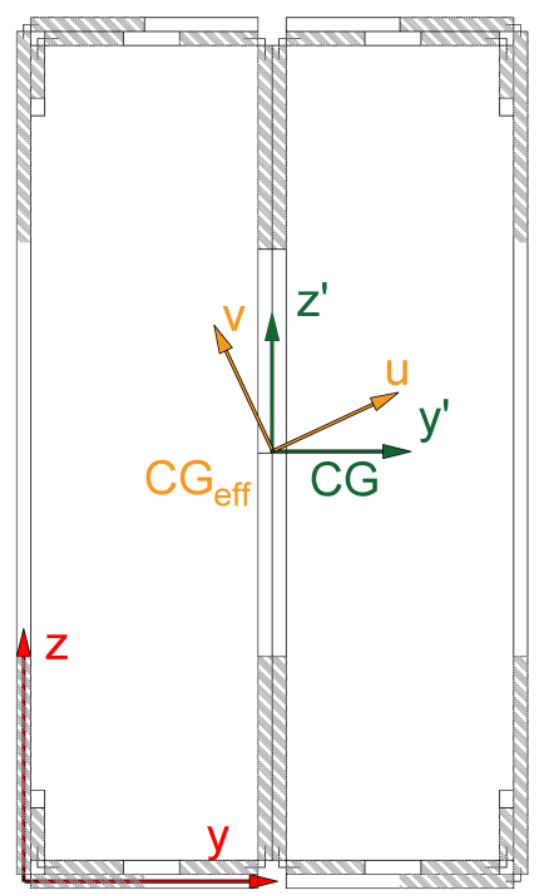

Figure C.11 Effective section scheme of the closed built-up 2R cross-section.

Effective cross-sectional area:

Effective gravity centre co-ordinate with respect to y-axis:

Effective gravity centre co-ordinate with respect to z-axis:

$$
\begin{gathered}
A_{\text {eff }}=1884.06 \mathrm{~mm}^{2} \\
Y_{\text {eff, }, G C}=44.25 \mathrm{~mm} \\
Z_{\text {eff, }, G C}=76.25 \mathrm{~mm}
\end{gathered}
$$

\section{Design buckling load - Pinned-end:}

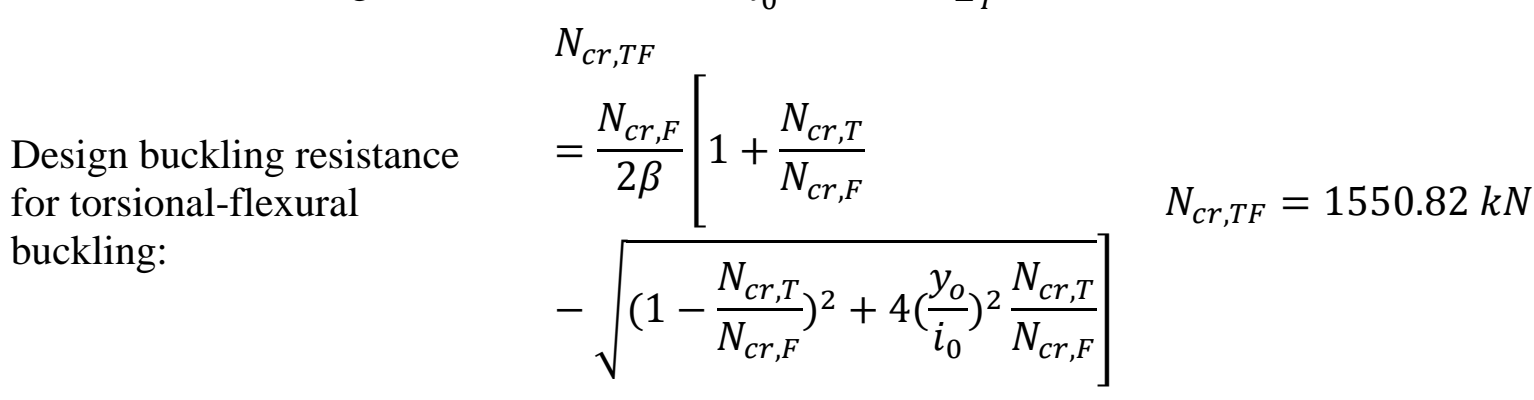

$$
\begin{aligned}
& N_{c, R d}=\frac{A_{e f f} f_{y b}}{\gamma_{M 0}} \quad N_{c, R d}=527.54 k N \\
& N_{c r, F}=\frac{\pi^{2} E I}{L^{2}} \quad N_{c r, F}=499.8 k N \\
& N_{c r, T}=\frac{1}{{i_{0}}^{2}}\left(G I_{t}+\frac{\pi^{2} E I_{w}}{L_{T}^{2}}\right) \quad N_{c r, T}=1550.82 k N \\
& N_{c, R d}=\frac{A_{e f f} f_{y b}}{\gamma_{M 0}} \quad N_{c, R d}=527.54 k N
\end{aligned}
$$


Where $\beta$ is:

$$
\beta=1-\left(\frac{y_{0}}{i_{0}}\right)^{2}
$$

$$
\beta=1
$$

Design buckling resistance:

$$
N_{c r}=\min \left(N_{c r, F} ; N_{c r, T} ; N_{c r, T F}\right)
$$

$$
N_{c r}=499.8 \mathrm{kN}
$$

Non-dimensional slenderness (clause 6.3.1.1 of EN 1993-11:2005):

Imperfection factor (Clause 6.3 of EN 1993-1-3:2006):

Capacity reduction factor:

$$
\Phi=0.5\left[1+\alpha(\bar{\lambda}-0.2)+\bar{\lambda}^{2}\right]
$$

Reduction factor for the relevant buckling mode:

$$
\begin{gathered}
\chi=\frac{1}{\Phi+\sqrt{\Phi^{2}-\bar{\lambda}^{2}}} \leq 1.0 \\
N_{b, R d}=\frac{\chi A_{e f f} f_{y}}{\gamma_{M 1}}
\end{gathered}
$$

Design value of the buckling resistance:

$$
\bar{\lambda}=\frac{A_{e f f} f_{y}}{N_{c r}}
$$

Curve $b$

$$
\alpha=0.34
$$

$$
\bar{\lambda}=1.03
$$

$$
\Phi=1.17
$$

$$
\chi=0.58
$$

$N_{b, R d}=305.57 \boldsymbol{k N}$

\section{Design buckling load - Semi-rigid:}

Design buckling resistance for flexural buckling:

Design buckling resistance for torsional buckling:

Design buckling resistance for torsional-flexural buckling:

Where $\beta$ is:

Design buckling resistance:

$$
\begin{aligned}
N_{c r, F}=\frac{\pi^{2} E I}{L^{2}} & N_{c r, F}=2289.66 \mathrm{KN} \\
N_{c r, T}=\frac{1}{i_{0}{ }^{2}}\left(G I_{t}+\frac{\pi^{2} E I_{w}}{L_{T}{ }^{2}}\right) & N_{c r, T}=1752.68 \mathrm{KN}
\end{aligned}
$$$$
N_{c r, T F}
$$$$
=\frac{N_{c r, F}}{2 \beta}\left[1+\frac{N_{c r, T}}{N_{c r, F}}\right.
$$$$
\left.-\sqrt{\left(1-\frac{N_{c r, T}}{N_{c r, F}}\right)^{2}+4\left(\frac{y_{o}}{i_{0}}\right)^{2} \frac{N_{c r, T}}{N_{c r, F}}}\right]
$$$$
N_{c r, T F}=1752.68 \mathrm{KN}
$$

$$
\beta=1-\left(\frac{y_{0}}{i_{0}}\right)^{2}
$$

$$
\beta=1
$$

$$
N_{c r}=\min \left(N_{c r, F} ; N_{c r, T} ; N_{c r, T F}\right)
$$


Non-dimensional slenderness

(clause 6.3.1.1 of EN 1993-1-

1:2005):

Imperfection factor (Clause

6.3 of EN 1993-1-3:2006):

Capacity reduction factor:

Reduction factor for the

relevant buckling mode:

Design value of the buckling resistance:

$$
\bar{\lambda}=\frac{A_{e f f} f_{y}}{N_{c r}}
$$$$
\bar{\lambda}=0.73
$$

Curve b

$$
\alpha=0.34
$$

$$
\Phi=0.5\left[1+\alpha(\bar{\lambda}-0.2)+\bar{\lambda}^{2}\right]
$$$$
\Phi=0.92
$$

$$
\begin{array}{cc}
\chi=\frac{1}{\Phi+\sqrt{\Phi^{2}-\bar{\lambda}^{2}}} \leq 1.0 & \chi=0.68 \\
N_{b, R d}=\frac{\chi A_{\text {eff }} f_{y}}{\gamma_{M 1}} & N_{b, R d}=\mathbf{4 4 3 . 4 2 ~ K N}
\end{array}
$$




\section{APPENDIX D IMPROVEMENT OF THE END-SUPPORT DEVICES}

It is important to show some improvements/modifications implemented in the test set-up introducing some significant differences in the behaviour of CFS lipped channel columns. Specifically, for the pin-ended situation it was observed that the friction between the steel cylinder and the end steel plate of the end-support device was very high. This translates in small differences between the global behaviour of a pinned-pinned CFS lipped channel column and fixed-fixed CFS lipped channel column. In order to address this situation and to assess the actual influence of the friction on the behaviour of CFS columns the end support device was modified. The steel cylinder was Teflon lined in order to reduce the friction between it and the end steel plate where the specimen was placed. It was observed that this small modification changed the behaviour of the CFS column as it can be seen in the Figure D.1 comparing the results obtained without Teflon (C_PP_30LL_K1) with the ones obtained using Teflon (C_PP_30LL_K1_TL) and with the one with semi-rigid (C_SR_30LL_K3) end support condition. It seems that friction may influence significantly the magnitude of the generated restraining forces and cause small increase in the recorded critical temperatures. For higher axial loads the influence of friction may be even bigger.

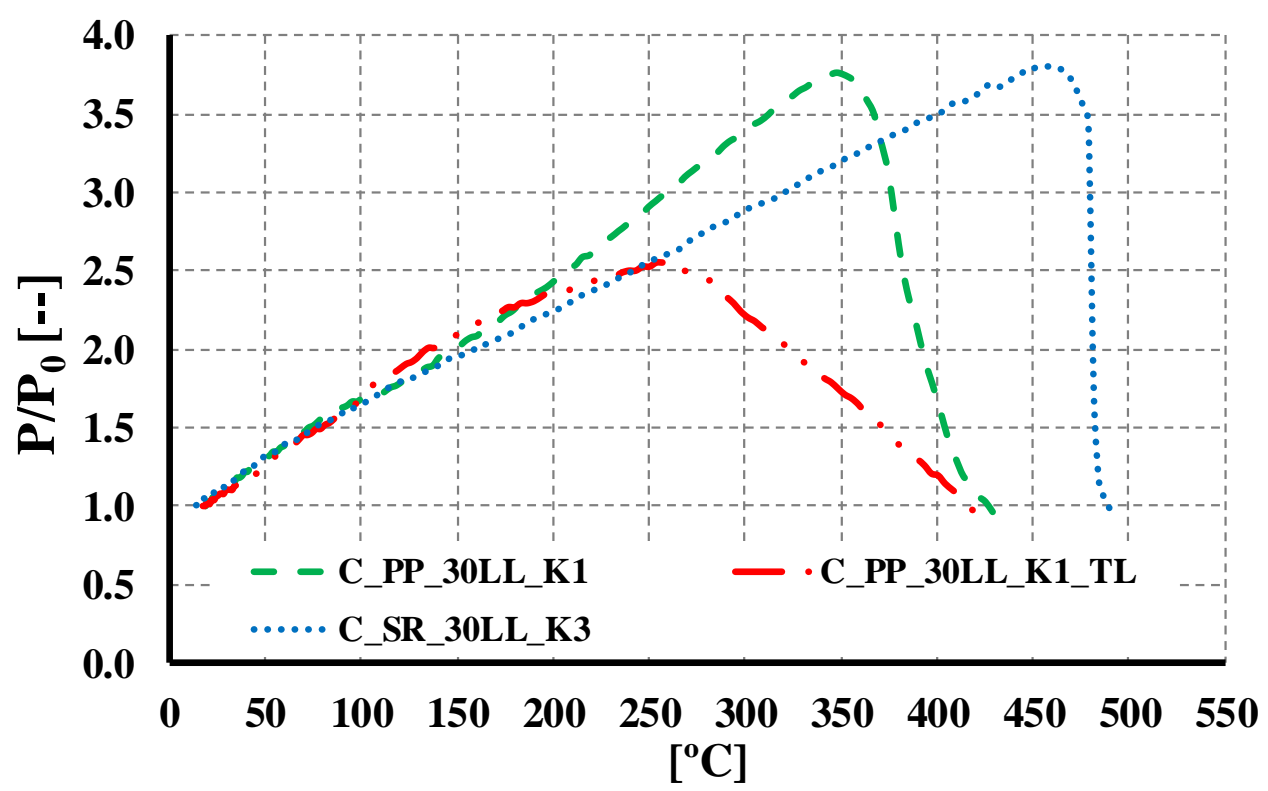

Figure D.1 Influence of Teflon (PTFE) in the hinge of the end-support device. 



\section{APPENDIX E BUCKLING TESTS AT AMBIENT TEMPERATURE}

\section{E.1 Pinned-ended Columns}

\section{E.1.1 Load versus axial shortening}
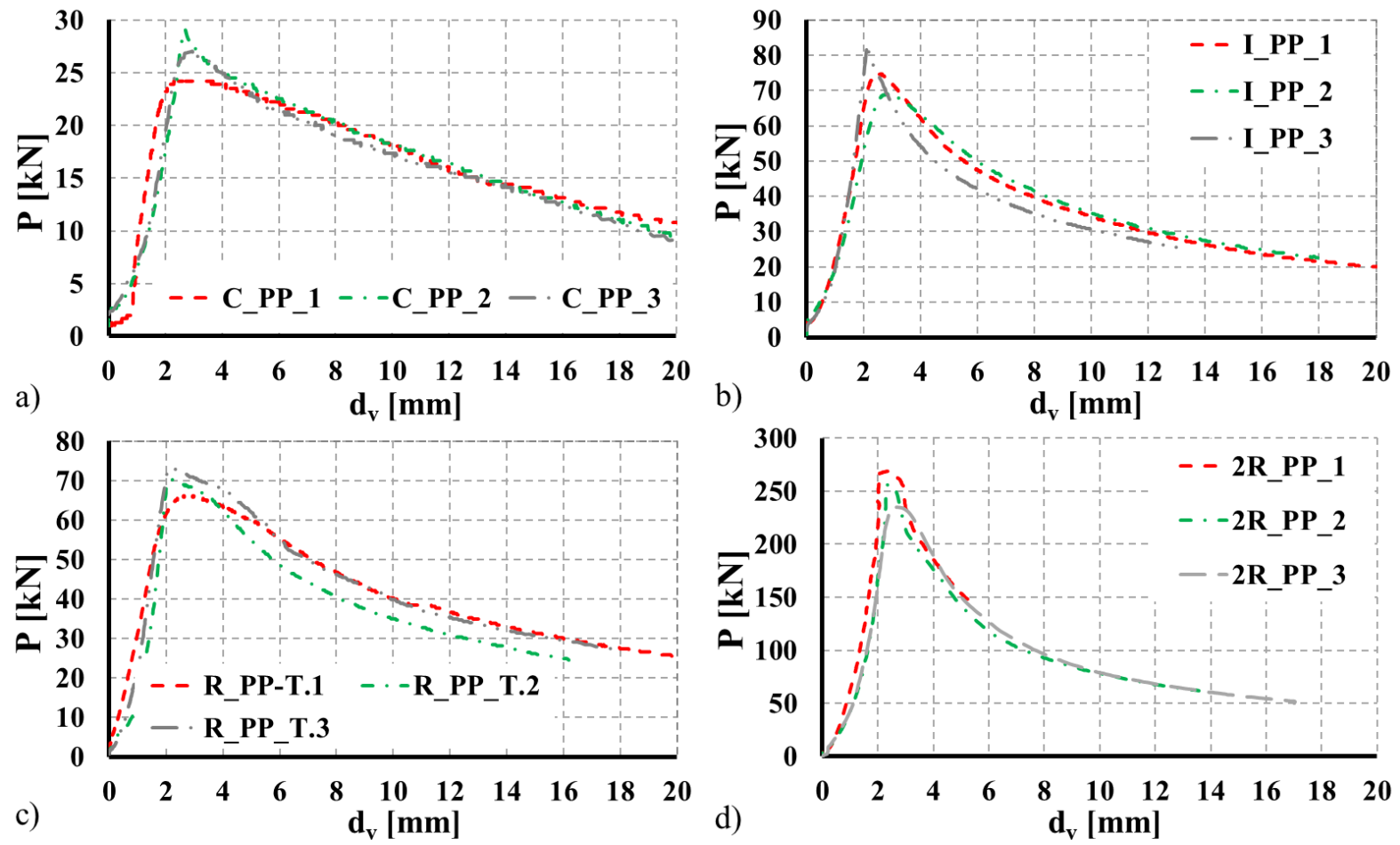

Figure E.1 Axial load versus axial shortening curves for all tested cross-sections considering the pinned-ended support condition. a) Columns with lipped channel cross-section. b) Columns with open built-up I cross-section. c) Columns with closed built-up R cross-section. d) Columns with closed built-up 2R cross-section. 


\section{E.1.2 Strain evolution}
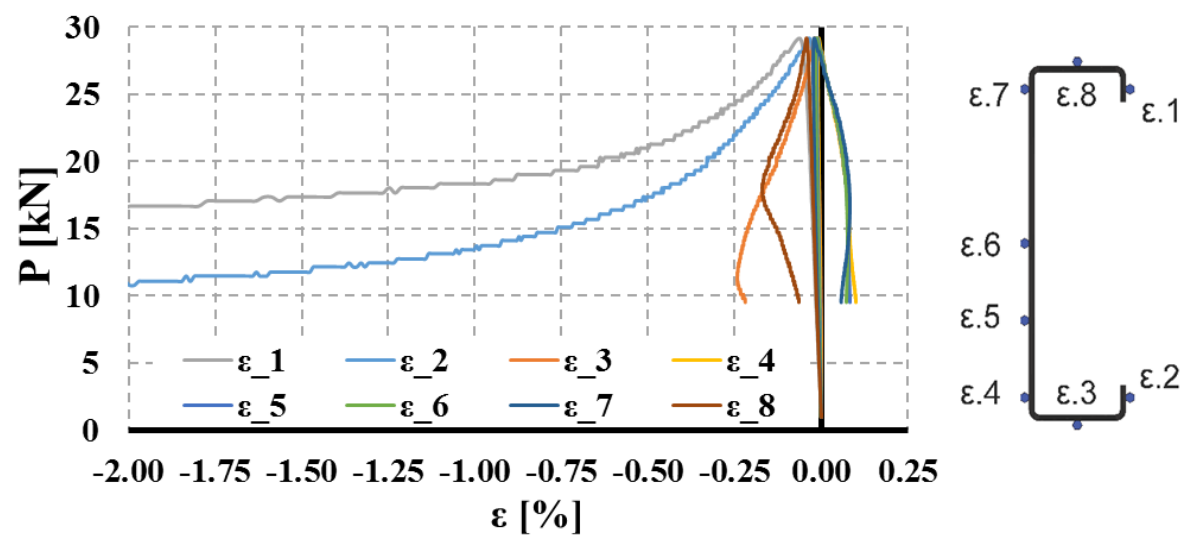

Figure E.2 Load-strain curves obtained from strain gauges placed in different points of the lipped channel cross-section. C_PP-2.
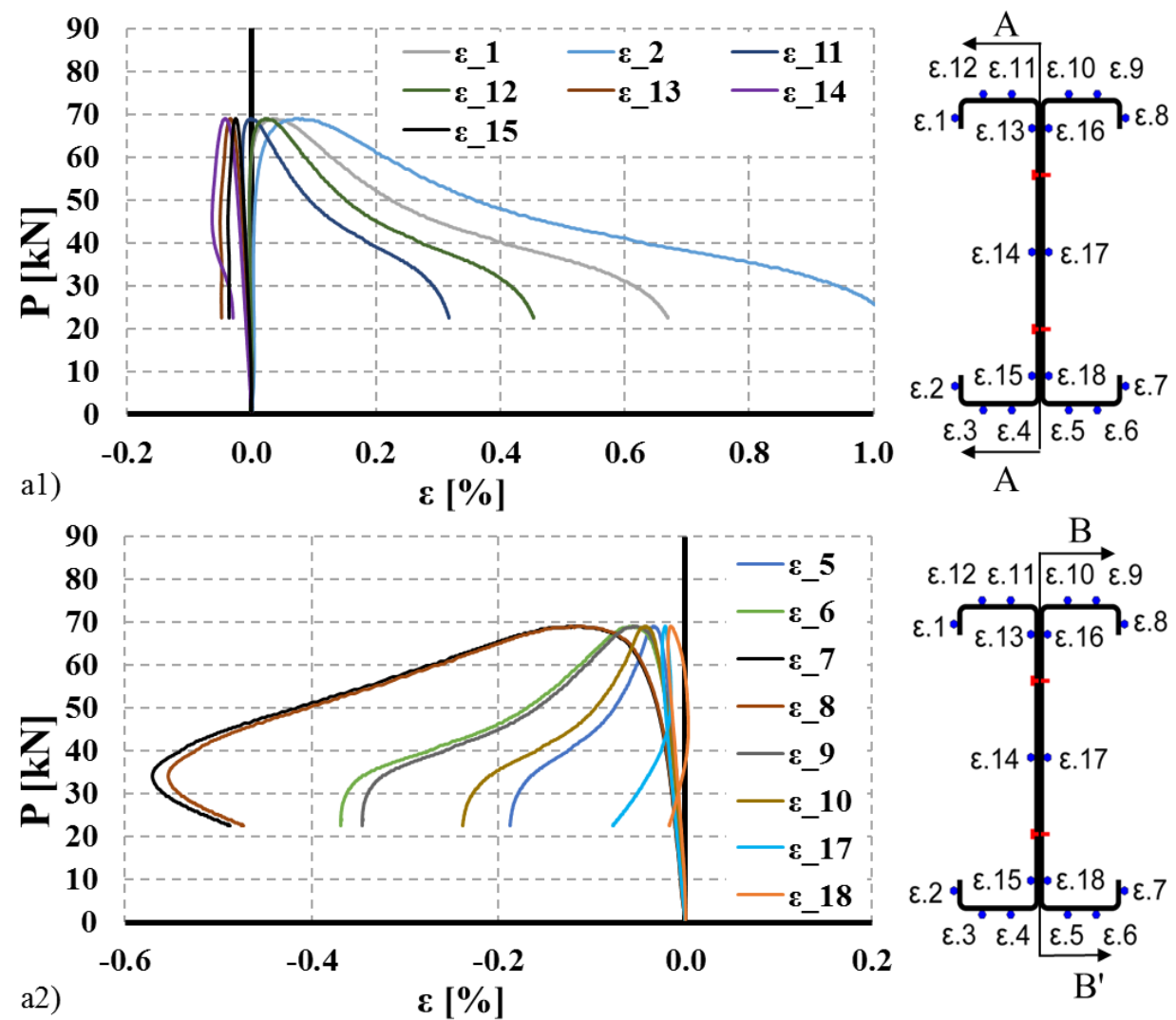

Figure E.3 Load-strain curves obtained from strain gauges placed in different points of the open built-up I cross-section. a1) and a2) I_PP-2. 

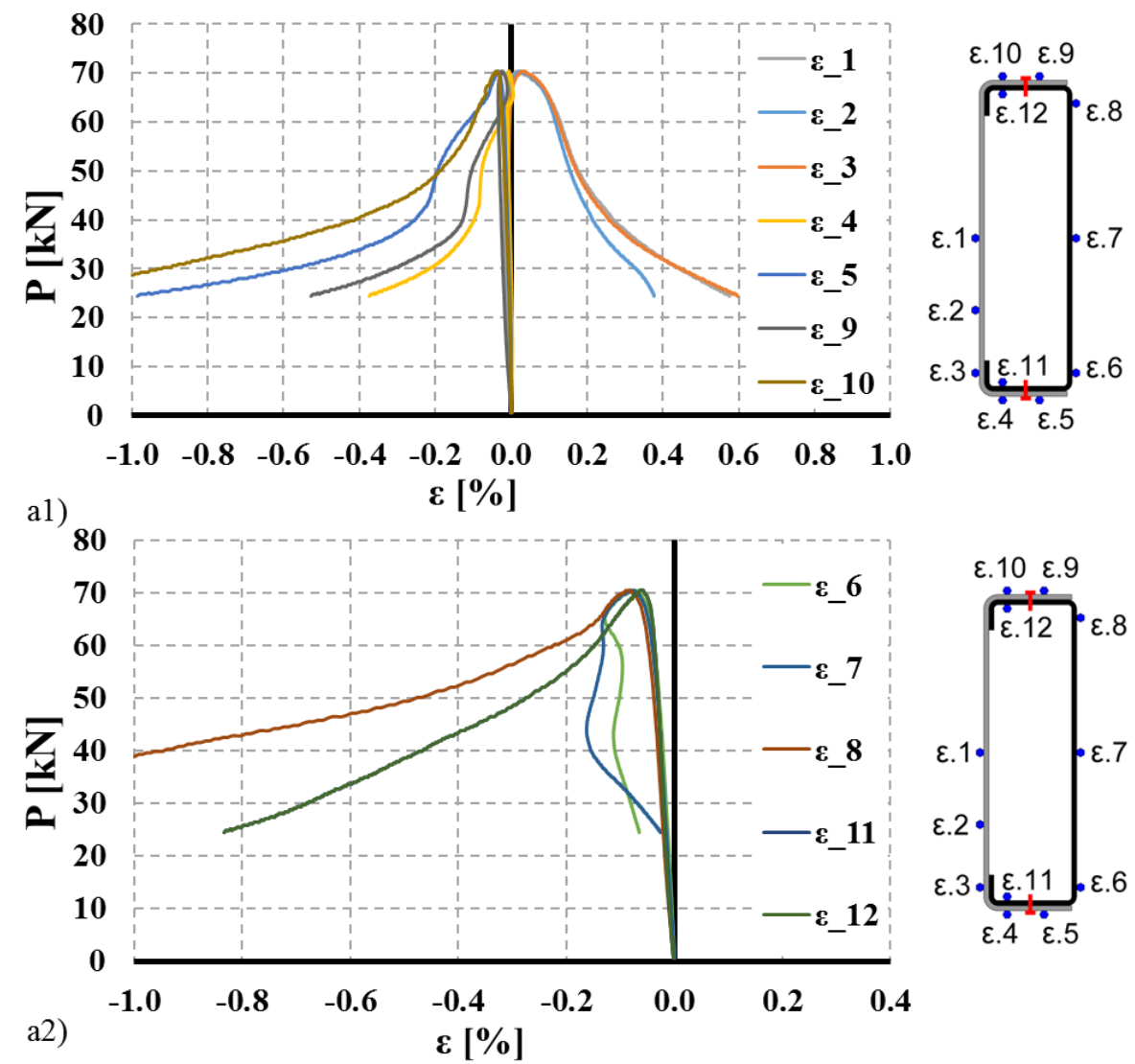

Figure E.4 Load-strain curves obtained from strain gauges placed in different points of the closed built-up R cross-section. a1) and a2) R_PP-2.
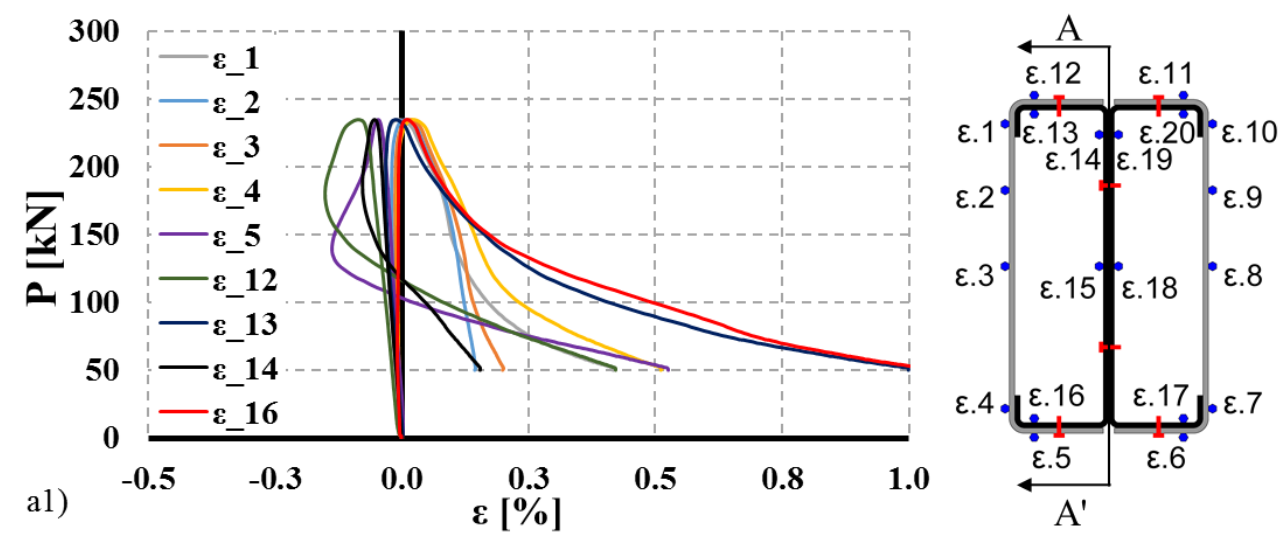

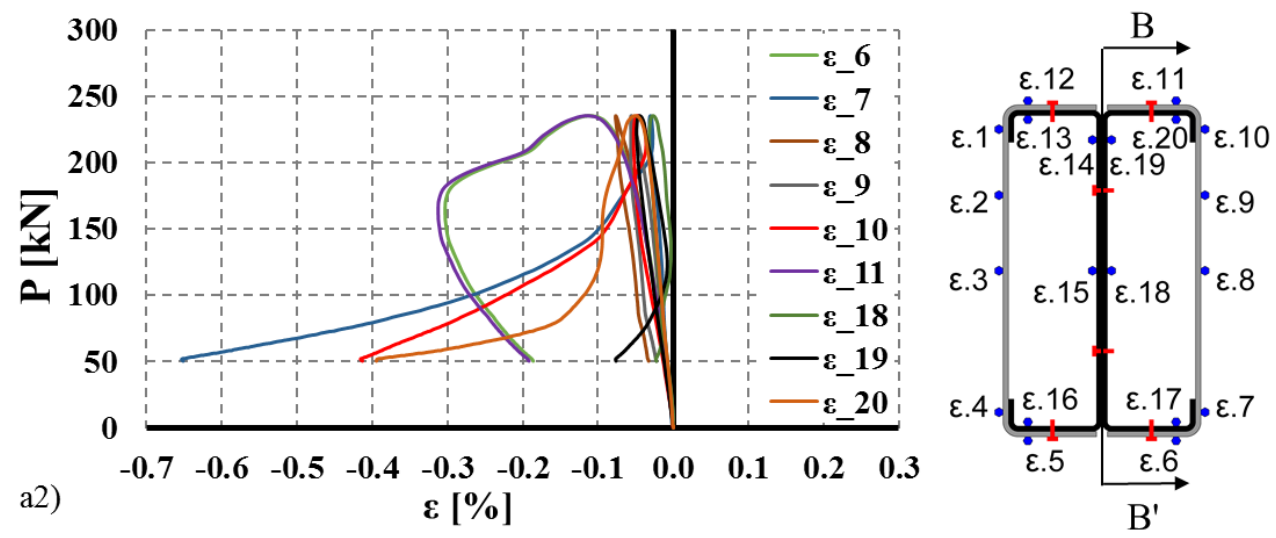

Figure E.5 Load-strain curves obtained from strain gauges placed in different points of the closed built-up 2R cross-section. a1) and a2) 2R_PP-3.

\section{E.2 Fixed-ended Columns}

\section{E.1.1 Load versus axial shortening}
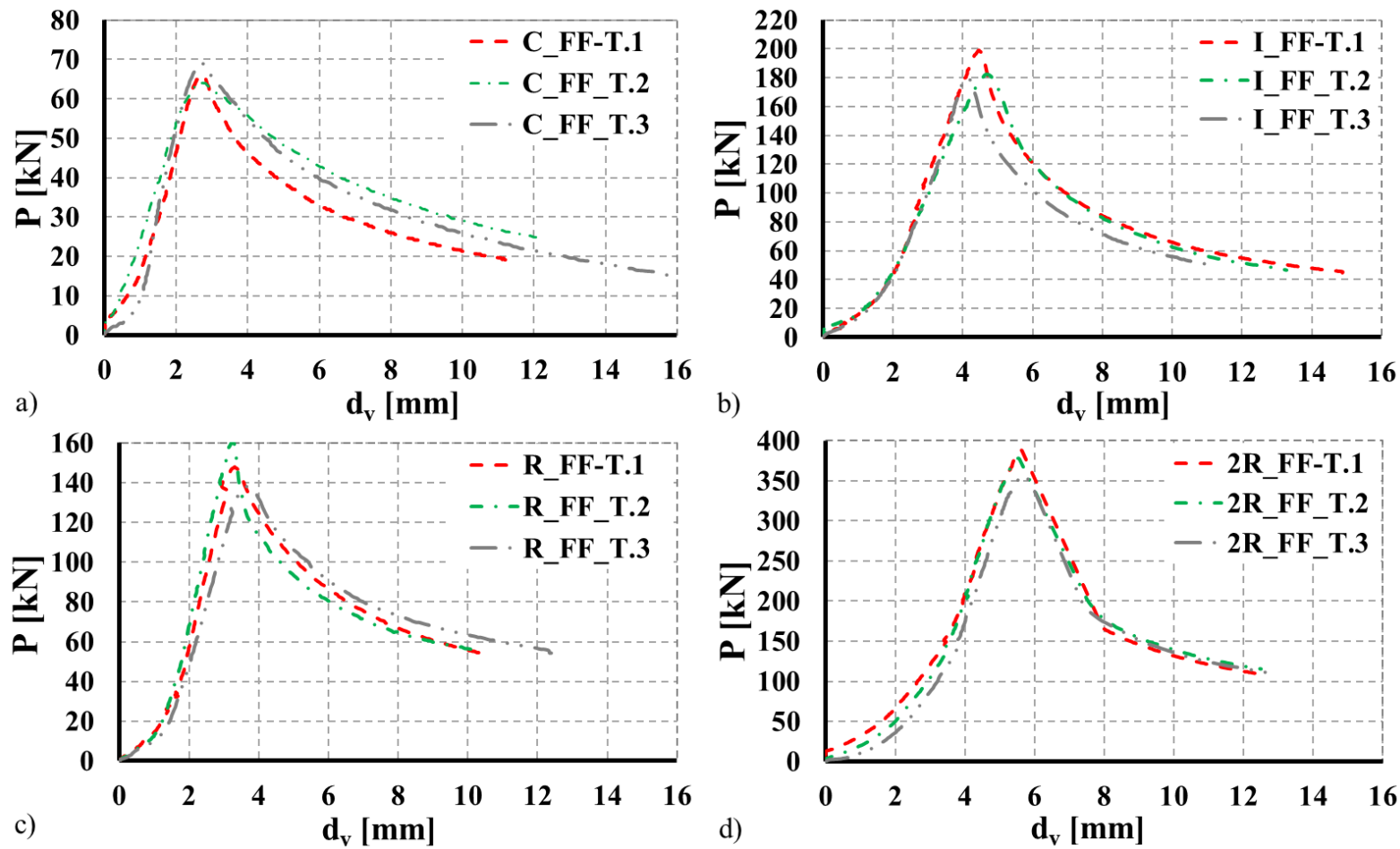

Figure E.6 Axial load versus axial shortening curves for all tested cross-sections considering the fixed-ended support condition. a) C. b) I. c) R. d) 2R columns. 


\section{E.2.2 Strain evolution}

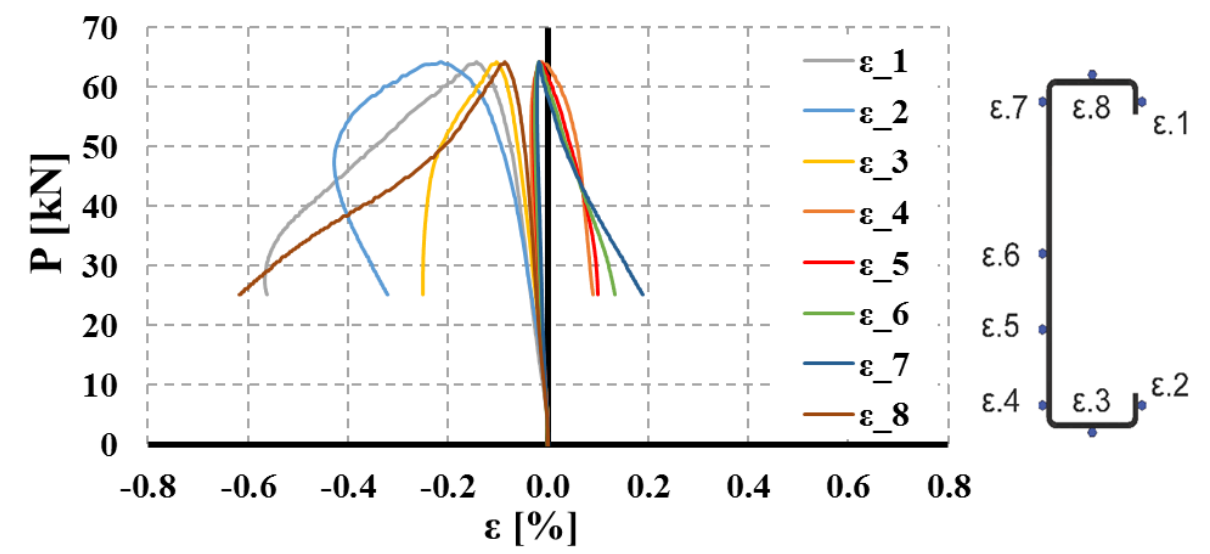

Figure E.7 Load-strain curves obtained from strain gauges placed in different points of the lipped channel cross-section. C_FF-2.
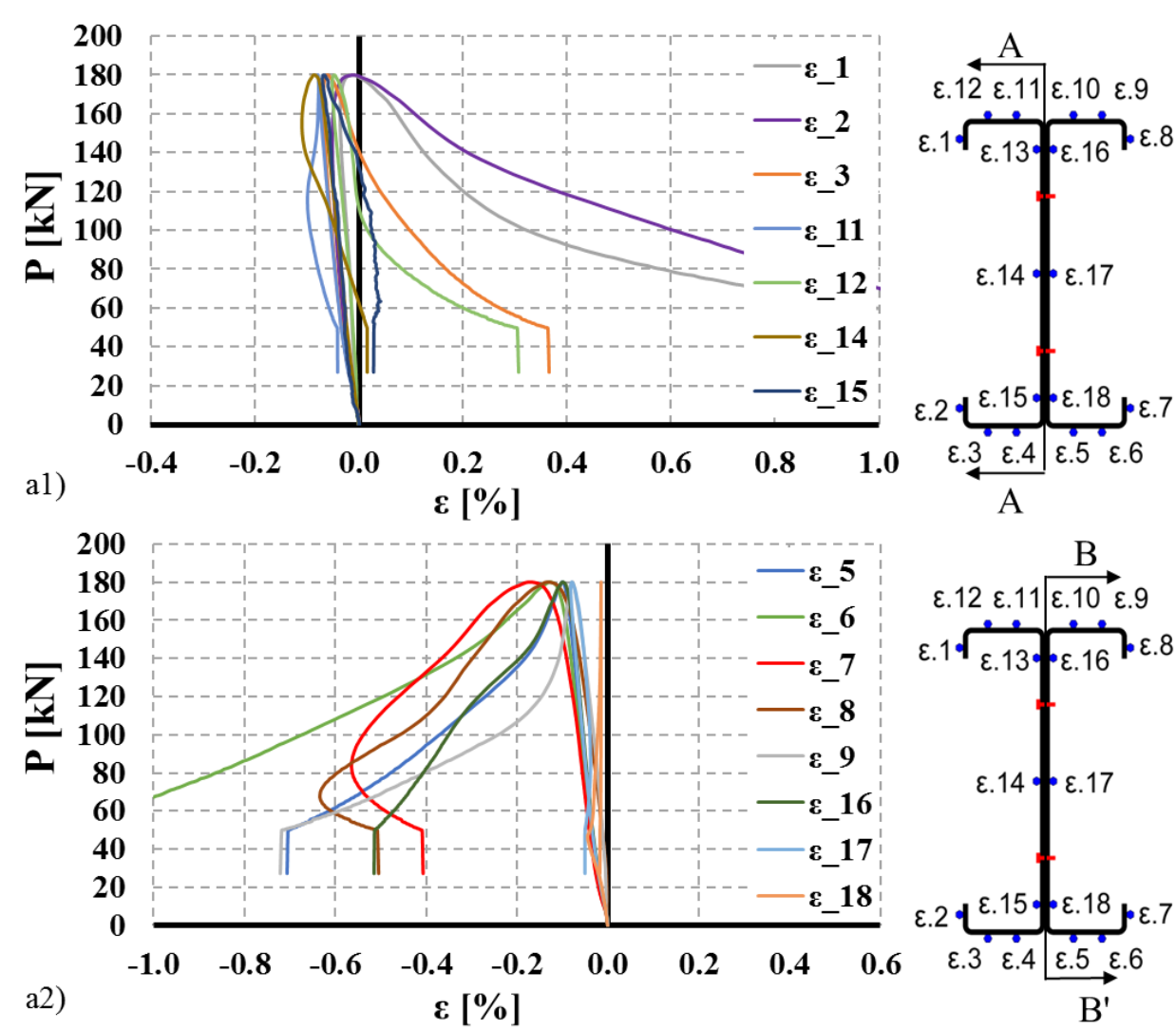

Figure E.8 Load-strain curves obtained from strain gauges placed in different points of the open built-up I cross-section. a1) and a2) I_FF-3. 

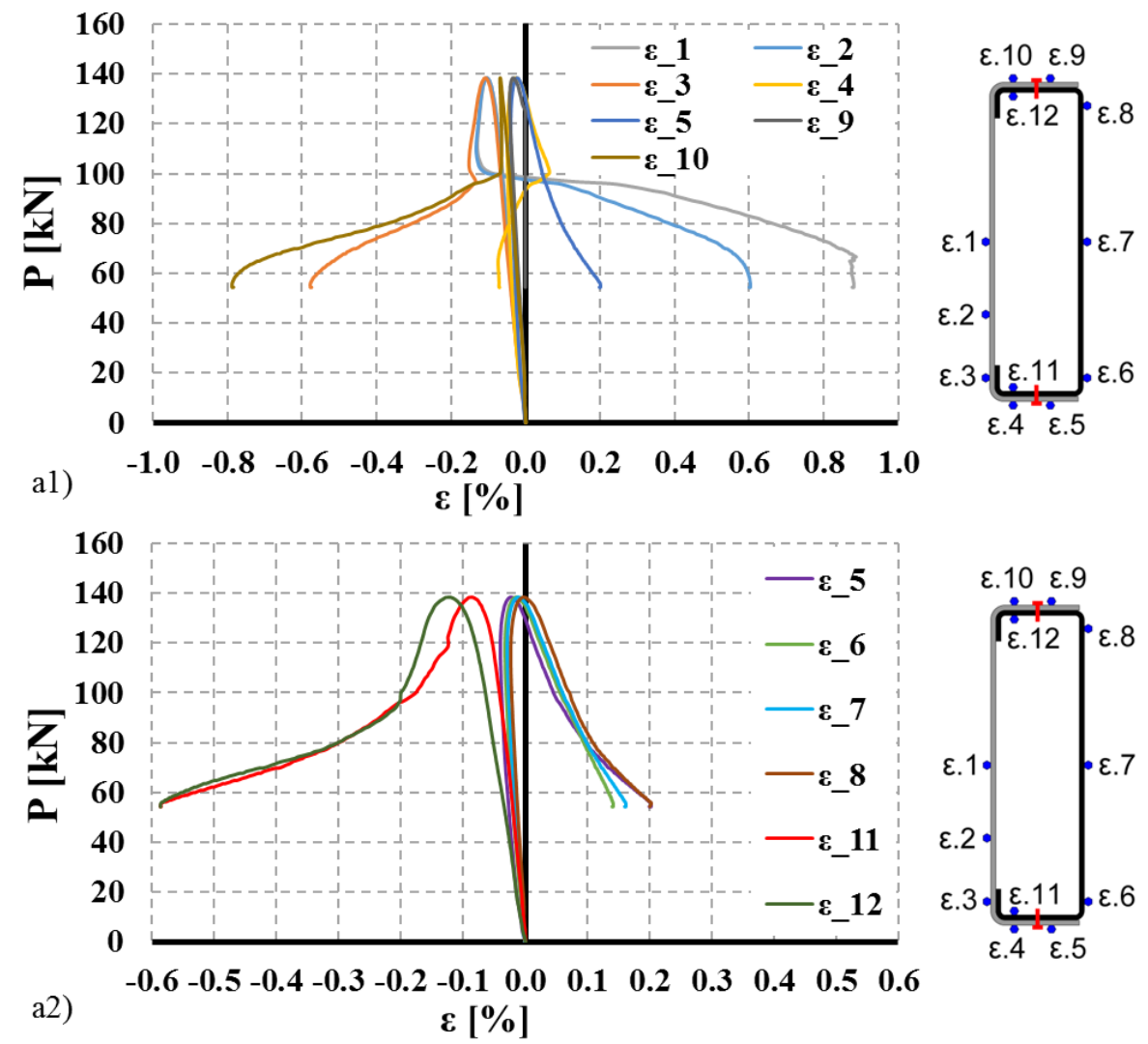

Figure E.9 Load-strain curves obtained from strain gauges placed in different points of the closed built-up R cross-section. a1) and a2) R_FF-3.
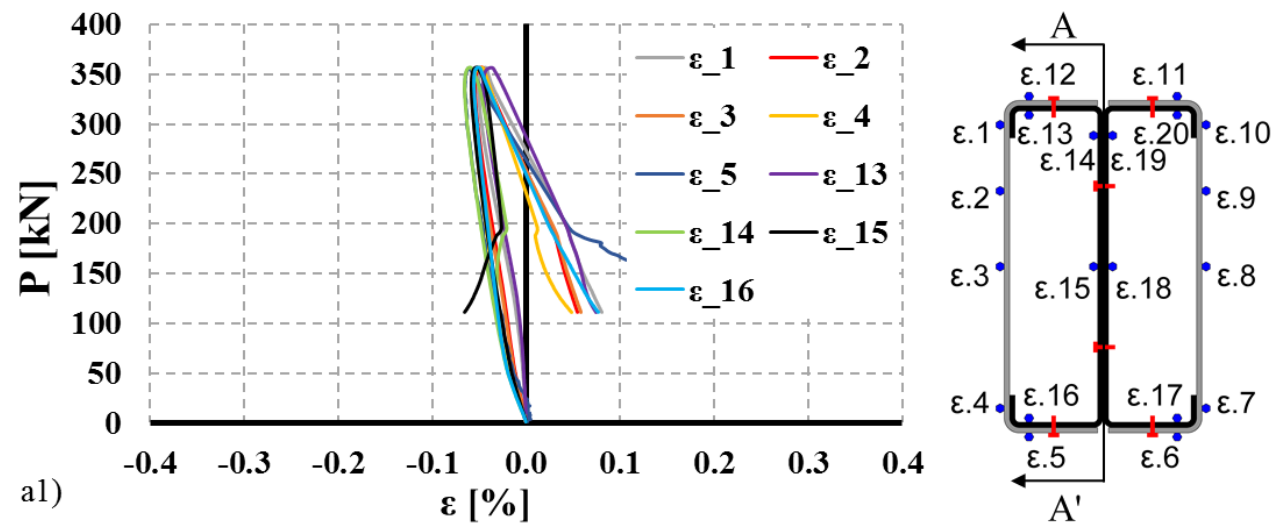

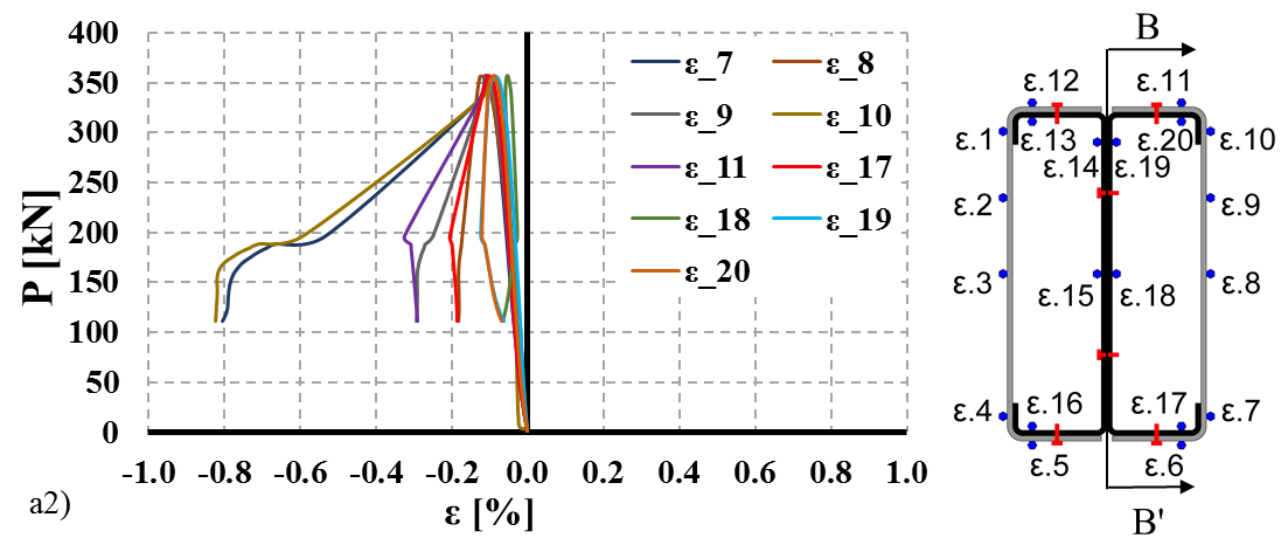

Figure E.10 Load-strain curves obtained from strain gauges placed in different points of the closed built-up 2R cross-section. a1) and a2) 2R_FF-3.

\section{E.3 Photographs of the Final Deformed Shapes}

\section{E.3.1 Pinned-ended columns}
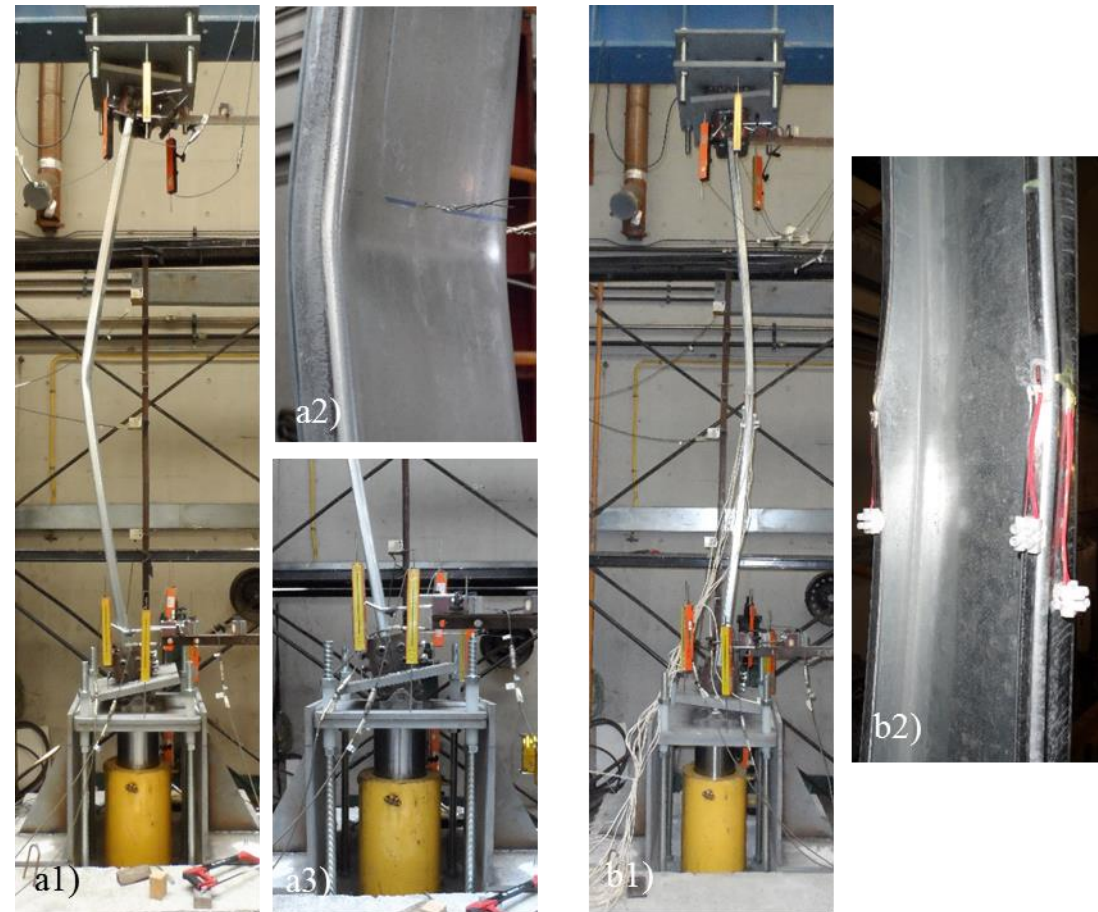

Figure E.11 Observed final deformed shape. a1), a2) and a3) C_PP-1.b1) and b2) C_PP_2. 

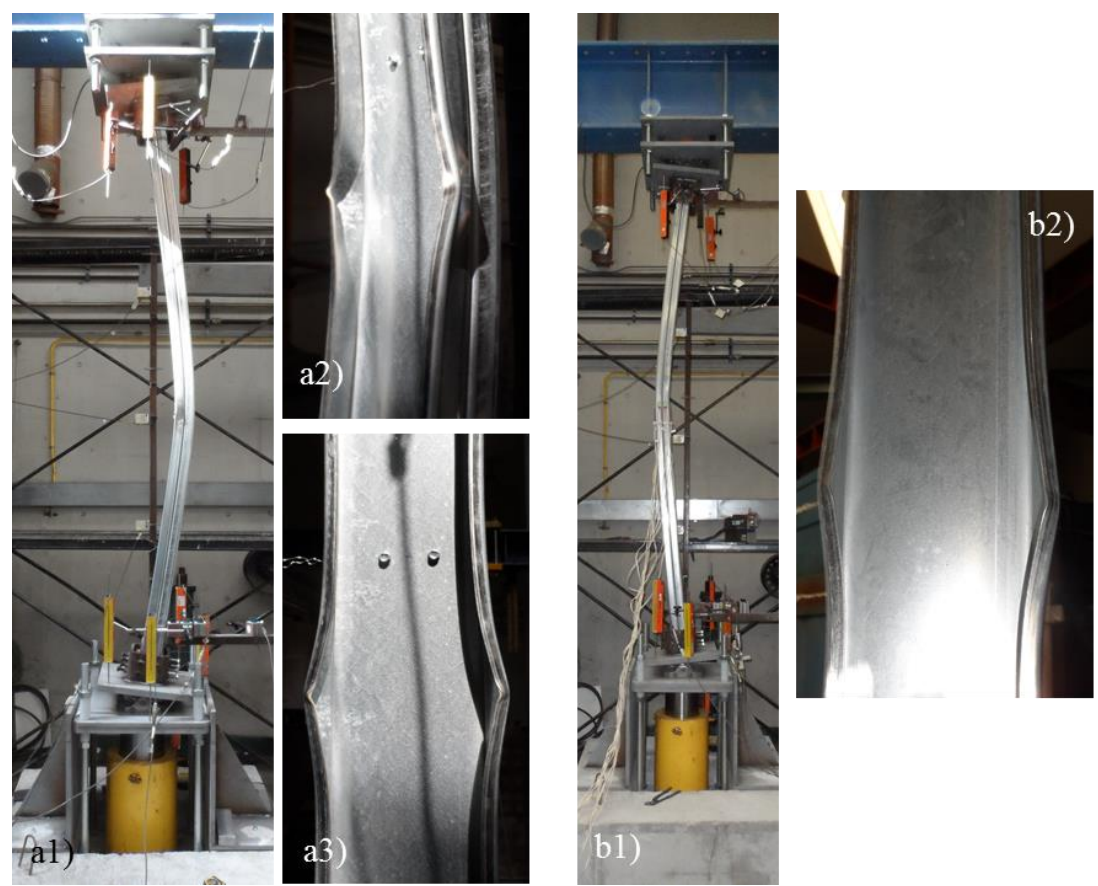

Figure E.12 Observed final deformed shape. a1), a2) and a3) I_PP-1. b1) and b2) I_PP-2.
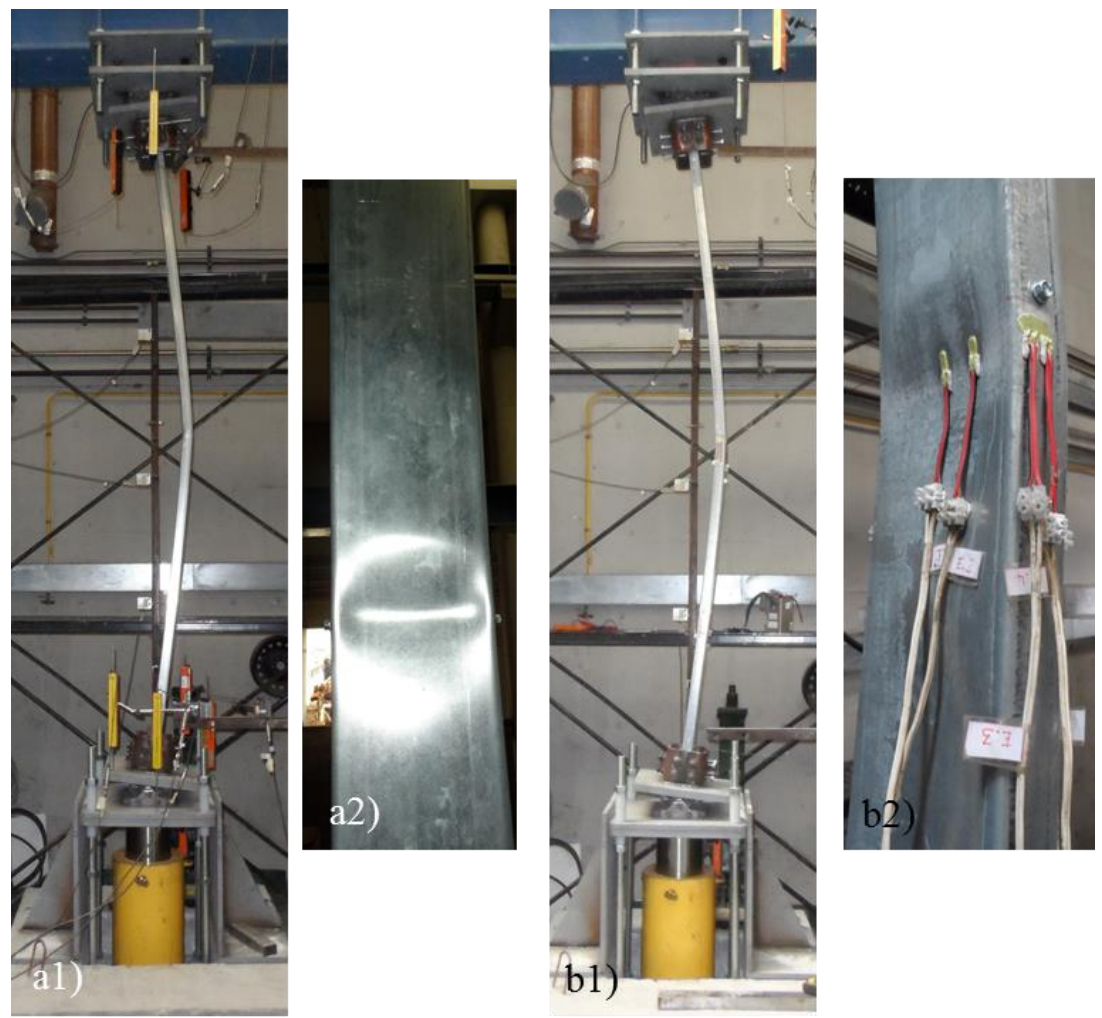

Figure E.13 Observed final deformed shape. a1) and a2) R_PP-1.b1) and b2) R_PP_2. 


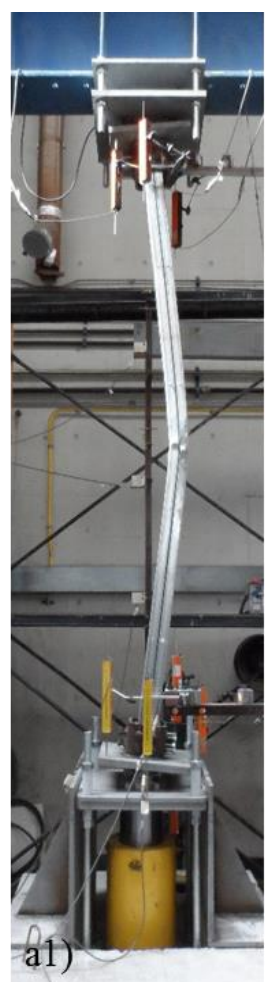

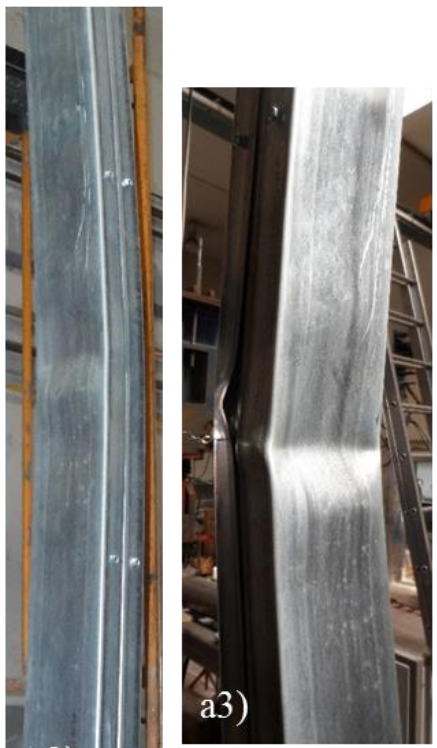

a2)
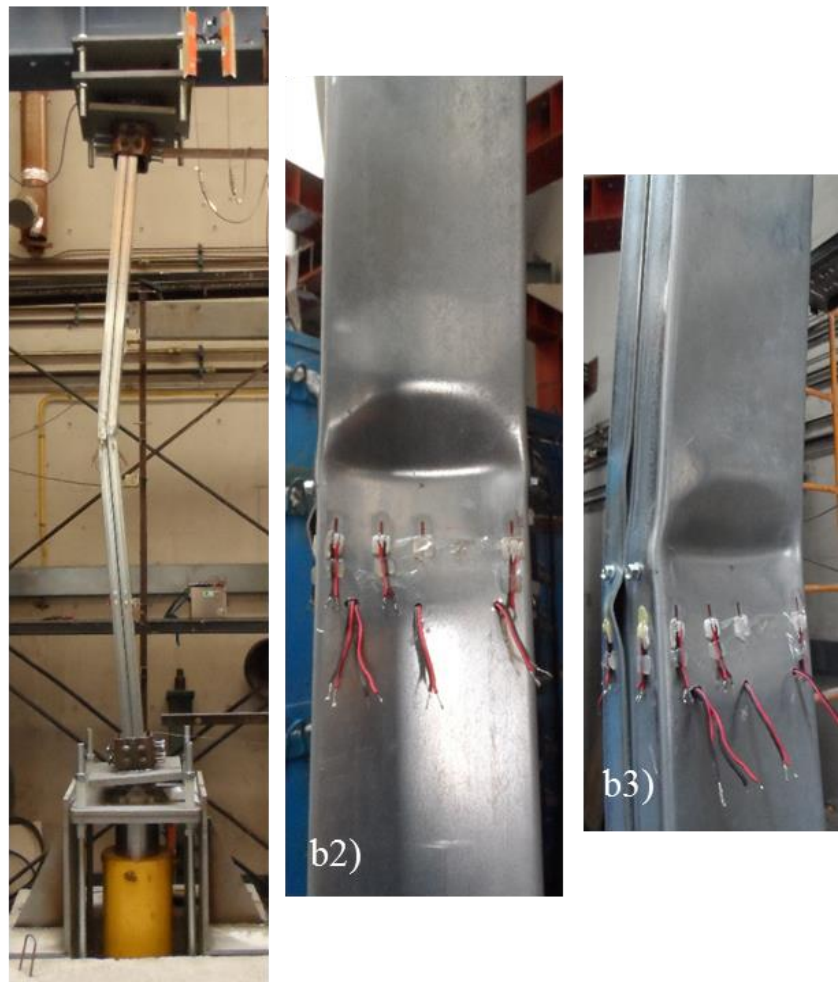

Figure E.14 Observed final deformed shape. a1), a2) and a3) 2R_PP-1. b1), b2) and b3) R_PP_3. 


\section{E.3.2 Fixed-ended columns}
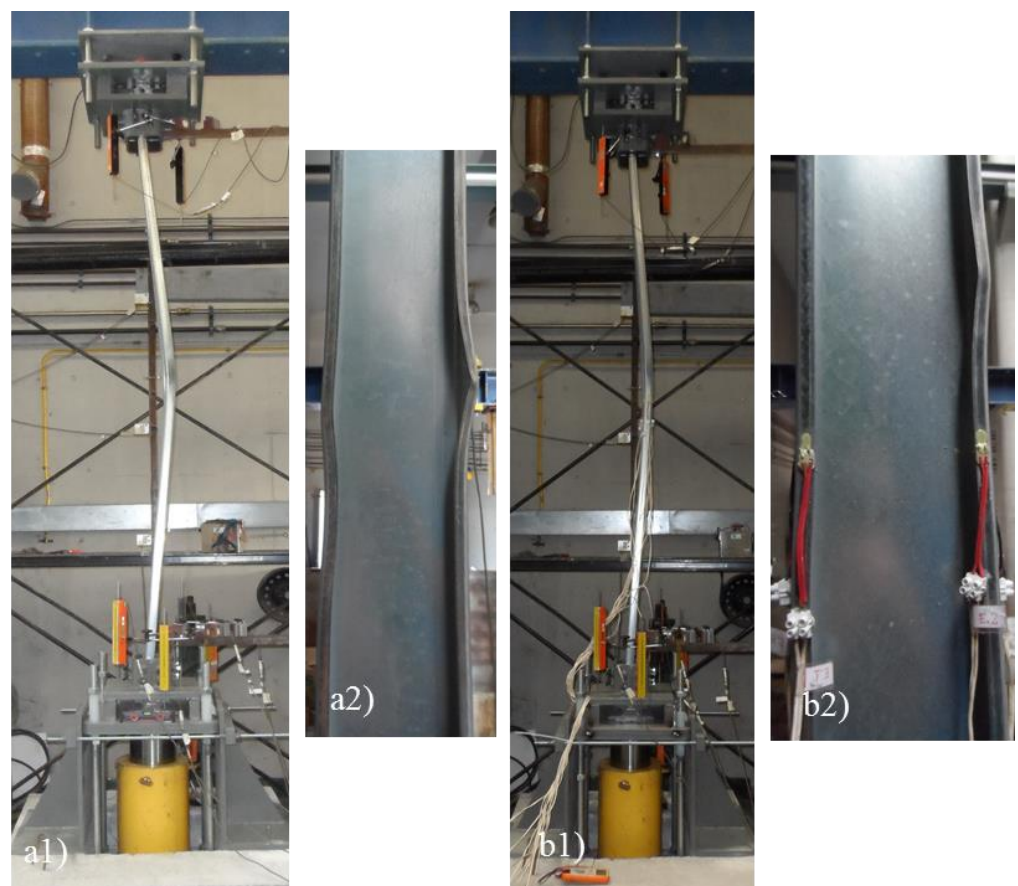

Figure E.15 Observed final deformed shape. a1) and a2) C_FF-1. b1) and b2) C_FF_2.
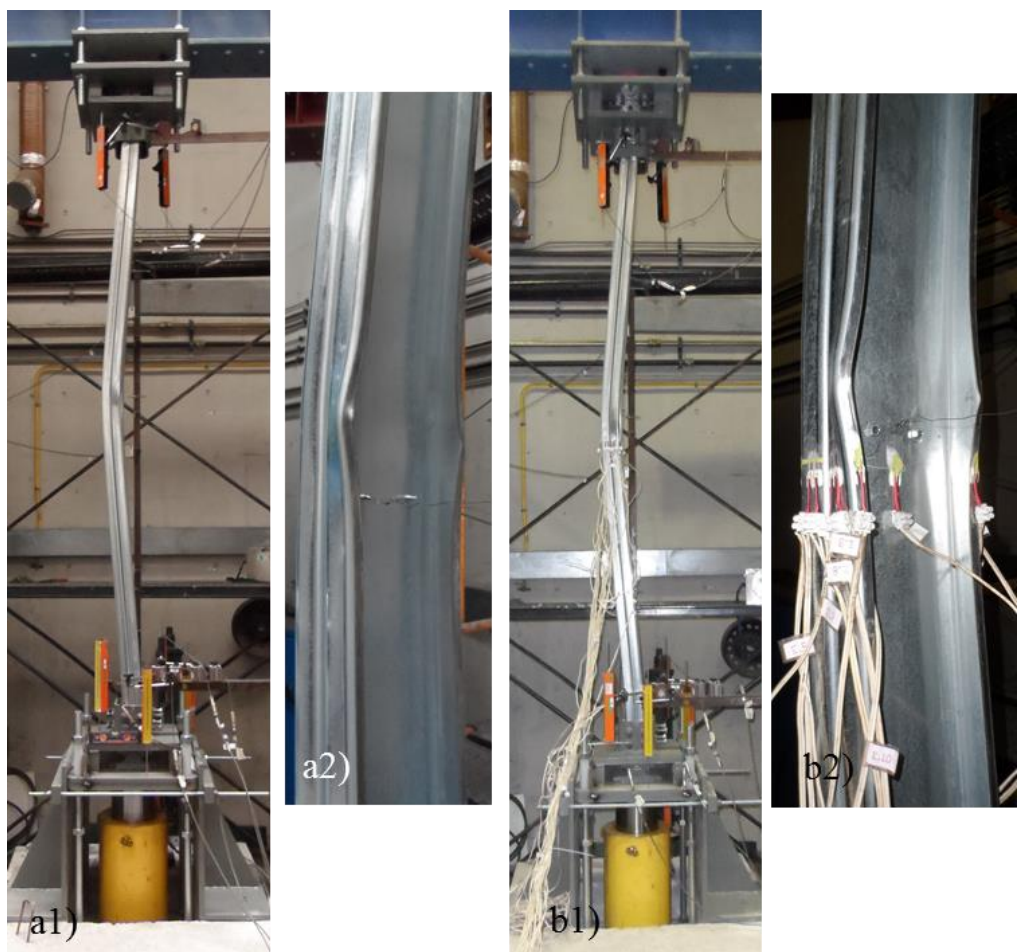

Figure E.16 Observed final deformed shape. a1) and a2) I_FF-1. b1) and b2) I_FF_3. 

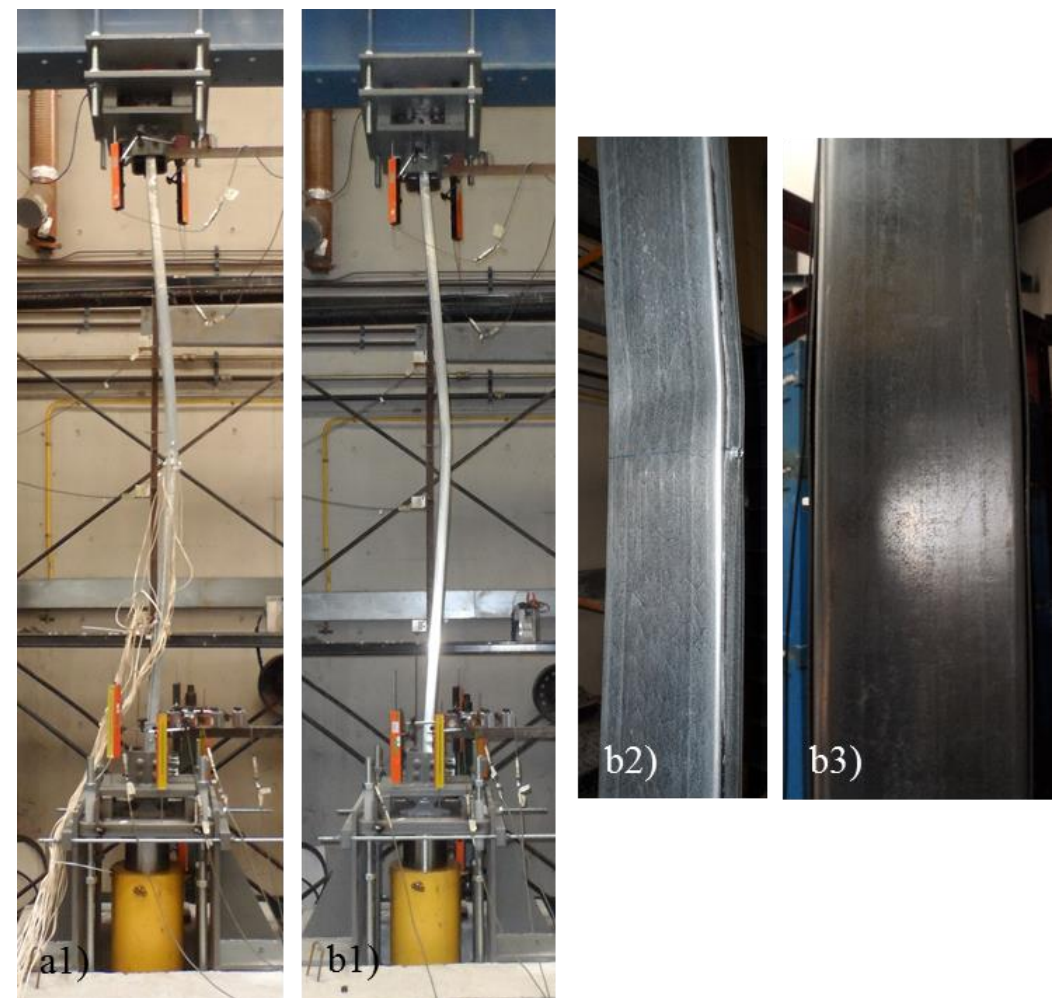

Figure E.17 Observed final deformed shape. a1) R_FF-3. b1), b2) and b3) R_FF_3.
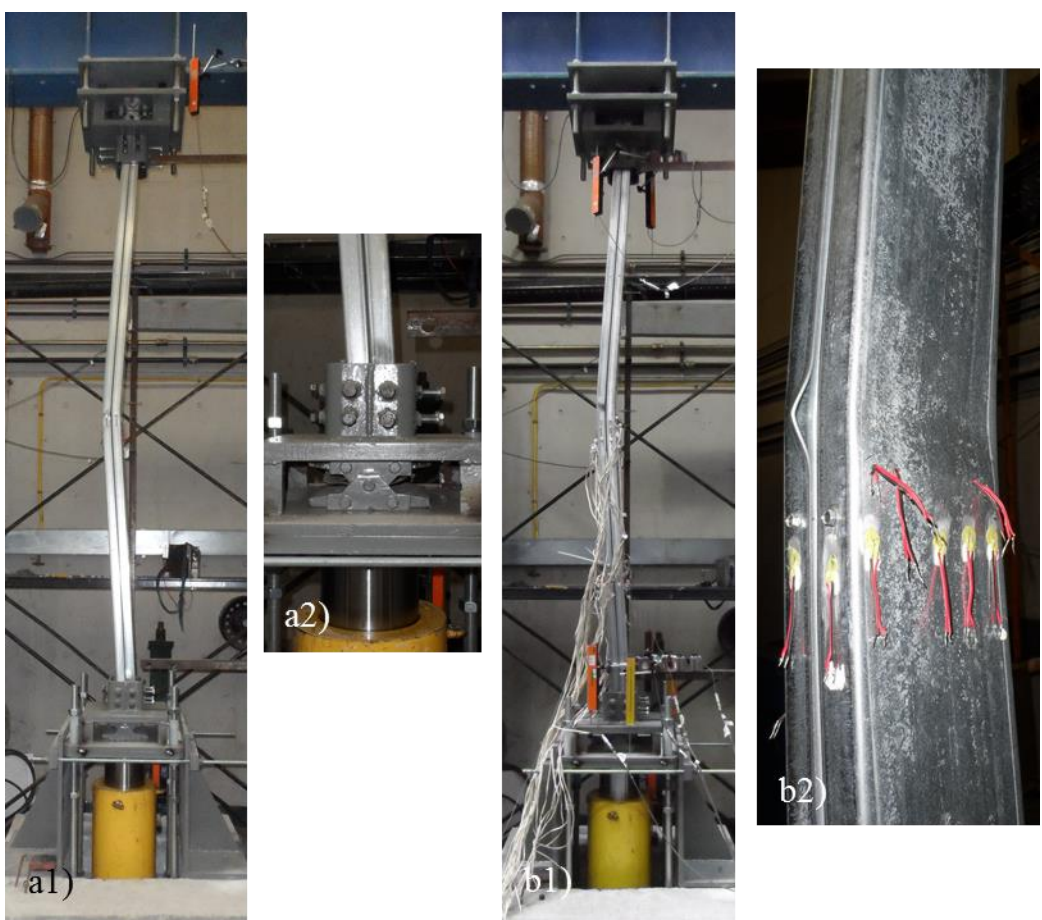

Figure E.18 Observed final deformed shape. a1) and a2) 2R_FF-1.b1) and b2) 2R_FF_3. 



\section{APPENDIX F FIRE RESISTANCE TESTS}

\section{F.1 Pinned-ended Columns}

\section{F.1.1 Lipped channel columns - C_PP_30LL_K1-i}

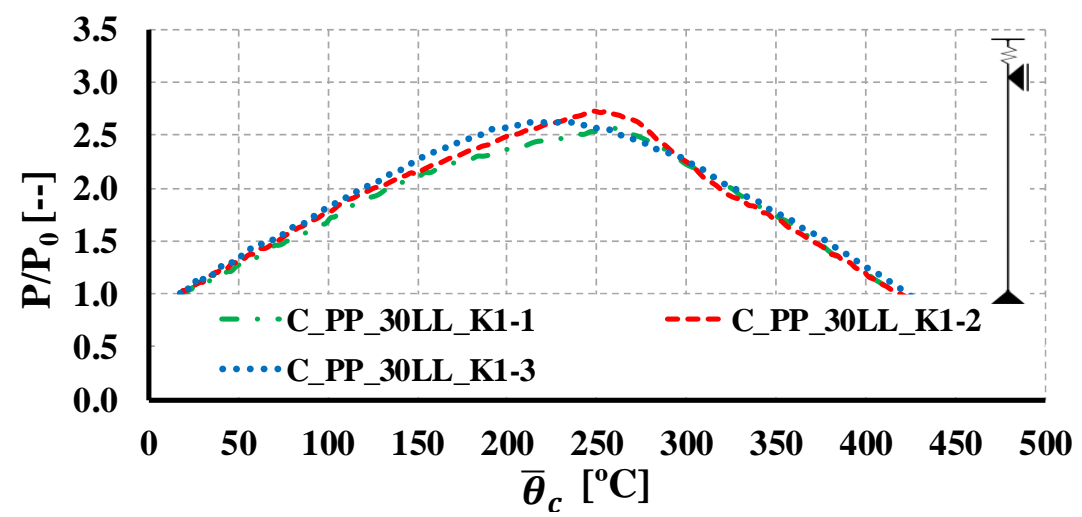

Figure F.1 Evolution of restraining forces with temperature increase for lipped channel columns considering a pinned-ended support condition a 30\% load level, and a K1 level of restraint to thermal elongation.

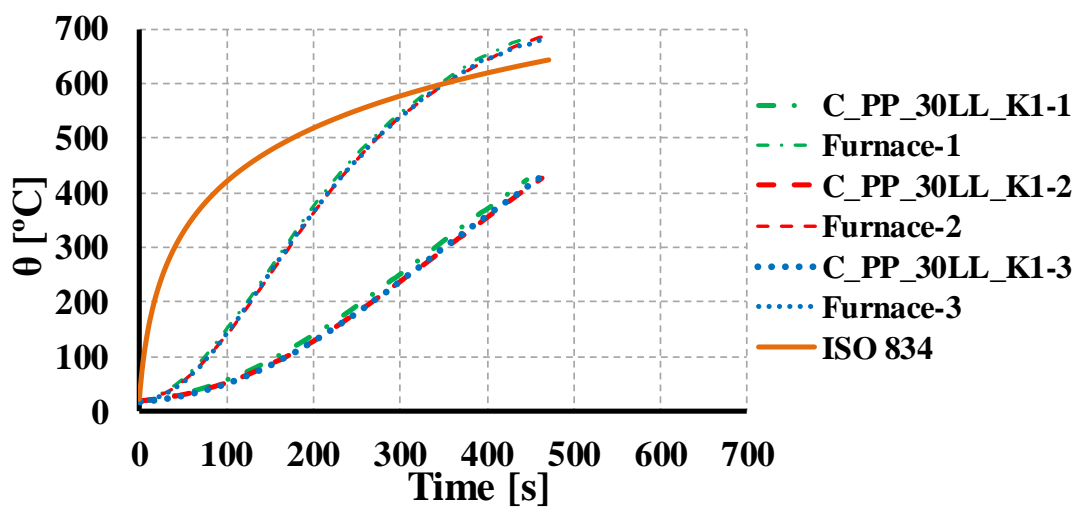

Figure F.2 Evolution of the mean temperature of the CFS column with temperature increase as well as the furnace temperature and ISO 834 fire curve. 


\section{F.1.2 Lipped channel columns - C_PP_50LL_K1-i}

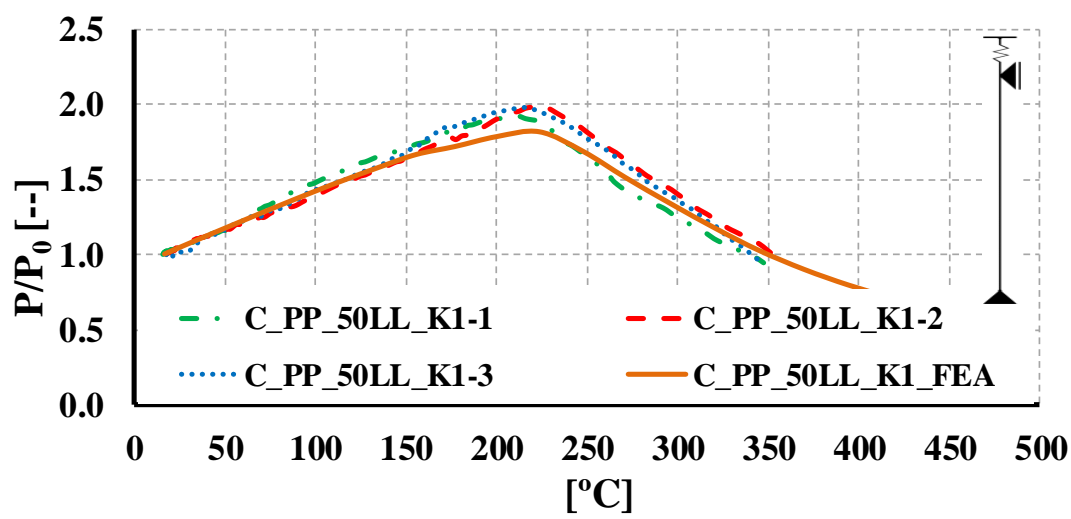

Figure F.3 Evolution of restraining forces with temperature increase for C columns.

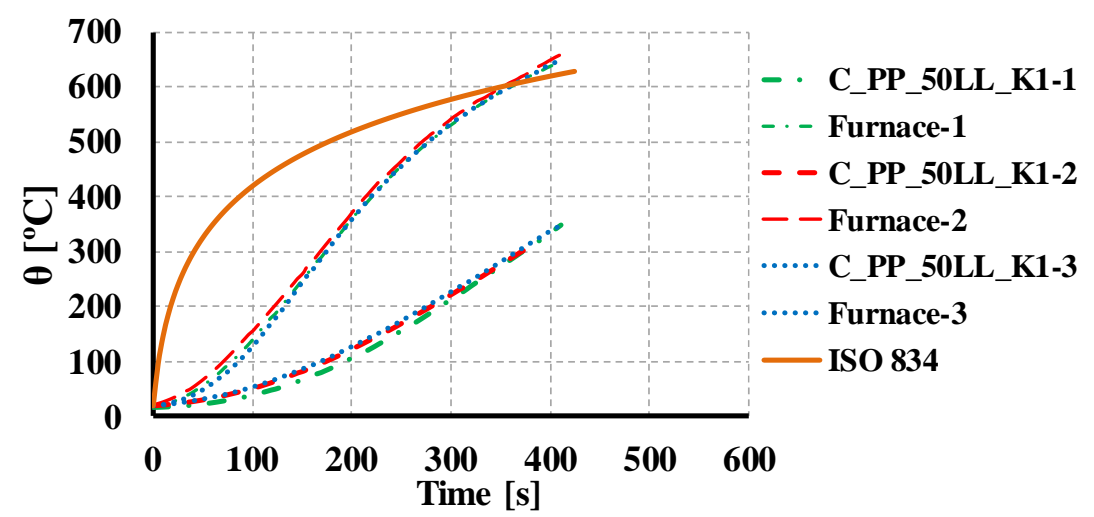

Figure F.4 Evolution of the mean temperature of the CFS column with temperature increase as well as the furnace temperature and ISO 834 fire curve.

\section{F.1.3 Lipped channel columns - C_PP_30LL_K2-i}

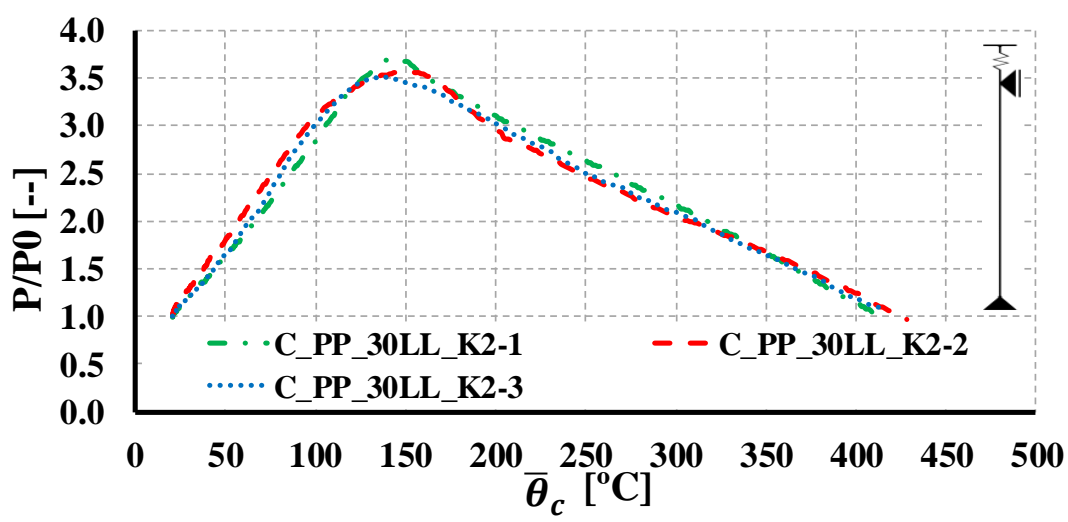

Figure F.5 Evolution of restraining forces with temperature increase for $\mathrm{C}$ columns. 


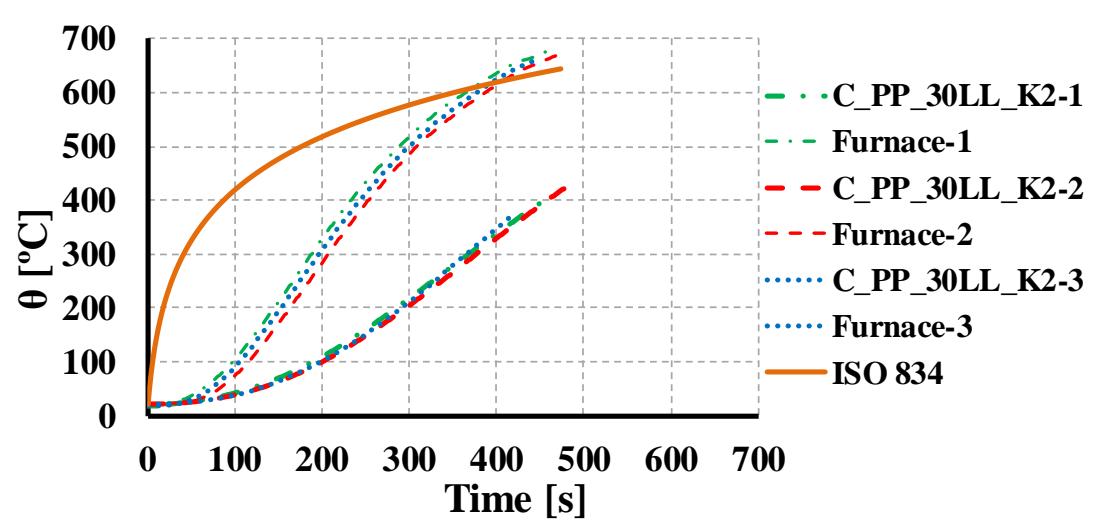

Figure F.6 Evolution of the mean temperature of the CFS column with temperature increase as well as the furnace temperature and ISO 834 fire curve.

\section{F.1.4 Lipped channel columns - C_PP_50LL_K2-i}

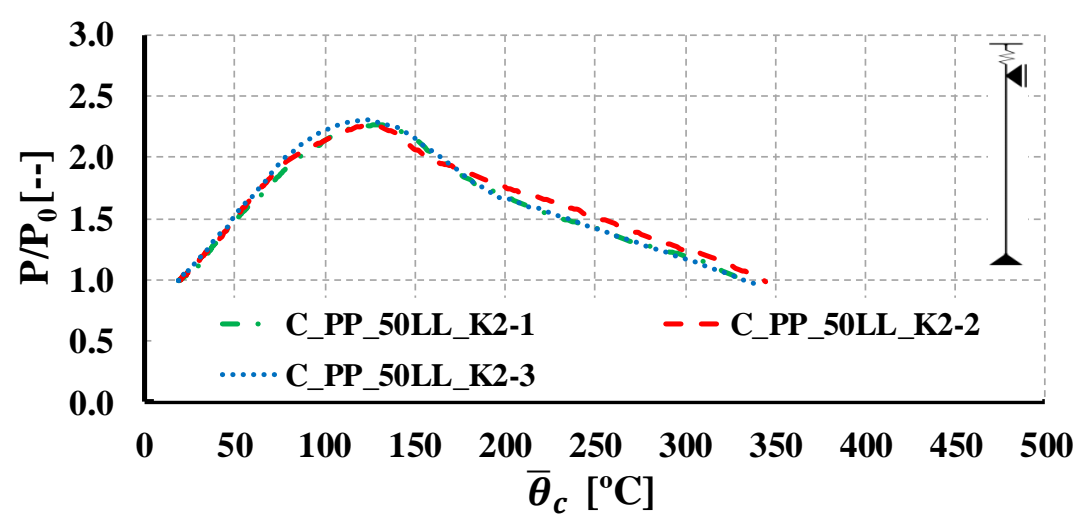

Figure F.7 Evolution of restraining forces with temperature increase for C columns.

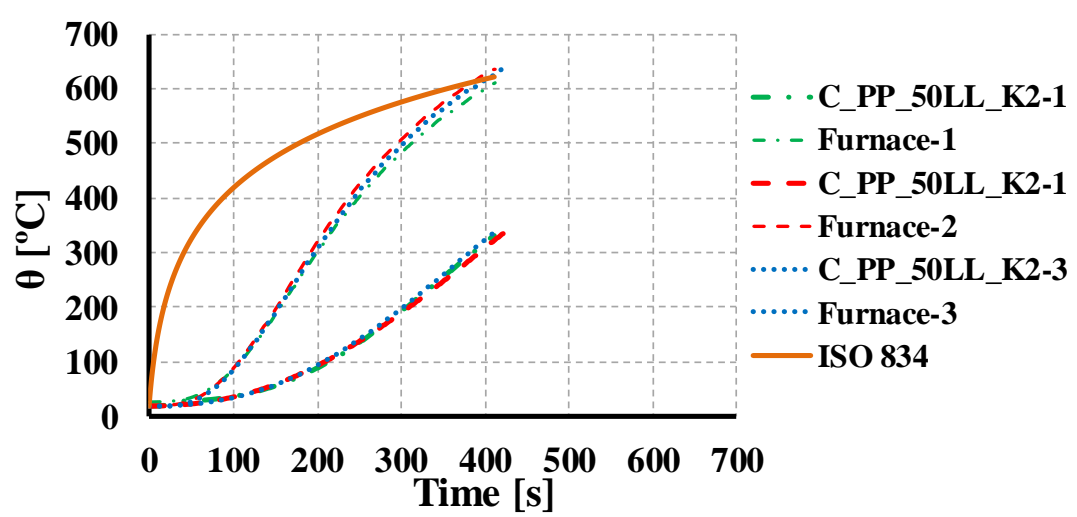

Figure F.8 Evolution of the mean temperature of the CFS column with temperature increase as well as the furnace temperature and ISO 834 fire curve. 


\section{F.1.5 Open built-up I columns - I_PP_30LL_K1-i}

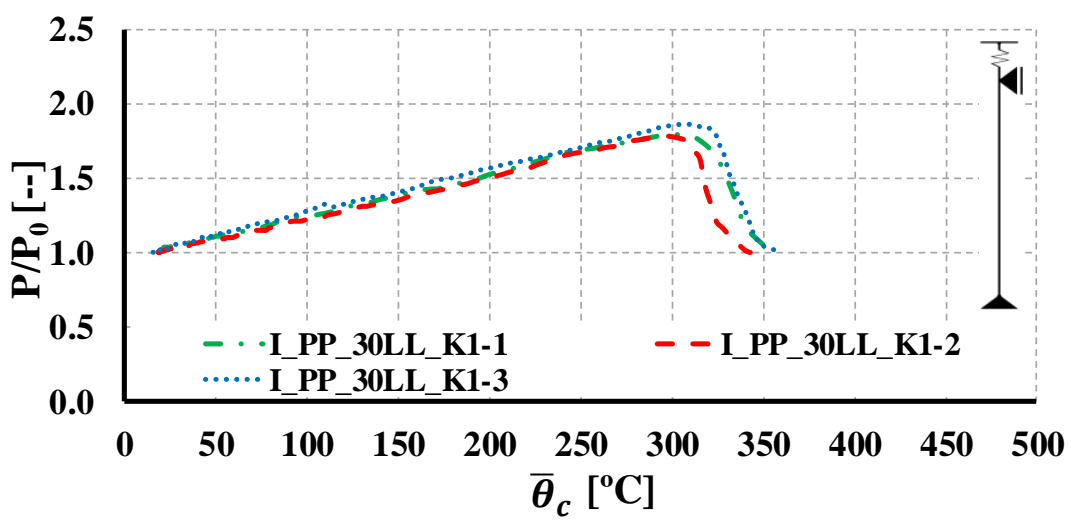

Figure F.9 Evolution of restraining forces with temperature increase for I columns.

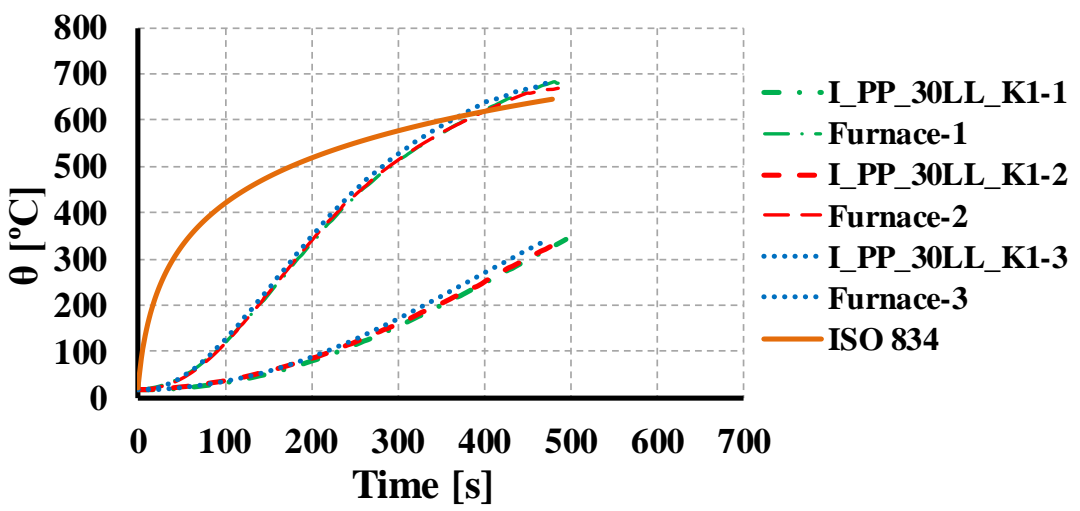

Figure F.10 Evolution of the mean temperature of the CFS column with temperature increase as well as the furnace temperature and ISO 834 fire curve.

\section{F.1.6 Open built-up I columns - I_PP_50LL_K1-i}

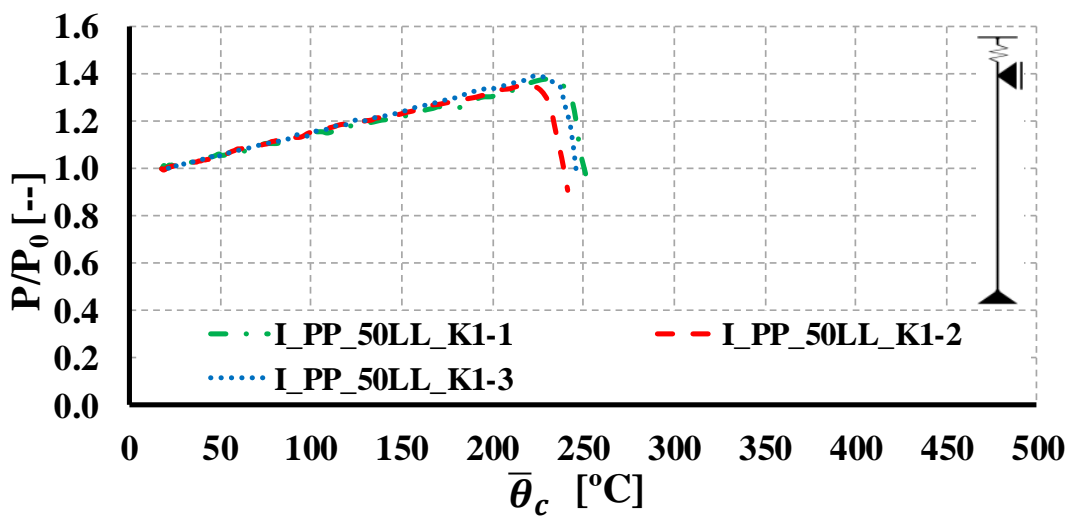

Figure F.11 Evolution of restraining forces with temperature increase for I columns. 


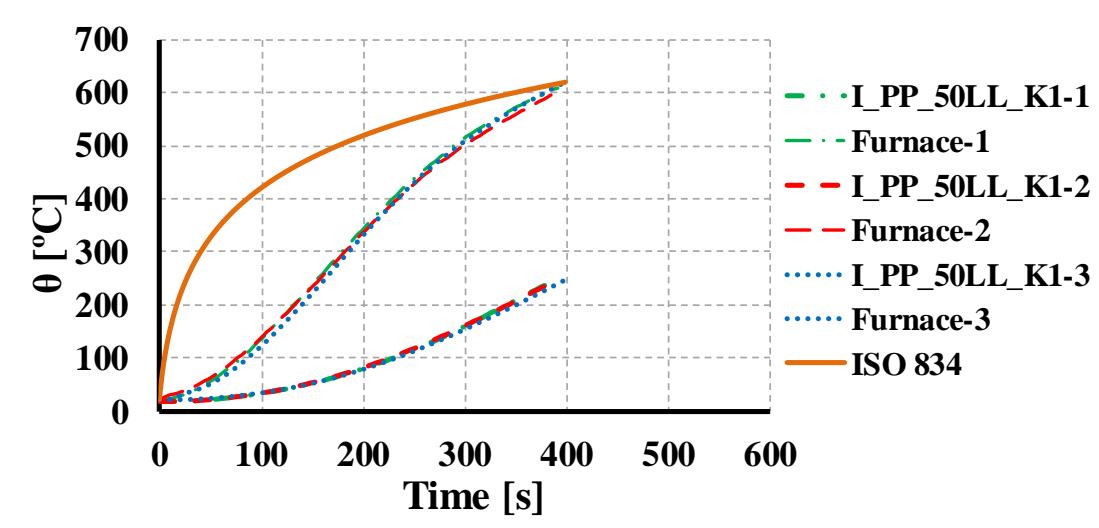

Figure F.12 Evolution of the mean temperature of the CFS column with temperature increase as well as the furnace temperature and ISO 834 fire curve.

\section{F.1.7 Open built-up I columns - I_PP_30LL_K2-i}

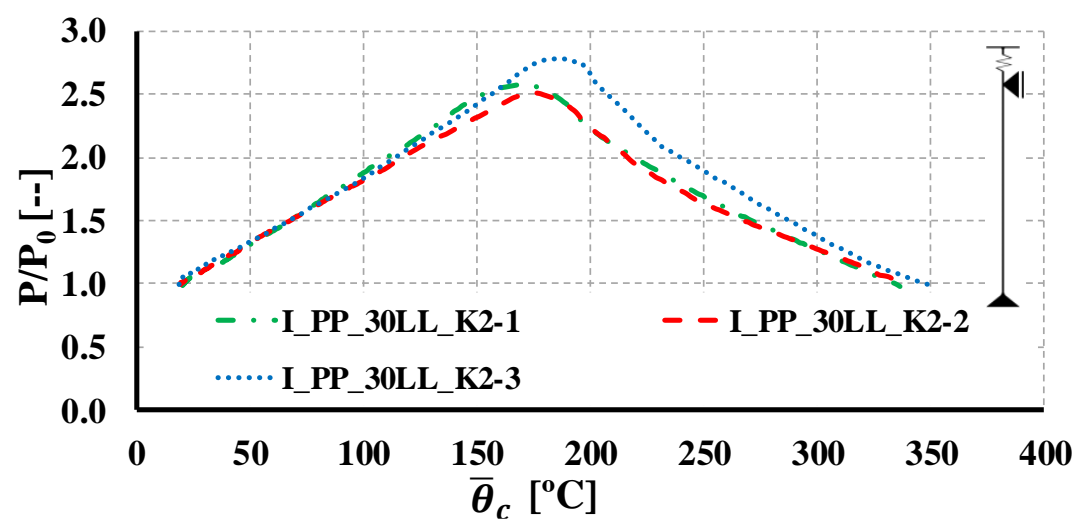

Figure F.13 Evolution of restraining forces with temperature increase for I columns.

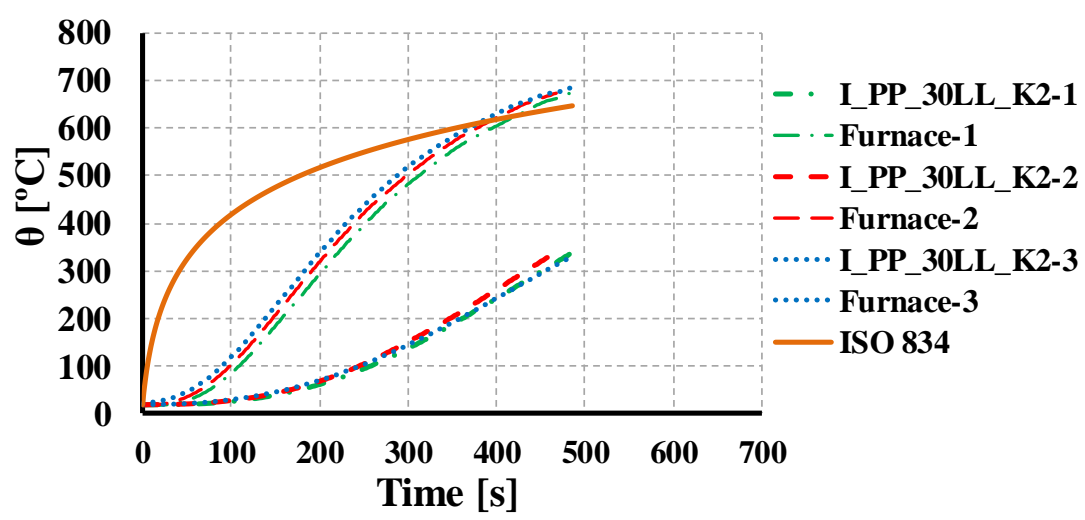

Figure F.14 Evolution of the mean temperature of the CFS column with temperature increase as well as the furnace temperature and ISO 834 fire curve. 


\section{F.1.8 Open built-up I columns - I_PP_50LL_K2-i}

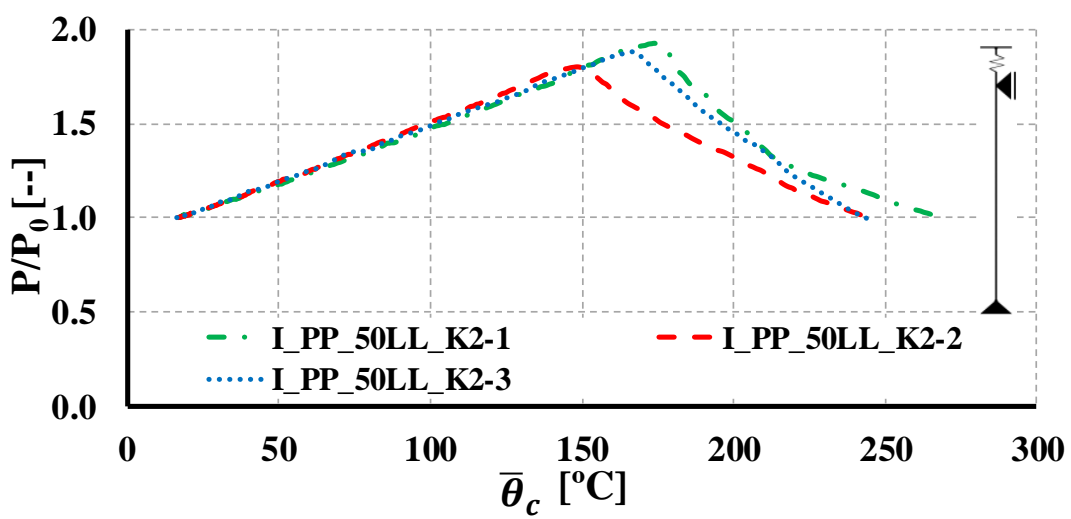

Figure F.15 Evolution of restraining forces with temperature increase for I columns.

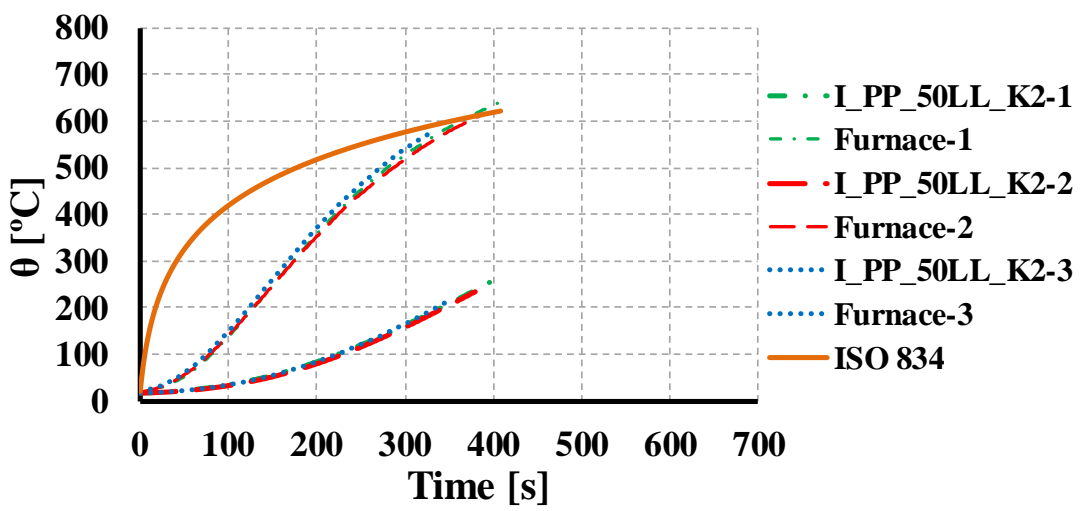

Figure F.16 Evolution of the mean temperature of the CFS column with temperature increase as well as the furnace temperature and ISO 834 fire curve.

\section{F.1.9 Closed built-up R columns - R_PP_30LL_K1-i}

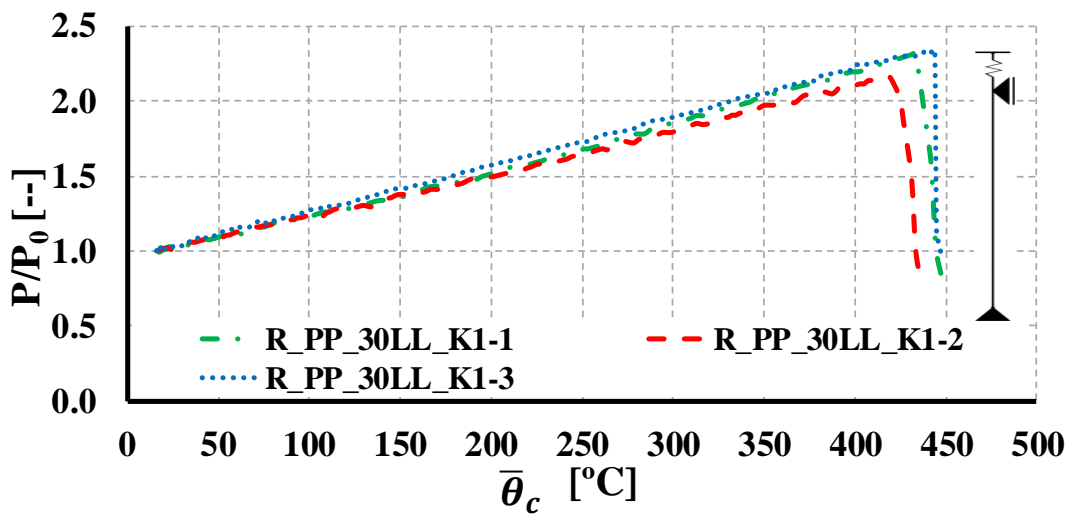

Figure F.17 Evolution of restraining forces with temperature increase for R columns. 


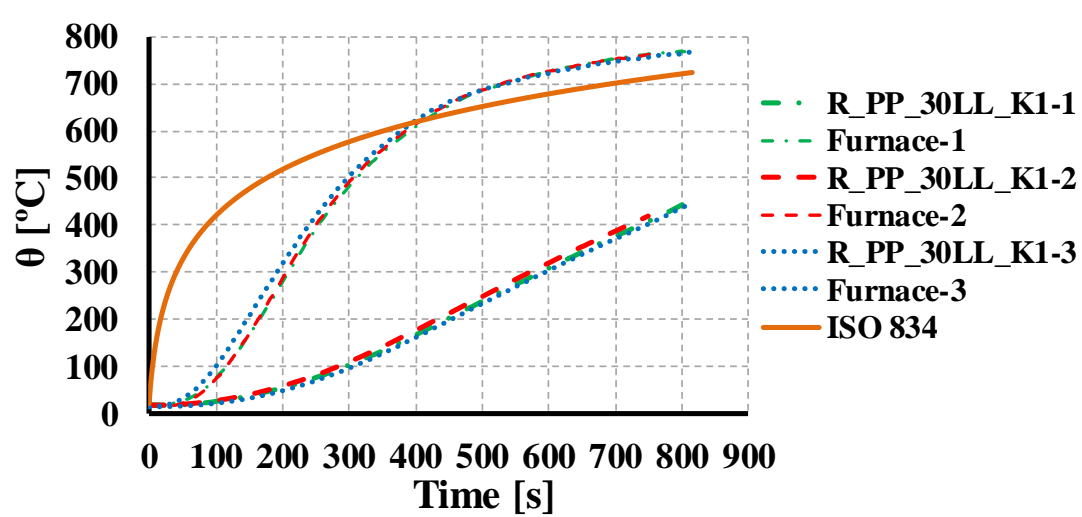

Figure F.18 Evolution of the mean temperature of the CFS column with temperature increase as well as the furnace temperature and ISO 834 fire curve.

\section{F.1.10 Closed built-up R columns - R_PP_50LL_K1-i}

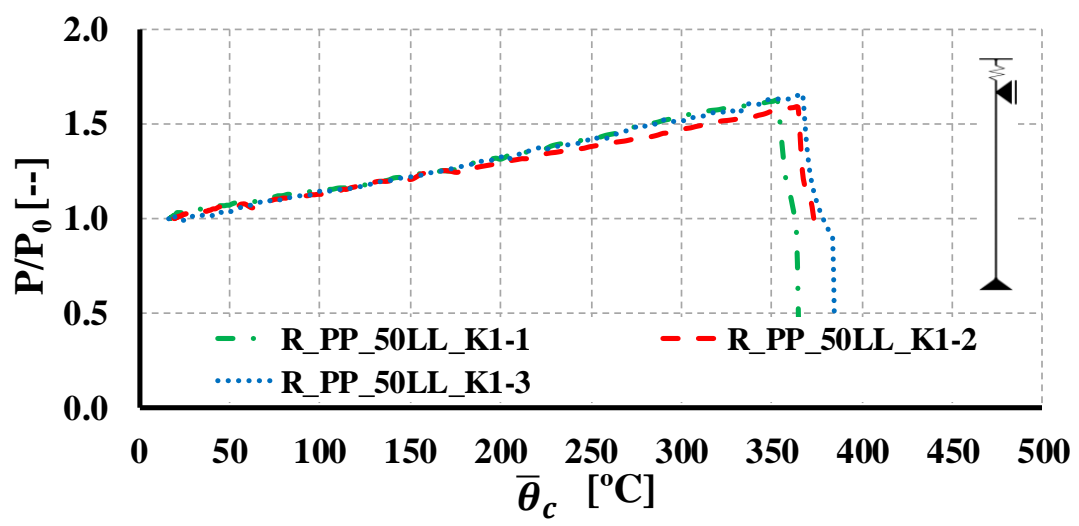

Figure F.19 Evolution of restraining forces with temperature increase for R columns.

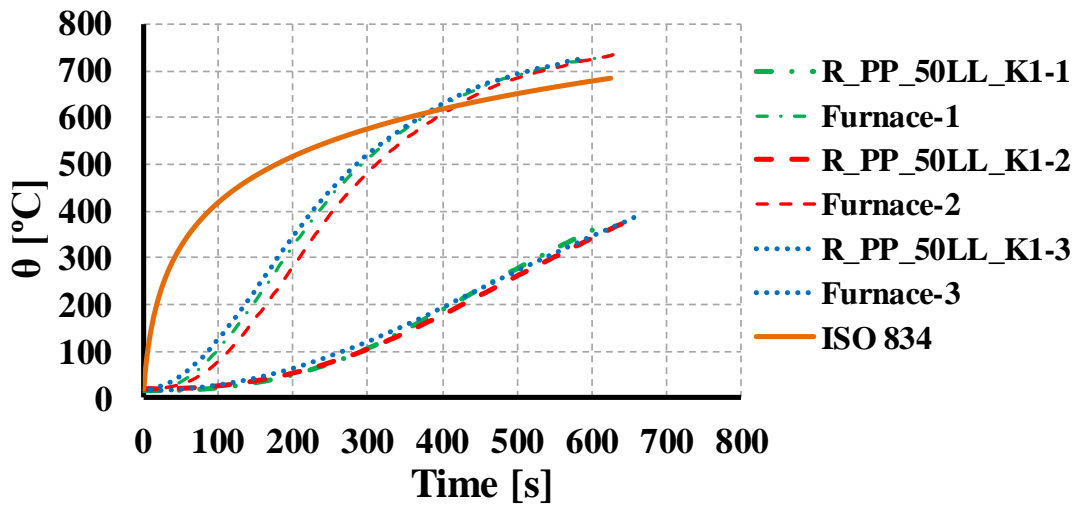

Figure F.20 Evolution of the mean temperature of the CFS column with temperature increase as well as the furnace temperature and ISO 834 fire curve. 


\section{F.1.11 Closed built-up R columns - R_PP_30LL_K2-i}

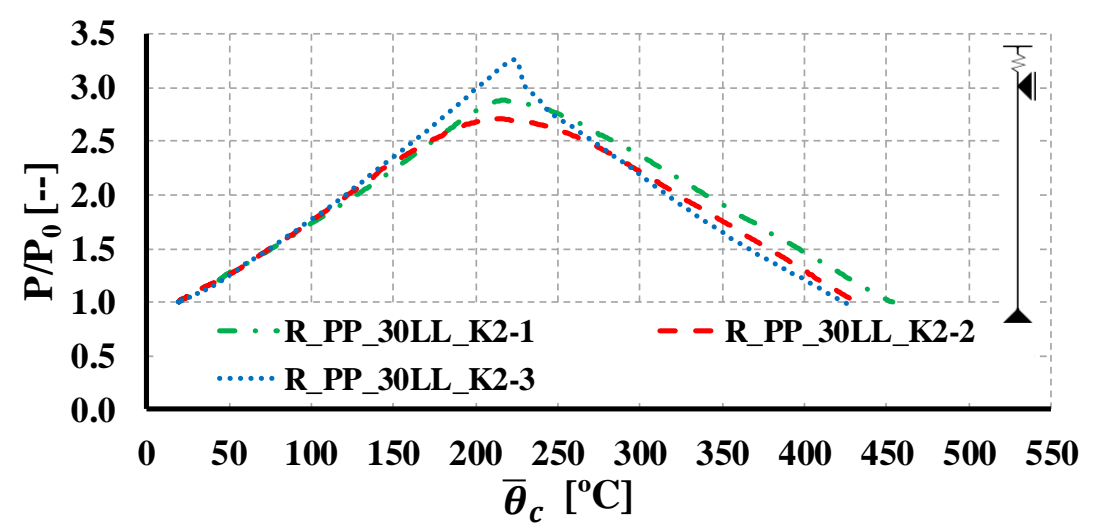

Figure F.21 Evolution of restraining forces with temperature increase for R columns.

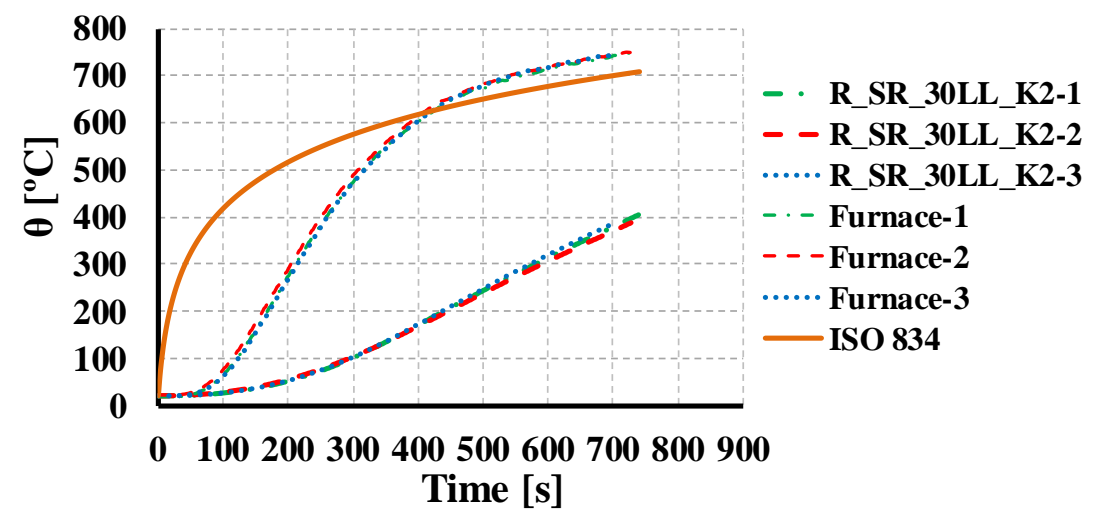

Figure F.22 Evolution of the mean temperature of the CFS column with temperature increase as well as the furnace temperature and ISO 834 fire curve.

\section{F.1.12 Closed built-up R columns - R_PP_50LL_K2-i}

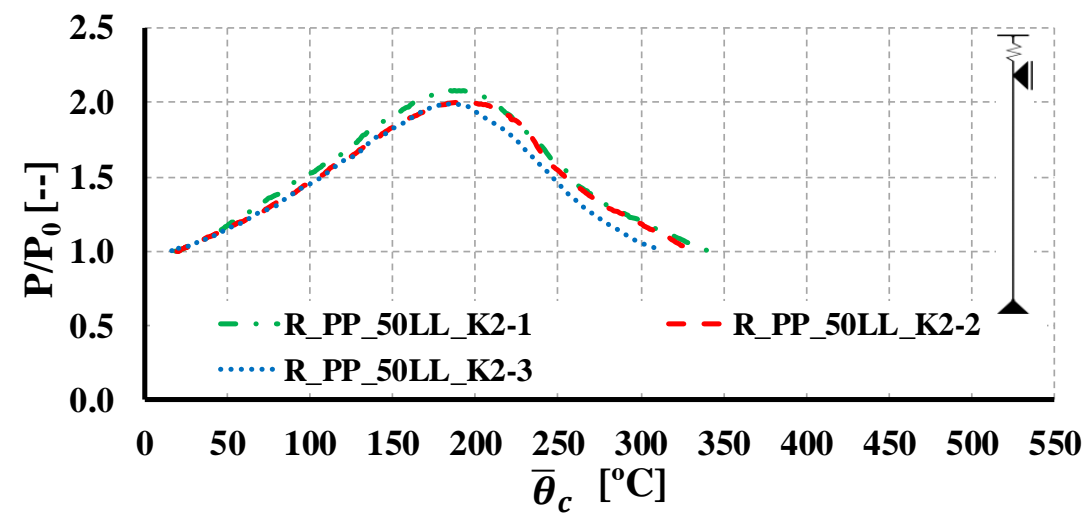

Figure F.23 Evolution of restraining forces with temperature increase for R columns. 


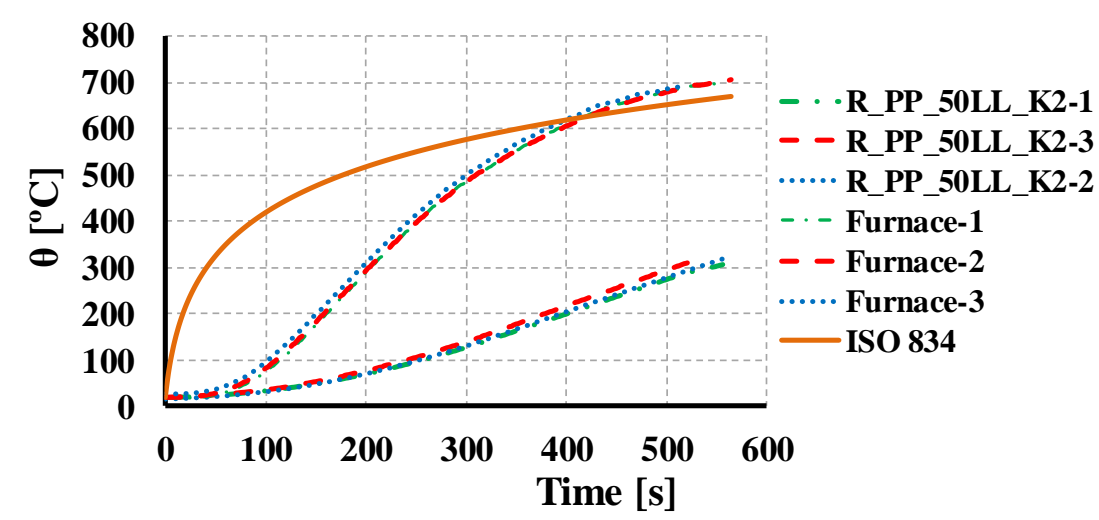

Figure F.24 Evolution of the mean temperature of the CFS column with temperature increase as well as the furnace temperature and ISO 834 fire curve.

\section{F.1.13 Closed built-up 2R columns - 2R_PP_30LL_K1-i}

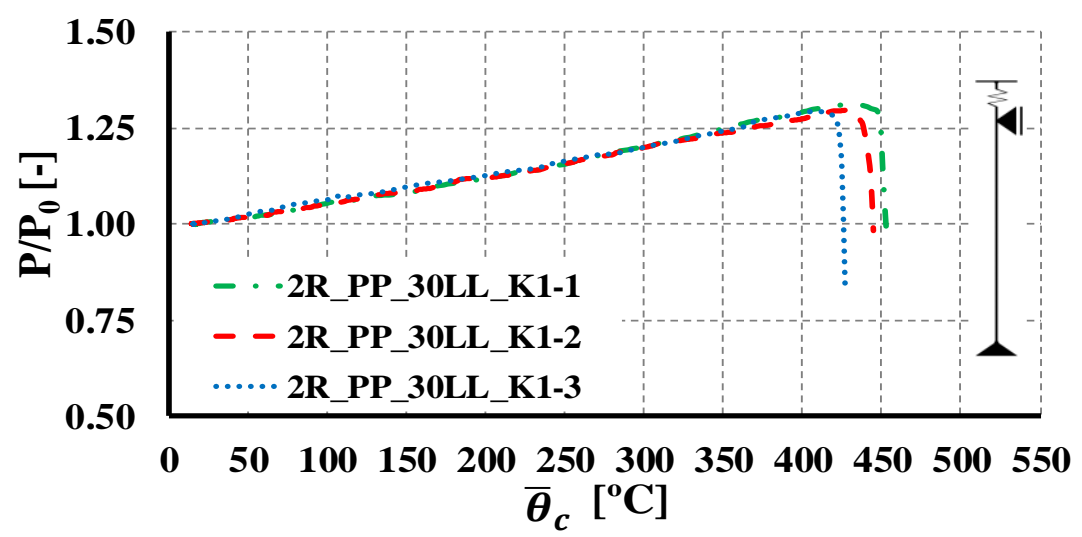

Figure F.25 Evolution of restraining forces with temperature increase for $2 \mathrm{R}$ columns.

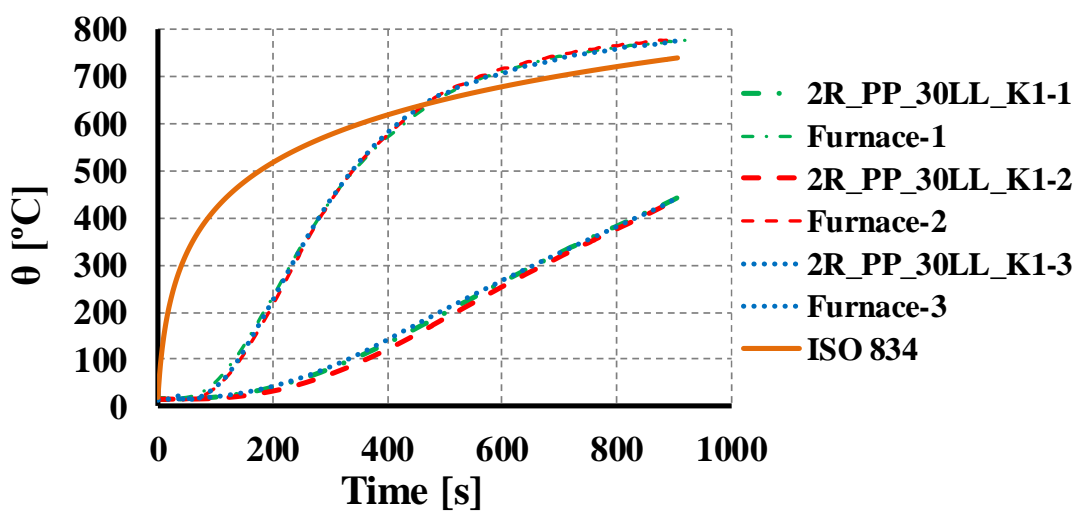

Figure F.26 Evolution of the mean temperature of the CFS column with temperature increase as well as the furnace temperature and ISO 834 fire curve. 


\section{F.1.14 Closed built-up 2R columns - 2R_PP_50LL_K1-i}

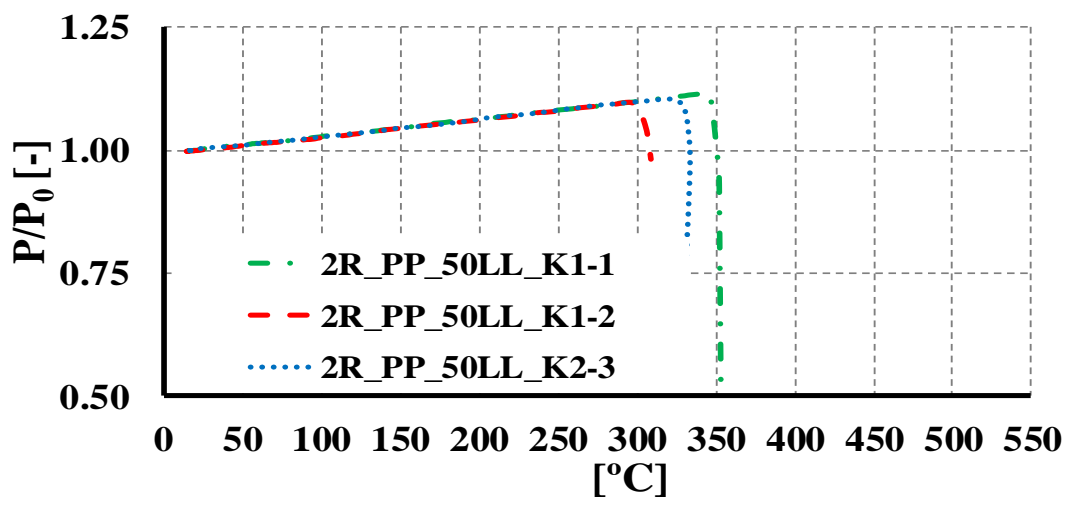

Figure F.27 Evolution of restraining forces with temperature increase for 2R columns.

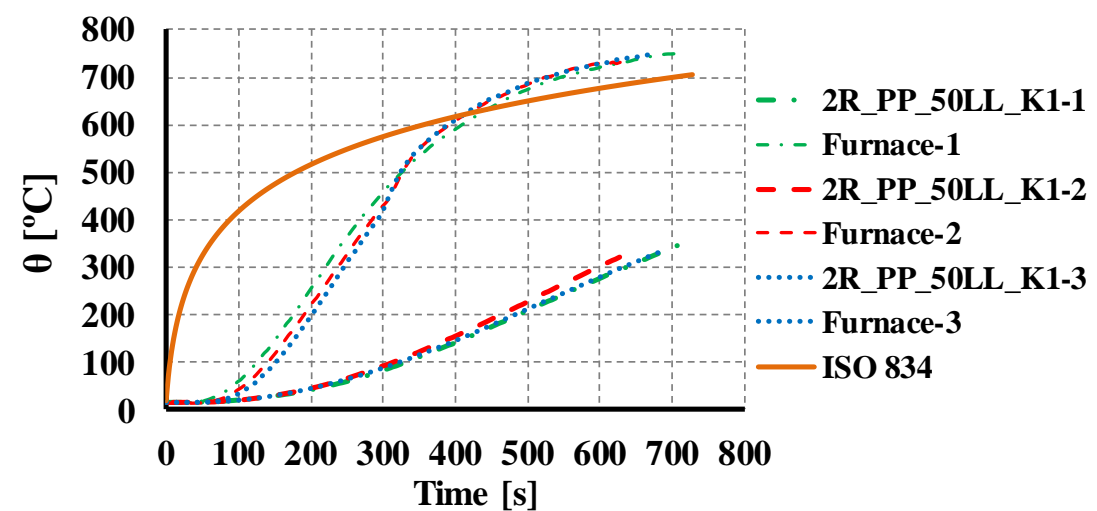

Figure F.28 Evolution of the mean temperature of the CFS column with temperature increase as well as the furnace temperature and ISO 834 fire curve.

\section{F.1.15 Closed built-up 2R columns - 2R_PP_30LL_K2-i}

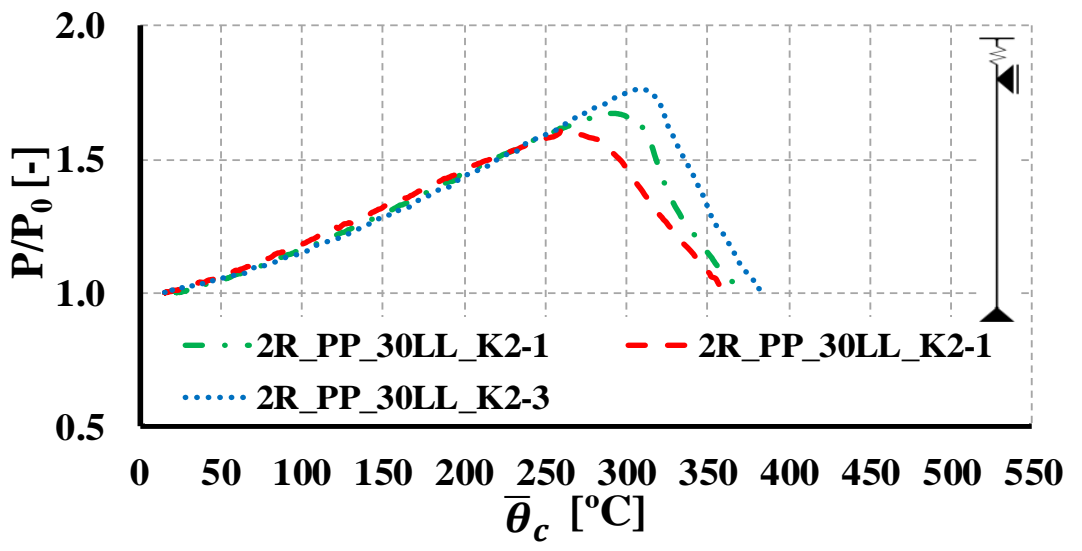

Figure F.29 Evolution of restraining forces with temperature increase for $2 \mathrm{R}$ columns. 


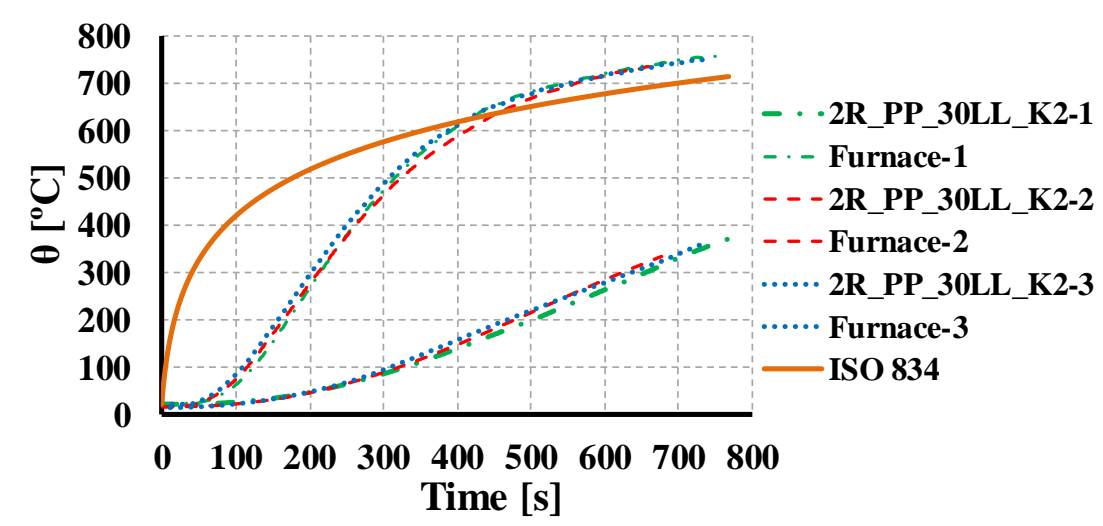

Figure F.30 Evolution of the mean temperature of the CFS column with temperature increase as well as the furnace temperature and ISO 834 fire curve.

\section{F.1.16 Closed built-up 2R columns - 2R_PP_50LL_K2-i}

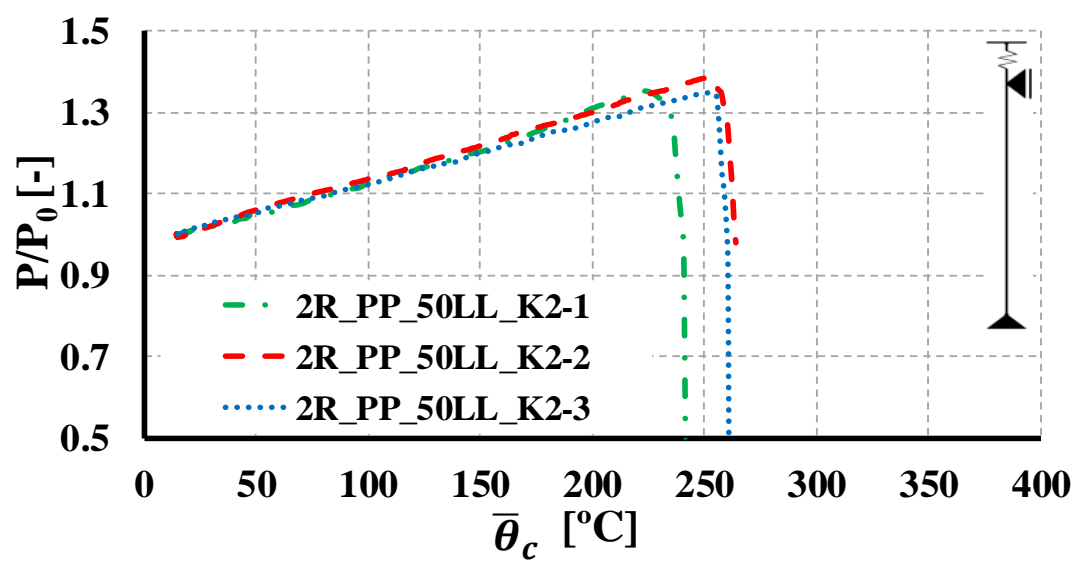

Figure F.31 Evolution of restraining forces with temperature increase for $2 \mathrm{R}$ columns.

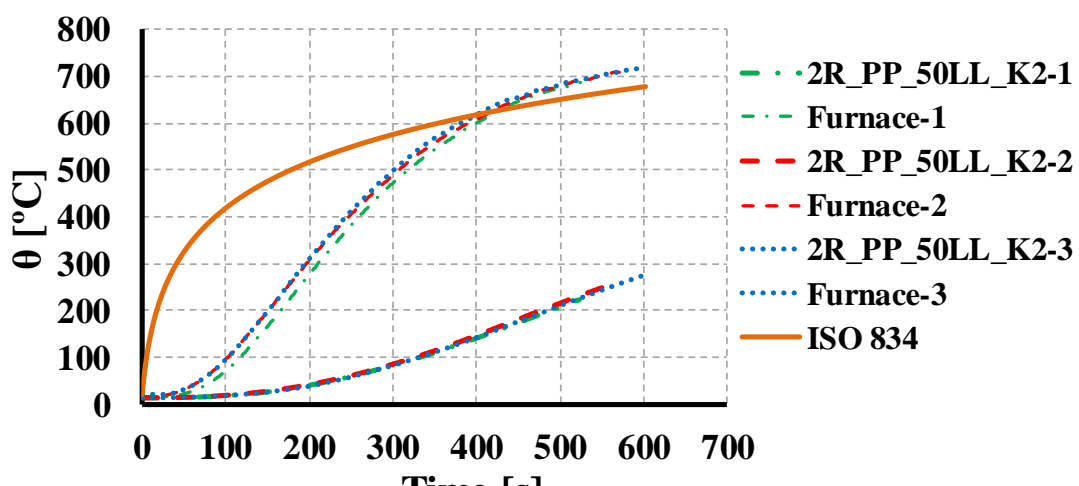

Time [s]

Figure F.32 Evolution of the mean temperature of the CFS column with temperature increase as well as the furnace temperature and ISO 834 fire curve. 


\section{F.2 Semi-rigid Columns}

\section{F.2.1 Lipped channel columns - C_SR_30LL_K3-i}

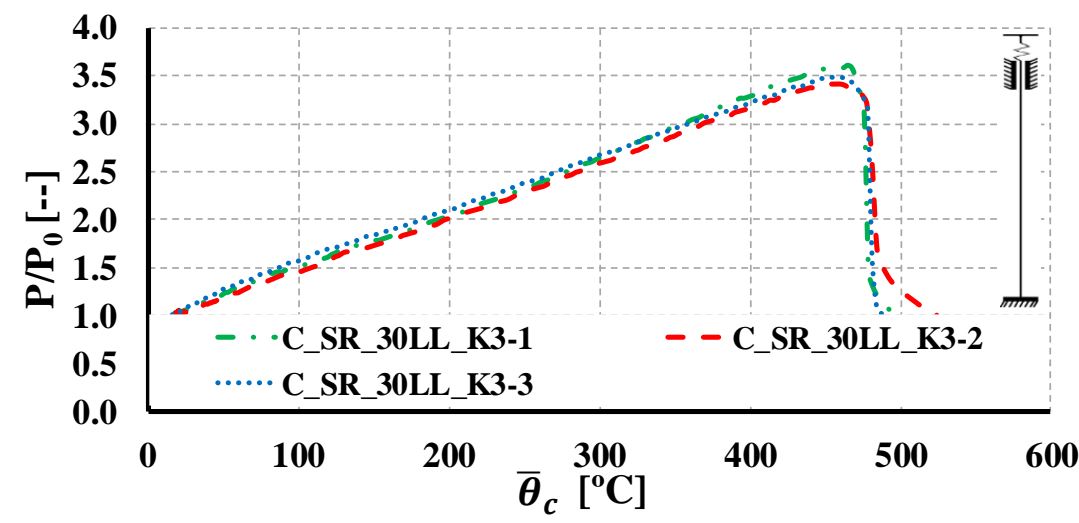

Figure F.33 Evolution of restraining forces with temperature increase for C columns.

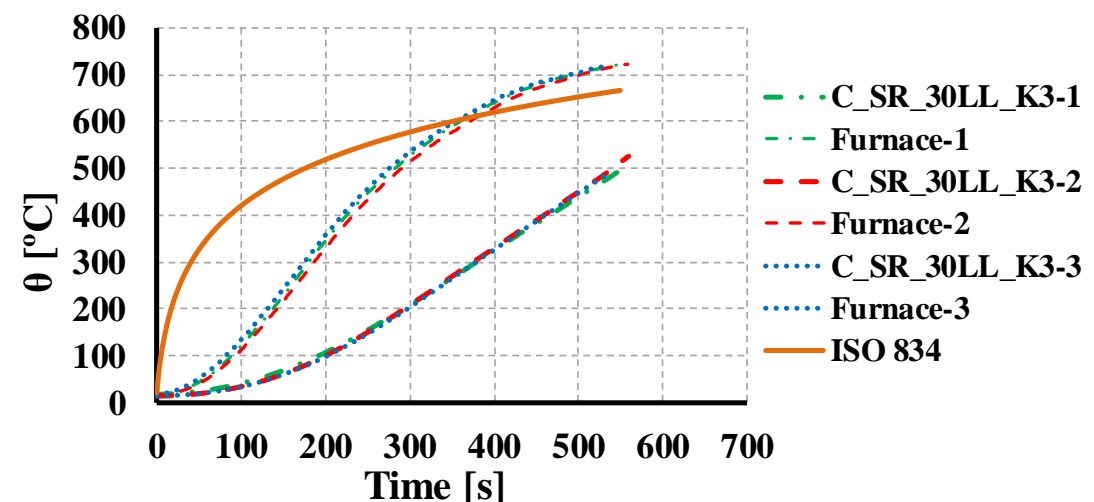

Figure F.34 Evolution of the mean temperature of the CFS column with temperature increase as well as the furnace temperature and ISO 834 fire curve. 


\section{F.2.2 Lipped channel columns - C_SR_50LL_K3-i}

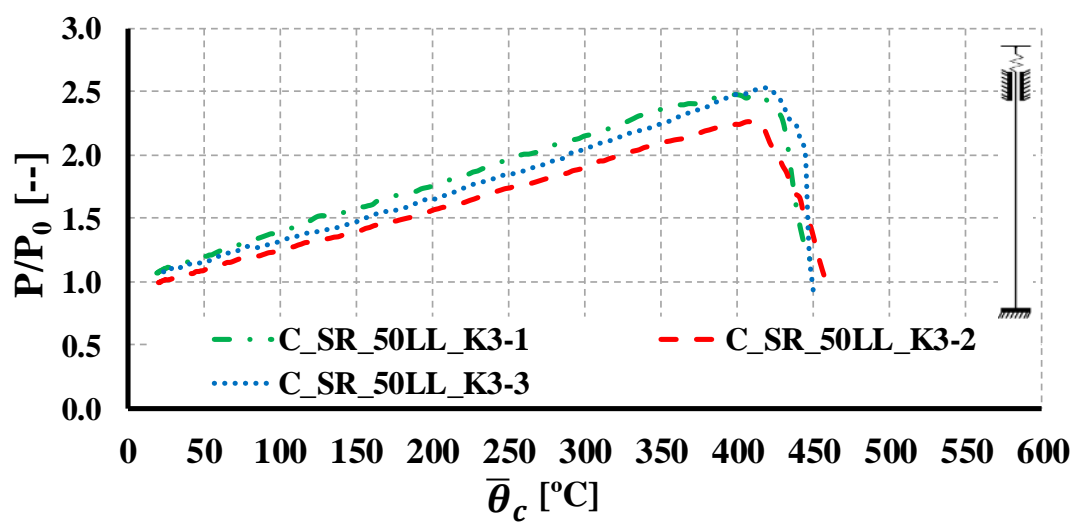

Figure F.35 Evolution of restraining forces with temperature increase for $\mathrm{C}$ columns.

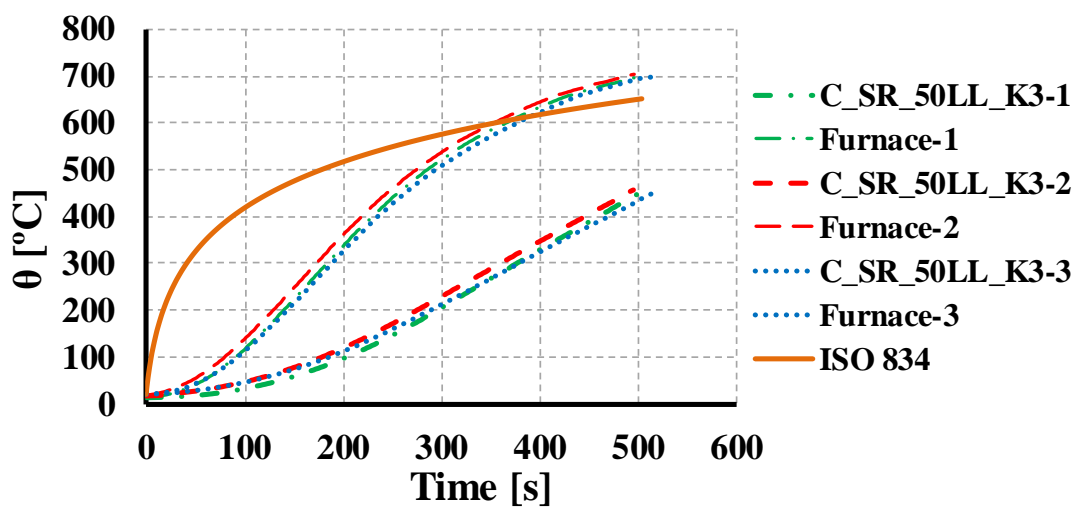

Figure F.36 Evolution of the mean temperature of the CFS column with temperature increase as well as the furnace temperature and ISO 834 fire curve.

\section{F.2.3 Lipped channel columns - C_SR_30LL_K4-i}

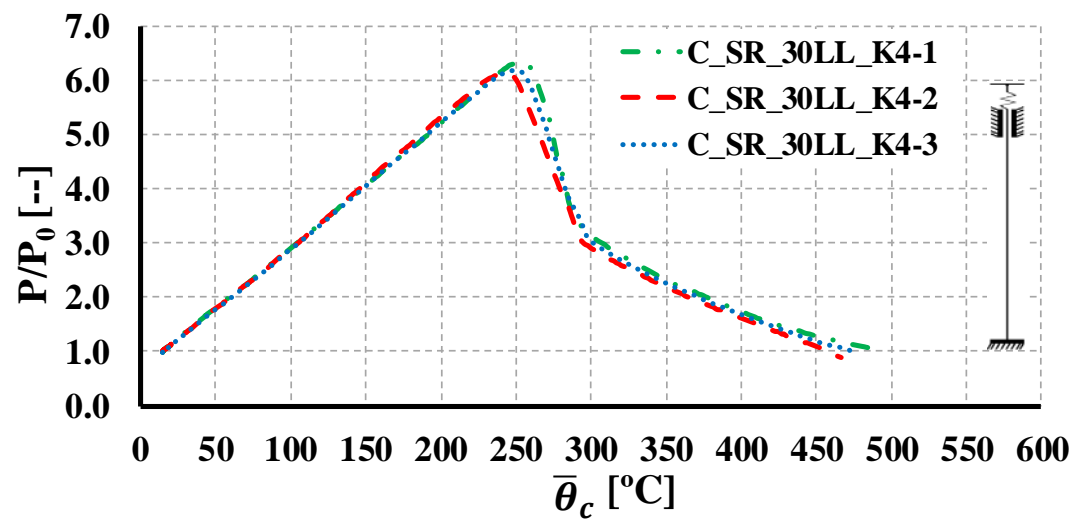

Figure F.37 Evolution of restraining forces with temperature increase for $\mathrm{C}$ columns. 


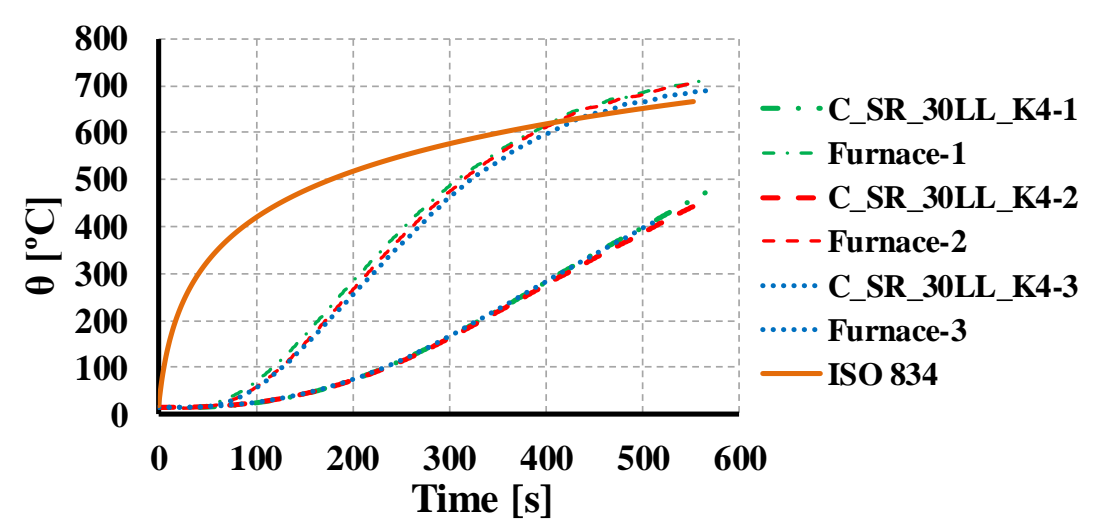

Figure F.38 Evolution of the mean temperature of the CFS column with temperature increase as well as the furnace temperature and ISO 834 fire curve.

\section{F.2.4 Lipped channel columns - C_SR_50LL_K4-i}

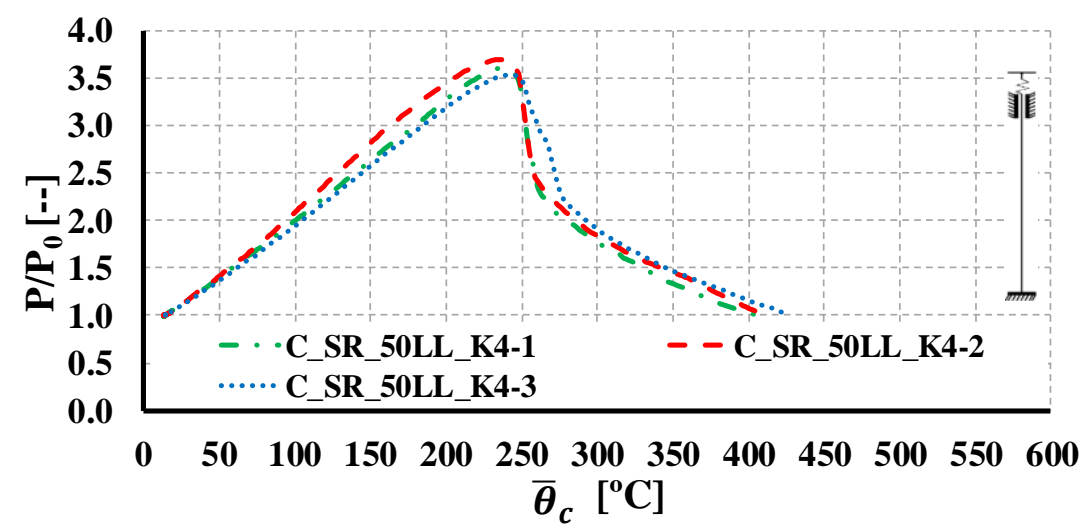

Figure F.39 Evolution of restraining forces with temperature increase for C columns.

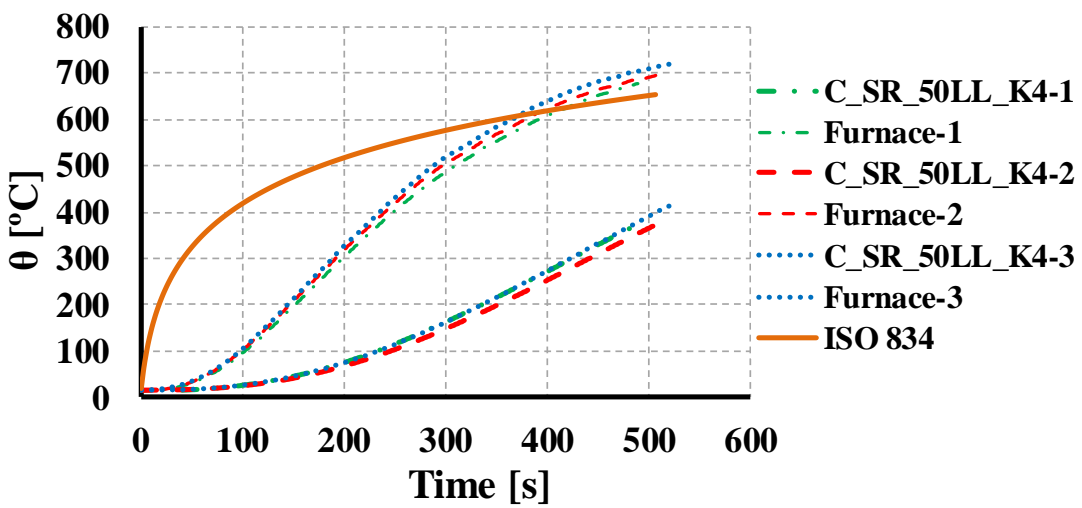

Figure F.40 Evolution of the mean temperature of the CFS column with temperature increase as well as the furnace temperature and ISO 834 fire curve. 


\section{F.2.5 Open built-up I columns - I_SR_30LL_K3-i}

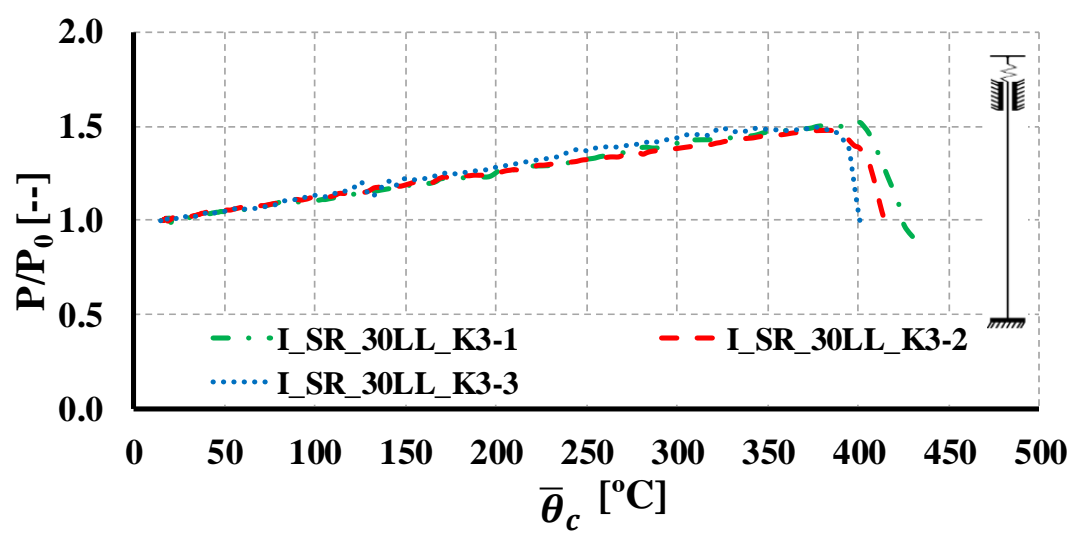

Figure F.41 Evolution of restraining forces with temperature increase for I columns.

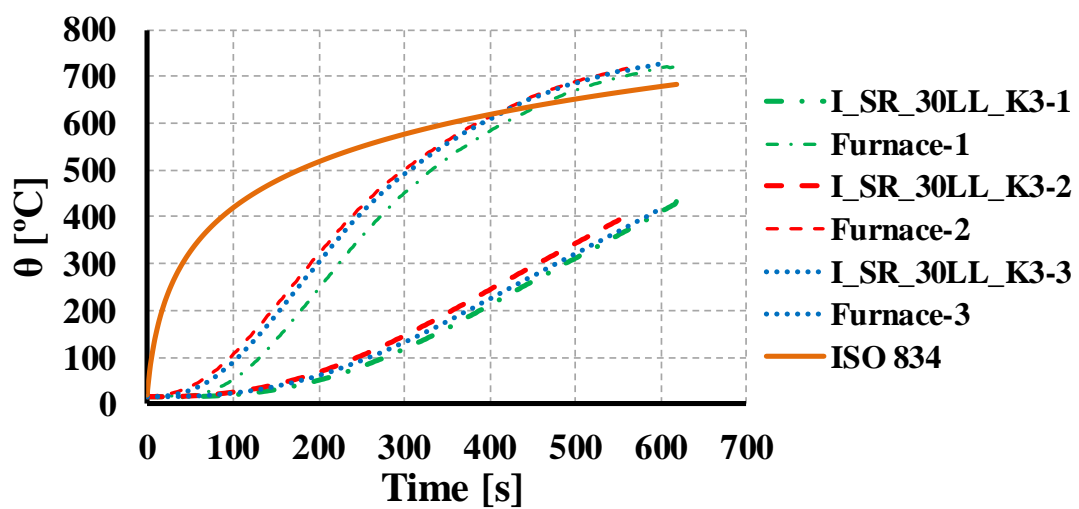

Figure F.42 Evolution of the mean temperature of the CFS column with temperature increase as well as the furnace temperature and ISO 834 fire curve.

\section{F.2.6 Open built-up I columns - I_SR_50LL_K3-i}

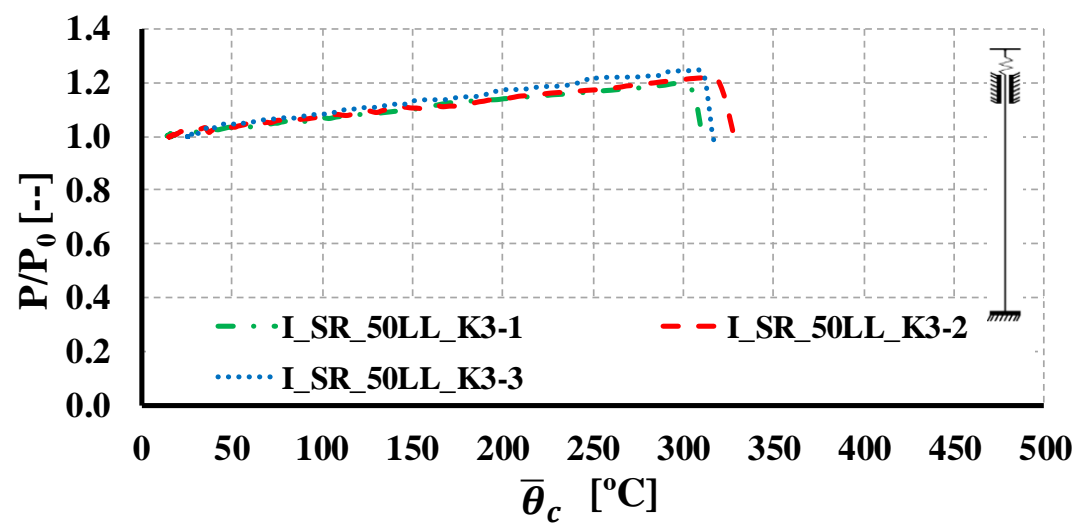

Figure F.43 Evolution of restraining forces with temperature increase for I columns. 


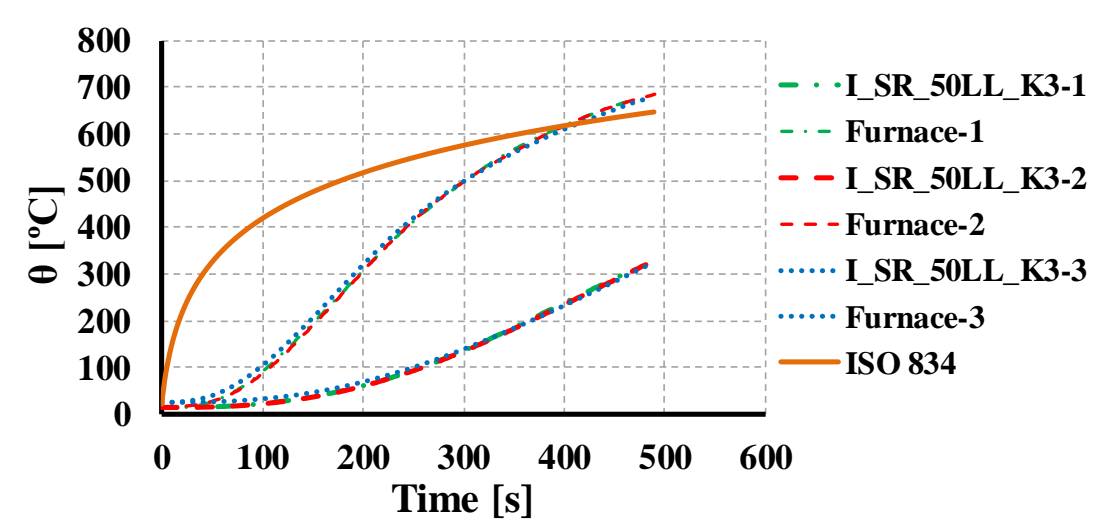

Figure F.44 Evolution of the mean temperature of the CFS column with temperature increase as well as the furnace temperature and ISO 834 fire curve.

\section{F.2.7 Open built-up I columns - I_SR_30LL_K4-i}

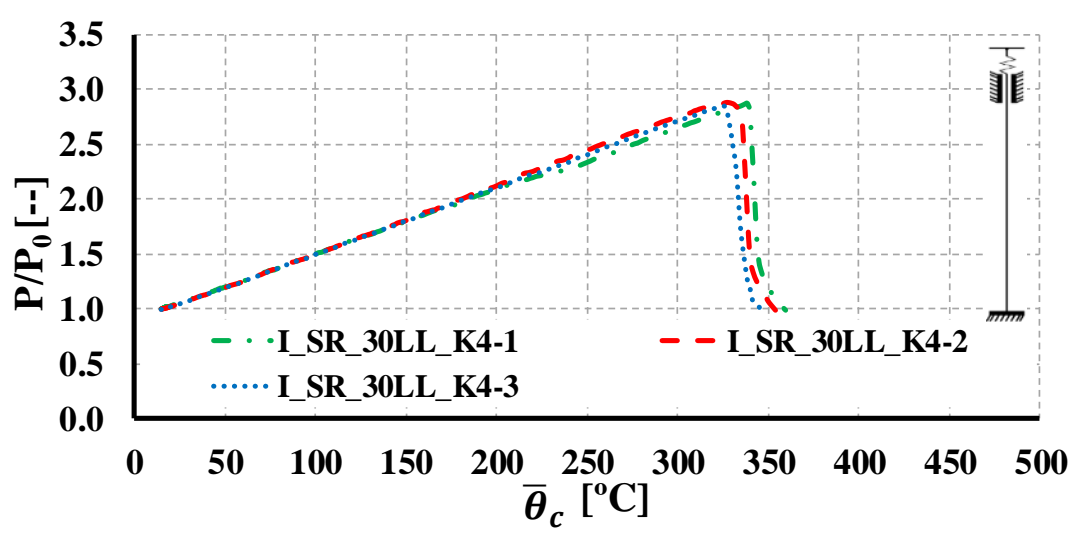

Figure F.45 Evolution of restraining forces with temperature increase for I columns.

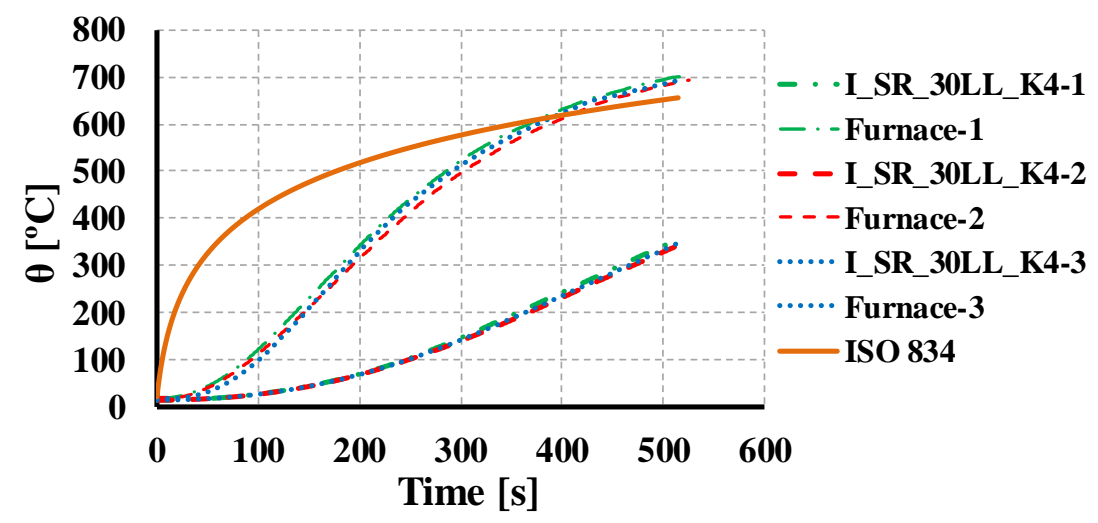

Figure F.46 Evolution of the mean temperature of the CFS column with temperature increase as well as the furnace temperature and ISO 834 fire curve. 


\section{F.2.8 Open built-up I columns - I_SR_50LL_K4-i}

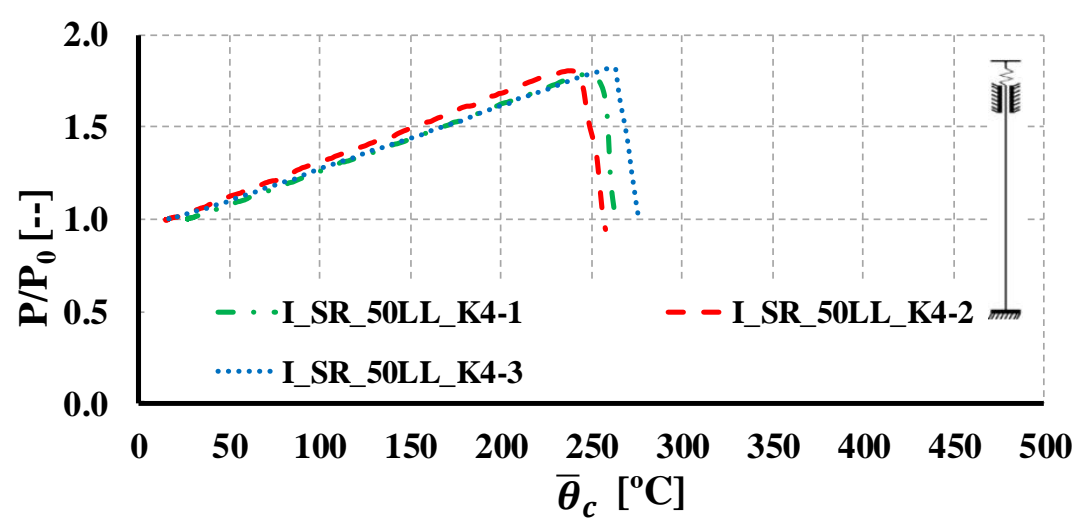

Figure F.47 Evolution of restraining forces with temperature increase for I columns.

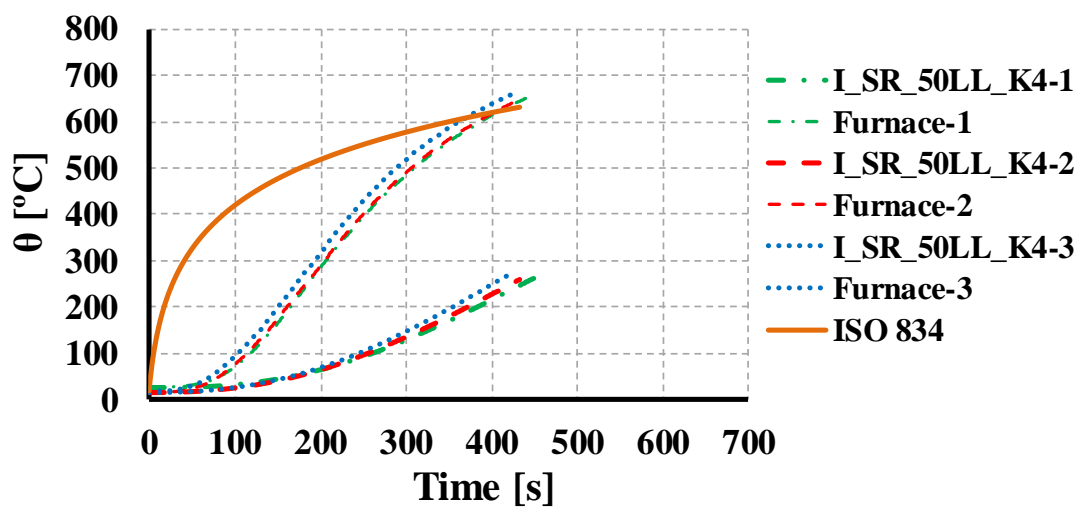

Figure F.48 Evolution of the mean temperature of the CFS column with temperature increase as well as the furnace temperature and ISO 834 fire curve.

\section{F.2.9 Closed built-up R columns - R_SR_30LL_K3-i}

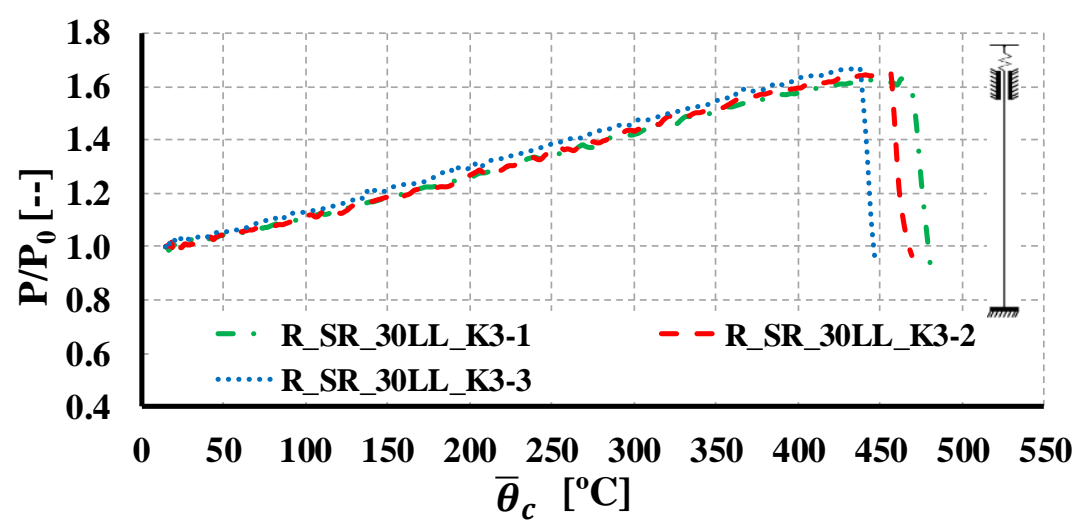

Figure F.49 Evolution of restraining forces with temperature increase for R columns. 


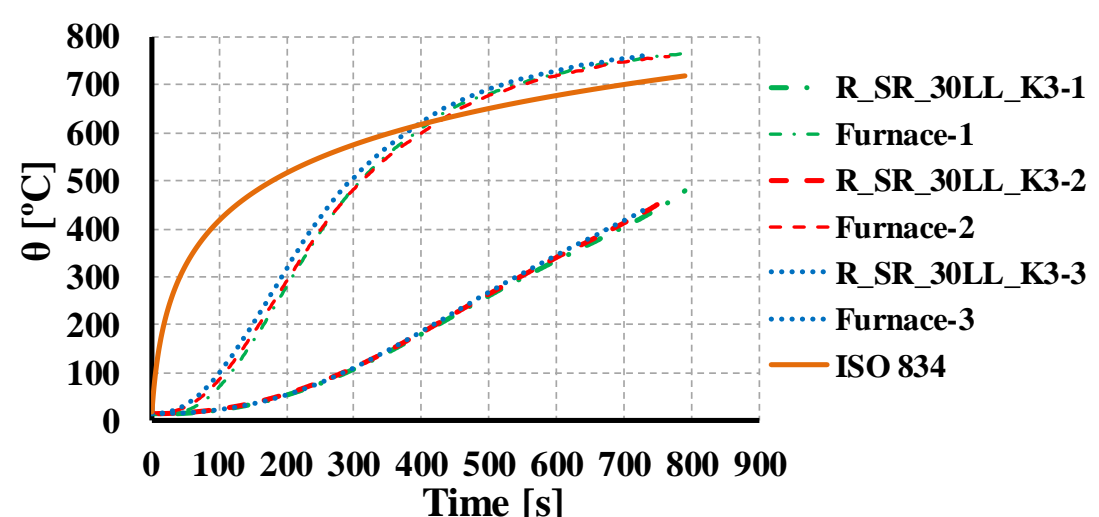

Figure F.50 Evolution of the mean temperature of the CFS column with temperature increase as well as the furnace temperature and ISO 834 fire curve.

\section{F.2.10 Closed built-up R columns - R_SR_30LL_K3-i}

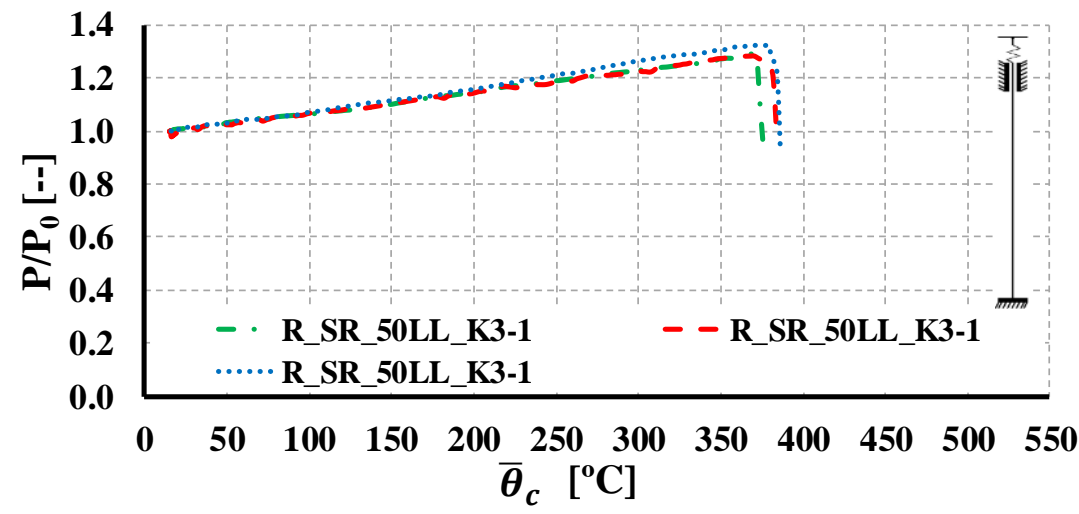

Figure F.51 Evolution of restraining forces with temperature increase for R columns.

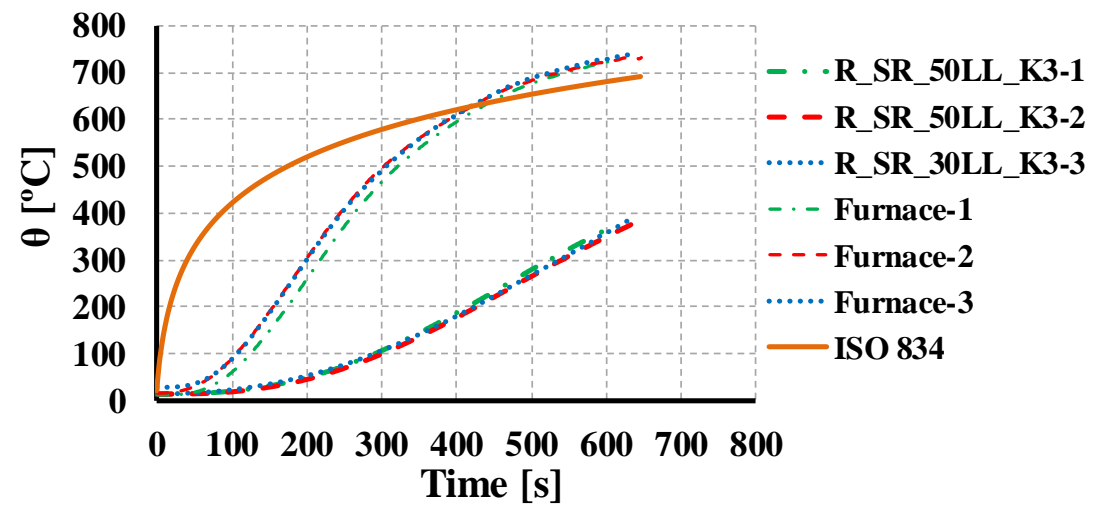

Figure F.52 Evolution of the mean temperature of the CFS column with temperature increase as well as the furnace temperature and ISO 834 fire curve. 


\section{F.2.11 Closed built-up R columns - R_SR_30LL_K4-i}

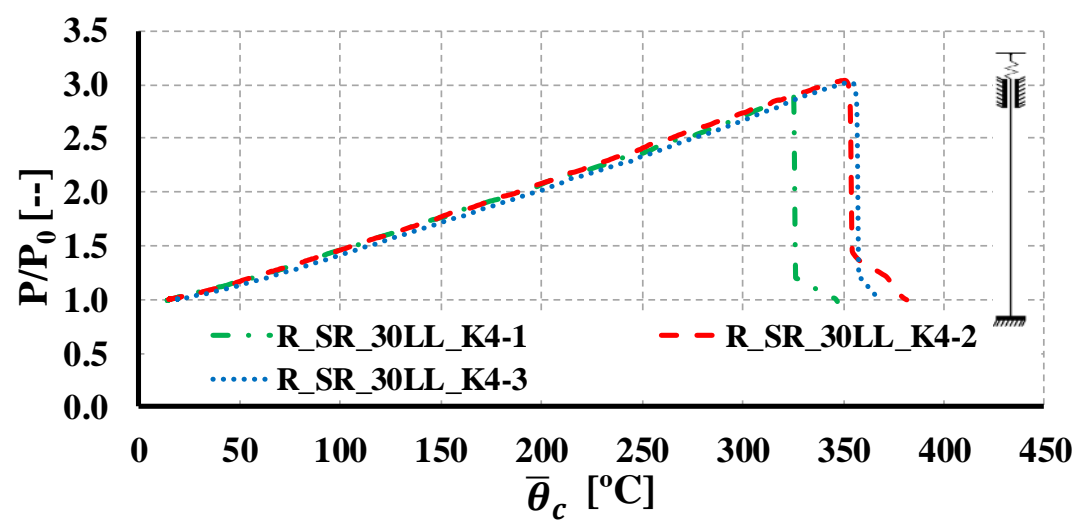

Figure F.53 Evolution of restraining forces with temperature increase for R columns.

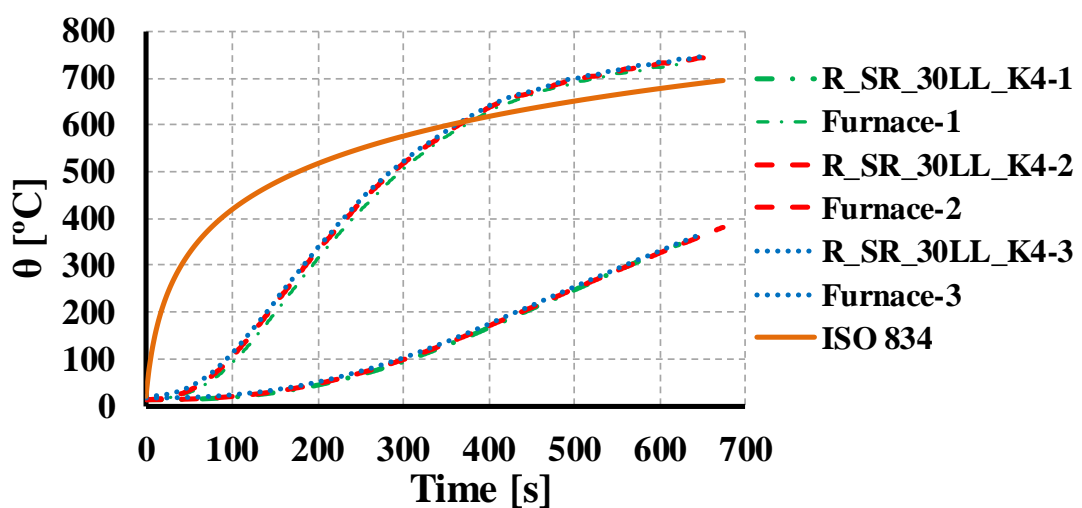

Figure F.54 Evolution of the mean temperature of the CFS column with temperature increase as well as the furnace temperature and ISO 834 fire curve.

\section{F.2.12 Closed built-up R columns - R_SR_50LL_K4-i}

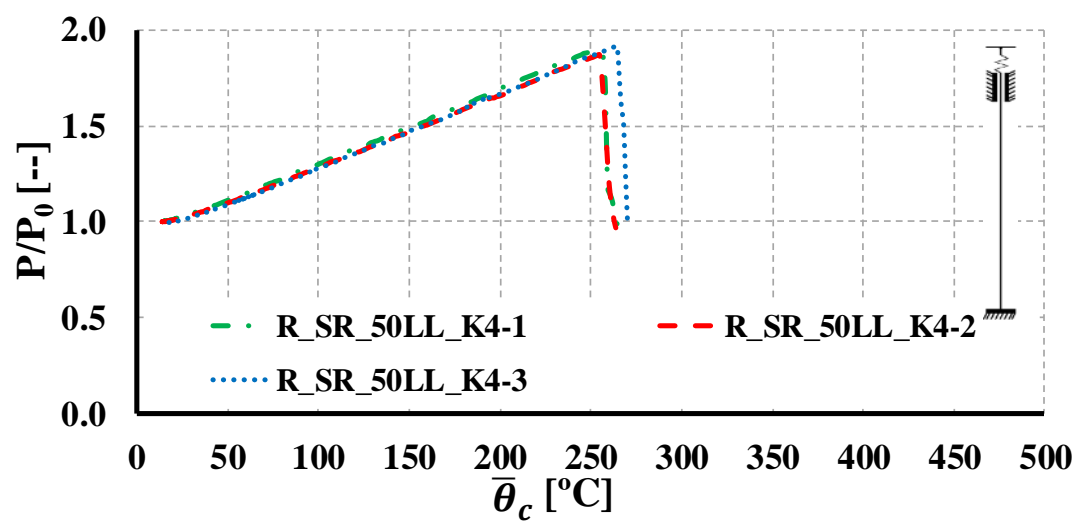

Figure F.55 Evolution of restraining forces with temperature increase for R columns. 


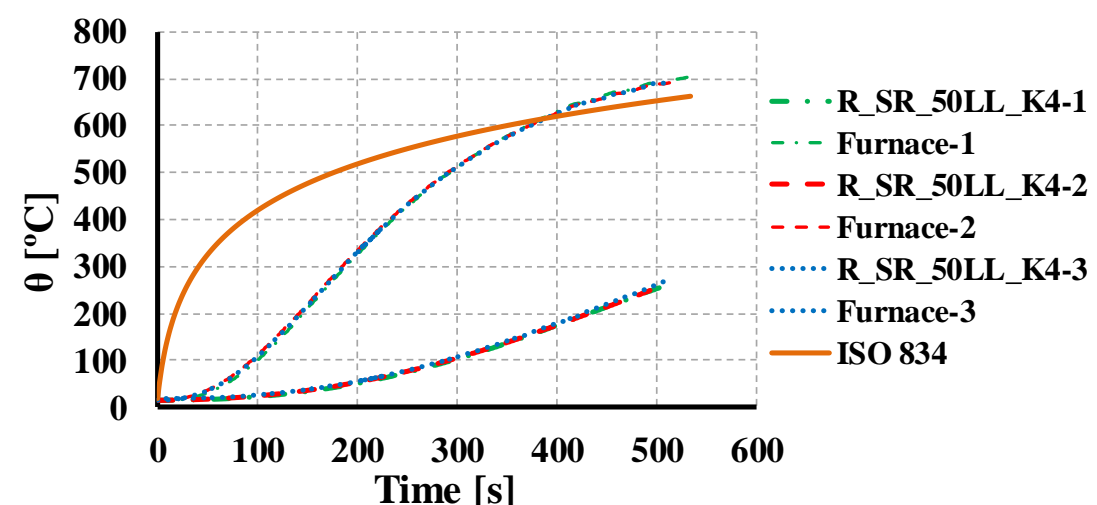

Figure F.56 Evolution of the mean temperature of the CFS column with temperature increase as well as the furnace temperature and ISO 834 fire curve.

\section{F.2.13 Closed built-up 2R columns - 2R_SR_30LL_K3-i}

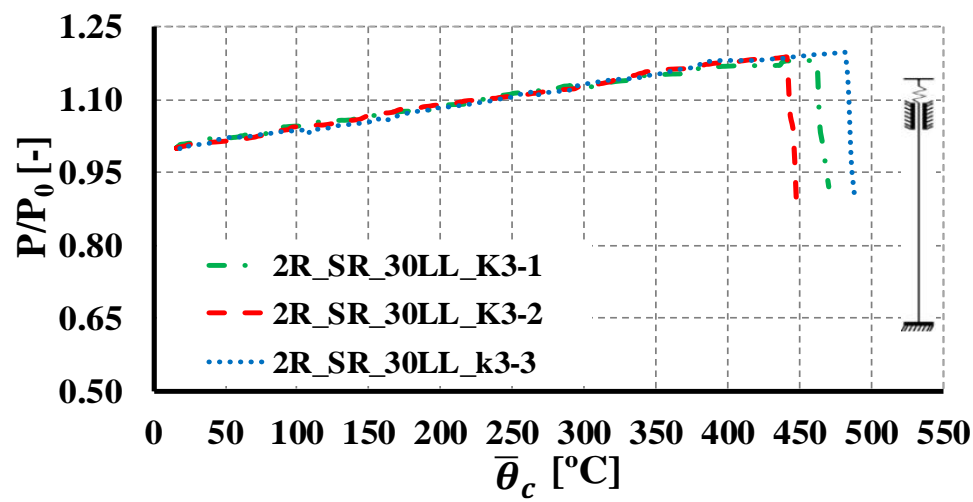

Figure F.57 Evolution of restraining forces with temperature increase for $2 \mathrm{R}$ columns.

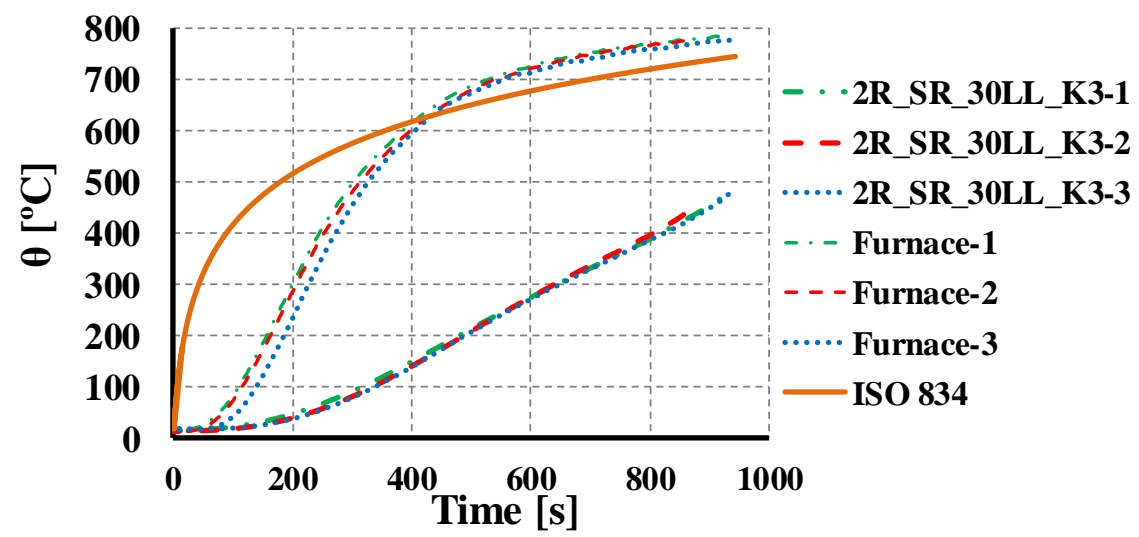

Figure F.58 Evolution of the mean temperature of the CFS column with temperature increase as well as the furnace temperature and ISO 834 fire curve. 


\section{F.2.14 Closed built-up 2R columns - 2R_SR_50LL_K3-i}

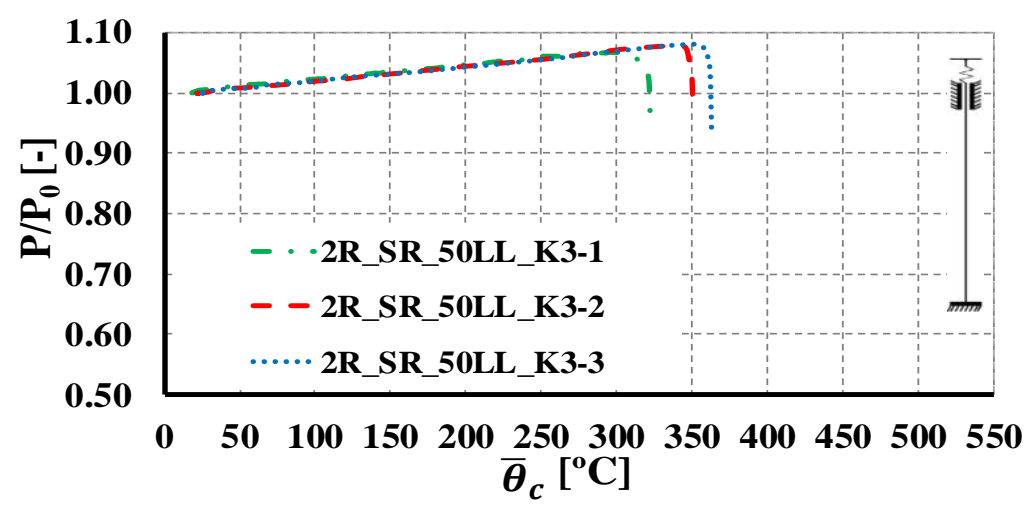

Figure F.59 Evolution of restraining forces with temperature increase for $2 \mathrm{R}$ columns.

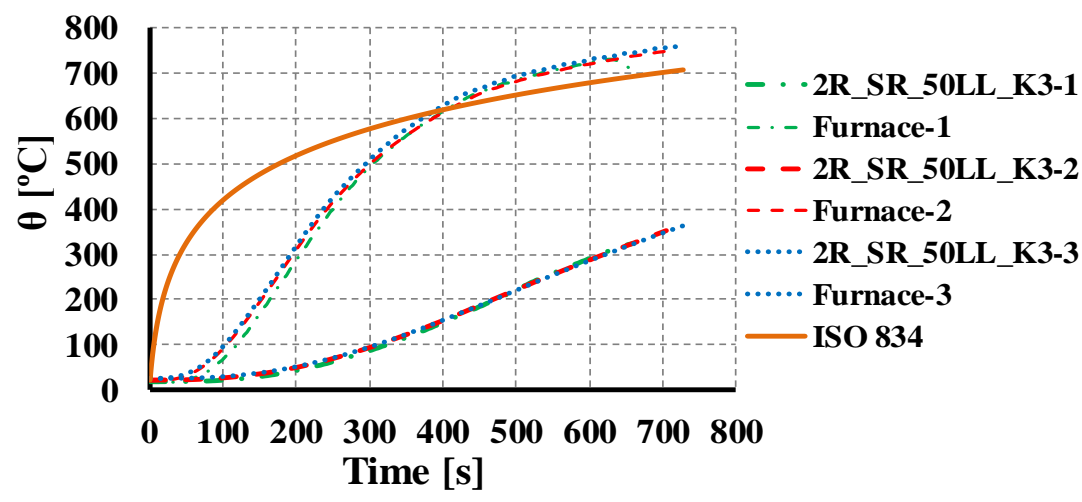

Figure F.60 Evolution of the mean temperature of the CFS column with temperature increase as well as the furnace temperature and ISO 834 fire curve.

\section{F.2.15 Closed built-up 2R columns - 2R_SR_30LL_K4-i}

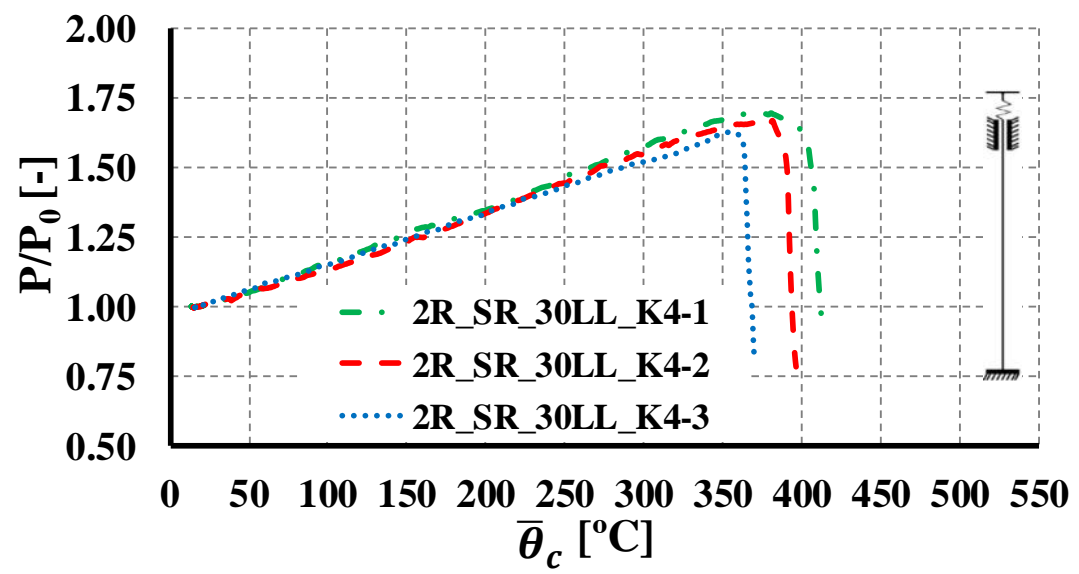

Figure F.61 Evolution of restraining forces with temperature increase for $2 \mathrm{R}$ columns. 


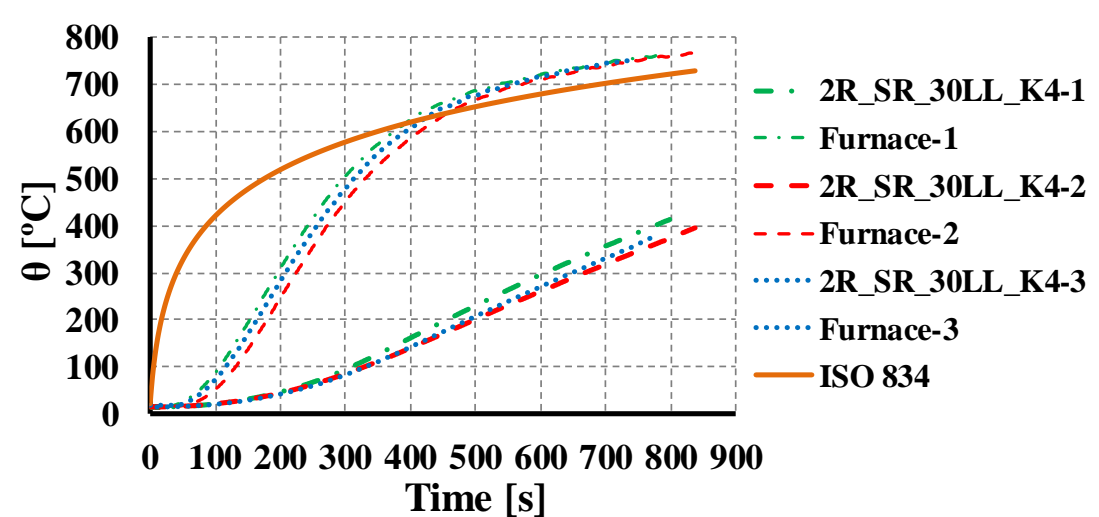

Figure F.62 Evolution of the mean temperature of the CFS column with temperature increase as well as the furnace temperature and ISO 834 fire curve.

\section{F.2.16 Closed built-up 2R columns - 2R_SR_50LL_K4-i}

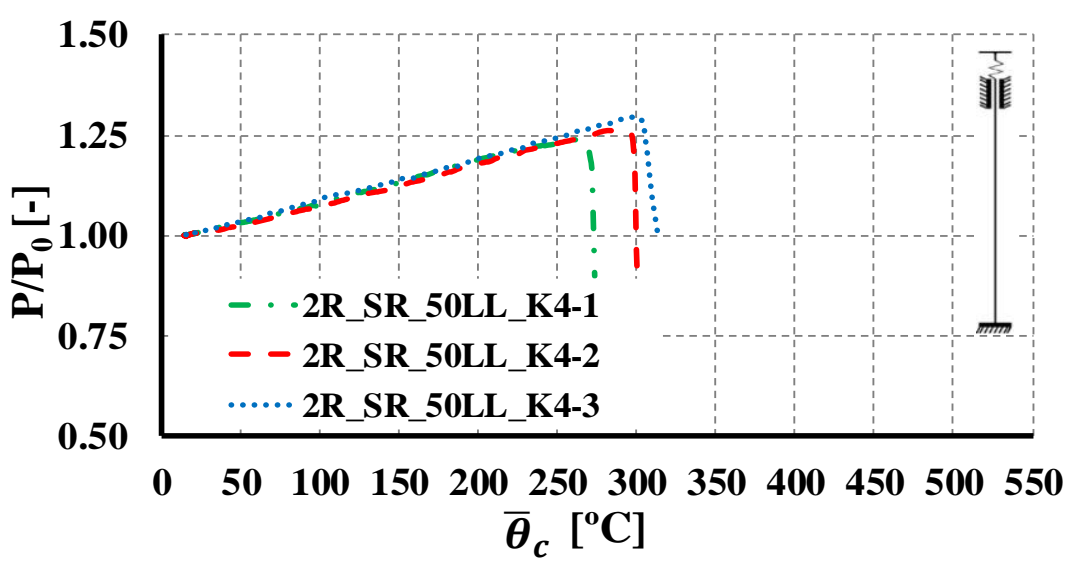

Figure F.63 Evolution of restraining forces with temperature increase for $2 \mathrm{R}$ columns.

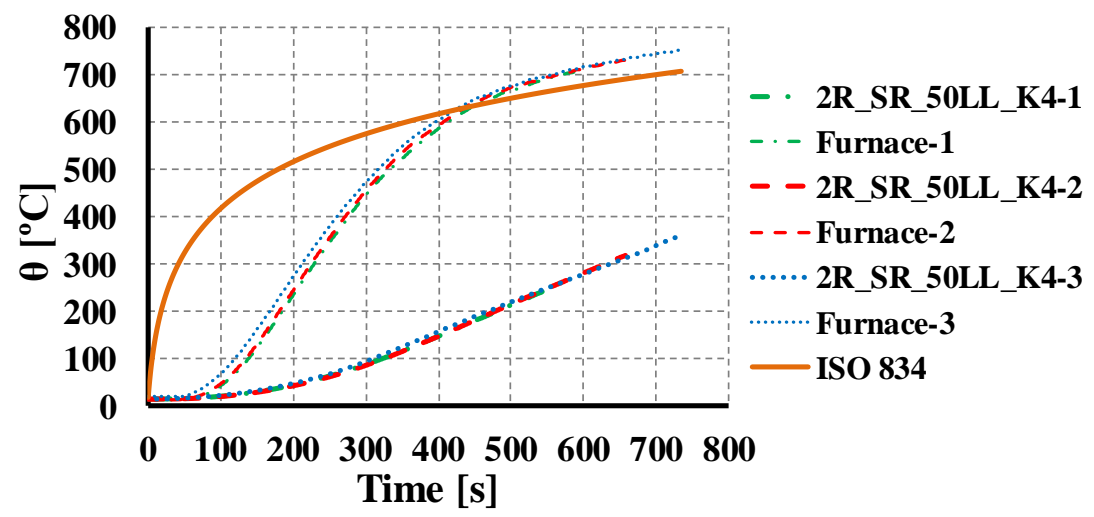

Figure F.64 Evolution of the mean temperature of the CFS column with temperature increase as well as the furnace temperature and ISO 834 fire curve. 


\section{APPENDIX G PHOTOGRAPHS OF COLUMNS AFTER FIRE CONDITIONS}

\section{G.1 Lipped Channel Columns}

\section{G.1.1 Pinned-end support condition and $\mathrm{K} 1$ level of restraint to thermal elongation}
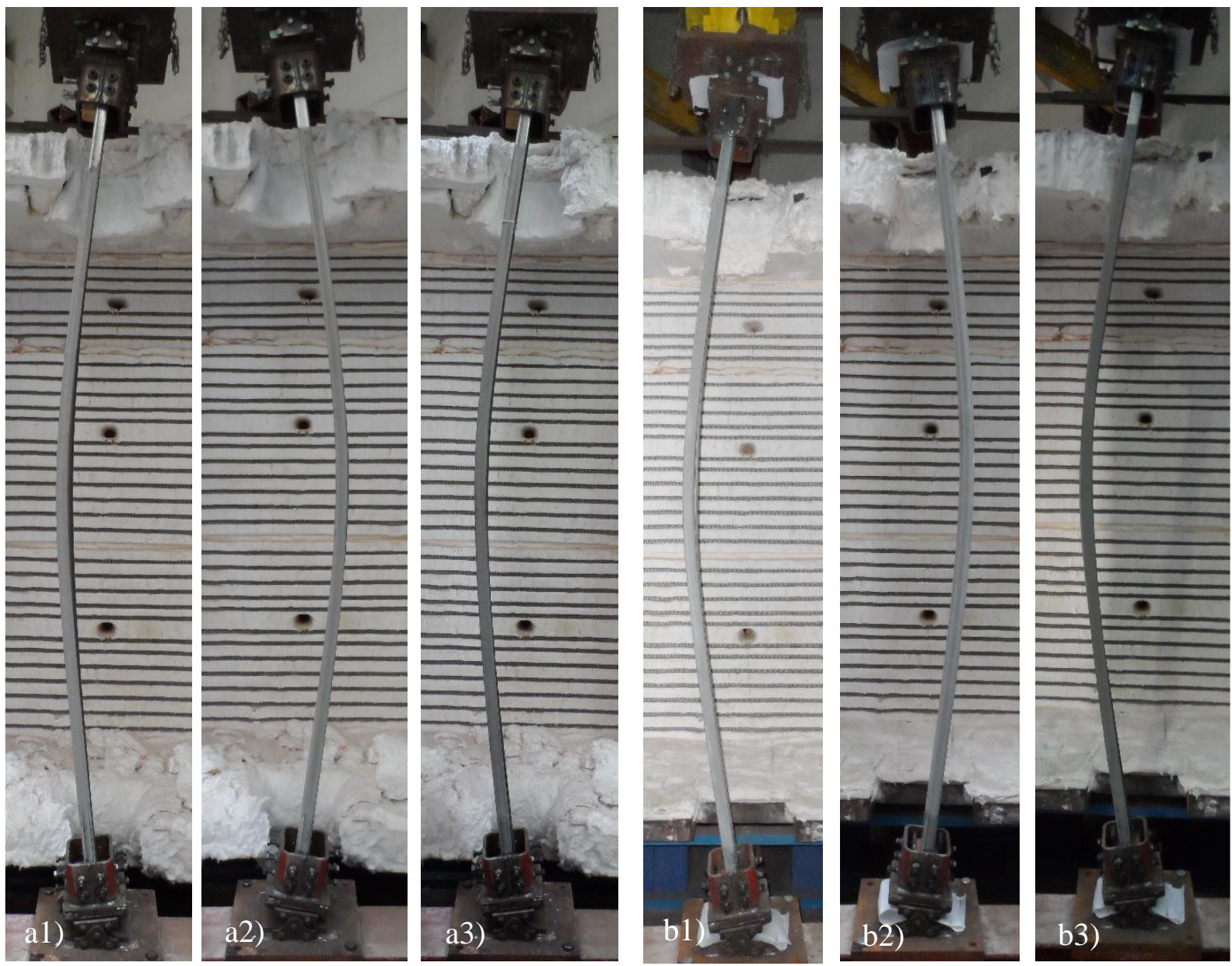

Figure G.1 Final deformed shapes for pinned-ended lipped channel columns. a1), a2) and a3) C_PP_30LL_K1-1, 2 and 3.b1), b2) and b3) C_PP_50LL_K1-1, 2 and 3. 


\section{G.1.2 Pinned-end support condition and K2 level of restraint to thermal elongation}
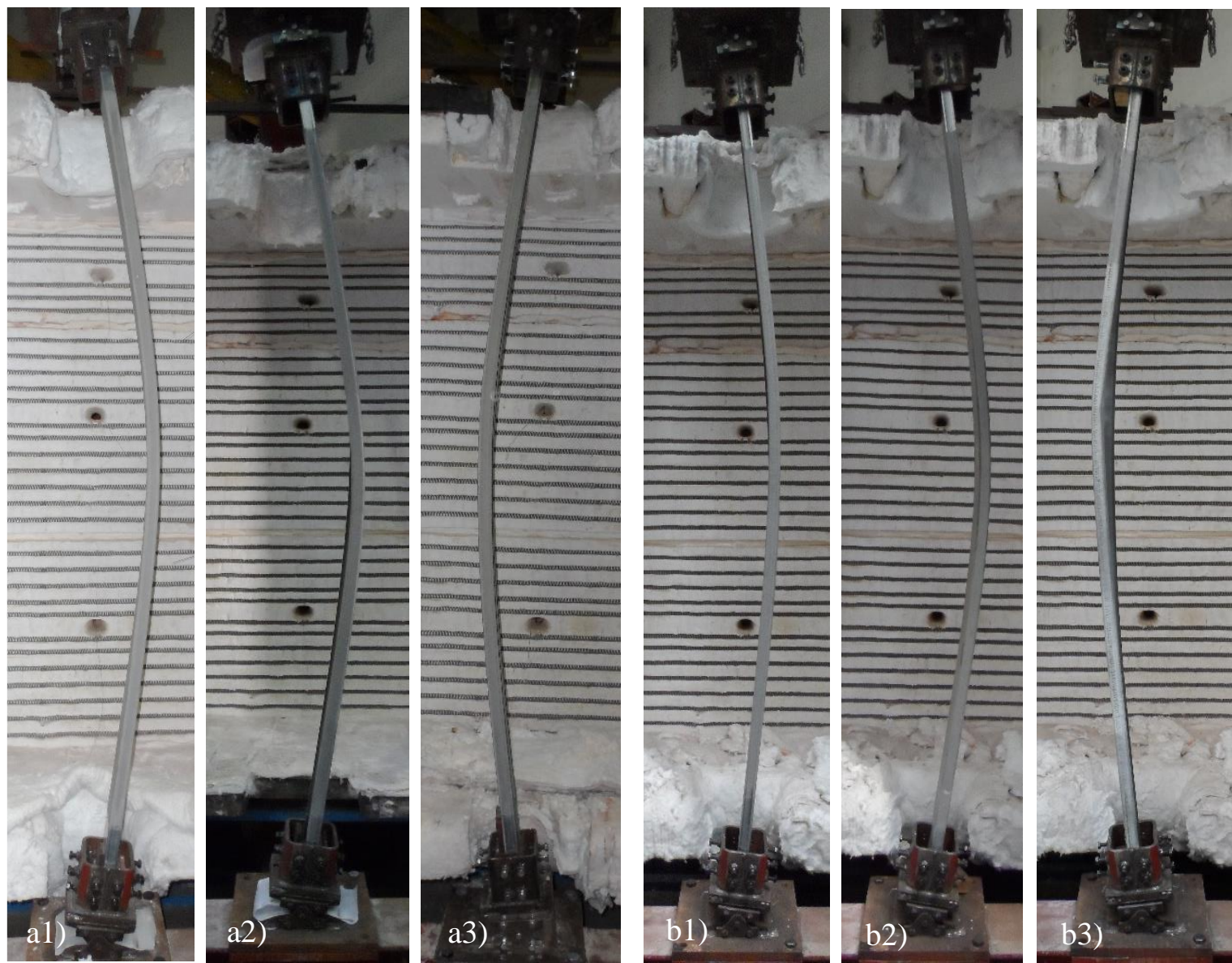

Figure G.2 Final deformed shapes for pinned-ended lipped channel columns. a1), a2) and a3) C_PP_30LL_K2-1, 2 and 3.b1), b2) and b3) C_PP_50LL_K2-1, 2 and 3. 


\section{G.1.3 Semi rigid end-support condition and K1 level of restraint to thermal elongation}
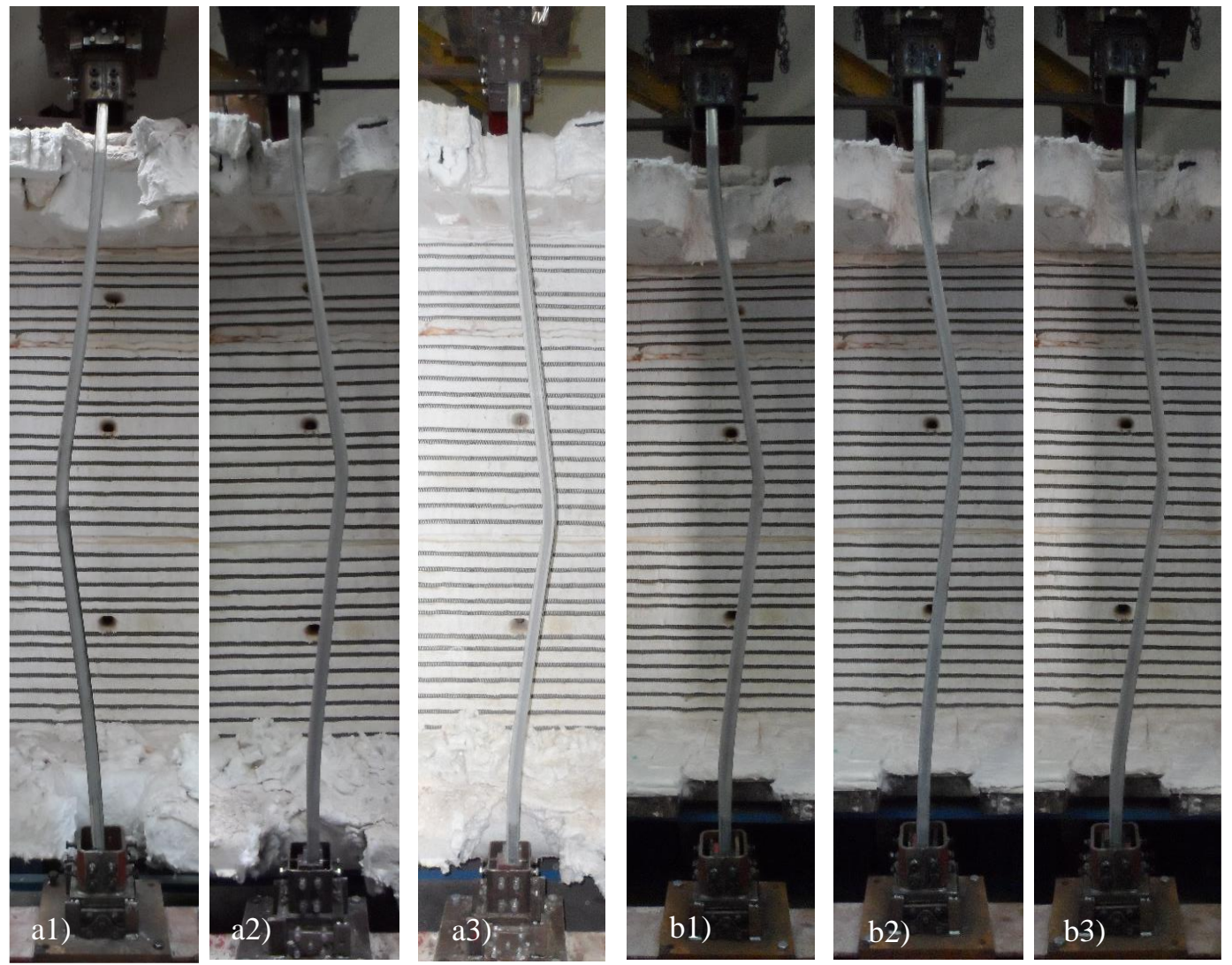

Figure G.3 Final deformed shapes for semi-rigid lipped channel columns. a1), a2) and a3) C_SR_30LL_K3-1, 2 and 3.b1), b2) and b3) C_SR_50LL_K3-1, 2 and 3.
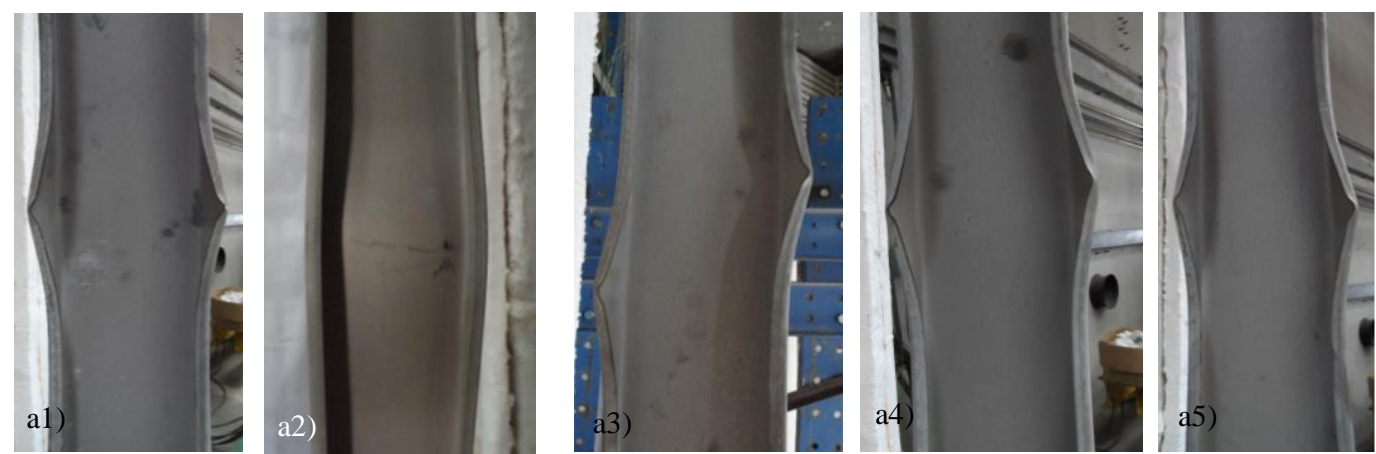

Figure G.4 Different configurations due to distortional buckling observed in the experimental tests. 


\section{G.1.4 Semi rigid end-support condition and K2 level of restraint to thermal elongation}
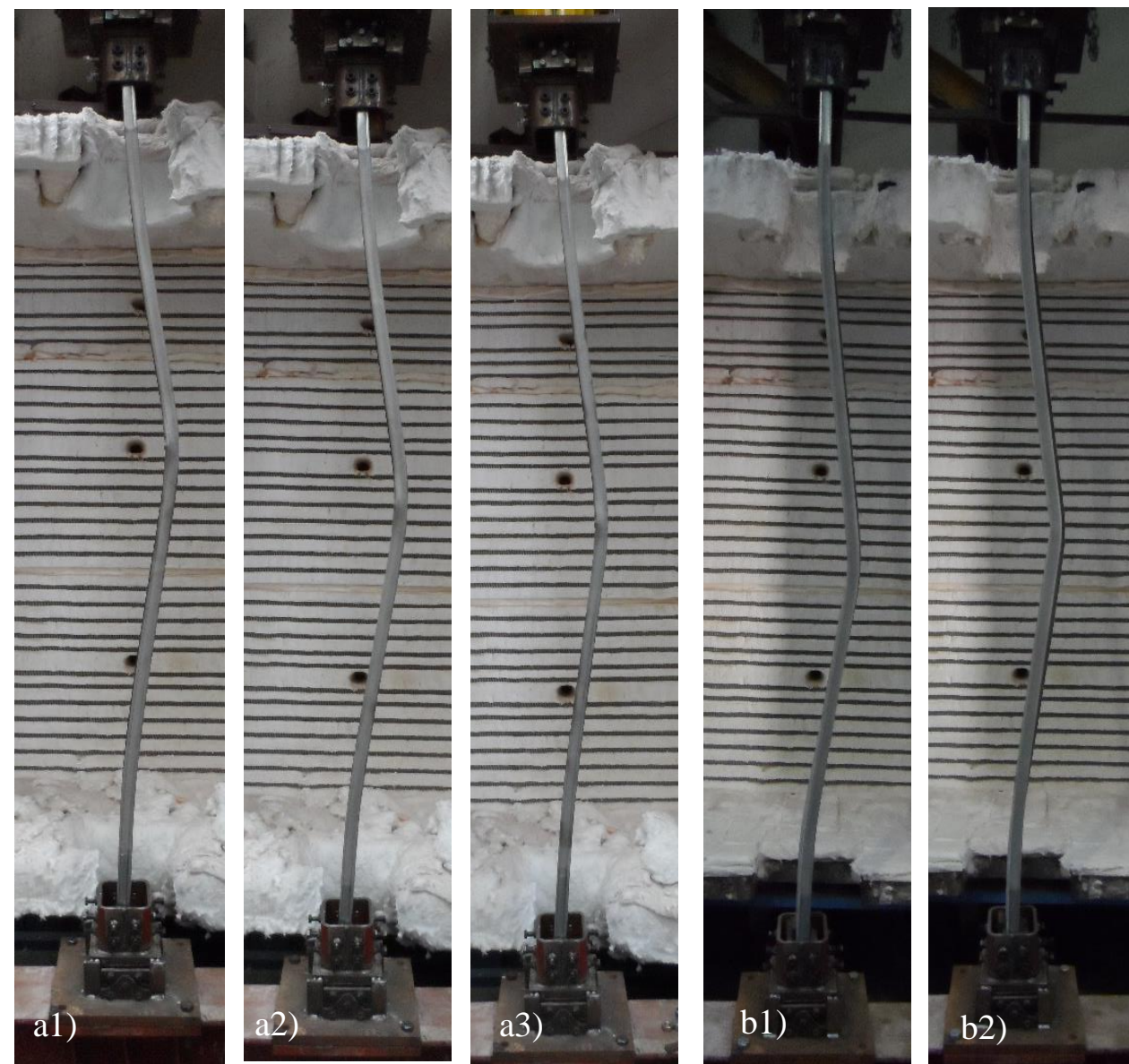

Figure G.5 Final deformed shapes for semi-rigid lipped channel columns. a1), a2) and a3) C_PP_30LL_K4-1, 2 and 3.b1), b2) C_PP_50LL_K4-1, 2. 


\section{G.2 Open Built-Up I Columns}

\section{G.2.1 Pinned-end support condition and K1 level of restraint to thermal elongation}
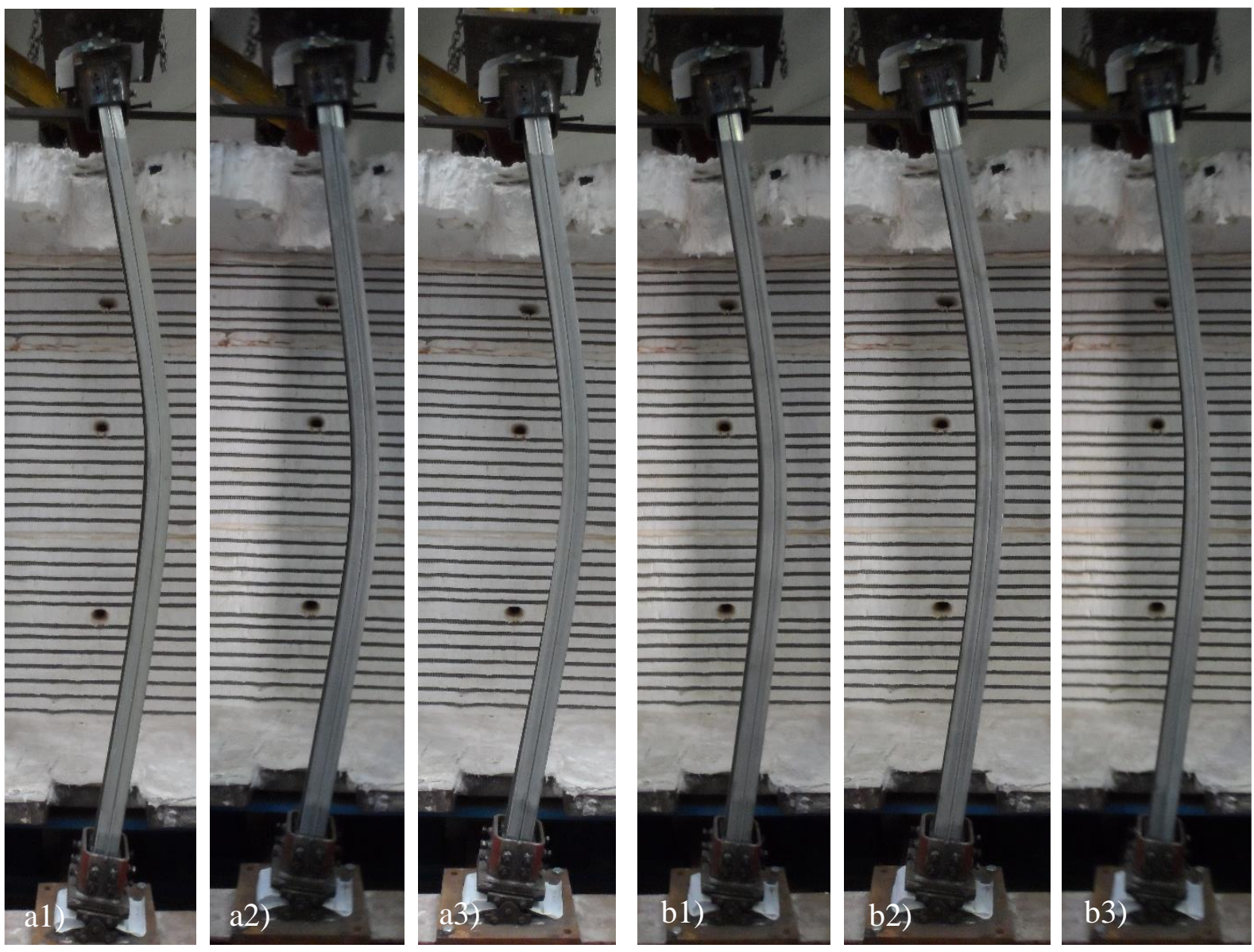

Figure G.6 Final deformed shapes for pinned-ended open built-up I columns. a1), a2) and a3) I_PP_30LL_K1-1, 2 and 3.b1), b2) I_PP_50LL_K1-1, 2 and 3. 


\section{G.2.2 Pinned-end support condition and K2 level of restraint to thermal elongation}
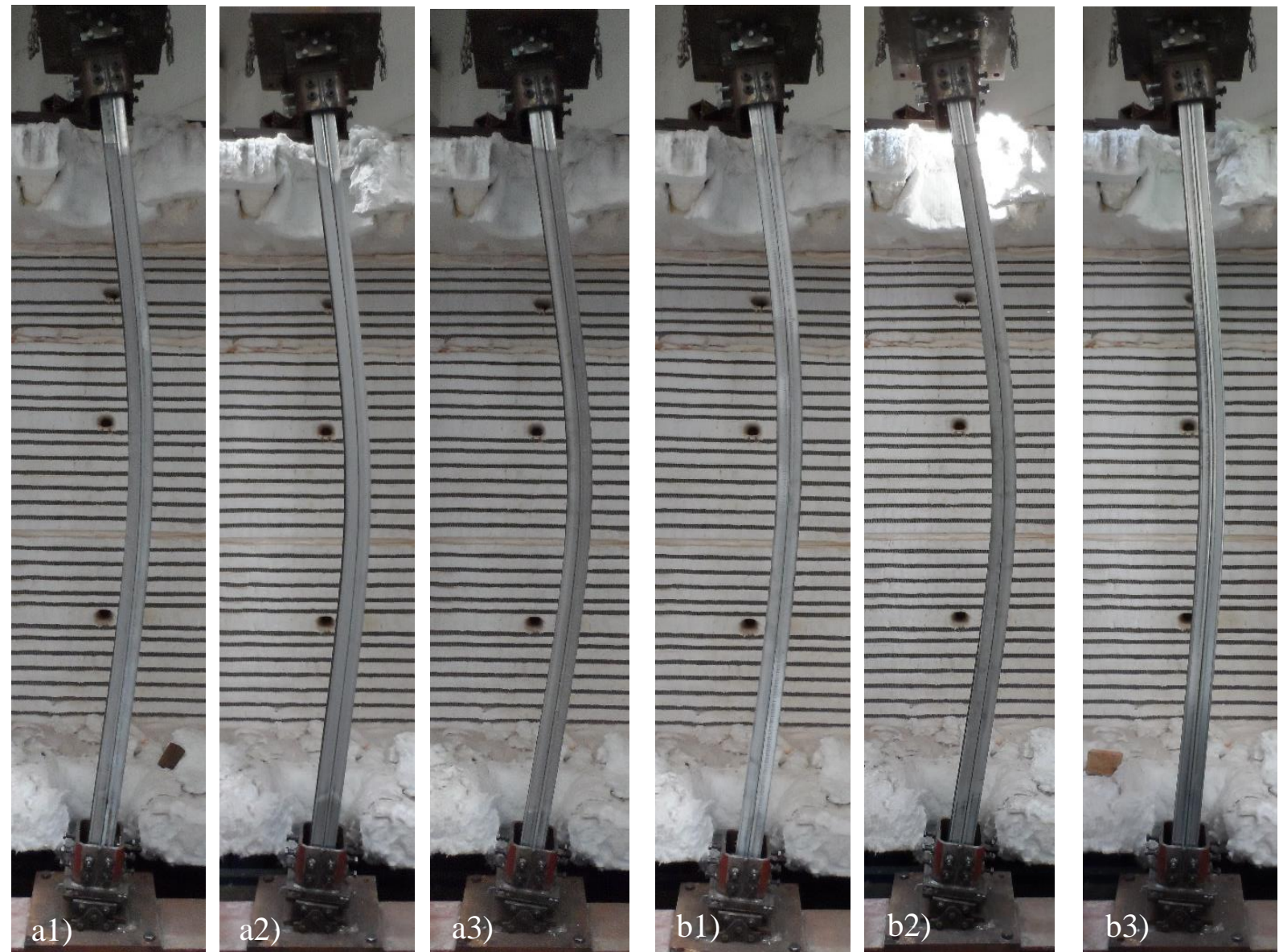

Figure G.7 Final deformed shapes for pinned-ended open built-up I columns. a1), a2) and a3) I_PP_30LL_K2-1, 2 and 3.b1), b2) I_PP_50LL_K2-1, 2 and 3. 


\section{G.2.3 Semi rigid end-support condition and K3 level of restraint to thermal elongation}
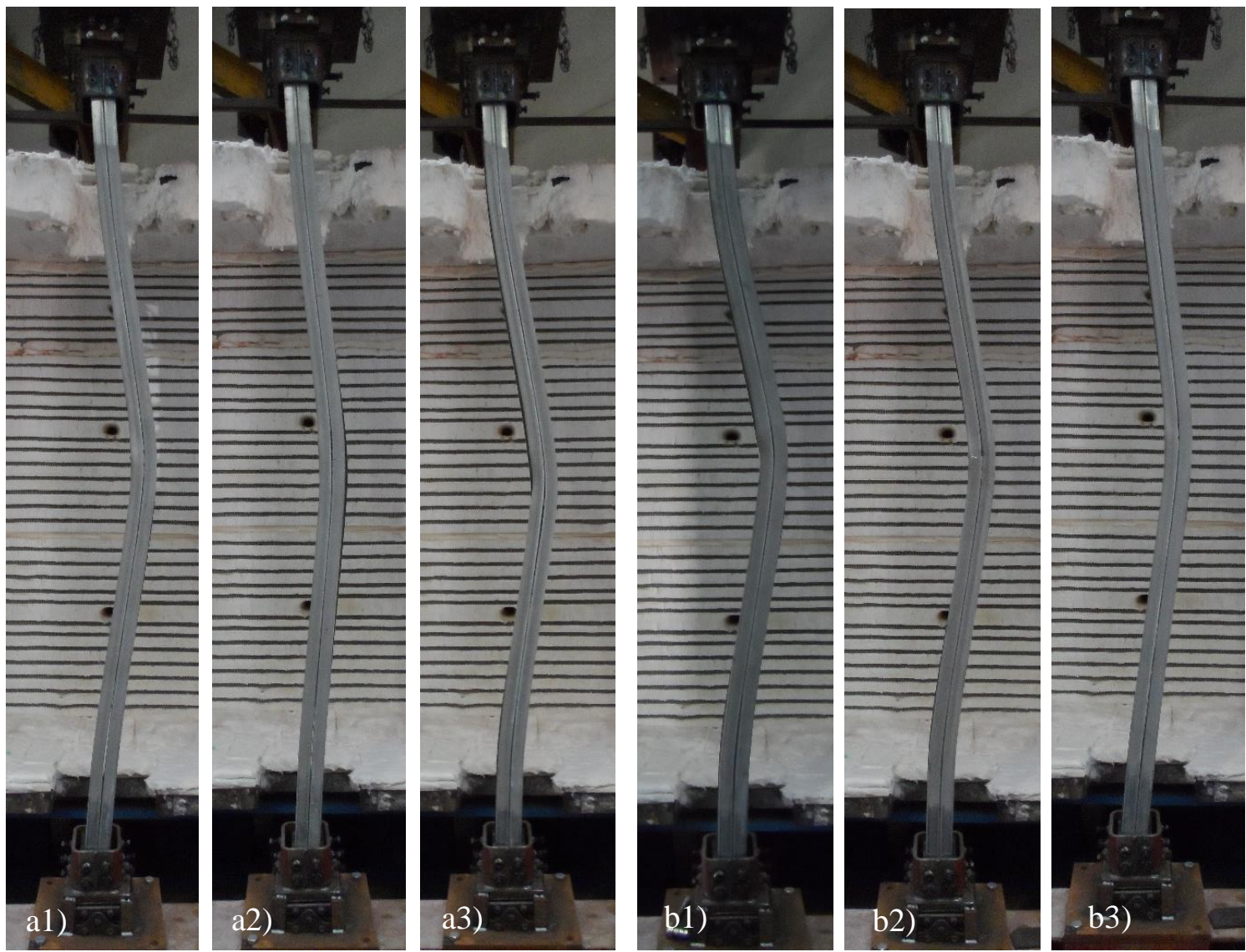

Figure G.8 Final deformed shapes for semi-rigid open built-up I columns. a1), a2) and a3) I_SR_30LL_K3-1, 2 and 3.b1), b2) I_FF_50LL_K3-1, 2 and 3.
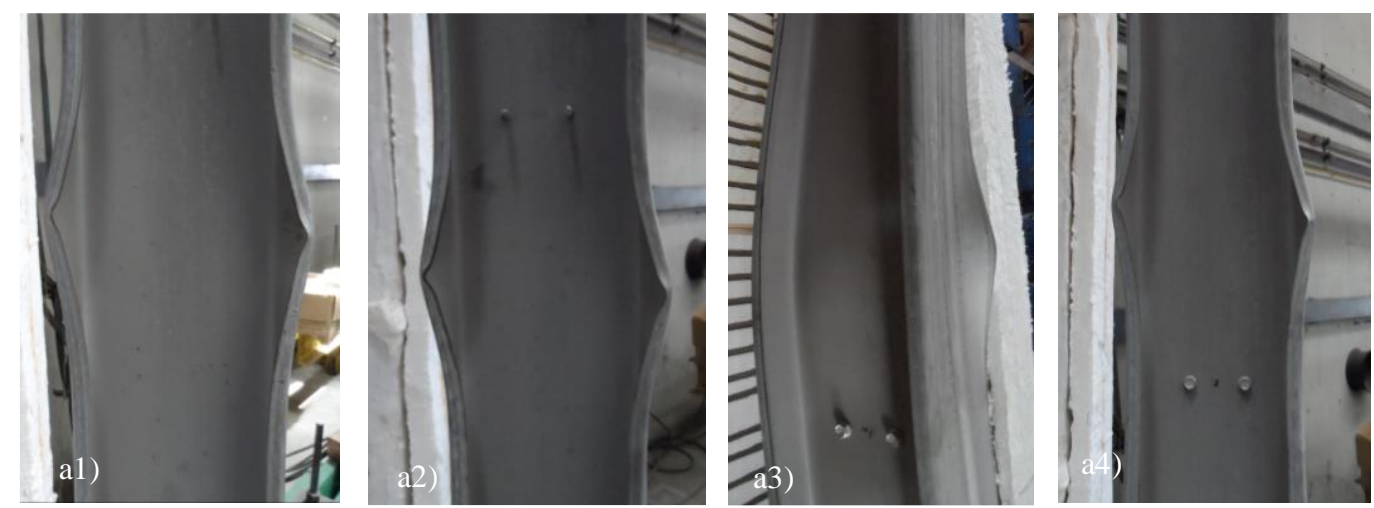

Figure G.9 Different configurations due to sectional buckling modes (distortional buckling) observed in the experimental tests. 


\section{G.2.4 Semi rigid end-support condition and K4 level of restraint to thermal elongation}
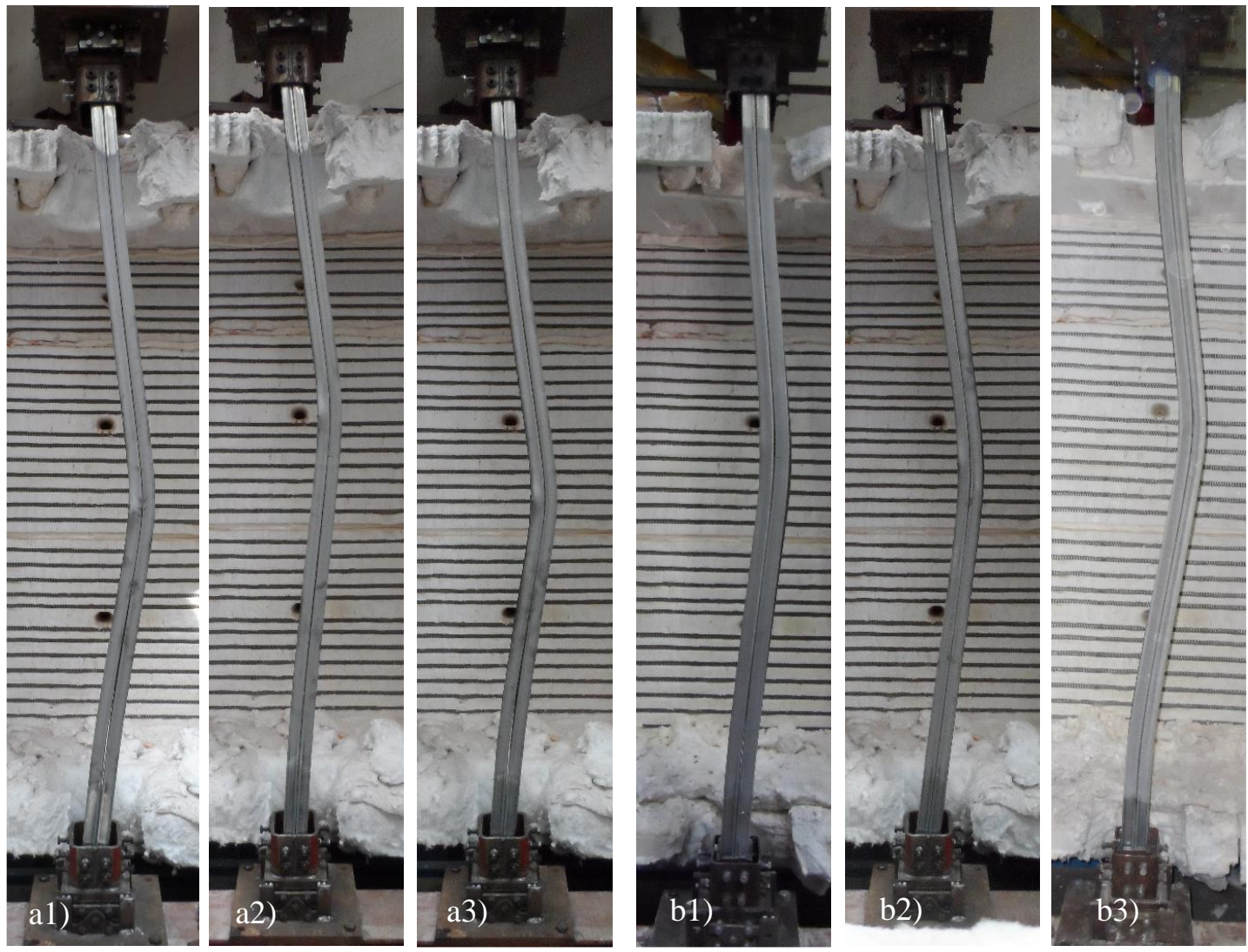

Figure G.10 Final deformed shapes for semi-rigid open built-up I columns. a1), a2) and a3) I_SR_30LL_K4-1, 2 and 3.b1), b2) I_FF_30LL_K4-1, 2 and 3.
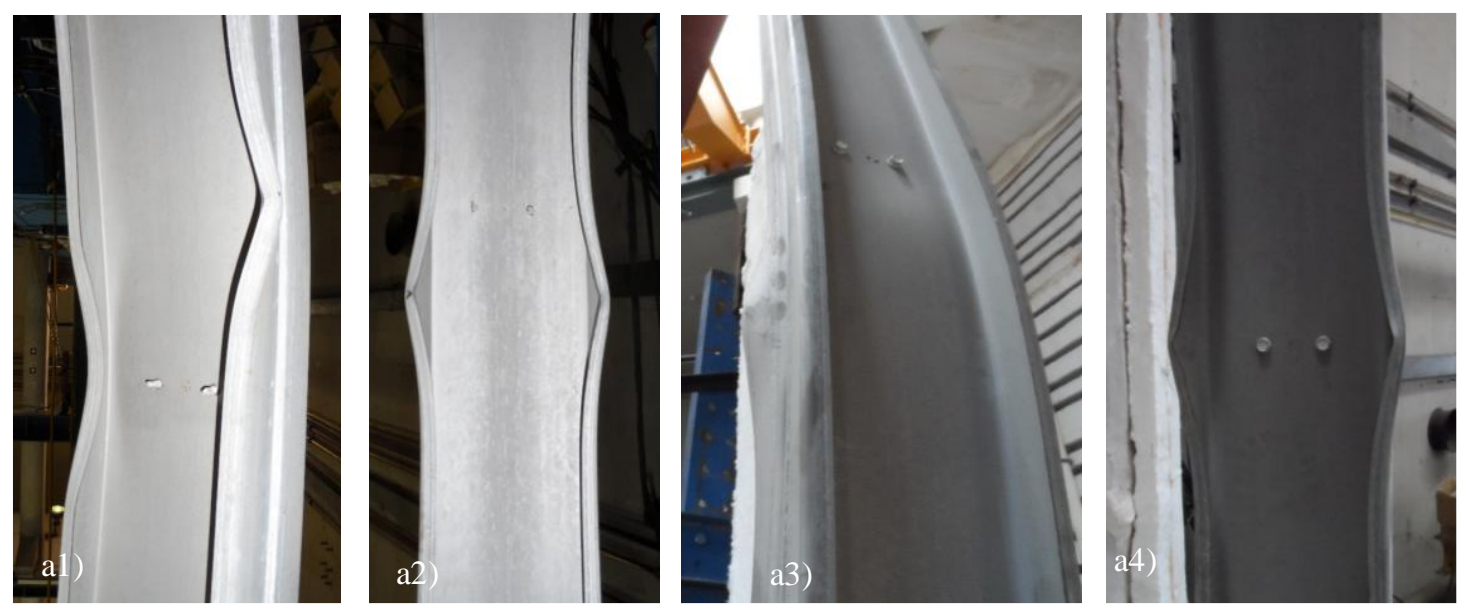

Figure G.11 Different configurations due to sectional buckling modes (distortional buckling) observed in the experimental tests. 


\section{G.3 Closed Built-Up R Columns}

\section{G.3.1 Pinned-end support condition and $\mathrm{K} 1$ level of restraint to thermal elongation}
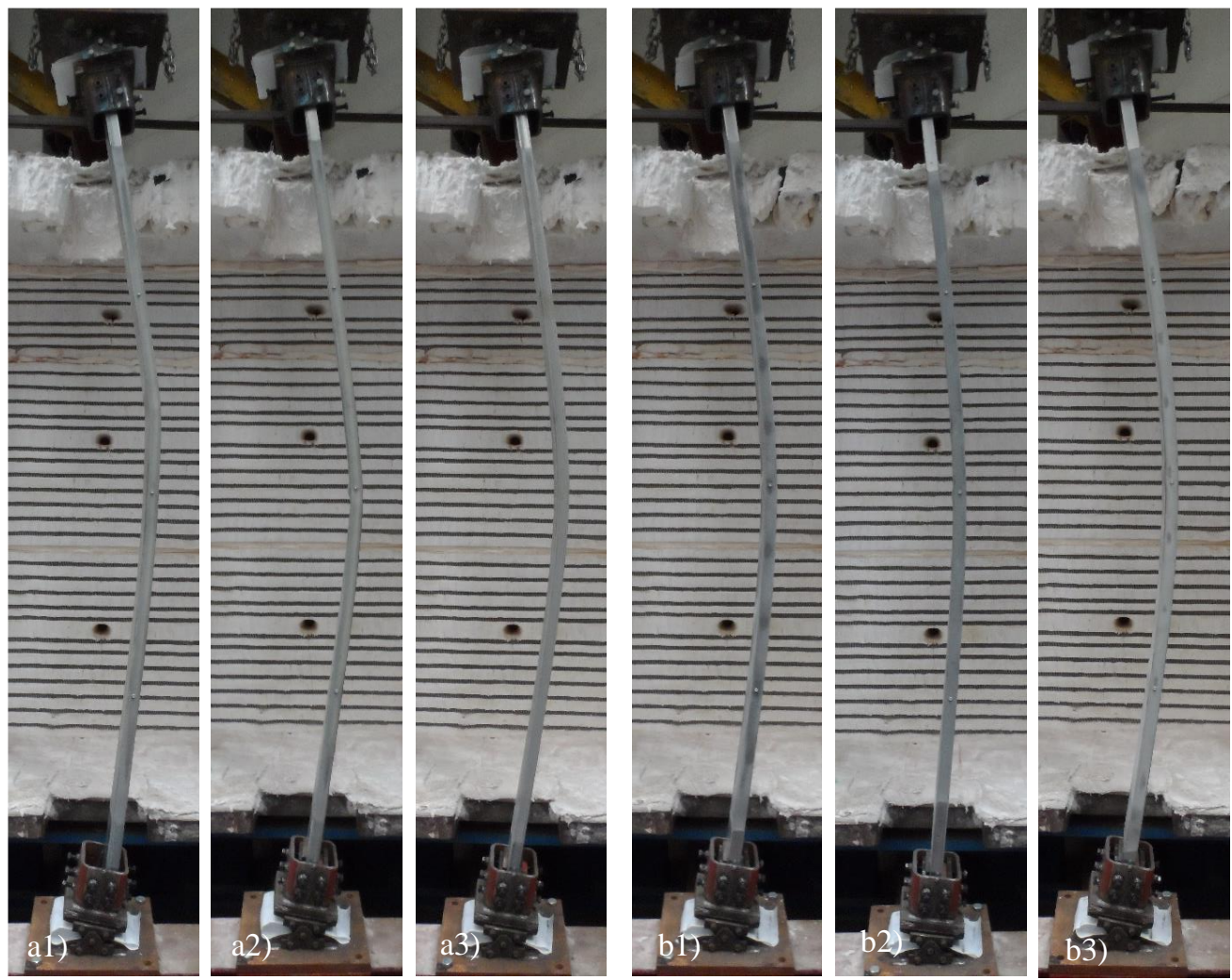

Figure G.12 Final deformed shapes for pinned-ended closed built-up R columns. a1), a2) and a3) R_PP_30LL_K1-1, 2 and 3.b1),b2) R_PP_50LL_K1-1, 2 and 3.
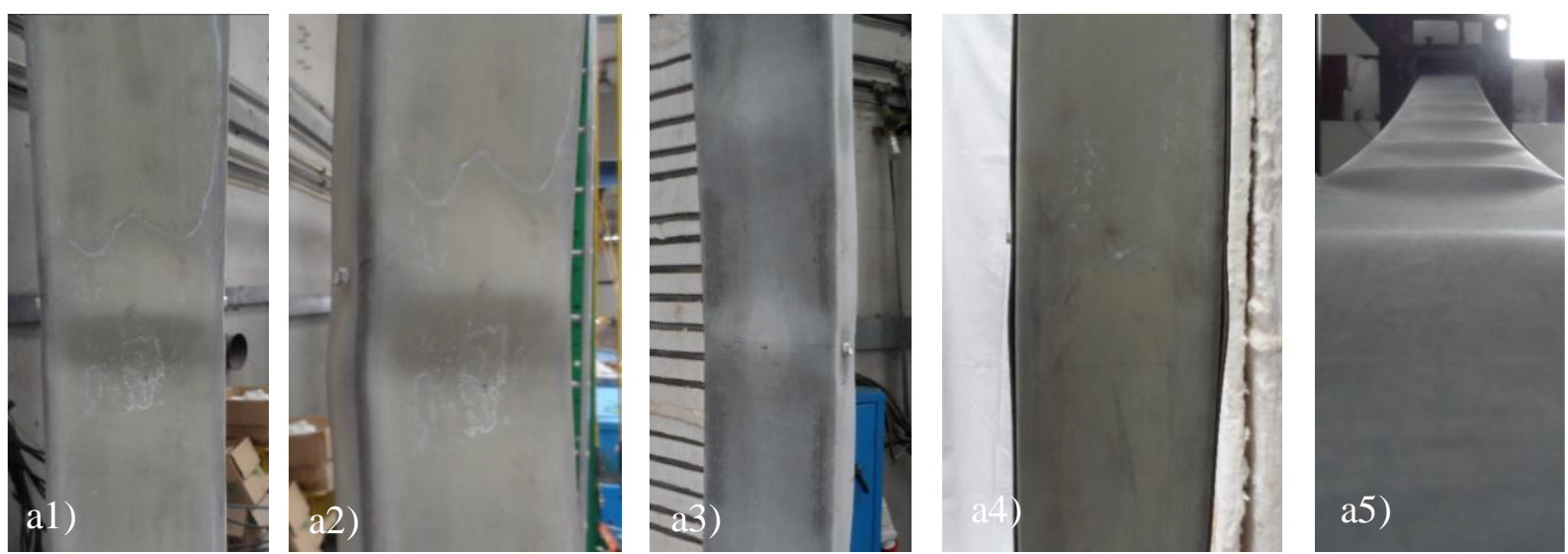

Figure G.13 Different configurations due to sectional buckling modes (local and distortional buckling) observed in the experimental tests. 


\section{G.3.2 Pinned-end support condition and K2 level of restraint to thermal elongation}
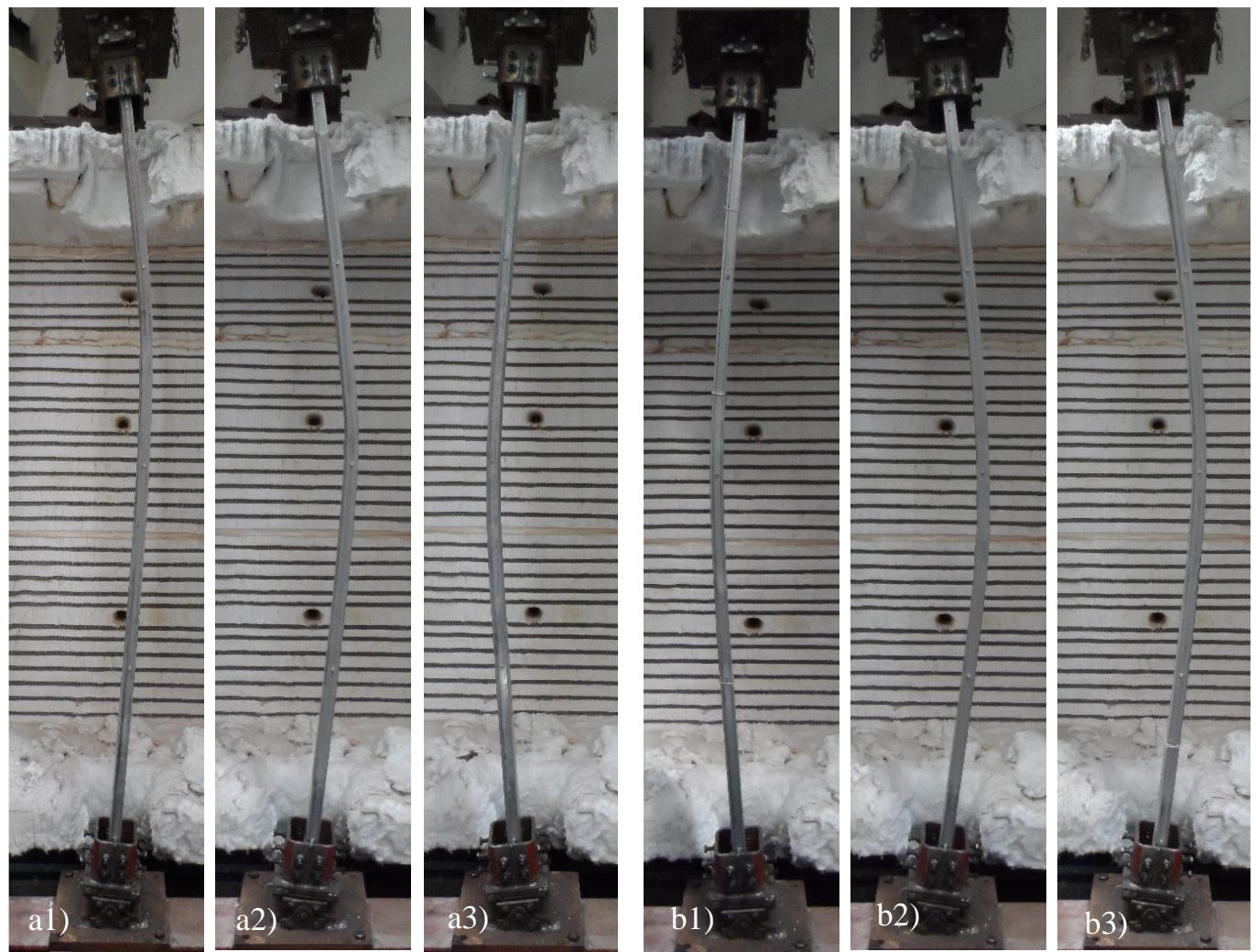

Figure G.14 Final deformed shapes for pinned-ended closed built-up R columns. a1), a2) and a3) R_PP_30LL_K2-1, 2 and 3.b1), b2) R_PP_50LL_K2-1, 2 and 3.
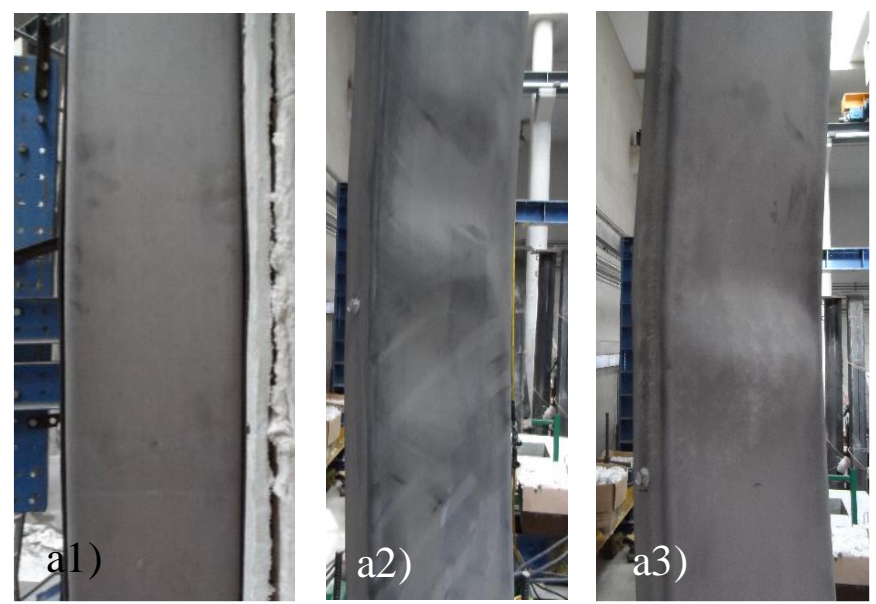

Figure G.15 Different configurations due to sectional buckling modes (local and distortional buckling) observed in the experimental tests. 


\section{G.3.3 Semi rigid end-support condition and K3 level of restraint to thermal elongation}
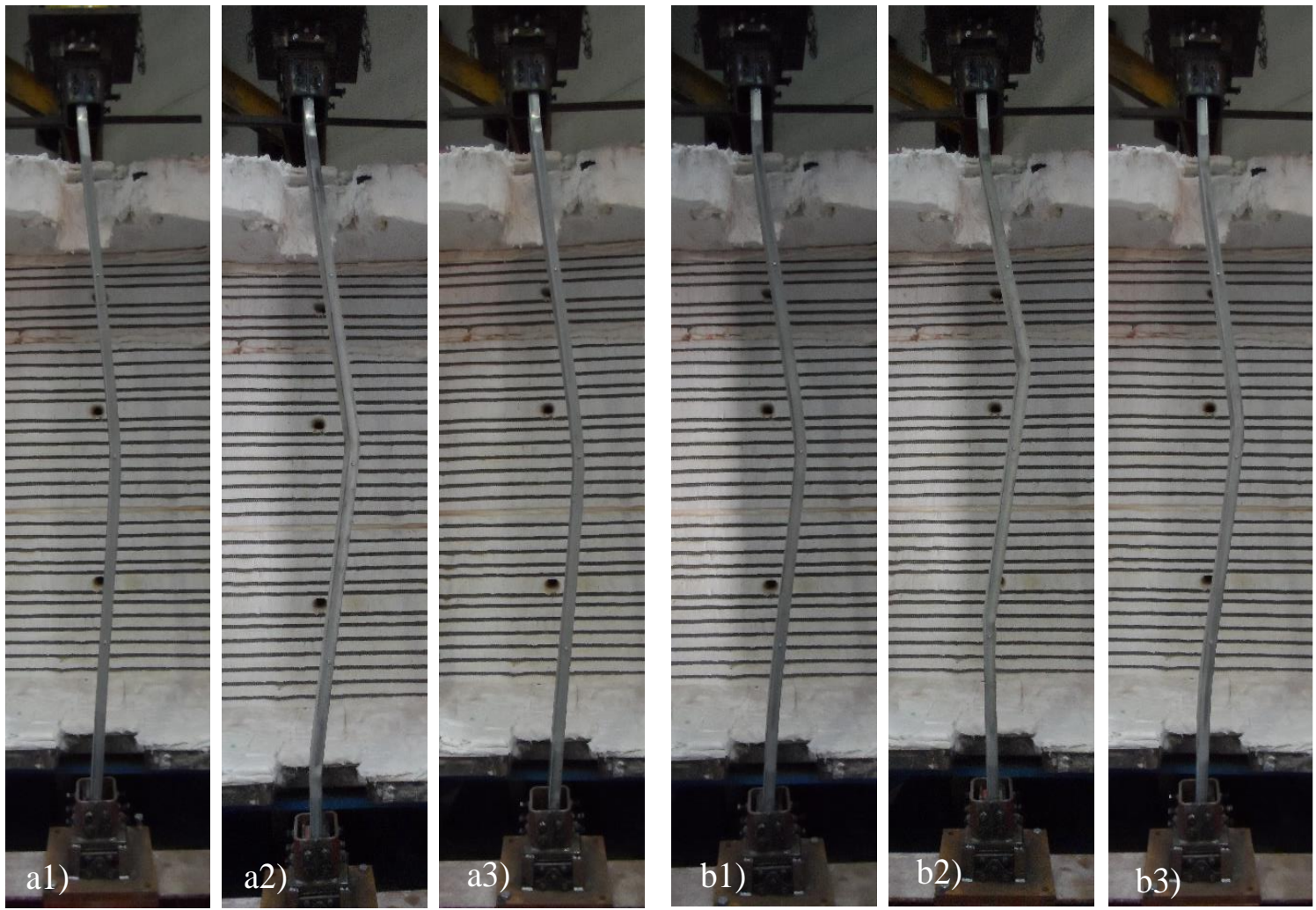

Figure G.16 Final deformed shapes for semi-rigid closed built-up R columns. a1), a2) and a3) R_SR_30LL_K3-1, 2 and 3.b1),b2) R_SR_50LL_K3-1, 2 and 3.
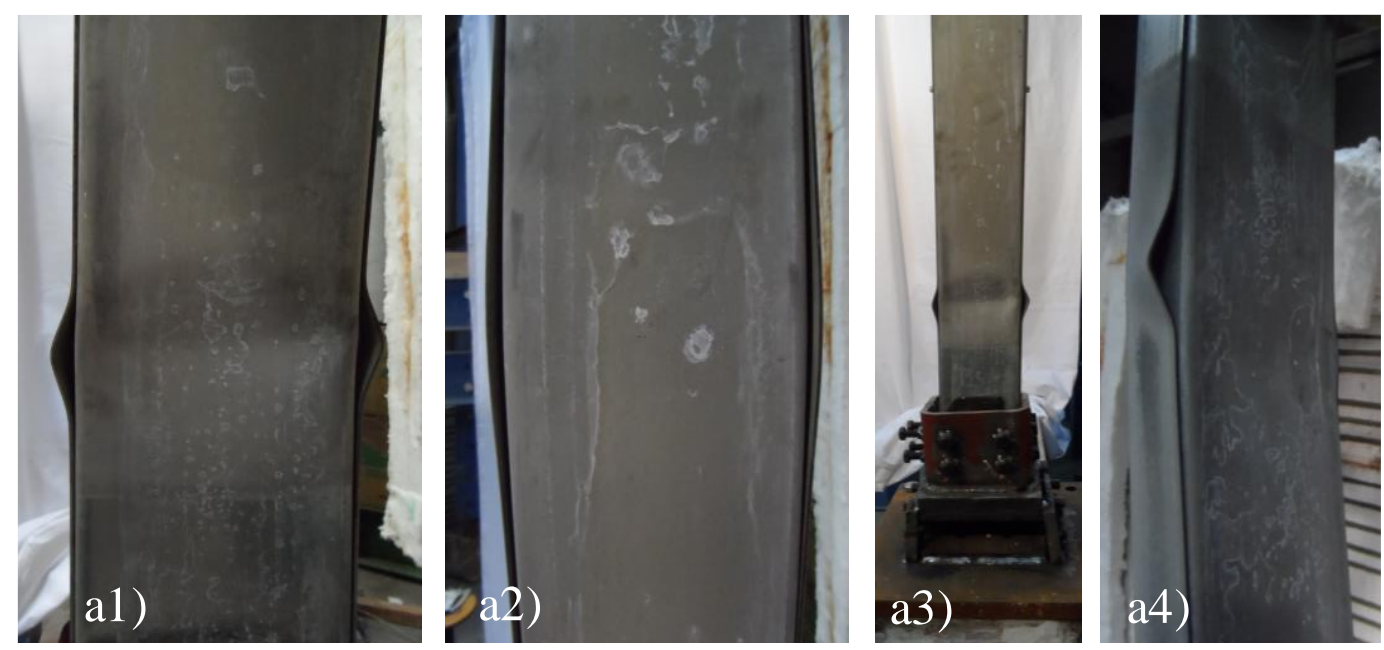

Figure G.17 Different configurations due to sectional buckling modes (local and distortional buckling) observed in the experimental tests. 


\section{G.3.4 Semi rigid end-support condition and K4 level of restraint to thermal elongation}
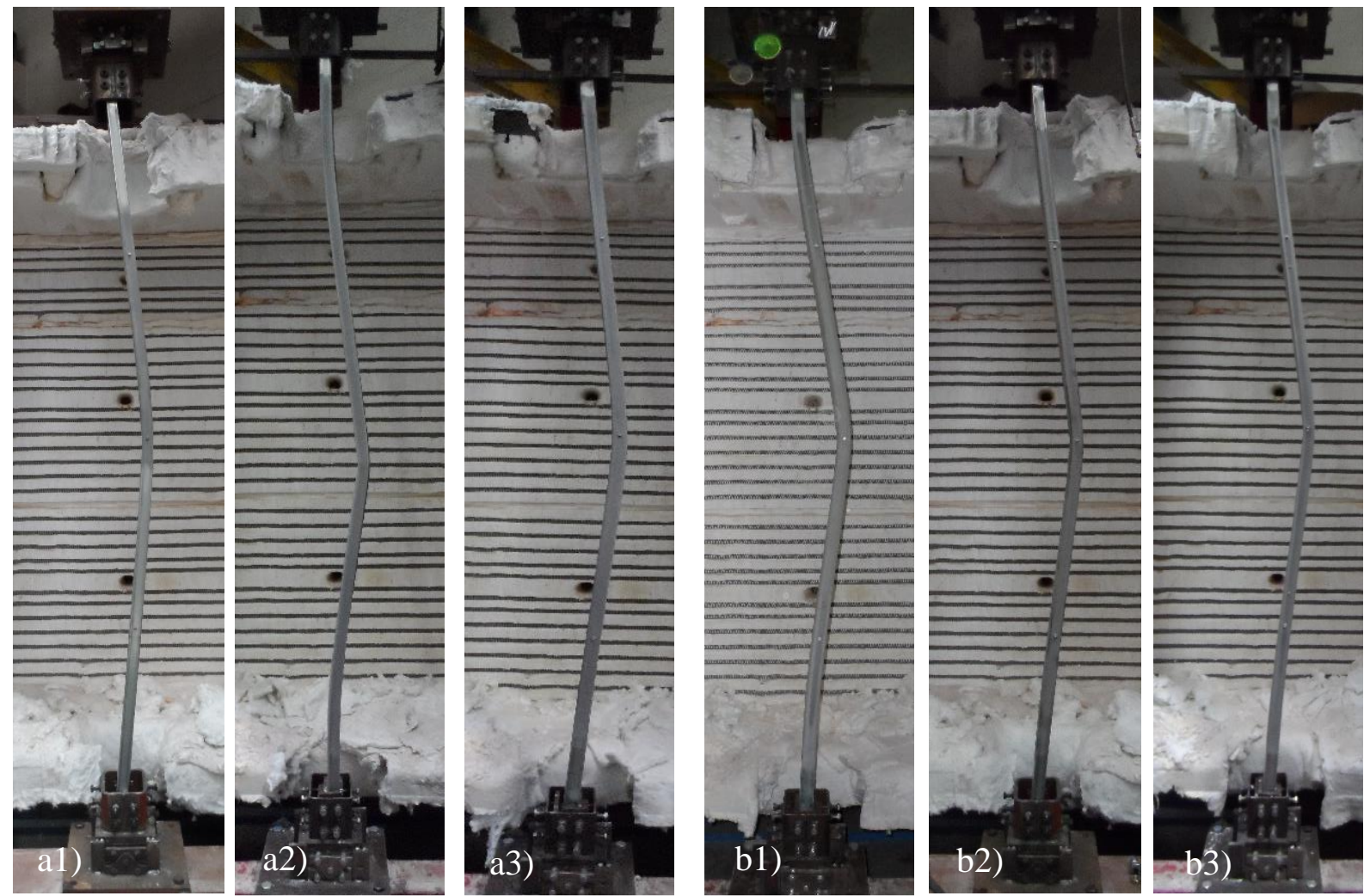

Figure G.18 Final deformed shapes for semi-rigid closed built-up R columns. a1), a2) and a3) R_SR_30LL_K4-1, 2 and 3.b1), b2) R_SR_50LL_K4-1, 2 and 3.
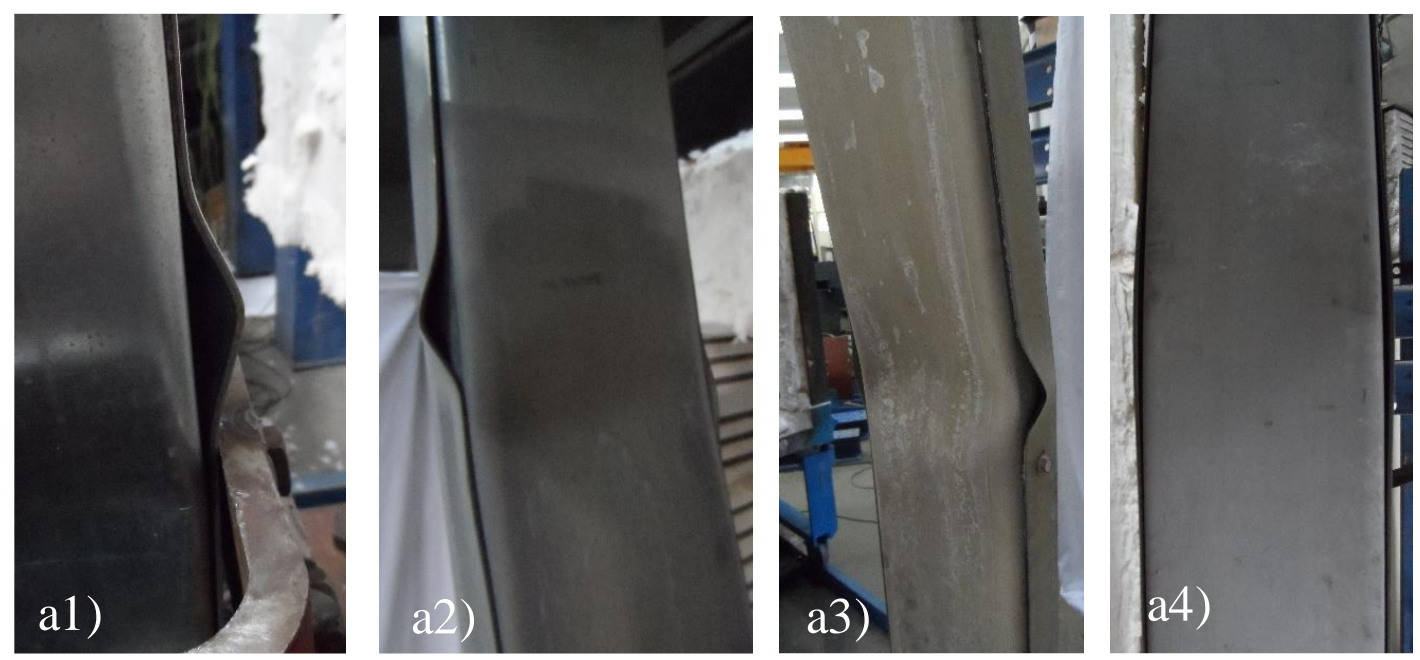

Figure G.19 Different configurations due to sectional buckling modes (local and distortional buckling) observed in the experimental tests. 


\section{G.4 Closed Built-Up 2R Columns}

\section{G.4.1 Pinned-end support condition and $\mathrm{K} 1$ level of restraint to thermal elongation}
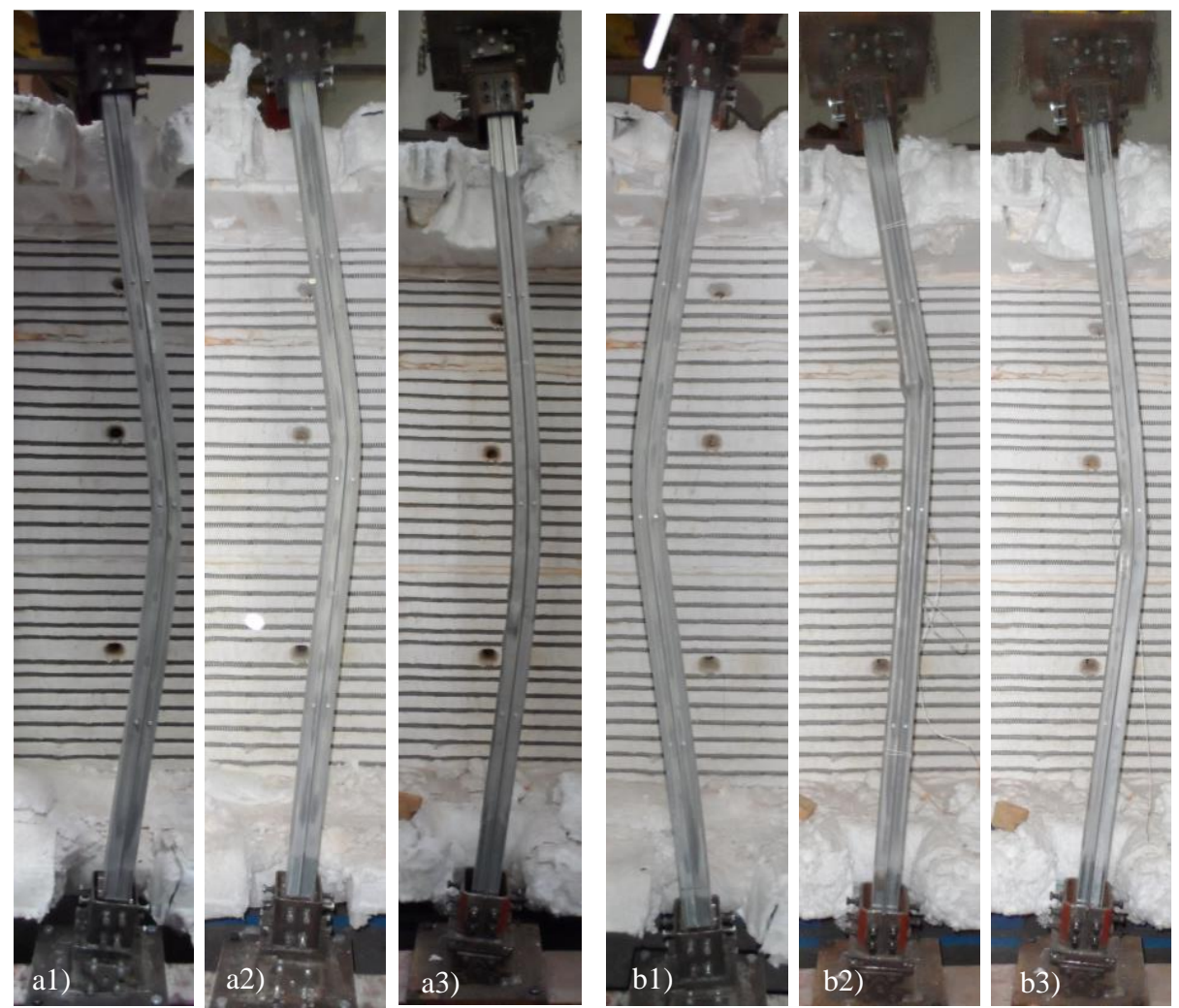

Figure G.20 Final deformed shapes for pinned-ended closed built-up R columns. a1), a2) and a3) 2R_PP_30LL_K1-1, 2 and 3.b1), b2) 2R_PP_50LL_K1-1, 2 and 3.
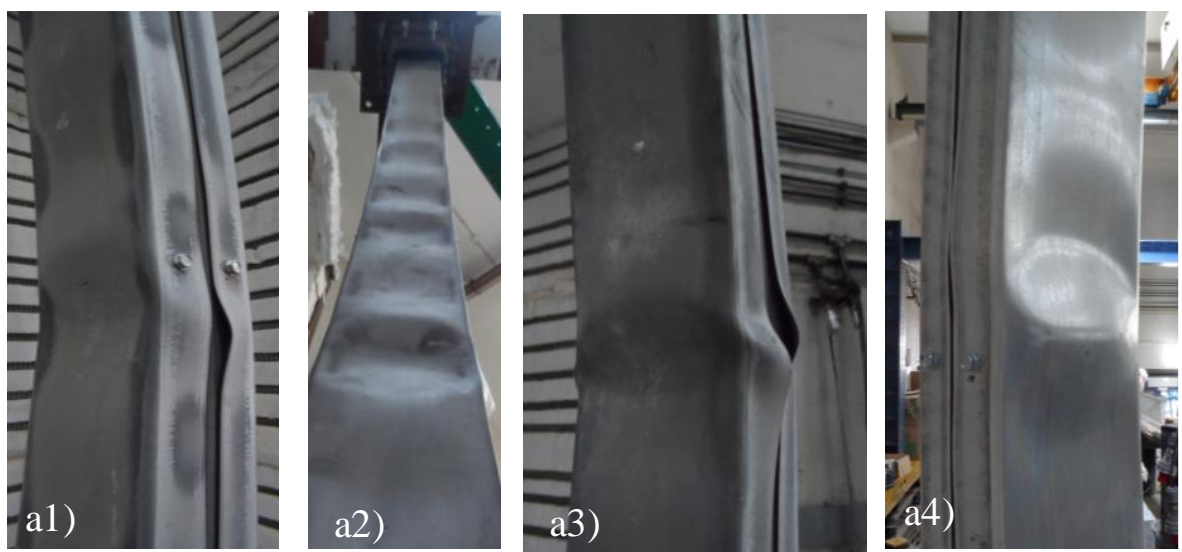

Figure G.21 Different configurations due to sectional buckling modes (local and distortional buckling) observed in the experimental tests. 


\section{G.4.2 Pinned-end support condition and K2 level of restraint to thermal elongation}
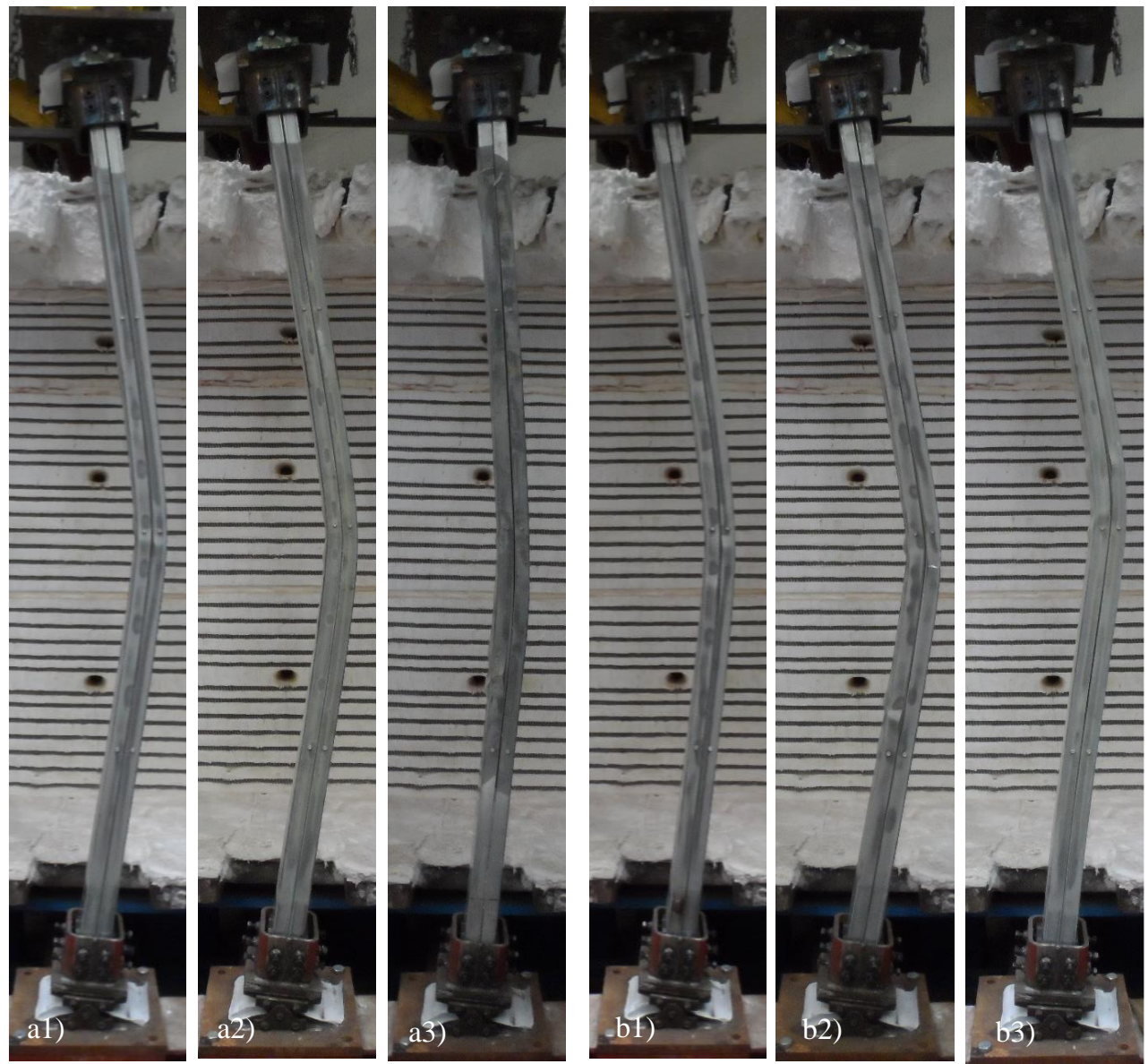

Figure G.22 Final deformed shapes for pinned-ended closed built-up R columns. a1), a2) and a3) 2R_PP_30LL_K2-1, 2 and 3.b1),b2) 2R_PP_50LL_K2-1, 2 and 3.
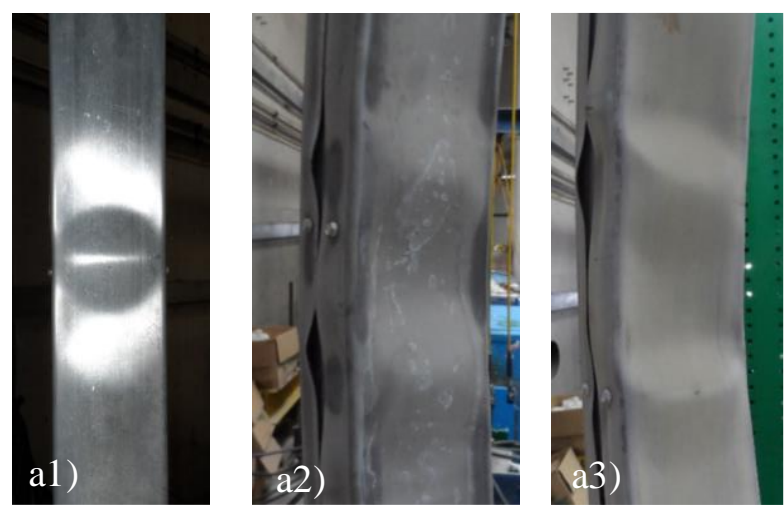

Figure G.23 Different configurations due to sectional buckling modes (local and distortional buckling) observed in the experimental tests. 


\section{G.4.3 Semi-rigid end-support condition and K3 level of restraint to thermal elongation}
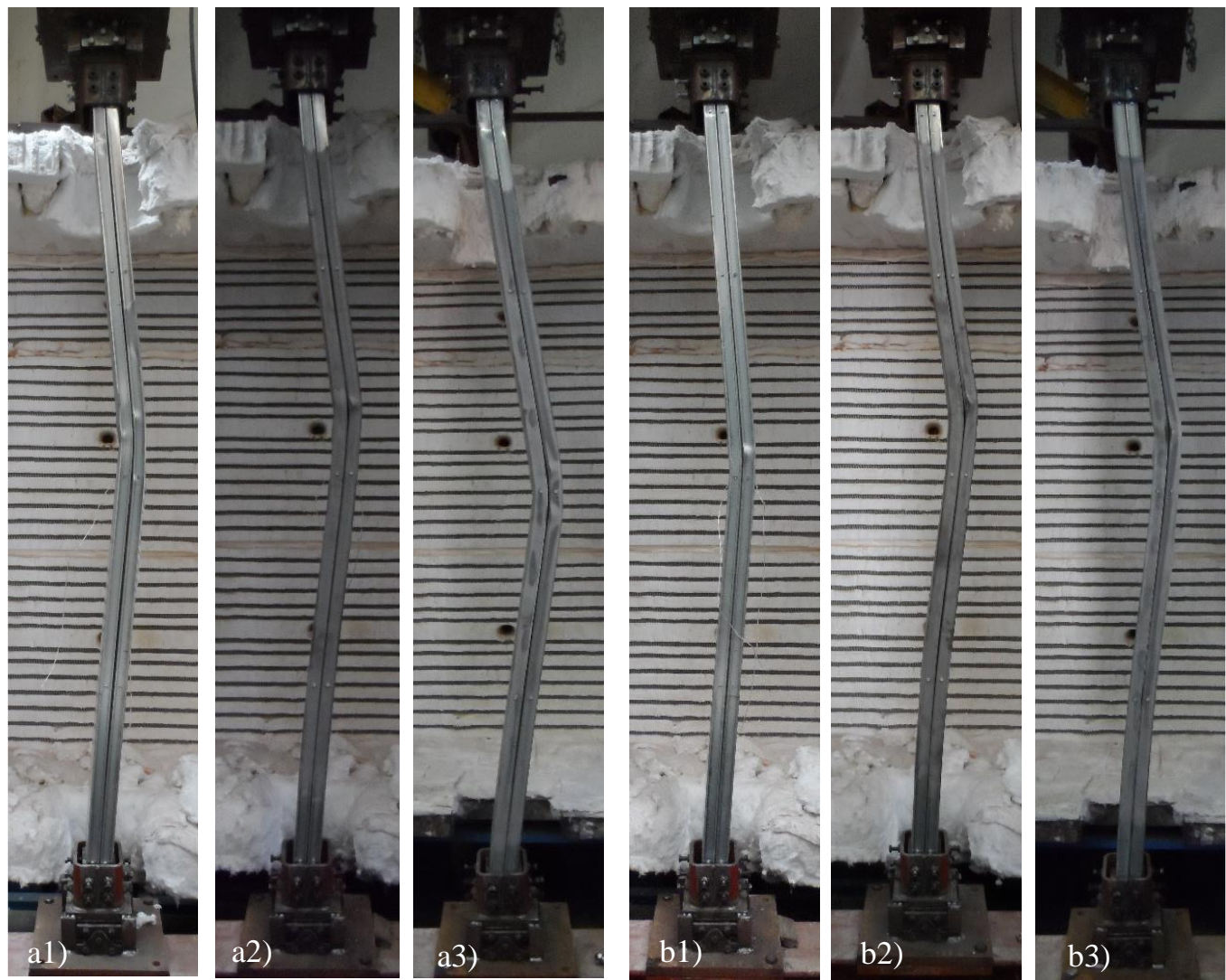

Figure G.24 Final deformed shapes for pinned-ended closed built-up R columns. a1), a2) and a3) 2R_SR_30LL_K3-1, 2 and 3.b1), b2) 2R_SR_50LL_K3-1, 2 and 3.
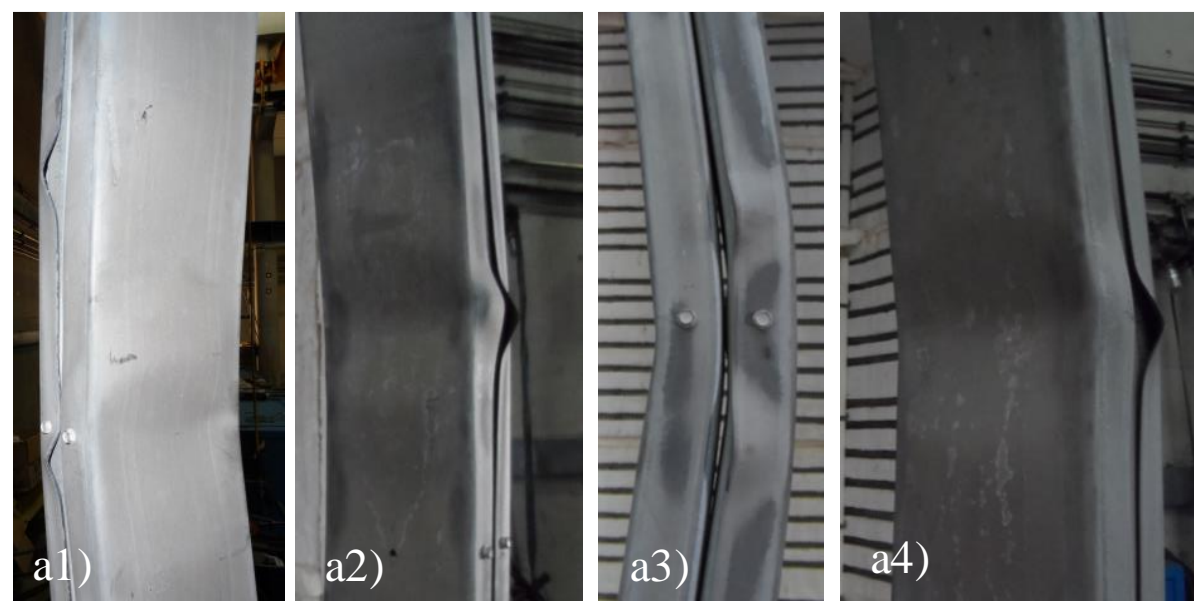

Figure G.25 Different configurations due to sectional buckling modes (local and distortional buckling) observed in the experimental tests. 


\section{G.4.4 Semi-rigid end-support condition and K4 level of restraint to thermal elongation}
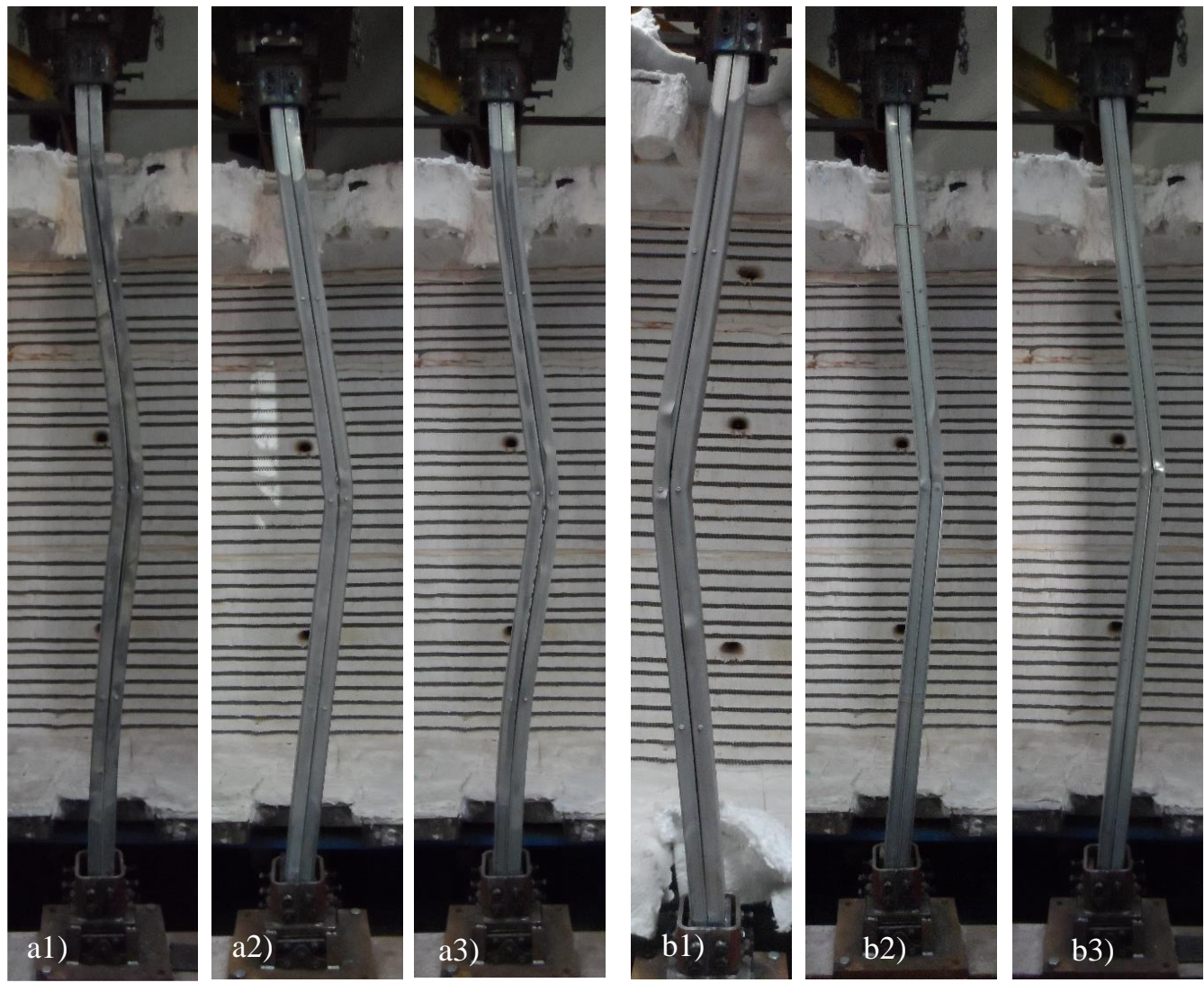

Figure G.26 Final deformed shapes for pinned-ended closed built-up R columns. a1), a2) and a3) 2R_SR_30LL_K4-1, 2 and 3. b1), b2) 2R_SR_50LL_K4-1, 2 and 3.
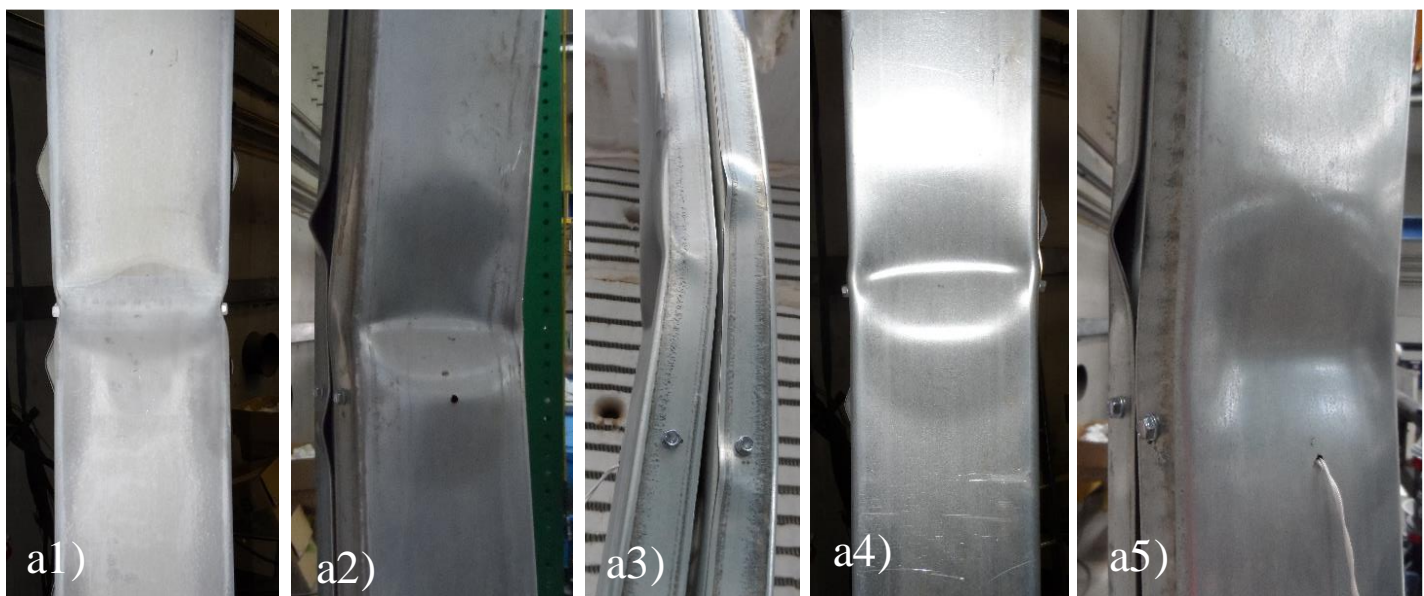

Figure G.27 Different configurations due to sectional buckling modes (local and distortional buckling) observed in the experimental tests. 


\section{APPENDIX H INFLUENCE OF MATERIAL PROPERTIES IN THE ACCURACY OF THE DEVELOPED FINITE ELEMENT MODEL}

In this Appendix a small comparison between experimental results and the FEA, undertaken using the determined material properties in this research and the material properties presented in the EN 1993-1-2:2005, is shown in Figure G.1. It is clear that the determined material properties in the scope of this research are way more accurate than the ones provided by the EN 1993-1-2:2005. The critical temperature and the maximum generated restraining forces are very similar. However, the peak load is reached for much lower temperatures when the material properties provided by the EN 1993-1-2:2005 are used in the finite element model.

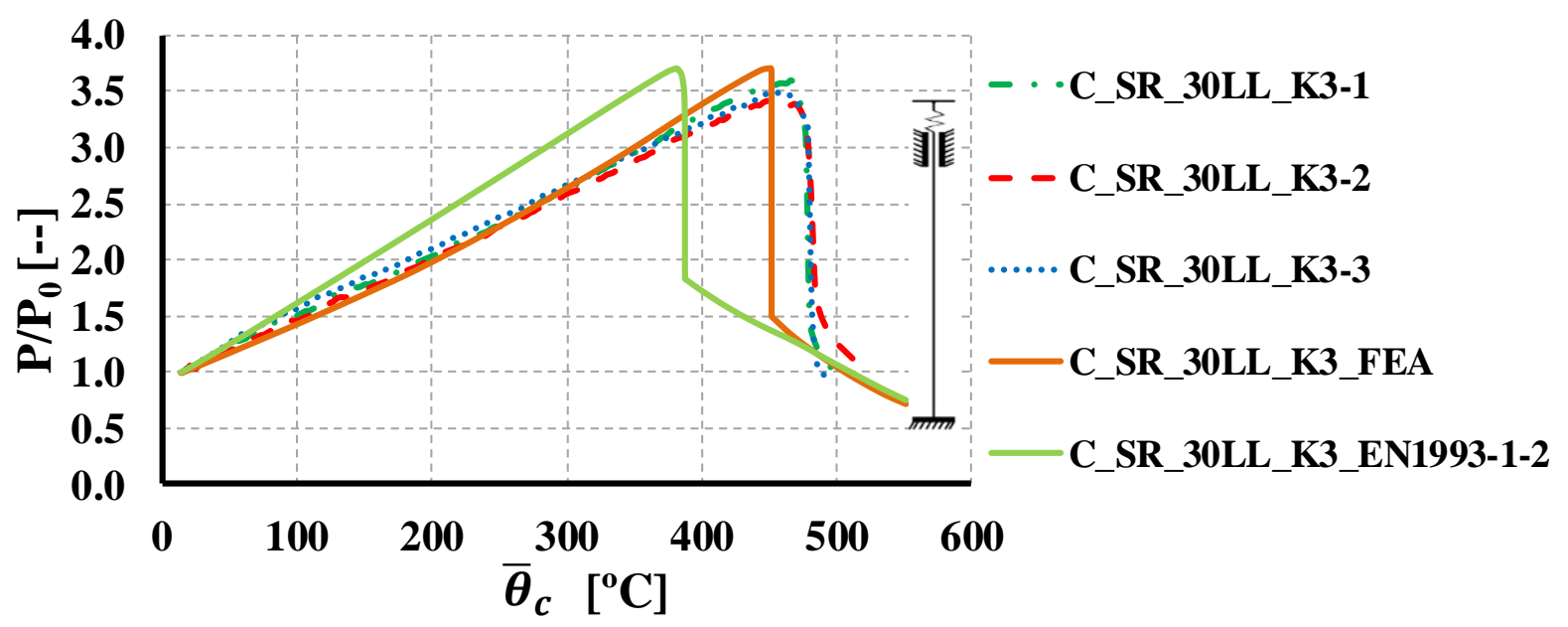

Figure H.1 Comparison between experimental and FEA using material properties assessed in experimental tests and using material properties presented in the EN 1993-1-2:2005. 